\title{
Rational Design of Synergistic Active Sites for Catalytic Ethene/2- Butene Cross-Metathesis in a Re-doped Y Zeolite Catalyst
}

Pu Zhao, ${ }^{1}$ Lin Ye, ${ }^{1}$ Guangchao Li, ${ }^{1},{ }^{2}$ Chen Huang, ${ }^{3}$ Simson Wu, ${ }^{1}$ Ping-Luen Ho, ${ }^{1}, 3$ Haokun Wang, ${ }^{1}$ Tatchamapan Yoskamtorn, ${ }^{1}$ Denis Sheptyakov, ${ }^{4}$ Giannantonio Cibin, ${ }^{5}$ Angus I. Kirkland, ${ }^{3}, 5$ Chiu C. Tang, ${ }^{5}$ Anmin Zheng, ${ }^{2}$ Wenjuan Xue, ${ }^{6}$ Donghai Mei, ${ }^{6,7}$ Kongkiat Suriye, ${ }^{8}$ and Shik Chi Edman Tsang 1 , *

1 Wolfson Catalysis Centre, Department of Chemistry, University of Oxford, Oxford OX1 3QR, U.K.

2 Wuhan Institute of Physics and Mathematics, Chinese Academy of Sciences, Wuhan, Hubei 430071, P.R. China.

3 Department of Materials, University of oxford, Oxford ox1 3PH, U.K.

4 Paul Scherrer Institute, 5232 Villigen PSI, Switzerland.

5 Diamond Light Source Ltd., Harwell Science and Innovation Campus, Didcot OX11 ODE, U.K.

6 School of Environmental Science and Engineering, Tiangong University, Tianjin 300387, P.R. China.

7 Physical and Computational Sciences Directorate \& Institute for Integrated Catalysis, Pacific Northwest National Laboratory, Richland, Washington 99354, U.S.A

8 SCG Chemicals Co., Ltd., Bangkok 10800, Thailand.

* edman.tsang@chem.ox.ac.uk 


\begin{tabular}{|c|c|c|c|}
\hline \multicolumn{4}{|c|}{601} \\
\hline & $23 . \overline{3} 8105 \overline{2}$ & 3.329261 & 1.245772 \\
\hline & 23.429115 & 1.235092 & 3.371499 \\
\hline & 23.408237 & 15.562931 & 13.44424 \\
\hline & 23.427902 & 13.390840 & 15.560746 \\
\hline & 11.210981 & 1.166880 & 15.712948 \\
\hline & 11.163646 & 15.568272 & 1.236791 \\
\hline & 11.228702 & 13.402249 & 3.372470 \\
\hline & 7.644799 & 3.354022 & 1.237276 \\
\hline & 7.656694 & 5.508635 & 3.350137 \\
\hline & 7.694562 & 15.429664 & 13.178194 \\
\hline & 19.989891 & 5.402070 & 15.4 \\
\hline & 19.908327 & 15.646921 & 1.26494 \\
\hline & 19.910027 & 17.801048 & 3.36 \\
\hline & 7.617369 & 3.380481 & 5.512033 \\
\hline & 7.573432 & 15.456122 & 17.682104 \\
\hline & 7.734858 & 13.237182 & \\
\hline i & 19.924833 & 3441 & 17.7 \\
\hline & 19.886723 & 1.223925 & 15.578953 \\
\hline & 19.943525 & 15.597158 & 5.412022 \\
\hline & 20.004940 & 13.381859 & 3.271973 \\
\hline & 23.462370 & 5963 & \\
\hline i & 23. & 17.7 & 15. \\
\hline i & 11.232 & 3.3 & 17.8 \\
\hline $\mathrm{Si}$ & 11.255160 & 5.524899 & 15.619492 \\
\hline $\mathrm{Si}$ & 11.255160 & 15.633813 & 5.465911 \\
\hline & 11.234528 & 17.757841 & 3.27124 \\
\hline$\dot{z}$ & $1.30^{\circ}$ & 23. & \\
\hline $\mathrm{Si}$ & 3.3 & 23. & 1.207902 \\
\hline$i$ & 1.240432 & 11.160975 & 15.583323 \\
\hline $\mathrm{Si}$ & 3.348681 & 11.219962 & 13.376761 \\
\hline $\mathrm{S}$ & 13.474101 & 23.372555 & $15.55103^{-}$ \\
\hline i & 15.6 & 23. & 13.409 \\
\hline $\mathrm{Si}$ & 13. & 11. & \\
\hline $\mathrm{Si}$ & 15.588904 & 11.1 & 1.2 \\
\hline $\mathrm{Si}$ & 1.342143 & 7.718595 & 3.317609 \\
\hline 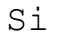 & 3.398687 & 7.668589 & 5.518344 \\
\hline $\mathrm{Si}$ & 1.265192 & 19.888906 & $15.60686^{7}$ \\
\hline $\mathrm{Si}$ & 01 & 19. & 17.77 \\
\hline i & 13 & & 15 \\
\hline $\mathrm{Si}$ & 15.666341 & 7.638488 & 17.796923 \\
\hline $\mathrm{Si}$ & 15.602741 & 19.961245 & 5.497226 \\
\hline $\mathrm{Si}$ & 5.544319 & 7.688251 & 3.39771 \\
\hline $\mathrm{Si}$ & 12 & 7. & 1.204991 \\
\hline $\mathrm{Si}$ & & 19. & 5.671438 \\
\hline $\mathrm{Si}$ & 3.3 & 19.88 & 13.4 \\
\hline $\mathrm{Si}$ & 17.861736 & 7.647955 & 15.638910 \\
\hline $\mathrm{S}$ & 15.677263 & 7.764959 & 13.52095 \\
\hline $\mathrm{Si}$ & 17.761967 & 9.916580 & 3.37732 \\
\hline $\mathrm{Si}$ & 15.620219 & 20.022417 & 1.244316 \\
\hline $\mathrm{Si}$ & 849 & 23. & 3.3 \\
\hline $\mathrm{Si}$ & 3.404270 & 23.400713 & $5.50717 \varepsilon$ \\
\hline $\mathrm{Si}$ & 5.454017 & 11.156848 & $15.59982 \varepsilon$ \\
\hline $\mathrm{Si}$ & 3.314939 & 11.179667 & 17.77895 \\
\hline $\mathrm{Si}$ & 17.726526 & 23.394159 & 15.624832 \\
\hline $\mathrm{Si}$ & 15.541570 & 23.365030 & 17.757113 \\
\hline $\mathrm{Si}$ & 17.817799 & 11.260986 & 3.28969 \\
\hline
\end{tabular}




\begin{tabular}{|c|c|c|c|}
\hline$i$ & 15.647649 & 11.214379 & 5.493099 \\
\hline & 3.371984 & 1.278058 & 23.367214 \\
\hline & 1.242617 & 3.363974 & 23.414549 \\
\hline & 3.355721 & 13.373363 & 11.212680 \\
\hline & 1.256939 & 15.544725 & 11.2643 \\
\hline & 15.651775 & 1.181202 & 112085 \\
\hline & 13.391811 & 3.227065 & 11.307 \\
\hline & 15.560989 & 13.449343 & 23.4 \\
\hline & 13.450070 & 15.575554 & 23.4822 \\
\hline & 3.348196 & 1.205962 & 7.65232 \\
\hline & 5.477321 & 3.333873 & 76110 \\
\hline & 3.296733 & 13.338164 & 19.9 \\
\hline & 17.782843 & 3.324649 & 19.841 \\
\hline & 15.652746 & 13.426281 & 7 . \\
\hline & 17.818769 & 15.572641 & 7.6566 \\
\hline & 3.319551 & 5.465911 & 7.6086 \\
\hline L & 1.237762 & 660 & \\
\hline 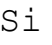 & 3.412 & 17.71 & 19.8 \\
\hline S & 1.250142 & 15.558319 & 19.87677 \\
\hline & 15.594003 & 5.447948 & 19.94401 \\
\hline & 13.415115 & 3.385821 & 19.96537 \\
\hline $\mathrm{Si}$ & 15.666583 & 17.773132 & 7.630721 \\
\hline $\mathrm{Si}$ & 13.472646 & 15.6 & \\
\hline i & 3.3 & 5. & 23. \\
\hline 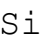 & 5.50305 & 3.386064 & 23.40872 \\
\hline $\mathrm{Si}$ & 5.515189 & 15.536714 & 11.08281 \\
\hline S & 17.930676 & 3.246728 & 11.21777 \\
\hline $\mathrm{Si}$ & 15.612937 & 17.7 & \\
\hline $\mathrm{Si}$ & 17.7272 & 15.6 & 23. \\
\hline $\mathrm{Si}$ & 1.645090 & 21.65 & 23.7 \\
\hline$C_{1} \rightarrow$ & 1.601638 & 23.779154 & 21.65634 \\
\hline $\mathrm{Si}$ & 1.615717 & 9.439178 & 11.57461 \\
\hline $\mathrm{Si}$ & 1.595327 & 11.5894 & 9.46054 \\
\hline $\mathrm{Si}$ & 13.8663 & 21.7 & 11. \\
\hline & 13.814 & 23.83 & 363 \\
\hline & 13.837008 & 9.417573 & 23.79226 \\
\hline S & 13.825112 & 11.625591 & $21.68717 \mathrm{C}$ \\
\hline $\mathrm{Si}$ & 17.474070 & 21.678188 & 23.77915 \\
\hline $\mathrm{Si}$ & 17.392021 & 19.532799 & 21.69372 \\
\hline S & 17. & 9. & 11. \\
\hline S & 17.481352 & 7.237472 & 3966 \\
\hline S & 5.061011 & 21.645418 & 11.63870 \\
\hline S & 5.091597 & 19.539595 & 9.48966 \\
\hline $\mathrm{Si}$ & 1 & 9.4 & 23. \\
\hline $\mathrm{Si}$ & & & 21 . \\
\hline S & 17.369446 & 21.69 & 19.54323 \\
\hline $\mathrm{Si}$ & 17.415812 & 23.855619 & 21.66993 \\
\hline $\mathrm{Si}$ & 17.407557 & 11.705212 & 9.48020 \\
\hline $\mathrm{Si}$ & 5.100579 & 21.602694 & 7.31078 \\
\hline $\mathrm{Si}$ & 5.097180 & 23.742258 & 9.43505 \\
\hline $\mathrm{Si}$ & 5.0 & 9.4 & 19. \\
\hline $\mathrm{Si}$ & 5.086499 & 11.632630 & 21.70561 \\
\hline S & 1.580276 & 21.629881 & 19.56726 \\
\hline$S$ & 1.627855 & 19.516779 & 21.61556 \\
\hline $\mathrm{Si}$ & 1.608920 & 9.463452 & 7.26733 \\
\hline $\mathrm{Si}$ & 1.623242 & 7.329715 & 9.39208 \\
\hline S: & 3.881916 & 21.736933 & 7.17630 \\
\hline & & 4250 & 9.344 \\
\hline
\end{tabular}




\begin{tabular}{|c|c|c|c|}
\hline i & 13.858611 & 7.200332 & 63624 \\
\hline 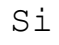 & 23.769688 & 1.592899 & 21.624784 \\
\hline & 21.672848 & 1.536097 & 23.712641 \\
\hline & 23.806099 & 13.836037 & 9.432138 \\
\hline & 21.692995 & 13.798167 & 11.615881 \\
\hline & 11.545485 & 1.667665 & 9.456656 \\
\hline i & 9.395241 & 1.687085 & 11.607871 \\
\hline & 11.684093 & 13.833365 & $21.72770^{\circ}$ \\
\hline & 9.461996 & 13.787002 & 23.80609 \\
\hline & 23.819937 & 17.346386 & 21.70124 \\
\hline & 21.696878 & 17.376728 & 19.56192 \\
\hline 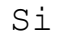 & 21.697365 & 5.167091 & 7.22946 \\
\hline i & 11.6 & 17.375273 & \\
\hline 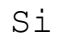 & 9.468307 & 17.394449 & $7.18770 \varepsilon$ \\
\hline & 11.600831 & 5.120969 & 21.642015 \\
\hline & 9.471949 & 5.119756 & 19.53207 \\
\hline & 19.533283 & 17.384983 & 21.69542 \\
\hline i & 21.722368 & 17.393236 & 23.818 \\
\hline $\mathrm{Si}$ & 19.605623 & 5.015375 & 9.460297 \\
\hline S & 21.747614 & 5.038436 & 11.634573 \\
\hline $\mathrm{Si}$ & 7.250338 & 17.362408 & 9.335768 \\
\hline L & 9.432382 & 17.221371 & 11.515384 \\
\hline i & 7.286263 & 5.152769 & 21.66823 \\
\hline i & 9.411990 & 5.1 & 23. \\
\hline$i$ & 19.556831 & 1.635623 & 21.597597 \\
\hline $\mathrm{Si}$ & 21.677217 & 1.614261 & 19.47842 \\
\hline $\mathrm{Si}$ & 19.562899 & 13.834579 & 9.421943 \\
\hline i & 7.271942 & 1.615232 & 9.42388 \\
\hline i & 9.421700 & 1.686356 & \\
\hline $\mathrm{Si}$ & 7.296216 & 13.71 & 21.62963 \\
\hline $\mathrm{Si}$ & 21.706347 & 23.740557 & 1.58488 \\
\hline $\mathrm{Si}$ & 23.842997 & 21.635221 & 1.609163 \\
\hline Si & 21.702219 & 11.590393 & 13.78457 \\
\hline$i$ & 23.818966 & 9.391600 & 13.79015 \\
\hline$i$ & 9.434323 & 23.82 & 13. \\
\hline $\mathrm{Si}$ & 9.467822 & 11.605928 & 1.66159 \\
\hline $\mathrm{Si}$ & 11.617580 & 9.464181 & 1.64509 \\
\hline Si & 21.686928 & 23.783768 & 17.37988 \\
\hline $\mathrm{Si}$ & 19.539839 & 21.702463 & 17.429647 \\
\hline $\mathrm{Si}$ & 21.772131 & $11.656 \mathrm{~s}$ & $4.89011 \varepsilon$ \\
\hline $\mathrm{Si}$ & 19.6 & 9. & 4 . \\
\hline $\mathrm{Si}$ & 9.380191 & 23.815323 & 5.208358 \\
\hline $\mathrm{Si}$ & 9.487727 & 11.531405 & 17.210691 \\
\hline $\mathrm{Si}$ & 7.253007 & 9.425827 & 17.35682 \\
\hline $\mathrm{Si}$ & 21.693724 & 19.534012 & 17.38595 \\
\hline $\mathrm{Si}$ & 23.834257 & 21.692268 & 17.36337 \\
\hline $\mathrm{Si}$ & 21.697609 & 7.371467 & 5.12315 \\
\hline $\mathrm{Si}$ & 23.776485 & 9.444518 & 5.09014 \\
\hline Si & 9.505691 & 19.547607 & 4.97265 \\
\hline $\mathrm{Si}$ & 11.600831 & 21.768976 & 5.10203 \\
\hline $\mathrm{Si}$ & 9.450830 & 7.288448 & 17.35172 \\
\hline $\mathrm{Si}$ & 11.635786 & 9.364169 & 17.38401 \\
\hline $\mathrm{Si}$ & 21.691782 & 19.527216 & 1.60770 \\
\hline $\mathrm{Si}$ & 19.570910 & 21.613375 & 1.64606 \\
\hline $\mathrm{Si}$ & 21.745186 & 7.194748 & 13.76782 \\
\hline $\mathrm{Si}$ & 19.590572 & 9.376792 & 13.85084 \\
\hline $\mathrm{Si}$ & 9.451558 & 19.569210 & 13.66368 \\
\hline Si & 7.241598 & 21.689840 & 13.81807 \\
\hline
\end{tabular}




\begin{tabular}{|c|c|c|c|}
\hline $\mathrm{Si}$ & 9.430197 & 7.298644 & 1.612076 \\
\hline $\mathrm{Si}$ & 7.328501 & 9.425584 & 1.653100 \\
\hline $\mathrm{Si}$ & 11.645740 & 21.726980 & 13.749375 \\
\hline i & 11.076499 & 3.352079 & 13.493522 \\
\hline$i$ & 20.059072 & 3.244058 & 13.37384 \\
\hline Si & 7.600377 & 1.224411 & 3.37368 \\
\hline$\Delta \perp$ & 23.389061 & 15.571912 & 17.73113 \\
\hline $\mathrm{Si}$ & 5.469795 & 15.483310 & 19.9134 \\
\hline i & 3.436555 & 17.691814 & 11.28793 \\
\hline$i$ & 13.864194 & 9.385531 & 19.53813 \\
\hline 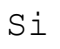 & 23.805614 & 5.152284 & 9.40932 \\
\hline 1 & 15.580408 & 1.256696 & 19.89910 \\
\hline Al & 15.850828 & 5.462756 & 11.32215 \\
\hline 1 & 17.506598 & 9.604731 & 7.1998 \\
\hline $\mathrm{L}$ & 9.529237 & 13.555665 & 19.58960 \\
\hline 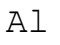 & 7.286021 & 21.634008 & 5.18117 \\
\hline 1 & 7.509347 & 17.649574 & 15.32795 \\
\hline Al & 23.387848 & 5.398671 & 3.41907 \\
\hline Al & 13.485996 & 19.842056 & 3.2904 \\
\hline Al & 21.777956 & 13.659075 & 7.2481 \\
\hline 1 & 13.879972 & 5.107861 & 13.3177 \\
\hline H. & 7.269029 & 18.344801 & 17.90081 \\
\hline 0 & 22.164650 & 0.346641 & 2.86488 \\
\hline 0 & 24.607161 & 24.540163 & \\
\hline 0 & 24.030396 & 2.099510 & 2.11553 \\
\hline 0 & 22.853807 & 2.185928 & 4.55221 \\
\hline 0 & 22.166836 & 12.477144 & 15.07889 \\
\hline 0 & 24.604733 & 12.400436 & 16.100 \\
\hline 0 & 24.014376 & 14.323227 & 14.33973 \\
\hline 0 & 22.914007 & 14.340462 & 16.78 \\
\hline 0 & 9.9 & 8815 & 15. \\
\hline 0 & 12.548997 & 24.615171 & 16.09357 \\
\hline 0 & 11.556894 & 2.115046 & 14.40017 \\
\hline 0 & 10.701942 & 2.096597 & 16.92667 \\
\hline 0 & 9.944575 & 12.479086 & 2950 \\
\hline 0 & 12.478843 & 9619 & 3. \\
\hline 0 & 11.723903 & 14.314731 & 2.11383 \\
\hline 0 & 10.780592 & 14.325897 & 4.62746 \\
\hline 0 & 8.929654 & 6.409223 & 2.87993 \\
\hline 0 & 6.413106 & 6.411407 & 3.88709 \\
\hline 0 & 7.081143 & 4.632322 & 2.09781 \\
\hline 0 & 8.181753 & 8284 & 4.552 \\
\hline 0 & 8.933052 & 18.805288 & 15.16142 \\
\hline 0 & 6.318678 & 18.625656 & 16.26543 \\
\hline 0 & 7.167318 & 16.757242 & 13.97440 \\
\hline 0 & 7.936823 & 16.860165 & 16.868 \\
\hline 0 & 21.309942 & 6.232018 & 15.00097 \\
\hline 0 & 18.800676 & 6.397085 & 16.010 \\
\hline 0 & 19.390549 & 4.472837 & 14.25428 \\
\hline 0 & 20.486547 & 4.554400 & 16.76112 \\
\hline 0 & 21.223766 & 18.619831 & 2.87338 \\
\hline 0 & 18.710861 & 18.711832 & 3.95457 \\
\hline 0 & 19.296366 & 16.854824 & 2.18519 \\
\hline 0 & 20.480965 & 16.849728 & 4.5565 \\
\hline 0 & 8.856102 & 0.335475 & 3.87665 \\
\hline 0 & 6.331786 & 24.692608 & 2.78138 \\
\hline 0 & 7.074589 & 2.118444 & 4.64615 \\
\hline 0 & 8.185394 & 2.185685 & 2.19903 \\
\hline
\end{tabular}




\begin{tabular}{|c|c|c|c|}
\hline 0 & 9.009274 & 12.313534 & 15.834321 \\
\hline 0 & 6.408737 & 12.388057 & 15.089577 \\
\hline 0 & 7.336027 & 14.171268 & 16.781031 \\
\hline 0 & 8.103104 & 14.251860 & 14.23850 \\
\hline o & 21.146332 & 0.338631 & 16.100128 \\
\hline 0 & 18.633909 & 24.610802 & 15.014811 \\
\hline 0 & 19.305590 & 2.185199 & 16.768650 \\
\hline 0 & 20.548933 & 2.036882 & 14.33269 \\
\hline 0 & 21.314796 & 12.472531 & \\
\hline 0 & 18.699209 & 12.551667 & 2.7852 \\
\hline 0 & 19.561686 & 14.240451 & 4.630137 \\
\hline 0 & 20.479752 & 14.411586 & 2.122571 \\
\hline 0 & 22.260294 & 6.569920 & 3.877139 \\
\hline 0 & 24.783152 & 6.407038 & 2.617044 \\
\hline 0 & 24.181870 & 4.550274 & 4.707088 \\
\hline 0 & 22.764961 & 4.489830 & 2.123056 \\
\hline 0 & 22.079933 & 18.621044 & 16.092360 \\
\hline 0 & 24.538219 & 18.719843 & 15.094916 \\
\hline 0 & 23.939367 & 16.848999 & 16.856766 \\
\hline 0 & 22.853321 & 16.767193 & 14.401876 \\
\hline 0 & 10.040460 & 6.507778 & 16.016867 \\
\hline 0 & 12.641240 & 6.318921 & 14.999032 \\
\hline O & 11.723175 & 4.567509 & 16.833706 \\
\hline 0 & 10.710681 & 4.695436 & 14.318130 \\
\hline 0 & 9.943604 & 18.619347 & 3.723724 \\
\hline 0 & 12.388541 & 18.692169 & 2.696180 \\
\hline 0 & 11.798426 & 16.857010 & 4.542263 \\
\hline 0 & 10.618681 & 16.779089 & 2.127669 \\
\hline 0 & 2.952762 & 22.165621 & 564 \\
\hline 0 & 3.971567 & 24.611774 & 24.532152 \\
\hline 0 & 2.108006 & 24.018988 & 2.032027 \\
\hline 0 & 4.536437 & 22.853807 & 2.184228 \\
\hline 0 & 2.877511 & 9.951614 & 12.475445 \\
\hline 0 & 3.880538 & 12.4671 & 12.4696 \\
\hline 0 & 2.118930 & 11.7 & 14. \\
\hline 0 & 4.558770 & 10.697574 & 14.332694 \\
\hline 0 & 15.172111 & 22.164650 & 12.400923 \\
\hline 0 & 16.177565 & 24.597450 & 12.473745 \\
\hline 0 & 14.321772 & 23.930141 & 14.249433 \\
\hline 0 & 16.777390 & 22 . & 14.4 \\
\hline 0 & 15.1 & 9.8 & 0 . \\
\hline 0 & 16.103285 & 12.462580 & 24.607405 \\
\hline 0 & 14.262055 & 11.710795 & 2.025715 \\
\hline 0 & 16.846087 & 10.711895 & 2.108249 \\
\hline 0 & 2.883094 & 8.925528 & 6.405096 \\
\hline 0 & 3.887577 & 6.4 & 765 \\
\hline 0 & 2.196366 & 7.086483 & 4.544447 \\
\hline 0 & 4.630622 & 8.245596 & 4.624554 \\
\hline 0 & 2.861732 & 21.073265 & 18.712559 \\
\hline 0 & 4.041964 & 18.626871 & 18.627598 \\
\hline 0 & 2.271374 & 19.288597 & 16.769379 \\
\hline 0 & 4.639604 & 20.553061 & 16.929590 \\
\hline 0 & 15.161186 & 8.942762 & 18.629784 \\
\hline 0 & 16.106440 & 6.406552 & 18.717659 \\
\hline 0 & 14.416926 & 7.081386 & 16.847057 \\
\hline 0 & 16.923765 & 8.103104 & 16.874729 \\
\hline 0 & 15.182305 & 21.322809 & 6.322805 \\
\hline ? & 16.184361 & 18.797764 & 6.49054 \\
\hline
\end{tabular}




$$
\begin{aligned}
& 14.326626 \\
& 16.838560 \\
& 3.865973 \\
& 2.777742 \\
& 4.640818 \\
& 2.210688 \\
& 3.800674 \\
& 2.950577 \\
& \text { 4. } 626496 \\
& 2.122571 \\
& 16.166397 \\
& 15.077197 \\
& 16.867691 \\
& 14.405275 \\
& 16.173437 \\
& 15.081567 \\
& 16.866476 \\
& 14.430763 \\
& 3.800189 \\
& 2.776529 \\
& 4.635478 \\
& 2.278899 \\
& 3.799460 \\
& 2.783811 \\
& 4.556342 \\
& 2.112376 \\
& 16.091631 \\
& 14.916984 \\
& 16.795109 \\
& 14.416442 \\
& 16.016380 \\
& 15.167498 \\
& 16.923765 \\
& 14.396779 \\
& 0.344942 \\
& 24.609102 \\
& 2.119173 \\
& 2.199036 \\
& 0.428204 \\
& 24.607891 \\
& 2.115288 \\
& 2.203405 \\
& 12.463307 \\
& 12.486610 \\
& 14.330267 \\
& 14.240208 \\
& 12.640026 \\
& 12.397767 \\
& 14.332936 \\
& 14.411345 \\
& 6.416748 \\
& 6.390531 \\
& 4.641304 \\
& 4.479635 \\
& 6.496853 \\
& 6.311881 \\
& 4.556585 \\
& 19.385210 \\
& 20.477324 \\
& 8.842994 \\
& 6.400969 \\
& 7.168531 \\
& 8.337111 \\
& 21.161625 \\
& 18.604296 \\
& 19.387636 \\
& 20.393818 \\
& 8.858287 \\
& 6.404125 \\
& 7.159550 \\
& 8.257733 \\
& 21.237604 \\
& 18.780287 \\
& 19.382053 \\
& 20.640450 \\
& 22.169748 \\
& 24.610802 \\
& 24.018017 \\
& 22.839972 \\
& 9.942390 \\
& 12.402864 \\
& 11.721476 \\
& 10.625963 \\
& 22.083332 \\
& 24.524143 \\
& 23.936939 \\
& 22.758408 \\
& 9.954527 \\
& 12.486854 \\
& 11.725117 \\
& 10.784233 \\
& 2.868529 \\
& 3.964042 \\
& 2.117959 \\
& 4.559498 \\
& 15.094673 \\
& 16.100370 \\
& 14.257443 \\
& 16.774233 \\
& 2.943052 \\
& 3.806257 \\
& 2.026443 \\
& 4.636448 \\
& 15.078652 \\
& 16.098185 \\
& 14.329296 \\
& 16.783943 \\
& 2.873384 \\
& 3.957731 \\
& 2.037610 \\
& 4.482062 \\
& 15.074526 \\
& 16.012011 \\
& 14.248947 \\
& 4.720681 \\
& 4.571635 \\
& 0.181089 \\
& 24.691879 \\
& 2.122814 \\
& 2.105336 \\
& 12.554094 \\
& 12.552153 \\
& 14.415956 \\
& 14.328568 \\
& 12.456025 \\
& 12.721104 \\
& 14.414500 \\
& 14.412557 \\
& 0.341544 \\
& 24.607647 \\
& 2.121600 \\
& 2.107763 \\
& 6.491028 \\
& 6.412864 \\
& 4.638148 \\
& 4.484489 \\
& 18.706978 \\
& 18.714745 \\
& 16.851185 \\
& 16.849485 \\
& 18.616919 \\
& 18.630270 \\
& 16.839775 \\
& 16.694370 \\
& 6.406309 \\
& 6.406795 \\
& 4.574306 \\
& 4.540078 \\
& 22.162708 \\
& 24.615171 \\
& 24.012920 \\
& 22.846523 \\
& 9.955499 \\
& 12.483212 \\
& 11.806922 \\
& 10.788603 \\
& 10.038033 \\
& 12.644639 \\
& 11.801825 \\
& 11.219234 \\
& 22.159796 \\
& 24.600849 \\
& 24.090355 \\
& 23.003824 \\
& 8.864597 \\
& 6.414320 \\
& 7.077502 \\
& 8.177870 \\
& 21.078848 \\
& 18.546038 \\
& 19.382296 \\
& 20.398916
\end{aligned}
$$




\begin{tabular}{|c|c|c|c|}
\hline 0 & 18.704792 & 2.937227 & 21.145847 \\
\hline 0 & 18.793396 & 3.963799 & 18.706734 \\
\hline 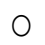 & 16.945612 & 2.124998 & 19.212132 \\
\hline 0 & 16.846087 & 4.551002 & 20.396490 \\
\hline O & 18.715958 & 15.167498 & 8.942762 \\
\hline O & 18.776644 & 16.033859 & 6.412135 \\
\hline 0 & 16.924007 & 14.327840 & 7.094737 \\
\hline 0 & 16.925949 & 16.855795 & 8.09606 \\
\hline 0 & 0.422864 & 3.884907 & 8.947374 \\
\hline 0 & 24.524143 & 2.691082 & 6.56409 \\
\hline 0 & 2.106064 & 4.558527 & 6.998367 \\
\hline 0 & 2.205105 & 2.182772 & 8.255791 \\
\hline 0 & 0.419951 & 16.092844 & 21.154827 \\
\hline 0 & 24.620996 & 15.012383 & 18.641922 \\
\hline 0 & 2.199521 & 16.778116 & 19.29879 \\
\hline 0 & 2.120629 & 14.321528 & 20.468100 \\
\hline 0 & 12.381745 & 3.804315 & 21.156042 \\
\hline 0 & 12.484912 & 2.866102 & 18. \\
\hline 0 & 14.241422 & 4.706602 & 19.389336 \\
\hline 0 & 14.338763 & 2.270403 & 20.553547 \\
\hline O & 12.569386 & 16.093330 & 8.926742 \\
\hline O & 12.484426 & 15.167983 & 6.424273 \\
\hline O & 14.414257 & 16.862837 & 7.084299 \\
\hline 0 & 14.418140 & 14.399693 & 550 \\
\hline 0 & 6.410921 & 3.879809 & 22.157854 \\
\hline 0 & 6.411650 & 2.785268 & 24.608860 \\
\hline 0 & 4.632565 & 4.637662 & 24.020687 \\
\hline O & 4.531582 & 2.257781 & 22.778313 \\
\hline 0 & 6.322805 & 16.088961 & 264 \\
\hline 0 & 6.502679 & 14.909701 & 12.221290 \\
\hline 0 & 4.706845 & 16.774477 & 11.796241 \\
\hline 0 & 4.543234 & 14.326140 & 10.621837 \\
\hline 0 & 18.702850 & 3.713771 & 9.856701 \\
\hline 0 & 18.968414 & 2.630396 & 12.311833 \\
\hline 0 & 17.105339 & 582 & 11. \\
\hline 0 & 16.858952 & 2.116745 & 10.704613 \\
\hline 0 & 18.630997 & 16.107168 & 22.160767 \\
\hline 0 & 18.712074 & 15.085935 & 24.605463 \\
\hline 0 & 16.856039 & 16.863077 & 24.024813 \\
\hline 0 & 16.770350 & 14.409401 & 22.923717 \\
\hline 0 & 2.867316 & 24.6 & 22 . \\
\hline 0 & 0.431117 & 0.417523 & 20.993402 \\
\hline 0 & 1.007639 & 22.923231 & 22.919592 \\
\hline 0 & 2.193453 & 22.756222 & 20.542622 \\
\hline 0 & 2.877511 & 12.469134 & 9.952828 \\
\hline 0 & 0.424077 & 12.560649 & 1839 \\
\hline 0 & 1.014436 & 10.693689 & 10.707768 \\
\hline 0 & 2.122086 & 10.637129 & 8.256763 \\
\hline 0 & 15.103413 & 24.701834 & 9.878305 \\
\hline 0 & 12.556279 & 0.513893 & 8.937422 \\
\hline 0 & 13.304180 & 22.925659 & 10.622079 \\
\hline 0 & 14.326626 & 23.007950 & 8.096307 \\
\hline 0 & 15.080594 & 12.558706 & 22.155184 \\
\hline 0 & 12.570844 & 12.552638 & 21.222069 \\
\hline 0 & 13.308791 & 10.700244 & 22.925659 \\
\hline 0 & 14.322013 & 10.632031 & 20.475866 \\
\hline 0 & 16.111052 & 18.629299 & 22.160767 \\
\hline ? & 18.622015 & 18.608908 & 21.14973 \\
\hline
\end{tabular}




\begin{tabular}{|c|c|c|c|}
\hline 0 & 18.036757 & 20.395519 & 22.925903 \\
\hline 0 & 16.855068 & 20.472469 & 20.487034 \\
\hline & 16.173195 & 6.411650 & 9.949915 \\
\hline & 18.708675 & 6.310668 & 9.011945 \\
\hline & 18.023647 & 8.273270 & 10.624992 \\
\hline & 16.931776 & 8.101890 & 8.167432 \\
\hline & 3.867186 & 18.616190 & 10.031235 \\
\hline & 6.322805 & 18.629299 & 8.936451 \\
\hline & 5.657681 & 20.395761 & 10.784719 \\
\hline & 4.469924 & 20.482420 & 8.346093 \\
\hline & 3.877139 & 6.488601 & 22.249613 \\
\hline & 6.319406 & 6.321834 & 21.074720 \\
\hline & 5.818864 & 8.275697 & 22.76 \\
\hline & 4.557798 & 8.181753 & 20.4 \\
\hline & 16.180719 & 24.767860 & 21.321 \\
\hline & 18.708191 & 0.417523 & 22.2 \\
\hline & 17.954222 & 22.935856 & 20.3 \\
\hline & 16.939301 & 22.845552 & 22.8 \\
\hline & 16.098429 & 12.553366 & 8.94 \\
\hline & 18.537298 & 12.73 & 10.0 \\
\hline & 18.034571 & 10.776223 & 8.3 \\
\hline & 16.933233 & 10.78 & 10.7 \\
\hline & 3.806985 & 24.598665 & 8.9 \\
\hline & 6.316979 & 0.42 & 9.9 \\
\hline & 5.721037 & 22.828804 & 8.2 \\
\hline & 4.556585 & 22.85 & 10.7 \\
\hline & 3.781012 & $12.47^{\circ}$ & 21.2 \\
\hline & 6.235416 & 12.64 & 22.2 \\
\hline & 5.649670 & 10.69 & 20.4 \\
\hline & 4.568965 & 10.70 & 22.9 \\
\hline & 2.941596 & 18.69 & 21.0 \\
\hline & 0.508310 & 18.53 & 22.2 \\
\hline & 0.933844 & 20.38 & 20.4 \\
\hline & 2.198065 & 20.48 & 22.7 \\
\hline & 2.860033 & 6.41 & 8.8 \\
\hline & 0.421893 & 6.39 & 9.9 \\
\hline & 1.010 & 8.24 & 8.1 \\
\hline & 2.187 & 8.25 & 10.6 \\
\hline & 15.170 & 18.62 & 8.9 \\
\hline 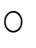 & 12.62 & 18.62 & 9.7 \\
\hline & 13.393267 & 20.48 & 8.0 \\
\hline O & 14.327 & 20.46 & 10.6 \\
\hline & 15.1677 & 6.33 & 21.2 \\
\hline O & 12.6366 & 6.25 & 22.1 \\
\hline 0 & 13.318501 & 8.09 & 20.3 \\
\hline 0 & 14.325 & 8.18 & 22.8 \\
\hline 0 & 22.171206 & 2.779199 & 24.6 \\
\hline O & 21.077 & 0.33 & 0.3 \\
\hline 0 & 22.9229 & 0.922435 & 22.84 \\
\hline O & 20.56 & 2.18 & 22.7 \\
\hline 0 & 22.151056 & 15.067244 & 12.5 \\
\hline O & 21.2291 & 12.55 & 12.566717 \\
\hline ค & 22.928816 & 13.244221 & 10.700486 \\
\hline 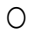 & 20.477810 & 14.33 & 10.695146 \\
\hline 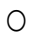 & 9.948215 & 3.034082 & 12.395096 \\
\hline O & 8.939363 & 0.593271 & 12.7 \\
\hline & 10.63 & 1.0967 & 10.7 \\
\hline & & 2.181073 & 10.6956 \\
\hline
\end{tabular}




\begin{tabular}{rrr}
9.849904 & 15.089090 & 24.684839 \\
8.931354 & 12.546083 & 0.440584 \\
10.795156 & 13.303937 & 23.003824 \\
8.251179 & 14.248947 & 22.828075 \\
22.172905 & 16.079494 & 18.689985 \\
21.224739 & 18.613035 & 18.629299 \\
22.925175 & 17.952036 & 20.479994 \\
20.477324 & 16.852156 & 20.481693 \\
22.153969 & 3.894859 & 6.333486 \\
21.144632 & 6.401940 & 6.329602 \\
22.921534 & 5.731961 & 8.170588 \\
20.556215 & 4.566295 & 8.241712 \\
9.945061 & 16.096729 & 6.327902 \\
9.029423 & 18.627598 & 6.248282 \\
10.695874 & 17.872173 & 8.174956 \\
8.181268 & 16.933718 & 8.090239 \\
9.946759 & 3.796062 & 18.708191 \\
8.935479 & 6.236145 & 18.464231 \\
10.704856 & 5.740700 & 20.398188 \\
8.255305 & 4.633535 & 20.476595 \\
21.233963 & 16.181448 & 24.771744 \\
22.330690 & 18.623472 & 0.417280 \\
20.476109 & 17.948397 & 22.919592 \\
22.924202 & 16.773991 & 22.917891 \\
21.403885 & 3.795819 & 12.633958 \\
22.252768 & 6.332272 & 12.484912 \\
20.409355 & 5.484846 & 10.800255 \\
22.915949 & 4.555129 & 10.628877 \\
9.020198 & 15.846701 & 12.308921 \\
9.773682 & 18.378542 & 12.654349 \\
8.188308 & 17.785999 & 10.628877 \\
10.791759 & 16.941000 & 10.694418 \\
8.928683 & 3.802616 & 24.609346 \\
9.957440 & 6.325232 & 0.431117 \\
8.165732 & 5.735602 & 22.915222 \\
10.624264 & 4.632322 & 22.836813 \\
21.236633 & 2.862946 & 18.539240 \\
22.328018 & 0.430631 & 18.549435 \\
20.404985 & 1.019290 & 20.313955 \\
23.007465 & 22.913036 & 1.010309 \\
22.830746 & 2.193453 & 20.461302 \\
21.327906 & 15.250031 & 6.326204 \\
22.339670 & 12.645610 & 5.999467 \\
20.393091 & 13.234998 & 8.179326 \\
23.002853 & 14.411101 & 8.186123 \\
8.850276 & 2.960773 & 6.493455 \\
10.032207 & 0.584775 & 6.314794 \\
8.264045 & 1.018562 & 8.266230 \\
10.631061 & 2.271617 & 8.271085 \\
8.866782 & 15.185947 & 18.717173 \\
10.026623 & 12.566231 & 18.292852 \\
10.707767 & 13.062405 & 20.395761 \\
24.767374 & 14.331723 & 20.552332 \\
\hline & 22.077505 & 2.861247 \\
2.8939 & 8.854646 & 12.548512
\end{tabular}




\begin{tabular}{|c|c|c|c|}
\hline 0 & 22.920076 & 10.635916 & 235239 \\
\hline 0 & 22.918861 & 8.160877 & 14.328811 \\
\hline 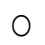 & 12.463551 & 22.163195 & 15.078168 \\
\hline 0 & 12.638812 & 21.303631 & 12.55045 \\
\hline 0 & 10.632275 & 22.913280 & 13.23087 \\
\hline 0 & 10.723062 & 20.412510 & 14.159616 \\
\hline 0 & 12.475930 & 9.940449 & 2.947422 \\
\hline 0 & 12.572058 & 8.935722 & 320 \\
\hline 0 & 10.708011 & 10.706554 & \\
\hline 0 & 10.630818 & 8.257490 & 2.116017 \\
\hline 0 & 18.704792 & 22.174118 & 16.104012 \\
\hline 0 & 18.608421 & 21.148273 & 18.6232 \\
\hline 0 & 20.470526 & 22.913765 & 18. \\
\hline 0 & 20.482420 & 20.482906 & 16.921095 \\
\hline 0 & 18.797035 & 10.022011 & 3. \\
\hline 0 & 18.710617 & 9.013886 & 6.159 \\
\hline 0 & 20.478781 & 10.858756 & 5.483874 \\
\hline 0 & 20.572481 & 8.341967 & \\
\hline 0 & 6.407523 & 22.176788 & 3.797276 \\
\hline 0 & 6.238572 & 20.984663 & 6.408979 \\
\hline 0 & 8.262345 & 22.925417 & 5.899213 \\
\hline 0 & 8.350462 & 20.481449 & 4.473809 \\
\hline 0 & 6.319649 & 9.863983 & 16.090902 \\
\hline 0 & 6.332272 & 8.948588 & 18. \\
\hline 0 & 8.185637 & 10.700486 & 17.787212 \\
\hline 0 & 8.183453 & 8.178112 & 16.857737 \\
\hline 0 & 24.855490 & 21.239546 & 16.177320 \\
\hline O & 0.424077 & 22.256165 & 18.622744 \\
\hline 0 & 23.004066 & 20.387506 & 72660 \\
\hline 0 & 22.858419 & 22.832687 & 16.772291 \\
\hline 0 & 24.606432 & 8.846393 & 3.801645 \\
\hline 0 & 0.502727 & 10.035362 & 6.234202 \\
\hline 0 & 22.913765 & 8.270113 & 5.812310 \\
\hline 0 & 22.924932 & 10.619652 & 4.387 \\
\hline 0 & 12.544385 & 4566 & 83 \\
\hline 0 & 12.643668 & 22.245728 & 6.247068 \\
\hline 0 & 10.872108 & 20.389692 & 5.483389 \\
\hline 0 & 10.701457 & 23.006979 & 4.634021 \\
\hline 0 & 12.468162 & 8.771870 & 16.095516 \\
\hline 0 & 12.644396 & 9.865440 & 610 \\
\hline 0 & 10.685922 & 8.1 & 17. \\
\hline 0 & 10.703885 & 10.536389 & 16.768894 \\
\hline 0 & 18.714260 & 21.157255 & 2.945480 \\
\hline 0 & 18.632696 & 22.244514 & 0.493988 \\
\hline 0 & 20.396732 & 20.330462 & 1.016135 \\
\hline 0 & 20.572966 & 22.755737 & 569 \\
\hline 0 & 18.713774 & 8.942034 & 15.164342 \\
\hline 0 & 18.622259 & 9.864955 & 12.640269 \\
\hline 0 & 20.462759 & 8.105045 & 13.309278 \\
\hline 0 & 20.491888 & 10.634945 & 14.323227 \\
\hline 0 & 6.509719 & 21.090500 & 15.160215 \\
\hline 0 & 6.241971 & 22.169506 & 12. \\
\hline 0 & 8.183696 & 20.471012 & 13.222132 \\
\hline 0 & 8.167189 & 22.915222 & 14.328811 \\
\hline 0 & 6.487387 & 8.932567 & 2.954462 \\
\hline 0 & 6.316494 & 9.951614 & 0.495687 \\
\hline 0 & 8.179568 & 8.180540 & 1.015892 \\
\hline 0 & 8.265744 & 10.632760 & 2.1830 \\
\hline
\end{tabular}




\begin{tabular}{|c|c|c|c|}
\hline O & 13.310005 & 5.305699 & 8.579857 \\
\hline 0 & 12.389028 & 6.404367 & 6.074476 \\
\hline 0 & 11.888728 & 7.840209 & 8.425714 \\
\hline 0 & 14.498975 & 7.671744 & 7.418560 \\
\hline 0 & 8.080286 & 18.128271 & 19.463617 \\
\hline 0 & 8.177384 & 19.877254 & 17.694727 \\
\hline 0 & 8.429597 & 20.906498 & 20.566170 \\
\hline 0 & 10.710681 & 19.796907 & 19.225969 \\
\hline $\mathrm{H}$ & 12.765284 & 22.157612 & 3.122927 \\
\hline $\mathrm{H}$ & 15.972929 & 7.897740 & 7.88293 \\
\hline $\mathrm{H}$ & 25.092896 & 6.110159 & 1.73199 \\
\hline $\mathrm{H}$ & 21.916807 & 16.001329 & 3326 \\
\hline $\mathrm{H}$ & 13.568287 & 5.346480 & 2632 \\
\hline $\mathrm{H}$ & 9.077244 & 15.978997 & 19.26893 \\
\hline $\mathrm{H}$ & 8.987670 & 19.402931 & 880 \\
\hline $\mathrm{H}$ & 12.212793 & 17.512424 & 20.41372 \\
\hline $\mathrm{H}$ & 12.856070 & 16.222713 & 19.26796 \\
\hline $\mathrm{H}$ & 14.748518 & 17.72725 & \\
\hline $\mathrm{H}$ & 14.103541 & 19.0 & 19. \\
\hline $\mathrm{Re}$ & 12.999534 & 6.8 & 7.6 \\
\hline $\mathrm{Re}$ & 9.021655 & 19.812929 & 19.41021 \\
\hline $\mathrm{C}$ & 12.968946 & 17.218702 & 19.68937 \\
\hline $\mathrm{C}$ & 13.983625 & 18.025105 & 19.36239 \\
\hline \multicolumn{4}{|c|}{601} \\
\hline \multicolumn{4}{|c|}{ USY } \\
\hline $\mathrm{Si}$ & $23 . \overline{3} 7838 \overline{0}$ & 3.332660 & 1.24067 \\
\hline $\mathrm{Si}$ & 23.417706 & 1.252569 & 3.36688 \\
\hline Si & 23.350224 & 15.568272 & \\
\hline $\mathrm{Si}$ & 23.393917 & 13.412 & 15. \\
\hline $\mathrm{Si}$ & 11.252490 & 1.240675 & 15.60274 \\
\hline $\mathrm{Si}$ & 11.173113 & 15.568999 & 1.23436 \\
\hline $\mathrm{Si}$ & 11.230886 & 13.395208 & 3.37101 \\
\hline $\mathrm{Si}$ & 7.644314 & 3.360333 & 12300 \\
\hline $\mathrm{Si}$ & 98 & 5 . & 3. \\
\hline $\mathrm{Si}$ & 7.677813 & 15.481126 & 13.2 \\
\hline $\mathrm{Si}$ & 20.025330 & 5.412022 & $15.50782^{\circ}$ \\
\hline $\mathrm{Si}$ & 19.905415 & 15.627745 & 1.23047 \\
\hline $\mathrm{Si}$ & 19.848368 & 17.790125 & 3.32659 \\
\hline Si & 7.620 & & $5 \quad 515$ \\
\hline $\mathrm{Si}$ & 8 & 15. & 17. \\
\hline $\mathrm{Si}$ & 7.674171 & 13.300295 & 15.51171 \\
\hline $\mathrm{Si}$ & 19.961002 & 3.389948 & 17.71535 \\
\hline $\mathrm{Si}$ & 19.951294 & 1.217128 & 15.59278 \\
\hline Si & 8576 & 15. & 5.41469 \\
\hline $\mathrm{Si}$ & 20. & 13.373119 & \\
\hline $\mathrm{Si}$ & 23. & 3. & 5 . \\
\hline $\mathrm{Si}$ & 23.376196 & 17.743519 & 15.60031 \\
\hline $\mathrm{Si}$ & 11.224574 & 3.353779 & 17.76342 \\
\hline $\mathrm{Si}$ & 11.235741 & 5.554756 & 15.55443 \\
\hline $\mathrm{Si}$ & 11.241324 & 15.624589 & 5.47100 \\
\hline $\mathrm{Si}$ & 11.178696 & 17. & 3.27440 \\
\hline $\mathrm{Si}$ & 1.294807 & 23.440767 & 3.34139 \\
\hline Si & 3.412523 & 23.483248 & 1.22611 \\
\hline $\mathrm{Si}$ & 1.226596 & 11.212194 & 15.57021 \\
\hline Si & 3.341156 & 11.230643 & 13.37967 \\
\hline $\mathrm{Si}$ & 13.467546 & 23.379837 & 15.51923 \\
\hline $\mathrm{Si}$ & 15.611723 & 23.339298 & 13.38428 \\
\hline
\end{tabular}




\begin{tabular}{|c|c|c|c|}
\hline i & 13.431377 & 11.229672 & 3.332902 \\
\hline i & 15.625317 & 11.190833 & 1.206205 \\
\hline $\mathrm{Si}$ & 1.334860 & 7.703787 & 3.329019 \\
\hline & 3.407668 & 7.659606 & 5.51251 \\
\hline & 1.273688 & 19.907598 & 15.58890 \\
\hline & 3.407425 & 19.904444 & 17748131 \\
\hline$i$ & 13.464877 & 7.662520 & 15 \\
\hline i & 15.646435 & 7.656209 & 17.810272 \\
\hline & 15.592303 & 19.960518 & 5.51033 \\
\hline S & 5.546260 & 7.666404 & $3.350 \varepsilon$ \\
\hline & 3.412523 & 7.644314 & 1.2047 \\
\hline i & 5.421732 & 20.045721 & 15.619 \\
\hline$i$ & 3.318823 & 19.947895 & 13.391325 \\
\hline 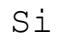 & 17.853725 & 7.628050 & 15.66658 \\
\hline & 15.614635 & 7.690436 & 13.49424 \\
\hline & 17.731138 & 19.953478 & 3.3816 \\
\hline & 15.591089 & 20.024845 & 1.2091 \\
\hline i & 5.509363 & 23.419405 & \\
\hline i & 3.418349 & 23.415 & 5.4 \\
\hline 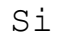 & 5.455231 & 11.164617 & 15.602741 \\
\hline $\mathrm{L}$ & 3.325863 & 11.223846 & 17.75856 \\
\hline i & 17.738420 & 23.390032 & 15.58842 \\
\hline 1 & 15.538414 & 23.374741 & 17.71730 \\
\hline i & 17.823381 & 11.266812 & \\
\hline i & 15.666826 & 11.229187 & 5.47707 \\
\hline $\mathrm{Si}$ & 3.394803 & 1.301847 & 23.40629 \\
\hline i & 1.240918 & 3.364460 & 23.41649 \\
\hline$i$ & 3.336544 & 13.412201 & 11.2444 \\
\hline i & 1.236548 & 15.597886 & 11 \\
\hline $\mathrm{Si}$ & 15.582593 & 1.221741 & 11.19496 \\
\hline $\mathrm{Si}$ & 13.374333 & 3.336544 & 11.27506 \\
\hline $\mathrm{Si}$ & 15.573126 & 13.435747 & 23.445137 \\
\hline Si & 13.461721 & 15.575068 & 23.46916 \\
\hline $\mathrm{Si}$ & 3.348438 & 1.213730 & 7.675 \\
\hline$i$ & 5.478777 & 3.3 & \\
\hline $\mathrm{Si}$ & 3.299404 & 13.361711 & 19.92677 \\
\hline $\mathrm{Si}$ & 17.780659 & 3.350866 & 19.78986 \\
\hline Si & 15.653960 & 13.419484 & 7.63727 \\
\hline $\mathrm{Si}$ & 17.817799 & 15.568272 & 7.65717 \\
\hline $\mathrm{Si}$ & 3.3025 & 5.446 & 7.595037 \\
\hline $\mathrm{Si}$ & 1.2 & 3.3 & 7 . \\
\hline Si & 3.418592 & 17.729681 & 19.895218 \\
\hline $\mathrm{Si}$ & 1.251841 & 15.580408 & 19.923134 \\
\hline $\mathrm{Si}$ & 15.587449 & 5.455716 & $19.94109^{\circ}$ \\
\hline $\mathrm{Si}$ & 13.391325 & 3.389462 & 19.91269 \\
\hline $\mathrm{Si}$ & 15.667069 & 17.768 & 7.630721 \\
\hline $\mathrm{Si}$ & 13.473860 & 15.627745 & 7.62198 \\
\hline $\mathrm{Si}$ & 3.382423 & 5.487759 & 23.40459 \\
\hline $\mathrm{Si}$ & 5.513732 & 3.392133 & 23.40702 \\
\hline $\mathrm{Si}$ & 5.501110 & 15.577496 & 11.09761 \\
\hline $\mathrm{Si}$ & 17.825567 & 3.275857 & 11.27093 \\
\hline $\mathrm{Si}$ & 15.609781 & 17.779688 & 23.41697 \\
\hline $\mathrm{Si}$ & 17.733564 & 15.616336 & 23.36600 \\
\hline $\mathrm{Si}$ & 1.638778 & 21.716785 & 23.76289 \\
\hline $\mathrm{Si}$ & 1.595812 & 23.795662 & 21.67915 \\
\hline $\mathrm{Si}$ & 1.601881 & 9.441362 & 11.576071 \\
\hline $\mathrm{Si}$ & 1.604794 & 11.601316 & $9.46563^{\circ}$ \\
\hline $\mathrm{Si}$ & 13.839677 & 21.664108 & 11.53310 \\
\hline
\end{tabular}




\begin{tabular}{|c|c|c|c|}
\hline L & 3.803023 & 23.851250 & 9.372908 \\
\hline $\mathrm{Si}$ & 13.838463 & 9.422428 & 23.806099 \\
\hline $\mathrm{Si}$ & 3.813946 & 11.623406 & 21.69275 \\
\hline $\mathrm{Si}$ & 17.441542 & 21.701248 & 23.771629 \\
\hline SI & 17.417267 & 19.575279 & 21.722126 \\
\hline $\mathrm{Si}$ & 17.359980 & 9.491611 & 11.634573 \\
\hline$i$ & 17.430376 & 7.274127 & 9.519284 \\
\hline S & 5.070964 & 21.694452 & 11.620251 \\
\hline 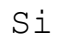 & 5.078489 & 19.571154 & 9.48287 \\
\hline 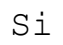 & 5.158595 & 9.443547 & 23.73376 \\
\hline i & 5.136020 & 7.277525 & 21.60900 \\
\hline i & 17.371874 & 21.747856 & 19.517 \\
\hline $\mathrm{Si}$ & 17.395178 & 23.911209 & 21.644447 \\
\hline i & 17.390566 & 11.680938 & 9.50739 \\
\hline 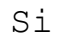 & 5.130436 & 21.630852 & 7.29621 \\
\hline 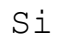 & 5.091840 & 23.799788 & 9.43043 \\
\hline $\mathrm{Si}$ & 5.089898 & 9.454228 & 19. \\
\hline $\mathrm{Si}$ & 5.085771 & 11.618793 & 21.69639 \\
\hline i & 1.591443 & 21.633766 & 19.60052 \\
\hline Si & 1.613290 & 19.567026 & 21.63740 \\
\hline $\mathrm{Si}$ & 1.650430 & 9.480930 & 7.2685 \\
\hline $\mathrm{Si}$ & 1.571780 & 7.307625 & 9.38771 \\
\hline $\mathrm{Si}$ & 13.880216 & 21.743486 & \\
\hline $\mathrm{Si}$ & 13.886284 & 19.5306 & 9 . \\
\hline Si & 13.857883 & 7.191107 & 21.70950 \\
\hline $\mathrm{Si}$ & 23.767502 & 1.596540 & 21.62721 \\
\hline $\mathrm{Si}$ & 21.667507 & 1.540466 & 23.70778 \\
\hline i & 23.820421 & $13 . \varepsilon$ & \\
\hline $\mathrm{Si}$ & 21.671877 & 13.809092 & 11.614182 \\
\hline $\mathrm{Si}$ & 11.544271 & 1.678589 & 9.46685 \\
\hline $\mathrm{Si}$ & 9.424128 & 1.725681 & 11.60107 \\
\hline Si & 11.682394 & 13.832395 & 21.72382 \\
\hline i & 9.472919 & 13.788215 & 23.81119 \\
\hline $\mathrm{Si}$ & 23.813381 & 17.378429 & 21. \\
\hline $\mathrm{Si}$ & 21.709017 & 17.410955 & $19.55221^{\circ}$ \\
\hline Si & 21.605850 & 5.138204 & 7.22654 \\
\hline $\mathrm{Si}$ & 11.674626 & 17.371147 & 9.37072 \\
\hline $\mathrm{Si}$ & 9.466851 & 17.394449 & 7.18867 \\
\hline $\mathrm{Si}$ & 11.615152 & 5.103977 & 21.67357 \\
\hline $\mathrm{Si}$ & 9.489183 & 5.099608 & 19.55318 \\
\hline $\mathrm{Si}$ & 19.524303 & 17.408772 & 21.7 \\
\hline $\mathrm{Si}$ & 21.682800 & 17.415083 & 23.83256 \\
\hline $\mathrm{Si}$ & 19.556345 & 5.089412 & 9.47364 \\
\hline $\mathrm{Si}$ & 21.779655 & 5.004209 & 11.63214 \\
\hline $\mathrm{Si}$ & 7.244268 & 17.368719 & 9.334068 \\
\hline $\mathrm{Si}$ & 9.429954 & 17.214817 & 11.50907 \\
\hline $\mathrm{Si}$ & 7.302770 & 5.141846 & $21.65415 \xi$ \\
\hline $\mathrm{Si}$ & 9.422428 & 5.105191 & 23.771872 \\
\hline $\mathrm{Si}$ & 19.560230 & 1.624699 & 21.584972 \\
\hline $\mathrm{Si}$ & 21.681101 & 1.612076 & 19.47987 \\
\hline $\mathrm{Si}$ & 19.545179 & 13.838463 & 9.42364 \\
\hline $\mathrm{Si}$ & 7.291361 & 1.626155 & 9.43068 \\
\hline $\mathrm{Si}$ & 9.429468 & 1.688056 & 7.31952 \\
\hline $\mathrm{Si}$ & 7.280195 & 13.730199 & 21.66022 \\
\hline $\mathrm{Si}$ & 21.692753 & 23.738859 & 1.57299 \\
\hline $\mathrm{Si}$ & 23.808285 & 21.651972 & 1.609649 \\
\hline $\mathrm{Si}$ & 21.682312 & 11.596947 & 13.78748 \\
\hline $\mathrm{Si}$ & 23.807070 & 9.418545 & 13.78991 \\
\hline
\end{tabular}




\begin{tabular}{|c|c|c|c|}
\hline L & 9.460540 & 23.833773 & 3.807878 \\
\hline $\mathrm{S}$ & 9.481173 & 11.600589 & 1.639992 \\
\hline & 11.614425 & 9.470736 & 1.637079 \\
\hline & 21.717270 & 23.773571 & 17.380371 \\
\hline & 19.540810 & 21.700035 & 17.413383 \\
\hline & 21.782326 & 11.644282 & 4.9022 \\
\hline & 19.636209 & 9.538218 & 5.029691 \\
\hline & 9.408349 & 23.851007 & 5.17485 \\
\hline & 9.444760 & 11.541115 & 17.27356 \\
\hline & 7.261018 & 9.417816 & 17.36604 \\
\hline & 21.691782 & 19.557318 & 17.39493 \\
\hline $\mathrm{Si}$ & 23.847122 & 21.679401 & 17.391779 \\
\hline $\mathrm{Si}$ & 21.694937 & 7.370982 & 5.12655 \\
\hline & 23.783281 & 9.442576 & 5.09669 \\
\hline S & 9.493795 & 19.552948 & 4.976778 \\
\hline 1 & 11.603745 & 21.767035 & 5.105191 \\
\hline $\mathrm{Si}$ & 9.443062 & 7.249852 & 17.356823 \\
\hline $\mathrm{Si}$ & 11.618551 & 9.347178 & 17.385468 \\
\hline 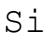 & 21.666294 & 19.542023 & 1.614504 \\
\hline $\mathrm{Si}$ & 19.550762 & 21.637892 & 1.64921 \\
\hline L & 21.750769 & 7.196933 & 13.75156 \\
\hline $\mathrm{Si}$ & 19.582319 & 9.352032 & 13.83433 \\
\hline $\mathrm{Si}$ & 9.449373 & 19.578192 & \\
\hline $\mathrm{Si}$ & 7.255435 & 21.7315 & 13.797683 \\
\hline i & 9.432867 & 7.299614 & 1.60358 \\
\hline 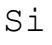 & 7.309082 & 9.436994 & 1.59678 \\
\hline 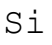 & 11.639914 & 21.710474 & 13.69354 \\
\hline$i$ & 11.198844 & 3.4 & 13. \\
\hline$i$ & 20.079948 & 3.232163 & 13.367294 \\
\hline$i$ & 7.642614 & 1.220527 & 3.373684 \\
\hline Si & 23.383965 & 15.601527 & 17.74497 \\
\hline Si & 5.429257 & 15.509284 & 20.03892 \\
\hline i & 3.418592 & 17.747644 & 11.28186 \\
\hline$i$ & 13.834579 & 9.370723 & 19.55489 \\
\hline i & 23.773329 & 5.1 & 9.39766 \\
\hline 1 & 15.596673 & 1.294079 & 19.85370 \\
\hline 1 & 15.596430 & 5.479748 & 11.322159 \\
\hline Al & 17.509752 & 9.559822 & 7.23625 \\
\hline 1 & 9.492582 & 13.645966 & 19.58329 \\
\hline 1 & 7.297430 & 21.650028 & 5.20496 \\
\hline 1 & 7.483616 & 17.695454 & 15.40514 \\
\hline Al & 23.367214 & 5.405225 & 3.40863 \\
\hline Al & 13.466334 & 19.830404 & 3.287752 \\
\hline Al & 21.786938 & 13.675824 & 7.23383 \\
\hline Al & 16.395792 & 4.456816 & 14.090677 \\
\hline Al & 6.100935 & 18.013695 & 18.00714 \\
\hline O & 22.171448 & 0.339116 & 2.85396 \\
\hline 0 & 24.620996 & 24.605219 & 3.95967 \\
\hline 0 & 24.027241 & 2.103879 & 2.10994 \\
\hline 0 & 22.836573 & 2.184714 & 4.548332 \\
\hline 0 & 22.155670 & 12.477387 & 15.08763 \\
\hline 0 & 24.607891 & 12.469134 & 16.09964 \\
\hline 0 & 23.941793 & 14.330267 & 14.32978 \\
\hline 0 & 22.909395 & 14.393380 & 16.78078 \\
\hline O & 10.023467 & 0.249543 & 15.16240 \\
\hline 0 & 12.548025 & 24.621483 & 16.021721 \\
\hline O & 11.634573 & 2.186656 & 14.32905 \\
\hline O & 10.702428 & 2.102180 & 16.85434 \\
\hline
\end{tabular}




$\begin{array}{rrr}9.945303 & 12.472775 & 2.941110 \\ 12.484183 & 12.468162 & 3.800674 \\ 11.724388 & 14.315217 & 2.113104 \\ 10.777679 & 14.319828 & 4.624554 \\ 8.942277 & 6.408737 & 2.871685 \\ 6.490542 & 6.477677 & 3.887334 \\ 7.079687 & 4.635963 & 2.101938 \\ 8.180782 & 4.555371 & 4.552458 \\ 8.930868 & 18.801891 & 15.163857 \\ 6.154824 & 18.635366 & 16.256456 \\ 7.172173 & 16.843658 & 13.991637 \\ 7.761075 & 16.936630 & 16.951923 \\ 21.316982 & 6.249253 & 15.000731 \\ 18.781013 & 6.330815 & 16.012739 \\ 19.460703 & 4.477935 & 14.255501 \\ 20.566170 & 4.555371 & 16.759668 \\ 21.157497 & 18.630026 & 2.862704 \\ 18.636824 & 18.696781 & 3.883450 \\ 19.288597 & 16.855553 & 2.113104 \\ 20.404257 & 16.861622 & 4.542506 \\ 8.937179 & 0.346884 & 3.807471 \\ 6.415049 & 24.626581 & 2.782355 \\ 7.082357 & 2.108977 & 4.639604 \\ 8.186123 & 2.191025 & 2.193696 \\ 8.938393 & 12.391212 & 15.932633 \\ 6.406309 & 12.398495 & 15.087877 \\ 7.171202 & 14.242393 & 16.847057 \\ 8.087568 & 14.339491 & 14.325655 \\ 21.225468 & 0.340815 & 16.093817 \\ 18.700666 & 24.601820 & 15.075012 \\ 19.381569 & 2.185928 & 16.768408 \\ 20.562284 & 2.028628 & 14.324684 \\ 21.315041 & 12.456268 & 3.548946 \\ 18.706247 & 12.551667 & 2.784054 \\ 19.629169 & 14.232198 & 4.631836 \\ 20.476839 & 14.410615 & 2.113590 \\ 22.254223 & 6.575017 & 3.875682 \\ 24.777082 & 6.399998 & 2.620686 \\ 24.110260 & 4.556585 & 4.711457 \\ 22.758650 & 4.489344 & 2.115288 \\ 22.161011 & 18.697023 & 16.093088 \\ 24.605946 & 18.701880 & 15.088606 \\ 23.944708 & 16.859194 & 16.857737 \\ 22.847494 & 16.780788 & 14.410615 \\ 9.962052 & 6.479861 & 16.012981 \\ 12.555308 & 6.408494 & 15.091275 \\ 11.708125 & 4.566295 & 16.769136 \\ 10.703642 & 4.720924 & 14.256229 \\ 9.873450 & 18.611336 & 3.717169 \\ 12.324215 & 18.709404 & 2.701520 \\ 11.729243 & 16.861622 & 4.542020 \\ 10.613583 & 16.777632 & 2.116260 \\ 2.953248 & 22.250097 & 0.253670 \\ 4.037837 & 24.694794 & 24.603035 \\ 2.101695 & 24.028698 & 2.035182 \\ 4.544205 & 22.916679 & 2.193210 \\ 2.870471 & 9.956470 & 12.477630 \\ 3.880295 & 12.478358 & 12.472775\end{array}$




\begin{tabular}{|c|c|c|c|}
\hline & 2.118930 & 11.794542 & 14.324684 \\
\hline 0 & 4.553672 & 10.701942 & 14.33633 \\
\hline & 15.093946 & 22.079205 & 12.48054 \\
\hline 0 & 16.103525 & 24.529238 & 12.390969 \\
\hline 0 & 14.340219 & 23.928686 & 14.245305 \\
\hline 0 & 16.848515 & 22.845068 & 14.3220 \\
\hline 0 & 15.174052 & 9.876848 & 0.3444 \\
\hline 0 & 16.166883 & 12.460879 & 24.60158 \\
\hline 0 & 14.317159 & 11.716621 & 2.0366 \\
\hline 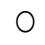 & 16.865021 & 10.706798 & 2.11771 \\
\hline 0 & 2.940625 & 8.93402 & \\
\hline 0 & 3.887820 & 6.406552 & 6.41529 \\
\hline 0 & 2.191754 & 7.083086 & 4.55367 \\
\hline 0 & 4.617029 & 8.185881 & 4.56775 \\
\hline 0 & 2.881152 & 20.977623 & 18.79703 \\
\hline 0 & 4.211157 & 18.5 & \\
\hline O & 2.283026 & 19.2 & 16.773262 \\
\hline O & 4.638876 & 20.576607 & 16.93420 \\
\hline 0 & 15.168468 & 8.936208 & 18.70722 \\
\hline O & 16.103525 & 6.407765 & 18.71692 \\
\hline O & 14.411101 & 7.162463 & 16. \\
\hline 0 & 16.9 & & 16. \\
\hline 0 & 15.1 & 21.3 & 6 . \\
\hline 0 & 16.182661 & 18.795094 & 6.49175 \\
\hline $\mathrm{O}$ & 14.325897 & 19.384481 & 4.71582 \\
\hline 0 & 16.852398 & 20.481693 & 4.62285 \\
\hline 0 & 3.8 & & \\
\hline 0 & 228 & 6. & 24. \\
\hline 0 & 4.637419 & 7.079444 & 2.11091 \\
\hline 0 & 2.211416 & 8.267200 & 2.103394 \\
\hline 0 & 3.797761 & 21.234934 & 12.548512 \\
\hline O & 2.88 & 18.6 & 12. \\
\hline 0 & 4.5 & 19. & 14. \\
\hline 0 & 2.1 & 20.4 & 14. \\
\hline 0 & 16.093330 & 8.855374 & 12.471317 \\
\hline 0 & 15.167254 & 6.302899 & 12.79611 \\
\hline 0 & 16.876431 & 7.163677 & 14.42421 \\
\hline 0 & 14.404 & 8.2 & 14.417 \\
\hline 0 & 16.0 & 21. & 0 . \\
\hline 0 & 15.082538 & 18.722269 & 24.618328 \\
\hline 0 & 16.854340 & 19.462887 & 2.105336 \\
\hline 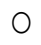 & 14.416685 & 20.630981 & 2.101452 \\
\hline 0 & 3.8747 & 22.152 & 6.39975 \\
\hline 0 & 2.7 & 24. & 13 \\
\hline 0 & 4.6 & 24.1 & 4.634749 \\
\hline 0 & 2.277443 & 22.849680 & 4.477935 \\
\hline 0 & 3.811112 & 9.951614 & 18.641678 \\
\hline 0 & 2.789880 & 12.465979 & 18.689014 \\
\hline 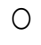 & 4.561925 & 11.7 & 16.841959 \\
\hline 0 & 2.1 & 10. & 16. \\
\hline 0 & 16.102312 & 22.071920 & 18.54530 \\
\hline 0 & 14.926208 & 24.514191 & 18.625414 \\
\hline 0 & 16.775204 & 23.938154 & 16.771078 \\
\hline 0 & 14.397265 & 22.757679 & 16.683203 \\
\hline 0 & 16. & 9.953556 & 6.3283 \\
\hline 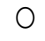 & 15.167254 & 12.482484 & 6.40825 \\
\hline 0 & 16.919395 & 11.802310 & 4.55852 \\
\hline & & 10.186 & \\
\hline
\end{tabular}




\begin{tabular}{|c|c|c|}
\hline 0.345185 & 2.867558 & 22.163439 \\
\hline 24.608374 & 3.965256 & 24.614685 \\
\hline 2.117716 & 2.121114 & 24.019472 \\
\hline 2.193938 & 4.550517 & 22.840212 \\
\hline 0.425048 & 15.158516 & 9.943847 \\
\hline 24.542347 & 16.107653 & 12.472775 \\
\hline 2.110676 & 14.328811 & 11.818089 \\
\hline 2.186656 & 16.833464 & 10.793943 \\
\hline 12.395582 & 2.939897 & 10.041188 \\
\hline 12.540015 & 3.872527 & 12.561133 \\
\hline 14.333423 & 2.109463 & 11.795028 \\
\hline 14.255501 & 4.560469 & 10.695874 \\
\hline 12.639055 & 15.080353 & 22.154940 \\
\hline 12.408447 & 16.098915 & 24.592840 \\
\hline 14.342647 & 14.330267 & 24.085014 \\
\hline 14.411829 & 16.783943 & 22.929300 \\
\hline 6.414805 & 2.871442 & 8.862656 \\
\hline 6.386405 & 3.954332 & 6.410193 \\
\hline 4.636691 & 2.033969 & 7.075317 \\
\hline 4.471867 & 4.478178 & 8.174714 \\
\hline 6.406795 & 15.100014 & 21.222795 \\
\hline 6.237116 & 16.176107 & 18.627113 \\
\hline 4.549060 & 14.330752 & 19.385937 \\
\hline 4.643974 & 16.857010 & 20.488731 \\
\hline 18.701880 & 2.884551 & 21.067682 \\
\hline 18.789511 & 3.978122 & 18.640707 \\
\hline 16.931776 & 2.183257 & 19.128141 \\
\hline 16.849241 & 4.554643 & 20.394062 \\
\hline 18.708920 & 15.173323 & 8.947374 \\
\hline 18.789753 & 16.020750 & 6.416262 \\
\hline 16.922308 & 14.327840 & 7.093038 \\
\hline 16.928619 & 16.855310 & 8.093394 \\
\hline 0.428932 & 3.884178 & 8.926984 \\
\hline 24.521957 & 2.693995 & 6.509962 \\
\hline 2.104122 & 4.550031 & 6.930883 \\
\hline 2.192724 & 2.192482 & 8.243653 \\
\hline 0.440341 & 16.099886 & 21.218912 \\
\hline 24.609346 & 15.085692 & 18.698238 \\
\hline 2.263849 & 16.772778 & 19.303162 \\
\hline 2.124027 & 14.331965 & 20.475624 \\
\hline 12.388057 & 3.799703 & 21.143175 \\
\hline 12.483698 & 2.872899 & 18.636 \\
\hline 14.239723 & 4.713399 & 19.386423 \\
\hline 14.328811 & 2.273073 & 20.479507 \\
\hline 12.569386 & 16.090902 & 8.924557 \\
\hline 12.482484 & 15.170653 & 6.418690 \\
\hline 14.413043 & 16.861135 & 7.083328 \\
\hline 14.418627 & 14.398236 & 8.095336 \\
\hline 6.411650 & 3.881266 & 22.155670 \\
\hline 6.411650 & 2.785753 & 24.609833 \\
\hline 4.632807 & 4.636448 & 24.019958 \\
\hline 4.536680 & 2.265791 & 22.770546 \\
\hline 6.320135 & 16.092360 & 9.783392 \\
\hline 6.496611 & 14.930820 & 12.234398 \\
\hline 4.710486 & 16.854340 & 11.791144 \\
\hline 4.478178 & 14.408673 & 10.631061 \\
\hline 18.544336 & 3.886606 & 9.949673 \\
\hline 18.878599 & 2.706618 & 12.3839 \\
\hline
\end{tabular}




\begin{tabular}{|c|c|c|c|}
\hline 0 & 16.857496 & 4.387148 & 12.056222 \\
\hline 0 & 16.855068 & 2.106792 & 10.708254 \\
\hline 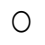 & 18.625900 & 16.100128 & 22.083572 \\
\hline 0 & 18.727854 & 15.084964 & 24.53263 \\
\hline 0 & 16.861380 & 16.864048 & 23.9444 \\
\hline 0 & 16.767679 & 14.404061 & 22.908667 \\
\hline 0 & 2.864645 & 24.680712 & 22.1571 \\
\hline 0 & 0.435486 & 0.421650 & 20 \\
\hline 0 & 0.939670 & 22.929787 & 22.90818 \\
\hline 0 & 2.200250 & 22.762291 & 20.56155 \\
\hline 0 & 2.869743 & 12.470833 & 10.0178 \\
\hline 0 & 0.427961 & 12.574486 & 8.939607 \\
\hline 0 & 1.012736 & 10.695631 & 10.702428 \\
\hline 0 & 2.177674 & 10.684950 & 8.24486 \\
\hline 0 & 15.093460 & 24.704988 & 9.86544 \\
\hline 0 & 12.548268 & 0.510738 & 8.93499 \\
\hline 0 & 13.312190 & 22.914494 & 10.62305 \\
\hline 0 & 14.325655 & 23.007708 & \\
\hline 0 & 15.077682 & 12.555551 & 22.158339 \\
\hline 0 & 12.566231 & 12.557250 & 21.22813 \\
\hline ? & 13.307578 & 10.699272 & 22.93294 \\
\hline O & 14.259627 & 10.641741 & 20.47441 \\
\hline 0 & 16.101099 & 18.703579 & 22.16076 \\
\hline 0 & 18.618618 & 18.624201 & 21 . \\
\hline 0 & 18.043552 & 20.387993 & 22.993872 \\
\hline 0 & 16.926191 & 20.568111 & 20.54747 \\
\hline 0 & 16.189459 & 6.408252 & 10.02419 \\
\hline O & 18.710133 & 6.405581 & 9.02165 \\
\hline 0 & 17.958107 & 850 & 10 . \\
\hline 0 & 16.857737 & 8.095094 & 8.174714 \\
\hline 0 & 3.803587 & 18.702122 & 10.030507 \\
\hline 0 & 6.305813 & 18.635366 & 8.943733 \\
\hline 0 & 5.653554 & 20.411539 & 10.792486 \\
\hline 0 & 4.474537 & 20.548933 & 8.356530 \\
\hline 0 & 3.878353 & 164 & 22 . \\
\hline 0 & 6.329602 & 6.319649 & 21.071808 \\
\hline 0 & 5.743127 & 8.265986 & 22.763504 \\
\hline 0 & 4.551245 & 8.186608 & 20.394304 \\
\hline 0 & 16.181204 & 24.858402 & 21.323050 \\
\hline 0 & 18.709404 & 6552 & 22 . \\
\hline 0 & 17.883341 & 23. & 20 . \\
\hline 0 & 16.941000 & 22.836329 & 22.766418 \\
\hline 0 & 16.102312 & 12.551667 & 8.943490 \\
\hline 0 & 18.533171 & 12.723532 & 10.037789 \\
\hline 0 & 18.029715 & 10.703156 & 8.419645 \\
\hline 0 & 16.857496 & 10.792001 & 10.8 \\
\hline 0 & 3.799460 & 24.686296 & 9.018742 \\
\hline 0 & 6.329844 & 0.431360 & 9.965937 \\
\hline 0 & 5.649427 & 22.922504 & 8.190249 \\
\hline 0 & 4.561197 & 22.911823 & 10.704856 \\
\hline 0 & 3.796304 & 12.474232 & 21.168421 \\
\hline 0 & 6.231047 & 12.635172 & 22.246214 \\
\hline 0 & 5.646514 & 10.694175 & 20.470526 \\
\hline 0 & 4.550759 & 10.707040 & 22.914736 \\
\hline 0 & 2.944266 & 18.796551 & 20.983448 \\
\hline 0 & 0.527002 & 18.541668 & 22.250584 \\
\hline 0 & 0.919279 & 20.388964 & 20.406927 \\
\hline 0 & 2.199036 & 20.553303 & 22.75670 \\
\hline
\end{tabular}




\begin{tabular}{|c|c|c|c|}
\hline 0 & 2.775800 & 6.337370 & 8.857801 \\
\hline 0 & 0.340087 & 6.404125 & 9.945788 \\
\hline 0 & 1.019048 & 8.262102 & 8.172286 \\
\hline 0 & 2.119901 & 8.244867 & 10.610912 \\
\hline O & 15.171138 & 18.624685 & 8.93742 \\
\hline 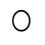 & 12.622549 & 18.619102 & 9.792616 \\
\hline 0 & 13.392054 & 20.479265 & 8.1004 \\
\hline 0 & 14.328568 & 20.412754 & 10.62960 \\
\hline 0 & 15.163371 & 6.335185 & 21.25289 \\
\hline 0 & 12.637114 & 6.241971 & 22.240873 \\
\hline 0 & 13.308550 & 8.106503 & 20.4552 \\
\hline 0 & 14.329538 & 8.167432 & 22.9157 \\
\hline 0 & 22.169506 & 2.778713 & 24. \\
\hline 0 & 21.072052 & 0.336689 & 0.34785 \\
\hline 0 & 22.922020 & 0.922678 & 22.84264 \\
\hline 0 & 20.556944 & 2.188598 & 22.740202 \\
\hline O & 22.079447 & 15.086178 & 12.560649 \\
\hline 0 & 21.226923 & 12.556765 & \\
\hline 0 & 22.933670 & 13.317287 & 10.705584 \\
\hline 0 & 20.464701 & 14.332452 & 10.691504 \\
\hline 0 & 9.960354 & 3.040151 & 12.399466 \\
\hline O & 8.936693 & 0.578949 & 12.66284 \\
\hline O & 10.624749 & 1.084832 & 10.695874 \\
\hline 0 & 8.167189 & 2.189811 & 10. \\
\hline 0 & 9.853788 & 15.092731 & 24.683868 \\
\hline 0 & 8.931110 & 12.552638 & 0.434515 \\
\hline 0 & 10.795885 & 13.304908 & 23.003096 \\
\hline 0 & 8.254820 & 14.248461 & 22.832687 \\
\hline 0 & 22.160280 & 16.094788 & 68 \\
\hline 0 & 21.163080 & 18.621531 & 18.617889 \\
\hline 0 & 23.010620 & 17.960775 & 20.391150 \\
\hline 0 & 20.556944 & 16.925707 & 20.559614 \\
\hline 0 & 22.081390 & 3.874712 & 6.327660 \\
\hline 0 & 21.134195 & 6.402668 & 6.327 \\
\hline 0 & 22.839485 & 5.652340 & 83 \\
\hline 0 & 20.398674 & 4.566781 & 8.187337 \\
\hline 0 & 9.937778 & 16.091873 & 6.335670 \\
\hline 0 & 9.028209 & 18.628084 & 6.251680 \\
\hline 0 & 10.693689 & 17.871445 & 8.174956 \\
\hline 0 & 8.176898 & 16.936630 & 38 \\
\hline 0 & 9.961810 & 3.7 & 18. \\
\hline 0 & 8.933295 & 6.246583 & 18.533171 \\
\hline 0 & 10.691990 & 5.738758 & 20.463243 \\
\hline 0 & 8.258946 & 4.564838 & 20.478294 \\
\hline 0 & 21.245615 & 16.174408 & 24.766159 \\
\hline 0 & 22.321707 & 18.630270 & 0.424320 \\
\hline 0 & 20.387993 & 17.953737 & 23.000910 \\
\hline 0 & 22.842398 & 16.851669 & 22.841913 \\
\hline 0 & 21.400244 & 3.714256 & 12.558464 \\
\hline 0 & 22.317581 & 6.247068 & 12.549968 \\
\hline 0 & 20.471741 & 5.488487 & 10.779378 \\
\hline 0 & 22.926146 & 4.478178 & 10.627419 \\
\hline 0 & 9.010245 & 15.849856 & 12.318388 \\
\hline 0 & 9.772953 & 18.377085 & 12.648279 \\
\hline 0 & 8.185394 & 17.786970 & 10.628148 \\
\hline 0 & 10.790302 & 16.938814 & 10.694175 \\
\hline 0 & 8.925528 & 3.802859 & 24.610802 \\
\hline 0 & 9.957926 & 6.322320 & 0.42383 \\
\hline
\end{tabular}




\begin{tabular}{|c|c|c|}
\hline 8.171558 & 5.731961 & 22.909153 \\
\hline 10.624992 & 4.560711 & 22.843855 \\
\hline 21.246101 & 2.862946 & 18.547493 \\
\hline 22.333603 & 0.427476 & 18.553318 \\
\hline 20.416153 & 1.009581 & 20.312014 \\
\hline 22.833174 & 2.193696 & 20.462030 \\
\hline 21.400002 & 15.257071 & 6.248767 \\
\hline 22.344040 & 12.647795 & 5.999467 \\
\hline 20.394304 & 13.305394 & 8.169859 \\
\hline 23.007950 & 14.417656 & 8.181268 \\
\hline 8.849305 & 2.957860 & 6.491999 \\
\hline 10.035119 & 0.588902 & 6.310182 \\
\hline 8.262589 & 1.017591 & 8.262831 \\
\hline 10.627663 & 2.268219 & 8.266472 \\
\hline 8.849791 & 15.253186 & 18.707220 \\
\hline 9.958169 & 12.569386 & 18.367619 \\
\hline 7.997995 & 13.218490 & 20.326336 \\
\hline 10.704127 & 14.337792 & 20.556944 \\
\hline 24.687996 & 22.160767 & 2.865131 \\
\hline 0.502484 & 21.063068 & 0.430874 \\
\hline 22.924688 & 22.850166 & 0.936514 \\
\hline 22.847010 & 20.482664 & 2.192482 \\
\hline 24.691635 & 9.886802 & 15.083993 \\
\hline 0.433302 & 8.862170 & 12.555551 \\
\hline 22.932941 & 10.690290 & 13.238396 \\
\hline 22.917164 & 8.173985 & 14.324441 \\
\hline 12.471317 & 22.162466 & 15.004616 \\
\hline 12.564046 & 21.224497 & 12.468648 \\
\hline 10.608485 & 22.851137 & 13.151492 \\
\hline 10.713594 & 20.401829 & 14.167142 \\
\hline 12.476173 & 9.949430 & 2.939654 \\
\hline 12.572785 & 8.935722 & 0.436215 \\
\hline 10.710196 & 10.705827 & 1.027301 \\
\hline 10.628391 & 8.263802 & 2.112133 \\
\hline 18.701393 & 22.169506 & 16.100128 \\
\hline 18.619831 & 21.156769 & 18.625414 \\
\hline 20.483877 & 22.907454 & 18.014423 \\
\hline 20.487034 & 20.486305 & 16.863077 \\
\hline 18.791695 & 10.033663 & 3.712315 \\
\hline 18.782955 & 9.002721 & 6.251194 \\
\hline 20.555973 & 10.795400 & 5.556941 \\
\hline 20.571266 & 8.338325 & 4.475265 \\
\hline 6.320863 & 22.162952 & 3.880052 \\
\hline 6.325475 & 20.985634 & 6.492484 \\
\hline 8.250450 & 22.994358 & 5.827846 \\
\hline 8.344637 & 20.491888 & 4.477450 \\
\hline 6.318921 & 9.872965 & 16.098185 \\
\hline 6.326931 & 8.940820 & 18.615463 \\
\hline 8.164034 & 10.694418 & 17.860037 \\
\hline 8.184181 & 8.177870 & 16.866234 \\
\hline 24.852093 & 21.229836 & 16.187031 \\
\hline 0.496416 & 22.257622 & 18.605995 \\
\hline 23.000669 & 20.397945 & 17.949610 \\
\hline 22.917891 & 22.838514 & 16.781515 \\
\hline 24.609346 & 8.845421 & 3.800917 \\
\hline 0.51680 & 10.032449 & 6.239543 \\
\hline 22.916193 & 8.268171 & 5.813281 \\
\hline 22.93609 & 10.617710 & 4.38690 \\
\hline
\end{tabular}




$\begin{array}{rrrr}\mathrm{O} & 12.545599 & 21.412624 & 3.719597 \\ \mathrm{O} & 12.642939 & 22.244028 & 6.248524 \\ \mathrm{O} & 10.870166 & 20.384838 & 5.479262 \\ \mathrm{O} & 10.709225 & 23.002367 & 4.631108 \\ \mathrm{O} & 12.474716 & 8.858287 & 16.092117 \\ \mathrm{O} & 12.635172 & 9.858400 & 18.548222 \\ \mathrm{O} & 10.700001 & 8.113056 & 17.959562 \\ \mathrm{O} & 10.635673 & 10.532263 & 16.842930 \\ \mathrm{O} & 18.714260 & 21.165995 & 2.951306 \\ \mathrm{O} & 18.604052 & 22.253252 & 0.494474 \\ \mathrm{O} & 20.405228 & 20.388721 & 1.009823 \\ \mathrm{O} & 20.564228 & 22.764719 & 2.194667 \\ \mathrm{O} & 18.795580 & 8.856831 & 15.177451 \\ \mathrm{O} & 18.538269 & 9.864470 & 12.706539 \\ \mathrm{O} & 20.478537 & 8.101647 & 13.233541 \\ \mathrm{O} & 20.488247 & 10.621837 & 14.315217 \\ \mathrm{O} & 6.501223 & 21.144390 & 15.162157 \\ \mathrm{O} & 6.241242 & 22.229950 & 12.651921 \\ \mathrm{O} & 8.170102 & 20.473440 & 13.225531 \\ \mathrm{O} & 8.186123 & 22.928087 & 14.326140 \\ \mathrm{O} & 6.408737 & 8.947374 & 2.862218 \\ \mathrm{O} & 6.329359 & 10.015699 & 0.422135 \\ \mathrm{O} & 8.175200 & 8.179083 & 1.010794 \\ \mathrm{O} & 8.263559 & 10.631304 & 2.116745 \\ \mathrm{O} & 13.145667 & 5.306185 & 8.506305 \\ \mathrm{O} & 12.465735 & 6.571620 & 6.060396 \\ \mathrm{O} & 11.718805 & 7.838026 & 8.429111 \\ \mathrm{O} & 14.419841 & 7.761803 & 7.674657 \\ \mathrm{O} & 8.937664 & 17.203409 & 20.154715 \\ \mathrm{O} & 7.076046 & 18.961132 & 19.050463 \\ \mathrm{O} & 8.514559 & 19.560957 & 21.585945 \\ \mathrm{O} & 9.107587 & 20.397703 & 18.871315 \\ \mathrm{H} & 12.797812 & 22.165136 & 3.149144 \\ \mathrm{H} & 15.854712 & 7.935609 & 7.954786 \\ \mathrm{H} & 25.065466 & 6.128366 & 1.721797 \\ \mathrm{H} & 21.923847 & 16.025604 & 6.548801 \\ \mathrm{H} & 13.600087 & 5.073148 & 9.394755 \\ \mathrm{H} & 9.040832 & 16.099400 & 19.350983 \\ \mathrm{H} & 9.291346 & 19.274517 & 15.943315 \\ \mathrm{H} & 11.331383 & 19.111391 & 20.663267 \\ \mathrm{H} & 11.131360 & 18.369802 & 19.041481 \\ \mathrm{H} & 11.048584 & 20.661324 & 18.088459 \\ \mathrm{H} & 10.765542 & 21.362862 & 19.712917 \\ \mathrm{Re} & 12.922583 & 6.896899 & 7.666161 \\ \mathrm{Re} & 8.661177 & 18.923748 & 20.034798 \\ \mathrm{C} & 10.777194 & 19.157270 & 19.717043 \\ \mathrm{C} & 10.556781 & 20.505482 & 19.058716\end{array}$

\begin{tabular}{lrrr}
601 & \multicolumn{3}{l}{} \\
USY_ReO3_C2H4_3 $\mathrm{E}=-7235.31730034186$ \\
$\mathrm{Si}$ & $23 . \overline{3} 7959 \overline{5}$ & 3.331446 & 1.238976 \\
$\mathrm{Si}$ & 23.419405 & 1.251841 & 3.366158 \\
$\mathrm{Si}$ & 23.352650 & 15.573369 & 13.438903 \\
$\mathrm{Si}$ & 23.398529 & 13.417057 & 15.570941 \\
$\mathrm{Si}$ & 11.220448 & 1.177318 & 15.634784 \\
$\mathrm{Si}$ & 11.168258 & 15.566087 & 1.225867 \\
$\mathrm{Si}$ & 11.228458 & 13.398123 & 3.367130 \\
$\mathrm{Si}$ & 7.641644 & 3.359362 & 1.238005
\end{tabular}




\begin{tabular}{|c|c|c|c|}
\hline Si & 7.676356 & 5.512762 & 6496 \\
\hline$i$ & 7.687523 & 15.441316 & 13.195673 \\
\hline $\mathrm{Si}$ & 20.025574 & 5.387505 & 15.490350 \\
\hline S & 19.908813 & 15.631385 & 1.225867 \\
\hline & 19.894976 & 17.796923 & 3.312026 \\
\hline$\perp$ & 7.616156 & 3.377325 & 5.509606 \\
\hline & 7.628778 & 15.462191 & 17.749830 \\
\hline & 7.679270 & 13.307093 & 15.486952 \\
\hline & 19.964401 & 3.387035 & 17.712204 \\
\hline & 19.955664 & 1.217128 & 15.593031 \\
\hline & 19.961245 & 15.596188 & 5.411051 \\
\hline & 20.018776 & 13.377246 & 3.264691 \\
\hline & 23.413578 & 3.358391 & \\
\hline i & 23.387848 & 17.750315 & 15.60079 \\
\hline & 11.223118 & 3.344311 & 17.78114 \\
\hline & 11.208553 & 5.555727 & 15.586720 \\
\hline L & 11.239625 & 15.625317 & 5.470281 \\
\hline$i$ & 11.177725 & 17.760754 & 3.271488 \\
\hline i & 1.296506 & 23.44 & 3.340185 \\
\hline $\mathrm{Si}$ & 3.410096 & 23.4 & 1.22 \\
\hline S & 1.225867 & 11.200300 & 15.58186 \\
\hline$S \perp$ & 3.350137 & 11.221662 & 13.37894 \\
\hline$i$ & 13.472646 & 23.360905 & 15.51583 \\
\hline i & 15.626531 & 23.3 & \\
\hline$i$ & 13.4287 & 11.2 & 974 \\
\hline $\mathrm{Si}$ & 15.626287 & 11.194231 & 1.203292 \\
\hline i & 1.335831 & 7.704030 & 3.32731 \\
\hline i & 3.406455 & 7.661064 & 5.51203 \\
\hline$i$ & 1.300148 & 19.907356 & 15.594245 \\
\hline$i$ & 3.401600 & 19.8 & 17.774834 \\
\hline i & 13.4775 & 7.6 & 15.64182 \\
\hline Si & 15.668768 & 7.662035 & 17.81003 \\
\hline $\mathrm{Si}$ & 15.598372 & 19.958090 & 5.504023 \\
\hline 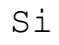 & 5.544561 & 7.668589 & 3.34868 \\
\hline$i$ & 3.413251 & 7 . & 35 \\
\hline$i$ & 5.4 & 20. & 15. \\
\hline $\mathrm{Si}$ & 3.383636 & 19.935757 & 13.424338 \\
\hline $\mathrm{Si}$ & 17.874844 & 7.597221 & 15.642551 \\
\hline $\mathrm{Si}$ & 15.685275 & 7.755006 & 13.53745 \\
\hline $\mathrm{Si}$ & 17.758812 & 19.965614 & 3.378782 \\
\hline $\mathrm{Si}$ & 15.598129 & 20.0 & 1.21130 \\
\hline $\mathrm{Si}$ & 5.504993 & 23.4 & 3.40 \\
\hline $\mathrm{Si}$ & 3.417864 & 23.412851 & 5.49382 \\
\hline $\mathrm{Si}$ & 5.455716 & 11.174812 & 15.60492 \\
\hline $\mathrm{Si}$ & 3.325135 & 11.236955 & 17.771435 \\
\hline $\mathrm{Si}$ & 17.749586 & 23.389790 & 15.59497 \\
\hline $\mathrm{Si}$ & 15.5 & 23.37 & 17.71851 \\
\hline Si & 17.8 & 11.2 & 3.30644 \\
\hline $\mathrm{Si}$ & 15.665612 & 11.232585 & 5.47222 \\
\hline Si & 3.390919 & 1.295778 & 23.40314 \\
\hline $\mathrm{Si}$ & 1.240918 & 3.362518 & 23.41552 \\
\hline $\mathrm{Si}$ & 3.338000 & 13.414386 & 11.24739 \\
\hline $\mathrm{Si}$ & 1.240675 & 15.603954 & 11.26705 \\
\hline Si & 15.647164 & 1.175861 & 11.19277 \\
\hline Si & 13.398850 & 3.234105 & 11.30443 \\
\hline $\mathrm{Si}$ & 15.571671 & 13.440360 & 23.444408 \\
\hline $\mathrm{Si}$ & 13.456139 & 15.571912 & 23.46334 \\
\hline $\mathrm{Si}$ & 3.345283 & 1.210332 & 7.67587 \\
\hline
\end{tabular}




\begin{tabular}{|c|c|c|c|}
\hline Si & 5.469067 & 3.333388 & 203 \\
\hline Si & 3.303045 & 13.366808 & 19.963430 \\
\hline $\mathrm{Si}$ & 17.789154 & 3.348196 & 19.788168 \\
\hline & 15.653476 & 13.425067 & 7.636061 \\
\hline & 17.820953 & 15.571184 & 7.655966 \\
\hline L & 3.298432 & 5.450133 & 7.5945 \\
\hline & 1.228537 & 3.334845 & 7.62271 \\
\hline & 3.394803 & 17.738420 & 19.88332 \\
\hline & 1.249656 & 15.582350 & 19.929 \\
\hline & 15.593760 & 5.455231 & 19.94473 \\
\hline & 13.399578 & 3.393831 & 19.92167 \\
\hline & 15.666341 & 17.769733 & 7.631691 \\
\hline & 13.469975 & 15.630900 & 7.6 \\
\hline 2 & 3.380723 & 5.488487 & 23.40338 \\
\hline & 5.507421 & 3.389705 & 2340775 \\
\hline & 5.502323 & 15.574583 & 11.09592 \\
\hline L & 17.930918 & 3.245028 & 11.22069 \\
\hline i & 15.608810 & 17.777018 & 23. \\
\hline i & 17.735508 & 15.618278 & 23.364302 \\
\hline $\mathrm{Si}$ & 1.639992 & 21.712416 & 23.7 \\
\hline S & 1.595084 & 23.786436 & 21.672361 \\
\hline S & 1.605036 & 9.442819 & 11.57752 \\
\hline L & 1.606007 & 11.603987 & 9.46855 \\
\hline i & 13.831667 & 21.674061 & \\
\hline$i$ & 13.8 & 23.8 & $9.3 .9-9$. \\
\hline 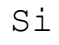 & 13.837492 & 9.427769 & 23.80828 \\
\hline 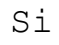 & 13.808848 & 11.632388 & 21.69396 \\
\hline 1 & 17.443241 & 21.698822 & 23.77065 \\
\hline$i$ & 17.418968 & 19.570667 & 21.72164 \\
\hline i & 17.449553 & 9.478745 & 11.5 \\
\hline i & 17.48 & 7.2 & 9.4 \\
\hline Si & 5.070721 & 21.689112 & 11.63044 \\
\hline$i$ & 5.078975 & 19.568968 & 9.48699 \\
\hline i & 5.158352 & 9.448159 & 23.72987 \\
\hline$i$ & $5.132 \varepsilon$ & 36 & 21. \\
\hline$i$ & $17.3^{\prime}$ & 21.7 & 19 . \\
\hline i & 17.398090 & 23.908539 & $21.64541 \varepsilon$ \\
\hline $\mathrm{Si}$ & 17.404888 & 11.695987 & 9.49598 \\
\hline $\mathrm{Si}$ & 5.128495 & 21.625755 & 7.29791 \\
\hline $\mathrm{Si}$ & 5.0833 & 23.787167 & 9.43262 \\
\hline$i$ & 5.088 & 9.464910 & 19.53813 \\
\hline$i$ & 5.090 & 11.6 & 21 . \\
\hline $\mathrm{Si}$ & 1.584888 & 21.639107 & 19.57600 \\
\hline $\mathrm{Si}$ & 1.605036 & 19.556345 & 21.63765 \\
\hline $\mathrm{Si}$ & 1.651158 & 9.483357 & 7.27097 \\
\hline $\mathrm{Si}$ & 1.571052 & 7.310538 & 9.38917 \\
\hline $\mathrm{Si}$ & 13.880 & 21.734991 & 7.17775 \\
\hline $\mathrm{Si}$ & 13.887 & 19.536440 & 9.34062 \\
\hline $\mathrm{Si}$ & 13.862009 & 7.189893 & 21.72018 \\
\hline Si & 23.770416 & 1.593627 & 21.62575 \\
\hline $\mathrm{Si}$ & 21.669691 & 1.540951 & 23.70584 \\
\hline $\mathrm{Si}$ & 23.824547 & 13.874147 & 9.43262 \\
\hline $\mathrm{Si}$ & 21.678431 & 13.810306 & 11.61976 \\
\hline Si & 11.563691 & 1.674462 & 9.44451 \\
\hline Si & 9.414660 & 1.694853 & 11.56563 \\
\hline $\mathrm{Si}$ & 11.666129 & 13.832881 & 21.71120 \\
\hline $\mathrm{Si}$ & 9.463210 & 13.790157 & 23.79129 \\
\hline $\mathrm{Si}$ & 23.817995 & 17.377214 & 21.68061 \\
\hline
\end{tabular}




\begin{tabular}{|c|c|c|c|}
\hline L & 21.714844 & 17.409500 & .550034 \\
\hline Si & 21.641048 & 5.147186 & 7.210770 \\
\hline $\mathrm{Si}$ & 11.671227 & 17.373089 & 9.376307 \\
\hline $\mathrm{Si}$ & 9.465152 & 17.393478 & 7.192564 \\
\hline & 11.614425 & 5.105433 & 21.679886 \\
\hline$i$ & 9.483600 & 5.102763 & 19.564842 \\
\hline Si & 19.530615 & 17.408043 & 21.713387 \\
\hline S & 21.689112 & 17.417997 & 23.828432 \\
\hline S & 19.611933 & 5.019259 & 9.46539 \\
\hline$\perp$ & 21.783783 & 4.997897 & 11.637971 \\
\hline$i$ & 7.243298 & 17.368475 & 9.337953 \\
\hline$i$ & 9.427526 & 17.217487 & 11.517326 \\
\hline i & 7.293546 & 5.143787 & 21.658283 \\
\hline 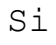 & 9.418787 & 5.106647 & 23.77478 \\
\hline i & 19.565084 & 1.622514 & 21.58424 \\
\hline i & 21.684986 & 1.609649 & 19.47818 \\
\hline $\mathrm{Si}$ & 19.555374 & 13.846475 & 9 . \\
\hline $\mathrm{Si}$ & 7.270728 & 1.615232 & 9.403008 \\
\hline i & 9.423885 & 1.686356 & 7.308353 \\
\hline Si & 7.285293 & 13.747677 & 21.613615 \\
\hline Si & 21.689354 & 23.754881 & 1.57542 \\
\hline$i$ & 23.813625 & 21.689596 & 1.59387 \\
\hline $\mathrm{Si}$ & 21.698580 & 11. & \\
\hline $\mathrm{Si}$ & 23.818966 & 9.3 & 13. \\
\hline Si & 9.430439 & 23.818966 & 13.80593 \\
\hline $\mathrm{Si}$ & 9.477531 & 11.607628 & 1.63368 \\
\hline $\mathrm{Si}$ & 11.611512 & 9.475104 & 1.63538 \\
\hline $\mathrm{Si}$ & 21.722853 & 23.768230 & 17. \\
\hline $\mathrm{Si}$ & 19.549791 & 21.696394 & 17.415569 \\
\hline $\mathrm{Si}$ & 21.785238 & 11.647681 & 4.901042 \\
\hline $\mathrm{Si}$ & 19.644705 & 9.541617 & 5.02120 \\
\hline Si & 9.407135 & 23.845181 & 5.17049 \\
\hline $\mathrm{Si}$ & 9.442819 & 11.591121 & 17.29710 \\
\hline $\mathrm{Si}$ & 7.254707 & 119 & 17.3713 \\
\hline $\mathrm{Si}$ & 21.695181 & 19.5 & 17.3 \\
\hline Si & 23.845909 & 21.671633 & 17.38255 \\
\hline $\mathrm{Si}$ & 21.700521 & 7.373652 & 5.12218 \\
\hline $\mathrm{Si}$ & 23.786436 & 9.444518 & 5.09621 \\
\hline $\mathrm{Si}$ & 9.492097 & 19.549063 & 4.977021 \\
\hline $\mathrm{Si}$ & 11.603987 & 21.7 & 5.10179 \\
\hline $\mathrm{Si}$ & 9.420730 & 9029 & 17.37381 \\
\hline $\mathrm{Si}$ & 11.592820 & 9.361985 & 17.39712 \\
\hline $\mathrm{Si}$ & 21.687654 & 19.558529 & 1.61013 \\
\hline $\mathrm{Si}$ & 19.555132 & 21.645903 & 1.64581 \\
\hline $\mathrm{Si}$ & 21.758537 & 7.186010 & 13.75495 \\
\hline $\mathrm{Si}$ & 19.613148 & 9.349848 & $13.83166^{\circ}$ \\
\hline $\mathrm{Si}$ & 9.436023 & 19.564114 & 13.660773 \\
\hline $\mathrm{Si}$ & 7.235530 & 21.708530 & 13.81273 \\
\hline $\mathrm{Si}$ & 9.430924 & 7.303499 & 1.60285 \\
\hline $\mathrm{Si}$ & 7.307868 & 9.441119 & $1.59532^{\circ}$ \\
\hline $\mathrm{Si}$ & 11.621222 & 21.711929 & 13.69548 \\
\hline $\mathrm{Si}$ & 11.088151 & 3.368829 & 13.46390 \\
\hline $\mathrm{Si}$ & 20.077763 & 3.219783 & 13.35855 \\
\hline $\mathrm{Si}$ & 7.640187 & 1.218099 & 3.37077 \\
\hline $\mathrm{Si}$ & 23.391733 & 15.602741 & 17.74910 \\
\hline $\mathrm{Si}$ & 5.459843 & 15.549095 & 19.961002 \\
\hline $\mathrm{Si}$ & 3.430001 & 17.747889 & 11.28429 \\
\hline $\mathrm{Si}$ & 13.841619 & 9.362713 & 19.57018 \\
\hline
\end{tabular}




\begin{tabular}{|c|c|c|c|}
\hline $\mathrm{Si}$ & 23.777212 & 5.118784 & 9.400338 \\
\hline $\mathrm{Al}$ & 15.605896 & 1.290438 & 19.853952 \\
\hline Al & 15.859566 & 5.467125 & 11.325071 \\
\hline 1 & 17.528446 & 9.588709 & 7.21344 \\
\hline 1 & 9.443304 & 13.781661 & 19.53304 \\
\hline 1 & 7.294032 & 21.646631 & 5.20447 \\
\hline Al & 7.495753 & 17.635983 & 15.4129 \\
\hline Al & 23.369400 & 5.404254 & 3.4052 \\
\hline 1 & 13.469247 & 19.824823 & 3.284 \\
\hline & 21.790579 & 13.678493 & 7.234802 \\
\hline & 13.865894 & 5.133593 & 13.30175 \\
\hline & 6.914619 & 18.143322 & 18.06564 \\
\hline 0 & 22.168535 & 0.343243 & 2.8605 \\
\hline 0 & 24.621725 & 24.605946 & 3.9604 \\
\hline 0 & 24.027727 & 2.103879 & 2.1099 \\
\hline 0 & 22.837299 & 2.184228 & 4.54760 \\
\hline 0 & 22.161011 & 12.474959 & 15.08545 \\
\hline 0 & 24.609346 & 12.465249 & 16.09745 \\
\hline 0 & 23.941551 & 14.329296 & 14.3285 \\
\hline 0 & 22.909153 & 14.394110 & 16.7800 \\
\hline 0 & 9.955013 & 0.258767 & 15.17235 \\
\hline 0 & 12.546570 & 24.613230 & 16.02196 \\
\hline 0 & 11.626319 & 2.118444 & 14.32832 \\
\hline 0 & 10.702671 & 2.104851 & 16. \\
\hline 0 & 9.945303 & 12.471804 & 2.94086 \\
\hline 0 & 12.483212 & 12.468891 & 3.80067 \\
\hline 0 & 11.724146 & 14.316672 & 2.11261 \\
\hline 0 & 10.778165 & 14.319344 & 4.62528 \\
\hline 0 & 8.943490 & 6.408494 & 2.87168 \\
\hline 0 & 6.490299 & 6.478405 & 3007 \\
\hline 0 & 7.079930 & 5720 & 2.10193 \\
\hline 0 & 8.181511 & 4.556585 & 4.55173 \\
\hline 0 & 8.929169 & 18.797035 & 15.16337 \\
\hline 0 & 6.245854 & 18.622744 & 16.27199 \\
\hline 0 & 7.177028 & 16.772778 & 14.0052 \\
\hline 0 & 7.916432 & 16.948040 & 16. \\
\hline 0 & 21.314554 & 6.246097 & 15.00194 \\
\hline 0 & 18.792181 & 6.323776 & 16.01468 \\
\hline 0 & 19.465315 & 4.466283 & 14.24579 \\
\hline 0 & 20.566170 & 4.554643 & 16.75918 \\
\hline 0 & 21.223282 & 18.624443 & 2.86440 \\
\hline 0 & 18.700909 & 18.723240 & 3.87592 \\
\hline 0 & 19.296366 & 16.860409 & 2.11480 \\
\hline 0 & 20.410812 & 16.861866 & 4.54080 \\
\hline 0 & 8.937907 & 0.348583 & 3.80844 \\
\hline 0 & 6.414563 & 24.625608 & 2.7823 \\
\hline 0 & 7.083086 & 2.108249 & 4.63984 \\
\hline 0 & 8.186366 & 2.191511 & 2.19418 \\
\hline 0 & 8.936451 & 12.388784 & 15.93069 \\
\hline 0 & 6.406795 & 12.392426 & 15.08545 \\
\hline 0 & 7.168046 & 14.333666 & 16.69582 \\
\hline 0 & 8.102860 & 14.252588 & 14.23244 \\
\hline 0 & 21.225468 & 0.340330 & 16.09551 \\
\hline 0 & 18.700909 & 24.602549 & 15.07574 \\
\hline 0 & 19.380840 & 2.184714 & 16.76913 \\
\hline 0 & 20.564713 & 2.026686 & 14.32735 \\
\hline 0 & 21.314312 & 12.456268 & 3.54870 \\
\hline 0 & 18.707220 & 12.552638 & 2.78405 \\
\hline
\end{tabular}




\begin{tabular}{|c|c|c|c|}
\hline O & 19.628925 & 14.232441 & 4.632079 \\
\hline $\mathrm{O}$ & 20.476595 & 14.412072 & 2.112618 \\
\hline 0 & 22.255438 & 6.574532 & 3.873983 \\
\hline 0 & 24.777569 & 6.400241 & 2.619957 \\
\hline $\mathrm{O}$ & 24.112444 & 4.558041 & 4.710001 \\
\hline 0 & 22.758408 & 4.487645 & 2.11504 \\
\hline 0 & 22.159554 & 18.692898 & 16.09017 \\
\hline O & 24.609589 & 18.709404 & 15.085 \\
\hline 0 & 23.944221 & 16.858952 & 16.8565 \\
\hline 0 & 22.848709 & 16.783215 & 14.41182 \\
\hline O & 9.961325 & 6.487872 & 16.0149 \\
\hline 0 & 12.566231 & 6.402668 & 15002431 \\
\hline 0 & 11.713951 & 4.565567 & 16.765982 \\
\hline 0 & 10.706798 & 4.718011 & 14.26351 \\
\hline 0 & 9.873936 & 18.610849 & 3.716684 \\
\hline O & 12.322758 & 18.709404 & 2.701520 \\
\hline 0 & 11.729486 & 16.861622 & 41 \\
\hline 0 & 10.613826 & 16.775932 & 2.114560 \\
\hline 0 & 2.951791 & 22.249369 & 0.253427 \\
\hline 0 & 4.037837 & 24.695036 & 24.603277 \\
\hline 0 & 2.103394 & 24.030153 & 2.035668 \\
\hline O & 4.543234 & 22.916920 & 2.192239 \\
\hline 0 & 2.874598 & 1857 & 12.4 \\
\hline 0 & 3.878595 & 12.476416 & 12.473989 \\
\hline 0 & 2.116260 & 11.736040 & 14.32225 \\
\hline 0 & 4.557556 & 10.698787 & 14.337306 \\
\hline 0 & 15.094916 & 22.086245 & 12.481271 \\
\hline 0 & 16.173679 & 24.517345 & 12.3 \\
\hline 0 & 14.344588 & 23.930872 & 14. \\
\hline 0 & 16.853127 & 22.841913 & 14.328568 \\
\hline 0 & 15.174780 & 9.877091 & 0.345185 \\
\hline 0 & 16.167368 & 12.462093 & 24.600849 \\
\hline 0 & 14.317886 & 11.716378 & 2.036639 \\
\hline 0 & 16.864292 & 10.706798 & 59 \\
\hline 0 & 2.940382 & 8.934266 & 6.411407 \\
\hline 0 & 3.887820 & 6.406795 & 6.415049 \\
\hline 0 & 2.191511 & 7.083086 & 4.553672 \\
\hline 0 & 4.617757 & 8.185637 & 4.568480 \\
\hline 0 & 2.861490 & 21.074236 & 18.717659 \\
\hline 0 & 4.0 & 18.626627 & 18. \\
\hline 0 & 2.277928 & 19.291025 & 16.772533 \\
\hline 0 & 4.637905 & 20.564713 & 16.936872 \\
\hline 0 & 15.172111 & 8.933295 & 18.703093 \\
\hline 0 & 16.104256 & 6.408494 & 18.713531 \\
\hline 0 & 14.408430 & 7.158336 & 16.848028 \\
\hline 0 & 16.932747 & 8.097278 & 16.867933 \\
\hline 0 & 15.178906 & 21.322565 & 6.32329 \\
\hline 0 & 16.182661 & 18.795094 & 6.492484 \\
\hline 0 & 14.326383 & 19.384966 & 4.716312 \\
\hline 0 & 16.858709 & 20.477324 & 4.618971 \\
\hline 0 & 3.878595 & 8.855617 & 0.253427 \\
\hline 0 & 2.778228 & 6.408737 & 24.618572 \\
\hline 0 & 4.637419 & 7.079201 & 2.11091 \\
\hline 0 & 2.212873 & 8.267443 & 2.103637 \\
\hline 0 & 3.801645 & 21.232506 & 12.550210 \\
\hline 0 & 2.951306 & 18.637552 & 12.565260 \\
\hline 0 & 4.631836 & 19.462402 & 14.406732 \\
\hline 0 & 2.172577 & 20.466642 & 14.34653 \\
\hline
\end{tabular}




$$
\begin{array}{rrr}
16.173922 & 8.850761 & 12.470105 \\
15.078168 & 6.405824 & 12.718919 \\
16.869633 & 7.101048 & 14.418627 \\
14.406488 & 8.258704 & 14.411586 \\
16.094545 & 21.238089 & 0.259981 \\
15.084236 & 18.722269 & 24.619541 \\
16.862837 & 19.464828 & 2.111162 \\
14.419354 & 20.627583 & 2.101209 \\
3.875440 & 22.152758 & 6.399998 \\
2.777499 & 24.536278 & 6.492727 \\
4.625768 & 24.101036 & 4.635235 \\
2.277686 & 22.849922 & 4.478421 \\
3.811840 & 9.951371 & 18.640951 \\
2.794249 & 12.468405 & 18.709404 \\
4.559741 & 11.792601 & 16.843901 \\
2.107763 & 10.703642 & 16.851669 \\
16.103041 & 22.071680 & 18.547251 \\
14.923538 & 24.512007 & 18.626143 \\
16.775204 & 23.939125 & 16.771078 \\
14.400178 & 22.759136 & 16.684418 \\
16.096001 & 9.953799 & 6.329359 \\
15.167983 & 12.481756 & 6.409465 \\
16.921581 & 11.800854 & 4.559255 \\
14.401634 & 10.786175 & 4.541049 \\
0.344457 & 2.867801 & 22.163679 \\
24.608860 & 3.965256 & 24.614445 \\
2.118444 & 2.121600 & 24.019716 \\
2.193938 & 4.550517 & 22.840212 \\
0.426262 & 15.160701 & 9.944818 \\
24.541620 & 16.107653 & 12.471804 \\
2.109948 & 14.329296 & 11.816632 \\
2.187870 & 16.841232 & 10.796128 \\
12.470590 & 2.947664 & 10.036090 \\
12.489523 & 3.868157 & 12.626432 \\
14.331723 & 2.026929 & 11.802795 \\
14.248704 & 4.640575 & 11.218991 \\
12.638571 & 15.075740 & 22.153484 \\
12.408689 & 16.099155 & 24.593082 \\
14.342403 & 14.330752 & 24.086714 \\
14.410858 & 16.783215 & 22.930029 \\
6.412135 & 2.873870 & 8.857073 \\
6.387861 & 3.955060 & 6.410436 \\
4.635963 & 2.032755 & 7.075075 \\
4.471138 & 4.480606 & 8.174471 \\
6.488843 & 15.167741 & 21.147303 \\
6.246339 & 16.105953 & 18.544336 \\
4.552458 & 14.319100 & 19.454878 \\
4.634264 & 16.853369 & 20.475138 \\
18.701637 & 2.882609 & 21.068653 \\
18.790724 & 3.977879 & 18.639494 \\
16.931047 & 2.184957 & 19.127657 \\
16.850698 & 4.553430 & 20.393091 \\
18.708920 & 15.174052 & 8.947618 \\
18.790724 & 16.020506 & 6.414563 \\
16.924007 & 14.327597 & 7.094008 \\
169393 & 16.855310 & 8.093637 \\
& 3.883450 & 8.926984 \\
2.6934 & 2.693510 & 6.508749
\end{array}
$$




\begin{tabular}{|c|c|c|c|}
\hline 0 & 2.104122 & 4.549545 & 6.930398 \\
\hline 0 & 2.193453 & 2.192724 & 8.243896 \\
\hline 0 & 0.429660 & 16.093088 & 21.228622 \\
\hline 0 & 24.604490 & 15.080110 & 18.70139 \\
\hline O & 2.205833 & 16.780544 & 19.30850 \\
\hline 0 & 2.111890 & 14.327840 & 20.481693 \\
\hline 0 & 12.387086 & 3.800189 & 21.1426 \\
\hline 0 & 12.488068 & 2.873142 & 18.63730 \\
\hline 0 & 14.241179 & 4.715098 & 19.38545 \\
\hline 0 & 14.329296 & 2.269432 & 20.47853 \\
\hline 0 & 12.569145 & 16.092844 & 8.9260 \\
\hline 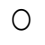 & 12.482484 & 15.170653 & 6. \\
\hline 0 & 14.413286 & 16.861622 & 7 . \\
\hline 0 & 14.417897 & 14.398964 & 8.09557 \\
\hline 0 & 6.411407 & 3.879567 & 22 \\
\hline 0 & 6.411407 & 2.785510 & 24.60934 \\
\hline 0 & 4.632807 & 4.636934 & 24.019958 \\
\hline 0 & 4.534981 & 2.264092 & 22.771030 \\
\hline 0 & 6.320620 & 16.0 & 80722 \\
\hline 0 & 6.493455 & 14.922811 & 12.22226 \\
\hline 0 & 4.715341 & 16.849241 & 11.78726 \\
\hline O & 4.475993 & 14.407702 & 10.62790 \\
\hline O & 18.705521 & 3.718140 & 9.864226 \\
\hline 0 & 18.963074 & 2.6 & 12. \\
\hline 0 & 17.106796 & 4.468711 & 11.893097 \\
\hline 0 & 16.857252 & 2.116502 & 10.70582 \\
\hline 0 & 18.626385 & 16.101099 & 22.083332 \\
\hline 0 & 18.727610 & 15.085692 & 24.53385 \\
\hline 0 & 16.861380 & 16.864048 & 23. \\
\hline 0 & 16.768894 & 14.4 & 22.90939 \\
\hline 0 & 2.862704 & 24.679014 & 22.15542 \\
\hline 0 & 0.435729 & 0.422378 & 20.991945 \\
\hline 0 & 0.938941 & 22.932941 & 22.91036 \\
\hline 0 & 2.195395 & 22.761806 & 20. \\
\hline 0 & 2.869986 & 12. & 10. \\
\hline 0 & 0.427718 & 12.574486 & 8.939607 \\
\hline 0 & 1.012251 & 10.694660 & 10.702186 \\
\hline 0 & 2.177917 & 10.686164 & 8.246081 \\
\hline 0 & 15.097101 & 24.704016 & 9.864955 \\
\hline 0 & 12.559919 & 1223 & 8. \\
\hline 0 & 13.309521 & 22.9 & 10.62572 \\
\hline 0 & 14.326383 & 23.005766 & 8.09533 \\
\hline 0 & 15.079382 & 12.554338 & 22.159069 \\
\hline 0 & 12.567202 & 12.556522 & 21.227894 \\
\hline 0 & 13.308550 & 10.698301 & 22.9319 \\
\hline 0 & 14.257929 & 10. & 20.475 \\
\hline 0 & 16.100613 & 18.7 & 22.16173 \\
\hline 0 & 18.619102 & 18.623230 & 21.15239 \\
\hline 0 & 18.043552 & 20.387506 & 22.99314 \\
\hline 0 & 16.926678 & 20.568596 & 20.549904 \\
\hline 0 & 16.174166 & 6.411893 & 9.950644 \\
\hline 0 & 18.710377 & 6.314309 & 9.01922 \\
\hline 0 & 18.021706 & 8.275211 & 10.62766 \\
\hline 0 & 16.924250 & 8.096550 & 8.16646 \\
\hline 0 & 3.805043 & 18.700180 & 10.02662 \\
\hline 0 & 6.307269 & 18.636095 & 8.943005 \\
\hline 0 & 5.655739 & 20.413725 & 10.790788 \\
\hline 0 & 4.475265 & 20.548206 & 8.35725 \\
\hline
\end{tabular}




$$
\begin{array}{rrr}
3.879081 & 6.410921 & 22.165380 \\
6.328874 & 6.320377 & 21.071808 \\
5.744098 & 8.264530 & 22.764719 \\
4.551973 & 8.187822 & 20.392605 \\
16.181204 & 24.857191 & 21.324020 \\
18.709162 & 0.417280 & 22.242086 \\
17.881399 & 23.076162 & 20.299391 \\
16.940029 & 22.836329 & 22.766903 \\
16.103041 & 12.549726 & 8.943975 \\
18.538511 & 12.728872 & 10.036333 \\
18.033844 & 10.705341 & 8.416732 \\
16.924250 & 10.780350 & 10.800497 \\
3.800189 & 24.686783 & 9.019227 \\
6.327417 & 0.428689 & 9.962052 \\
5.649670 & 22.922747 & 8.188551 \\
4.562411 & 22.908667 & 10.707283 \\
3.780283 & 12.477872 & 21.225468 \\
6.234446 & 12.653378 & 22.240631 \\
5.643844 & 10.698301 & 20.475138 \\
4.554158 & 10.707283 & 22.915707 \\
2.882123 & 18.707949 & 21.064039 \\
0.505640 & 18.548222 & 22.258350 \\
0.927775 & 20.391634 & 20.402557 \\
2.195637 & 20.553547 & 22.755737 \\
2.775558 & 6.337855 & 8.858287 \\
0.341786 & 6.404610 & 9.947488 \\
1.019048 & 8.262102 & 8.171558 \\
2.121357 & 8.244867 & 10.610185 \\
15.170653 & 18.627113 & 8.939607 \\
12.627403 & 18.622015 & 9.794315 \\
13.391568 & 20.479752 & 8.099706 \\
14.330509 & 20.458633 & 10.613826 \\
15.164585 & 6.333728 & 21.252653 \\
12.638328 & 6.240028 & 22.242815 \\
13.312919 & 8.099463 & 20.464701 \\
14.330752 & 8.167917 & 22.919592 \\
22.169264 & 2.779199 & 24.603277 \\
21.067196 & 0.341544 & 0.346399 \\
22.921776 & 0.922920 & 22.842398 \\
20.556459 & 2.189811 & 22.740202 \\
22.080660 & 15.086906 & 12.561863 \\
21.232021 & 12.552395 & 12.564775 \\
23.008921 & 17.960291 & 20.391634 \\
20.557186 & 16.924736 & 20.557673 \\
22.088186 & 3.873255 & 6.321349 \\
21.140747 & 6.406309 & 6.324261 \\
20.468342 & 13.317045 & 10.704856 \\
9.946274 & 3.031655 & 12.388057 \\
8.935966 & 0.584532 & 12.652892 \\
10.689077 & 1.100610 & 10.710924 \\
8.189279 & 2.187384 & 10.641013 \\
9.849904 & 15.090548 & 24.680471 \\
8.930625 & 12.555551 & 0.431602 \\
10.798313 & 13.304180 & 23.006495 \\
& &
\end{array}
$$




$\begin{array}{rrr}22.853079 & 5.660594 & 8.178355 \\ 20.470526 & 4.559255 & 8.181996 \\ 9.938021 & 16.092360 & 6.336398 \\ 9.028937 & 18.627842 & 6.252894 \\ 10.695631 & 17.871931 & 8.174956 \\ 8.176656 & 16.936872 & 8.092667 \\ 9.961082 & 3.798732 & 18.698967 \\ 8.933295 & 6.242456 & 18.531958 \\ 10.694418 & 5.737544 & 20.462273 \\ 8.257248 & 4.564110 & 20.480478 \\ 21.247313 & 16.177320 & 24.765915 \\ 22.325350 & 18.634880 & 0.423592 \\ 20.387266 & 17.954222 & 23.002367 \\ 22.840212 & 16.850456 & 22.841913 \\ 21.405342 & 3.713528 & 12.559919 \\ 22.321707 & 6.245368 & 12.548268 \\ 20.473682 & 5.485816 & 10.781321 \\ 22.926874 & 4.477935 & 10.625478 \\ 9.014615 & 15.847672 & 12.310619 \\ 9.772468 & 18.379755 & 12.651678 \\ 8.187094 & 17.787941 & 10.627419 \\ 10.789816 & 16.940758 & 10.695389 \\ 8.925528 & 3.802373 & 24.611044 \\ 9.958169 & 6.322320 & 0.424320 \\ 8.172286 & 5.731961 & 22.907696 \\ 10.623536 & 4.560226 & 22.844339 \\ 21.246342 & 2.862946 & 18.548706 \\ 22.333603 & 0.427476 & 18.552591 \\ 20.416393 & 1.009823 & 20.311043 \\ 22.832445 & 2.193938 & 20.463730 \\ 21.399757 & 15.257071 & 6.249010 \\ 22.344284 & 12.648038 & 6.000681 \\ 20.394547 & 13.305394 & 8.168888 \\ 23.008921 & 14.417897 & 8.182482 \\ 8.849791 & 2.961015 & 6.492484 \\ 10.037789 & 0.588659 & 6.309454 \\ 8.266230 & 1.014436 & 8.256519 \\ 10.631789 & 2.271860 & 8.270356 \\ 8.847606 & 15.249546 & 18.711588 \\ 9.962538 & 12.629103 & 18.366161 \\ 8.011346 & 13.148823 & 20.325850 \\ 10.702671 & 14.338763 & 20.558157 \\ 24.690424 & 22.164650 & 2.865131 \\ 0.500542 & 21.069139 & 0.430146 \\ 22.943623 & 22.911823 & 0.936514 \\ 22.835358 & 20.542137 & 2.186413 \\ 24.690666 & 9.874179 & 15.091033 \\ 0.437671 & 8.857558 & 12.554338 \\ 22.919348 & 10.641499 & 13.236939 \\ 22.917650 & 8.165005 & 14.328082 \\ 12.469862 & 22.163923 & 14.999760 \\ 12.558706 & 21.233234 & 12.471317 \\ 10.625721 & 22.905998 & 13.155619 \\ 10.708982 & 20.409111 & 14.161072 \\ 12.476416 & 9.948702 & 2.939897 \\ 12.573515 & 8.935479 & 0.437671 \\ 10.709225 & 10.706554 & 1.027301 \\ 10.629604 & 8.263316 & 2.112618\end{array}$




\begin{tabular}{|c|c|c|c|}
\hline & 18.702850 & 22.167807 & 16.102798 \\
\hline & 18.620073 & 21.156769 & 18.624929 \\
\hline & 20.482664 & 22.910124 & 18.016607 \\
\hline & 20.487276 & 20.484121 & 16.862593 \\
\hline & 18.791210 & 10.033905 & 3.711101 \\
\hline & 18.789511 & 9.004419 & 6.243912 \\
\hline & 20.557430 & 10.796856 & 5.556456 \\
\hline & 20.572481 & 8.337597 & 4.47283 \\
\hline & 6.321349 & 22.163923 & 3.87980 \\
\hline & 6.325475 & 20.985634 & 6.492727 \\
\hline & 8.250208 & 22.994358 & 5.828816 \\
\hline & 8.345850 & 20.492617 & 4.4776 \\
\hline & 6.318193 & 9.873450 & 16.09818 \\
\hline & 6.327417 & 8.941548 & 18.61691 \\
\hline & 8.172529 & 10.698545 & 17.863434 \\
\hline & 8.178841 & 8.182482 & 16.865992 \\
\hline & 24.852093 & 21.229836 & 16.181204 \\
\hline & 0.432331 & 22.259079 & 18.628084 \\
\hline & 22.994843 & 20.395519 & 17.946211 \\
\hline & 22.915222 & 22.837057 & 16.776419 \\
\hline & 24.609589 & 8.845179 & 3.800674 \\
\hline & 0.517534 & 10.032934 & 6.239786 \\
\hline & 22.917164 & 8.270113 & 5.812552 \\
\hline & 22.935856 & 10.617710 & 4.386906 \\
\hline & 12.547054 & 21.411654 & 3.718626 \\
\hline & 12.642697 & 22.244757 & 6.247797 \\
\hline & 10.869680 & 20.384838 & 5.479505 \\
\hline & 10.710681 & 23.002611 & 4.631351 \\
\hline & 12.460879 & 8.854403 & 16.088234 \\
\hline & 12.639298 & 9.791160 & 18.548464 \\
\hline & 10.701215 & 8.101404 & 17.955193 \\
\hline & 10.626934 & 10.539545 & 16.852882 \\
\hline & 18.703335 & 21.222069 & 2.956646 \\
\hline & 18.605995 & 22.254467 & 0.489861 \\
\hline & 20.402800 & 20.386295 & 1.016377 \\
\hline & 20.569082 & 22.769089 & 2.191996 \\
\hline & 18.787569 & 8.854403 & 15.159973 \\
\hline & 18.624201 & 9.862527 & 12.645367 \\
\hline & 20.485092 & 8.093880 & 13.234027 \\
\hline & 20.490675 & 10.625235 & 14.320801 \\
\hline & 6.500494 & 21.148760 & 15.171381 \\
\hline & 6.245612 & 22.232862 & 12.651194 \\
\hline & 8.173257 & 20.475866 & 13.225531 \\
\hline & 8.176898 & 22.922747 & 14.322256 \\
\hline & 6.409950 & 8.947374 & 2.862461 \\
\hline & 6.329602 & 10.014486 & 0.422378 \\
\hline & 8.174714 & 8.179326 & 1.011037 \\
\hline & 8.265501 & 10.631304 & 2.116988 \\
\hline & 13.225287 & 5.382164 & 8.750265 \\
\hline & 12.474959 & 6.333243 & 6.164777 \\
\hline & 11.719291 & 7.839724 & 8.419888 \\
\hline & 14.413286 & 7.760346 & 7.664219 \\
\hline & 7.332628 & 18.139679 & 19.882111 \\
\hline & 7.931726 & 19.626499 & 18.125599 \\
\hline & 8.767500 & 20.905527 & 20.901157 \\
\hline & 11.035475 & 21.656340 & 18.956762 \\
\hline & 12.806793 & 22.162952 & 3.15035 \\
\hline & & & \\
\hline
\end{tabular}




$\begin{array}{rrrr}\mathrm{H} & 25.067165 & 6.128366 & 1.721312 \\ \mathrm{H} & 21.935257 & 16.023663 & 6.533023 \\ \mathrm{H} & 13.545227 & 5.425616 & 9.678768 \\ \mathrm{H} & 7.394528 & 17.415812 & 20.537525 \\ \mathrm{H} & 9.145699 & 19.322094 & 15.970258 \\ \mathrm{H} & 11.064848 & 18.775673 & 20.831976 \\ \mathrm{H} & 10.555810 & 17.718029 & 19.410213 \\ \mathrm{H} & 12.289017 & 23.149958 & 19.531828 \\ \mathrm{H} & 11.006103 & 22.540178 & 20.789738 \\ \mathrm{Re} & 12.935692 & 6.873838 & 7.713982 \\ \mathrm{Re} & 8.778181 & 19.607079 & 19.827250 \\ \mathrm{C} & 10.317190 & 18.572496 & 20.057859 \\ \mathrm{C} & 11.450814 & 22.464199 & 19.776758\end{array}$

601

USY ReO3 $\mathrm{C} 2 \mathrm{H} 4 \quad 4 \mathrm{E}$

$\mathrm{Si}-23 . \overline{3} 7959 \overline{5}$

$E=-7235.31558141639$

Si 23.419405

$3.331203 \quad 1.238490$

$\begin{array}{ll}1.251356 & 3.365673\end{array}$

Si 23.352165

Si 23.398045

Si 11.221418

15.573126

13.418028

1.179260

13.439874

15.567785

15.571912

11.169957

11.228702

13.398364

3.358876

5.512033

7.676114

7.682668

15.460977

5.387505

20.025330

19.909784

15.632113

19.895218

17.797649

3.377082

7.615670

7.536535

15.460249

7.673201

13.298596

3.386792

19.963915

1.216886

19.955664

15.596673

13.377489

3.357905

20.019018

23.413822

23.384449

17.747644

3.338485

5.554028

11.221662

15.626044

15.635269

1.229508

3.368343

1.237276

3.346011

13.190089

15.491078

1.225624

3. 311541

5.508878

17.734295

15.512440

17.713417

15.594003

5.410565

3.264448

5.509606

15.602499

17.786484

15.603227

5.470524

3.272459

11.177725

17.762451

3.339457

1.296749

23.442223

23.479362

3.410338

11.200542

3.347953

11.221904

13.473860

23.363573

23.333231

15.627258

11.232828

13.428708

11.193746

7.703787

7.661064

1.220527

15.582350

13.377732

15.516323

13.394238

3.329989

1.204020

3.327077

5.511791

$19.908813 \quad 15.596673$

$19.898859 \quad 17.772890$

1.280971

3.384607

7.686794

7.661549

15.672652

19.958090

15.656388

17.812700

5.503537

7.668103

3.348681 


\begin{tabular}{|c|c|c|c|}
\hline $8 i$ & 3.413008 & 7.645285 & 1.201593 \\
\hline Si & 5.424159 & 20.030670 & 15.616577 \\
\hline $\mathrm{Si}$ & 3.315910 & 19.947651 & 13.403463 \\
\hline $\mathrm{Si}$ & 17.871931 & 7.594552 & 15.638425 \\
\hline & 15.686974 & 7.735343 & 13.520710 \\
\hline $\mathrm{Si}$ & 17.759296 & 19.965858 & 3.378296 \\
\hline i & 15.598615 & 20.022903 & 1.211303 \\
\hline i & 5.504751 & 23.416735 & 3.405 \\
\hline S & 3.417864 & 23.412607 & 5.493 \\
\hline$\perp$ & 5.450861 & 11.173598 & 15.6051 \\
\hline i & 3.322707 & 11.239140 & 17.7738 \\
\hline i & 17.750072 & 23.390032 & 15.596 \\
\hline i & 15.545210 & 23.373283 & 17.72142 \\
\hline 2 & 17.826294 & 11.269240 & 3.306443 \\
\hline i & 15.665612 & 11.233070 & 5.47198 \\
\hline i & 3.390919 & 1.294807 & 23.40289 \\
\hline $\mathrm{Si}$ & 1.240918 & 3.361789 & 23.415279 \\
\hline $\mathrm{Si}$ & 3.337029 & 13.4 & 11.247150 \\
\hline i & 1.238490 & 15.601527 & 11.26729 \\
\hline Si & 15.650561 & 1.171735 & 11.191561 \\
\hline Si & 13.402006 & 3.220026 & 11.28283 \\
\hline i & 15.573369 & 13.440603 & 23.44465 \\
\hline $\mathrm{Si}$ & 13.459294 & 15 & 23. \\
\hline $\mathrm{Si}$ & 3.3 & 1.2 & 7. \\
\hline Si & 5.469067 & 3.333388 & 7.60596 \\
\hline $\mathrm{Si}$ & 3.303773 & 13.371178 & 19.96513 \\
\hline $\mathrm{Si}$ & 17.788183 & 3.346982 & 19.78841 \\
\hline i & 15.6 & 13.2 & 7.6 \\
\hline $\mathrm{Si}$ & 1198 & 15.5 & 5723 \\
\hline $\mathrm{Si}$ & 3.298432 & 5.44 & 7.59430 \\
\hline $\mathrm{Si}$ & 1.228537 & 3.334602 & 7.622467 \\
\hline $\mathrm{Si}$ & 3.384122 & 17.736237 & 19.889151 \\
\hline i & 1.248443 & 15.580651 & 19.93138 \\
\hline $\mathrm{Si}$ & 15.593031 & 5. & 19 \\
\hline $\mathrm{Si}$ & 13.399 & 3.3 & 19.92167 \\
\hline $\mathrm{Si}$ & 15.666341 & 17.769733 & 7.631448 \\
\hline $\mathrm{Si}$ & 13.469975 & 15.631143 & 7.622952 \\
\hline $\mathrm{Si}$ & 3.380723 & 5.487516 & 23.40362 \\
\hline $\mathrm{Si}$ & 5.507421 & 8734 & 23.407511 \\
\hline $\mathrm{Si}$ & 5.49 & 15.5 & 11.09859 \\
\hline $\mathrm{Si}$ & 17.935045 & 3.24 & 11.22020 \\
\hline $\mathrm{Si}$ & 15.610752 & 17.779203 & 23.416491 \\
\hline $\mathrm{Si}$ & 17.737207 & 15.619248 & 23.364302 \\
\hline $\mathrm{Si}$ & 1.640477 & 21.711687 & 23.75172 \\
\hline $\mathrm{Si}$ & 1.595327 & 23.785709 & 21.672848 \\
\hline $\mathrm{Si}$ & 1.604308 & 9.442333 & 11.57704 \\
\hline $\mathrm{Si}$ & 1.605765 & 11.603745 & 9.468307 \\
\hline $\mathrm{Si}$ & 13.831908 & 21.673819 & 11.545241 \\
\hline $\mathrm{Si}$ & 13.813218 & 23.833044 & 9.37145 \\
\hline $\mathrm{Si}$ & 13.837735 & 9.426069 & 23.808769 \\
\hline $\mathrm{Si}$ & 13.811033 & 11.630203 & 21.69493 \\
\hline $\mathrm{Si}$ & 17.443241 & 21.699551 & 23.77041 \\
\hline $\mathrm{Si}$ & 17.419939 & 19.571880 & 21.72139 \\
\hline $\mathrm{Si}$ & 17.448339 & 9.478988 & 11.59913 \\
\hline $\mathrm{Si}$ & 17.484995 & 7.242812 & 9.48869 \\
\hline $\mathrm{Si}$ & 5.067080 & 21.688383 & 11.629718 \\
\hline $\mathrm{Si}$ & 5.077761 & 19.568239 & 9.48796 \\
\hline $\mathrm{Si}$ & 5.158110 & 9.447674 & 23.73012 \\
\hline
\end{tabular}




$\begin{array}{lrrr} & & & \\ \mathrm{Si} & 5.133107 & 7.282622 & 21.609005 \\ \mathrm{Si} & 17.376244 & 21.744457 & 19.519205 \\ \mathrm{Si} & 17.397362 & 23.909510 & 21.645418 \\ \mathrm{Si} & 17.404888 & 11.696230 & 9.496223 \\ \mathrm{Si} & 5.128009 & 21.625511 & 7.297915 \\ \mathrm{Si} & 5.083101 & 23.787167 & 9.432138 \\ \mathrm{Si} & 5.085286 & 9.464910 & 19.540081 \\ \mathrm{Si} & 5.086499 & 11.634088 & 21.704163 \\ \mathrm{Si} & 1.584646 & 21.639591 & 19.577465 \\ \mathrm{Si} & 1.606007 & 19.555861 & 21.638136 \\ \mathrm{Si} & 1.651158 & 9.483114 & 7.270728 \\ \mathrm{Si} & 1.571295 & 7.310296 & 9.388929 \\ \mathrm{Si} & 13.879972 & 21.734749 & 7.177028 \\ \mathrm{Si} & 13.887984 & 19.536440 & 9.340380 \\ \mathrm{Si} & 13.862252 & 7.187466 & 21.720184 \\ \mathrm{Si} & 23.770901 & 1.593142 & 21.625511 \\ \mathrm{Si} & 21.669691 & 1.540709 & 23.705359 \\ \mathrm{Si} & 23.823820 & 13.873418 & 9.432382 \\ \mathrm{Si} & 21.678431 & 13.810306 & 11.620008 \\ \mathrm{Si} & 11.559807 & 1.670092 & 9.439906 \\ \mathrm{Si} & 9.414175 & 1.696309 & 11.566604 \\ \mathrm{Si} & 11.674383 & 13.834579 & 21.722126 \\ \mathrm{Si} & 9.464910 & 13.790400 & 23.801245 \\ \mathrm{Si} & 23.819937 & 17.377699 & 21.681101 \\ \mathrm{Si} & 21.716055 & 17.409500 & 19.550276 \\ \mathrm{Si} & 21.641775 & 5.147186 & 7.210041 \\ \mathrm{Si} & 11.670984 & 17.373329 & 9.376549 \\ \mathrm{Si} & 9.464666 & 17.393721 & 7.192564 \\ \mathrm{Si} & 11.614667 & 5.103977 & 21.679401 \\ \mathrm{Si} & 9.484086 & 5.100336 & 19.565813 \\ \mathrm{Si} & 19.532070 & 17.408527 & 21.713142 \\ \mathrm{Si} & 21.690327 & 17.418482 & 23.827703 \\ \mathrm{Si} & 19.613634 & 5.019502 & 9.464910 \\ \mathrm{Si} & 21.784510 & 4.997897 & 11.638456 \\ \mathrm{Si} & 7.243541 & 17.368961 & 9.339167 \\ \mathrm{Si} & 9.428497 & 17.219673 & 11.519997 \\ \mathrm{Si} & 7.293788 & 5.142817 & 21.658768 \\ \mathrm{Si} & 9.418787 & 5.105919 & 23.774300 \\ \mathrm{Si} & 19.564598 & 1.622514 & 21.584488 \\ \mathrm{Si} & 21.684986 & 1.609891 & 19.478909 \\ \mathrm{Si} & 19.555616 & 13.846718 & 9.424613 \\ \mathrm{Si} & 7.270242 & 1.615232 & 9.403008 \\ \mathrm{Si} & 9.421943 & 1.685628 & 7.306654 \\ \mathrm{Si} & 7.260533 & 13.768796 & 21.648087 \\ \mathrm{Si} & 21.689596 & 23.754881 & 1.574936 \\ \mathrm{Si} & 23.814596 & 21.689596 & 1.593142 \\ \mathrm{Si} & 21.698580 & 11.591121 & 13.787244 \\ \mathrm{Si} & 23.818480 & 9.399853 & 13.797924 \\ \mathrm{Si} & 9.431410 & 23.820663 & 13.806420 \\ \mathrm{Si} & 9.477775 & 11.605443 & 1.636836 \\ \mathrm{Si} & 11.611269 & 9.474133 & 1.635865 \\ \mathrm{Si} & 21.722126 & 23.768959 & 17.380127 \\ \mathrm{Si} & 19.550034 & 21.696636 & 17.416054 \\ \mathrm{Si} & 21.785480 & 11.647681 & 4.900799 \\ \mathrm{Si} & 19.645433 & 9.541617 & 5.020958 \\ \mathrm{Si} & 9.406650 & 23.844696 & 5.169762 \\ \mathrm{Si} & 9.436023 & 11.558836 & 17.275747 \\ \mathrm{Si} & 7.247424 & 9.430924 & 17.369690\end{array}$




$\begin{array}{rrrr}\text { Si } & 21.694452 & 19.550276 & 17.393721 \\ \mathrm{Si} & 23.842026 & 21.673574 & 17.388138 \\ \mathrm{Si} & 21.701006 & 7.373410 & 5.122183 \\ \mathrm{Si} & 23.786436 & 9.444275 & 5.095967 \\ \mathrm{Si} & 9.491611 & 19.549549 & 4.977021 \\ \mathrm{Si} & 11.603745 & 21.762905 & 5.101550 \\ \mathrm{Si} & 9.420972 & 7.263203 & 17.372358 \\ \mathrm{Si} & 11.599617 & 9.353731 & 17.395178 \\ \mathrm{Si} & 21.688383 & 19.559015 & 1.609891 \\ \mathrm{Si} & 19.555616 & 21.646145 & 1.645575 \\ \mathrm{Si} & 21.758295 & 7.186010 & 13.755444 \\ \mathrm{Si} & 19.612663 & 9.349848 & 13.831667 \\ \mathrm{Si} & 9.436265 & 19.564598 & 13.671454 \\ \mathrm{Si} & 7.237229 & 21.712900 & 13.806420 \\ \mathrm{Si} & 9.430682 & 7.302285 & 1.602609 \\ \mathrm{Si} & 7.307140 & 9.440392 & 1.595327 \\ \mathrm{Si} & 11.620978 & 21.713387 & 13.694757 \\ \mathrm{Si} & 11.102716 & 3.366401 & 13.452497 \\ \mathrm{Si} & 20.078733 & 3.219783 & 13.360010 \\ \mathrm{Si} & 7.639944 & 1.217614 & 3.370043 \\ \mathrm{Si} & 23.392218 & 15.602256 & 17.750801 \\ \mathrm{Si} & 5.452075 & 15.539385 & 20.038681 \\ \mathrm{Si} & 3.417135 & 17.751286 & 11.289388 \\ \mathrm{Si} & 13.843319 & 9.360529 & 19.570423 \\ \mathrm{Si} & 23.777456 & 5.118542 & 9.400096 \\ \mathrm{Al} & 15.602741 & 1.288981 & 19.854923 \\ \mathrm{Al} & 15.874617 & 5.456930 & 11.300069 \\ \mathrm{Al} & 17.529417 & 9.590652 & 7.211984 \\ \mathrm{Al} & 9.481416 & 13.682863 & 19.568726 \\ \mathrm{Al} & 7.293303 & 21.646631 & 5.203989 \\ \mathrm{Al} & 7.495511 & 17.648361 & 15.327952 \\ \mathrm{Al} & 23.369400 & 5.403769 & 3.404755 \\ \mathrm{Al} & 13.469247 & 19.825550 & 3.284596 \\ \mathrm{Al} & 21.790337 & 13.678979 & 7.234559 \\ \mathrm{Al} & 13.845988 & 5.154469 & 13.195914 \\ \mathrm{Al} & 6.728676 & 18.315428 & 17.897419 \\ \mathrm{O} & 22.168535 & 0.343243 & 2.860519 \\ \mathrm{O} & 24.621969 & 24.605946 & 3.960401 \\ \mathrm{O} & 24.027483 & 2.104122 & 2.109948 \\ \mathrm{O} & 22.837299 & 2.184228 & 4.547604 \\ \mathrm{O} & 22.161011 & 12.474959 & 15.085450 \\ \mathrm{O} & 24.609346 & 12.465249 & 16.097458 \\ \mathrm{O} & 23.941793 & 14.329053 & 14.328568 \\ \mathrm{O} 0 & 22.908182 & 14.394110 & 16.780302 \\ \mathrm{O} & 9.955984 & 0.259495 & 15.171867 \\ \mathrm{O} & 12.547054 & 24.613716 & 16.020992 \\ \mathrm{O} & 11.629232 & 2.117716 & 14.325169 \\ \mathrm{O} & 10.701700 & 2.104365 & 16.851427 \\ \mathrm{O} & 9.945546 & 12.472047 & 2.941839 \\ \mathrm{O} & 12.483212 & 12.468648 & 3.800674 \\ \mathrm{O} & 11.724874 & 14.316188 & 2.113104 \\ \mathrm{O} & 10.778407 & 14.319587 & 4.625525 \\ \mathrm{O} 0 & 8.943490 & 6.408737 & 2.871928 \\ \mathrm{O} & 6.490542 & 6.478405 & 3.887334 \\ \mathrm{O} & 7.079930 & 4.635720 & 2.101938 \\ \mathrm{O} & 8.181511 & 4.556828 & 4.551973 \\ \mathrm{O} 0 & 8.933052 & 18.794851 & 15.165071 \\ \mathrm{O} & 6.239300 & 18.649931 & 16.105467\end{array}$




\begin{tabular}{|c|c|c|c|}
\hline 0 & 7.171445 & 16.839046 & 04248 \\
\hline 0 & 7.755006 & 16.853369 & 16.876431 \\
\hline . & 21.314554 & 6.245854 & 15.002188 \\
\hline 0 & 18.791939 & 6.323776 & 16.01419 \\
\hline 0 & 19.465559 & 4.466526 & 14.24554 \\
\hline 0 & 20.566170 & 4.554400 & 16.759426 \\
\hline 0 & 21.223526 & 18.624685 & 2.864160 \\
\hline 0 & 18.700909 & 18.723240 & 3.87592 \\
\hline 0 & 19.296122 & 16.860409 & 2.11480 \\
\hline 0 & 20.410812 & 16.861866 & 4.54080 \\
\hline 0 & 8.937907 & 0.348583 & 3.80844 \\
\hline 0 & 6.414320 & 24.625608 & 2 \\
\hline 0 & 7.083086 & 2.108249 & \\
\hline 0 & 8.186366 & 2.191511 & 2.194424 \\
\hline 0 & 8.943975 & 12.384901 & 15.92607 \\
\hline 0 & 6.407765 & 12.393154 & 15.08253 \\
\hline O & 7.179941 & 14.171268 & 16.85579 \\
\hline 0 & 8.088297 & 14.336578 & 2499 \\
\hline 0 & 21.225224 & 0.340330 & 95516 \\
\hline 0 & 18.700909 & 24.602549 & 15.075497 \\
\hline 0 & 19.380840 & 2.184957 & 16.769136 \\
\hline O & 20.564713 & 2.026443 & 14.327597 \\
\hline O & 21.314070 & 12.456268 & 3.548704 \\
\hline 0 & 18.707220 & 12.552638 & \\
\hline 0 & 19.628925 & 14.232441 & 4.632079 \\
\hline 0 & 20.476595 & 14.412072 & 2.112376 \\
\hline 0 & 22.255680 & 6.574290 & 3.873741 \\
\hline O & 24.777327 & 6.400241 & 2.619957 \\
\hline 0 & 24.112444 & 4.558041 & 01 \\
\hline 0 & 22.758408 & 4.487160 & 2.115046 \\
\hline 0 & 22.157368 & 18.691685 & 16.09090 \\
\hline 0 & 24.604248 & 18.705036 & 15.085692 \\
\hline 0 & 23.943737 & 16.857981 & 16.85701 \\
\hline 0 & 22.847254 & 16.782001 & 14 \\
\hline 0 & 9.957684 & 33746 & \\
\hline 0 & 12.563318 & 6.403154 & 15.073312 \\
\hline 0 & 11.712252 & 4.558284 & 16.774477 \\
\hline 0 & 10.775009 & 4.721409 & 14.257686 \\
\hline 0 & 9.873693 & 18.611336 & 3.717169 \\
\hline 0 & 12.323485 & 18.709404 & 20 \\
\hline 0 & 11.729486 & 16.861866 & 020 \\
\hline 0 & 10.614068 & 16.776661 & 2.115774 \\
\hline 0 & 2.951549 & 22.249613 & 0.253427 \\
\hline 0 & 4.038080 & 24.695278 & 24.603764 \\
\hline 0 & 2.103637 & 24.030396 & 2.035911 \\
\hline 0 & 4.542991 & 22.916920 & 82 \\
\hline 0 & 2.874355 & 9.951614 & 12.473261 \\
\hline 0 & 3.878838 & 12.476416 & 12.473989 \\
\hline 0 & 2.116260 & 11.736283 & 14.322499 \\
\hline 0 & 4.557313 & 10.699272 & 14.336092 \\
\hline 0 & 15.095160 & 22.086245 & 12.481271 \\
\hline 0 & 16.174408 & 24.516132 & 12.39849 \\
\hline 0 & 14.345075 & 23.931356 & 14.242393 \\
\hline 0 & 16.853611 & 22.841427 & 14.329296 \\
\hline 0 & 15.174537 & 9.877091 & 0.34518 \\
\hline 0 & 16.167126 & 12.461609 & 24.600607 \\
\hline 0 & 14.317886 & 11.716378 & 2.036639 \\
\hline ? & 16.864292 & 10.706798 & 2.11795 \\
\hline
\end{tabular}




\begin{tabular}{|c|c|c|c|}
\hline 0 & 2.940382 & 8.934023 & 6.411407 \\
\hline 0 & 3.887820 & 6.406795 & 6.415049 \\
\hline 0 & 2.191511 & 7.083086 & 4.553672 \\
\hline 0 & 4.617757 & 8.185637 & 4.568722 \\
\hline O & 2.859548 & 21.082975 & 18.714987 \\
\hline O & 3.965498 & 18.626627 & 18.627356 \\
\hline 0 & 2.271617 & 19.296366 & 16.760641 \\
\hline 0 & 4.640332 & 20.555489 & 16.932018 \\
\hline 0 & 15.173323 & 8.934508 & 18.704063 \\
\hline 0 & 16.105711 & 6.408494 & 18.715231 \\
\hline 0 & 14.413286 & 7.159793 & 16.857252 \\
\hline 0 & 16.933718 & 8.097035 & 16.866476 \\
\hline 0 & 15.179150 & 21.322565 & 6.323047 \\
\hline 0 & 16.182661 & 18.795338 & 6.49248 \\
\hline 0 & 14.326383 & 19.384966 & 4.71631 \\
\hline 0 & 16.858709 & 20.477324 & 4.618728 \\
\hline 0 & 3.878353 & 8.855374 & 0.253670 \\
\hline 0 & 2.778228 & 6.408494 & 24.618328 \\
\hline 0 & 4.637177 & 7.079201 & 2.110919 \\
\hline 0 & 2.212873 & 8.267443 & 2.103637 \\
\hline 0 & 3.794605 & 21.234205 & 12.547054 \\
\hline 0 & 2.880667 & 18.639009 & 12.556279 \\
\hline O & 4.547360 & 19.469198 & 14.396051 \\
\hline 0 & 2.107521 & 20.479265 & 26140 \\
\hline 0 & 16.173922 & 8.851490 & 12.471804 \\
\hline 0 & 15.083750 & 6.414320 & 12.651194 \\
\hline 0 & 16.867691 & 7.091824 & 14.414257 \\
\hline 0 & 14.402120 & 8.190735 & 14.404790 \\
\hline 0 & 16.094545 & 21.238089 & \\
\hline 0 & 15.084236 & 18.722269 & 24.619783 \\
\hline 0 & 16.862837 & 19.464588 & 2.111162 \\
\hline 0 & 14.419354 & 20.627583 & 2.101209 \\
\hline 0 & 3.875197 & 22.152758 & 6.400241 \\
\hline 0 & 2.777499 & 24.536278 & 6.492727 \\
\hline 0 & 4.626010 & 24. & 35 \\
\hline 0 & 2.277443 & 22.849922 & 4.478421 \\
\hline 0 & 3.811598 & 9.951371 & 18.639980 \\
\hline 0 & 2.794492 & 12.468891 & 18.710377 \\
\hline 0 & 4.559741 & 11.792843 & 16.843658 \\
\hline 0 & 2.108249 & 10. & 16. \\
\hline 0 & 16.1 & 22.0 & 18. \\
\hline 0 & 14.925480 & 24.514673 & 18.624685 \\
\hline 0 & 16.774961 & 23.938639 & 16.770836 \\
\hline 0 & 14.400420 & 22.757923 & 16.683933 \\
\hline 0 & 16.095758 & 9.954041 & 6.329359 \\
\hline 0 & 15.167983 & 12.481756 & 708 \\
\hline 0 & 16.921581 & 11.800854 & 4.559012 \\
\hline 0 & 14.401634 & 10.786175 & 4.541049 \\
\hline 0 & 0.344214 & 2.867801 & 22.163679 \\
\hline 0 & 24.609102 & 3.965498 & 24.614445 \\
\hline 0 & 2.118687 & 2.121600 & 24.019958 \\
\hline 0 & 2.193696 & 4.550517 & 22.840212 \\
\hline 0 & 0.425776 & 15.159245 & 9.944575 \\
\hline 0 & 24.540892 & 16.107168 & 12.472047 \\
\hline 0 & 2.109705 & 14.328325 & 11.817846 \\
\hline 0 & 2.187627 & 16.833950 & 10.793215 \\
\hline 0 & 12.469376 & 2.944266 & 10.023952 \\
\hline ? & 12.477387 & 3.865001 & 12.56720 \\
\hline
\end{tabular}




\begin{tabular}{|c|c|c|c|}
\hline & 14.334150 & 2.024987 & 11.801339 \\
\hline & 14.250889 & 4.636934 & 11.215107 \\
\hline & 12.639542 & 15.078896 & 22.154455 \\
\hline & 12.409175 & 16.099155 & 24.593809 \\
\hline & 14.342162 & 14.330509 & 24.085499 \\
\hline & 14.411829 & 16.783943 & 22.929787 \\
\hline & 6.412135 & 2.873870 & 8.857073 \\
\hline & 6.387618 & 3.955060 & 6.41043 \\
\hline & 4.635963 & 2.032755 & $7.0750^{-}$ \\
\hline & 4.471138 & 4.480848 & 8.17447 \\
\hline & 6.409950 & 15.140553 & 21.25872 \\
\hline & 6.336156 & 16.008612 & 18.70090 \\
\hline & 4.549545 & 14.322499 & 19.46458 \\
\hline & 4.626981 & 16.860165 & 20.47732 \\
\hline & 18.701637 & 2.882609 & 21.068653 \\
\hline & 18.790239 & 3.977879 & 18.63973 \\
\hline & 16.930319 & 2.184228 & 19.128141 \\
\hline & 16.851185 & 4.553187 & 20.39357 \\
\hline & 18.708920 & 15.174052 & 8.947618 \\
\hline & 18.790968 & 16.020506 & 6.41456 \\
\hline & 16.924007 & 14.327597 & 7.0940 \\
\hline & 16.927893 & 16.855310 & 637 \\
\hline & 0.429903 & 3.883450 & 8.926984 \\
\hline & 24.524143 & 2.693510 & 6.50874 \\
\hline & 2.104122 & 4.549545 & 6.930398 \\
\hline & 2.193453 & 2.192724 & 8.24389 \\
\hline & 0.427961 & 16.092117 & 21.22 \\
\hline & 24.602791 & 15.077925 & 18.701880 \\
\hline & 2.198793 & 16.775204 & 19.310202 \\
\hline & 2.109220 & 14.325897 & 20.483391 \\
\hline & 12.387086 & 3.800674 & 21.14220 \\
\hline & 12.489038 & 2.872413 & 18.638279 \\
\hline & 14.240694 & 4.715098 & 19.385452 \\
\hline & 14.330752 & 2.270889 & 20.479994 \\
\hline & 12.568902 & 16.092844 & 8.926256 \\
\hline & 12.482484 & 15.170653 & 6.418690 \\
\hline & 14.413529 & 16.861622 & 7.08405 \\
\hline & 14.417897 & 14.398964 & 8.095579 \\
\hline & 6.410921 & 3.879809 & 22.157854 \\
\hline & 6.411164 & 2.785510 & 24.609346 \\
\hline & 4.633050 & 4.636934 & 24.019958 \\
\hline & 4.534738 & 2.264092 & 22.771030 \\
\hline & 6.320135 & 16.092117 & 9.782907 \\
\hline & 6.494184 & 14.918441 & 12.223475 \\
\hline & 4.710001 & 16.853853 & 11.789930 \\
\hline & 4.476236 & 14.408430 & 10.629119 \\
\hline & 18.705763 & 3.718140 & 9.863498 \\
\hline & 18.964287 & 2.622385 & 12.314746 \\
\hline & 17.109465 & 4.465555 & 11.892612 \\
\hline & 16.857496 & 2.116260 & 10.70534 \\
\hline & 18.626385 & 16.101099 & 22.08308 \\
\hline & 18.727610 & 15.085692 & 24.534094 \\
\hline & 16.861135 & 16.863808 & 23.944950 \\
\hline & 16.769136 & 14.404790 & 22.909395 \\
\hline & 2.862461 & 24.679499 & 22.155426 \\
\hline & 0.435972 & 0.422378 & 20.991945 \\
\hline & 0.938456 & 22.933428 & 22.91133 \\
\hline & 2 . & 22.76 & \\
\hline
\end{tabular}




\begin{tabular}{|c|c|c|c|}
\hline 0 & 2.870229 & 12.470833 & 10.019098 \\
\hline 0 & 0.427476 & 12.573999 & 8.939363 \\
\hline 0 & 1.012493 & 10.694660 & 10.702186 \\
\hline 0 & 2.178160 & 10.685922 & 8.24608 \\
\hline o & 15.096858 & 24.703289 & 9.86447 \\
\hline 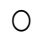 & 12.559678 & 0.509524 & 8.931839 \\
\hline 0 & 13.309521 & 22.923475 & 10.625721 \\
\hline 0 & 14.326626 & 23.005280 & 8 . \\
\hline 0 & 15.078896 & 12.555065 & 22.15882 \\
\hline 0 & 12.565989 & 12.557493 & 21.22765 \\
\hline 0 & 13.308064 & 10.698301 & 22.93197 \\
\hline 0 & 14.256958 & 10.639799 & 20.47441 \\
\hline 0 & 16.100857 & 18.704063 & 22.161011 \\
\hline 0 & 18.619102 & 18.622988 & 21.15239 \\
\hline 0 & 18.043310 & 20.387506 & 22.99338 \\
\hline 0 & 16.925707 & 20.568354 & 20.54917 \\
\hline 0 & 16.176836 & 6.413349 & 9.940691 \\
\hline 0 & 18.711588 & 6.314066 & \\
\hline 0 & 18.021219 & 8.275211 & 10.627905 \\
\hline 0 & 16.925465 & 8.098248 & 8.16476 \\
\hline 0 & 3.803344 & 18.700909 & 10.030749 \\
\hline O & 6.306540 & 18.635851 & 8.94373 \\
\hline O & 5.654768 & 20.413481 & 10.791273 \\
\hline 0 & 4.475265 & 20.547234 & \\
\hline 0 & 3.879081 & 6.410679 & 22.165621 \\
\hline 0 & 6.328874 & 6.320377 & 21.071808 \\
\hline 0 & 5.744341 & 8.264530 & 22.764719 \\
\hline 0 & 4.551730 & 8.187094 & 20.393091 \\
\hline 0 & 16.181690 & 24.857674 & 21 . \\
\hline 0 & 18.709404 & 0.417038 & 22.242329 \\
\hline 0 & 17.881639 & 23.076162 & 20.29963 \\
\hline 0 & 16.940271 & 22.836329 & 22.766903 \\
\hline 0 & 16.102798 & 12.549726 & 8.943975 \\
\hline 0 & 18.538511 & 12.729115 & 10.036333 \\
\hline 0 & 18.033844 & 10.705341 & \\
\hline 0 & 16.923765 & 10.780107 & 10.800497 \\
\hline 0 & 3.800189 & 24.68678 & 9.018985 \\
\hline 0 & 6.327417 & 0.428447 & 9.962296 \\
\hline 0 & 5.649427 & 22.922504 & 8.188793 \\
\hline 0 & 4.561440 & 22 . & 10. \\
\hline 0 & 3.779312 & 12.4 & 21.2 \\
\hline 0 & 6.238329 & 12.639542 & 22.244757 \\
\hline 0 & 5.644330 & 10.698059 & 20.474895 \\
\hline 0 & 4.552944 & 10.705584 & 22.916435 \\
\hline 0 & 2.877511 & 18.7069 & 21.070351 \\
\hline 0 & 0.504183 & 18.54 & 323 \\
\hline 0 & 0.927532 & 20.392363 & 20.402800 \\
\hline 0 & 2.194909 & 20.554518 & 22.756222 \\
\hline 0 & 2.775558 & 6.33785 & 8.858287 \\
\hline 0 & 0.341786 & 6.404610 & 9.947488 \\
\hline 0 & 1.018805 & 8.262102 & 8.171558 \\
\hline 0 & 2.121600 & 8.244625 & 10.609941 \\
\hline 0 & 15.170897 & 18.627113 & 8.939607 \\
\hline 0 & 12.627403 & 18.622015 & 9.794315 \\
\hline 0 & 13.391568 & 20.479752 & 8.099706 \\
\hline 0 & 14.330509 & 20.458633 & 10.613826 \\
\hline 0 & 15.165071 & 6.333486 & 21.252897 \\
\hline ? & 12.637841 & 6.240271 & 22.243057 \\
\hline
\end{tabular}




\begin{tabular}{|c|c|c|c|}
\hline 0 & 13.312919 & 8.099706 & 20.465429 \\
\hline 0 & 14.330752 & 8.167917 & 22.919348 \\
\hline O & 22.169264 & 2.779199 & 24.603277 \\
\hline 0 & 21.067196 & 0.341544 & 0.34639 \\
\hline 0 & 22.922020 & 0.922920 & 22.842640 \\
\hline 0 & 20.556459 & 2.189811 & 22.739958 \\
\hline 0 & 22.080660 & 15.086663 & 12.561863 \\
\hline 0 & 21.231777 & 12.552395 & 12. \\
\hline 0 & 22.933670 & 13.316802 & 10.70485 \\
\hline 0 & 20.468342 & 14.331965 & 10.69490 \\
\hline O & 9.945788 & 3.032383 & 12.3899 \\
\hline O & 8.935966 & 0.584532 & 12.6524 \\
\hline 0 & 10.689077 & 1.1 & 10. \\
\hline 0 & 8.189279 & 2.187870 & 10.64077 \\
\hline 0 & 9.853302 & 15.090304 & 24.6838 \\
\hline 0 & 8.931596 & 12.553366 & 0.43378 \\
\hline 0 & 10.797585 & 13.304423 & 23.005037 \\
\hline 0 & 8.254820 & 14.247976 & 22 . \\
\hline 0 & 22.155426 & 16.094301 & 18.688772 \\
\hline 0 & 21.161139 & 18.620317 & 18.61837 \\
\hline 0 & 23.008678 & 17.960049 & 20.391634 \\
\hline O & 20.556944 & 16.924250 & 20.557186 \\
\hline 0 & 22.088428 & 3.873255 & 6.321349 \\
\hline 0 & 21.140507 & 309 & \\
\hline 0 & 22.853079 & 5.660837 & 8.178355 \\
\hline 0 & 20.470770 & 4.559012 & 8.181753 \\
\hline 0 & 9.937778 & 16.092360 & 6.336641 \\
\hline O & 9.028937 & 18.627842 & 6.253137 \\
\hline 0 & 10.695631 & 17.871931 & \\
\hline 0 & 8.176170 & 16.936872 & 8.092909 \\
\hline 0 & 9.958897 & 3.797276 & 18.700666 \\
\hline 0 & 8.933295 & 6.242213 & 18.533171 \\
\hline 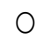 & 10.693932 & 5.738029 & 20.462517 \\
\hline 0 & 8.256519 & 4.564353 & 20.480 \\
\hline 0 & 21.247557 & 16.1 & 24. \\
\hline $\mathrm{O}$ & 22.325350 & 18.634880 & 0.423592 \\
\hline 0 & 20.387266 & 17.954222 & 23.002853 \\
\hline 0 & 22.840212 & 16.850456 & 22.841669 \\
\hline 0 & 21.405342 & 3.713528 & 12.559919 \\
\hline 0 & 22.321707 & 12 & 12. \\
\hline 0 & 20.473925 & 5.4 & 10 . \\
\hline 0 & 22.926874 & 4.478178 & 10.625478 \\
\hline 0 & 9.018499 & 15.842817 & 12.309405 \\
\hline 0 & 9.771982 & 18.381210 & 12.655077 \\
\hline $\mathrm{O}$ & 8.187822 & 17.787455 & 10.628148 \\
\hline 0 & 10.789816 & 16.941000 & 10.696116 \\
\hline 0 & 8.925528 & 3.802616 & 24.611288 \\
\hline 0 & 9.958411 & 6.322320 & 0.424077 \\
\hline 0 & 8.172286 & 5.731961 & 22.907940 \\
\hline 0 & 10.623778 & 4.560711 & 22.844339 \\
\hline $\mathrm{O}$ & 21.246342 & 2.862946 & 18.548950 \\
\hline $\mathrm{O}$ & 22.333603 & 0.427718 & 18.55307 \\
\hline 0 & 20.416153 & 1.009823 & 20.311285 \\
\hline 0 & 22.832445 & 2.193938 & 20.463972 \\
\hline 0 & 21.399757 & 15.257071 & 6.248767 \\
\hline $\mathrm{O}$ & 22.344284 & 12.648038 & 6.000681 \\
\hline 0 & 20.394547 & 13.305394 & 8.168888 \\
\hline 0 & 23.008678 & 14.417897 & 8.18248 \\
\hline
\end{tabular}




\begin{tabular}{|c|c|c|c|}
\hline o & 8.849305 & 2.961501 & 6.492242 \\
\hline 0 & 10.037789 & 0.588174 & 6.309211 \\
\hline 0 & 8.265744 & 1.014436 & 8.256519 \\
\hline O & 10.629848 & 2.271617 & 8.268414 \\
\hline 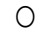 & 8.935722 & 15.256101 & 18.625656 \\
\hline 0 & 9.959382 & 12.569145 & 18.374172 \\
\hline 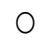 & 7.997995 & 13.232083 & 20.323908 \\
\hline 0 & 10.706069 & 14.339005 & 20.5557 \\
\hline 0 & 24.690180 & 22.164650 & 2.865374 \\
\hline 0 & 0.500542 & 21.069139 & 0.430389 \\
\hline U & 22.943623 & 22.912066 & 0.936 \\
\hline O & 22.835115 & 20.543108 & 2.186656 \\
\hline 0 & 24.690910 & 9.874179 & 15.0907 \\
\hline 0 & 0.437428 & 8.857558 & 12.55433 \\
\hline 0 & 22.919348 & 10.641499 & 13.236939 \\
\hline 0 & 22.917650 & 8.165005 & 14.328082 \\
\hline 0 & 12.470590 & 22.164165 & \\
\hline 0 & 12.559192 & 21.233477 & 12. \\
\hline 0 & 10.626206 & 22.907454 & 13.155134 \\
\hline O & 10.711410 & 20.411297 & 14.161315 \\
\hline 0 & 12.476416 & 9.948702 & 2.940139 \\
\hline O & 12.573272 & & \\
\hline 0 & 10.709710 & 10.706312 & 1.027786 \\
\hline 0 & 10.629362 & 8.263074 & 2.112618 \\
\hline 0 & 18.703335 & 22.167807 & 16.102798 \\
\hline 0 & 18.619831 & 21.156769 & 18.624929 \\
\hline 0 & 20.481936 & 22.910608 & 18.01757 \\
\hline 0 & 20.486790 & 20. & 16. \\
\hline 0 & 18.791210 & 10.033905 & 3.711101 \\
\hline 0 & 18.789753 & 9.004663 & 6.243670 \\
\hline 0 & 20.557430 & 10.796856 & 5.556456 \\
\hline 0 & 20.572481 & 8.337354 & 4.47283 \\
\hline 0 & 6.32 & 22 & 52 \\
\hline $\mathrm{O}$ & 6.325475 & 5634 & 2970 \\
\hline 0 & 8.250208 & 22.994114 & 5.829060 \\
\hline 0 & 8.345850 & 20.493101 & 4.478178 \\
\hline 0 & 6.317950 & 9.874179 & 16.096729 \\
\hline 0 & 6.325475 & 8.9 & 18. \\
\hline 0 & 8.1 & 10. & 21 \\
\hline 0 & 8.179083 & 026 & 16.865263 \\
\hline 0 & 24.842140 & 21.232021 & 16.184361 \\
\hline 0 & 0.431117 & 22.260050 & 18.629541 \\
\hline 0 & 22.992657 & 20.395275 & 17.947426 \\
\hline 0 & 22.913036 & 22.837543 & 16.777145 \\
\hline 0 & 24.609589 & 8.845179 & 3.80067 \\
\hline 0 & 0.517534 & 10.032934 & 6.23978 \\
\hline 0 & 22.917164 & 8.270113 & 5.812552 \\
\hline 0 & 22.935856 & 10.617710 & 4.386906 \\
\hline 0 & 12.547054 & 21.411654 & 3.718626 \\
\hline 0 & 12.642697 & 22.244757 & 6.24755 \\
\hline 0 & 10.869923 & 20.384594 & 5.47950 \\
\hline 0 & 10.710681 & 23.002611 & 4.631351 \\
\hline 0 & 12.467677 & 8.858287 & 16.087990 \\
\hline 0 & 12.638812 & 9.792130 & 18.549921 \\
\hline 0 & 10.700486 & 8.100433 & 17.954464 \\
\hline $\mathrm{O}$ & 10.632760 & 10.532506 & 16.846329 \\
\hline 0 & 18.703335 & 21.222311 & 2.95664 \\
\hline & 18 & 2 & \\
\hline
\end{tabular}




$\begin{array}{rrrr}\mathrm{O} & 20.402800 & 20.386051 & 1.016377 \\ \mathrm{O} & 20.569082 & 22.769331 & 2.192239 \\ \mathrm{O} & 18.785383 & 8.853432 & 15.158516 \\ \mathrm{O} & 18.624201 & 9.862770 & 12.645123 \\ \mathrm{O} & 20.485092 & 8.093880 & 13.234027 \\ \mathrm{O} & 20.490433 & 10.624992 & 14.320801 \\ \mathrm{O} & 6.491999 & 21.158712 & 15.161429 \\ \mathrm{O} & 6.243184 & 22.234562 & 12.647795 \\ \mathrm{O} & 8.174228 & 20.476353 & 13.226743 \\ \mathrm{O} & 8.177870 & 22.923717 & 14.321528 \\ \mathrm{O} & 6.409708 & 8.947132 & 2.862704 \\ \mathrm{O} & 6.329844 & 10.014243 & 0.422378 \\ \mathrm{O} & 8.174956 & 8.179326 & 1.011037 \\ \mathrm{O} & 8.265016 & 10.630575 & 2.117473 \\ \mathrm{O} & 13.225044 & 5.382407 & 8.748808 \\ \mathrm{O} & 12.474232 & 6.332757 & 6.164049 \\ \mathrm{O} & 11.719049 & 7.839724 & 8.420131 \\ \mathrm{O} & 14.413043 & 7.760590 & 7.664219 \\ \mathrm{O} & 9.032336 & 17.516066 & 19.725296 \\ \mathrm{O} & 8.194619 & 19.391764 & 17.874357 \\ \mathrm{O} & 7.922501 & 19.726025 & 20.908926 \\ \mathrm{O} & 9.585553 & 20.529757 & 19.199266 \\ \mathrm{H} & 12.798539 & 22.161495 & 3.144531 \\ \mathrm{H} & 15.945984 & 7.930511 & 7.939008 \\ \mathrm{H} & 25.067650 & 6.127395 & 1.721555 \\ \mathrm{H} & 21.936954 & 16.023420 & 6.530110 \\ \mathrm{H} & 13.543042 & 5.436054 & 9.676341 \\ \mathrm{H} & 9.160991 & 16.141396 & 19.139791 \\ \mathrm{H} & 9.057581 & 19.307774 & 16.011768 \\ \mathrm{H} & 11.021397 & 19.040995 & 21.354122 \\ \mathrm{H} & 11.520967 & 18.592400 & 19.689856 \\ \mathrm{H} & 11.514898 & 21.277657 & 18.830050 \\ \mathrm{H} & 10.418172 & 22.361759 & 19.749571 \\ \mathrm{Re} & 12.934962 & 6.875295 & 7.712040 \\ \mathrm{Re} & 8.907806 & 19.260681 & 19.614119 \\ \mathrm{C} & 10.943960 & 19.305346 & 20.292109 \\ \mathrm{C} & 10.739326 & 21.321108 & 19.607079\end{array}$

$\begin{array}{lrrr}601 & & & \\ \text { USY_ReO3_C2H4 } 5 & E & = & -7235.38071108852 \\ \text { Si } & 23.381292 & 3.329504 & 1.245772 \\ \text { Si } & 23.429358 & 1.235334 & 3.371742 \\ \text { Si } & 23.408482 & 15.562931 & 13.444243 \\ \text { Si } & 23.428387 & 13.390840 & 15.560504 \\ \text { Si } & 11.210011 & 1.165424 & 15.712948 \\ \text { Si } & 11.162432 & 15.566330 & 1.238490 \\ \text { Si } & 11.228216 & 13.401520 & 3.373927 \\ \text { Si } & 7.644799 & 3.354264 & 1.237276 \\ \text { Si } & 7.656451 & 5.508635 & 3.350380 \\ \text { Si } & 7.694077 & 15.433547 & 13.180865 \\ \text { Si } & 19.990131 & 5.402070 & 15.484524 \\ \text { Si } & 19.909540 & 15.646921 & 1.265920 \\ \text { Si } & 19.910755 & 17.800806 & 3.362275 \\ \text { Si } & 7.617126 & 3.380481 & 5.512519 \\ \text { Si } & 7.544303 & 15.446170 & 17.684530 \\ \text { Si } & 7.735101 & 13.237424 & 15.444471 \\ \text { Si } & 19.925076 & 3.373441 & 17.724098 \\ \text { Si } & 19.886965 & 1.223925 & 15.578710\end{array}$




$\begin{array}{lrrr} & & & \\ \mathrm{Si} & 19.943768 & 15.596915 & 5.412750 \\ \mathrm{Si} & 20.005182 & 13.381616 & 3.272459 \\ \mathrm{Si} & 23.462614 & 3.356206 & 5.512762 \\ \mathrm{Si} & 23.347795 & 17.730896 & 15.609052 \\ \mathrm{Si} & 11.231857 & 3.334359 & 17.837946 \\ \mathrm{Si} & 11.254917 & 5.524899 & 15.619492 \\ \mathrm{Si} & 11.254917 & 15.633813 & 5.467368 \\ \mathrm{Si} & 11.234284 & 17.757597 & 3.272702 \\ \mathrm{Si} & 1.307915 & 23.402170 & 3.329019 \\ \mathrm{Si} & 3.396502 & 23.419889 & 1.207661 \\ \mathrm{Si} & 1.240918 & 11.160975 & 15.583323 \\ \mathrm{Si} & 3.349166 & 11.219962 & 13.377246 \\ \mathrm{Si} & 13.472159 & 23.371826 & 15.550066 \\ \mathrm{Si} & 15.622161 & 23.369400 & 13.409288 \\ \mathrm{Si} & 13.412930 & 11.227973 & 3.340913 \\ \mathrm{Si} & 15.588663 & 11.189862 & 1.211545 \\ \mathrm{Si} & 1.342628 & 7.718595 & 3.317367 \\ \mathrm{Si} & 3.398687 & 7.668831 & 5.518587 \\ \mathrm{Si} & 1.266406 & 19.888906 & 15.606867 \\ \mathrm{Si} & 3.398929 & 19.883081 & 17.778231 \\ \mathrm{Si} & 13.508328 & 7.644072 & 15.636725 \\ \mathrm{Si} & 15.666341 & 7.638488 & 17.796194 \\ \mathrm{Si} & 15.602741 & 19.961245 & 5.498439 \\ \mathrm{Si} & 5.544075 & 7.688251 & 3.397716 \\ \mathrm{Si} & 3.406455 & 7.691649 & 1.204506 \\ \mathrm{Si} & 5.464941 & 19.970470 & 15.670467 \\ \mathrm{Si} & 3.368101 & 19.881140 & 13.440603 \\ \mathrm{Si} & 17.861979 & 7.647955 & 15.638425 \\ \mathrm{Si} & 15.677022 & 7.764959 & 13.520951 \\ \mathrm{Si} & 17.762695 & 19.916822 & 3.378539 \\ \mathrm{Si} & 15.621433 & 20.023632 & 1.246743 \\ \mathrm{Si} & 5.509606 & 23.397074 & 3.376111 \\ \mathrm{Si} & 3.404270 & 23.400471 & 5.507664 \\ \mathrm{Si} & 5.454503 & 11.157820 & 15.599586 \\ \mathrm{Si} & 3.314939 & 11.179909 & 17.778231 \\ \mathrm{Si} & 17.726768 & 23.394159 & 15.623618 \\ \mathrm{Si} & 15.541813 & 23.366243 & 17.755169 \\ \mathrm{Si} & 17.818041 & 11.260743 & 3.290179 \\ \mathrm{Si} & 15.647892 & 11.214379 & 5.493585 \\ \mathrm{Si} & 3.371742 & 1.278058 & 23.367214 \\ \mathrm{Si} & 1.242859 & 3.363974 & 23.414549 \\ \mathrm{Si} & 3.356449 & 13.373847 & 11.212923 \\ \mathrm{Si} & 1.257667 & 15.544725 & 11.264627 \\ \mathrm{Si} & 15.651775 & 1.180959 & 11.208553 \\ \mathrm{Si} & 13.391325 & 3.226580 & 11.307351 \\ \mathrm{Si} & 15.562446 & 13.447158 & 23.442951 \\ \mathrm{Si} & 13.449827 & 15.572883 & 23.483974 \\ \mathrm{Si} & 3.348438 & 1.205477 & 7.652810 \\ \mathrm{Si} & 5.477321 & 3.333873 & 7.611544 \\ \mathrm{Si} & 3.283625 & 13.336464 & 19.943525 \\ \mathrm{Si} & 17.783087 & 3.325135 & 19.841572 \\ \mathrm{Si} & 15.652505 & 13.426281 & 7.635575 \\ \mathrm{Si} & 17.818769 & 15.572641 & 7.657179 \\ \mathrm{Si} & 3.319551 & 5.465911 & 7.608873 \\ \mathrm{Si} & 1.238005 & 3.332660 & 7.662520 \\ \mathrm{Si} & 3.415679 & 17.716574 & 19.862690 \\ \mathrm{Si} & 1.249656 & 15.561475 & 19.875799 \\ \mathrm{Si} & 15.594003 & 5.447948 & 19.943768\end{array}$




\begin{tabular}{|c|c|c|c|}
\hline$i$ & 3.414629 & 3.385821 & .964886 \\
\hline & 15.666341 & 17.772890 & 7.63144 \\
\hline & 3.472159 & 15.632599 & 7.625622 \\
\hline & 3.372227 & 5.505479 & 23.44319 \\
\hline & 5.502809 & 3.386307 & 23.408724 \\
\hline & 5.516160 & 15.536714 & 11.083782 \\
\hline & 17.930433 & 3.246485 & 11.217777 \\
\hline & 15.616093 & 17.776047 & 23.462370 \\
\hline & 17.729681 & 15.619492 & 23.42814 \\
\hline & 1.645332 & 21.652943 & 23.74589 \\
\hline & 1.601881 & 23.778667 & 21.65585 \\
\hline & 1.615960 & & \\
\hline & 1.595812 & 11.589908 & 9.460182 \\
\hline & 13.864923 & 21.702219 & 11.549854 \\
\hline & 13.814432 & 23.839598 & 9.374122 \\
\hline & 13.836764 & 9.416845 & 23.79250 \\
\hline & 13. & 11. & \\
\hline S & 17.2 & 21.6 & 23. \\
\hline & 17.393963 & 19.534012 & 21.692753 \\
\hline & 17.450768 & 9.480687 & 11.59112 \\
\hline & 17.481352 & 7.237229 & 9.48991 \\
\hline & 5.062710 & 21.645174 & 11.64015 \\
\hline & 5.0 & 19.5 & \\
\hline & 5.1 & 9. & 23. \\
\hline & 5.14573 & 7.298401 & 21.63400 \\
\hline $\mathrm{Si}$ & 17.370417 & 21.692753 & 19.54178 \\
\hline & 17.416296 & 23.856590 & 21.669691 \\
\hline $\mathrm{Si}$ & 17. & 2 & \\
\hline $\mathrm{Si}$ & 5.7 & 21.6 & 7 . \\
\hline & 5.0971 & 23.7 & 5780 \\
\hline & 5.097423 & 9.458840 & 19.556831 \\
\hline $\mathrm{Si}$ & 5.0648 & 11.639670 & 21.70173 \\
\hline & $1.5 \varepsilon$ & 21.628910 & 19.566 \\
\hline $\mathrm{Si}$ & 1.6 & 19. & 21. \\
\hline & 1.6091 & 9.4 & 7.2 \\
\hline & 1.623485 & 7.329958 & 9.39232 \\
\hline$C$ & 13.881916 & 21.736933 & 7.17727 \\
\hline S & 13.889197 & 19.542509 & 9.34499 \\
\hline $\mathrm{Si}$ & $13 . \varepsilon$ & 46 & 21.663 \\
\hline S & 23. & 1. & 21. \\
\hline & 21.6 & 1.536339 & 23.71264 \\
\hline & 23.806585 & 13.836278 & 9.43262 \\
\hline S & 21.693481 & 13.798410 & 11.61636 \\
\hline $\mathrm{Si}$ & 11.5 & 1.6 & \\
\hline $\mathrm{Si}$ & & & \\
\hline S & 11.681 & 13.831667 & 21.72600 \\
\hline $\mathrm{Si}$ & 9.4 & 13.786031 & 23.80512 \\
\hline S & 23.821150 & 17.346870 & 21.70100 \\
\hline$S$ & 21.698093 & 17.376488 & 19.56071 \\
\hline $\mathrm{Si}$ & 21.697365 & 091 & 7.22970 \\
\hline $\mathrm{Si}$ & 11.6 & 17. & 9 . \\
\hline $\mathrm{Si}$ & 9.467580 & 17.394449 & 7.18940 \\
\hline$S$ & 11.600346 & 5.120969 & 21.64177 \\
\hline $\mathrm{C}$ & 9.471706 & 5.119756 & 19.53158 \\
\hline $\mathrm{Si}$ & 19.535469 & 17.384741 & 21.69518 \\
\hline $\mathrm{Si}$ & 21.723581 & 17.393236 & 23.818722 \\
\hline$c:$ & 19.605623 & 5.015375 & 9.46029 \\
\hline & 21.74 & 5.038436 & 11.6345 \\
\hline
\end{tabular}




\begin{tabular}{|c|c|c|c|}
\hline $\mathrm{Si}$ & 7.251065 & 17.362650 & 9.337953 \\
\hline $\mathrm{Si}$ & 9.432624 & 17.226227 & 11.522182 \\
\hline $\mathrm{Si}$ & 7.285778 & 5.153012 & 21.667994 \\
\hline $\mathrm{Si}$ & 9.411747 & 5.116115 & 23.779882 \\
\hline $\mathrm{Si}$ & 19.557318 & 1.635865 & 21.597353 \\
\hline $\mathrm{Si}$ & 21.677702 & 1.614504 & 19.478182 \\
\hline $\mathrm{Si}$ & 19.563385 & 13.834336 & 9.422186 \\
\hline $\mathrm{Si}$ & 7.271942 & 1.614989 & 9.424613 \\
\hline $\mathrm{Si}$ & 9.421700 & 1.686356 & 7.312723 \\
\hline $\mathrm{Si}$ & 7.290390 & 13.718547 & 21.628183 \\
\hline $\mathrm{Si}$ & 21.707075 & 23.740801 & 1.584888 \\
\hline $\mathrm{Si}$ & 23.843725 & 21.635221 & 1.608920 \\
\hline $\mathrm{Si}$ & 21.702705 & 11.590393 & 13.784574 \\
\hline $\mathrm{Si}$ & 23.819450 & 9.391600 & 13.790157 \\
\hline $\mathrm{Si}$ & 9.433595 & 23.820179 & 13.874147 \\
\hline $\mathrm{Si}$ & 9.467337 & 11.604958 & 1.661839 \\
\hline $\mathrm{Si}$ & 11.617337 & 9.463938 & 1.645332 \\
\hline $\mathrm{Si}$ & 21.687654 & 23.783768 & 17.379642 \\
\hline $\mathrm{Si}$ & 19.540810 & 21.702705 & 17.428919 \\
\hline $\mathrm{Si}$ & 21.772615 & 11.656662 & 4.890361 \\
\hline $\mathrm{Si}$ & 19.617273 & 9.552298 & 4.970710 \\
\hline $\mathrm{Si}$ & 9.379948 & 23.815567 & 5.208843 \\
\hline $\mathrm{Si}$ & 9.486513 & 11.532377 & 17.208750 \\
\hline $\mathrm{Si}$ & 7.252279 & 9.426556 & 17.357067 \\
\hline $\mathrm{Si}$ & 21.694695 & 19.533770 & 17.385712 \\
\hline $\mathrm{Si}$ & 23.834986 & 21.692024 & 17.3 \\
\hline $\mathrm{Si}$ & 21.697851 & 7.371225 & 5.123397 \\
\hline $\mathrm{Si}$ & 23.776970 & 9.444518 & 5.0 \\
\hline $\mathrm{Si}$ & 9.504720 & 19.547363 & 4.973622 \\
\hline $\mathrm{Si}$ & 11.600102 & 21.769218 & 5.1 \\
\hline $\mathrm{Si}$ & 9.450101 & 7.288692 & 17.351484 \\
\hline $\mathrm{Si}$ & 11.634815 & 9.364169 & 17.3 \\
\hline $\mathrm{Si}$ & 21.692268 & 19.527458 & 1.607949 \\
\hline $\mathrm{Si}$ & 19.571880 & 21.613375 & 1.6 \\
\hline $\mathrm{Si}$ & 21.745428 & 7.194992 & 13.767825 \\
\hline $\mathrm{Si}$ & 19.590815 & 9.377035 & 13.8 \\
\hline $\mathrm{Si}$ & 9.446945 & 19.562899 & 13.687962 \\
\hline $\mathrm{Si}$ & 7.244512 & 21.685957 & 13.81 \\
\hline $\mathrm{Si}$ & 9.429468 & 7.298401 & 1.612076 \\
\hline $\mathrm{Si}$ & 7.327530 & 9.425342 & 1.6 \\
\hline $\mathrm{Si}$ & 11.642584 & 21.724310 & 13.750346 \\
\hline $\mathrm{Si}$ & 11.075771 & 3.351351 & 13.49 \\
\hline $\mathrm{Si}$ & 20.059072 & 3.244058 & 13.373606 \\
\hline $\mathrm{Si}$ & 7.600134 & 1.224654 & $3.3^{7}$ \\
\hline $\mathrm{Si}$ & 23.389790 & 15.571671 & 17.730410 \\
\hline $\mathrm{Si}$ & 5.458144 & 15.474086 & 19.919250 \\
\hline $\mathrm{Si}$ & 3.437041 & 17.692055 & 11.288174 \\
\hline $\mathrm{Si}$ & 13.863952 & 9.384803 & 19.536926 \\
\hline $\mathrm{Si}$ & 23.805857 & 5.152284 & 9.409320 \\
\hline Al & 15.580408 & 1.257667 & 19.898617 \\
\hline Al & 15.850828 & 5.462756 & 11.322401 \\
\hline Al & 17.506598 & 9.604245 & 7.200089 \\
\hline Al & 9.523168 & 13.559305 & 19.584261 \\
\hline Al & 7.285293 & 21.634008 & 5.181656 \\
\hline Al & 7.524155 & 17.648361 & 15.334750 \\
\hline Al & 23.388090 & 5.398671 & 3.419077 \\
\hline Al & 13.484782 & 19.842787 & 3.29236 \\
\hline Al & 21.778442 & 13.658345 & 7.24888 \\
\hline
\end{tabular}




\begin{tabular}{|c|c|c|c|}
\hline Al & 13.878517 & 5.107618 & 13.314860 \\
\hline Al & 7.259561 & 18.341644 & 17.893536 \\
\hline 0 & 22.164650 & 0.346641 & 2.865131 \\
\hline O & 24.607161 & 24.540163 & 3.95408 \\
\hline 0 & 24.030153 & 2.099510 & 2.115531 \\
\hline 0 & 22.853565 & 2.185928 & 4.55221 \\
\hline 0 & 22.166836 & 12.477144 & 15.07889 \\
\hline O & 24.604733 & 12.400436 & 16.1008 \\
\hline 0 & 24.014376 & 14.323227 & 14.33973 \\
\hline O & 22.913765 & 14.340462 & 16.78030 \\
\hline 0 & 9.945061 & 0.248815 & 15.252702 \\
\hline O & 12.549239 & 24.615414 & 16.0935 \\
\hline 0 & 11.556894 & 2.115046 & 14.4009 \\
\hline O & 10.701942 & 2.096597 & 16.926678 \\
\hline 0 & 9.944332 & 12.479086 & 2.950092 \\
\hline O & 12.479086 & 12.469619 & 3.80722 \\
\hline 0 & 11.724146 & 14.314003 & 2.11407 \\
\hline O & 10.780592 & 14.326140 & 4.627467 \\
\hline O & 8.929654 & 6.408979 & 2.880181 \\
\hline O & 6.413106 & 6.411407 & 3.88709 \\
\hline 0 & 7.081143 & 4.632322 & 2.09781 \\
\hline O & 8.181753 & 4.558284 & 4.55221 \\
\hline 0 & 8.934508 & 18.803347 & 15.17308 \\
\hline 0 & 6.320377 & 18.6 & 16.268351 \\
\hline O & 7.166833 & 16.7 & 13.97877 \\
\hline 0 & 7.924928 & 16.847057 & 16.87643 \\
\hline 0 & 21.309942 & 6.232018 & 15.00097 \\
\hline 0 & 18.800676 & 6.397085 & 16.01006 \\
\hline O & 19.390549 & 4.472837 & 14.254288 \\
\hline O & 20.486547 & 4.55 & 16.76088 \\
\hline 0 & 21.223766 & 18.619 & 2.87338 \\
\hline 0 & 18.710861 & 18.711588 & 3.95457 \\
\hline 0 & 19.296366 & 16.854824 & 2.18519 \\
\hline 0 & 20.480965 & 16.849728 & 4.55658 \\
\hline 0 & 8.856102 & 0.335475 & 3.87665 \\
\hline O & 6.331786 & 24.6 & 2.78138 \\
\hline O & 7.074589 & 2.118687 & 4.646401 \\
\hline 0 & 8.185394 & 2.185685 & 2.19903 \\
\hline 0 & 9.009760 & 12.312804 & 15.83262 \\
\hline O & 6.408979 & 12.386843 & 15.08812 \\
\hline O & 7.337241 & 14.163015 & 16.77641 \\
\hline 0 & 8.104074 & 14.25 & 14.23680 \\
\hline 0 & 21.146332 & 0.338631 & 16.10037 \\
\hline 0 & 18.634153 & 24.610802 & 15.01456 \\
\hline 0 & 19.305590 & 2.185199 & 16.76889 \\
\hline 0 & 20.548933 & 2.036882 & 14.33269 \\
\hline 0 & 21.315041 & 12.47 & 3.54263 \\
\hline 0 & 18.699209 & 12.551667 & 2.78526 \\
\hline 0 & 19.561686 & 14.240451 & 4.63038 \\
\hline 0 & 20.479752 & 14.411345 & 2.12305 \\
\hline 0 & 22.260294 & 6.569920 & 3.87713 \\
\hline O & 24.783152 & 6.407038 & 2.61704 \\
\hline 0 & 24.181870 & 4.550274 & 4.70708 \\
\hline 0 & 22.764961 & 4.490073 & 2.12305 \\
\hline 0 & 22.079933 & 18.620802 & 16.09236 \\
\hline O & 24.538219 & 18.720085 & 15.09491 \\
\hline 0 & 23.939125 & 16.848999 & 16.85676 \\
\hline 0 & 22.853321 & 16.767193 & 14.40187 \\
\hline
\end{tabular}




$$
\begin{array}{rrr}
10.040460 & 6.508020 & 16.017109 \\
12.641240 & 6.318921 & 14.999275 \\
11.723175 & 4.567509 & 16.833950 \\
10.710681 & 4.695436 & 14.318130 \\
9.943604 & 18.619589 & 3.723481 \\
12.388541 & 18.692411 & 2.695694 \\
11.798426 & 16.856525 & 4.542263 \\
10.619166 & 16.779573 & 2.126455 \\
2.952762 & 22.165621 & 0.265564 \\
3.971567 & 24.611774 & 24.532152 \\
2.108006 & 24.018988 & 2.032027 \\
4.536437 & 22.853807 & 2.183986 \\
2.877511 & 9.951614 & 12.475445 \\
3.880538 & 12.467191 & 12.469619 \\
2.118687 & 11.728029 & 14.327353 \\
4.558770 & 10.697330 & 14.332936 \\
15.172111 & 22.164650 & 12.400680 \\
16.177807 & 24.597450 & 12.473989 \\
14.322013 & 23.930386 & 14.249675 \\
16.777145 & 22.838028 & 14.405033 \\
15.168225 & 9.868353 & 0.341544 \\
16.103285 & 12.462093 & 24.607647 \\
14.261812 & 11.711038 & 2.025715 \\
16.846329 & 10.711895 & 2.108492 \\
2.883094 & 8.925528 & 6.405096 \\
3.887577 & 6.407280 & 6.407765 \\
2.196366 & 7.086483 & 4.544447 \\
4.630622 & 8.245596 & 4.624554 \\
2.861975 & 21.073509 & 18.712318 \\
4.040993 & 18.626871 & 18.627113 \\
2.271374 & 19.289082 & 16.769379 \\
4.639847 & 20.552816 & 16.929590 \\
15.161429 & 8.942762 & 18.629784 \\
16.106440 & 6.406795 & 18.717659 \\
14.416926 & 7.081386 & 16.847057 \\
16.924007 & 8.103104 & 16.874729 \\
15.182062 & 21.323050 & 6.322562 \\
16.184361 & 18.797522 & 6.490542 \\
14.326869 & 19.385210 & 4.720681 \\
16.837833 & 20.477324 & 4.571635 \\
3.866458 & 8.843237 & 0.180846 \\
2.777985 & 6.400969 & 24.691879 \\
16.8640818 & 7.168531 & 2.122814 \\
14.429550 & 20.640205 & 2.107521 \\
3.800431 & 22.169506 & 6.491028 \\
2.776529 & 24.610802 & 6.412864 \\
2.210931 & 8.337111 & 2.105093 \\
3.800674 & 21.161625 & 12.554094 \\
2.950335 & 18.604538 & 12.552153 \\
4.626253 & 19.387152 & 14.415712 \\
16.122571 & 20.394062 & 14.328082 \\
15.077439 & 8.858287 & 12.456268 \\
14.867691 & 6.404125 & 12.720860 \\
16.173195 & 21.2373650 & 14.414500 \\
& & \\
15.081080 & 18.781258 & 24.641301 \\
\hline
\end{array}
$$




$$
\begin{aligned}
& 4.635478 \\
& 24.018259 \\
& 22.839972 \\
& 9.942390 \\
& 3.798975 \\
& 2.781141 \\
& 4.556342 \\
& 12.400923 \\
& 11.721232 \\
& 10.625478 \\
& 2.112133 \\
& 16.091146 \\
& 14.917227 \\
& 16.796324 \\
& 14.416199 \\
& 16.016623 \\
& 15.167498 \\
& 16.923523 \\
& 14.397265 \\
& 0.344942 \\
& 24.609346 \\
& 2.119173 \\
& 2.199036 \\
& 0.428204 \\
& 24.607891 \\
& 2.115046 \\
& 2.203405 \\
& 12.463307 \\
& 12.486368 \\
& 14.330509 \\
& 14.239964 \\
& 12.640026 \\
& 12.397038 \\
& 14.333423 \\
& 14.410131 \\
& 6.416748 \\
& 6.390531 \\
& 4.641304 \\
& 4.479635 \\
& 6.496368 \\
& 6.249010 \\
& 4.547360 \\
& 4.631593 \\
& 18.704792 \\
& 18.793636 \\
& 16.945612 \\
& 16.846087 \\
& 18.715958 \\
& 18.776403 \\
& 16.923765 \\
& 16.925949 \\
& 0.422864 \\
& 24.524143 \\
& 2.106064 \\
& 2.205347 \\
& 0.419222 \\
& 22.083817 \\
& 24.523899 \\
& 23.936939 \\
& 22.758408 \\
& 9.954527 \\
& 12.486854 \\
& 11.725359 \\
& 10.784233 \\
& 2.868529 \\
& \text { 3. } 964042 \\
& 2.117959 \\
& 4.559498 \\
& 15.094916 \\
& 16.100370 \\
& 14.257200 \\
& 16.774477 \\
& 2.943052 \\
& 3.805772 \\
& 2.026201 \\
& 4.636691 \\
& 15.081080 \\
& 16.098429 \\
& 14.329053 \\
& 16.785885 \\
& 2.873384 \\
& 3.957731 \\
& 2.037610 \\
& 4.482062 \\
& 15.074283 \\
& 15.933118 \\
& 14.238509 \\
& 16.776661 \\
& 2.937227 \\
& 3.963799 \\
& 2.125241 \\
& 4.551002 \\
& 15.167498 \\
& 16.033859 \\
& 14.327840 \\
& 16.855795 \\
& 3.884907 \\
& 2.691082 \\
& 4.558527 \\
& 2.182529 \\
& 16.093330 \\
& 24.620514 \\
& 2.199521 \\
& 2.114318 \\
& 12.381745 \\
& 12.484669 \\
& 14.241422 \\
& 15.012869 \\
& 16.779331 \\
& 14.323713 \\
& 3.804315 \\
& 2.866102 \\
& 4.706602 \\
& 4.638148 \\
& 4.484732 \\
& 18.706491 \\
& 18.713045 \\
& 16.851185 \\
& 16.848999 \\
& 18.616919 \\
& 18.630754 \\
& 16.839775 \\
& 16.694370 \\
& 6.406309 \\
& 6.406795 \\
& 4.574063 \\
& 4.540078 \\
& 22.162708 \\
& 24.615171 \\
& 24.012920 \\
& 22.846523 \\
& 9.955741 \\
& 12.483212 \\
& 11.806922 \\
& 10.788603 \\
& 10.037789 \\
& 12.644639 \\
& 11.801825 \\
& 11.219477 \\
& 22.156883 \\
& 24.600121 \\
& 24.089869 \\
& 23.000425 \\
& 8.864841 \\
& 6.414320 \\
& 7.077502 \\
& 8.178112 \\
& 21.079819 \\
& 18.522490 \\
& 19.390549 \\
& 20.400858 \\
& 21.145847 \\
& 18.706734 \\
& 19.212132 \\
& 20.396490 \\
& 8.942762 \\
& 6.412135 \\
& 7.094737 \\
& 8.096065 \\
& 8.947132 \\
& 6.564095 \\
& 6.998367 \\
& 8.255791 \\
& 21.154827 \\
& 18.641436 \\
& 19.298792 \\
& 20.469799 \\
& 21.156042 \\
& 18.705521 \\
& 19.389336 \\
& 20.553547
\end{aligned}
$$




\begin{tabular}{|c|c|c|c|}
\hline O & 12.568902 & 16.093817 & 8.927227 \\
\hline O & 12.484669 & 15.167983 & 6.424273 \\
\hline 0 & 14.414257 & 16.862837 & 7.084542 \\
\hline 0 & 14.418140 & 14.399935 & 8.096550 \\
\hline 0 & 6.411164 & 3.879809 & 22.157854 \\
\hline 0 & 6.411650 & 2.785268 & 24.60886 \\
\hline 0 & 4.632322 & 4.637662 & 24.02068 \\
\hline O & 4.531582 & 2.257538 & 22.7783 \\
\hline 0 & 6.322562 & 16.089205 & 9.77926 \\
\hline O & 6.503165 & 14.910430 & 12.22177 \\
\hline O & 4.706845 & 16.774477 & 11.795999 \\
\hline 0 & 4.543477 & 14.326383 & 10.621837 \\
\hline 0 & 18.702850 & 3.713771 & 9.85670 \\
\hline 0 & 18.968414 & 2.630396 & 12.31183 \\
\hline ? & 17.105339 & 4.469682 & 11.89067 \\
\hline O & 16.858952 & 2.116745 & 10.70461 \\
\hline 0 & 18.630754 & 16.107410 & 22.160524 \\
\hline 0 & 18.712318 & 15.085692 & 24.606190 \\
\hline 0 & 16.856525 & 16.862106 & 24.02505 \\
\hline 0 & 16.769865 & 14.409887 & 22.923475 \\
\hline o & 2.867316 & 24.696249 & 22.08381 \\
\hline O & 0.431117 & 0.417280 & 20.993402 \\
\hline O & 1.00 & 22.923475 & 22. \\
\hline O & 2.193696 & 22.756466 & 11893 \\
\hline 0 & 2.877754 & 12.469376 & 9.953071 \\
\hline 0 & 0.424077 & 12.560649 & 8.931839 \\
\hline 0 & 1.014436 & 10.693689 & 10.70776 \\
\hline 0 & 2.122086 & 10.637372 & 05 \\
\hline 0 & 15.103656 & 1834 & \\
\hline 0 & 12.556279 & 0.513893 & 8.937422 \\
\hline 0 & 13.304180 & 22.925659 & 10.622079 \\
\hline 0 & 14.326626 & 23.008194 & 8.096307 \\
\hline 0 & 15.080353 & 12.558949 & 22.154699 \\
\hline 0 & 12.570844 & 12.552880 & 21. \\
\hline 0 & 13.308791 & 0244 & 22.926146 \\
\hline 0 & 14.322013 & 10.632031 & 20.475624 \\
\hline 0 & 16.109352 & 18.628813 & 22.160040 \\
\hline 0 & 18.621288 & 18.607450 & 21.149973 \\
\hline 0 & 18.036512 & 20.395519 & 22.926388 \\
\hline 0 & 16.855068 & 20.472954 & 034 \\
\hline $\mathrm{O}$ & 16.173195 & 6.411650 & 9.949915 \\
\hline 0 & 18.708920 & 6.310910 & 9.011945 \\
\hline 0 & 18.023647 & 8.273270 & 10.624749 \\
\hline 0 & 16.931776 & 8.101890 & 8.167675 \\
\hline $\mathrm{O}$ & 3.867186 & 18.616190 & 10.030993 \\
\hline 0 & 6.322805 & 18.629055 & 8.936451 \\
\hline 0 & 5.657681 & 20.395761 & 10.784476 \\
\hline 0 & 4.469924 & 20.482420 & 8.346335 \\
\hline 0 & 3.877382 & 6.488601 & 22.249613 \\
\hline 0 & 6.319406 & 6.321834 & 21.074720 \\
\hline 0 & 5.818864 & 8.276424 & 22.763504 \\
\hline 0 & 4.557798 & 8.182239 & 20.403044 \\
\hline 0 & 16.180233 & 24.767618 & 21.321352 \\
\hline 0 & 18.708191 & 0.417766 & 22.239902 \\
\hline 0 & 17.954222 & 22.935856 & 20.399645 \\
\hline $\mathrm{O}$ & 16.939058 & 22.845552 & 22.839727 \\
\hline $\mathrm{O}$ & 16.098429 & 12.553366 & 8.9 \\
\hline ( & 18.537298 & 12.730085 & 10.04118 \\
\hline
\end{tabular}




$$
\begin{array}{rrr}
18.034571 & 10.776223 & 8.343180 \\
16.933474 & 10.780350 & 10.785447 \\
3.807228 & 24.598423 & 8.942519 \\
6.316979 & 0.423835 & 9.962296 \\
5.721037 & 22.829046 & 8.253364 \\
4.556828 & 22.853079 & 10.703642 \\
3.734647 & 12.466220 & 21.234447 \\
6.226435 & 12.642454 & 22.242086 \\
5.646029 & 10.703156 & 20.479507 \\
4.565324 & 10.702913 & 22.921776 \\
2.941596 & 18.699938 & 21.060642 \\
0.508310 & 18.536083 & 22.249613 \\
0.933844 & 20.380955 & 20.402557 \\
2.198065 & 20.487034 & 22.760107 \\
2.860033 & 6.410193 & 8.855131 \\
0.421893 & 6.399513 & 9.955984 \\
1.010309 & 8.248752 & 8.179568 \\
2.187627 & 8.257490 & 10.613826 \\
15.169926 & 18.629055 & 8.940820 \\
12.628860 & 18.623714 & 9.795286 \\
13.393024 & 20.480965 & 8.099948 \\
14.327840 & 20.467371 & 10.619894 \\
15.167741 & 6.334942 & 21.245615 \\
12.636627 & 6.254108 & 22.167807 \\
13.318501 & 8.099948 & 20.390663 \\
14.325412 & 8.184423 & 22.855991 \\
22.171206 & 2.779199 & 24.606432 \\
21.077635 & 0.331348 & 0.354166 \\
22.922991 & 0.922435 & 22.842884 \\
20.561069 & 2.187384 & 22.748941 \\
22.151056 & 15.067244 & 12.550939 \\
21.229107 & 12.552395 & 12.566717 \\
22.928816 & 13.244221 & 10.700486 \\
20.477810 & 14.330509 & 10.695389 \\
9.948215 & 3.034082 & 12.395096 \\
8.939607 & 0.593028 & 12.726687 \\
10.630818 & 1.096726 & 10.703400 \\
8.164276 & 2.181073 & 10.695631 \\
9.849661 & 15.089090 & 24.683868 \\
8.9318967060 & 12.546083 & 0.441069 \\
10.704613 & 5.740700 & 20.398188 \\
8.255305 & 4.633535 & 20.476595
\end{array}
$$




$$
\begin{array}{rrr}
21.233963 & 16.181690 & 24.771502 \\
22.330690 & 18.623472 & 0.417280 \\
20.476109 & 17.948397 & 22.919834 \\
22.924202 & 16.774233 & 22.917650 \\
21.403885 & 3.795819 & 12.633958 \\
22.252768 & 6.332272 & 12.484912 \\
20.409355 & 5.484846 & 10.800255 \\
22.915949 & 4.555129 & 10.628877 \\
9.019712 & 15.847187 & 12.309164 \\
9.772225 & 18.381454 & 12.659931 \\
8.189279 & 17.786484 & 10.629604 \\
10.790545 & 16.941729 & 10.695874 \\
8.928683 & 3.802616 & 24.609346 \\
9.957440 & 6.325232 & 0.431117 \\
8.165490 & 5.735602 & 22.915222 \\
10.624264 & 4.632322 & 22.836813 \\
21.236633 & 2.862946 & 18.539240 \\
22.328018 & 0.430631 & 18.549435 \\
20.404985 & 1.019290 & 20.313955 \\
22.830746 & 2.193453 & 20.461546 \\
21.327906 & 15.249789 & 6.326446 \\
22.339428 & 12.645853 & 5.999467 \\
20.393091 & 13.234998 & 8.179326 \\
23.002853 & 14.411101 & 8.186366 \\
8.850519 & 2.960773 & 6.493213 \\
10.032207 & 0.584775 & 6.314794 \\
8.264045 & 1.018562 & 8.266230 \\
10.631061 & 2.271617 & 8.270842 \\
8.852219 & 15.187889 & 18.720327 \\
10.026623 & 12.566717 & 18.292610 \\
8.091209 & 13.061919 & 20.397217 \\
10.706312 & 14.332936 & 20.550632 \\
24.767374 & 22.077747 & 2.861490 \\
0.498358 & 21.000927 & 0.421893 \\
23.007465 & 22.913036 & 1.010309 \\
22.847254 & 20.491646 & 2.188841 \\
24.689938 & 9.858886 & 15.083993 \\
0.444711 & 8.854646 & 12.548512 \\
22.920076 & 10.635916 & 13.234998 \\
22.918861 & 8.161120 & 14.328811 \\
12.464035 & 22.162708 & 15.077682 \\
12.639055 & 21.304844 & 12.551182 \\
10.632517 & 22.913521 & 13.231112 \\
10.725489 & 20.411053 & 14.160344 \\
12.476173 & 9.940206 & 2.947422 \\
12.572058 & 8.935722 & 0.432088 \\
10.708011 & 10.706798 & 1.088716 \\
10.630818 & 8.257490 & 2.115774 \\
18.704550 & 22.173876 & 16.104012 \\
18.608665 & 21.148516 & 18.623230 \\
20.470526 & 22.914007 & 18.031416 \\
20.482664 & 20.482664 & 16.921337 \\
18.797035 & 10.022011 & 3.630995 \\
18.710617 & 9.014130 & 6.159437 \\
20.478781 & 10.858756 & 5.483874 \\
6.407523 & 22.176788 & 3.797033 \\
6.238572 & 20.984663 & 6.408979
\end{array}
$$




\begin{tabular}{|c|c|c|c|}
\hline 0 & 8.262345 & 22.925417 & 5.899213 \\
\hline & 8.350462 & 20.481693 & 4.473566 \\
\hline 0 & 6.319891 & 9.864226 & 16.09066 \\
\hline 0 & 6.332029 & 8.948831 & 18.61521 \\
\hline 0 & 8.186123 & 10.700244 & 17.78769 \\
\hline U & 8.183210 & 8.178597 & 16.857737 \\
\hline 0 & 24.855490 & 21.239546 & 16.177078 \\
\hline 0 & 0.424077 & 22.256165 & 18.6229 \\
\hline o & 23.004066 & 20.387506 & 17.8726 \\
\hline 0 & 22.858419 & 22.832445 & 16.7725 \\
\hline 0 & 24.606432 & 8.846393 & 3.8014 \\
\hline 0 & 0.502727 & 10.035362 & 6.2344 \\
\hline 0 & 22.913765 & 8.270113 & 5.8123 \\
\hline 0 & 22.925175 & 10.619652 & 4.3876 \\
\hline 0 & 12.544142 & 21.414808 & 3.7188 \\
\hline o & 12.643668 & 22.245728 & 6.2473 \\
\hline c & 10.872108 & 20.389450 & 5.483146 \\
\hline 0 & 10.701457 & 23.006979 & \\
\hline 0 & 12.468405 & 8.772355 & 16.09551 \\
\hline 0 & 12.644396 & 9.865440 & 18.54361 \\
\hline O & 10.685922 & 8.168646 & 17.96636 \\
\hline 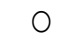 & 10.703642 & 10.536633 & 16.7691 \\
\hline O & 18.714016 & 21.157497 & 2 . \\
\hline 0 & 18.632696 & 22.244514 & 9447 \\
\hline 0 & 20.396732 & 20.330219 & 1.01613 \\
\hline 0 & 20.573208 & 22.755980 & 2.18956 \\
\hline 0 & 18.713774 & 8.942034 & 15.16434 \\
\hline 0 & 18.622259 & 9.864955 & 12.6402 \\
\hline 0 & 20.462759 & 8.105045 & 13 \\
\hline 0 & 20.492130 & 10.635187 & 14. \\
\hline 0 & 6.508020 & 21.091471 & 15.16167 \\
\hline 0 & 6.242941 & 22.169020 & 12.653620 \\
\hline 0 & 8.185394 & 20.468584 & 13.224317 \\
\hline 0 & 8.16 & 22.915464 & 14. \\
\hline 0 & 6.487387 & 8.932324 & 462 \\
\hline 0 & 6.316250 & 9.951857 & 0.49520 \\
\hline 0 & 8.179568 & 8.180782 & 1.015892 \\
\hline 0 & 8.265501 & 10.632275 & 2.183015 \\
\hline 0 & 13.310005 & 5.306185 & 8.580100 \\
\hline 0 & 12.388784 & 6.404367 & 476 \\
\hline 0 & 11.888728 & 7.839967 & 8.425714 \\
\hline 0 & 14.498975 & 7.671987 & 7.418560 \\
\hline 0 & 7.763988 & 18.049135 & 19.538139 \\
\hline 0 & 8.434694 & 19.637180 & 17.699823 \\
\hline 0 & 8.505819 & 20.570780 & 20.814739 \\
\hline 0 & 10.713108 & 20.217585 & 19.04172 \\
\hline $\mathrm{H}$ & 12.774508 & 22.161253 & 3.13118 \\
\hline $\mathrm{H}$ & 15.972929 & 7.897498 & 7.883176 \\
\hline $\mathrm{H}$ & 25.093140 & 6.110159 & 1.731993 \\
\hline $\mathrm{H}$ & 21.916807 & 16.001087 & 6.534722 \\
\hline $\mathrm{H}$ & 13.567801 & 5.345752 & 9.526809 \\
\hline $\mathrm{H}$ & 8.947374 & 15.952538 & 19.340302 \\
\hline $\mathrm{H}$ & 9.019227 & 19.328892 & 16.05958 \\
\hline $\mathrm{H}$ & 10.490511 & 18.335575 & 21.52064 \\
\hline $\mathrm{H}$ & 10.652180 & 17.367262 & 20.025574 \\
\hline $\mathrm{H}$ & 12.363053 & 18.920835 & 19.200237 \\
\hline 11 & 12.092876 & 19.998142 & 20.613262 \\
\hline 7 & 12.999775 & 6.843009 & 7.60086 \\
\hline
\end{tabular}




$\begin{array}{rrrr}\text { Re } & 9.030879 & 19.545179 & 19.588631 \\ \mathrm{C} & 10.581055 & 18.386066 & 20.428532 \\ \mathrm{C} & 11.606900 & 19.366276 & 19.859049\end{array}$

601

USY ReO3 C2H4 $5 \mathrm{E}=-7235.38071108852$

$\mathrm{Si}-23 . \overline{3} 8493 \overline{5}$

$3.309842 \quad 1.236791$

$\begin{array}{ll}\text { Si } & 23.435184 \\ \text { Si } & 23.427658\end{array}$

$1.270532 \quad 3.352808$

Si 23.417706

15.570457

13.432591

13.419970

15.569486

1.212273

15.635269

15.559047

11.159276

11.214136

13.395452

3. 342127

5.506207

15.524092

5.451590

1.215429

3.364460

1.231936

3. 349166

7.674171

19.890606

19.903957

15.628959

19.907843

7.615427

17.789640

3.351594

7.532893

15.452724

7.651354

13.287672

3. 416650

19.922407

1.242617

19.958332

15.596673

20.007124

13.380158

3.317609

23.448050

23.375467

17.743761

3. 338243

5.513004

11.222147

11.238654

11.192533

15.605896

17.721914

23.442951

1. 292622

23.479120

3.396259

11.187677

1.180717

11.216321

23.388819

23.381779

11.235255

11.200300

7.650625

7.613729

13.250289

15.500060

1.227566

3. 320765

5. 482661

17.712690

15.498846

17.732838

15.635755

5. 404983

3.271488

5.548203

15.607596

17.798864

15.605655

5.458144

3. 275614

3. 340670

1. 221012

15.611237

13.399578

15.551037

13.398850

3. 331203

1. 189698

3. 323192

5.485331

19.873615

15.645221

17.770948

15.566330

17.772890

5.494798

3. 322464

1.173191

15.636725

13.417542

15.675079

13.448856

3. 351109

1. 206690

3. 387278

5.511791

$11.147381 \quad 15.606139$ 


\begin{tabular}{|c|c|c|c|}
\hline $\mathrm{Si}$ & 3.319794 & 11.201514 & 3619 \\
\hline i & 17.723614 & 23.385422 & 15.623375 \\
\hline S & 15.549095 & 23.369400 & 17.745703 \\
\hline & 17.820469 & 11.268026 & 3.295034 \\
\hline & 15.664398 & 11.235013 & 5.4714 \\
\hline & 3.393589 & 1.298934 & 23.40775 \\
\hline$i$ & 1.236548 & 3.359847 & $23 \quad 412$ \\
\hline i & 3.322464 & 13.386471 & 11.22603 \\
\hline & 1.250870 & 15.608081 & 11.25418 \\
\hline S & 15.602741 & 1.229266 & 11.23865 \\
\hline & 13.374333 & 3.322464 & 11.28404 \\
\hline i & 15.574340 & 13.448370 & 23.36988 \\
\hline$i$ & 13.436718 & 15.604441 & 23.440281 \\
\hline 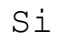 & 3.310812 & 1.199408 & 7.6382 \\
\hline & 5.438481 & 3.302074 & 6275 \\
\hline S & 3.299404 & 13.367051 & 19.90517 \\
\hline & 17.758812 & 3.381695 & 19.80151 \\
\hline i & 15.653719 & 13.423853 & \\
\hline $\mathrm{Si}$ & 17.820227 & 15.571427 & 7.65499 \\
\hline 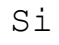 & 3.276100 & 5.446734 & 7.58945 \\
\hline $\mathrm{L}$ & 1.203777 & 3.319066 & 7.67028 \\
\hline 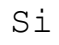 & 3.441895 & 17.769733 & 19.91997 \\
\hline i & 1.285583 & 15.617791 & 19.8799 \\
\hline i & 15.562202 & 5.491642 & \\
\hline i & 13.399335 & 3.393831 & 19.93672 \\
\hline $\mathrm{Si}$ & 15.665128 & 17.773863 & 7.63096 \\
\hline Si & 13.469975 & 15.630172 & 7.62149 \\
\hline i & 3.366401 & 5.489458 & 23.41018 \\
\hline i & 5.507178 & 3.396 & 23. \\
\hline $\mathrm{Si}$ & 5.514461 & 15.553465 & 11.10927 \\
\hline Si & 17.825323 & 3.277556 & 11.26729 \\
\hline $\mathrm{Si}$ & 15.596673 & 17.779444 & 23.411880 \\
\hline Si & 17.730167 & 15.612694 & 23.35434 \\
\hline $\mathrm{Si}$ & 1.633195 & 21.719940 & 23.758 \\
\hline$i$ & 1.593142 & 23.7 & 21. \\
\hline $\mathrm{Si}$ & 1.597269 & 9.451558 & 11.58044 \\
\hline $\mathrm{Si}$ & 1.593627 & 11.589421 & 9.45228 \\
\hline Si & 13.854485 & 21.709503 & 11.54330 \\
\hline $\mathrm{Si}$ & 13.800595 & 23.844938 & 9.39305 \\
\hline $\mathrm{Si}$ & 13.8 & $9.426^{\circ}$ & 23.80512 \\
\hline $\mathrm{Si}$ & 13.8 & 11.6 & 21 . \\
\hline $\mathrm{Si}$ & 17.423578 & 21.730621 & 23.767502 \\
\hline $\mathrm{Si}$ & 17.407316 & 19.572124 & 21.698580 \\
\hline $\mathrm{Si}$ & 17.335705 & 9.477046 & 11.60908 \\
\hline $\mathrm{Si}$ & 17.451494 & 7.308 & 9.49768 \\
\hline $\mathrm{Si}$ & 5.065623 & 21.70 & 11.65083 \\
\hline Si & 5.085528 & 19.573095 & 9.51030 \\
\hline $\mathrm{Si}$ & 5.130922 & 9.425584 & 23.72744 \\
\hline Si & 5.134321 & 7.269271 & 21.61070 \\
\hline $\mathrm{Si}$ & 17.369204 & 21.693966 & 19.531828 \\
\hline $\mathrm{Si}$ & 17.396391 & 23.870670 & 21.639107 \\
\hline $\mathrm{Si}$ & 17.394449 & 11.693560 & 9.49088 \\
\hline $\mathrm{Si}$ & 5.089170 & 21.602694 & 7.30374 \\
\hline $\mathrm{Si}$ & 5.089412 & 23.772842 & 9.43189 \\
\hline $\mathrm{Si}$ & 5.093539 & 9.451073 & 19.54954 \\
\hline $\mathrm{Si}$ & 5.057856 & 11.611998 & 21.68935 \\
\hline $\mathrm{Si}$ & 1.575907 & 21.646873 & 19.58062 \\
\hline Si & 1.608920 & 19.569939 & 21.64250 \\
\hline
\end{tabular}




\begin{tabular}{|c|c|c|c|}
\hline Si & 1.589501 & 9.424856 & 9224 \\
\hline . & 1.571538 & 7.284322 & 9.417816 \\
\hline $\mathrm{Si}$ & 13.880216 & 21.741787 & 7.186981 \\
\hline & 13.886284 & 19.543480 & 9.354702 \\
\hline & 13.849144 & 7.229947 & 21.69275 \\
\hline & 23.755365 & 1.599696 & 21.60827 \\
\hline S & 21.641048 & 1.520075 & 23.681572 \\
\hline & 23.821878 & 13.872206 & $9.42849^{\circ}$ \\
\hline & 21.689112 & 13.817101 & 11.618 \\
\hline & 11.547184 & 1.650187 & $9.467 \mathrm{C}$ \\
\hline & 9.398640 & 1.689269 & 11.5952 \\
\hline $\mathrm{Si}$ & 11.639670 & 13.819044 & 21.6978 \\
\hline i & 9.426312 & 13.767825 & 23.7 \\
\hline S & 23.814596 & 17.374544 & 21.669 \\
\hline & 21.706104 & 17.397120 & 19. \\
\hline & 21.634251 & 5.139175 & 7.257 \\
\hline & 11.683122 & 17.380613 & 9.39232 \\
\hline L & 9.466851 & 17.3 & \\
\hline $\mathrm{Si}$ & 11.612725 & 5.1 & 21.66799 \\
\hline S & 9.483843 & 5.104948 & 19.564842 \\
\hline $\mathrm{Si}$ & 19.533283 & 17.401005 & 21.707317 \\
\hline S & 21.688141 & 17.417025 & 23.82988 \\
\hline$\perp$ & 19.539110 & 5.108832 & 9.46806 \\
\hline i & 21.700035 & 5.0 & 11.6 \\
\hline i & 7.250094 & 17.3 & 9.398640 \\
\hline $\mathrm{Si}$ & 9.445246 & 17.215546 & 11.564177 \\
\hline i & 7.304227 & 5.141603 & 21.661438 \\
\hline i & 9.433838 & 5.111745 & 23.77915 \\
\hline i & 19.543964 & 1.647760 & 21. \\
\hline $\mathrm{Si}$ & 21.674061 & 1.61 & 19.452692 \\
\hline $\mathrm{Si}$ & 19.550520 & 13.842105 & 9.428740 \\
\hline $\mathrm{Si}$ & 7.261261 & 1.626155 & 9.38892 \\
\hline $\mathrm{Si}$ & 9.414417 & 1.661596 & 7.28359 \\
\hline i & 7.290633 & 13.665629 & 21.628 \\
\hline $\mathrm{Si}$ & 21.670 & 23.7 & 1.5 \\
\hline $\mathrm{Si}$ & 23.804886 & 21.698580 & 1.592899 \\
\hline $\mathrm{Si}$ & 21.653185 & 11.614182 & 13.806664 \\
\hline $\mathrm{Si}$ & 23.779398 & 9.389900 & 13.83627 \\
\hline $\mathrm{Si}$ & 9.459569 & 23.819206 & 13.82681 \\
\hline $\mathrm{Si}$ & 9.460 & 11.5 & 1.608192 \\
\hline $\mathrm{Si}$ & 11.6 & 9. & 1 . \\
\hline $\mathrm{Si}$ & 21.694210 & 23.766047 & 17.372358 \\
\hline $\mathrm{Si}$ & 19.535469 & 21.680130 & 17.409742 \\
\hline $\mathrm{Si}$ & 21.764849 & 11.635786 & 4.89424 \\
\hline $\mathrm{Si}$ & 19.612177 & 9.5 & 4.96245 \\
\hline $\mathrm{Si}$ & 9.345720 & 23. & 879 \\
\hline $\mathrm{Si}$ & 9.442333 & 11.553981 & 17.18593 \\
\hline $\mathrm{Si}$ & 7.256163 & 9.420730 & 17.363621 \\
\hline $\mathrm{Si}$ & 21.674789 & 19.529886 & 17.37648 \\
\hline $\mathrm{Si}$ & 23.835714 & 21.680130 & 17.36847 \\
\hline $\mathrm{Si}$ & 21.674545 & 7.318064 & 5.12825 \\
\hline $\mathrm{Si}$ & 23.742498 & 9.4 & 5.12339 \\
\hline $\mathrm{Si}$ & 9.484571 & 19.499300 & 5.00493 \\
\hline $\mathrm{Si}$ & 11.585538 & 21.723339 & 5.11660 \\
\hline $\mathrm{Si}$ & 9.448402 & 7.256891 & 17.39954 \\
\hline $\mathrm{Si}$ & 11.609813 & 9.374607 & 17.33109 \\
\hline $\mathrm{Si}$ & 21.685957 & 19.561443 & 1.60989 \\
\hline $\mathrm{Si}$ & 19.555132 & 21.651730 & 1.64363 \\
\hline
\end{tabular}




\begin{tabular}{|c|c|c|c|}
\hline Si & 21.673334 & 7.252522 & 13.759329 \\
\hline Si & 19.525032 & 9.430924 & 13.865651 \\
\hline$i$ & 9.490397 & 19.592272 & 153 \\
\hline i & 7.302528 & 21.688383 & 13.803994 \\
\hline 1 & 9.443304 & 7.290148 & 1.600 \\
\hline $\mathrm{Si}$ & 7.304955 & 9.413446 & 605 \\
\hline$i$ & 11.677782 & 21.717270 & 13.745492 \\
\hline $\mathrm{Si}$ & 11.179909 & 3.380481 & 13.42 \\
\hline $\mathrm{Si}$ & 20.009068 & 3.291636 & 13.398 \\
\hline$i$ & 7.606203 & 1.175861 & 3.322 \\
\hline $\mathrm{Si}$ & 23.405325 & 15.602499 & 17.741 \\
\hline Si & 5.441394 & 15.512440 & 20 . \\
\hline $\mathrm{Si}$ & 3.424903 & 17.750557 & 11.26851 \\
\hline$i$ & 13.797683 & 9.410534 & 19.51944 \\
\hline $\mathrm{Si}$ & 23.751968 & 5.151798 & 9.41830 \\
\hline 1 & 15.595216 & 1.300390 & 19.83089 \\
\hline 1 & 15.609295 & 5.485816 & 11.295 \\
\hline 1 & 17.512424 & 9.599390 & 7.20615 \\
\hline 1 & 9.491368 & 13.555422 & 19.575 \\
\hline 1 & 7.258105 & 21.601967 & 5.1581 \\
\hline 1 & 7.553770 & 17.709534 & 15.4063 \\
\hline 1 & 23.393917 & 5.338955 & 3.4314 \\
\hline Al & 13.4 & 19.816084 & \\
\hline Al & 21.787182 & 13.672425 & 7.23431 \\
\hline Al & 16.331465 & 4.474294 & 14.06761 \\
\hline 1 & 6.316979 & 17.990149 & 18.01053 \\
\hline 0 & 22.168049 & 0.421893 & 2.78381 \\
\hline 0 & 24.615414 & 24.604004 & 3.960 \\
\hline 0 & 24.098608 & 2.112376 & \\
\hline 0 & 22.838757 & 2.182772 & 4.55051 \\
\hline 0 & 22.167564 & 12.491708 & 15.08957 \\
\hline 0 & 24.601820 & 12.465249 & 16.16639 \\
\hline 0 & 24.017775 & 14.326140 & 14.33435 \\
\hline 0 & 22.914251 & 14.402605 & 1 \\
\hline 0 & 10.0 & 0.249300 & 15.17308 \\
\hline 0 & 12.552880 & 24.616386 & 16.09648 \\
\hline 0 & 11.645982 & 2.108492 & 14.33196 \\
\hline 0 & 10.703400 & 2.107035 & 16.86113 \\
\hline 0 & 9.946517 & 12.478601 & 2.8796 \\
\hline 0 & 12.475687 & 12.473261 & 989 \\
\hline 0 & 11.718320 & 14.319100 & 2.11019 \\
\hline 0 & 10.712866 & 14.308419 & 4.61897 \\
\hline 0 & 8.930140 & 6.407765 & 2.87144 \\
\hline 0 & 6.409465 & 6.418447 & 3.87738 \\
\hline 0 & 7.085270 & 4.620427 & 2.09902 \\
\hline 0 & 8.179326 & 4.558770 & 4.55221 \\
\hline 0 & 8.931110 & 18.877384 & 15.17939 \\
\hline 0 & 6.227162 & 18.632696 & 16.26956 \\
\hline 0 & 7.170474 & 16.880556 & 14.00183 \\
\hline 0 & 7.758890 & 16.859194 & 16.93614 \\
\hline O & 21.152399 & 6.332272 & 9587 \\
\hline 0 & 18.629055 & 6.336156 & 16.01735 \\
\hline 0 & 19.308502 & 4.542506 & 14.23899 \\
\hline 0 & 20.479265 & 4.548332 & 16.69558 \\
\hline 0 & 21.227409 & 18.625414 & 2.86294 \\
\hline 0 & 18.707949 & 18.717173 & 3.87738 \\
\hline 0 & 19.298307 & 16.860409 & 2.11868 \\
\hline 0 & 20.470284 & 16.855795 & 4.53910 \\
\hline
\end{tabular}




$$
\begin{aligned}
& \begin{array}{lll}
8.855860 & 0.260466 & 3.794363
\end{array} \\
& \begin{array}{lll}
6.333486 & 24.614927 & 2.777014
\end{array} \\
& \begin{array}{lll}
7.087698 & 2.111890 & 4.566295
\end{array} \\
& \begin{array}{lll}
8.181753 & 2.110191 & 2.119901
\end{array} \\
& \begin{array}{lll}
8.938393 & 12.386600 & 15.842090
\end{array} \\
& \begin{array}{lll}
6.388103 & 12.386356 & 15.082294
\end{array} \\
& \begin{array}{lll}
7.165619 & 14.163257 & 16.871574
\end{array} \\
& \begin{array}{lll}
8.020571 & 14.412072 & 14.399207
\end{array} \\
& \begin{array}{lll}
21.145847 & 0.338145 & 16.102554
\end{array} \\
& \begin{array}{lll}
18.636095 & 24.617842 & 15.077682
\end{array} \\
& \begin{array}{lll}
19.301220 & 2.180102 & 16.845600
\end{array} \\
& \begin{array}{lll}
20.479507 & 2.118930 & 14.404790
\end{array} \\
& \begin{array}{lll}
21.313583 & 12.468648 & 3.547733
\end{array} \\
& \begin{array}{lll}
18.704792 & 12.553123 & 2.780655
\end{array} \\
& \begin{array}{lll}
19.563385 & 14.240451 & 4.627710
\end{array} \\
& 20.474653 \quad 14.412315 \quad 2.114560 \\
& \begin{array}{lll}
22.331417 & 6.566522 & 3.891461
\end{array} \\
& \begin{array}{lll}
24.782667 & 6.327417 & 2.613889
\end{array} \\
& \begin{array}{lll}
24.180656 & 4.477935 & 4.711457
\end{array} \\
& \begin{array}{lll}
22.757923 & 4.469439 & 2.113347
\end{array} \\
& \begin{array}{lll}
22.092556 & 18.618132 & 16.089447
\end{array} \\
& \begin{array}{lll}
24.600365 & 18.708920 & 15.100500
\end{array} \\
& \begin{array}{lll}
23.949804 & 16.865263 & 16.860651
\end{array} \\
& \begin{array}{lll}
22.917891 & 16.774719 & 14.405033
\end{array} \\
& \begin{array}{lll}
9.945303 & 6.408252 & 16.096243
\end{array} \\
& \begin{array}{lll}
12.483455 & 6.419175 & 15.094916
\end{array} \\
& \begin{array}{lll}
11.722446 & 4.570179 & 16.839291
\end{array} \\
& \begin{array}{lll}
10.702428 & 4.635478 & 14.333179
\end{array} \\
& \begin{array}{lll}
9.861071 & 18.466902 & 3.809898
\end{array} \\
& \begin{array}{lll}
12.306008 & 18.707949 & 2.704190
\end{array} \\
& \begin{array}{lll}
11.804252 & 16.771807 & 4.474051
\end{array} \\
& \begin{array}{lll}
10.605330 & 16.769865 & 2.099996
\end{array} \\
& \begin{array}{llr}
2.947664 & 22.249126 & 0.247844
\end{array} \\
& \begin{array}{lll}
4.037837 & 24.691153 & 24.606674
\end{array} \\
& \begin{array}{lll}
2.095869 & 24.034765 & 2.035425
\end{array} \\
& \begin{array}{lll}
4.478178 & 22.844826 & 2.201221
\end{array} \\
& \begin{array}{lll}
2.872899 & 9.947245 & 12.474959
\end{array} \\
& \begin{array}{lll}
3.802373 & 12.464765 & 12.483698
\end{array} \\
& \begin{array}{lll}
2.033240 & 11.721961 & 14.322256
\end{array} \\
& \begin{array}{lll}
4.476722 & 10.699759 & 14.395081
\end{array} \\
& \begin{array}{lll}
15.166284 & 22.168049 & 12.397767
\end{array} \\
& \begin{array}{lll}
16.176350 & 24.608374 & 12.475203
\end{array} \\
& \begin{array}{lll}
14.324198 & 23.930141 & 14.248704
\end{array} \\
& \begin{array}{lll}
16.776903 & 22.840942 & 14.403334
\end{array} \\
& \begin{array}{lll}
15.171381 & 9.873936 & 0.343971
\end{array} \\
& \begin{array}{lll}
16.102070 & 12.454568 & 24.534336
\end{array} \\
& \begin{array}{lll}
14.313517 & 11.719776 & 2.035182
\end{array} \\
& \begin{array}{lll}
16.855068 & 10.718206 & 2.104365
\end{array} \\
& \begin{array}{lll}
2.858091 & 8.865569 & 6.403397
\end{array} \\
& \begin{array}{lll}
3.878838 & 6.402425 & 6.410193
\end{array} \\
& \begin{array}{lll}
2.122814 & 6.995697 & 4.557556
\end{array} \\
& \begin{array}{lll}
4.483033 & 8.174228 & 4.474294
\end{array} \\
& \begin{array}{lll}
2.867073 & 21.061127 & 18.723484
\end{array} \\
& \begin{array}{lll}
4.223780 & 18.617405 & 18.621531
\end{array} \\
& \begin{array}{lll}
2.277443 & 19.221355 & 16.847786
\end{array} \\
& \begin{array}{lll}
4.644702 & 20.568111 & 16.922066
\end{array} \\
& \begin{array}{lll}
15.084964 & 8.942034 & 18.630512
\end{array} \\
& \begin{array}{llll}
0 & 16.093330 & 6.427914 & 18.701880
\end{array}
\end{aligned}
$$




$$
\begin{aligned}
& 14.413286 \\
& 7.162949 \\
& 8.178112 \\
& \begin{array}{r}
16.931532 \\
15.178422
\end{array} \\
& 21.322565 \\
& 18.798735 \\
& 16.183876 \\
& 14.324198 \\
& 16.838076 \\
& 3.798247 \\
& 2.708802 \\
& 4.638391 \\
& 2.194181 \\
& 3.788537 \\
& 2.951306 \\
& 4.631593 \\
& 2.194181 \\
& 16.016138 \\
& 15.179877 \\
& 16.856766 \\
& 14.323713 \\
& 16.092117 \\
& 15.085692 \\
& 16.938087 \\
& 14.428578 \\
& 3.792178 \\
& 2.704190 \\
& 4.628195 \\
& 2.270403 \\
& 3.791207 \\
& 2.789880 \\
& 4.566781 \\
& 2.105579 \\
& 16.090176 \\
& 14.918441 \\
& 16.834677 \\
& 14.420568 \\
& 16.082407 \\
& 15.167254 \\
& 16.926678 \\
& 14.399693 \\
& 0.339844 \\
& 24.605219 \\
& 2.116745 \\
& 2.191025 \\
& 0.423106 \\
& 24.608374 \\
& 2.112861 \\
& 2.190297 \\
& 19.382296 \\
& 20.471254 \\
& 8.849791 \\
& 6.405338 \\
& 7.085756 \\
& 8.259675 \\
& 21.230806 \\
& 18.630754 \\
& 19.459490 \\
& 20.400373 \\
& 8.847120 \\
& 6.252166 \\
& 7.325346 \\
& 8.257005 \\
& 21.233477 \\
& 18.717415 \\
& 19.462160 \\
& 20.566410 \\
& 22.170961 \\
& 24.599634 \\
& 24.016075 \\
& 22.842154 \\
& 9.958654 \\
& 12.485397 \\
& 11.729972 \\
& 10.691748 \\
& 22.082361 \\
& 24.520985 \\
& 23.932327 \\
& 22.758650 \\
& 9.960596 \\
& 12.486126 \\
& 11.797455 \\
& 10.789816 \\
& 2.864403 \\
& 3.965013 \\
& 2.120143 \\
& 4.545904 \\
& 15.161429 \\
& 16.168339 \\
& 14.328325 \\
& 16.844387 \\
& 2.871442 \\
& \text { 3. } 804315 \\
& \text { 2. } 111647 \\
& 4.558284 \\
& 14.342403 \\
& 14.251132 \\
& 12.559919 \\
& 12.405777 \\
& 14.400420 \\
& 14.336578 \\
& 6.341011 \\
& 6.327660 \\
& 4.568965 \\
& 4.466283
\end{aligned}
$$




\begin{tabular}{|c|c|c|c|}
\hline & 6.561667 & 15.081080 & 21.064770 \\
\hline 0 & 6.234202 & 16.172224 & 18.613762 \\
\hline O & 4.558770 & 14.329053 & 19.383511 \\
\hline 0 & 4.654169 & 16.849728 & 20.496744 \\
\hline 0 & 18.704550 & 2.943781 & 21.073023 \\
\hline 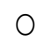 & 18.797035 & 4.049489 & 18.700422 \\
\hline 0 & 16.940758 & 2.193210 & 19.13008 \\
\hline O & 16.782972 & 4.552216 & 20.3979 \\
\hline O & 18.709646 & 15.173809 & 8.947132 \\
\hline 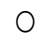 & 18.792910 & 16.025362 & 6.41310 \\
\hline O & 16.924250 & 14. & 7.092309 \\
\hline 0 & 16.927162 & 16.857010 & 8.092909 \\
\hline 0 & 0.401259 & 3.899957 & 8.95587 \\
\hline O & 24.518801 & 2.618744 & 6.573076 \\
\hline 0 & 2.035668 & 4.560954 & 6.992298 \\
\hline O & 2.193938 & 2.189083 & \\
\hline 0 & 0.426019 & 16.103041 & 21.156527 \\
\hline 0 & 24.687265 & 15.089334 & 18.62856 \\
\hline 0 & 2.266277 & 16.849728 & 19.302191 \\
\hline O & 2.191511 & 14.406246 & 20.469313 \\
\hline V & 12.389028 & 3.796062 & 21. \\
\hline 0 & 12.470105 & 2 . & 18. \\
\hline 0 & 14.230984 & 4.7 & 19.388365 \\
\hline 0 & 14.337306 & 2.272588 & 20.484848 \\
\hline o & 12.568902 & 16.096243 & 8.927712 \\
\hline O & 12.478115 & 15.166284 & 6.418690 \\
\hline 0 & 14.41 & 16. & \\
\hline 0 & 14.417170 & 0906 & 8.094365 \\
\hline 0 & 6.410436 & 3.881994 & 22.158339 \\
\hline 0 & 6.407523 & 2.786481 & 24.606190 \\
\hline 0 & 4.626010 & 4.641789 & 24.021172 \\
\hline 0 & 4.534495 & 2.265548 & 22.772488 \\
\hline 0 & 6.337370 & 16. & 34 \\
\hline 0 & 6.488843 & 14.993205 & 12.297997 \\
\hline 0 & 4.715584 & 16.849970 & 11.722932 \\
\hline 0 & 4.546632 & 14.326383 & 10.700244 \\
\hline 0 & 18.542639 & 3.892918 & 9.946517 \\
\hline 0 & 18.870344 & 2 . & 12.381745 \\
\hline 0 & 16.8 & 4. & 12.048697 \\
\hline 0 & 16.856766 & 2.108977 & 10.703885 \\
\hline 0 & 18.627113 & 16.101583 & 22.082361 \\
\hline 0 & 18.720814 & 15.086421 & 24.530939 \\
\hline 8 & 16.849728 & 16.85652 & 23.937910 \\
\hline 0 & 16.7 & 14. & 22.837786 \\
\hline 0 & 2.864160 & 24.679014 & 22.157612 \\
\hline 0 & 0.433544 & 0.423106 & 20.989759 \\
\hline 0 & 0.936757 & 22.932457 & 22.905025 \\
\hline 0 & 2.194667 & 22.762291 & 20.557430 \\
\hline 0 & 2.873870 & 12.476658 & 9.955013 \\
\hline 0 & 0.425776 & 12.5 & 8.936208 \\
\hline 0 & 1.012736 & 10.702671 & 10.70437 \\
\hline 0 & 2.113104 & 10.620137 & 8.255305 \\
\hline 0 & 15.085935 & 24.686054 & 9.938749 \\
\hline 0 & 12.555065 & 0.499571 & 8.928683 \\
\hline 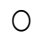 & 13.238153 & 22.916193 & 10.624992 \\
\hline 0 & 14.325655 & 23.006495 & 8.109900 \\
\hline 0 & 15.080353 & 12.563561 & 22.08454 \\
\hline & 12 & 12 & 2 \\
\hline
\end{tabular}




\begin{tabular}{|c|c|c|c|}
\hline & 13.315346 & 10.703642 & 22.919834 \\
\hline & 14.249190 & 10.704370 & 20.397461 \\
\hline & 16.103525 & 18.701151 & 22.164410 \\
\hline & 18.623714 & 18.621288 & 21.153130 \\
\hline & 18.037241 & 20.469070 & 22.912066 \\
\hline & 16.856525 & 20.477566 & 20.478781 \\
\hline & 16.188730 & 6.481318 & 10.030507 \\
\hline & 18.694839 & 6.405338 & 8.946404 \\
\hline & 18.028017 & 8.348278 & 10.622322 \\
\hline & 16.864534 & 8.171315 & 8.185881 \\
\hline & 3.800917 & 18.709890 & 10.029536 \\
\hline & 6.318678 & 18.623230 & 9.022383 \\
\hline & 5.654768 & 20.467857 & 10.782534 \\
\hline & 4.470896 & 20.481936 & 8.341480 \\
\hline & 3.875440 & 6.409223 & 22.170235 \\
\hline & 6.331301 & 6.317707 & 21.073265 \\
\hline & 5.740214 & 8.261617 & 22.760592 \\
\hline & 4.552701 & 8.180540 & 20.396732 \\
\hline & 16.181448 & 24.778055 & 21.239304 \\
\hline & 18.706978 & 0.420679 & 22.171934 \\
\hline & 17.955193 & 22.927601 & 20.398188 \\
\hline & 16.874973 & 22.918621 & 22.846767 \\
\hline & 16.097458 & 12.556765 & 8.943005 \\
\hline & 18.535357 & 12.726687 & 10.040460 \\
\hline & 18.034328 & 10.781806 & 8.345365 \\
\hline & 16.860409 & 10.790059 & 10.783262 \\
\hline & 3.794848 & 24.610802 & 8.933052 \\
\hline & 6.320377 & 0.427476 & 9.954285 \\
\hline & 5.659623 & 22.832203 & 8.253849 \\
\hline & 4.558284 & 22.924202 & 10.729615 \\
\hline & 3.779798 & 12.473261 & 21.154585 \\
\hline & 6.167933 & 12.657990 & 22.238932 \\
\hline & 5.649185 & 10.694903 & 20.475382 \\
\hline & 4.548089 & 10.706312 & 22.917406 \\
\hline & 2.953248 & 18.790724 & 21.051661 \\
\hline & 0.519719 & 18.540939 & 22.249126 \\
\hline & 0.928989 & 20.388964 & 20.400131 \\
\hline & 2.194909 & 20.557186 & 22.759378 \\
\hline & 2.780413 & 6.332757 & 8.868725 \\
\hline & 0.344699 & 6.398784 & 10.021525 \\
\hline & 1.005697 & 8.183210 & 8.173500 \\
\hline & 2.114803 & 8.251422 & 10.616982 \\
\hline & 15.166284 & 18.628084 & 8.941063 \\
\hline & 12.634201 & 18.622501 & 9.858157 \\
\hline ) & 13.390111 & 20.475624 & 8.103104 \\
\hline & 14.331723 & 20.472954 & 10.618923 \\
\hline & 15.169683 & 6.405338 & 21.234692 \\
\hline 0 & 12.640997 & 6.250224 & 22.174118 \\
\hline 0 & 13.301752 & 8.179326 & 20.469313 \\
\hline 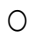 & 14.327111 & 8.170344 & 22.920319 \\
\hline 0 & 22.179216 & 2.714628 & 24.613472 \\
\hline 0 & 20.996315 & 0.339602 & 0.331591 \\
\hline o & 22.916193 & 0.920250 & 22.831717 \\
\hline 0 & 20.567625 & 2.191025 & 22.682913 \\
\hline ) & 22.157125 & 15.081567 & 12.557493 \\
\hline 0 & 21.233963 & 12.562347 & 12.561863 \\
\hline 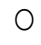 & 22.937553 & 13.314375 & 10.701457 \\
\hline & 20.472954 & 14.334394 & 10.698545 \\
\hline
\end{tabular}




\begin{tabular}{|c|c|c|}
\hline .9 & 2.959802 & 12.4679 \\
\hline .931354 & 0.512437 & 12.640754 \\
\hline 10.624264 & 1.082404 & 10.699272 \\
\hline 8.17956 & 2.182529 & 63251 \\
\hline 9.864470 & 15.087877 & 610315 \\
\hline 8.862170 & 12.552638 & 0.42771 \\
\hline 10.706798 & 13.233297 & 22.923 \\
\hline 8.18515 & 14.247490 & \\
\hline 22.17533 & 16.086777 & 18.69 \\
\hline 21.16089 & 15948 & 18.623 \\
\hline 23.00212 & 17. & \\
\hline $20 \cdot$ & 16.86502 & \\
\hline 22.1 & 3.870827 & \\
\hline 21.073994 & 6.332515 & 6.37 \\
\hline $22 . \varepsilon$ & 33660 & 8.185 \\
\hline 20.471012 & 5925 & \\
\hline 9.9 & 16. & \\
\hline 9.0 & 19 & \\
\hline 10.70631 & 17.871689 & 8.185 \\
\hline 8.196075 & 16.927649 & 8.160 \\
\hline 9.949430 & 3.800917 & 18.705 \\
\hline & & \\
\hline 10.6 & 5.7 & 20. \\
\hline 8.2562 & 4.561683 & 20.483 \\
\hline 21.245129 & 16.175623 & 24.7634 \\
\hline 22.325350 & 18.634153 & 0.422 \\
\hline 20 . & 17. & 22 . \\
\hline $22 . \varepsilon$ & 16. & 22 . \\
\hline 21.3 & 3.7 & 12. \\
\hline 22.2289 & 6.318921 & 12.5465 \\
\hline 20.39236 & 5.570292 & 10.7888 \\
\hline $22 . \varepsilon$ & 52701 & 10 \\
\hline & 1 & \\
\hline 9.7 & 18. & 12. \\
\hline 8.1 & 17.778959 & 10.7043 \\
\hline 10.784233 & 16.939785 & 10.70461 \\
\hline 8.92 & 3.807228 & 24.6120 \\
\hline 10. & & \\
\hline 8. & & 22 \\
\hline 10.6 & 2168 & 22 . \\
\hline 21.233477 & 2.877511 & 18.5336 \\
\hline 22.32583 & 0.423592 & 18.54093 \\
\hline 20.41 & 1.0 & 20 . \\
\hline $22 . \varepsilon$ & & \\
\hline 21.3 & 15. & 6.246 \\
\hline 22.3333 & 12.637598 & 5.9965 \\
\hline 20.39503 & 13.306365 & 8.1720 \\
\hline 23.0084 & 14.417413 & 8.1793 \\
\hline 8.8 & & 6.4844 \\
\hline 10. & 0.5 & 6.2378 \\
\hline & 1.019533 & 8.1834 \\
\hline 10.617467 & 2.192724 & 8.25821 \\
\hline 8.758275 & 15.180848 & 18.78756 \\
\hline 9.95210 & 12.64925 & 18.219 \\
\hline & & \\
\hline 10.708254 & 14.331238 & 20.48533 \\
\hline 24.68 & 22.164894 & 2.862 \\
\hline & 21. & \\
\hline
\end{tabular}




$$
\begin{array}{rrr}
22.934156 & 22.919348 & 0.932145 \\
22.834387 & 20.544807 & 2.184714 \\
24.609346 & 9.870538 & 15.164827 \\
0.422864 & 8.930625 & 12.572785 \\
22.847494 & 10.620137 & 13.310248 \\
22.926874 & 8.103346 & 14.332694 \\
12.472047 & 22.164410 & 15.080837 \\
12.649736 & 21.238089 & 12.551424 \\
10.625478 & 22.841913 & 13.218246 \\
10.784962 & 20.388721 & 14.172726 \\
12.474716 & 9.951857 & 2.936498 \\
12.569873 & 8.938878 & 0.433059 \\
10.705098 & 10.705098 & 1.016863 \\
10.629119 & 8.265016 & 2.114318 \\
18.704792 & 22.167807 & 16.097700 \\
18.612549 & 21.142691 & 18.617405 \\
20.496744 & 22.856478 & 18.022676 \\
20.482664 & 20.476353 & 16.856281 \\
18.794607 & 10.021282 & 3.634150 \\
18.714502 & 8.999323 & 6.165991 \\
20.494072 & 10.794186 & 5.480234 \\
20.549419 & 8.272056 & 4.466283 \\
6.322320 & 22.089643 & 3.793391 \\
6.235173 & 20.980293 & 6.415291 \\
8.185394 & 22.936340 & 5.831001 \\
8.362842 & 20.466158 & 4.479877 \\
6.320135 & 9.863012 & 16.091146 \\
6.324504 & 8.950530 & 18.616190 \\
8.171073 & 10.708011 & 17.789640 \\
8.183453 & 8.172286 & 16.925949 \\
24.865200 & 21.223282 & 16.187031 \\
0.428447 & 22.253738 & 18.623714 \\
22.992901 & 20.381195 & 17.874357 \\
22.908424 & 22.846039 & 16.770836 \\
24.603521 & 8.774297 & 3.875440 \\
0.431845 & 10.021525 & 6.305570 \\
22.837786 & 8.268657 & 5.827360 \\
22.932215 & 10.612126 & 4.388848 \\
12.541957 & 21.405342 & 3.724694 \\
12.634929 & 22.234806 & 6.246339 \\
10.881818 & 20.315413 & 5.477078 \\
10.634945 & 22.916435 & 4.634992 \\
12.477872 & 8.922372 & 16.027061 \\
12.564046 & 9.870052 & 18.545307 \\
10.696116 & 8.167432 & 17.948397 \\
10.628877 & 10.542701 & 16.762096 \\
18.709890 & 21.226194 & 2.956646 \\
18.605267 & 22.253252 & 0.486706 \\
20.402800 & 20.388237 & 1.015164 \\
20.572481 & 22.774672 & 2.192724 \\
18.800676 & 8.930383 & 15.248818 \\
18.455736 & 9.865197 & 12.729357 \\
20.480965 & 8.252150 & 13.234998 \\
20.399158 & 10.719663 & 14.325169 \\
6.589582 & 21.071566 & 15.171138 \\
6.230319 & 22.169506 & 12.716006 \\
8.248508 & 20.489702 & 13.156348 \\
8.183938 & 22.922504 & 14.331965
\end{array}
$$




$\begin{array}{rrrr}\mathrm{O} & 6.403397 & 8.927955 & 2.873627 \\ \mathrm{O} & 6.317464 & 9.946759 & 0.422621 \\ \mathrm{O} & 8.182482 & 8.170344 & 1.010552 \\ \mathrm{O} & 8.256034 & 10.621351 & 2.112133 \\ \mathrm{O} & 13.312433 & 5.229962 & 8.423043 \\ \mathrm{O} & 12.473502 & 6.492727 & 6.055784 \\ \mathrm{O} & 11.809350 & 7.747967 & 8.433967 \\ \mathrm{O} & 14.485868 & 7.750879 & 7.585812 \\ \mathrm{O} & 8.597578 & 17.524076 & 19.899830 \\ \mathrm{O} & 8.849548 & 19.897646 & 17.693998 \\ \mathrm{O} & 7.157123 & 20.325850 & 20.066597 \\ \mathrm{O} & 10.623293 & 17.363621 & 17.530630 \\ \mathrm{H} & 12.794656 & 22.170719 & 3.172690 \\ \mathrm{H} & 15.868548 & 7.979546 & 7.931968 \\ \mathrm{H} & 25.033909 & 6.083700 & 1.695581 \\ \mathrm{H} & 21.953218 & 16.011768 & 6.489814 \\ \mathrm{H} & 13.677766 & 5.011491 & 9.355916 \\ \mathrm{H} & 8.956599 & 16.046482 & 19.287140 \\ \mathrm{H} & 9.169730 & 19.361420 & 16.028519 \\ \mathrm{H} & 9.488698 & 18.589245 & 21.494671 \\ \mathrm{H} & 10.578870 & 18.267849 & 20.010765 \\ \mathrm{H} & 12.412331 & 16.411814 & 17.321383 \\ \mathrm{H} & 12.197986 & 18.080935 & 16.455023 \\ \mathrm{Re} & 13.010456 & 6.836941 & 7.630963 \\ \mathrm{Re} & 8.213310 & 19.345156 & 19.191498 \\ \mathrm{C} & 9.567833 & 18.373930 & 20.421978 \\ \mathrm{C} & 11.766141 & 17.283514 & 17.094416 \\ \mathrm{H} & & & \end{array}$

\begin{tabular}{lrrr} 
G07 & \multicolumn{4}{l}{$r$} \\
USY_ReO3_C $4 \mathrm{H} 8{ }^{1} \mathrm{E}=-7249.21408600677$ \\
$\mathrm{Si}$ & 23.380566 & 3.327805 & 1.238976 \\
$\mathrm{Si}$ & 23.416250 & 1.257424 & 3.371499 \\
$\mathrm{Si}$ & 23.406296 & 15.563902 & 13.393753 \\
$\mathrm{Si}$ & 23.403625 & 13.407347 & 15.544482 \\
$\mathrm{Si}$ & 11.224089 & 1.205962 & 15.627745 \\
$\mathrm{Si}$ & 11.195930 & 15.585021 & 1.222712 \\
$\mathrm{Si}$ & 11.232828 & 13.395208 & 3.370043 \\
$\mathrm{Si}$ & 7.646499 & 3.361061 & 1.235092 \\
$\mathrm{Si}$ & 7.662277 & 5.506936 & 3.352565 \\
$\mathrm{Si}$ & 7.679270 & 15.499331 & 13.258301 \\
$\mathrm{Si}$ & 19.952507 & 5.471980 & 15.503700 \\
$\mathrm{Si}$ & 19.907843 & 15.637697 & 1.270290 \\
$\mathrm{Si}$ & 19.911240 & 17.800077 & 3.351837 \\
$\mathrm{Si}$ & 7.623681 & 3.376597 & 5.518102 \\
$\mathrm{Si}$ & 7.552556 & 15.526519 & 17.687201 \\
$\mathrm{Si}$ & 7.670774 & 13.307093 & 15.519722 \\
$\mathrm{Si}$ & 19.937700 & 3.388491 & 17.730167 \\
$\mathrm{Si}$ & 19.950565 & 1.224168 & 15.621918 \\
$\mathrm{Si}$ & 19.940613 & 15.594488 & 5.409837 \\
$\mathrm{Si}$ & 19.995716 & 13.360981 & 3.273430 \\
$\mathrm{Si}$ & 23.457029 & 3.344554 & 5.539464 \\
$\mathrm{Si}$ & 23.349009 & 17.730896 & 15.538657 \\
$\mathrm{Si}$ & 11.274823 & 3.341641 & 17.767065 \\
$\mathrm{Si}$ & 11.236712 & 5.543833 & 15.551280 \\
$\mathrm{Si}$ & 11.239625 & 15.642551 & 5.444064 \\
$\mathrm{Si}$ & 11.192289 & 17.789640 & 3.232163 \\
$\mathrm{Si}$ & 1.267377 & 23.409695 & 3.371256
\end{tabular}




$\begin{array}{lrrr} & & & \\ \mathrm{Si} & 3.364702 & 23.436882 & 1.221741 \\ \mathrm{Si} & 1.223683 & 11.193746 & 15.575554 \\ \mathrm{Si} & 3.361546 & 11.223118 & 13.377975 \\ \mathrm{Si} & 13.459294 & 23.345367 & 15.539628 \\ \mathrm{Si} & 15.638910 & 23.325462 & 13.408074 \\ \mathrm{Si} & 13.410259 & 11.234042 & 3.357177 \\ \mathrm{Si} & 15.589391 & 11.199815 & 1.223440 \\ \mathrm{Si} & 1.318111 & 7.705971 & 3.311784 \\ \mathrm{Si} & 3.364945 & 7.610330 & 5.494313 \\ \mathrm{Si} & 1.270775 & 19.893276 & 15.588177 \\ \mathrm{Si} & 3.400629 & 19.877985 & 17.718758 \\ \mathrm{Si} & 13.449827 & 7.670045 & 15.576525 \\ \mathrm{Si} & 15.640853 & 7.674900 & 17.772890 \\ \mathrm{Si} & 15.596430 & 19.962460 & 5.512276 \\ \mathrm{Si} & 5.524656 & 7.656209 & 3.357905 \\ \mathrm{Si} & 3.413980 & 7.666890 & 1.192611 \\ \mathrm{Si} & 5.433869 & 20.039167 & 15.611723 \\ \mathrm{Si} & 3.316881 & 19.946438 & 13.390840 \\ \mathrm{Si} & 17.812458 & 7.698689 & 15.621190 \\ \mathrm{Si} & 15.578466 & 7.696747 & 13.442545 \\ \mathrm{Si} & 17.756384 & 19.962944 & 3.384365 \\ \mathrm{Si} & 15.594731 & 20.030186 & 1.220527 \\ \mathrm{Si} & 5.458629 & 23.411880 & 3.385821 \\ \mathrm{Si} & 3.348924 & 23.408237 & 5.540434 \\ \mathrm{Si} & 5.469067 & 11.150294 & 15.598615 \\ \mathrm{Si} & 3.327562 & 11.191561 & 17.763908 \\ \mathrm{Si} & 17.757841 & 23.397800 & 15.621190 \\ \mathrm{Si} & 15.569242 & 23.350950 & 17.736721 \\ \mathrm{Si} & 17.805902 & 11.228216 & 3.318338 \\ \mathrm{Si} & 15.666341 & 11.228944 & 5.481690 \\ \mathrm{Si} & 3.374412 & 1.284126 & 23.368429 \\ \mathrm{Si} & 1.241403 & 3.363246 & 23.415279 \\ \mathrm{Si} & 3.358633 & 13.405648 & 11.229429 \\ \mathrm{Si} & 1.257910 & 15.603470 & 11.255403 \\ \mathrm{Si} & 15.625560 & 1.187756 & 11.222876 \\ \mathrm{Si} & 13.407347 & 3.272459 & 11.286717 \\ \mathrm{Si} & 15.572155 & 13.435506 & 23.425716 \\ \mathrm{Si} & 13.487696 & 15.593517 & 23.448534 \\ \mathrm{Si} & 3.342127 & 1.216643 & 7.664705 \\ \mathrm{Si} & 5.481933 & 3.331203 & 7.614214 \\ \mathrm{Si} & 3.295762 & 13.350301 & 19.893276 \\ \mathrm{Si} & 17.776047 & 3.381695 & 19.853466 \\ \mathrm{Si} & 15.650561 & 13.422882 & 7.637032 \\ \mathrm{Si} & 17.813915 & 15.572155 & 7.654994 \\ \mathrm{Si} & 3.313240 & 5.444550 & 7.578530 \\ \mathrm{Si} & 1.225139 & 3.318095 & 7.655966 \\ \mathrm{Si} & 3.400871 & 17.727497 & 19.894976 \\ \mathrm{Si} & 1.231208 & 15.583564 & 19.859291 \\ \mathrm{Si} & 15.583564 & 5.498682 & 19.934786 \\ \mathrm{Si} & 13.414145 & 3.387763 & 19.934301 \\ \mathrm{Si} & 15.665370 & 17.774347 & 7.637032 \\ \mathrm{Si} & 13.474344 & 15.640367 & 7.623923 \\ \mathrm{Si} & 3.382423 & 5.489458 & 23.406054 \\ \mathrm{Si} & 5.510334 & 3.388977 & 23.407024 \\ \mathrm{Si} & 5.522714 & 15.595702 & 11.126263 \\ \mathrm{Si} & 17.857851 & 3.287995 & 11.226031 \\ \mathrm{Si} & 15.646921 & 17.793524 & 23.441010 \\ \mathrm{Si} & 17.732838 & 15.629443 & 23.429358\end{array}$




\begin{tabular}{|c|c|c|c|}
\hline$i$ & 1.642419 & 21.683771 & 23.736673 \\
\hline $\mathrm{Si}$ & 1.616203 & 23.792505 & 21.61604 \\
\hline i & 1.671306 & 9.440392 & 11.572673 \\
\hline $\mathrm{Si}$ & 1.663781 & 11.604715 & 9.448888 \\
\hline $\mathrm{Si}$ & 13.875361 & 21.668236 & 11.55568 \\
\hline $\mathrm{Si}$ & 13.834336 & 23.851492 & 9.3879 \\
\hline$i$ & 13.848903 & 9.429954 & 23.812168 \\
\hline$i$ & 13.812004 & 11.631417 & $21.67 \varepsilon$ \\
\hline i & 17.444212 & 21.697123 & 23.775 \\
\hline$S I$ & 17.426977 & 19.570423 & 21.726 \\
\hline $\mathrm{Si}$ & 17.336433 & 9.464423 & 11.5913 \\
\hline $\mathrm{Si}$ & 17.385712 & 7.242812 & \\
\hline$i$ & 5.066351 & 21.681587 & 11.61806 \\
\hline$i$ & 5.087471 & 19.549305 & 9.486 \\
\hline $\mathrm{Si}$ & 5.152041 & 9.468551 & 23.735 \\
\hline i & 5.147672 & 7.288692 & 21.644 \\
\hline $\mathrm{Si}$ & 17.374060 & 21.739117 & 19.521 \\
\hline $\mathrm{Si}$ & 17.397362 & 23.908052 & 21.6553 \\
\hline $\mathrm{Si}$ & 17.389595 & 11.679238 & 9.498 \\
\hline $\mathrm{Si}$ & 5.090869 & 21.603664 & 7.30252 \\
\hline $\mathrm{Si}$ & 5.097666 & 23.773087 & 9.4311 \\
\hline $\mathrm{Si}$ & 5.114658 & 9.470007 & 19.55950 \\
\hline $\mathrm{Si}$ & 5.109075 & 11.635301 & 21. \\
\hline $\mathrm{Si}$ & 1.573480 & 21.610462 & 19.57309 \\
\hline $\mathrm{Si}$ & 1.601153 & 19.542023 & 21.6269 \\
\hline $\mathrm{Si}$ & 1.612319 & 9.451315 & 7.27024 \\
\hline $\mathrm{Si}$ & 1.594356 & 7.337241 & 9.3743 \\
\hline $\mathrm{Si}$ & 13.879488 & 21.743729 & 7 \\
\hline $\mathrm{Si}$ & 13.860796 & 19.523575 & 9.34499 \\
\hline $\mathrm{Si}$ & 13.853999 & 7.229947 & 21.69372 \\
\hline $\mathrm{Si}$ & 23.767746 & 1.595327 & 21.62769 \\
\hline $\mathrm{Si}$ & 21.669935 & 1.538039 & 23.70900 \\
\hline $\mathrm{Si}$ & 23.846880 & 13.867593 & 9.4248 \\
\hline $\mathrm{Si}$ & 21.709988 & 13.788700 & 11.564 \\
\hline $\mathrm{Si}$ & 11.553495 & 1.645332 & 9.47486 \\
\hline $\mathrm{Si}$ & 9.418787 & 1.711602 & 11.61903 \\
\hline $\mathrm{Si}$ & 11.677782 & 13.849874 & 21.72261 \\
\hline $\mathrm{Si}$ & 9.459569 & 13.796469 & 23.81168 \\
\hline $\mathrm{Si}$ & 23.796875 & 17.348085 & 21.69153 \\
\hline $\mathrm{Si}$ & 21.675760 & 17.369446 & 19.53085 \\
\hline $\mathrm{Si}$ & 21.681829 & 5.177772 & 7.24135 \\
\hline $\mathrm{Si}$ & 11.641370 & 17.358038 & 9.36853 \\
\hline $\mathrm{Si}$ & 9.439421 & 17.377459 & 7.18212 \\
\hline $\mathrm{Si}$ & 11.608356 & 5.119027 & 21.63473 \\
\hline $\mathrm{Si}$ & 9.490154 & 5.087956 & 19.53158 \\
\hline $\mathrm{Si}$ & 19.529886 & 17.385954 & 21.69566 \\
\hline $\mathrm{Si}$ & 21.714600 & 17.393963 & 23.82673 \\
\hline $\mathrm{Si}$ & 19.554403 & 5.067808 & 9.45107 \\
\hline $\mathrm{Si}$ & 21.739845 & 5.047903 & 11.62656 \\
\hline $\mathrm{Si}$ & 7.253736 & 17.352697 & 9.33212 \\
\hline $\mathrm{Si}$ & 9.438692 & 17.224043 & 11.51854 \\
\hline $\mathrm{Si}$ & 7.301799 & 5.140874 & 21.64930 \\
\hline $\mathrm{Si}$ & 9.416845 & 5.117571 & 23.77260 \\
\hline $\mathrm{Si}$ & 19.548334 & 1.646789 & 21.59759 \\
\hline $\mathrm{Si}$ & 21.669449 & 1.630525 & 19.49395 \\
\hline $\mathrm{Si}$ & 19.544693 & 13.819044 & 9.40131 \\
\hline $\mathrm{Si}$ & 7.294032 & 1.622029 & 9.42898 \\
\hline $\mathrm{Si}$ & 9.429468 & 1.666451 & 7.30010 \\
\hline
\end{tabular}




\begin{tabular}{|c|c|c|c|}
\hline Si & 7.296216 & 13.764911 & 21.650028 \\
\hline Si & 21.701248 & 23.752939 & 1.584888 \\
\hline & 23.820908 & 21.652943 & 1.613533 \\
\hline & 21.707802 & 11.597432 & 13.755201 \\
\hline & 23.836443 & 9.390629 & 13.78069 \\
\hline & 9.467580 & 23.835472 & 13.858 \\
\hline$S 1$ & 9.467822 & 11.597190 & 1.6079 \\
\hline & 11.616609 & 9.473890 & 1647517 \\
\hline & 21.713142 & 23.788378 & 17.4 \\
\hline & 19.546392 & 21.698822 & 17.41799 \\
\hline & 21.758295 & 11.636758 & 4.89691 \\
\hline & 19.610235 & 9.478745 & 5.0066 \\
\hline $\mathrm{Si}$ & 9.359800 & 23.835472 & 5.15543 \\
\hline i & 9.461025 & 11.552281 & 17.2992 \\
\hline - & 7.269271 & 9.418787 & 17.369 \\
\hline . & 21.669691 & 19.501728 & 17.33376 \\
\hline Si & 23.825033 & 21.642990 & 17.38498 \\
\hline i & 21.704163 & 7.337726 & 5.102520 \\
\hline Si & 23.783524 & 9.450344 & \\
\hline $\mathrm{Si}$ & 9.490154 & 19.5 & 4.97702 \\
\hline $\mathrm{Si}$ & 11.582624 & 21.771160 & 5.1185 \\
\hline Si & 9.446218 & 7.245239 & 17.35196 \\
\hline $\mathrm{Si}$ & 11.615638 & 9.345720 & 17.38012 \\
\hline $\mathrm{Si}$ & 21.677460 & 19.543238 & \\
\hline $\mathrm{Si}$ & 19.554403 & 21.64 & 1.64945 \\
\hline Si & 21.718969 & 7.241113 & 13.74160 \\
\hline $\mathrm{Si}$ & 19.557558 & 9.414417 & 13.78918 \\
\hline $\mathrm{Si}$ & 9.442333 & 19.585960 & 13.62776 \\
\hline $\mathrm{Si}$ & 7.282137 & 21.701248 & 13.781903 \\
\hline $\mathrm{Si}$ & 9.427769 & 7.301072 & \\
\hline $\mathrm{Si}$ & 7.301557 & 9.4 & 1.6 \\
\hline $\mathrm{Si}$ & 11.663217 & 21.704647 & 13.74379 \\
\hline Si & 11.191319 & 3.415436 & 13.39132 \\
\hline $\mathrm{Si}$ & 20.059315 & 3.297462 & 13.40176 \\
\hline $\mathrm{Si}$ & 7.590909 & 9 & 3.362 \\
\hline $\mathrm{Si}$ & 23.368914 & 15.5 & 17.67700 \\
\hline Si & 5.430713 & 15.515595 & 20.02630 \\
\hline $\mathrm{Si}$ & 3.421504 & 17.750072 & 11.28477 \\
\hline $\mathrm{Si}$ & 13.840648 & 9.407135 & 19.53692 \\
\hline $\mathrm{Si}$ & 23.802700 & 5.149856 & 9.40883 \\
\hline 1 & 15.598857 & 1.2 & 19.90954 \\
\hline Al & 15.612937 & 5.47 & 11.27579 \\
\hline Al & 17.523590 & 9.552054 & 7.22484 \\
\hline Al & 9.520497 & 13.650578 & 19.58887 \\
\hline Al & 7.256406 & 21.650028 & 5.16029 \\
\hline Al & 7.495025 & 17.728710 & 15.39325 \\
\hline Al & 23.404 & 5.3 & 3.42004 \\
\hline Al & 13.472159 & 19.8 & 3.29988 \\
\hline Al & 21.779413 & 13.655433 & 7.24791 \\
\hline Al & 16.462790 & 4.505365 & 14.03703 \\
\hline Al & 6.037821 & 17.990877 & 17.98529 \\
\hline 0 & 22.165380 & 0.349797 & 2.86585 \\
\hline 0 & 24.612017 & 24.602306 & 3.96015 \\
\hline 0 & 24.028212 & 2.102180 & 2.11091 \\
\hline 0 & 22.842154 & 2.194424 & 4.55585 \\
\hline O & 22.164894 & 12.474232 & 15.06554 \\
\hline 0 & 24.612261 & 12.463307 & 16.09600 \\
\hline 0 & 24.001509 & 14.338763 & 14.32856 \\
\hline
\end{tabular}




\begin{tabular}{|c|c|c|}
\hline 22.850653 & 14.339491 & 16.773020 \\
\hline 9.948944 & 0.247358 & 15.248575 \\
\hline 12.546813 & 24.611044 & 16.016138 \\
\hline 11.558836 & 2.118444 & 14.319587 \\
\hline 10.709952 & 2.104851 & 16.864779 \\
\hline 9.956470 & 12.482970 & 2.882366 \\
\hline 12.473989 & 12.470833 & 3.867914 \\
\hline 11.725844 & 14.326626 & 2.116017 \\
\hline 10.773796 & 14.319828 & 4.622855 \\
\hline 8.930140 & 6.410921 & 2.877754 \\
\hline 6.419661 & 6.417233 & 3.886121 \\
\hline 7.080657 & 4.634506 & 2.098296 \\
\hline 8.180782 & 4.556585 & 4.552701 \\
\hline 8.852946 & 18.871559 & 15.099286 \\
\hline 6.147057 & 18.630754 & 16.262281 \\
\hline 7.088426 & 16.781515 & 14.077326 \\
\hline 7.761803 & 16.946827 & 16.944641 \\
\hline 21.238575 & 6.324261 & 14.999760 \\
\hline 18.717899 & 6.403639 & 16.014196 \\
\hline 19.381325 & 4.552944 & 14.245791 \\
\hline 20.479507 & 4.543234 & 16.706507 \\
\hline 21.225224 & 18.622501 & 2.866345 \\
\hline 18.704306 & 18.723000 & 3.884421 \\
\hline 19.297577 & 16.850212 & 2.180344 \\
\hline 20.477324 & 16.852640 & 4.554158 \\
\hline 8.864112 & 0.331591 & 3.798489 \\
\hline 6.317221 & 24.686783 & 2.785753 \\
\hline 7.077745 & 2.114803 & 4.643974 \\
\hline 8.180054 & 2.189326 & 2.192967 \\
\hline 8.946646 & 12.459181 & 16.004244 \\
\hline 6.409950 & 12.396067 & 15.091518 \\
\hline 7.157365 & 14.244093 & 16.851669 \\
\hline 8.093637 & 14.329538 & 14.331965 \\
\hline 21.233721 & 0.339844 & 16.100370 \\
\hline 18.709162 & 24.607405 & 15.077197 \\
\hline 19.377199 & 2.118444 & 16.853127 \\
\hline 20.546021 & 2.119173 & 14.398236 \\
\hline 21.313583 & 12.467677 & 3.550160 \\
\hline 18.719357 & 12.483455 & 2.785753 \\
\hline 19.559744 & 14.237538 & 4.626253 \\
\hline 20.474653 & 14.402848 & 2.128154 \\
\hline 22.252768 & 6.497339 & 3.879567 \\
\hline 24.773928 & 6.395872 & 2.612918 \\
\hline 24.181627 & 4.534495 & 4.721409 \\
\hline 22.763264 & 4.482305 & 2.117230 \\
\hline 22.083332 & 18.626385 & 16.016138 \\
\hline 24.589926 & 18.703579 & 15.083750 \\
\hline 23.937910 & 16.838076 & 16.780788 \\
\hline 22.847010 & 16.779573 & 14.331965 \\
\hline 9.966422 & 6.475977 & 16.010313 \\
\hline 12.548755 & 6.404610 & 15.087149 \\
\hline 11.714922 & 4.564353 & 16.767679 \\
\hline 10.710439 & 4.707573 & 14.252346 \\
\hline 9.884374 & 18.616674 & 3.712557 \\
\hline 12.380774 & 18.704792 & 2.697151 \\
\hline 11.724874 & 16.848757 & 4.472352 \\
\hline 10.622079 & 16.848999 & 2.031541 \\
\hline 2.947422 & 22.176788 & 0.26192 \\
\hline
\end{tabular}




\begin{tabular}{|c|c|c|c|}
\hline 0 & 3.963799 & 24.611044 & 24.534336 \\
\hline 0 & 2.105336 & 24.018501 & 2.094170 \\
\hline 0 & 4.482790 & 22.853079 & 2.196366 \\
\hline 0 & 2.945723 & 9.943847 & 12.469134 \\
\hline O & 3.877382 & 12.476901 & 12.47326 \\
\hline 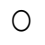 & 2.120386 & 11.729972 & 14.320314 \\
\hline 0 & 4.564838 & 10.699515 & 14.3380 \\
\hline 0 & 15.168955 & 22.0 & 12. \\
\hline 0 & 16.174892 & 24.534580 & 12.45044 \\
\hline 0 & 14.330509 & 23.853920 & 14.24433 \\
\hline 0 & 16.844145 & 22.843126 & 14.38537 \\
\hline 0 & 15.179877 & 9.887529 & 0.344457 \\
\hline 0 & 16.102798 & 12.467677 & 24.607891 \\
\hline 0 & 14.254530 & 11.715164 & 2.03178 \\
\hline 0 & 16.846329 & 10.710439 & 2.11310 \\
\hline 0 & 2.871442 & 8.851733 & 6.415049 \\
\hline 0 & 3.887334 & 6.329359 & 6.334214 \\
\hline 0 & 2.122328 & 7.072162 & \\
\hline 0 & 4.555129 & 8.180297 & 4.550031 \\
\hline 0 & 2.864160 & 20.909655 & 18.797035 \\
\hline 0 & 4.215769 & 18.529045 & 18.535112 \\
\hline 0 & 2.279870 & 19.221600 & 16.763554 \\
\hline O & 4.636691 & 20.573936 & 16.926920 \\
\hline 0 & 15.164827 & 8.936936 & 18.699451 \\
\hline 0 & 16.100857 & 6.429370 & 18.692169 \\
\hline 0 & 14.426880 & 7.167561 & 16.783215 \\
\hline 0 & 16.921337 & 8.180540 & 16.879099 \\
\hline 0 & 15.175994 & 21.324751 & 6.325232 \\
\hline 0 & 16.183147 & 18.7 & 98 \\
\hline 0 & 14.327353 & 19.388123 & 4.718497 \\
\hline 0 & 16.857981 & 20.477324 & 4.621884 \\
\hline 0 & 3.861603 & 8.916789 & 0.267263 \\
\hline 0 & 2.781626 & 6.418690 & 24.615658 \\
\hline 0 & 4.632322 & 7.084 & 2.100724 \\
\hline 0 & 2.209231 & 8.2 & 209 \\
\hline 0 & 3.792178 & 21.232506 & 12.548268 \\
\hline 0 & 2.880424 & 18.635851 & 12.558949 \\
\hline 0 & 4.550274 & 19.467743 & 14.407459 \\
\hline 0 & 2.114075 & 20.471983 & 14.334879 \\
\hline 0 & 16.029245 & 8.85 & 12. \\
\hline 0 & 15.167498 & 6.307 & 12. \\
\hline 0 & 16.843416 & 7.177756 & 14.396779 \\
\hline 0 & 14.334150 & 8.259432 & 14.333908 \\
\hline 0 & 16.095272 & 21.237362 & 0.260952 \\
\hline 0 & 15.081567 & 18.731251 & 24.625122 \\
\hline 0 & 16.863077 & 19.465073 & 2.113104 \\
\hline 0 & 14.418627 & 20.643118 & 2.105093 \\
\hline 0 & 3.792906 & 22.168049 & 6.487872 \\
\hline 0 & 2.710987 & 24.598179 & 6.472094 \\
\hline 0 & 4.555371 & 24.024084 & 4.634749 \\
\hline 0 & 2.192724 & 22.837543 & 4.557070 \\
\hline 0 & 3.801645 & 9.960596 & 18.712074 \\
\hline 0 & 2.793278 & 12.475687 & 18.634880 \\
\hline 0 & 4.569208 & 11.725602 & 16.847057 \\
\hline 0 & 2.104608 & 10.691990 & 16.847786 \\
\hline 0 & 16.097458 & 22.009050 & 18.542152 \\
\hline 0 & 14.998304 & 24.512976 & 18.640951 \\
\hline ? & 16.848515 & 23.933298 & 16.84972 \\
\hline
\end{tabular}




$$
\begin{aligned}
& 14.416926 \\
& 16.102554 \\
& 15.166284 \\
& 16.916241 \\
& 14.404790 \\
& 0.345670 \\
& 24.609589 \\
& 2.117230 \\
& 2.194424 \\
& 0.421893 \\
& 24.612743 \\
& 2.118444 \\
& 2.195880 \\
& 12.464035 \\
& 12.546813 \\
& 14.338034 \\
& 14.322742 \\
& 12.639783 \\
& 12.465249 \\
& 14.336822 \\
& 14.483439 \\
& 6.416262 \\
& 6.389075 \\
& 4.636691 \\
& 4.474294 \\
& 6.485202 \\
& 6.233475 \\
& 4.554400 \\
& 4.634021 \\
& 18.702850 \\
& 18.800920 \\
& 16.937843 \\
& 16.857010 \\
& 18.713774 \\
& 18.776403 \\
& 16.920366 \\
& 16.926435 \\
& 0.418737 \\
& 24.522926 \\
& \text { 2.091985 } \\
& 2.201706 \\
& 0.422864 \\
& 24.612501 \\
& 2.198793 \\
& 2.117959 \\
& 12.382473 \\
& 12.552638 \\
& 14.248219 \\
& 14.331723 \\
& 12.565989 \\
& 12.480542 \\
& 14.417170 \\
& 14.414257 \\
& 6.410193 \\
& \begin{array}{r}
22.763020 \\
9.953799
\end{array} \\
& 12.483455 \\
& 11.801581 \\
& 10.786175 \\
& 2.867073 \\
& 3.964285 \\
& 2.115046 \\
& 4.551245 \\
& 15.167983 \\
& 16.167610 \\
& 14.319828 \\
& 16.838804 \\
& 2.868772 \\
& 3.794120 \\
& 2.026929 \\
& 4.469924 \\
& 15.090790 \\
& 16.098671 \\
& 14.326140 \\
& 16.773262 \\
& 2.870471 \\
& 3.955546 \\
& 2.034940 \\
& 4.480120 \\
& 15.163371 \\
& 16.182905 \\
& 14.330995 \\
& 16.855553 \\
& 2.948878 \\
& 3.977150 \\
& 2.192482 \\
& 4.624797 \\
& 15.166527 \\
& 16.034586 \\
& 14.328811 \\
& 16.857010 \\
& 3.884178 \\
& 2.629910 \\
& 4.543477 \\
& 2.189326 \\
& 16.097944 \\
& 15.086178 \\
& 16.840017 \\
& 14.337792 \\
& 3.800674 \\
& 2.870229 \\
& 4.718011 \\
& 2.273316 \\
& 16.096243 \\
& 15.239108 \\
& 16.865505 \\
& 14.402362 \\
& 3.880780 \\
& 6.411893 \quad 2.785510 \\
& 4.632565 \quad 4.635963 \\
& 4.533767 \\
& 6.319891 \\
& 2.257538 \\
& 16.696796 \\
& 6.328874 \\
& 6.408737 \\
& 4.559255 \\
& 4.542748 \\
& 22.162952 \\
& 24.613716 \\
& 24.012434 \\
& 22.840942 \\
& 9.948459 \\
& 12.480300 \\
& 11.801825 \\
& 10.783990 \\
& 10.032934 \\
& 12.563075 \\
& 11.804009 \\
& 10.700972 \\
& 22.153728 \\
& 24.604490 \\
& 24.026756 \\
& 22.929300 \\
& 8.864597 \\
& 6.412621 \\
& 7.076531 \\
& 8.176656 \\
& 21.157984 \\
& 18.618132 \\
& 19.386181 \\
& 20.482180 \\
& 21.146088 \\
& 18.702122 \\
& 19.215288 \\
& 20.397703 \\
& 8.939121 \\
& 6.411164 \\
& 7.093281 \\
& 8.097035 \\
& 8.942519 \\
& 6.564823 \\
& 6.989871 \\
& 8.264772 \\
& 21.153372 \\
& 18.619589 \\
& 19.295151 \\
& 20.398432 \\
& 21.139776 \\
& 18.626143 \\
& 19.394192 \\
& 20.543837 \\
& 8.924557 \\
& 6.409950 \\
& 7.084299 \\
& 8.092667 \\
& 22.154940 \\
& 24.608131 \\
& 24.019716 \\
& 22.777828 \\
& 9.791888
\end{aligned}
$$




\begin{tabular}{|c|c|c|c|}
\hline & 6.494426 & 14.989322 & 12.290229 \\
\hline 0 & 4.709272 & 16.861866 & 11.795999 \\
\hline 0 & 4.538379 & 14.393623 & 10.696116 \\
\hline 0 & 18.615704 & 3.793877 & 9.877334 \\
\hline 0 & 18.886852 & 2.776286 & 12.387570 \\
\hline 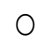 & 16.941971 & 4.465798 & 11.972475 \\
\hline O & 16.862837 & 2.112376 & 10.718206 \\
\hline 0 & 18.634880 & 16.105227 & 22.1544 \\
\hline 0 & 18.712559 & 15.084722 & 24.60473 \\
\hline & 16.927893 & 16.930805 & 24.012920 \\
\hline O & 16.774477 & 14. & 22.925175 \\
\hline 0 & 2.871442 & 24.695518 & 22.080904 \\
\hline 0 & 0.430389 & 0.420922 & 20.9856 \\
\hline O & 1.009338 & 22.910124 & 22.84822 \\
\hline 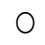 & 2.204619 & 22.767389 & 20.48218 \\
\hline 0 & 2.953248 & 12.476658 & \\
\hline 0 & 0.519234 & 12.626918 & 8.933052 \\
\hline 0 & 1.093085 & 10.704127 & 10.703 \\
\hline O & 2.184471 & 10.626692 & 8.25093 \\
\hline O & 15.155360 & 24.687265 & 9.8695 \\
\hline 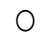 & 12.564046 & 0.492532 & 8.938 \\
\hline 0 & 13.3 & 22. & 10. \\
\hline 0 & 14.32 & 23. & 8.100919 \\
\hline 0 & 15.081808 & 12.555065 & 22.14037 \\
\hline o & 12.556765 & 12.566960 & 21.230322 \\
\hline O & 13.311219 & 10.698301 & 22.92323 \\
\hline 0 & 14.2 & 10. & 20. \\
\hline 0 & 16.1 & 18. & 22. \\
\hline 0 & 18.623959 & 18.616434 & 21.154827 \\
\hline 0 & 18.047436 & 20.385323 & 22.99751 \\
\hline 0 & 16.932261 & 20.564713 & 20.556702 \\
\hline 0 & 16.102554 & 6.2 & 2876 \\
\hline 0 & 18.6 & 6 & 72 \\
\hline 0 & 17.94 & 8.2 & 10.624749 \\
\hline 0 & 16.850212 & 8.100433 & 8.165732 \\
\hline 0 & 3.805529 & 18.697510 & 10.033177 \\
\hline 0 & 6.318678 & 18.62541 & 8.94154 \\
\hline 0 & 5.65 & 20. & $10.78^{\circ}$ \\
\hline 0 & 4.4 & 20.4 & 995 \\
\hline 0 & 3.880052 & 6.412135 & 22.165865 \\
\hline 0 & 6.326446 & 6.324990 & 21.074236 \\
\hline 0 & 5.747497 & 8.248752 & 22.83171 \\
\hline 0 & 4.557556 & 8.2 & 20.475866 \\
\hline 0 & 16.17 & 24.8 & 21.38 \\
\hline 0 & 18.711588 & 0.419708 & 22.241358 \\
\hline 0 & 17.873144 & 23.077862 & 20.299877 \\
\hline 0 & 16.943670 & 22.830017 & 22.770786 \\
\hline 0 & 16.101583 & 12.553123 & 8.941063 \\
\hline 0 & 18.529045 & 12.722318 & 10.038760 \\
\hline 0 & 18.031172 & 10. & 8.41212 \\
\hline 0 & 16.854582 & 10.787389 & 10.78957 \\
\hline 0 & 3.799703 & 24.607891 & 8.946889 \\
\hline 0 & 6.323290 & 0.428447 & 9.956226 \\
\hline 0 & 5.663749 & 22.831474 & 8.252150 \\
\hline 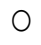 & 4.559741 & 22.9 & 10.716750 \\
\hline $\mathrm{O}$ & 3.796547 & 12.475930 & 21.15142 \\
\hline 0 & 6.236630 & 12.665515 & 22.23407 \\
\hline & 5 & 10.1 & \\
\hline
\end{tabular}




\begin{tabular}{|c|c|c|c|}
\hline & 4.556342 & 10.698787 & 22.852835 \\
\hline 0 & 2.946936 & 18.795822 & 20.983448 \\
\hline 0 & 0.514136 & 18.523460 & 22.242086 \\
\hline O & 0.910783 & 20.383623 & 20.411783 \\
\hline 0 & 2.197822 & 20.488731 & 22.765446 \\
\hline 0 & 2.796434 & 6.416262 & 8.777452 \\
\hline 0 & 0.416552 & 6.396114 & 9.962781 \\
\hline 0 & 0.942340 & 8.263559 & 8.1832 \\
\hline O & 2.191268 & 8.249479 & 10.59780 \\
\hline 0 & 15.158516 & 18.625414 & 8.943005 \\
\hline 0 & 12.565746 & 18.629541 & 9.784605 \\
\hline 0 & 13.389869 & 20.478050 & 8.1018 \\
\hline 0 & 14.322742 & 20.409111 & 10.63324 \\
\hline O & 15.172111 & 6.405581 & 21.234692 \\
\hline 0 & 12.641240 & 6.253137 & 22.168293 \\
\hline 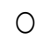 & 13.307093 & 8.180054 & 20.4712 \\
\hline 0 & 14.327353 & 8.171558 & 22.922504 \\
\hline 0 & 22.170477 & 2.777985 & 24.60303 \\
\hline 0 & 21.074720 & 0.334018 & 0.35004 \\
\hline O & 22.923231 & 0.920736 & 22.843126 \\
\hline U & 20.560343 & 2.189326 & 22.747000 \\
\hline 0 & 22.165136 & $15.0^{\circ}$ & 12.477 \\
\hline 0 & 21.239059 & 12.567688 & 12.547784 \\
\hline 0 & 22.996300 & 13.246892 & 10.70048 \\
\hline 0 & 20.500383 & 14.324441 & 10.63664 \\
\hline O & 9.953314 & 3.040636 & 12.394368 \\
\hline 0 & 8.9 & & 12. \\
\hline 0 & 10.620866 & 1.0 & 10.704370 \\
\hline 0 & 8.167675 & 2.180830 & 10.699272 \\
\hline 0 & 9.863983 & 15.092247 & 24.687752 \\
\hline 0 & 8.870910 & 12.558221 & 0.421164 \\
\hline 0 & 10.785933 & 13.308791 & 22.999939 \\
\hline 0 & 8.2 & 14.314 & 22. \\
\hline 0 & 22.162708 & 16.095 & 18.631968 \\
\hline 0 & 21.158226 & 18.548222 & 18.545065 \\
\hline 0 & 22.916193 & 17.948397 & 20.455961 \\
\hline 0 & 20.479023 & 16.852640 & 20.479994 \\
\hline 0 & 22.167564 & 38 & 6.401 \\
\hline 0 & 21.1 & 6.4 & 6.327175 \\
\hline 0 & 22.915222 & 5.733175 & 8.175927 \\
\hline 0 & 20.474167 & 4.633535 & 8.198260 \\
\hline 0 & 9.940934 & 16.094059 & 6.32450 \\
\hline 0 & 9.020926 & 18.623472 & 6.249738 \\
\hline 0 & 10.632517 & 17.8 & 8.193405 \\
\hline 0 & 8.170344 & 16.865505 & 8.090967 \\
\hline 0 & 10.023952 & 3.792906 & 18.709404 \\
\hline 0 & 8.928926 & 6.242456 & 18.524675 \\
\hline 0 & 10.702913 & 5.740942 & 20.405956 \\
\hline 0 & 8.262345 & 4.565324 & 20.473196 \\
\hline 0 & 21.233963 & 16.178291 & 24.77271 \\
\hline 0 & 22.324619 & 18.627598 & 0.41703 \\
\hline 0 & 20.477079 & 17.948153 & 22.917650 \\
\hline 0 & 22.922020 & 16.776661 & 22.916435 \\
\hline 0 & 21.400972 & 3.799946 & 12.632987 \\
\hline 0 & 22.245485 & 6.334942 & 12.491708 \\
\hline 0 & 20.402557 & 5.484118 & 10.790545 \\
\hline 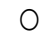 & 22.912794 & 4.555129 & 10.63130 \\
\hline & & & \\
\hline
\end{tabular}




\begin{tabular}{rrr}
9.781207 & 18.385096 & 12.641483 \\
8.192192 & 17.782843 & 10.624749 \\
10.782291 & 16.868177 & 10.693689 \\
8.928683 & 3.806257 & 24.606918 \\
9.955013 & 6.326689 & 0.429903 \\
8.166218 & 5.733903 & 22.905998 \\
10.628877 & 4.631351 & 22.835115 \\
21.247799 & 2.873142 & 18.543610 \\
22.327047 & 0.420922 & 18.608421 \\
20.400616 & 1.024388 & 20.318083 \\
22.831717 & 2.199521 & 20.469070 \\
21.327177 & 15.249546 & 6.325232 \\
22.332632 & 12.639055 & 5.997525 \\
20.391876 & 13.230142 & 8.169859 \\
23.007708 & 14.408430 & 8.186366 \\
8.851733 & 2.956161 & 6.494912 \\
10.023952 & 0.594970 & 6.247068 \\
8.262102 & 1.013950 & 8.258461 \\
10.633002 & 2.197579 & 8.264045 \\
8.867996 & 15.256342 & 18.641436 \\
10.029536 & 12.568416 & 18.384609 \\
8.011104 & 13.226501 & 20.319538 \\
10.704127 & 14.406488 & 20.561556 \\
24.703775 & 22.099838 & 2.884308 \\
0.496173 & 21.066954 & 0.420436 \\
22.997513 & 22.922260 & 1.004483 \\
22.847494 & 20.489702 & 2.184957 \\
24.689938 & 9.871509 & 15.088848 \\
0.505883 & 8.861443 & 12.560891 \\
22.926874 & 10.633002 & 13.231112 \\
22.923231 & 8.166946 & 14.321285 \\
12.475203 & 22.093527 & 15.089334 \\
12.640269 & 21.235420 & 12.547541 \\
10.689320 & 22.921289 & 13.233541 \\
10.710439 & 20.406927 & 14.157675 \\
12.467920 & 9.952585 & 2.952519 \\
12.636870 & 8.936451 & 0.503455 \\
10.708739 & 10.702428 & 1.017106 \\
10.627419 & 8.264530 & 2.111647 \\
18.711832 & 22.164650 & 16.102312 \\
18.623472 & 21.155313 & 18.627598 \\
20.479507 & 22.910608 & 18.024618 \\
20.486790 & 20.483635 & 16.860651 \\
18.711588 & 9.946517 & 3.713285 \\
18.786112 & 8.934752 & 6.250467 \\
20.486790 & 10.792973 & 5.479262 \\
20.559614 & 8.280551 & 4.465070 \\
6.327417 & 22.169020 & 3.799703 \\
6.238572 & 20.986605 & 6.410193 \\
8.184181 & 23.000669 & 5.820563 \\
8.342209 & 20.493587 & 4.478663 \\
6.327902 & 9.864470 & 16.098915 \\
6.324990 & 8.939363 & 18.615704 \\
8.169617 & 10.700001 & 17.863920 \\
\hline 1805037 & 8.177627 & 16.867691 \\
\hline & 20.315168 & 17.867804
\end{tabular}




\begin{tabular}{|c|c|c|c|}
\hline O & 22.910124 & 22.836329 & 16.842203 \\
\hline 0 & 24.608374 & 8.853918 & 3.790964 \\
\hline o & 0.507096 & 10.034634 & 6.23493 \\
\hline o & 22.914494 & 8.263559 & 5.753323 \\
\hline O & 22.927359 & 10.623051 & 4.385449 \\
\hline 0 & 12.477630 & 21.408497 & 3.707945 \\
\hline 0 & 12.641968 & 22.243057 & 6.245612 \\
\hline 0 & 10.864340 & 20.379982 & 5.48581 \\
\hline 0 & 10.687378 & 23.001883 & 4.63280 \\
\hline O & 12.473745 & 8.860957 & 16.08823 \\
\hline 0 & 12.628132 & 9.862285 & 18.54021 \\
\hline 0 & 10.700001 & 8.111357 & 17.957863 \\
\hline 0 & 10.631304 & 10.532263 & 16.84001 \\
\hline 0 & 18.705036 & 21.216244 & 2.958102 \\
\hline 0 & 18.609636 & 22.253496 & 0.492046 \\
\hline 0 & 20.403770 & 20.385080 & 1.015406 \\
\hline 0 & 20.573936 & 22.766418 & 2.190783 \\
\hline O & 18.717659 & 8.939363 & 15.105841 \\
\hline 0 & 18.529772 & 9.867140 & 12.62934 \\
\hline C & 20.478294 & 8.178597 & 13.229898 \\
\hline 0 & 20.463730 & 10.685193 & 14.248704 \\
\hline 0 & 6.574775 & 21.072052 & 15.162642 \\
\hline 0 & 6.238087 & 22.171448 & 12.657019 \\
\hline 0 & 8.181026 & 20.479023 & 13.141782 \\
\hline $\mathrm{O}$ & 8.185394 & 22.915464 & 14.322256 \\
\hline $\mathrm{O}$ & 6.405581 & 8.934752 & 2.868772 \\
\hline O & 6.327417 & 10.025652 & 0.425291 \\
\hline 0 & 8.173015 & 0782 & 1.011280 \\
\hline 0 & 8.261860 & 10.628148 & 2.115046 \\
\hline o & 13.305879 & 5.305456 & 8.505091 \\
\hline P & 12.389028 & 6.415776 & 6.075204 \\
\hline 0 & 11.802795 & 7.823217 & 8.425956 \\
\hline 0 & 14.424452 & 48 & 424 \\
\hline 0 & 9.615897 & 17.616804 & 19.306803 \\
\hline 0 & 9.438935 & 19.722141 & 17.385712 \\
\hline 0 & 7.091339 & 18.970842 & 18.973997 \\
\hline o & 9.437236 & 20.234821 & 20.142820 \\
\hline $\mathrm{H}$ & 12.707753 & 22.163923 & 3.131666 \\
\hline $\mathrm{H}$ & 15.862237 & 83 & 96770 \\
\hline $\mathrm{H}$ & 25.023472 & 6.154097 & 1.694367 \\
\hline $\mathrm{H}$ & 21.901272 & 16.007399 & 6.549772 \\
\hline $\mathrm{H}$ & 13.706653 & 5.037465 & 9.408349 \\
\hline $\mathrm{H}$ & 9.314650 & 16.127802 & 18.956034 \\
\hline $\mathrm{H}$ & 9.227261 & 19.283741 & 15.982639 \\
\hline $\mathrm{H}$ & 11.633359 & 17.394449 & 21.549776 \\
\hline $\mathrm{H}$ & 11.729972 & 20.450378 & 21.694452 \\
\hline $\mathrm{H}$ & 14.011298 & 19.790596 & 20.671764 \\
\hline $\mathrm{H}$ & 12.808978 & 19.962460 & 19.402931 \\
\hline $\mathrm{H}$ & 13.254659 & 18.334362 & 19.988920 \\
\hline $\mathrm{H}$ & 9.472677 & 17.797407 & 22.778070 \\
\hline $\mathrm{H}$ & 9.920300 & 19.500757 & 23.071793 \\
\hline $\mathrm{H}$ & 10.736413 & 18.187986 & 23.936453 \\
\hline $\operatorname{Re}$ & 12.957538 & 6.848835 & 7.625380 \\
\hline $\mathrm{Re}$ & 8.916789 & 19.155087 & 18.949722 \\
\hline $\mathrm{C}$ & 11.352016 & 18.393106 & 21.883308 \\
\hline C & 11.989468 & 19.453178 & 21.338345 \\
\hline C & 13.062890 & 19.369675 & 20.297449 \\
\hline . & 10.314277 & 18.482437 & 22.961344 \\
\hline
\end{tabular}




\begin{tabular}{|c|c|c|c|}
\hline \multicolumn{4}{|c|}{607} \\
\hline $\mathrm{Si}$ & $23 . \overline{3} 8177 \overline{9}$ & 3.327319 & 1.244316 \\
\hline & 23.426931 & 1.240918 & 3.377325 \\
\hline & 23.393188 & 15.561960 & 13.398607 \\
\hline & 23.386877 & 13.412444 & 15.566330 \\
\hline & 11.237440 & 1.214458 & 15.639881 \\
\hline & 11.174812 & 15.583564 & \\
\hline & 11.231857 & 13.403463 & 3.368586 \\
\hline & 7.634604 & 3.353779 & 1.216643 \\
\hline & 7.658636 & 5.501110 & 3.34625 \\
\hline & 7.755978 & 15.484282 & 13.220675 \\
\hline & 19.994259 & & \\
\hline & 19.906 & 15. & \\
\hline & 19.882839 & $17.803^{-}$ & 3. \\
\hline & 7.626593 & 3.371256 & 5. \\
\hline & 7.643828 & 15.527004 & 17.73210 \\
\hline & 7.751608 & 13. & 15 \\
\hline i & 19.91 & & \\
\hline i & 19. & & 15. \\
\hline & 19.934 & 15.5 & 5 . \\
\hline Si & 20.009308 & 13.377246 & 3.281683 \\
\hline 1 & 23.425 & 3. & \\
\hline i & 23. & 17. & \\
\hline$i$ & 11.2 & & 17. \\
\hline i & 11.28 & 5 . & 15. \\
\hline & 11.242 & 15 . & 5. \\
\hline $\mathrm{Si}$ & 11.188 & 17. & 3. \\
\hline i & 74 & 23.405083 & 3.334116 \\
\hline i & 1 & & \\
\hline i & 2 & $1+{ }^{2}$ & 15. \\
\hline & 3.40 & 11. & 13. \\
\hline 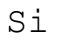 & 13.472 & 23. & 15 \\
\hline . & 15.619 & 23. & 13. \\
\hline $\mathrm{Si}$ & 13.429 & 58 & 3873 \\
\hline i & 15.610 & 1. & 1 . \\
\hline i & $1+2>$ & 7 . & 3. \\
\hline & 3.379 & 7. & 5 . \\
\hline S & 1.287 & 19.8 & 5 . \\
\hline$i$ & 34 & 99 & 17. \\
\hline $\mathrm{Si}$ & & & 02 \\
\hline i & & & 17. \\
\hline i & 15.5 & 19. & 5 . \\
\hline & 5.525870 & 7.6615 & 3.37926 \\
\hline $\mathrm{Si}$ & 3.404 & 7. & 1 . \\
\hline $\mathrm{Si}$ & 5.471 & 9 . & 366 \\
\hline $\mathrm{Si}$ & & & 12 \\
\hline $\mathrm{Si}$ & 17.8 & & 15. \\
\hline $\mathrm{Si}$ & 15.5 & 7. & 13. \\
\hline S & 17.723 & 19. & 3 \\
\hline $\mathrm{Si}$ & 15.609052 & 0.005911 & $1.23120 \varepsilon$ \\
\hline $\mathrm{Si}$ & 5.500867 & 23.367214 & 3.37611 \\
\hline $\mathrm{Si}$ & & 23. & \\
\hline $\mathrm{Si}$ & & 11.1 & \\
\hline $\mathrm{Si}$ & & 11.215592 & 17.7233 \\
\hline & & 23.390762 & \\
\hline & & 381052 & \\
\hline
\end{tabular}




\begin{tabular}{|c|c|c|c|}
\hline i & 17.820469 & 11.267298 & 3.305958 \\
\hline Si & 15.665612 & 11.235255 & 5.475136 \\
\hline $\mathrm{Si}$ & 3.359362 & 1.280485 & 23.367943 \\
\hline $\mathrm{Si}$ & 1.240918 & 3.366401 & 23.414307 \\
\hline & 3.366401 & 13.370934 & 11.229429 \\
\hline $\mathrm{Si}$ & 1.275387 & 15.554436 & 11.257831 \\
\hline $\mathrm{Si}$ & 15.619248 & 1.223683 & 11.203699 \\
\hline L & 13.382830 & 3.343098 & 11.237 \\
\hline S & 15.563416 & 13.418270 & 23.391247 \\
\hline S & 13.454440 & 15.565116 & 23.416491 \\
\hline i & 3.349895 & 1.214458 & 7.64868 \\
\hline i & 5.503294 & 3.325620 & \\
\hline i & 3.304015 & 13.360253 & 19.895947 \\
\hline 2 & 17.776289 & 3.362032 & 19.85370 \\
\hline 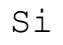 & 15.649106 & 13.416814 & 7.65062 \\
\hline i & 17.824839 & 15.567785 & 7.71908 \\
\hline $\mathrm{Si}$ & 3.332174 & 5.438239 & 7 . \\
\hline $\mathrm{Si}$ & 1.233392 & 3.3338 & 7.664462 \\
\hline $\mathrm{Si}$ & 3.451120 & 17.720942 & 19.888180 \\
\hline Si & 1.248200 & 15.581622 & 19.861719 \\
\hline Si & 15.572398 & 5.465911 & 19.93770 \\
\hline $\mathrm{Si}$ & 13.400549 & 3.394803 & 19.95542 \\
\hline $\mathrm{Si}$ & 15.669739 & 17.77 & 7 . \\
\hline $\mathrm{Si}$ & 13.479442 & 15.6386 & 7.62149 \\
\hline Si & 3.377325 & 5.5108 & 23.38226 \\
\hline $\mathrm{Si}$ & 5.505479 & 3.388006 & 23.34585 \\
\hline $\mathrm{Si}$ & 5.551601 & 15.538898 & 11.11412 \\
\hline $\mathrm{Si}$ & 17.827265 & 3.3 & 11. \\
\hline $\mathrm{Si}$ & 15.615606 & 17.757113 & 23.443438 \\
\hline $\mathrm{Si}$ & 17.728224 & 15.6107 & 23.4 \\
\hline $\mathrm{Si}$ & 1.643148 & 21.679401 & 23.73230 \\
\hline Si & 1.613775 & 23.787167 & 21.61264 \\
\hline i & 1.673005 & 9.440634 & 11.58359 \\
\hline $\mathrm{Si}$ & 1.667422 & 11.588208 & 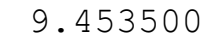 \\
\hline $\mathrm{Si}$ & 13.867593 & 21.703192 & 11.54597 \\
\hline Si & 13.832395 & 23.861931 & 9.381162 \\
\hline $\mathrm{Si}$ & 13.836764 & 9.415631 & 23.787651 \\
\hline $\mathrm{Si}$ & 13.812004 & 11.622921 & 21.66289 \\
\hline $\mathrm{Si}$ & 17.482809 & 21.680857 & $23.77405^{\circ}$ \\
\hline $\mathrm{Si}$ & 17.393236 & 19.538382 & 21.68886 \\
\hline $\mathrm{Si}$ & 17.362408 & 9.482872 & 11.61588 \\
\hline $\mathrm{Si}$ & 17.448339 & 7.316607 & 9.496466 \\
\hline $\mathrm{Si}$ & 5.065381 & 21.682072 & 11.616123 \\
\hline $\mathrm{Si}$ & 5.099851 & 19.543238 & 9.48408 \\
\hline $\mathrm{Si}$ & 5.181413 & 9.435780 & 23.73181 \\
\hline $\mathrm{Si}$ & 5.153012 & 7.303499 & 21.61459 \\
\hline $\mathrm{Si}$ & 17.336433 & 21.700521 & 19.52309 \\
\hline $\mathrm{Si}$ & 17.412897 & 23.857077 & 21.66289 \\
\hline $\mathrm{Si}$ & 17.386683 & 11.678753 & 9.507632 \\
\hline $\mathrm{Si}$ & 5.101307 & 21.607307 & 7.31005 \\
\hline $\mathrm{Si}$ & 5.107861 & 23.768959 & 9.42242 \\
\hline $\mathrm{Si}$ & 5.080431 & 9.451558 & 19.52406 \\
\hline $\mathrm{Si}$ & 5.027269 & 11.624134 & 21.69202 \\
\hline $\mathrm{Si}$ & 1.576878 & 21.619686 & 19.54639 \\
\hline $\mathrm{Si}$ & 1.610862 & 19.541052 & 21.61459 \\
\hline $\mathrm{Si}$ & 1.628826 & 9.420001 & 7.304955 \\
\hline $\mathrm{Si}$ & 1.618873 & 7.274612 & 9.43238 \\
\hline $\mathrm{Si}$ & 13.877546 & 21.747370 & 7.18382 \\
\hline
\end{tabular}




\begin{tabular}{|c|c|c|c|}
\hline i & 13.893324 & 19.549063 & 9.342808 \\
\hline Si & 13.815160 & 7.233831 & 21.653185 \\
\hline $\mathrm{Si}$ & 23.767260 & 1.595327 & 21.624784 \\
\hline $\mathrm{Si}$ & 21.671148 & 1.537068 & 23.711185 \\
\hline & 23.854891 & 13.847445 & 9.422428 \\
\hline $\mathrm{Si}$ & 21.726252 & 13.765882 & 11.590150 \\
\hline$i$ & 11.545241 & 1.660625 & 9.475104 \\
\hline L & 9.423642 & 1.716700 & 11.62219 \\
\hline S & 11.675839 & 13.828268 & 21.71532 \\
\hline$\perp$ & 9.467337 & 13.779477 & 23.8165 \\
\hline$\perp$ & 23.809982 & 17.346870 & 21.70197 \\
\hline i & 21.685957 & 17.389595 & 19.56023 \\
\hline i & 21.670906 & 5.179956 & 7.25931 \\
\hline 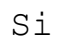 & 11.687248 & 17.382313 & 9.34620 \\
\hline 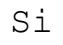 & 9.460054 & 17.380371 & 7.191107 \\
\hline i & 11.581654 & 5.159323 & 21.63765 \\
\hline $\mathrm{Si}$ & 9.477046 & 5.115386 & \\
\hline $\mathrm{Si}$ & 19.528915 & 17.385225 & 21.699064 \\
\hline i & 21.717270 & 17.394207 & 23.826975 \\
\hline Si & 19.550762 & 5.111988 & 9.48602 \\
\hline Si & 21.724068 & 5.052515 & 11.65205 \\
\hline $\mathrm{Si}$ & 7.252765 & 17.347601 & 9.32654 \\
\hline $\mathrm{Si}$ & 9.455441 & 17.247589 & 11. \\
\hline $\mathrm{Si}$ & 7.297187 & 5.1 & 21.64736 \\
\hline Si & 9.410776 & 5.116843 & 23.77502 \\
\hline $\mathrm{Si}$ & 19.551733 & 1.639992 & 21.59565 \\
\hline $\mathrm{Si}$ & 21.673819 & 1.614746 & 19.4776 \\
\hline i & 19.548820 & 13. & R \\
\hline $\mathrm{Si}$ & 9614 & 1.619359 & 262 \\
\hline $\mathrm{Si}$ & 9.427526 & 1.662325 & 7.296702 \\
\hline $\mathrm{Si}$ & 7.272184 & 13.689417 & 21.67139 \\
\hline $\mathrm{Si}$ & 21.701977 & 23.747839 & 1.58731 \\
\hline i & 23.834257 & 21.648331 & 1.61935 \\
\hline $\mathrm{Si}$ & 21.696152 & 11.576556 & 13. \\
\hline $\mathrm{Si}$ & 23.835228 & 9.3 & 13. \\
\hline $\mathrm{Si}$ & 9.464181 & 23.846151 & 13.86103 \\
\hline $\mathrm{Si}$ & 9.466851 & 11.595976 & 1.668150 \\
\hline $\mathrm{Si}$ & 11.620736 & 9.461511 & 1.64606 \\
\hline $\mathrm{Si}$ & 21.671633 & 23.770658 & 17.38401 \\
\hline $\mathrm{Si}$ & 19.525274 & 21.678188 & $17.39857^{\circ}$ \\
\hline $\mathrm{Si}$ & 21.813883 & 11.641855 & $4.88307 \xi$ \\
\hline $\mathrm{Si}$ & 19.637665 & 9.534092 & 5.01149 \\
\hline $\mathrm{Si}$ & 9.359315 & 23.825277 & 5.16199 \\
\hline $\mathrm{Si}$ & 9.474861 & 11.536018 & 17.29929 \\
\hline $\mathrm{Si}$ & 7.268786 & 9.418059 & 17.367990 \\
\hline $\mathrm{Si}$ & 21.688141 & 19.524303 & 17.34323 \\
\hline $\mathrm{Si}$ & 23.822849 & 21.684015 & 17.35124 \\
\hline $\mathrm{Si}$ & 21.700764 & 7.371953 & 5.12388 \\
\hline $\mathrm{Si}$ & 23.811197 & 9.445004 & 5.13140 \\
\hline $\mathrm{Si}$ & 9.485299 & 19.531342 & 4.977992 \\
\hline $\mathrm{Si}$ & 11.571459 & 21.729164 & 5.12800 \\
\hline $\mathrm{Si}$ & 9.443547 & 7.259076 & $17.31992^{\circ}$ \\
\hline $\mathrm{Si}$ & 11.590150 & 9.358344 & 17.35415 \\
\hline $\mathrm{Si}$ & 21.695423 & 19.508039 & 1.63610 \\
\hline $\mathrm{Si}$ & 19.558289 & 21.630367 & 1.652372 \\
\hline $\mathrm{Si}$ & 21.719212 & 7.219994 & 13.765882 \\
\hline $\mathrm{Si}$ & 19.538868 & 9.386744 & 13.84258 \\
\hline $\mathrm{Si}$ & 9.474861 & 19.621887 & 13.58625 \\
\hline
\end{tabular}




\begin{tabular}{|c|c|c|c|}
\hline$i$ & 7.275340 & 21.707075 & 13.787729 \\
\hline Si & 9.430197 & 7.297187 & 1.612562 \\
\hline $8 i$ & 7.312480 & 9.419758 & 1.663053 \\
\hline L & 11.679238 & 21.751497 & 13.737724 \\
\hline 1 & 11.223846 & 3.388491 & 13.400308 \\
\hline $\mathrm{Si}$ & 20.042322 & 3.303530 & 13.398607 \\
\hline i & 7.629750 & 1.213002 & 3.365188 \\
\hline$i$ & 23.362602 & 15.601770 & 17.71487 \\
\hline i & 5.470524 & 15.508313 & 20.01100 \\
\hline i & 3.466170 & 17.695454 & 11.28768 \\
\hline $\mathrm{Si}$ & 13.805449 & 9.421215 & 19.49808 \\
\hline Si & 23.784981 & 5.134806 & 9.4243 \\
\hline 1 & 15.578953 & 1.284612 & 19.89303 \\
\hline L & 15.567543 & 5.522714 & 11.23986 \\
\hline L & 17.522135 & 9.591137 & 7.21853 \\
\hline 1 & 9.488698 & 13.549110 & 19.64057 \\
\hline 1 & 7.261018 & 21.636435 & 5.166 \\
\hline 1 & 7.595037 & 17.677734 & 15.45588 \\
\hline $\mathrm{L}$ & 23.369156 & 5.398671 & 3.41179 \\
\hline 1 & 13.458566 & 19.863905 & 3.28896 \\
\hline Al & 21.790094 & 13.674125 & 7.23601 \\
\hline Al & 16.392637 & 4.471138 & 14.01736 \\
\hline Al & 6.231047 & 18.061516 & 18. \\
\hline 0 & 22.164894 & 0.348826 & 2.86634 \\
\hline 0 & 24.605219 & 24.542589 & 3.95554 \\
\hline 0 & 24.029911 & 2.099267 & 2.11723 \\
\hline 0 & 22.846767 & 2.186171 & 4.55779 \\
\hline 0 & 22.158583 & 12.469619 & 15.08132 \\
\hline 0 & 24.611530 & 12.468405 & 16.09284 \\
\hline 0 & 23.942280 & 14.330267 & 14.33123 \\
\hline 0 & 22.847010 & 14.389740 & 16.76962 \\
\hline 0 & 9.958897 & 0.253427 & 15.24857 \\
\hline 0 & 12.543657 & 24.617355 & 16.09260 \\
\hline 0 & 11.633 & 2.100724 & 14. \\
\hline 0 & 10.711410 & 2.123299 & 16.86356 \\
\hline 0 & 9.945303 & 12.480542 & 2.94887 \\
\hline 0 & 12.482970 & 12.470590 & 3.79751 \\
\hline 0 & 11.720019 & 14.331238 & 2.10922 \\
\hline 0 & 10.782048 & 14.321772 & 4.62698 \\
\hline 0 & 8.928198 & 6.408009 & 2070 \\
\hline 0 & 6.415291 & 6.408979 & 3.88345 \\
\hline 0 & 7.077745 & 4.628923 & 2.09028 \\
\hline 0 & 8.181026 & 4.555371 & 4.55003 \\
\hline 0 & 9.010489 & 18.799463 & 15.09758 \\
\hline 0 & 6.239543 & 18.623472 & 16.27636 \\
\hline 0 & 7.243541 & 16.780060 & 14.08023 \\
\hline 0 & 7.927841 & 16.958235 & 17.01965 \\
\hline 0 & 21.318439 & 6.239786 & 15.00437 \\
\hline 0 & 18.788540 & 6.411650 & 16.01808 \\
\hline 0 & 19.383024 & 4.555857 & 14.25307 \\
\hline 0 & 20.480478 & 4.544205 & 16.75821 \\
\hline 0 & 21.241972 & 18.547735 & 2.86780 \\
\hline 0 & 18.719114 & 18.785870 & 3.88417 \\
\hline 0 & 19.284470 & 16.852156 & 2.17597 \\
\hline 0 & 20.398916 & 16.855068 & 4.56362 \\
\hline 0 & 8.930140 & 0.342515 & 3.79533 \\
\hline 0 & 6.401940 & 24.609833 & 2.78259 \\
\hline 0 & 7.082357 & 2.106307 & 4.63644 \\
\hline
\end{tabular}




\begin{tabular}{|c|c|c|c|}
\hline & 8.175685 & 2.190540 & 2.187627 \\
\hline O & 9.017042 & 12.473989 & 16.009098 \\
\hline 0 & 6.492727 & 12.397767 & 091033 \\
\hline 0 & 7.246939 & 14.325897 & 16.774233 \\
\hline 0 & 8.181026 & 14.305264 & 14.271279 \\
\hline 0 & 21.142448 & 0.333776 & 16.100613 \\
\hline 0 & 18.633425 & 24.612017 & 15.011169 \\
\hline O & 19.303162 & 2.119415 & 16.85142 \\
\hline O & 20.479023 & 2.115046 & 14.40042 \\
\hline 0 & 21.319651 & 12.466949 & 3.54773 \\
\hline O & 18.706734 & 12.549968 & 2.783326 \\
\hline 0 & 19.559015 & 14.239723 & 4.634992 \\
\hline 0 & 20.480965 & 14.410858 & 2.12985 \\
\hline O & 22.257380 & 6.574047 & 3.87422 \\
\hline 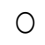 & 24.774899 & 6.400969 & 2.6214 \\
\hline 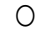 & 24.111717 & 4. & \\
\hline O & 22.760107 & 4.486188 & 2.116260 \\
\hline 0 & 22.084059 & 18.627842 & 16.028759 \\
\hline 0 & 24.590654 & 18.703335 & 15.087877 \\
\hline 0 & 23.932814 & 16.860409 & 16.83395 \\
\hline O & 22.855991 & 16.785643 & 14.34021 \\
\hline 0 & 10.0 & 6 . & 16. \\
\hline 0 & 12.556279 & 6.4 & 15.019179 \\
\hline 0 & 11.719534 & 4.630865 & 16.855795 \\
\hline 0 & 10.774038 & 4.633293 & 14.327840 \\
\hline O & 9.877334 & 18.608908 & 3.7079 \\
\hline O & 12.375918 & 18. & \\
\hline 0 & 11.725 & 16.8 & 4.471624 \\
\hline 0 & 10.620866 & 16.848270 & 2.029599 \\
\hline 0 & 2.950820 & 22.170961 & 0.257068 \\
\hline 0 & 3.969625 & 24.613958 & 24.52826 \\
\hline 0 & 2.0 & 24.0 & 2.036 \\
\hline 0 & 4.4 & 22. & 95 \\
\hline 0 & 2.95 & 9.9 & 12.473745 \\
\hline 0 & 3.888548 & 12.466706 & 12.476901 \\
\hline 0 & 2.180587 & 11.727058 & 14.336578 \\
\hline 0 & 4.634021 & 10.704856 & 14.328325 \\
\hline O & 15.17 & 22.163 & 12.40 \\
\hline 0 & 16.1 & 24. & 12.465249 \\
\hline 0 & 14.323470 & 23.931599 & 14.247733 \\
\hline 0 & 16.773020 & 22.841183 & 14.399935 \\
\hline 0 & 15.167012 & 9.858157 & 0.341786 \\
\hline 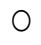 & 16.103041 & 12.396794 & 24.525599 \\
\hline 0 & 14.3 & 11.7 & 2.037610 \\
\hline 0 & 16.860651 & 10.709952 & 2.111890 \\
\hline 0 & 2.868529 & 8.859500 & 6.407523 \\
\hline 0 & 3.895102 & 6.407280 & 6.477677 \\
\hline 0 & 2.193696 & 6.995939 & 4.550274 \\
\hline 0 & 4.555129 & 8.177141 & 4.565809 \\
\hline 0 & 2.866345 & 20.9 & 18.71717 \\
\hline 0 & 4.230092 & 18.543365 & 18.53705 \\
\hline 0 & 2.280599 & 19.216259 & 16.773504 \\
\hline 0 & 4.631836 & 20.559614 & 16.856766 \\
\hline 0 & 15.094673 & 8.939849 & 18.619831 \\
\hline 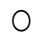 & 16.097700 & 6.418932 & 18.714016 \\
\hline $\mathrm{O}$ & 14.340219 & 7.083813 & 16.85045 \\
\hline 0 & 16.857737 & 8.097035 & 16.85895 \\
\hline & 15 & 21.323 & \\
\hline
\end{tabular}




$$
\begin{aligned}
& 16.179506 \\
& 18.799221 \\
& 6.485687 \\
& 14.262540 \\
& 19.389578 \\
& 20.477324 \\
& 8.843722 \\
& 6.407038 \\
& 7.151539 \\
& 8.266715 \\
& 21.228865 \\
& 18.616434 \\
& 19.394432 \\
& 20.399887 \\
& 8.850276 \\
& 6.320135 \\
& 7.090853 \\
& 8.257976 \\
& 21.232992 \\
& 18.730766 \\
& 19.382540 \\
& 20.633894 \\
& 22.170477 \\
& 24.612017 \\
& 24.018988 \\
& 22.841427 \\
& 9.945546 \\
& 12.477387 \\
& 11.789930 \\
& 10.704370 \\
& 22.080660 \\
& 24.530453 \\
& 23.930386 \\
& 22.764235 \\
& 9.959625 \\
& 12.488068 \\
& 11.803038 \\
& 10.788360 \\
& 2.867316 \\
& 3.964285 \\
& 2.114803 \\
& 4.554400 \\
& 15.155117 \\
& 16.101583 \\
& 14.250646 \\
& 16.760155 \\
& 2.939654 \\
& 3.799460 \\
& 2.105093 \\
& 4.560469 \\
& 15.087877 \\
& 16.093088 \\
& 14.322013 \\
& 16.783701 \\
& 2.868772 \\
& 3.953847 \\
& \text { 2. } 042707 \\
& 4.489344 \\
& 4.642760 \\
& 4.533524 \\
& 6.487872 \\
& 6.304113 \\
& 4.559741 \\
& 4.734033 \\
& 4.553430 \\
& 0.184001 \\
& 24.619783 \\
& 2.100481 \\
& 2.103879 \\
& 12.551182 \\
& 12.542443 \\
& 14.343133 \\
& 14.354299 \\
& 12.469862 \\
& 12.723289 \\
& 14.399935 \\
& 14.419111 \\
& 0.340330 \\
& 24.613958 \\
& \text { 2. } 110191 \\
& 2.102180 \\
& 6.490299 \\
& 6.412135 \\
& 4.635235 \\
& 4.485218 \\
& 18.630026 \\
& 18.627842 \\
& 16.839533 \\
& 16.842688 \\
& 18.626871 \\
& 18.630270 \\
& 16.858952 \\
& 16.690971 \\
& 6.325475 \\
& 6.409708 \\
& 4.556828 \\
& 4.542263 \\
& 22.161982 \\
& 24.614927 \\
& 24.015589 \\
& 22.837299 \\
& 9.946032 \\
& 12.478601 \\
& 11.801339 \\
& 10.789574 \\
& 10.026866 \\
& 12.558949 \\
& 11.722690 \\
& 10.618923 \\
& 22.085758 \\
& 24.527056 \\
& 24.017046 \\
& 22.927116 \\
& 8.869938 \\
& 6.407280 \\
& 7.076531 \\
& 8.185881 \\
& 21.152399 \\
& \begin{array}{ll}
16.182661 & 18.620073 \\
14.336578 & 19.387636
\end{array}
\end{aligned}
$$




\begin{tabular}{|c|c|c|c|}
\hline 0 & 4.705631 & 16.865505 & 20.479994 \\
\hline O & 18.704306 & 2.943052 & 21.146818 \\
\hline 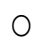 & 18.794607 & 3.972053 & 18.709404 \\
\hline 0 & 16.932747 & 2.181073 & 19.21164 \\
\hline O & 16.834921 & 4.576975 & 20.40474 \\
\hline 0 & 18.707705 & 15.176237 & 9.019712 \\
\hline 0 & 18.802134 & 16.017593 & 6.483988 \\
\hline 0 & 16.932018 & 14.326626 & $7.1627 \mathrm{C}$ \\
\hline 0 & 16.926678 & 16.861622 & 8.10237 \\
\hline 0 & 0.417280 & 3.881751 & 8.95150 \\
\hline 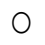 & 24.522926 & 2.692053 & 6.5626 \\
\hline 0 & 2.112133 & 4.557070 & 7.0056 \\
\hline 0 & 2.202920 & 2.183743 & 8.255791 \\
\hline 0 & 0.427718 & 16.099400 & 21.15021 \\
\hline 0 & 24.616386 & 15.086663 & 18.62565 \\
\hline 0 & 2.278414 & 16.777145 & 19.29879 \\
\hline O & 2.114318 & 14.329053 & 20.399158 \\
\hline 0 & 12.393639 & 3.869128 & \\
\hline 0 & 12.473745 & 2.867316 & 18.701151 \\
\hline 0 & 14.242149 & 4.701504 & 19.38059 \\
\hline 0 & 14.327353 & 2.276472 & 20.555002 \\
\hline O & 12.574970 & 16.092604 & 8.923100 \\
\hline O & 12.482970 & 15.239593 & 6.410436 \\
\hline 0 & 14.419841 & 16.867207 & \\
\hline 0 & 14.413286 & 14.400178 & 8.093394 \\
\hline 0 & 6.404367 & 3.871556 & 22.085274 \\
\hline 0 & 6.422574 & 2.790123 & 24.542589 \\
\hline O & 4.637662 & 4.643246 & 23.944950 \\
\hline 0 & 4.486188 & 2.272102 & 22 . \\
\hline 0 & 6.319649 & 16.084106 & 9.783149 \\
\hline 0 & 6.588612 & 14.936161 & 12.231728 \\
\hline 0 & 4.723109 & 16.777874 & 11.806680 \\
\hline 0 & 4.559741 & 14.334637 & 10.697330 \\
\hline 0 & 18.539240 & 3.881023 & $9.87 \varepsilon$ \\
\hline 0 & 18.875685 & 94 & 12. \\
\hline 0 & 16.860165 & 4.464342 & 11.972718 \\
\hline 0 & 16.871574 & 2.119658 & 10.707525 \\
\hline 0 & 18.630754 & 16.106440 & 22.161011 \\
\hline 0 & 18.713289 & 15.083750 & 24.605219 \\
\hline 0 & 16.856281 & 16. & 24. \\
\hline 0 & 16.772291 & 14.2 & 22 . \\
\hline 0 & 2.867558 & 24.692366 & 22.079447 \\
\hline 0 & 0.429418 & 0.420679 & 20.985147 \\
\hline 0 & 1.009095 & 22.908911 & 22.848709 \\
\hline 0 & 2.201706 & 22.765690 & 20.478781 \\
\hline 0 & 2.952277 & 12. & 9.947730 \\
\hline 0 & 0.503941 & 12.579097 & 8.933295 \\
\hline 0 & 1.093571 & 10.699030 & 10.709467 \\
\hline 0 & 2.190783 & 10.616253 & 8.258704 \\
\hline 0 & 15.157302 & 24.686296 & 9.869809 \\
\hline 0 & 12.563075 & 0.507582 & 8.940092 \\
\hline 0 & 13.309278 & 22.925903 & 10.623051 \\
\hline 0 & 14.326383 & 23.009649 & 8.099220 \\
\hline 0 & 15.084479 & 12.561863 & 22.082117 \\
\hline 0 & 12.558464 & 12.556036 & 21.227409 \\
\hline 0 & 13.316074 & 10.704370 & 22.919592 \\
\hline 0 & 14.251132 & 10.701942 & 20.395519 \\
\hline ? & 16.110323 & 18.631241 & 22.15736 \\
\hline
\end{tabular}




\begin{tabular}{|c|c|c|c|}
\hline & 18.622015 & 18.608908 & 21.148516 \\
\hline O & 18.036026 & 20.392363 & 22.923231 \\
\hline 0 & 16.854340 & 20.474653 & 20.483150 \\
\hline 0 & 16.174652 & 6.479376 & 9.959382 \\
\hline & 18.706491 & 6.413835 & 9.012916 \\
\hline 0 & 18.019764 & 8.347549 & 10.622564 \\
\hline 0 & 16.867447 & 8.169131 & 8.179326 \\
\hline U & 3.874469 & 18.621288 & 10.030022 \\
\hline O & 6.327902 & 18.625172 & 8.93572 \\
\hline 0 & 5.651370 & 20.404741 & 10.786903 \\
\hline U & 4.470410 & 20.483391 & $8.3456 c$ \\
\hline O & 3.868886 & 6.483502 & 22.17581 \\
\hline 0 & 6.322805 & 6.325475 & 21.07059 \\
\hline 0 & 5.813766 & 8.274968 & 22.76059 \\
\hline 0 & 4.552701 & 8.190493 & 20.393576 \\
\hline 0 & 16.179993 & 24.770042 & 21.31261 \\
\hline 0 & 18.706978 & 0 . & 22 . \\
\hline 0 & 17.950823 & 22.928572 & 20.391876 \\
\hline 0 & 16.937843 & 22.846283 & 22.83778 \\
\hline O & 16.095516 & 12.544869 & 8.950288 \\
\hline 0 & 18.526617 & 12.717462 & 10.040945 \\
\hline 0 & 18.034328 & 10.710439 & 674 \\
\hline 0 & 16.852640 & 6418 & 10.798799 \\
\hline 0 & 3.803587 & 24.603035 & 8.936936 \\
\hline 0 & 6.324261 & 0.430146 & 9.958897 \\
\hline O & 5.720552 & 22.829290 & 8.255063 \\
\hline 0 & 4.560711 & 22.910608 & 10.711166 \\
\hline 0 & 3.724937 & 12. & 21. \\
\hline $\mathrm{O}$ & 6.157738 & 12.651678 & 22.247669 \\
\hline 0 & 5.580245 & 10.701942 & 20.466642 \\
\hline 0 & 4.554643 & 10.702186 & 22.923962 \\
\hline 0 & 2.950820 & 18.786840 & 20.972282 \\
\hline 0 & 0.5 & $1 \varepsilon$ & 22. \\
\hline $\mathrm{O}$ & 0.924619 & 20 . & 20.398432 \\
\hline 0 & 2.198308 & 20.486790 & 22.761320 \\
\hline 0 & 2.855907 & 6.329602 & 8.935237 \\
\hline 0 & 0.426505 & 6.338341 & 10.029293 \\
\hline 0 & 1.020504 & 8.1 & 8 . \\
\hline 0 & 2.1 & & 10. \\
\hline 0 & 15.170897 & 18.630026 & 8.940820 \\
\hline 0 & 12.631773 & 18.625656 & 9.789460 \\
\hline 0 & 13.392054 & 20.481449 & 8.098492 \\
\hline 0 & 14.330509 & 20.464945 & 10.619409 \\
\hline 0 & 15.094916 & 6.329602 & 21.240030 \\
\hline 0 & 12.560406 & 6884 & 22.170477 \\
\hline 0 & 13.304180 & 8.166218 & 20.403770 \\
\hline 0 & 14.325897 & 8.186123 & 22.856962 \\
\hline 0 & 22.171448 & 2.777742 & 24.604977 \\
\hline 0 & 21.076422 & 0.332319 & 0.352710 \\
\hline 0 & 22.922991 & 0.921706 & 22.84191 \\
\hline 0 & 20.560099 & 2.186899 & 22.747242 \\
\hline 0 & 22.159554 & 15.079139 & 12.476416 \\
\hline 0 & 21.240274 & 12.550453 & 12.567688 \\
\hline 0 & 23.000910 & 13.240823 & 10.704127 \\
\hline 0 & 20.486305 & 14.258900 & 10.697330 \\
\hline $\mathrm{O}$ & 9.970063 & 3.038209 & 12.40699 \\
\hline 0 & 8.938878 & 0.593757 & 12.72086 \\
\hline & 10 & & \\
\hline
\end{tabular}




$$
\begin{array}{rrr}
8.171801 & 2.182529 & 10.702186 \\
9.848448 & 15.090548 & 24.681927 \\
8.930383 & 12.542443 & 0.446410 \\
10.796614 & 13.309034 & 23.005524 \\
8.248994 & 14.239723 & 22.834871 \\
22.167078 & 16.091389 & 18.697752 \\
21.228136 & 18.619347 & 18.611820 \\
22.924932 & 17.954222 & 20.478537 \\
20.476353 & 16.855553 & 20.482420 \\
22.146202 & 3.880295 & 6.409465 \\
21.147545 & 6.401454 & 6.328146 \\
22.920563 & 5.733418 & 8.178597 \\
20.485819 & 4.638148 & 8.258461 \\
9.942390 & 16.094301 & 6.325232 \\
9.018499 & 18.619102 & 6.258720 \\
10.690290 & 17.876059 & 8.165005 \\
8.181996 & 16.867933 & 8.092423 \\
9.952828 & 3.788537 & 18.702850 \\
8.928683 & 6.235659 & 18.462532 \\
10.691748 & 5.752837 & 20.391392 \\
8.262102 & 4.632565 & 20.467857 \\
21.232506 & 16.179506 & 24.772470 \\
22.329233 & 18.626627 & 0.418009 \\
20.477079 & 17.948639 & 22.919106 \\
22.924202 & 16.775204 & 22.919348 \\
21.398060 & 3.792906 & 12.642939 \\
22.247913 & 6.342467 & 12.494622 \\
20.371000 & 5.491642 & 10.854630 \\
22.849195 & 4.550031 & 10.617710 \\
9.095935 & 15.927050 & 12.387086 \\
9.792859 & 18.460346 & 12.562104 \\
8.183210 & 17.781385 & 10.623536 \\
10.782534 & 16.935902 & 10.636401 \\
8.929411 & 3.803830 & 24.607891 \\
9.954285 & 6.325961 & 0.429660 \\
8.156994 & 5.731476 & 22.913280 \\
10.622808 & 4.633293 & 22.835842 \\
21.240517 & 2.865131 & 18.540939 \\
22.319523 & 0.424563 & 18.547493 \\
20.404015 & 1.019290 & 20.314198 \\
22.850653 & 20.488491 & 2.190783 \\
24.692366 & 9.883888 & 15.086906
\end{array}
$$




\begin{tabular}{|c|c|c|c|}
\hline 0 & 0.504426 & 8.861685 & 12.564775 \\
\hline 0 & 22.923717 & 10.632031 & 13.233541 \\
\hline O & 22.917891 & 8.168888 & 14.321042 \\
\hline 0 & 12.473502 & 22.162952 & 15.083022 \\
\hline O & 12.646824 & 21.241732 & 12.54972 \\
\hline 0 & 10.682280 & 22.934156 & 13.224560 \\
\hline 0 & 10.706554 & 20.470770 & 14.1620 \\
\hline 0 & 12.480785 & 9.946032 & 2 \\
\hline 0 & 12.571330 & 8.937907 & 3014 \\
\hline 0 & 10.707525 & 10.705827 & 1.08968 \\
\hline 0 & 10.630333 & 8.258461 & 2.11553 \\
\hline 0 & 18.706491 & 22.174603 & 16.084835 \\
\hline 0 & 18.536327 & 21.146088 & 51620 \\
\hline 0 & 20.482906 & 22.850166 & 18.03093 \\
\hline 0 & 20.481936 & 20.474895 & 16.85725 \\
\hline o & 18.785383 & 10.031478 & 3.70527 \\
\hline 0 & 18.790968 & 9.011459 & 6.243912 \\
\hline O & 20.564468 & 10.796371 & \\
\hline 0 & 20.572481 & 8.331771 & 4.472109 \\
\hline 0 & 6.322076 & 22.091585 & 3.79290 \\
\hline 0 & 6.239786 & 20.982964 & 6.41359 \\
\hline 0 & 8.172286 & 23.004553 & 5.818622 \\
\hline 0 & 8.349977 & 20.489702 & 4.483519 \\
\hline 0 & 6.328874 & 9.864712 & 16. \\
\hline O & 6.329359 & 8.944704 & 18.616674 \\
\hline 0 & 8.170830 & 10.698301 & 17.85639 \\
\hline 0 & 8.176656 & 8.176898 & 16.855310 \\
\hline O & 24.865200 & 21.225224 & 16.176836 \\
\hline 0 & 0.428447 & 22.249369 & 22 \\
\hline O & 22.997997 & 20.377312 & 17.863192 \\
\hline 0 & 22.859390 & 22.832203 & 16.770107 \\
\hline 0 & 24.692122 & 8.844935 & 3.878838 \\
\hline 0 & 0.500057 & 10.031235 & 6.315279 \\
\hline 0 & 22.915949 & 8.271570 & \\
\hline 0 & 23.001154 & 10.629362 & \\
\hline $\mathrm{O}$ & 12.475203 & 21.408497 & 3.709159 \\
\hline 0 & 12.633715 & 22.238932 & 6.242456 \\
\hline 0 & 10.879633 & 20.319054 & 5.486059 \\
\hline 0 & 10.627419 & 22.920563 & 4.636934 \\
\hline 0 & 12.475687 & 34 & 16 \\
\hline 0 & 12.559678 & 450 & 18. \\
\hline 0 & 10.629848 & 8.179326 & 17.958591 \\
\hline 0 & 10.629604 & 10.535904 & 16.764280 \\
\hline 0 & 18.630754 & 21.244400 & 2.929944 \\
\hline 0 & 18.702608 & 22.240631 & 0.421 \\
\hline 0 & 20.389692 & 20.320267 & 84 \\
\hline 0 & 20.573208 & 22.759378 & 2.192482 \\
\hline 0 & 18.700422 & 8.935722 & 15.172352 \\
\hline 0 & 18.551376 & 9.874421 & 12.655320 \\
\hline 0 & 20.407169 & 8.096550 & 13.313161 \\
\hline $\mathrm{O}$ & 20.484606 & 10.625235 & 14.317159 \\
\hline 0 & 6.579145 & 21.071566 & 15.159487 \\
\hline 0 & 6.240028 & 22.171934 & 12.654349 \\
\hline 0 & 8.179083 & 20.476839 & 13.150764 \\
\hline 0 & 8.188308 & 22.917406 & 14.322499 \\
\hline $\mathrm{O}$ & 6.424030 & 8.940578 & 2.9449 \\
\hline $\mathrm{O}$ & 279 & 9.953071 & 32 \\
\hline 0 & 8.175200 & 8.182724 & 1.02268 \\
\hline
\end{tabular}




$\begin{array}{rrrr}\mathrm{O} & 8.260160 & 10.627177 & 2.186413 \\ \mathrm{O} & 13.235725 & 5.219039 & 8.265259 \\ \mathrm{O} & 12.468162 & 6.659979 & 5.965240 \\ \mathrm{O} & 11.805708 & 7.754035 & 8.431053 \\ \mathrm{O} & 14.487082 & 7.754278 & 7.587512 \\ \mathrm{O} & 8.678411 & 16.941729 & 20.559614 \\ \mathrm{O} & 7.240385 & 18.949238 & 19.126200 \\ \mathrm{O} & 8.179568 & 19.386423 & 21.919720 \\ \mathrm{O} & 9.507632 & 19.985521 & 19.371372 \\ \mathrm{H} & 12.687847 & 22.178972 & 3.146716 \\ \mathrm{H} & 15.874617 & 7.974935 & 7.932453 \\ \mathrm{H} & 25.041433 & 6.152154 & 1.709417 \\ \mathrm{H} & 21.939627 & 16.020021 & 6.495154 \\ \mathrm{H} & 13.609553 & 4.995955 & 9.184537 \\ \mathrm{H} & 8.918002 & 15.931904 & 19.567997 \\ \mathrm{H} & 9.494524 & 19.181545 & 15.860780 \\ \mathrm{H} & 11.103202 & 17.874844 & 20.065142 \\ \mathrm{H} & 10.859727 & 20.867903 & 20.677345 \\ \mathrm{H} & 12.920155 & 20.197681 & 19.400503 \\ \mathrm{H} & 11.810321 & 21.111620 & 18.360823 \\ \mathrm{H} & 11.914944 & 19.331320 & 18.216873 \\ \mathrm{H} & 10.907306 & 17.584518 & 22.572706 \\ \mathrm{H} & 10.897110 & 19.350740 & 22.780497 \\ \mathrm{H} & 12.343633 & 18.517878 & 22.144989 \\ \mathrm{Re} & 12.990309 & 6.881606 & 7.566392 \\ \mathrm{Re} & 8.589082 & 18.682461 & 20.442368 \\ \mathrm{C} & 10.748549 & 18.692656 & 20.709875 \\ \mathrm{C} & 10.849532 & 20.022417 & 19.974110 \\ \mathrm{C} & 11.928781 & 20.173649 & 18.921322 \\ \mathrm{C} & 11.240596 & 18.533899 & 22.134066 \\ & & & \end{array}$

\begin{tabular}{lrrr} 
607 & & & \\
USY_ReO3_C $4 \mathrm{H} 8$ & \multicolumn{3}{l}{$\mathrm{E}=-7249.18733153525$} \\
$\mathrm{Si}$ & 23.382507 & 3.327319 & 1.244316 \\
$\mathrm{Si}$ & 23.427416 & 1.240432 & 3.377325 \\
$\mathrm{Si}$ & 23.394402 & 15.565844 & 13.403220 \\
$\mathrm{Si}$ & 23.385662 & 13.418756 & 15.570941 \\
$\mathrm{Si}$ & 11.232585 & 1.218828 & 15.635755 \\
$\mathrm{Si}$ & 11.173841 & 15.582108 & 1.217371 \\
$\mathrm{Si}$ & 11.231857 & 13.401278 & 3.370771 \\
$\mathrm{Si}$ & 7.633148 & 3.353536 & 1.216643 \\
$\mathrm{Si}$ & 7.657908 & 5.501352 & 3.346739 \\
$\mathrm{Si}$ & 7.745782 & 15.418739 & 13.207566 \\
$\mathrm{Si}$ & 19.993530 & 5.451347 & 15.515595 \\
$\mathrm{Si}$ & 19.909540 & 15.642795 & 1.270532 \\
$\mathrm{Si}$ & 19.884296 & 17.802990 & 3.350866 \\
$\mathrm{Si}$ & 7.625865 & 3.371499 & 5.510577 \\
$\mathrm{Si}$ & 7.511046 & 15.475786 & 17.726282 \\
$\mathrm{Si}$ & 7.747967 & 13.284516 & 15.484524 \\
$\mathrm{Si}$ & 19.922407 & 3.368101 & 17.736237 \\
$\mathrm{Si}$ & 19.873371 & 1.225139 & 15.609052 \\
$\mathrm{Si}$ & 19.935514 & 15.587934 & 5.435811 \\
$\mathrm{Si}$ & 20.010038 & 13.377489 & 3.281683 \\
$\mathrm{Si}$ & 23.429600 & 3.354022 & 5.539464 \\
$\mathrm{Si}$ & 23.368185 & 17.734535 & 15.565116 \\
$\mathrm{Si}$ & 11.239625 & 3.366401 & 17.794252 \\
$\mathrm{Si}$ & 11.280649 & 5.544804 & 15.572398 \\
$\mathrm{Si}$ & 11.243024 & 15.645464 & 5.448677
\end{tabular}




\begin{tabular}{|c|c|c|c|}
\hline i & 11.183793 & 17.789883 & 3.236775 \\
\hline . & 1.305974 & 23.404354 & 3.332902 \\
\hline & 3.378539 & 23.429115 & 1.201593 \\
\hline & 1.223683 & 11.203213 & 58259 \\
\hline & 3.365673 & 11.218748 & .3908 \\
\hline & 13.475801 & 23.402412 & 15.53914 \\
\hline i & 15.626774 & 23.385422 & $13 \quad 389$ \\
\hline i & 13.431377 & 11.228944 & - \\
\hline & 15.610995 & 11.169714 & 207 \\
\hline S & 1.358164 & 7.680968 & 3.34188 \\
\hline & 3.379267 & 7.603047 & 25 \\
\hline 5 & 1.295778 & 19.898617 & $15.59^{\circ}$ \\
\hline i & 3.416407 & $19 . \varepsilon$ & 17. \\
\hline S & 13.459294 & 7.650140 & 15.582108 \\
\hline & 15.601527 & 7.632905 & 17. \\
\hline & 15.559047 & 19.952265 & 5.49989 \\
\hline & 5.524656 & 7.661306 & 3.37926 \\
\hline$\perp$ & 3.404998 & & \\
\hline $\mathrm{Si}$ & 5.522714 & 19.940369 & 15.67556 \\
\hline 2 & 3.411067 & 19.822151 & 13.45274 \\
\hline 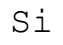 & 17.817799 & 7.668103 & 15.63478 \\
\hline S & 15.595216 & 7.676356 & 13.46196 \\
\hline$\perp$ & 17.726526 & 19.965858 & 3.36106 \\
\hline i & 15.610752 & 20. & \\
\hline i & 5.498925 & 23.366243 & 3.37538 \\
\hline $\mathrm{Si}$ & 3.406697 & 23.403625 & 5.50790 \\
\hline $\mathrm{Si}$ & 5.492857 & 11.173113 & 15.60128 \\
\hline i & 3.357177 & 11.237440 & 17.75201 \\
\hline i & 17.734295 & 23.3 & 15. \\
\hline $\mathrm{Si}$ & 15.554677 & 23.385906 & 17.74424 \\
\hline $\mathrm{Si}$ & 17.821924 & 11.267541 & 3.307900 \\
\hline $\mathrm{Si}$ & 15.668283 & 11.231857 & 5.479020 \\
\hline $\mathrm{Si}$ & 3.357662 & 1.278058 & 23.36721 \\
\hline $\mathrm{Si}$ & 1.241160 & 3.3656 & 23.4143 \\
\hline i & 3.363003 & 13.3 & 11.23 \\
\hline $\mathrm{Si}$ & 1.273931 & 15.554920 & 11.25855 \\
\hline $\mathrm{Si}$ & 15.662457 & 1.225139 & 11.18864 \\
\hline $\mathrm{Si}$ & 13.397636 & 3.329261 & $11.24156^{\circ}$ \\
\hline $\mathrm{Si}$ & 15.567543 & 13.417057 & 23.39173 \\
\hline $\mathrm{Si}$ & 13.457838 & 15.5 & 23.41988 \\
\hline $\mathrm{Si}$ & 3.3 & 1.2 & 7.65548 \\
\hline $\mathrm{Si}$ & 5.501595 & 3.323192 & 7.61372 \\
\hline $\mathrm{Si}$ & 3.302802 & 13.376032 & 19.96391 \\
\hline $\mathrm{Si}$ & 17.779203 & 3.362518 & 19.85370 \\
\hline $\mathrm{Si}$ & 15.649590 & 13.416 & 7.65062 \\
\hline $\mathrm{Si}$ & 17.826538 & 15.566571 & \\
\hline $\mathrm{Si}$ & 3.332174 & 5.438239 & 7.65499 \\
\hline $\mathrm{Si}$ & 1.235092 & 3.331689 & 7.664462 \\
\hline $\mathrm{Si}$ & 3.402085 & 17.738420 & 19.87531 \\
\hline $\mathrm{Si}$ & 1.248928 & 15.575797 & 19.87701 \\
\hline $\mathrm{Si}$ & 15.573612 & 5.467854 & 19.93697 \\
\hline $\mathrm{Si}$ & 13.400549 & 7716 & 19.95614 \\
\hline $\mathrm{Si}$ & 15.670711 & 17.774103 & 7.63339 \\
\hline $\mathrm{Si}$ & 13.477986 & 15.639639 & 7.62635 \\
\hline $\mathrm{Si}$ & 3.376839 & 5.511062 & 23.38153 \\
\hline $\mathrm{Si}$ & 5.503051 & 3.387278 & 23.346092 \\
\hline $\mathrm{Si}$ & 5.546503 & 15.533801 & 11.10805 \\
\hline $\mathrm{Si}$ & 17.854454 & 3.318581 & 11.25176 \\
\hline
\end{tabular}




\begin{tabular}{|c|c|c|c|}
\hline i & 15.618521 & 17.758083 & 4651 \\
\hline$i$ & 17.733324 & 15.610995 & 23.427658 \\
\hline $\mathrm{Si}$ & 1.643148 & 21.676731 & 23.726965 \\
\hline S & 1.612562 & 23.784494 & 1.613375 \\
\hline & 1.671063 & 9.440634 & 11.584081 \\
\hline$\perp$ & 1.666937 & 11.588450 & 9.453743 \\
\hline i & 13.868807 & 21.702705 & 11.541358 \\
\hline & 13.835793 & 23.864115 & 9.380191 \\
\hline & 13.836764 & 9.415146 & 23.789349 \\
\hline & 13.813461 & 11.620978 & 21.66338 \\
\hline & 17.484509 & 21.681101 & 23.77624 \\
\hline & 17.397848 & 19.535955 & 21.689840 \\
\hline & 17.362892 & 9.484571 & 11.615638 \\
\hline i & 17.445911 & 7.304713 & 9.51612 \\
\hline & 5.108832 & 21.637405 & 11.641127 \\
\hline & 5.114172 & 19.546877 & 9.494524 \\
\hline & 5.183355 & 9.436023 & 23.731091 \\
\hline$i$ & 5.151798 & 7.305926 & 21.613619 \\
\hline i & 17.342987 & 21.699064 & 19.525032 \\
\hline $\mathrm{Si}$ & 17.414841 & 23.8 & 21.66556 \\
\hline $\mathrm{Si}$ & 17.387409 & 11.676568 & 9.50617 \\
\hline$\perp$ & 5.097908 & 21.605850 & 7.31563 \\
\hline$i$ & 5.103977 & 23.746628 & 9.440634 \\
\hline$i$ & 5.077761 & 2725 & \\
\hline$i$ & 5.03 & 11.6 & 21.7 \\
\hline i & 1.579063 & 21.624784 & 19.54736 \\
\hline i & 1.599939 & 19.532799 & 21.621872 \\
\hline i & 1.628826 & 9.420730 & 7.30544 \\
\hline$i$ & 1.619601 & 7.275826 & 9.432382 \\
\hline$i$ & 13.877060 & 21.746643 & 3340 \\
\hline i & 13.890654 & 19.544935 & 159 \\
\hline Si & 13.815403 & 7.233588 & 21.65391 \\
\hline Si & 23.769688 & 1.593870 & 21.625027 \\
\hline i & 21.673334 & 1.536339 & 23.71191 \\
\hline$i$ & 23.855135 & 13.847689 & 01020 \\
\hline $\mathrm{Si}$ & 21.728193 & 8310 & $11.59257 \varepsilon$ \\
\hline$i$ & 11.544999 & 1.660625 & 9.474861 \\
\hline $\mathrm{Si}$ & 9.420486 & 1.714515 & 11.621464 \\
\hline $\mathrm{Si}$ & 11.675112 & 13.826569 & 21.71775 \\
\hline $\mathrm{Si}$ & 9.458597 & 13.778020 & 23.814596 \\
\hline $\mathrm{Si}$ & 23.814838 & 17.347841 & 21.70270 \\
\hline $\mathrm{Si}$ & 21.693239 & 17.389109 & 19.55925 \\
\hline $\mathrm{Si}$ & 21.671148 & 5.179714 & 7.25907 \\
\hline Si & 11.680695 & 17.374544 & 9.37485 \\
\hline $\mathrm{Si}$ & 9.464910 & 17.369204 & 7.211984 \\
\hline $\mathrm{Si}$ & 11.580197 & 5.159809 & 21.638863 \\
\hline $\mathrm{Si}$ & 9.474619 & 5.116600 & 19.51022 \\
\hline $\mathrm{Si}$ & 19.536 & 17.384010 & 21.69760 \\
\hline $\mathrm{Si}$ & 21.722368 & 17.393478 & 23.82382 \\
\hline Si & 19.570183 & 5.148157 & 9.49500 \\
\hline $\mathrm{Si}$ & 21.738146 & 5.107861 & 11.645982 \\
\hline $\mathrm{Si}$ & 7.255435 & 17.346630 & 9.335768 \\
\hline $\mathrm{Si}$ & 9.446218 & 17.215546 & $11.52266^{\circ}$ \\
\hline Si & 7.294274 & 5.155925 & 21.64833 \\
\hline Si & 9.409320 & 5.116843 & 23.77624 \\
\hline $\mathrm{Si}$ & 19.555616 & 1.640477 & 21.595898 \\
\hline $\mathrm{Si}$ & 21.676731 & 1.614261 & 19.47866 \\
\hline $\mathrm{Si}$ & 19.550520 & 13.820500 & 9.43432 \\
\hline
\end{tabular}




\begin{tabular}{|c|c|c|c|}
\hline Si & 7.293061 & 1.613775 & 9.433352 \\
\hline Si & 9.425584 & 1.662325 & 7.296702 \\
\hline & 7.257377 & 13.747191 & 21.645174 \\
\hline & 21.703918 & 23.747355 & 1.587073 \\
\hline & 23.836443 & 21.646873 & 1.617417 \\
\hline & 21.699551 & 11.580197 & 13.784331 \\
\hline - & 23.837414 & 9.395484 & 13.7870 \\
\hline & 9.470736 & 23.835714 & 13.8566 \\
\hline i & 9.465880 & 11.594034 & 1.66936 \\
\hline & 11.620251 & 9.461025 & 1.647032 \\
\hline & 21.677460 & 23.768230 & 17.38352 \\
\hline & 19.534740 & 21.675516 & 17.3988 \\
\hline 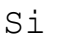 & 21.813883 & 11.641612 & $4.8830^{\circ}$ \\
\hline $\mathrm{Si}$ & 19.636452 & 9.533849 & 5.014404 \\
\hline & 9.358586 & 23.826248 & 5.16199 \\
\hline $\mathrm{Si}$ & 9.473405 & 11.528250 & 17.295410 \\
\hline Si & 7.260047 & 9.418302 & 17.36799 \\
\hline Si & 21.696152 & 19.521633 & 17.34347 \\
\hline Si & 23.831102 & 21.685471 & 17.353668 \\
\hline $\mathrm{Si}$ & 21.700764 & 7.3 & 5.1238 \\
\hline $\mathrm{Si}$ & 23.811197 & 9.445246 & 5.13140 \\
\hline Si & 9.485542 & 19.520176 & 5.01319 \\
\hline $\mathrm{Si}$ & 11.570003 & 21.728680 & 5.12946 \\
\hline $\mathrm{Si}$ & 9.440392 & 7.2 & 17.319927 \\
\hline $\mathrm{Si}$ & 11.591364 & 9.3 & 17.353910 \\
\hline Si & 21.697851 & 19.507069 & 1.63489 \\
\hline $\mathrm{Si}$ & 19.561443 & 21.629639 & 1.65237 \\
\hline $\mathrm{Si}$ & 21.718241 & 7.236986 & 13.77243 \\
\hline $\mathrm{Si}$ & 19.539352 & 9.393542 & 13.842589 \\
\hline $\mathrm{Si}$ & 9.468551 & 19.573580 & 13.649121 \\
\hline $\mathrm{Si}$ & 7.3 & 21.6 & 13.8 \\
\hline $\mathrm{Si}$ & 9.429468 & 7.296459 & 1.613047 \\
\hline Si & 7.311266 & 9.418545 & 1.663781 \\
\hline $\mathrm{Si}$ & 11.671227 & 21.742273 & 13.72752 \\
\hline $\mathrm{Si}$ & 11.223846 & 433 & 13.39666 \\
\hline $\mathrm{Si}$ & 20.0 & 3.3 & 13.3 \\
\hline Si & 7.628778 & 1.212759 & 3.36518 \\
\hline $\mathrm{Si}$ & 23.373283 & 15.601527 & $17.72312^{\circ}$ \\
\hline $\mathrm{Si}$ & 5.467854 & 15.570457 & 19.96925 \\
\hline $\mathrm{Si}$ & 3.468354 & 17.684288 & 11.28599 \\
\hline Si & 13.805693 & $9.41 \mathrm{~s} \quad-9$ & 19.49711 \\
\hline $\mathrm{Si}$ & $23.7 \varepsilon$ & 5.1 & 9.42291 \\
\hline Al & 15.582836 & 1.289224 & 19.89521 \\
\hline Al & 15.588663 & 5.490429 & 11.28865 \\
\hline Al & 17.514608 & 9.564435 & 7.230675 \\
\hline Al & 9.488213 & 13.511727 & $19.65635^{\circ}$ \\
\hline Al & 7.2 & 21.6 & 5.17850 \\
\hline Al & 7.579 & 17.6 & 15.37747 \\
\hline Al & 23.369400 & 5.398913 & 3.41155 \\
\hline Al & 13.457109 & 19.863661 & 3.29017 \\
\hline Al & 21.791065 & 13.673396 & 7.23674 \\
\hline Al & 16.438272 & 4.417734 & 14.09990 \\
\hline Al & 7.281895 & 18.346256 & 17.91756 \\
\hline 0 & 22.164650 & 0.349069 & 2.86658 \\
\hline 0 & 24.605463 & 24.542347 & 3.95578 \\
\hline 0 & 24.029911 & 2.099267 & 2.11723 \\
\hline 0 & 22.846767 & 2.186171 & 4.55779 \\
\hline 0 & 22.159554 & 12.470833 & 15.08229 \\
\hline
\end{tabular}




$\begin{array}{rrr}24.606674 & 12.470346 & 16.097700 \\ 23.941793 & 14.330024 & 14.330995 \\ 22.845797 & 14.396051 & 16.769865 \\ 9.959140 & 0.251728 & 15.247118 \\ 12.540502 & 24.614685 & 16.092604 \\ 11.634330 & 2.101209 & 14.324198 \\ 10.712137 & 2.121843 & 16.864779 \\ 9.945061 & 12.480785 & 2.948393 \\ 12.483455 & 12.470346 & 3.797518 \\ 11.719776 & 14.330995 & 2.109705 \\ 10.782048 & 14.322256 & 4.626981 \\ 8.928198 & 6.407765 & 2.879453 \\ 6.415049 & 6.409223 & 3.883693 \\ 7.077987 & 4.629166 & 2.090528 \\ 8.180782 & 4.555371 & 4.550274 \\ 8.952958 & 18.803104 & 15.153662 \\ 6.405581 & 18.629784 & 16.333164 \\ 7.168531 & 16.689514 & 14.065674 \\ 8.008190 & 16.863321 & 16.951437 \\ 21.318680 & 6.240271 & 15.000974 \\ 18.785383 & 6.413349 & 16.015896 \\ 19.379141 & 4.553915 & 14.253317 \\ 20.479994 & 4.545176 & 16.756027 \\ 21.241972 & 18.548222 & 2.867801 \\ 18.719357 & 18.785141 & 3.884178 \\ 19.283499 & 16.851669 & 2.176218 \\ 20.398674 & 16.855068 & 4.563868 \\ 8.930383 & 0.342515 & 3.795091 \\ 6.401940 & 24.609346 & 2.782597 \\ 7.082600 & 2.106307 & 4.636448 \\ 8.175685 & 2.190540 & 2.187627 \\ 9.024811 & 12.463551 & 16.006428 \\ 6.480590 & 12.380774 & 15.085450 \\ 7.248638 & 14.247733 & 16.772049 \\ 8.180540 & 14.242879 & 14.250404 \\ 21.142691 & 0.334018 & 16.100613 \\ 18.635366 & 24.614685 & 15.010684 \\ 19.303162 & 2.120872 & 16.848999 \\ 20.482664 & 2.120143 & 14.395323 \\ 21.319410 & 12.466706 & 3.547733 \\ 18.706491 & 12.549968 & 2.783568 \\ 19.559015 & 14.239723 & 4.634992 \\ 20.480722 & 14.410858 & 2.129853 \\ 22.257380 & 6.574047 & 3.874469 \\ 24.774899 & 6.401212 & 2.621414 \\ 24.111473 & 4.551973 & 4.716797 \\ 22.759865 & 4.486188 & 2.116260 \\ 22.085758 & 18.627598 & 16.027548 \\ 24.597937 & 18.708920 & 15.089577 \\ 23.933784 & 16.860893 & 16.837589 \\ 22.860846 & 16.789768 & 14.342890 \\ 10.025167 & 6.492727 & 16.006670 \\ 12.556279 & 6.407280 & 15.020151 \\ 11.719534 & 4.630865 & 16.855068 \\ 10.775738 & 4.632807 & 14.327353 \\ 12.371064 & 18.703335 & 2.692781 \\ 11.724631 & 16.849241 & 4.471867\end{array}$




\begin{tabular}{|c|c|c|c|}
\hline O & 10.620137 & 16.850456 & 2.029599 \\
\hline 0 & 2.950092 & 22.170719 & 0.257311 \\
\hline O & 3.970111 & 24.614202 & 24.52899 \\
\hline O & 2.098782 & 24.022629 & 2.03129 \\
\hline 0 & 4.488616 & 22.856234 & 2.194909 \\
\hline 0 & 2.948393 & 9.944575 & 12.47277 \\
\hline 0 & 3.883450 & 12.465979 & 12.48005 \\
\hline 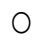 & 2.119658 & 11.729243 & 14.32080 \\
\hline 0 & 4.575519 & 10.706554 & 14.3504 \\
\hline 0 & 15.170167 & 22.163195 & 12.40432 \\
\hline O & 16.176350 & 24.610802 & 12.465006 \\
\hline 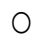 & 14.326383 & 23.932570 & 14.244821 \\
\hline 0 & 16.772533 & 22.841427 & 14.4021 \\
\hline 0 & 15.167012 & 9.858400 & $0.3417 \varepsilon$ \\
\hline 0 & 16.103041 & 12.396310 & 24.52584 \\
\hline 0 & 14.315701 & 11.715164 & 2.03761 \\
\hline 0 & 16.861135 & 10.709952 & 2.112376 \\
\hline 0 & 2.868287 & 8.859500 & 6.407523 \\
\hline 0 & 3.895102 & 6.407038 & 6.47767 \\
\hline 0 & 2.193696 & 6.995939 & 4.55027 \\
\hline 0 & 4.555129 & 8.177141 & 4.56629 \\
\hline O & 2.868772 & 21.068653 & 18.704792 \\
\hline 0 & 4.040993 & 18.624201 & 32696 \\
\hline 0 & 2.281327 & 19.293938 & 16.772533 \\
\hline 0 & 4.708301 & 20.492617 & 16.945612 \\
\hline 0 & 15.092247 & 8.936208 & 18.616674 \\
\hline 0 & 16.096972 & 6.420146 & 18.713531 \\
\hline 0 & 14.409160 & 0415 & 32730 \\
\hline 0 & 16.872547 & 8.107230 & 16.866962 \\
\hline 0 & 15.174294 & 21.323050 & 6.324261 \\
\hline 0 & 16.179262 & 18.798977 & 6.485930 \\
\hline 0 & 14.262540 & 19.389336 & 4.733790 \\
\hline 0 & 16.773748 & 20.477324 & 4.552944 \\
\hline 0 & 3.863060 & 8.8 & 73 \\
\hline 0 & 2.780170 & 7038 & 24.619783 \\
\hline 0 & 4.638391 & 7.151783 & 2.100238 \\
\hline 0 & 2.207775 & 8.266715 & 2.103879 \\
\hline 0 & 3.879324 & 21.077393 & 12.555308 \\
\hline 0 & 2.955918 & 18.549677 & 12.571571 \\
\hline 0 & 4.6359 & 19.310444 & 14.483439 \\
\hline 0 & 2.190297 & 20.395033 & 14.337063 \\
\hline 0 & 16.099155 & 8.854646 & 12.464035 \\
\hline 0 & 15.094189 & 6.321834 & 12.732270 \\
\hline 0 & 16.861866 & 7.152025 & 14.398964 \\
\hline 0 & 14.407946 & 8.265016 & 14.409160 \\
\hline 0 & 16.178535 & 21.232748 & 0.34008 \\
\hline 0 & 15.077682 & 18.731495 & 24.613716 \\
\hline 0 & 16.858952 & 19.382296 & 2.110434 \\
\hline 0 & 14.425423 & 20.632681 & 2.101938 \\
\hline 0 & 3.799946 & 22.169991 & 6.491271 \\
\hline 0 & 2.777742 & 24.611530 & 6.414320 \\
\hline 0 & 4.638876 & 24.018501 & 4.635478 \\
\hline 0 & 2.279628 & 22.841427 & 4.485703 \\
\hline 0 & 3.806500 & 9.946517 & 18.630512 \\
\hline 0 & 2.863432 & 12.471076 & 18.699209 \\
\hline 0 & 4.623097 & 11.794299 & 16.851912 \\
\hline 0 & 2.119658 & 10.712623 & 16.848757 \\
\hline , & 16.024876 & 22.079447 & 18.62638 \\
\hline
\end{tabular}




\begin{tabular}{|c|c|c|c|}
\hline & 14.920139 & 24.528755 & 18.630512 \\
\hline & 16.842445 & 23.931112 & 16.856281 \\
\hline & 14.413043 & 22.763264 & 16.692913 \\
\hline & 16.097700 & 9.955499 & 6.327902 \\
\hline & 15.169683 & 12.487339 & 6.409950 \\
\hline & 16.918182 & 11.802552 & 4.556828 \\
\hline & 14.403334 & 10.788117 & 4.543477 \\
\hline & 0.343971 & 2.867801 & 22.162224 \\
\hline & 24.608860 & 3.964285 & 24.614927 \\
\hline & 2.110676 & 2.115288 & 24.016075 \\
\hline & 2.193210 & 4.554643 & 22.837299 \\
\hline & 0.425776 & 15.154633 & 9.945788 \\
\hline & 24.619055 & 16.101583 & 12.47811 \\
\hline & 2.109220 & 14.250889 & 11.80133 \\
\hline & 2.258994 & 16.756271 & 10.783262 \\
\hline & 12.390483 & 2.934799 & 10.032449 \\
\hline & 12.558949 & 3.793877 & 12.557250 \\
\hline & 14.400420 & 2.120872 & 11.722204 \\
\hline & 14.257929 & 4.559255 & 10.62887 \\
\hline & 12.641726 & 15.089819 & 22.085274 \\
\hline & 12.390483 & 16.094059 & 24.525841 \\
\hline & 14.334879 & 14.320801 & 24.016804 \\
\hline & 14.411101 & 16.785643 & 22.926630 \\
\hline & 6.426700 & 2.867073 & 8.871152 \\
\hline & 6.396357 & 3.952876 & 6.408009 \\
\hline & 4.642517 & 2.038824 & 7.078473 \\
\hline & 4.533524 & 4.488373 & 8.185637 \\
\hline & 6.573319 & 15.165556 & 21.073994 \\
\hline & 6.245126 & 16.091873 & 18.546764 \\
\hline & 4.560469 & 14.326626 & 19.469929 \\
\hline & 4.635235 & 16.854097 & 20.480965 \\
\hline & 18.704063 & 2.942324 & 21.147058 \\
\hline & 18.794607 & 3.972053 & 18.708433 \\
\hline & 16.933233 & 2.181558 & 19.210918 \\
\hline & 16.833464 & 4.575034 & 20.404015 \\
\hline & 18.707705 & 15.175994 & 9.019956 \\
\hline & 18.802376 & 16.017593 & 6.483746 \\
\hline & 16.931532 & 14.326869 & 7.162463 \\
\hline & 16.927162 & 16.861135 & 02375 \\
\hline & 0.417523 & 3.881023 & 8.951015 \\
\hline & 24.522926 & 2.692053 & 6.563124 \\
\hline & 2.112376 & 4.556585 & 7.006135 \\
\hline & 2.204862 & 2.181801 & 8.256519 \\
\hline & 0.418737 & 16.097214 & 21.157497 \\
\hline & 24.617113 & 15.077197 & 18.631968 \\
\hline & 2.214572 & 16.779331 & 19.298792 \\
\hline & 2.105336 & 14.331723 & 20.470284 \\
\hline & 12.393639 & 3.869128 & 21.152643 \\
\hline & 12.473502 & 2.866830 & 18.700909 \\
\hline 0 & 14.242393 & 4.701990 & 19.380354 \\
\hline & 14.326140 & 2.275744 & 20.553303 \\
\hline & 12.571330 & 16.092360 & 8.928926 \\
\hline & 12.483212 & 15.240322 & 6.412135 \\
\hline & 14.418627 & 16.866962 & 7.085756 \\
\hline & 14.413043 & 14.400420 & 8.093637 \\
\hline & 6.404367 & 3.871070 & 22.085758 \\
\hline & 6.422574 & 2.790123 & 24.54210 \\
\hline & 4 & 4.643 .30 & 23.9449 \\
\hline
\end{tabular}




\begin{tabular}{|c|c|c|c|}
\hline 0 & 4.485460 & 2.271860 & 22.761806 \\
\hline 0 & 6.321349 & 16.082407 & 9.783392 \\
\hline . & 6.577202 & 14.916013 & 12.218134 \\
\hline 0 & 4.728207 & 16.773020 & 11.80400 \\
\hline $\mathrm{O}$ & 4.555857 & 14.331481 & 10.69417 \\
\hline O & 18.538755 & 3.959187 & 9.938021 \\
\hline 0 & 18.961132 & 2.784054 & 12.317660 \\
\hline 0 & 16.852398 & 4.391032 & 12.0 \\
\hline 0 & 16.936630 & 2.102909 & 10.70388 \\
\hline 0 & 18.630512 & 16.107653 & 22.15979 \\
\hline 0 & 18.713289 & 15.083508 & 24.6059 \\
\hline 0 & 16.856039 & 16.856766 & 24.021416 \\
\hline 0 & 2049 & 6975 & 22. \\
\hline 0 & 2.866830 & 24.693335 & 22.079933 \\
\hline 0 & 0.428689 & 0.421650 & 20. \\
\hline 0 & 1.007639 & 22.912794 & 22.8525 \\
\hline O & 2.199764 & 22.772972 & 20.483877 \\
\hline 0 & 2.952034 & 12.468162 & \\
\hline 0 & 0.503455 & 12.579097 & 3052 \\
\hline 0 & 1.092842 & 10.698787 & 10.708739 \\
\hline 0 & 2.190783 & 10.616253 & 8.258946 \\
\hline O & 15.163614 & 24.688969 & 9.86811 \\
\hline O & 12.564532 & 0.507582 & 8.941063 \\
\hline 0 & 13.310976 & 22.927116 & 10. \\
\hline 0 & 14.326869 & 23.009649 & 8.099706 \\
\hline 0 & 15.083750 & 12.562591 & 22.08187 \\
\hline 0 & 12.557250 & 12.557007 & 21.225952 \\
\hline O & 13.315831 & 10.704370 & 22.919348 \\
\hline 0 & 14.250889 & 10.701700 & 20. \\
\hline 0 & 16.109837 & 18.631725 & 22.156397 \\
\hline 0 & 18.621531 & 18.606480 & 21.148031 \\
\hline 0 & 18.035786 & 20.392847 & 22.923231 \\
\hline 0 & 16.853369 & 20.473925 & 20.482664 \\
\hline 0 & 16.184847 & 6.478162 & 10.031478 \\
\hline 0 & 18.706247 & 273 & \\
\hline 0 & 18.022676 & 8.351191 & 10.622322 \\
\hline 0 & 16.866720 & 8.107230 & 8.171558 \\
\hline 0 & 3.879809 & 18.626143 & 10.036819 \\
\hline C & 6.332272 & 18.620802 & 8.935237 \\
\hline 0 & 5.7 & 20. & 10. \\
\hline 0 & 4.472837 & 20.4 & 8 . \\
\hline 0 & 3.869371 & 6.483502 & 22.175331 \\
\hline 0 & 6.322562 & 6.325961 & 21.070351 \\
\hline 0 & 5.814495 & 8.273512 & 22.761320 \\
\hline 0 & 4.553672 & 8.193648 & 20.39 \\
\hline 0 & 16.179262 & 24.7 & 21. \\
\hline 0 & 18.707220 & 0.417280 & 22.234806 \\
\hline 0 & 17.949852 & 22.929543 & 20.391392 \\
\hline 0 & 16.937601 & 22.845797 & 22.837543 \\
\hline 0 & 16.096001 & 12.544869 & 8.950288 \\
\hline 0 & 18.526617 & 12.716491 & 10.039489 \\
\hline 0 & 18.030445 & 10.704127 & 8.417217 \\
\hline 0 & 16.852640 & 10.787389 & 10.798070 \\
\hline 0 & 3.807956 & 24.594782 & 8.945189 \\
\hline 0 & 6.319649 & 0.424563 & 9.964480 \\
\hline 0 & 5.720309 & 22.829290 & 8.256276 \\
\hline 0 & 3965 & 22.843126 & 10.713594 \\
\hline 0 & 3.711101 & 12.473261 & 21.23905 \\
\hline
\end{tabular}




\begin{tabular}{|c|c|c|c|}
\hline 0 & 6.145843 & 12.721590 & 22.242573 \\
\hline 0 & 5.578060 & 10.711410 & 20.476109 \\
\hline . & 4.566052 & 10.704613 & 22.926630 \\
\hline 0 & 2.884308 & 18.700909 & 21.05772 \\
\hline O & 0.499329 & 18.537054 & 22.25373 \\
\hline O & 0.927532 & 20.391876 & 20.398916 \\
\hline 0 & 2.195395 & 20.489462 & 22.762291 \\
\hline 0 & 2.856149 & 6.329359 & 8. \\
\hline 0 & 0.426990 & 6.338826 & 10.02880 \\
\hline 0 & 1.020504 & 8.176413 & 8.18927 \\
\hline 0 & 2.191754 & 8.248752 & 10.61382 \\
\hline 0 & 15.170410 & 18.629784 & 8.940820 \\
\hline 0 & 12.630074 & 18.624443 & 9.79552 \\
\hline 0 & 13.391325 & 20.481207 & 8.09922 \\
\hline 0 & 14.330509 & 20.463488 & 10. \\
\hline 0 & 15.094916 & 6.329359 & 21.240030 \\
\hline 0 & 12.560649 & 6.336641 & 22.170477 \\
\hline 0 & 13.304180 & 8.165976 & 20.4040 \\
\hline 0 & 14.325897 & 8.186608 & 22.856962 \\
\hline 0 & 22.171448 & 2.777985 & 24.60521 \\
\hline 0 & 21.076178 & 0.332319 & 0.35295 \\
\hline 0 & 22.922747 & 0.921706 & 22.84215 \\
\hline O & 20.560343 & 2.187141 & 22.748213 \\
\hline 0 & 22.160767 & 15.080837 & 12.477144 \\
\hline 0 & 21.240274 & 12.551182 & 12.567202 \\
\hline 0 & 23.000669 & 13.240823 & 10.70388 \\
\hline 0 & 20.486547 & 14.258657 & 10.69781 \\
\hline 0 & 9.969092 & 3.036510 & 12.40723 \\
\hline 0 & 8.938393 & 0.589630 & 12. \\
\hline 0 & 10.621351 & 1.078763 & 10.705827 \\
\hline 0 & 8.170102 & 2.180102 & 10.701942 \\
\hline 0 & 9.849175 & 15.092247 & 24.677557 \\
\hline 0 & 8.930140 & 12.542928 & 0.44713 \\
\hline 0 & 10.794671 & 13.310492 & 23.004 \\
\hline 0 & 8.250693 & 14.247733 & 04 \\
\hline 0 & 22.166836 & 16.090660 & 18.696539 \\
\hline 0 & 21.226194 & 18.618132 & 18.610123 \\
\hline 0 & 22.923475 & 17.954708 & 20.479507 \\
\hline 0 & 20.477079 & 16.855795 & 20.480478 \\
\hline 0 & 22.145231 & 3.879 & 6.40 \\
\hline 0 & 21.14 & 6.4 & 6 . \\
\hline 0 & 22.920563 & 5.733418 & 8.178355 \\
\hline 0 & 20.484606 & 4.639604 & 8.262831 \\
\hline 0 & 9.942390 & 16.095272 & 6.325961 \\
\hline 0 & 9.022868 & 18.628084 & 6.313 \\
\hline 0 & 10.695874 & 17.868774 & 311 \\
\hline 0 & 8.181026 & 16.862593 & 8.098977 \\
\hline 0 & 9.952585 & 3.788537 & 18.70333 \\
\hline 0 & 8.928926 & 6.235659 & 18.46228 \\
\hline 0 & 10.692475 & 5.751624 & 20.390905 \\
\hline 0 & 8.261860 & 4.632565 & 20.468828 \\
\hline 0 & 21.232506 & 16.180477 & 24.772470 \\
\hline 0 & 22.328747 & 18.626627 & 0.418251 \\
\hline 0 & 20.476353 & 17.948881 & 22.920319 \\
\hline 0 & 22.922020 & 16.773748 & 22.918861 \\
\hline 0 & 21.403885 & 3.866215 & 12.650950 \\
\hline 0 & 22.254953 & 6.404125 & 12.479571 \\
\hline 0 & 20.390421 & 5.565923 & 10.85050 \\
\hline
\end{tabular}




\begin{tabular}{|c|c|c|}
\hline 22.848225 & 4.554886 & 10.618438 \\
\hline 9.090837 & 15.861023 & 12.383444 \\
\hline 9.786790 & 18.377813 & 12.654591 \\
\hline 8.195348 & 17.773619 & 10.635430 \\
\hline 10.795885 & 16.939058 & 10.693204 \\
\hline 8.929411 & 3.803587 & 24.608131 \\
\hline 9.954285 & 6.325961 & 0.429660 \\
\hline 8.157479 & 5.731476 & 22.912794 \\
\hline 10.622564 & 4.633293 & 22.836329 \\
\hline 21.240517 & 2.865131 & 18.541182 \\
\hline 22.319036 & 0.425291 & 18.547493 \\
\hline 20.403770 & 1.019533 & 20.313713 \\
\hline 22.830261 & 2.192482 & 20.460089 \\
\hline 21.394417 & 15.248575 & 6.246583 \\
\hline 22.343554 & 12.644882 & 5.998739 \\
\hline 20.389935 & 13.308064 & 8.165247 \\
\hline 23.013775 & 14.406004 & 8.191707 \\
\hline 8.851975 & 2.953733 & 6.493213 \\
\hline 10.026866 & 0.590115 & 6.247068 \\
\hline 8.261860 & 1.011765 & 8.259432 \\
\hline 10.627905 & 2.196851 & 8.259918 \\
\hline 8.773568 & 15.170410 & 18.800434 \\
\hline 10.036333 & 12.478358 & 18.445539 \\
\hline 8.011589 & 13.059734 & 20.404015 \\
\hline 10.686649 & 14.341189 & 20.555002 \\
\hline 24.766159 & 22.078962 & 2.866345 \\
\hline 0.496416 & 21.065012 & 0.417280 \\
\hline 23.002367 & 22.918135 & 1.012008 \\
\hline 22.850166 & 20.489218 & 2.191025 \\
\hline 24.691879 & 9.876121 & 15.092975 \\
\hline 0.503455 & 8.861443 & 12.565503 \\
\hline 22.924202 & 10.632275 & 13.233784 \\
\hline 22.919834 & 8.173500 & 14.327111 \\
\hline 12.473018 & 22.159796 & 15.082538 \\
\hline 12.649493 & 21.239788 & 12.549726 \\
\hline 10.697330 & 22.937311 & 13.221161 \\
\hline 10.706069 & 20.463243 & 14.165685 \\
\hline 12.481028 & 9.946032 & 2.944509 \\
\hline 12.570844 & 8.938149 & 0.430146 \\
\hline 10.707768 & 10.706312 & 1.089687 \\
\hline 10.630090 & 8.258219 & 2.115531 \\
\hline 18.706491 & 22.173389 & 16.087019 \\
\hline 18.535841 & 21.146574 & 18.549677 \\
\hline 20.482180 & 22.852350 & 18.030687 \\
\hline 20.482906 & 20.472469 & 16.857496 \\
\hline 18.785383 & 10.030993 & 3.706003 \\
\hline 18.789026 & 9.010245 & 6.245612 \\
\hline 20.564228 & 10.796128 & 5.488972 \\
\hline 20.571995 & 8.332013 & 4.472595 \\
\hline 6.323776 & 22.091341 & 3.793391 \\
\hline 6.235902 & 20.982721 & 6.418204 \\
\hline 8.172044 & 23.007950 & 5.820078 \\
\hline 8.348278 & 20.484848 & 4.550031 \\
\hline 6.324990 & 9.866168 & 16.098185 \\
\hline 6.327417 & 8.946889 & 18.620073 \\
\hline 8.170588 & 10.696845 & 17.856396 \\
\hline 8.175200 & 8.178355 & 16.855310 \\
\hline 24.858160 & 21.236147 & 16.16906 \\
\hline
\end{tabular}




\begin{tabular}{|c|c|c|c|}
\hline O & 0.419708 & 22.253252 & 18.612791 \\
\hline 0 & 22.997271 & 20.380711 & 17.863434 \\
\hline 0 & 22.858419 & 22.831717 & 16.76986 \\
\hline 0 & 24.692122 & 8.844693 & 3.87883 \\
\hline 0 & 0.499814 & 10.031235 & 6.31503 \\
\hline 0 & 22.915949 & 8.271570 & 5.8130 \\
\hline 0 & 23.000910 & 10.629119 & 4.3898 \\
\hline 0 & 12.475203 & 21.408741 & 3.70 \\
\hline 0 & 12.634201 & 22.239902 & 6.2424 \\
\hline 0 & 10.884974 & 20.317110 & 5.4892 \\
\hline$c$ & 10.627177 & 22.920319 & 4.6371 \\
\hline 0 & 12.479329 & 8.845664 & 16.0926 \\
\hline 0 & 12.559919 & 9.873208 & 18.54506 \\
\hline 0 & 10.629604 & 8.178597 & 17.9585 \\
\hline 0 & 10.633002 & 10.533962 & 16.76428 \\
\hline$c$ & 18.630270 & 21.244886 & 2.9287 \\
\hline O & 18.702850 & 22.240873 & 0 . \\
\hline 0 & 20.389692 & 20.319782 & 1.095027 \\
\hline 0 & 20.573208 & 22.759865 & 2.19272 \\
\hline 0 & 18.707220 & 8.943248 & $15.1747 \varepsilon$ \\
\hline 0 & 18.550648 & 9.876606 & 12.65580 \\
\hline 0 & 20.403044 & 8.099706 & 13.315104 \\
\hline 0 & 20.486063 & 10.628148 & 16431 \\
\hline 0 & 6.643714 & 20.992916 & 15.171138 \\
\hline 0 & 6.238572 & 22.167807 & 12.72086 \\
\hline 0 & 8.178841 & 20.405472 & 13.14712 \\
\hline 0 & 8.258946 & 22.838270 & 14.32565 \\
\hline 0 & 6.424273 & 8.940092 & \\
\hline 0 & 6.316494 & 2343 & \\
\hline 0 & 8.175685 & 8.182724 & 1.022932 \\
\hline 0 & 8.260160 & 10.626692 & 2.18689 \\
\hline 0 & 13.227229 & 5.304243 & 8.417703 \\
\hline 0 & 12.471560 & 6.580601 & 5.997040 \\
\hline 0 & 11.808136 & 7.833170 & 02 \\
\hline 0 & 14.485381 & 7.755734 & 7.583870 \\
\hline 0 & 7.499880 & 18.364948 & 19.634752 \\
\hline 0 & 8.696860 & 19.475510 & 17.793039 \\
\hline 0 & 8.354345 & 20.657440 & 20.817411 \\
\hline 0 & 10.605572 & 20.818624 & 19.215044 \\
\hline $\mathrm{H}$ & 12.701926 & 22.182856 & 368 \\
\hline $\mathrm{H}$ & 15.865149 & 7.935123 & 7.933667 \\
\hline $\mathrm{H}$ & 25.039005 & 6.154097 & 1.707961 \\
\hline $\mathrm{H}$ & 21.943024 & 16.018564 & 6.491999 \\
\hline $\mathrm{H}$ & 13.636013 & 5.066109 & 9.329700 \\
\hline $\mathrm{H}$ & 8.815806 & 15.839175 & 19.517750 \\
\hline $\mathrm{H}$ & 9.123365 & 19.306074 & 16.01104 \\
\hline $\mathrm{H}$ & 10.660918 & 17.668268 & 20.053490 \\
\hline $\mathrm{H}$ & 12.168614 & 21.921177 & 18.656971 \\
\hline $\mathrm{H}$ & 13.274565 & 19.797150 & 17.913925 \\
\hline $\mathrm{H}$ & 12.286102 & 20.414938 & 16.611835 \\
\hline $\mathrm{H}$ & 11.658361 & 19.022789 & 93502 \\
\hline $\mathrm{H}$ & 10.096776 & 17.392750 & 22.506437 \\
\hline $\mathrm{H}$ & 10.030749 & 19.171593 & 22.70646 \\
\hline 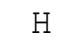 & 11.566361 & 18.326838 & 22.265390 \\
\hline $\operatorname{Re}$ & 12.978900 & 6.898841 & 7.595037 \\
\hline $\operatorname{Re}$ & 8.987185 & 19.577465 & 19.701750 \\
\hline C & 10.149695 & 18.432917 & 20.6683 \\
\hline$C$ & 11.645496 & 20.958689 & 18.5314 \\
\hline
\end{tabular}




$\begin{array}{llll}\text { C } & 12.219591 & 19.961731 & 17.620447 \\ \text { C } & 10.471577 & 18.361307 & 22.117073\end{array}$

\begin{tabular}{|c|c|c|c|}
\hline \multicolumn{4}{|c|}{607} \\
\hline \\
\hline & $23 . \overline{3} 8226 \overline{5}$ & 3.327562 & 80 \\
\hline & 23.427416 & 1.240432 & 3.377810 \\
\hline & 23.395372 & 15.566087 & 13.400792 \\
\hline & 23.387119 & 13.419726 & 15.569727 \\
\hline & 11.248849 & 1.261794 & 15.583079 \\
\hline & 11.162675 & 15.578953 & 1.1 \\
\hline & 11.230400 & 13.405162 & 3.367858 \\
\hline & 7.633633 & 3.353779 & 1.217857 \\
\hline & 7.658150 & 5.501352 & 3.347224 \\
\hline & 7.783407 & 15.500788 & 13.256600 \\
\hline & 19.998871 & 5.447948 & 15.5 \\
\hline & 19.908569 & 15.642 & 1.2 \\
\hline & 19.883810 & 17.802990 & \\
\hline & 7.625865 & 3.371256 & 5.511062 \\
\hline & 7.541875 & 15.534529 & 17.702251 \\
\hline & 7.757919 & 13. & \\
\hline & 19.921 & 3.3 & 17. \\
\hline & 19.870701 & 1.22 & 15. \\
\hline & 19.935272 & 15.587690 & 5.436296 \\
\hline & 20.009794 & 13.377489 & 3.281926 \\
\hline & 23.430086 & 3.354022 & \\
\hline & 23.3 & 17. & 15. \\
\hline & 11.24 & 3. & 17. \\
\hline & 11.280649 & 5.5 & 15. \\
\hline & 11.240839 & $15.64^{\circ}$ & 9405 \\
\hline & 11.187192 & 17.782843 & 3.228765 \\
\hline & 1.306702 & 23. & \\
\hline & 3.37 & 23. & \\
\hline & 1.249656 & 11.22 & 15 \\
\hline & 3.405969 & 11.210 & 5499 \\
\hline & 13.472887 & 23.473780 & 15.520451 \\
\hline & 15.623618 & 23.412365 & 13.401763 \\
\hline & 13.42 & 11. & $\$ 73$ \\
\hline & 15.6 & 11.1 & 1. \\
\hline & 1.357921 & 7.680968 & 2613 \\
\hline & 3.379510 & 7.603290 & 5.525627 \\
\hline & 1.299419 & 19.897888 & 15.593517 \\
\hline & 08 & 19. & 366 \\
\hline & 13.4 & & \\
\hline & 15.6 & 7. & 17. \\
\hline & 15.558319 & 19.952021 & 5.500381 \\
\hline & 5.525384 & 1791 & 79510 \\
\hline & 3.404512 & 7.665190 & 3387 \\
\hline & 5.508149 & 19.98 & 15.6 \\
\hline & 3.432671 & $19 . \varepsilon$ & 6915 \\
\hline & 17.819498 & 7.656451 & 15. \\
\hline & 15.608567 & 7.681211 & 64877 \\
\hline 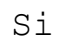 & 17.725311 & 19.965858 & 3.361546 \\
\hline & 15.609781 & 20.005669 & 1.233635 \\
\hline & & 23.3 & \\
\hline & 3.407911 & 23.402899 & 5.509606 \\
\hline & & 11.178452 & 15.602983 \\
\hline & & 11.2337 & \\
\hline
\end{tabular}




\begin{tabular}{|c|c|c|c|}
\hline i & 7.734535 & 23.390518 & 508324 \\
\hline & 15.559047 & 23.400471 & 17.747160 \\
\hline & 17.821198 & 11.267541 & 3.307414 \\
\hline & 5.667312 & 11.232828 & 5.478049 \\
\hline & 3.358148 & 1.279271 & 23.3681 \\
\hline & 1.240918 & 3.366158 & 23.4145 \\
\hline & 3.372956 & 13.376761 & 11.229187 \\
\hline & 1.280485 & 15.559775 & 11.25831 \\
\hline & 15.619733 & 1.243102 & 11.196 \\
\hline & 3.390111 & 3.358876 & 11.237 \\
\hline & 15.565116 & 13.418270 & 23.3919 \\
\hline & 13.454440 & 15.565358 & 23.4 \\
\hline & 3.354022 & 1.205234 & 7.6 \\
\hline & 5.501595 & 3.323192 & 7.613 \\
\hline & 3.294791 & 13.366080 & 19.96173 \\
\hline & 17.778959 & 3.363488 & 19.8544 \\
\hline L & 15.647892 & 13.418028 & \\
\hline 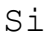 & 17.825567 & 15.5 & 7. \\
\hline & 3.332417 & 5.437996 & 7.654 \\
\hline & 1.236548 & 3.330718 & 7.66421 \\
\hline & 3.422961 & 17.734051 & 19.86026 \\
\hline i & 1.256939 & 15.578466 & 19.8719 \\
\hline 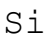 & 15.572155 & 5.467125 & 19. \\
\hline 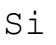 & 13.3 & 3.3 & 19. \\
\hline & 15.669739 & 17.774590 & 7.63339 \\
\hline $\mathrm{Si}$ & 13.475558 & 15.641337 & 7.62440 \\
\hline S & 3.376839 & 5.511062 & 23.38226 \\
\hline i & & & \\
\hline L & 5.5 & 15.5 & 11. \\
\hline & 17.827023 & 3.337515 & 11.27360 \\
\hline $\mathrm{Si}$ & 15.615849 & 17.756870 & 23.44440 \\
\hline Si & 17.731138 & 15.610752 & 23.42814 \\
\hline L & 1.643633 & 21.676003 & 23.72817 \\
\hline 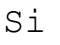 & 1.612319 & 23. & 21. \\
\hline & 1.673 & 9.4 & 11.58456 \\
\hline & 1.669364 & 11.591121 & 9.45495 \\
\hline $\mathrm{Si}$ & 13.892111 & 21.709503 & 11.53431 \\
\hline $\mathrm{Si}$ & 13.836521 & 23.867758 & 9.37703 \\
\hline 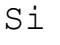 & 13.8362 & 9.4166 & 23.7893 \\
\hline $\mathrm{Si}$ & 13 & 11. & 21. \\
\hline S & 17.484024 & 21.680372 & 23.77648 \\
\hline & 17.396149 & 19.535955 & 21.69008 \\
\hline $\mathrm{Si}$ & 17.407557 & 9.494039 & 11.61515 \\
\hline Si & 17.4 & 7.3 & 9.51321 \\
\hline $\mathrm{Si}$ & 5.143059 & 21.645 & 11.63578 \\
\hline $\mathrm{Si}$ & 5.1 & 19.5 & 9. \\
\hline $\mathrm{Si}$ & 5.182141 & 9.432624 & 23.73400 \\
\hline Si & 5.152041 & 7.304713 & 21.61434 \\
\hline $\mathrm{Si}$ & 17.339588 & 21.699551 & 19.52357 \\
\hline $\mathrm{Si}$ & 17.414598 & 23.857561 & 21.66580 \\
\hline Si & 17.3 & 11.6 & \\
\hline Si & 5.101792 & 21.603422 & 7.3 \\
\hline$S=$ & 5.117814 & 23.750753 & 9.43019 \\
\hline$S$ & 5.077033 & 9.455441 & 19.52624 \\
\hline Si & 5.023628 & 11.624863 & 21.70294 \\
\hline$S$ & 1.572751 & 21.629154 & 19.54979 \\
\hline S: & 1.596540 & 19.535711 & 21.61628 \\
\hline & .62 & 9.42145 & 7.306 \\
\hline
\end{tabular}




\begin{tabular}{|c|c|c|c|}
\hline $\mathrm{Si}$ & 1.619601 & 7.275826 & 9.432624 \\
\hline $\mathrm{Si}$ & 13.877060 & 21.746643 & 7.183582 \\
\hline & 13.894538 & 19.547850 & 9.342079 \\
\hline $\mathrm{Si}$ & 13.815160 & 7.234316 & 21.654642 \\
\hline & 23.768959 & 1.594599 & 21.625511 \\
\hline & 21.672848 & 1.536582 & 23.712400 \\
\hline 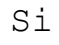 & 23.857561 & 13.849874 & 9.424371 \\
\hline & 21.728680 & 13.768066 & 11.591849 \\
\hline i & 11.546699 & 1.664752 & 9.476075 \\
\hline & 9.419758 & 1.717185 & 11.61806 \\
\hline & 11.673655 & 13.828996 & 21.710474 \\
\hline & 9.452529 & 13.776321 & 23.78012 \\
\hline $\mathrm{Si}$ & 23.812895 & 17.347357 & 21.70221 \\
\hline$i$ & 21.691053 & 17.389109 & 19.559259 \\
\hline & 21.670420 & 5.179228 & 7.259804 \\
\hline S & 11.676568 & 17.394449 & 9.345963 \\
\hline$\perp$ & 9.463938 & 17.391537 & 7.19984 \\
\hline$i$ & 11.580440 & 5.159323 & 21.638620 \\
\hline$i$ & 9.475590 & & 19.509010 \\
\hline Si & 19.534254 & 17.3 & 21.698336 \\
\hline i & 21.720911 & 17.393478 & 23.825033 \\
\hline$\perp$ & 19.558529 & 5.112959 & 9.496709 \\
\hline $\mathrm{Si}$ & 21.733534 & 5.051058 & 11.65423 \\
\hline $\mathrm{Si}$ & 7.265630 & 17.3 & 9.344022 \\
\hline $\mathrm{Si}$ & $9.47-2$. & 17.2 & 11.526065 \\
\hline $\mathrm{Si}$ & 7.295002 & 5.155925 & 21.648087 \\
\hline $\mathrm{Si}$ & 9.409562 & 5.117085 & 23.77648 \\
\hline Si & 19.555132 & 1.640477 & 21.59614 \\
\hline $\mathrm{Si}$ & 21.676489 & 1.613775 & 19.478666 \\
\hline$i$ & 19.551247 & 13.821957 & 9.43359 \\
\hline $\mathrm{Si}$ & 7.25 & 1.6 & 9.431410 \\
\hline Si & 9.426798 & 1.662082 & 7.29694 \\
\hline $\mathrm{Si}$ & 7.247910 & 13.688689 & 21.62429 \\
\hline$i$ & 21.703676 & 23.747112 & 1.58755 \\
\hline $\mathrm{Si}$ & 23.836199 & 21.6 & 1.61814 \\
\hline $\mathrm{Si}$ & 21.7 & 11.5 & 13.7 \\
\hline $\mathrm{Si}$ & 23.842026 & 9.405436 & 13.788943 \\
\hline $\mathrm{Si}$ & 9.483600 & 23.822121 & 13.818072 \\
\hline $\mathrm{Si}$ & 9.464666 & 11.601316 & 1.66159 \\
\hline $\mathrm{Si}$ & 11.619279 & 9.462967 & 1.646789 \\
\hline $\mathrm{Si}$ & 21.677460 & 23.767502 & 17.38474 \\
\hline $\mathrm{Si}$ & 19.533 & 21.6 & 17.399061 \\
\hline $\mathrm{Si}$ & 21.814125 & 11.641855 & 4.88332 \\
\hline Si & 19.636936 & 9.534092 & 5.01343 \\
\hline $\mathrm{Si}$ & 9.358586 & 23.825033 & 5.162479 \\
\hline $\mathrm{Si}$ & 9.472434 & 11.531649 & 17.29492 \\
\hline Si & 7.2634 & 9.418302 & 17.36920 \\
\hline $\mathrm{Si}$ & 21.69 & 19.521 & 17.34274 \\
\hline $\mathrm{Si}$ & 23.829647 & 21.684256 & 17.35148 \\
\hline Si & 21.700764 & 7.372196 & 5.12412 \\
\hline $\mathrm{Si}$ & 23.811440 & 9.445489 & 5.13189 \\
\hline $\mathrm{Si}$ & 9.483843 & 19.530857 & 4.97702 \\
\hline $\mathrm{Si}$ & 11.570245 & 21.728680 & 5.12898 \\
\hline Si & 9.441119 & 7.260775 & $17.31992^{\circ}$ \\
\hline Si & 11.590150 & 9.356887 & 17.35391 \\
\hline $\mathrm{Si}$ & 21.697123 & 19.507069 & 1.63538 \\
\hline $\mathrm{Si}$ & 19.560472 & 21.629396 & 1.65285 \\
\hline Ci & 21.748341 & 7.214169 & 13.77292 \\
\hline
\end{tabular}




\begin{tabular}{|c|c|c|c|}
\hline Si & 19.572367 & 9.380919 & 13.845988 \\
\hline Si & 9.503991 & 19.567026 & 13.711993 \\
\hline & 7.313451 & 21.651972 & 13.811033 \\
\hline 1 & 9.429711 & 7.297430 & 1.613290 \\
\hline L & 7.312723 & 9.420486 & 1.663781 \\
\hline & 11.685549 & 21.759022 & 13.664657 \\
\hline Si & 11.224817 & 3.419077 & 13.367294 \\
\hline . & 20.049606 & 3.303287 & $13.4046^{\circ}$ \\
\hline $\mathrm{Si}$ & 7.629021 & 1.212273 & 3.36591 \\
\hline i & 23.371826 & 15.602499 & 17.72142 \\
\hline Si & 5.449162 & 15.548852 & 19.95566 \\
\hline$\perp$ & 3.477822 & 17.699581 & 11.28841 \\
\hline $\mathrm{Si}$ & 13.805449 & 9.420486 & 19.49784 \\
\hline i & 23.787893 & 5.134321 & 9.422671 \\
\hline & 15.583564 & 1.292137 & 19.89910 \\
\hline 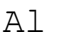 & 15.588904 & 5.514461 & 11.28162 \\
\hline L & 17.516550 & 9.569047 & 7.22776 \\
\hline 1 & 9.479473 & 13.499590 & 19.663883 \\
\hline Al & 7.259561 & 21.634979 & 5.16781 \\
\hline Al & 7.714225 & 17.775805 & 15.46656 \\
\hline L & 23.369400 & 5.398913 & 3.41179 \\
\hline - & 13.456382 & 19.861963 & 3.28993 \\
\hline Al & 21.792036 & 13.673882 & 7.23747 \\
\hline Al & 16.428078 & 4.548089 & 14. \\
\hline Al & 6.804898 & 18.17 & 18.10253 \\
\hline 0 & 22.164650 & 0.349069 & 2.86658 \\
\hline 0 & 24.605219 & 24.542347 & 3.95554 \\
\hline 0 & 24.029911 & 2.099267 & 2.11723 \\
\hline O & 22.846767 & 2.186413 & 4.557798 \\
\hline 0 & 22.161011 & 12.471804 & 15.08326 \\
\hline 0 & 24.611288 & 12.4 & 16.0 \\
\hline 0 & 23.942038 & 14.330752 & 14.33050 \\
\hline 0 & 22.846283 & 14.396051 & 16.77035 \\
\hline 0 & 10.028564 & 0.247358 & 15.17963 \\
\hline 0 & 12.554580 & 24.690910 & 16.09333 \\
\hline O & 11.642826 & 2.1 & 14. \\
\hline 0 & 10.707283 & 2.192239 & $16.78345^{\circ}$ \\
\hline 0 & 9.944090 & 12.481271 & 2.94620 \\
\hline 0 & 12.481998 & 12.471560 & 3.79654 \\
\hline 0 & 11.714193 & 14.332936 & 2.10460 \\
\hline 0 & 10.781806 & 14.322 & 4.62698 \\
\hline 0 & 8.928440 & 6.40 & 2.87921 \\
\hline 0 & 6.415049 & 6.409223 & 3.88369 \\
\hline 0 & 7.077987 & 4.629166 & 2.09052 \\
\hline 0 & 8.181026 & 4.555371 & 4.55027 \\
\hline 0 & 9.092052 & 18.865004 & 15.19080 \\
\hline 0 & 6.329359 & 18.6 & 16.34772 \\
\hline 0 & 7.326560 & 16.7 & 14.17102 \\
\hline 0 & 8.005034 & 17.023291 & 17.10898 \\
\hline 0 & 21.321352 & 6.238815 & 15.00558 \\
\hline 0 & 18.789997 & 6.410436 & 16.01783 \\
\hline 0 & 19.383024 & 4.556828 & 14.25404 \\
\hline 0 & 20.480965 & 4.543719 & 16.75894 \\
\hline 0 & 21.241972 & 18.547979 & 2.86780 \\
\hline 0 & 18.719357 & 18.785383 & 3.88417 \\
\hline 0 & 19.283983 & 16.851912 & 2.17597 \\
\hline 0 & 20.398916 & 16.855068 & 4.56386 \\
\hline 0 & 8.930383 & 0.342515 & 3.79533 \\
\hline
\end{tabular}




\begin{tabular}{|c|c|c|c|}
\hline 0 & 6.401940 & 24.609589 & 2.782597 \\
\hline 0 & 7.082357 & 2.106550 & 4.636448 \\
\hline 0 & 8.175685 & 2.190297 & 2.187627 \\
\hline 0 & 9.019227 & 12.470105 & 16.010069 \\
\hline O & 6.488843 & 12.389998 & 15.09079 \\
\hline 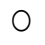 & 7.256891 & 14.342647 & 16.706749 \\
\hline 0 & 8.190978 & 14.251860 & 236324 \\
\hline 0 & 21.143175 & 0.332805 & 16.102070 \\
\hline 0 & 18.635124 & 24.613716 & 15.01092 \\
\hline 0 & 19.302433 & 2.119658 & 16.85191 \\
\hline 0 & 20.481449 & 2.114075 & 14.40381 \\
\hline 0 & 21.319410 & 12.466706 & 3.547733 \\
\hline 0 & 18.706734 & 12.549968 & 2.783568 \\
\hline 0 & 19.559015 & 14.239723 & 4.63499 \\
\hline 0 & 20.480722 & 14.410858 & 2.129853 \\
\hline 0 & 22.257137 & 6.573804 & 3.874712 \\
\hline O & 24.774899 & 6.400969 & 2.621657 \\
\hline 0 & 24.111473 & 51730 & 16797 \\
\hline 0 & 22.759865 & 4.486431 & 2.116502 \\
\hline 0 & 22.086000 & 18.627356 & 16.027548 \\
\hline 0 & 24.599152 & 18.708433 & 15.090062 \\
\hline 0 & 23.934027 & 16.861622 & 16.837833 \\
\hline O & 22.861332 & 16.789284 & 14.342890 \\
\hline 0 & 10.024681 & 6.4 & 16. \\
\hline 0 & 12.557735 & 6.408979 & 15.016510 \\
\hline 0 & 11.724874 & 4.641789 & 16.854582 \\
\hline 0 & 10.772097 & 4.634506 & 14.329053 \\
\hline 0 & 9.877819 & 18.608421 & 3.705760 \\
\hline 0 & 12.373734 & 18.701151 & 25 \\
\hline 0 & 11.724631 & 16.849241 & 4.470896 \\
\hline 0 & 10.618196 & 16.842445 & 2.026443 \\
\hline 0 & 2.950577 & 22.170477 & 0.256825 \\
\hline 0 & 3.969868 & 24.613958 & 24.52875 \\
\hline 0 & 2.098296 & 24.022387 & 2 . \\
\hline 0 & 4.488859 & 22.8 & \\
\hline 0 & 2.951306 & 9.944575 & 12.474232 \\
\hline 0 & 3.890247 & 12.466949 & 12.475930 \\
\hline 0 & 2.185199 & 11.734098 & 14.336092 \\
\hline 0 & 4.633050 & 10.704370 & 14.329538 \\
\hline 0 & 15.155117 & 22. & 12.467 \\
\hline 0 & 16.172951 & 24. & 12. \\
\hline 0 & 14.336578 & 24.014376 & 14.242636 \\
\hline 0 & 16.770592 & 22.846283 & 14.404061 \\
\hline 0 & 15.167012 & 9.858643 & 0.341786 \\
\hline 0 & 16.103041 & 12.396553 & 24.525599 \\
\hline 0 & 14.315701 & 11.715 & 2.037610 \\
\hline 0 & 16.860893 & 10.709952 & 2.112376 \\
\hline 0 & 2.868287 & 8.859743 & 6.40776 \\
\hline 0 & 3.894859 & 6.407038 & 6.477677 \\
\hline 0 & 2.193696 & 6.995939 & 4.550274 \\
\hline 0 & 4.555129 & 8.177141 & 4.566295 \\
\hline 0 & 2.865131 & 21.062342 & 18.713531 \\
\hline 0 & 4.131780 & 18.629299 & 18.62395 \\
\hline 0 & 2.281812 & 19.284712 & 16.779573 \\
\hline 0 & 4.649314 & 20.565926 & 16.933960 \\
\hline 0 & 15.092489 & 8.936208 & 18.61594 \\
\hline 0 & 16.096972 & 6.420146 & 18.713045 \\
\hline ? & 14.409401 & 7.080657 & 16.78127 \\
\hline
\end{tabular}




$$
\begin{array}{rrr}
16.870846 & 8.104560 & 16.860165 \\
15.174537 & 21.323294 & 6.324019 \\
16.179506 & 18.798977 & 6.485687 \\
14.262783 & 19.389336 & 4.733790 \\
16.773504 & 20.477566 & 4.553187 \\
3.861846 & 8.842508 & 0.185215 \\
2.779927 & 6.406795 & 24.620028 \\
4.638633 & 7.151297 & 2.100966 \\
2.207047 & 8.266472 & 2.104122 \\
3.884664 & 21.158468 & 12.559678 \\
3.023644 & 18.614248 & 12.558706 \\
4.634749 & 19.383511 & 14.483439 \\
2.187627 & 20.400131 & 14.336335 \\
16.163727 & 8.846393 & 12.479086 \\
15.094189 & 6.326689 & 12.735426 \\
16.859922 & 7.085513 & 14.405033 \\
14.414257 & 8.265744 & 14.407217 \\
16.178291 & 21.232748 & 0.340087 \\
15.077925 & 18.731737 & 24.613716 \\
16.858952 & 19.382540 & 2.110434 \\
14.425423 & 20.633410 & 2.102180 \\
3.800917 & 22.169020 & 6.491028 \\
2.777985 & 24.611288 & 6.414320 \\
4.638391 & 24.019230 & 4.635478 \\
2.279870 & 22.841669 & 4.486188 \\
3.806015 & 9.945061 & 18.629784 \\
2.861490 & 12.467434 & 18.695326 \\
4.624797 & 11.793571 & 16.848757 \\
2.120629 & 10.713351 & 16.849485 \\
16.021721 & 22.082602 & 18.622501 \\
14.921838 & 24.534336 & 18.636095 \\
16.847786 & 23.933540 & 16.858223 \\
14.406004 & 22.832445 & 16.681990 \\
16.097458 & 9.955984 & 6.327660 \\
15.169926 & 12.487339 & 6.410436 \\
16.918425 & 11.802552 & 4.557070 \\
14.402848 & 10.788360 & 4.542991 \\
0.344214 & 2.867801 & 22.162224 \\
24.608860 & 3.964285 & 24.614927 \\
2.110434 & 2.115288 & 24.016075 \\
2.193210 & 4.554400 & 22.837299 \\
0.427476 & 15.157302 & 9.947245 \\
24.620028 & 16.101828 & 12.477872 \\
2.112133 & 14.251617 & 11.799882 \\
2.262878 & 16.766708 & 10.791759 \\
12.388298 & 2.944994 & 10.030507 \\
12.557493 & 3.874226 & 12.544628 \\
14.331481 & 2.108006 & 11.725602 \\
14.307448 & 4.546875 & 10.620380 \\
12.641726 & 15.088363 & 22.083572 \\
12.392426 & 16.094059 & 24.526085 \\
14.334394 & 14.321528 & 24.016317 \\
14.412072 & 16.784185 & 22.925903 \\
1.505835 & 15.086663 & 21.066711 \\
\hline & 3.969500 & 8.869210 \\
\hline
\end{array}
$$




\begin{tabular}{|c|c|c|c|}
\hline 0 & 6.162107 & 16.097944 & 18.462288 \\
\hline 0 & 4.547360 & 14.318130 & 19.455606 \\
\hline 0 & 4.645430 & 16.852882 & 20.479507 \\
\hline 0 & 18.704306 & 2.942567 & 21.14705 \\
\hline o & 18.794607 & 3.972538 & 18.70892 \\
\hline 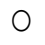 & 16.933718 & 2.182287 & 19.211403 \\
\hline 0 & 16.833220 & 4.575762 & 20.4040 \\
\hline 0 & 18.707705 & 15.175994 & 9.01971 \\
\hline 0 & 18.802376 & 16.017593 & 6.4837 \\
\hline 0 & 16.931776 & 14.326383 & 7.1629 \\
\hline 0 & 16.927162 & 16.861380 & 8.1021 \\
\hline 0 & 0.418251 & 3.881023 & 8.949802 \\
\hline 0 & 24.523170 & 2.691810 & 6.56312 \\
\hline 0 & 2.112376 & 4.556585 & 7.0058 \\
\hline 0 & 2.205590 & 2.180830 & 8.25651 \\
\hline 0 & 0.423592 & 16.100370 & 21.152643 \\
\hline O & 24.619783 & 15.081567 & 18.631725 \\
\hline 0 & 2.263364 & 16.763309 & 19. \\
\hline 0 & 2.105821 & 14.331965 & 20.470284 \\
\hline 0 & 12.393154 & 3.868643 & 21.151428 \\
\hline 0 & 12.469134 & 2.866102 & 18.69435 \\
\hline 0 & 14.241665 & 4.700776 & 19.37938 \\
\hline O & 14.324441 & 2.276958 & 20.552816 \\
\hline 0 & 12.566960 & 16.099155 & 8.921401 \\
\hline 0 & 12.483212 & 15.239350 & 6.410193 \\
\hline 0 & 14.419354 & 16.866720 & 7.08478 \\
\hline 0 & 14.412315 & 14.401634 & 8.093880 \\
\hline 0 & 6.404610 & 3.871070 & 22.085516 \\
\hline 0 & 6.423059 & 2.790123 & 24 . \\
\hline 0 & 4.637662 & 4.643731 & 23.944950 \\
\hline 0 & 4.486431 & 2.271617 & 22.76180 \\
\hline 0 & 6.325961 & 16.081923 & 9.790189 \\
\hline 0 & 6.575989 & 15.003159 & 12.297270 \\
\hline 0 & 4.724080 & 16.777632 & 11.805 \\
\hline 0 & 4.572849 & 14.3 & 700 \\
\hline 0 & 18.544580 & 3.899229 & 9.936808 \\
\hline 0 & 18.880054 & 2.782840 & 12.386843 \\
\hline 0 & 16.864534 & 4.466283 & 12.053552 \\
\hline 0 & 16.852156 & 2.178645 & 10.710196 \\
\hline 0 & 18.630512 & 16.107 & 22 . \\
\hline 0 & 18.713289 & 15.0 & 24. \\
\hline 0 & 16.856281 & 16.857252 & 24.02141 \\
\hline 0 & 16.772291 & 14.406488 & 22.919348 \\
\hline 0 & 2.867316 & 24.69333 & 22.080175 \\
\hline 0 & 0.428447 & $0.421 \varepsilon$ & 85876 \\
\hline 0 & 1.008124 & 22.9 & 22. \\
\hline 0 & 2.199764 & 22.773458 & 20.484362 \\
\hline 0 & 2.953976 & 12.470346 & 9.94894 \\
\hline 0 & 0.504183 & 12.580068 & 8.933537 \\
\hline 0 & 1.093813 & 10.699272 & 10.708982 \\
\hline 0 & 2.190783 & 10.616982 & 8.259432 \\
\hline 0 & 15.163128 & 24.692122 & 9.86859 \\
\hline 0 & 12.564046 & 0.509767 & 8.941063 \\
\hline 0 & 13.318744 & 22.930515 & 10.620866 \\
\hline 0 & 14.327353 & 23.010620 & 8.098492 \\
\hline 0 & 15.083750 & 12.562347 & 22.081875 \\
\hline 0 & 12.557735 & 12.557493 & 21.220854 \\
\hline ? & 13.315589 & 10.704856 & 22.91862 \\
\hline
\end{tabular}




\begin{tabular}{|c|c|c|c|}
\hline 0 & 14.251132 & 10.701700 & 20.395033 \\
\hline 0 & 16.109837 & 18.631483 & 22.156641 \\
\hline 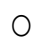 & 18.621773 & 18.607695 & 21.148031 \\
\hline 0 & 18.035786 & 20.393091 & 22.92299 \\
\hline 0 & 16.853127 & 20.474653 & 20.48218 \\
\hline 0 & 16.186304 & 6.487387 & 10.025652 \\
\hline 0 & 18.708675 & 6.407765 & 9.0163 \\
\hline 0 & 18.032629 & 8.349006 & 10.61868 \\
\hline 0 & 16.874973 & 8.109173 & 8.17034 \\
\hline 0 & 3.879809 & 18.626627 & 10.0290 \\
\hline O & 6.334214 & 18.618132 & 8.9342 \\
\hline 0 & 5.730748 & 20.391876 & 10.7781 \\
\hline 0 & 4.474537 & 20.482180 & 8 . \\
\hline 0 & 3.868886 & 6.483260 & 22.17557 \\
\hline 0 & 6.322805 & 6.325475 & 21.0703 \\
\hline 0 & 5.814495 & 8.273026 & 22.76132 \\
\hline O & 4.552944 & 8.190493 & 20.393818 \\
\hline 0 & 16.179749 & 24.768829 & 21. \\
\hline 0 & 18.707220 & 0.417038 & 22.23480 \\
\hline 0 & 17.948881 & 22.929543 & 20.39066 \\
\hline 0 & 16.937843 & 22.845552 & 22.83778 \\
\hline O & 16.095516 & 12.544385 & 8.95077 \\
\hline 0 & 18.527344 & 12.717220 & 10.039246 \\
\hline 0 & 18.030687 & 10.704370 & 431 \\
\hline 0 & 16.858467 & 10.785689 & 10.801954 \\
\hline 0 & 3.811598 & 24.592596 & 8.945189 \\
\hline 0 & 6.324504 & 0.430146 & 9.959867 \\
\hline O & 5.723950 & 22.829290 & 8.248023 \\
\hline 0 & 4.629409 & 22.849680 & 10. \\
\hline 0 & 3.711343 & 12.473261 & 21.239304 \\
\hline 0 & 6.157738 & 12.656048 & 22.247185 \\
\hline 0 & 5.573933 & 10.705098 & 20.471012 \\
\hline 0 & 4.557070 & 10.697330 & 22.92930 \\
\hline 0 & 2.874598 & 18.716444 & 20.995 \\
\hline 0 & 0.503455 & 18.535841 & 22. \\
\hline 0 & 0.923406 & 20.394304 & 20.398432 \\
\hline 0 & 2.196851 & 20.486063 & 22.759378 \\
\hline 0 & 2.855907 & 6.329602 & 8.935237 \\
\hline 0 & 0.426747 & 6.338583 & 10.029051 \\
\hline 0 & 1.02 & 8.17 & 22 \\
\hline 0 & 2.19 & 8.2 & 10.6 \\
\hline 0 & 15.171867 & 18.629541 & 8.940092 \\
\hline 0 & 12.635656 & 18.627842 & 9.791403 \\
\hline 0 & 13.392539 & 20.482180 & 8.098735 \\
\hline 0 & 14.344104 & 20.466158 & 10.615525 \\
\hline 0 & 15.094431 & 6.329359 & 21. \\
\hline 0 & 12.560649 & 6.336398 & 22.17023 \\
\hline 0 & 13.304423 & 8.165976 & 20.40352 \\
\hline 0 & 14.325655 & 8.186608 & 22.857449 \\
\hline 0 & 22.171448 & 2.777985 & 24.605219 \\
\hline 0 & 21.076422 & 0.332077 & 0.352953 \\
\hline 0 & 22.922747 & 0.921706 & 22.842154 \\
\hline 0 & 20.560343 & 2.186899 & 22.747971 \\
\hline 0 & 22.160524 & 15.080837 & 12.477144 \\
\hline 0 & 21.240030 & 12.551424 & 12.56744 \\
\hline 0 & 23.000910 & 13.241066 & 10.703885 \\
\hline 0 & 20.486547 & 14.258900 & 10.697816 \\
\hline 0 & 9.963752 & 3.045006 & 12.39752 \\
\hline
\end{tabular}




\begin{tabular}{|c|c|c|}
\hline 8.934752 & 0.594728 & 12.716734 \\
\hline 10.622808 & 1.082162 & 10.704856 \\
\hline 8.170102 & 2.183257 & 10.700244 \\
\hline 9.856215 & 15.100014 & 24.616144 \\
\hline 8.929897 & 12.550210 & 0.435244 \\
\hline 10.798555 & 13.300538 & 22.995813 \\
\hline 8.258219 & 14.240694 & 22.764961 \\
\hline 22.167564 & 16.090660 & 18.697510 \\
\hline 21.227409 & 18.618618 & 18.610607 \\
\hline 22.923962 & 17.954708 & 20.479265 \\
\hline 20.476839 & 16.855795 & 20.481207 \\
\hline 22.145231 & 3.879809 & 6.409465 \\
\hline 21.147058 & 6.400969 & 6.328874 \\
\hline 22.919834 & 5.733175 & 8.179083 \\
\hline 20.483635 & 4.636691 & 8.261374 \\
\hline 9.944332 & 16.099886 & 6.330815 \\
\hline 9.017285 & 18.619347 & 6.253865 \\
\hline 10.689563 & 17.934559 & 8.165490 \\
\hline 8.187579 & 16.871817 & 8.097278 \\
\hline 9.954285 & 3.787080 & 18.701151 \\
\hline 8.929169 & 6.235173 & 18.462288 \\
\hline 10.692233 & 5.750896 & 20.390421 \\
\hline 8.262102 & 4.632079 & 20.468100 \\
\hline 21.232506 & 16.179993 & 24.772470 \\
\hline 22.328989 & 18.626385 & 0.418009 \\
\hline 20.476595 & 17.948881 & 22.919834 \\
\hline 22.922747 & 16.773991 & 22.918861 \\
\hline 21.399517 & 3.792178 & 12.643909 \\
\hline 22.257380 & 6.336156 & 12.498749 \\
\hline 20.383381 & 5.495770 & 10.859241 \\
\hline 22.852108 & 4.548089 & 10.616738 \\
\hline 9.101033 & 15.929962 & 12.388298 \\
\hline 9.866653 & 18.450151 & 12.625947 \\
\hline 8.199231 & 17.785271 & 10.638343 \\
\hline 10.780592 & 16.940271 & 10.635916 \\
\hline 8.929411 & 3.803587 & 24.607891 \\
\hline 9.954285 & 6.326204 & 0.429903 \\
\hline 8.157722 & 5.731476 & 22.912794 \\
\hline 10.622564 & 4.633050 & 22.836086 \\
\hline 21.240761 & 2.864888 & 18.541668 \\
\hline 22.319523 & 0.425048 & 18.547979 \\
\hline 20.403770 & 1.019533 & 20.313713 \\
\hline 22.830261 & 2.192482 & 20.459846 \\
\hline 21.394661 & 15.248575 & 6.246583 \\
\hline 22.343554 & 12.644882 & 5.998982 \\
\hline 20.390179 & 13.308064 & 8.165247 \\
\hline 23.014019 & 14.406246 & 8.192677 \\
\hline 8.852461 & 2.953733 & 6.492970 \\
\hline 10.026866 & 0.589873 & 6.246583 \\
\hline 8.263074 & 1.012251 & 8.258461 \\
\hline 10.628633 & 2.197579 & 8.259432 \\
\hline 8.763130 & 15.166527 & 18.797035 \\
\hline 10.037062 & 12.479086 & 18.444811 \\
\hline 8.010375 & 12.985939 & 20.397217 \\
\hline 10.677182 & 14.338520 & 20.552090 \\
\hline 24.766645 & 22.078962 & 2.866102 \\
\hline 0.496658 & 21.064526 & 0.416795 \\
\hline 23. & 22.918135 & 1.01200 \\
\hline
\end{tabular}




\begin{tabular}{|c|c|c|c|}
\hline 0 & 22.850409 & 20.488976 & 2.190783 \\
\hline 0 & 24.686054 & 9.933167 & 15.082538 \\
\hline 0 & 0.504912 & 8.863870 & 12.567445 \\
\hline 0 & 22.921534 & 10.632760 & 13.23087 \\
\hline 0 & 22.931000 & 8.173015 & 14.32735 \\
\hline 0 & 12.478358 & 22.245972 & 15.008499 \\
\hline 0 & 12.643668 & 21.238089 & 12.482242 \\
\hline 0 & 10.691748 & 22.932457 & 13.14056 \\
\hline 0 & 10.778407 & 20.475138 & 14.15913 \\
\hline 0 & 12.480785 & 9.946274 & 2.94426 \\
\hline 0 & 12.570601 & 8.938393 & 0.4301 \\
\hline 0 & 10.706554 & 10.707283 & 1.0884 \\
\hline 0 & 0333 & 58704 & 152 \\
\hline 0 & 18.706491 & 22.173147 & 16.08677 \\
\hline 0 & 18.534628 & 21.146818 & 18.54967 \\
\hline 0 & 20.482180 & 22.851379 & 18.03117 \\
\hline O & 20.482664 & 20.472712 & 16.85749 \\
\hline 0 & 18.785383 & 10.031235 & \\
\hline 0 & 18.789753 & 0489 & 6.24512 \\
\hline 0 & 20.564468 & 10.796128 & 5.48872 \\
\hline 0 & 20.571995 & 8.331771 & 4.47259 \\
\hline 0 & 6.322562 & 22.091827 & 3.79242 \\
\hline 0 & 6.239543 & 20.981993 & 6.41286 \\
\hline 0 & 8.172044 & 23.004309 & \\
\hline 0 & 8.350220 & 20.490433 & 4.481334 \\
\hline 0 & 6.327660 & 9.865197 & 16.10085 \\
\hline O & 6.328388 & 8.944704 & 18.61934 \\
\hline O & 8.171315 & 10.697330 & 17.856880 \\
\hline 0 & 8.175927 & 8.177870 & 16. \\
\hline 0 & 24.860344 & 21.233721 & 71009 \\
\hline 0 & 0.419222 & 22.253983 & 18.61084 \\
\hline 0 & 22.997271 & 20.380224 & 17.863434 \\
\hline 0 & 22.858662 & 22.831474 & 16.769621 \\
\hline 0 & 24.691879 & 8.844935 & \\
\hline 0 & 0.500057 & 10.031235 & 79 \\
\hline 0 & 22.915949 & 8.271570 & 5.813281 \\
\hline 0 & 23.000910 & 10.629119 & 4.38981 \\
\hline 0 & 12.475203 & 21.408497 & 3.70915 \\
\hline 0 & 12.634201 & 22.239660 & 6.242213 \\
\hline 0 & 10.879633 & 20.318567 & 5 . \\
\hline 0 & 10.627663 & 22.9 & 4 . \\
\hline 0 & 12.479571 & 8.845907 & 16.091873 \\
\hline 0 & 12.559919 & 9.872965 & 18.544580 \\
\hline 0 & 10.630090 & 8.178841 & 17.958591 \\
\hline 0 & 10.631789 & 10.534690 & 16.763554 \\
\hline 0 & 18.630270 & 21.244644 & 216 \\
\hline 0 & 18.702850 & 22.240873 & 0.421650 \\
\hline 0 & 20.389692 & 20.320024 & 1.09502 \\
\hline 0 & 20.572966 & 22.759865 & 2.192724 \\
\hline 0 & 18.705763 & 8.934994 & 15.164342 \\
\hline 0 & 18.615463 & 9.874421 & 12.639542 \\
\hline 0 & 20.461060 & 8.108201 & 31243 \\
\hline 0 & 20.490675 & 10.630575 & 14.32395 \\
\hline 0 & 6.574532 & 21.074480 & 15.17308 \\
\hline 0 & 6.322076 & 22.167078 & 12.65313 \\
\hline 0 & 8.192192 & 20.394791 & 13.225044 \\
\hline 0 & 385 & 22.846283 & 14.327353 \\
\hline 0 & 6.425244 & 8.940578 & 2.94572 \\
\hline
\end{tabular}




\begin{tabular}{|c|c|c|c|}
\hline 0 & 6.316008 & 9.952343 & 0.492046 \\
\hline 0 & 8.175685 & 8.182967 & 1.023174 \\
\hline 0 & 8.260889 & 10.629848 & 2.183986 \\
\hline 0 & 13.301752 & 5.295990 & 8.341238 \\
\hline 0 & 12.470833 & 6.657309 & 5.98344 \\
\hline 0 & 11.812991 & 7.828558 & 8.42765 \\
\hline 0 & 14.492179 & 7.767386 & 758435 \\
\hline 0 & 7.412735 & 17.777502 & 19.810501 \\
\hline 0 & 7.585327 & 19.884296 & $18.44529^{\circ}$ \\
\hline 0 & 7.667375 & 20.154957 & 21.58910 \\
\hline 0 & 10.790545 & 19.135908 & 16.94269 \\
\hline $\mathrm{H}$ & 12.707994 & 22.183342 & 3.16128 \\
\hline $\mathrm{H}$ & 15.874132 & 7.938037 & 7.9351 \\
\hline $\mathrm{H}$ & 25.039978 & 6.153368 & 1.70868 \\
\hline $\mathrm{H}$ & 21.942539 & 16.019051 & 6.49297 \\
\hline $\mathrm{H}$ & 13.686018 & 5.055185 & $9.25517^{\circ}$ \\
\hline $\mathrm{H}$ & 8.943733 & 15.903260 & 19.42307 \\
\hline $\mathrm{H}$ & 9.980259 & 19. & 4598 \\
\hline $\mathrm{H}$ & 10.416959 & 18.7 & 19. \\
\hline $\mathrm{H}$ & 10.851717 & 21.1 & 16.5 \\
\hline $\mathrm{H}$ & 12.184393 & 21.329847 & 18.717899 \\
\hline $\mathrm{H}$ & 13.248105 & 20.932957 & 17.40852 \\
\hline $\mathrm{H}$ & 12.749504 & 19.602953 & 18.53559 \\
\hline $\mathrm{H}$ & 11.2 & 18.2 & 21. \\
\hline $\mathrm{H}$ & 10.3 & 19. & 22 . \\
\hline $\mathrm{H}$ & 11.76 & 19.845942 & $21.25289^{\circ}$ \\
\hline $\mathrm{Re}$ & 12.987882 & 6.916319 & 7.58459 \\
\hline $\mathrm{Re}$ & 8.129321 & 19.448324 & 20.1 \\
\hline $\mathrm{C}$ & 9.9 & 19. & \\
\hline $\mathrm{C}$ & 11.2 & 20.2 & 17. \\
\hline $\mathrm{C}$ & 12.4 & 20.5 & 18. \\
\hline $\mathrm{C}$ & 10.881089 & 19.225725 & 21.49588 \\
\hline \multicolumn{4}{|c|}{607} \\
\hline \multirow{2}{*}{\multicolumn{2}{|c|}{ USY }} & $=-7249.1$ & 57223 \\
\hline & & 3.327319 & 1.2 \\
\hline $\mathrm{Si}$ & 23.427172 & 1.240432 & 3.37732 \\
\hline $\mathrm{Si}$ & 23.393431 & 15.565358 & 13.40249 \\
\hline $\mathrm{Si}$ & 23.385662 & 13.419242 & 15.57045 \\
\hline $\mathrm{Si}$ & 11.23 & 0284 & 15.63357 \\
\hline Si & 11. & 15 & 1 . \\
\hline $\mathrm{Si}$ & 11.233799 & 13.402977 & 3.37247 \\
\hline $\mathrm{Si}$ & 7.633148 & 3.354022 & 1.21737. \\
\hline $\mathrm{Si}$ & 7.657908 & 5.501595 & 3.34698 \\
\hline Si & 7.7 & 15.448112 & 13.21727 \\
\hline $\mathrm{Si}$ & 19.996443 & 5.449162 & 15.51705 \\
\hline $\mathrm{Si}$ & 19.9 & 15.6 & 1.270 \\
\hline $\mathrm{Si}$ & 19.8840 & 17.802990 & 3.35062 \\
\hline $\mathrm{Si}$ & 7.625622 & 3.371742 & $5.51057^{\circ}$ \\
\hline $\mathrm{Si}$ & 7.599406 & 15.491321 & 17.761967 \\
\hline $\mathrm{Si}$ & 7.748694 & 13.311705 & $15.49544^{\circ}$ \\
\hline Si & 19.9 & 7130 & 17.73647 \\
\hline $\mathrm{Si}$ & 19.869972 & 1.220770 & 15.61366 \\
\hline $\mathrm{Si}$ & 19.935272 & 15.587934 & 5.43556 \\
\hline $\mathrm{Si}$ & 20.010038 & 13.377732 & 3.28119 \\
\hline $\mathrm{Si}$ & 23.428629 & 3.354507 & 5.53994 \\
\hline $\mathrm{Si}$ & 23.365273 & 17.732838 & 15.56511 \\
\hline $\mathrm{Si}$ & 11.240839 & 3.368343 & 17.79328 \\
\hline
\end{tabular}




\begin{tabular}{|c|c|c|c|}
\hline i & 11.280407 & 5.545532 & 5.571912 \\
\hline $\mathrm{Si}$ & 11.242053 & 15.647406 & 5.448677 \\
\hline $\mathrm{Si}$ & 11.189134 & 17.787455 & 3.231435 \\
\hline $\mathrm{Si}$ & 1.305488 & 23.404354 & 3.333145 \\
\hline Si & 3.378053 & 23.429358 & 1.202078 \\
\hline $\mathrm{Si}$ & 1.248443 & 11.228458 & 15.577252 \\
\hline$i$ & 3.405241 & 11.209525 & 13.386956 \\
\hline 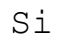 & 13.479442 & 23.400471 & 15.54084 \\
\hline 1 & 15.625803 & 23.384691 & 13.39084 \\
\hline 1 & 3.431621 & 11.229429 & 3.33533 \\
\hline$i$ & 15.611237 & 11.170199 & 1.20110 \\
\hline$i$ & 1.35767 & 7.680968 & \\
\hline i & 3.379267 & 7.603047 & 5.525384 \\
\hline i & 1.288496 & 19.897404 & 15.59812 \\
\hline i & 3.396987 & 19.884052 & 17.775805 \\
\hline i & 13.453469 & 7.648684 & 15.59545 \\
\hline $\mathrm{Si}$ & 15.588177 & 7.630721 & 17. \\
\hline $\mathrm{Si}$ & 15.559533 & 19.952507 & 5.499896 \\
\hline i & 5.524170 & 7.661064 & 3.379510 \\
\hline Si & 3.404512 & 7.665433 & 1.18265 \\
\hline Si & 5.468339 & 20.017321 & 15.65201 \\
\hline $\mathrm{Si}$ & 3.384607 & 19.860020 & 13.41997 \\
\hline $\mathrm{Si}$ & 17.813187 & 7.6 & \\
\hline $\mathrm{Si}$ & 15.597644 & 7.669 & 13.46779 \\
\hline Si & 17.726282 & 19.965858 & 3.36057 \\
\hline $\mathrm{Si}$ & 15.610752 & 20.005669 & 1.23217 \\
\hline $\mathrm{Si}$ & 5.498197 & 23.366972 & 3.37562 \\
\hline $\mathrm{Si}$ & 3.406455 & 23.404112 & 21 \\
\hline $\mathrm{Si}$ & 5.509606 & 11.178452 & 15.60468 \\
\hline $\mathrm{Si}$ & 3.360090 & 11.236226 & 17.751286 \\
\hline $\mathrm{Si}$ & 17.732594 & 23.389305 & 15.608567 \\
\hline Si & 15.553949 & 23.384449 & 17.74424 \\
\hline $\mathrm{Si}$ & 17.821924 & 11.267541 & 3.30717 \\
\hline $\mathrm{Si}$ & 15.668526 & 11.232100 & 4782 \\
\hline $\mathrm{Si}$ & 3.357420 & 1.278786 & $23.36770=$ \\
\hline Si & 1.240675 & 3.365916 & 23.414307 \\
\hline $\mathrm{Si}$ & 3.364702 & 13.370450 & 11.229429 \\
\hline $\mathrm{Si}$ & 1.273203 & 15.552735 & $11.25928^{\circ}$ \\
\hline $\mathrm{Si}$ & 15.619005 & 1.225867 & 11.202727 \\
\hline $\mathrm{Si}$ & 13.382101 & 3.341884 & 11.23671 \\
\hline $\mathrm{Si}$ & 15.567058 & 13.417542 & 23.391247 \\
\hline $\mathrm{Si}$ & 13.459294 & 15.563902 & 23.419647 \\
\hline $\mathrm{Si}$ & 3.351109 & 1.209846 & 7.65281 \\
\hline $\mathrm{Si}$ & 5.501110 & 3.324164 & 7.61348 \\
\hline $\mathrm{Si}$ & 3.305715 & 13.375304 & 19.96124 \\
\hline $\mathrm{Si}$ & 17.779688 & 3.362518 & 19.85370 \\
\hline $\mathrm{Si}$ & 15.649590 & 13.416328 & 7.650868 \\
\hline $\mathrm{Si}$ & 17.826780 & 15.566814 & 7.71908 \\
\hline $\mathrm{Si}$ & 3.331689 & 5.438481 & 7.65499 \\
\hline $\mathrm{Si}$ & 1.234121 & 3.332660 & 7.664462 \\
\hline $\mathrm{Si}$ & 3.399172 & 17.735992 & 19.87531 \\
\hline $\mathrm{Si}$ & 1.247472 & 15.573612 & 19.87725 \\
\hline $\mathrm{Si}$ & 15.574340 & 5.467611 & 19.93794 \\
\hline $\mathrm{Si}$ & 13.402006 & 3.398687 & 19.95614 \\
\hline $\mathrm{Si}$ & 15.671196 & 17.774834 & 7.63363 \\
\hline $\mathrm{Si}$ & 13.478714 & 15.639639 & 7.622952 \\
\hline $\mathrm{Si}$ & 3.376354 & 5.511305 & 23.38202 \\
\hline $\mathrm{Si}$ & 5.503051 & 3.388006 & 23.34658 \\
\hline
\end{tabular}




\begin{tabular}{|c|c|c|c|}
\hline i & 5.547232 & 15.535743 & 11.105387 \\
\hline Si & 17.818769 & 3.321493 & 11.269968 \\
\hline $\mathrm{Si}$ & 15.617064 & 17.757597 & 23.4 \\
\hline $\mathrm{Si}$ & 17.731867 & 15.611480 & 23.427658 \\
\hline & 1.642662 & 21.677217 & 23.728178 \\
\hline $\mathrm{Si}$ & 1.611833 & 23.786194 & 21.615074 \\
\hline i & 1.672763 & 9.441848 & 11.585780 \\
\hline i & 1.667180 & 11.588208 & 9.45 \\
\hline S & 13.868564 & 21.702948 & 11.542814 \\
\hline S & 13.832881 & 23.862417 & 9.3799 \\
\hline I & 13.837249 & 9.415874 & 23.788864 \\
\hline i & 13.81321 & 11.621949 & 21.663624 \\
\hline i & 17.484509 & 21.680372 & 23.775270 \\
\hline S & 17.396635 & 19.535711 & 21.689112 \\
\hline 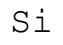 & 17.363863 & 9.483114 & 11.616366 \\
\hline i & 17.450523 & 7.299614 & 9.51758 \\
\hline $\mathrm{Si}$ & 5.063438 & 21.648573 & 11.628 \\
\hline $\mathrm{Si}$ & 5.096938 & 19.541536 & 9.484328 \\
\hline i & 5.181899 & 9.434566 & 23.732790 \\
\hline Si & 5.151798 & 7.305684 & 21.61434 \\
\hline Si & 17.341290 & 21.698093 & 19.52430 \\
\hline i & 17.415083 & 23.857077 & 21.66459 \\
\hline $\mathrm{Si}$ & 17.387167 & 11.676810 & \\
\hline $\mathrm{Si}$ & 5.1 & 21.6 & 7.31126 \\
\hline Si & 5.10300 & 23.751238 & 9.43359 \\
\hline $\mathrm{Si}$ & 5.076790 & 9.456899 & 19.52673 \\
\hline $\mathrm{Si}$ & 5.027997 & 11.628018 & 21.70343 \\
\hline i & 1.573237 & 21.628183 & 19.551491 \\
\hline $\mathrm{Si}$ & 1.598 & 19.533283 & $21.62332^{-}$ \\
\hline $\mathrm{Si}$ & 1.6285 & 9.420486 & 7.305440 \\
\hline $\mathrm{Si}$ & 1.618873 & 7.275583 & 9.432867 \\
\hline Si & 13.877546 & 21.746643 & 7.18334 \\
\hline i & 13.893324 & 19.547607 & 9.341837 \\
\hline $\mathrm{Si}$ & 13.815 & 7.2 & 21.653427 \\
\hline $\mathrm{Si}$ & 23.768717 & 1.5943 & $21.62502^{\circ}$ \\
\hline $\mathrm{Si}$ & 21.672848 & 1.536339 & 23.711914 \\
\hline $\mathrm{Si}$ & 23.854649 & 13.846959 & 9.423157 \\
\hline $\mathrm{Si}$ & 21.727707 & 13.767582 & 11.59233 \\
\hline $\mathrm{Si}$ & 11.544271 & 1.660625 & 9.473648 \\
\hline $\mathrm{Si}$ & 9.421457 & 1.717185 & 11.61855 \\
\hline $\mathrm{Si}$ & 11.674626 & 13.823656 & 21.72139 \\
\hline $\mathrm{Si}$ & 9.460297 & 13.772923 & 23.827461 \\
\hline Si & 23.813381 & 17.347357 & 21.70294 \\
\hline $\mathrm{Si}$ & 21.691782 & 17.389351 & 19.55974 \\
\hline $\mathrm{Si}$ & 21.668720 & 5.179228 & 7.26101 \\
\hline $\mathrm{Si}$ & 11.678995 & 17.392750 & 9.34062 \\
\hline $\mathrm{Si}$ & 9.464910 & 17.392265 & $7.19547^{\circ}$ \\
\hline $\mathrm{Si}$ & 11.580925 & 5.160294 & 21.639107 \\
\hline $\mathrm{Si}$ & 9.474861 & 5.117571 & 19.509981 \\
\hline $\mathrm{Si}$ & 19.534985 & 17.384254 & 21.697609 \\
\hline $\mathrm{Si}$ & 21.721395 & 17.393478 & 23.824062 \\
\hline $\mathrm{Si}$ & 19.554403 & 5.108104 & 9.49865 \\
\hline $\mathrm{Si}$ & 21.731350 & 5.054457 & 11.65690 \\
\hline $\mathrm{Si}$ & 7.256891 & 17.361921 & 9.33188 \\
\hline $\mathrm{Si}$ & 9.448402 & 17.284000 & 11.48164 \\
\hline $\mathrm{Si}$ & 7.294274 & 5.156410 & 21.648331 \\
\hline $\mathrm{Si}$ & 9.409562 & 5.117328 & 23.77624 \\
\hline $\mathrm{Si}$ & 19.555132 & 1.639992 & 21.59589 \\
\hline
\end{tabular}




\begin{tabular}{|c|c|c|c|}
\hline Si & 21.676247 & 1.614018 & 19.478182 \\
\hline Si & 19.550520 & 13.820744 & 9.434080 \\
\hline & 7.293061 & 1.614261 & 9.432624 \\
\hline & 9.425342 & 1.662082 & 7.296459 \\
\hline & 7.243783 & 13.726315 & 21.68255 \\
\hline & 21.703676 & 23.747112 & 1.58707 \\
\hline$S 1$ & 23.835714 & 21.646873 & 1.6176 \\
\hline & 21.698093 & 11.579954 & 13.784817 \\
\hline Si & 23.838627 & 9.403737 & 13. \\
\hline & 9.460782 & 23.845181 & 13.85739 \\
\hline & 9.470250 & 11.588694 & 1.68538 \\
\hline & 11.621464 & 9.460540 & $1.6467 \varepsilon$ \\
\hline $\mathrm{Si}$ & 21.676247 & 23.767746 & 17.38449 \\
\hline $\mathrm{Si}$ & 19.532799 & 21.675276 & 17.398819 \\
\hline & 21.813883 & 11.641612 & $4.88307 \varepsilon$ \\
\hline L & 19.636936 & 9.533849 & 5.01367 \\
\hline & 9.358344 & 23.825520 & 5.16199 \\
\hline Si & 9.473890 & 11.534561 & 17.301477 \\
\hline Si & 7.263689 & 9.418787 & 17.369690 \\
\hline $\mathrm{Si}$ & 21.694210 & 19.521875 & 17.34371 \\
\hline $\mathrm{Si}$ & 23.828190 & 21.685713 & 17.35463 \\
\hline $\mathrm{Si}$ & 21.700521 & 7.371710 & 5.124368 \\
\hline $\mathrm{Si}$ & 23.811197 & 9.445246 & 5.13165 \\
\hline $\mathrm{Si}$ & 9.484328 & 19.533527 & \\
\hline $\mathrm{Si}$ & 11.570973 & 21.729408 & 5 . \\
\hline Si & 9.441605 & 7.262231 & $17.31992^{\circ}$ \\
\hline $\mathrm{Si}$ & 11.589664 & 9.358829 & 17.35391 \\
\hline $\mathrm{Si}$ & 21.697365 & 19.506824 & 1.63489 \\
\hline $\mathrm{Si}$ & 19.560957 & 21.629639 & 1.65212 \\
\hline $\mathrm{Si}$ & 21.723339 & 7.225577 & $13.76903^{\circ}$ \\
\hline $\mathrm{Si}$ & 19.54 & 9.388929 & 13. \\
\hline $\mathrm{Si}$ & 9.483843 & 19.603924 & 13.61149 \\
\hline $\mathrm{Si}$ & 7.251794 & 21.717028 & 13.80884 \\
\hline $\mathrm{Si}$ & 9.429468 & 7.297430 & 1.61304 \\
\hline $\mathrm{Si}$ & 7.310781 & 9.418302 & 01 \\
\hline $\mathrm{Si}$ & 11.676568 & 21.7 & 13. \\
\hline Si & 11.222147 & 3.392133 & 13.396422 \\
\hline $\mathrm{Si}$ & 20.047905 & 3.304015 & 13.40394 \\
\hline $\mathrm{Si}$ & 7.628536 & 1.212759 & 3.36543 \\
\hline $\mathrm{Si}$ & 23.372068 & 15.601285 & 17.72288 \\
\hline Si & 5.466640 & 15.544239 & 20.03164 \\
\hline $\mathrm{Si}$ & 3.466413 & 17.683 & 11.28 \\
\hline $\mathrm{Si}$ & 13.805449 & 9.421215 & 19.49760 \\
\hline $\mathrm{Si}$ & 23.785709 & 5.134806 & 9.42412 \\
\hline Al & 15.584293 & 1.288010 & 19.89400 \\
\hline Al & 15.567058 & 5.502323 & 11.27652 \\
\hline Al & 17.515823 & 9.566377 & 7.22970 \\
\hline Al & 9.494524 & 13.557120 & 19.63887 \\
\hline Al & 7.258590 & 21.636679 & 5.16709 \\
\hline Al & 7.545516 & 17.645206 & 15.35902 \\
\hline Al & 23.369156 & 5.398671 & 3.41179 \\
\hline Al & 13.459780 & 19.863419 & 3.28969 \\
\hline Al & 21.790821 & 13.673882 & 7.23650 \\
\hline Al & 16.412300 & 4.479635 & 14.07635 \\
\hline Al & 7.191350 & 18.346014 & 17.93455 \\
\hline 0 & 22.164650 & 0.349069 & 2.866587 \\
\hline 0 & 24.605219 & 24.542589 & 3.95578 \\
\hline 0 & 24.029669 & 2.099267 & 2.11723 \\
\hline
\end{tabular}




\begin{tabular}{|c|c|c|}
\hline 22.846767 & 2.186413 & 4.557798 \\
\hline 22.159796 & 12.470833 & 15.082538 \\
\hline 24.611044 & 12.476416 & 16.096729 \\
\hline 23.942038 & 14.330267 & 14.330509 \\
\hline 22.845552 & 14.395808 & 16.769865 \\
\hline 9.960596 & 0.251485 & 15.249546 \\
\hline 12.540015 & 24.614202 & 16.094059 \\
\hline 1.633844 & 2.101938 & 14.323227 \\
\hline 10.712623 & 2.120386 & 16.865749 \\
\hline 9.947973 & 12.480300 & 2.956889 \\
\hline 12.484912 & 12.470346 & 3.798489 \\
\hline 11.720262 & 14.330509 & 2.110676 \\
\hline 10.782048 & 14.323470 & 4.628438 \\
\hline 8.928440 & 6.407765 & 2.879210 \\
\hline 6.415049 & 6.409223 & 3.883693 \\
\hline 7.077987 & 4.629166 & 2.090528 \\
\hline 8.181026 & 4.555371 & 4.550031 \\
\hline 9.017771 & 18.718143 & 15.083750 \\
\hline 6.403882 & 18.718872 & 16.273933 \\
\hline 7.086727 & 16.691942 & 14.077083 \\
\hline 7.938522 & 16.927406 & 16.963091 \\
\hline 21.319410 & 6.240999 & 15.004616 \\
\hline 18.788055 & 6.411650 & 16.018080 \\
\hline 19.383511 & 4.556585 & 14.254288 \\
\hline 20.480722 & 4.544447 & 16.758455 \\
\hline 21.241972 & 18.548222 & 2.867801 \\
\hline 18.719357 & 18.785141 & 3.884178 \\
\hline 19.283499 & 16.851669 & 2.176218 \\
\hline 20.398674 & 16.855310 & 4.563868 \\
\hline 8.930140 & 0.342757 & 3.795091 \\
\hline 6.401940 & 24.609589 & 2.782355 \\
\hline 7.082600 & 2.106307 & 4.636448 \\
\hline 8.175927 & 2.190540 & 2.187627 \\
\hline 9.019227 & 12.471560 & 16.010798 \\
\hline 6.488601 & 12.392183 & 15.091518 \\
\hline 7.248153 & 14.317645 & 16.763796 \\
\hline 8.180782 & 14.257929 & 14.244334 \\
\hline 21.142448 & 0.333048 & 16.101828 \\
\hline 18.634153 & 24.612986 & 15.011169 \\
\hline 19.302433 & 2.119658 & 16.851669 \\
\hline 20.481207 & 2.114075 & 14.403334 \\
\hline 21.319410 & 12.466706 & 3.547733 \\
\hline 18.706491 & 12.549968 & 2.783568 \\
\hline 19.559015 & 14.239480 & 4.634992 \\
\hline 20.480722 & 14.411101 & 2.129611 \\
\hline 22.257137 & 6.573804 & 3.874712 \\
\hline 24.774899 & 6.400969 & 2.621657 \\
\hline 24.111231 & 4.551973 & 4.717040 \\
\hline 22.759865 & 4.486431 & 2.116260 \\
\hline 22.085030 & 18.627598 & 16.028032 \\
\hline 24.594782 & 18.707220 & 15.089090 \\
\hline 23.933540 & 16.860409 & 16.837349 \\
\hline 22.859148 & 16.788555 & 14.342403 \\
\hline 10.025167 & 6.492727 & 16.006914 \\
\hline 12.556522 & 6.407523 & 15.019423 \\
\hline 11.719534 & 4.631351 & 16.854824 \\
\hline 10.774038 & 4.632807 & 14.328568 \\
\hline 7577 & 18.609879 & 3.706488 \\
\hline
\end{tabular}




$\begin{array}{rrr}12.375918 & 18.703093 & 2.692296 \\ 11.724874 & 16.849970 & 4.471867 \\ 10.621351 & 16.848999 & 2.028871 \\ 2.950092 & 22.170719 & 0.257311 \\ 3.970111 & 24.614202 & 24.528997 \\ 2.098539 & 24.022629 & 2.031298 \\ 4.488373 & 22.856478 & 2.194667 \\ 2.951306 & 9.944332 & 12.474232 \\ 3.890004 & 12.466949 & 12.475930 \\ 2.184714 & 11.733613 & 14.336335 \\ 4.633778 & 10.704856 & 14.329538 \\ 15.170167 & 22.163439 & 12.404320 \\ 16.169312 & 24.612261 & 12.464521 \\ 14.325897 & 23.932814 & 14.245064 \\ 16.772778 & 22.841427 & 14.401391 \\ 15.167012 & 9.858400 & 0.342029 \\ 16.103285 & 12.396310 & 24.525841 \\ 14.315945 & 11.714922 & 2.037610 \\ 16.860893 & 10.709952 & 2.112618 \\ 2.868287 & 8.859500 & 6.407765 \\ 3.894859 & 6.407038 & 6.477677 \\ 2.193696 & 6.995939 & 4.550274 \\ 4.555129 & 8.177141 & 4.566537 \\ 2.863674 & 21.070351 & 18.711103 \\ 4.045605 & 18.627598 & 18.629055 \\ 2.275744 & 19.288111 & 16.772049 \\ 4.643974 & 20.553547 & 16.931047 \\ 15.094673 & 8.939849 & 18.619589 \\ 16.097700 & 6.419175 & 18.713774 \\ 14.339977 & 7.083813 & 16.850698 \\ 16.857981 & 8.097035 & 16.858709 \\ 15.174537 & 21.323294 & 6.324019 \\ 16.179749 & 18.798977 & 6.485687 \\ 14.263026 & 19.389336 & 4.734518 \\ 16.773748 & 20.477566 & 4.552944 \\ 3.862331 & 8.842508 & 0.184972 \\ 2.779684 & 6.406795 & 24.620028 \\ 4.638391 & 7.150812 & 2.100724 \\ 2.207289 & 8.266472 & 2.104122 \\ 3.805772 & 21.154343 & 12.549239 \\ 2.956646 & 18.559143 & 12.566474 \\ 4.641061 & 19.384966 & 14.418384 \\ 2.180830 & 20.396975 & 14.340705 \\ 16.101099 & 8.850761 & 12.470105 \\ 15.088848 & 6.322320 & 12.732027 \\ 16.858709 & 7.090611 & 14.400906 \\ 14.411829 & 8.259190 & 14.419841 \\ 16.178535 & 21.233234 & 0.340087 \\ 15.077925 & 18.731251 & 24.613958 \\ 16.858952 & 19.382540 & 2.110434 \\ 14.426394 & 20.633894 & 2.101695 \\ 3.800431 & 22.170235 & 6.490299 \\ 2.777499 & 24.611530 & 6.413349 \\ 4.638633 & 24.019230 & 4.635478 \\ 3.805772 & 92.841427 & 4.485218 \\ 2.864403 & 12.470590 & 18.697996 \\ 4.624311 & 11.793571 & 16.848757\end{array}$




\begin{tabular}{|c|c|c|c|}
\hline & 2.121114 & 10.714080 & 16.849970 \\
\hline 0 & 16.025604 & 22.080904 & 18.62735 \\
\hline 0 & 14.920139 & 24.528997 & 18.630754 \\
\hline 0 & 16.842688 & 23.931356 & 16.857010 \\
\hline 0 & 14.415228 & 22.765446 & 16.693157 \\
\hline 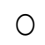 & 16.097700 & 9.955499 & 6.3281 \\
\hline 0 & 15.169926 & 12.487097 & 6.41019 \\
\hline 0 & 16.918911 & 11.802552 & $4.5570^{\circ}$ \\
\hline O & 14.403576 & 10.787874 & 4.5434 \\
\hline 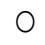 & 0.343971 & 2.867801 & 22.16222 \\
\hline O & 24.608860 & 3.964285 & 24.615171 \\
\hline 0 & 2.110676 & 2.115531 & 24.016075 \\
\hline 0 & 2.193210 & 4.554400 & 22.83729 \\
\hline O & 0.425776 & 15.153662 & 9.94578 \\
\hline 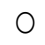 & 24.618572 & 16.101341 & 12.47811 \\
\hline 0 & 2.109948 & 14.249918 & \\
\hline 0 & 2.257052 & 16.754814 & 10.784476 \\
\hline O & 12.384415 & 2.939168 & 10.02638 \\
\hline 0 & 12.554580 & 3.799217 & 12.55870 \\
\hline $\mathrm{O}$ & 14.337306 & 2.104365 & 11.7231 \\
\hline O & 14.251375 & 4.558284 & 10.6211 \\
\hline 0 & 12.6 & 15. & 22 \\
\hline 0 & 12.391697 & 16. & 24.526327 \\
\hline 0 & 14.335608 & 14.320314 & 24.017288 \\
\hline 0 & 14.412315 & 16.784185 & 22.926874 \\
\hline 0 & 6.426457 & 2.867316 & 8.870424 \\
\hline O & 6.3 & & 65 \\
\hline 0 & 4.6 & 2. & 7.077502 \\
\hline 0 & 4.533524 & 4.489101 & 8.185881 \\
\hline 0 & 6.414078 & 15.097101 & 21.241001 \\
\hline 0 & 6.403639 & 16.099155 & 18.71110 \\
\hline 0 & 4.561197 & 14. & 19.459 \\
\hline $\mathrm{O}$ & 4.6 & 16. & 20 \\
\hline 0 & 18.704306 & 2324 & 21.147058 \\
\hline 0 & 18.794851 & 3.972053 & 18.70892 \\
\hline 0 & 16.933474 & 2.181801 & 19.210918 \\
\hline 0 & 16.833464 & 4.574306 & 20.404499 \\
\hline 0 & 18.707 & 15.17 & 9.01 \\
\hline 0 & 18.8 & 16. & 3502 \\
\hline 0 & 16.931532 & 14.326869 & 7.16270 \\
\hline 0 & 16.927162 & 16.861135 & 8.102375 \\
\hline 0 & 0.417523 & 3.881751 & 8.95101 \\
\hline 0 & 24.522686 & 2.692296 & 6.56288 \\
\hline 0 & 2.112 & 28 & \\
\hline 0 & 2.204134 & 2.182772 & 8.256034 \\
\hline 0 & 0.419222 & 16.097458 & 21.157255 \\
\hline 0 & 24.616869 & 15.077197 & 18.63172 \\
\hline 0 & 2.215057 & 16.778845 & 19.29757 \\
\hline 0 & 2.107521 & 14.331723 & 20.469313 \\
\hline 0 & 12.393397 & 3.868643 & 21.15239 \\
\hline 0 & 12.472775 & 2.866345 & 18.70163 \\
\hline 0 & 14.242636 & 4.701990 & 19.380354 \\
\hline 0 & 14.325897 & 2.275015 & 20.553061 \\
\hline 0 & 12.567202 & 16.097944 & 8.92091 \\
\hline 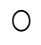 & 12.4 & 15.239836 & 6.41092 \\
\hline $\mathrm{O}$ & 14.419598 & 16.867447 & 7.08527 \\
\hline 0 & 14.412557 & 14.400906 & 8.09388 \\
\hline & & & \\
\hline
\end{tabular}




\begin{tabular}{|c|c|c|c|}
\hline 0 & 6.423059 & 2.790123 & 24.542105 \\
\hline 0 & 4.637662 & 4.643731 & 23.944950 \\
\hline . & 4.486431 & 2.271617 & 22.761806 \\
\hline 0 & 6.326931 & 16.091146 & 9.78436 \\
\hline O & 6.575746 & 14.914314 & 12.21546 \\
\hline O & 4.726993 & 16.773504 & 11.803281 \\
\hline 0 & 4.556342 & 14.333423 & 10.6912 \\
\hline 0 & 18.542639 & 3.891218 & 9 \\
\hline 0 & 18.879084 & 2.782355 & 12.38660 \\
\hline 0 & 16.858467 & 4.457059 & 12.04384 \\
\hline 0 & 16.869633 & 2.128154 & 10.7133 \\
\hline 0 & 18.630754 & 16.107410 & 22.160040 \\
\hline 0 & 18.713289 & 15.083750 & 24.605705 \\
\hline 0 & 16.855795 & 16.857010 & 24.0216 \\
\hline 0 & 16.772291 & 14.406732 & 22.9195 \\
\hline 0 & 2.867316 & 24.693821 & 22.08017 \\
\hline O & 0.428447 & 0.421893 & 20.985876 \\
\hline 0 & 1.007639 & 22.912550 & 22 . \\
\hline 0 & 2.199521 & 4429 & 20.485092 \\
\hline 0 & 2.952034 & 12.468162 & 9.94748 \\
\hline 0 & 0.503455 & 12.578369 & 8.933052 \\
\hline O & 1.093328 & 10.698787 & 10.70946 \\
\hline O & 2.190783 & 10.616253 & 8.258946 \\
\hline 0 & 15.158274 & 24.686783 & \\
\hline 0 & 12.563318 & 0.507582 & 8.940092 \\
\hline 0 & 13.309763 & 22.926388 & 10.623293 \\
\hline 0 & 14.326626 & 23.009649 & 8.099220 \\
\hline 0 & 15.084479 & 12.562104 & 22.082117 \\
\hline 0 & 12.558464 & 12.555794 & 21 . \\
\hline 0 & 13.316074 & 10.704127 & 22.9 \\
\hline 0 & 14.250889 & 10.701942 & 20.39551 \\
\hline 0 & 16.110323 & 18.631725 & 22.156883 \\
\hline 0 & 18.622015 & 18.607450 & 21.14827 \\
\hline 0 & 18.036026 & 20.392847 & 22 . \\
\hline 0 & 16.853853 & 20. & 91 \\
\hline 0 & 16.182661 & 6.482532 & 10.027351 \\
\hline 0 & 18.707949 & 6.407038 & 9.01728 \\
\hline 0 & 18.023890 & 8.350220 & 10.62232 \\
\hline 0 & 16.873032 & 8.106259 & 8.172044 \\
\hline 0 & 3.872527 & 18.620 & 10. \\
\hline 0 & 6.324747 & 18.6 & 8 . \\
\hline 0 & 5.661079 & 20.394547 & 10.78326 \\
\hline 0 & 4.469682 & 20.482906 & 8.345608 \\
\hline 0 & 3.869128 & 6.483260 & 22.175573 \\
\hline 0 & 6.322562 & 6.325717 & 21.070 \\
\hline 0 & 5.814495 & 3026 & 22 . \\
\hline 0 & 4.552944 & 493 & 20.39357 \\
\hline 0 & 16.179262 & 24.768101 & 21.31188 \\
\hline 0 & 18.706978 & 0.417280 & 22.23480 \\
\hline 0 & 17.950094 & 22.929058 & 20.391392 \\
\hline 0 & 16.937601 & 22.845797 & 22.837786 \\
\hline 0 & 16.095758 & 12.544628 & 8.95053 \\
\hline 0 & 18.526373 & 12.716491 & 10.039489 \\
\hline 0 & 18.030445 & 10.704127 & 8.417703 \\
\hline 0 & 16.852398 & 10.786175 & 10.799041 \\
\hline 0 & 3.806500 & 24.598665 & 8.9415 \\
\hline 0 & 6.319649 & 0.424805 & 9.962052 \\
\hline 0 & 5.721037 & 22.828562 & 8.25433 \\
\hline
\end{tabular}




\begin{tabular}{|c|c|c|c|}
\hline & 4.556342 & 22.855507 & 10.701215 \\
\hline 0 & 3.714014 & 12.474716 & 21.238333 \\
\hline 0 & 6.167448 & 12.645853 & 22.250097 \\
\hline 0 & 5.575147 & 10.705098 & 20.470770 \\
\hline 0 & 4.556585 & 10.697574 & 22.928087 \\
\hline 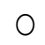 & 2.885036 & 18.701880 & 21.0 \\
\hline $\mathrm{O}$ & 0.499814 & 18.536327 & 22.253738 \\
\hline 0 & 0.926561 & 20.391392 & 20.39988 \\
\hline O & 2.195880 & 20.489702 & 22.762291 \\
\hline 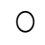 & 2.855907 & 6.329844 & 8.935479 \\
\hline O & 0.426505 & 6.338583 & 10.029051 \\
\hline 0 & 1.020504 & 8.176413 & 8.189522 \\
\hline 0 & 2.191268 & 8.249237 & 10.614553 \\
\hline O & 15.170897 & 18.629784 & 8.940820 \\
\hline 0 & 12.632501 & 18.626143 & 9.79261 \\
\hline 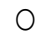 & 13.391811 & 20.481449 & 8.098735 \\
\hline 0 & 14.330752 & 20.464458 & 10.619166 \\
\hline O & 15.094673 & 6.329359 & 21.240274 \\
\hline 0 & 12.560891 & 6.336884 & 22.170235 \\
\hline O & 13.304423 & 8.166218 & 20.403528 \\
\hline 0 & 14.325897 & 8.186123 & 22.857449 \\
\hline 0 & 22.171448 & 2 . & 24.605219 \\
\hline 0 & 21.076178 & 0.3 & 0.352953 \\
\hline 0 & 22.922747 & 0.921706 & 22.842154 \\
\hline 0 & 20.560343 & 2.186899 & 22.747971 \\
\hline O & 22.160280 & 15.080353 & 12.476901 \\
\hline 0 & 21.2 & 12. & 12. \\
\hline 0 & 23.0 & 13.2 & 10.704127 \\
\hline 0 & 20.486305 & 14.258657 & 10.697816 \\
\hline 0 & 9.968122 & 3.037966 & 12.406748 \\
\hline 0 & 8.939363 & 0.594728 & 12.720619 \\
\hline 0 & 10.621837 & 1.079491 & 10.705098 \\
\hline 0 & 8.169859 & 3 & 10. \\
\hline 0 & 9.850633 & 15. & 24.680229 \\
\hline 0 & 8.930383 & 12.559192 & 0.493745 \\
\hline 0 & 10.793215 & 13.308064 & 23.009165 \\
\hline 0 & 8.252635 & 14.239964 & 22.841183 \\
\hline 0 & 22.167 & 16.090 & 18.697023 \\
\hline 0 & 21.2 & 18. & 18. \\
\hline 0 & 22.923717 & 17.954708 & 20.479265 \\
\hline 0 & 20.476839 & 16.855553 & 20.480965 \\
\hline 0 & 22.144018 & 3.880052 & 6.409708 \\
\hline 0 & 21.147058 & 6.400 & 6.328874 \\
\hline 0 & 22.9 & 5. & 8.178841 \\
\hline 0 & 20.482664 & 4.636691 & 8.262102 \\
\hline 0 & 9.944332 & 16.099642 & 6.330815 \\
\hline 0 & 9.016800 & 18.620317 & 6.253379 \\
\hline 0 & 10.688106 & 17.934559 & 8.165732 \\
\hline 0 & 8.188551 & 16.874245 & 8.098492 \\
\hline 0 & 9.952585 & 3.7 & 18.70333 \\
\hline 0 & 8.928926 & 6.235416 & 18.46180 \\
\hline 0 & 10.692719 & 5.750896 & 20.390905 \\
\hline 0 & 8.261860 & 4.632079 & 20.468584 \\
\hline 0 & 21.232506 & 16.180233 & 24.772470 \\
\hline 0 & 22.328747 & 18.626627 & 0.418009 \\
\hline $\mathrm{O}$ & 20.476353 & 17.948881 & 22.920076 \\
\hline 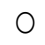 & 22.921776 & 16.773748 & 22.919348 \\
\hline & 21 & & 12 \\
\hline
\end{tabular}




\begin{tabular}{rrr}
22.250584 & 6.344652 & 12.499720 \\
20.381926 & 5.495041 & 10.858271 \\
22.851137 & 4.549545 & 10.618438 \\
9.023354 & 15.994776 & 12.394125 \\
9.859371 & 18.471272 & 12.564532 \\
8.186608 & 17.851782 & 10.619894 \\
10.772824 & 16.935902 & 10.632275 \\
8.929654 & 3.803344 & 24.607891 \\
9.954527 & 6.325961 & 0.429903 \\
8.157479 & 5.731476 & 22.912794 \\
10.622564 & 4.633050 & 22.836086 \\
21.240517 & 2.865131 & 18.541668 \\
22.319281 & 0.425048 & 18.547735 \\
20.403770 & 1.019533 & 20.313955 \\
22.830017 & 2.192724 & 20.459846 \\
21.394176 & 15.248575 & 6.246583 \\
22.343554 & 12.644882 & 5.998739 \\
20.389935 & 13.308064 & 8.165247 \\
23.013535 & 14.405761 & 8.191463 \\
8.851975 & 2.953733 & 6.492970 \\
10.026381 & 0.589630 & 6.246826 \\
8.261860 & 1.011765 & 8.259190 \\
10.626934 & 2.196609 & 8.258946 \\
8.934023 & 15.176478 & 18.706734 \\
10.033420 & 12.477387 & 18.454279 \\
8.005034 & 13.146152 & 20.394304 \\
10.698301 & 14.339248 & 20.559858 \\
24.766159 & 22.078718 & 2.866345 \\
0.496173 & 21.064770 & 0.417523 \\
23.002367 & 22.918377 & 1.012008 \\
22.850166 & 20.488976 & 2.191025 \\
24.685324 & 9.931709 & 15.082051 \\
0.504426 & 8.863870 & 12.568416 \\
22.920563 & 10.633245 & 13.230871 \\
22.922020 & 8.173500 & 14.323713 \\
12.472531 & 22.164165 & 15.085692 \\
12.648038 & 21.241732 & 12.550210 \\
10.684464 & 22.935127 & 13.226258 \\
10.709467 & 20.471983 & 14.171996 \\
12.481028 & 9.946032 & 2.944752 \\
12.571330 & 8.937664 & 0.429903 \\
10.707768 & 10.705584 & 1.090900 \\
10.630575 & 8.257976 & 2.115774 \\
18.706734 & 22.173389 & 16.086777 \\
18.536083 & 21.146574 & 18.550163 \\
20.481936 & 22.851379 & 18.031172 \\
20.482664 & 20.472712 & 16.857252 \\
18.785383 & 10.030993 & 3.706003 \\
18.789511 & 9.010245 & 6.245368 \\
20.564228 & 10.796128 & 5.488972 \\
20.572237 & 8.332013 & 4.472595 \\
6.322076 & 22.092312 & 3.792421 \\
6.239543 & 20.982721 & 6.413835 \\
8.172286 & 23.004066 & 5.818864 \\
8.349491 & 20.491159 & 4.481576 \\
6.327660 & 9.865440 & 16.100613 \\
8.170588 & 8.944704 & 18.619347 \\
& 10.697574 & 17.857122 \\
\hline & &
\end{tabular}




\begin{tabular}{|c|c|c|c|}
\hline 0 & 8.175685 & 8.177870 & 553 \\
\hline & 24.857918 & 21.236391 & 16.171738 \\
\hline & 0.418251 & 22.253983 & 18.613035 \\
\hline c & 22.997026 & 20.379740 & 17.863434 \\
\hline & 22.857933 & 22.831959 & 16.7698 \\
\hline 0 & 24.692122 & 8.844935 & 3.8790 \\
\hline 0 & 0.499814 & 10.031235 & 6.315279 \\
\hline 0 & 22.915949 & 8.271570 & 58130 \\
\hline 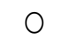 & 23.000910 & 10.629119 & 4.3898 \\
\hline 0 & 12.475203 & 21.408497 & 3.7089 \\
\hline 0 & 12.633958 & 22.239174 & 6.2424 \\
\hline 0 & 10.879875 & 20.319054 & 5.4853 \\
\hline 0 & 10.627419 & 22.920805 & 4.6369 \\
\hline 0 & 12.475687 & 8.849791 & 16.09041 \\
\hline 0 & 12.559919 & 9.873208 & 18.5448 \\
\hline 0 & 10.630333 & 8.178841 & 17.9585 \\
\hline 0 & 10.629848 & 10.535904 & 16.7647 \\
\hline 0 & 18.630512 & 21.244886 & 2.928973 \\
\hline 0 & 18.702850 & 22.240873 & 0 . \\
\hline 0 & 20.389692 & 20.320024 & 1.0 \\
\hline 0 & 20.572966 & 22.759865 & 2.19272 \\
\hline c & 18.700909 & 8.936451 & 15.17211 \\
\hline 0 & 18.551863 & 9.874664 & 12.6562 \\
\hline O & 20.408140 & 8.096792 & 13.313646 \\
\hline 0 & 20.485092 & 10.626206 & 14.31764 \\
\hline 0 & 6.499281 & 21.158226 & $15.1662 \varepsilon$ \\
\hline 0 & 6.241485 & 22.170235 & 12.6487 \\
\hline 0 & 8.176413 & 20.468100 & 13.2298 \\
\hline 0 & 8.189036 & 22.920563 & 14.32419 \\
\hline 0 & 6.423544 & 8.939607 & 2 \\
\hline 0 & 6.316250 & 9.952100 & 0 . \\
\hline 0 & 8.175442 & 8.182482 & 1.0229 \\
\hline 0 & 8.259675 & 10.623536 & 2.18981 \\
\hline 0 & 13.235969 & 5.218554 & 8.2657 \\
\hline 0 & 12.467677 & 6.659736 & 5. \\
\hline 0 & 11.804980 & 3550 & 8 . \\
\hline 0 & 14.485381 & 7.754278 & 7.5877 \\
\hline 0 & 9.095207 & 17.357552 & 20.13553 \\
\hline 0 & 8.600976 & 19.480610 & 17.93601 \\
\hline 0 & 7.577316 & 19.733793 & 21.003839 \\
\hline 0 & 9.519041 & 21.230564 & 19.71728 \\
\hline $\mathrm{H}$ & 12.707267 & 22.182856 & 3. \\
\hline $\mathrm{H}$ & 15.874373 & 7.933182 & 7.93803 \\
\hline $\mathrm{H}$ & 25.039492 & 6.153611 & 1.7084 \\
\hline $\mathrm{H}$ & 21.943024 & 16.018808 & 6.4915 \\
\hline $\mathrm{H}$ & 13.612709 & 4.999111 & 9.183809 \\
\hline $\mathrm{H}$ & 9.141814 & 15.973900 & 19.3102 \\
\hline $\mathrm{H}$ & 9.283821 & 19.191984 & 15. \\
\hline $\mathrm{H}$ & 11.079655 & 18.050592 & 20.0845 \\
\hline $\mathrm{H}$ & 10.584939 & 22.875168 & 19.3619 \\
\hline $\mathrm{H}$ & 12.539044 & 21.451706 & 18.72785 \\
\hline $\mathrm{H}$ & 11.492566 & 21.578176 & 35218 \\
\hline $\mathrm{H}$ & 11.448144 & 20.061499 & 18.3052 \\
\hline $\mathrm{H}$ & 9.393542 & 18.061029 & 22.7178 \\
\hline $\mathrm{H}$ & 10.981343 & 18.864277 & 22.5256 \\
\hline $\mathrm{H}$ & 10.865067 & 17.094173 & 22.425846 \\
\hline $\mathrm{Re}$ & 12.989096 & 6.880877 & 7.56663 \\
\hline $\mathrm{Re}$ & 8.650253 & 19.233252 & 19.8257 \\
\hline
\end{tabular}




$\begin{array}{llll}\text { C } & 10.161347 & 18.099627 & 20.688513 \\ \text { C } & 10.502648 & 21.790094 & 19.180574 \\ \text { C } & 11.540629 & 21.150702 & 18.357180 \\ \text { C } & 10.348504 & 18.035055 & 22.179459\end{array}$

\begin{tabular}{|c|c|c|c|}
\hline \multicolumn{4}{|c|}{607} \\
\hline \\
\hline & $23 . \overline{3} 8080 \overline{8}$ & 3.327805 & 1.2 \\
\hline & 23.416491 & 1.257181 & 3.371499 \\
\hline & 23.408482 & 15.562202 & 13.395208 \\
\hline & 23.404112 & 13.402977 & 15.543268 \\
\hline & 11.222632 & 1.210089 & 15.625560 \\
\hline & 11.193260 & 15.5872 & 1255 \\
\hline & 11.232585 & 13.396422 & 3.368829 \\
\hline & 7.646013 & 3.360818 & 1.234849 \\
\hline & 7.661791 & 5.506936 & 3.352322 \\
\hline & 7.689222 & 15.539385 & 13.275050 \\
\hline & 19.951778 & 5.47 & 15. \\
\hline & 19.908569 & 15. & 775 \\
\hline & 19.911726 & 17.799835 & 2079 \\
\hline & 7.623195 & 3.376354 & 5.518102 \\
\hline & 7.614456 & 15.592060 & 50072 \\
\hline & 7. & 13 & \\
\hline & 19.9 & & 17. \\
\hline & 19.950565 & 1.2 & 15.6 \\
\hline & 19.941097 & 15.594245 & 5.410323 \\
\hline & 19.995716 & 13.360740 & 3915 \\
\hline & 23.457516 & 3. & \\
\hline & 23.3 & 17. & \\
\hline & 11.2 & & 17. \\
\hline & 11.235498 & 5.5 & 15.5 \\
\hline & 11.239383 & 15.643279 & 5.443821 \\
\hline & 11.191804 & 17. & 949 \\
\hline & 1.26 & 23 & 771 \\
\hline & 3.3 & 23. & 01 \\
\hline & 1.240918 & 11. & 15.5 \\
\hline & 3.405726 & 11.2 & 13. \\
\hline & 13.473617 & 23. & 15. \\
\hline-1 & 15.642308 & 23.335173 & 13.377246 \\
\hline 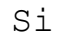 & 13.409775 & 11.2 & \\
\hline & 15 . & 11. & \\
\hline & 1.318111 & 7.7 & 784 \\
\hline & 3.364945 & 7.610330 & 4555 \\
\hline 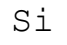 & 1.247 & 19. & 319 \\
\hline $\mathrm{Si}$ & 3.3 & 19. & 940 \\
\hline Si & 13.449100 & & \\
\hline & 15.6 & & 17. \\
\hline & 15.596673 & 19. & 2519 \\
\hline & 5.524413 & 7.656451 & 662 \\
\hline $\mathrm{Si}$ & 3.413980 & 7.666646 & 1.192611 \\
\hline $\mathrm{Si}$ & 5.423431 & 20.043051 & -5.616820 \\
\hline $\mathrm{Si}$ & & 19. & 97 \\
\hline S: & 17.811003 & & 15.655417 \\
\hline SI & 15.575311 & 7.708156 & 13.442545 \\
\hline$\Delta \perp$ & 17.757355 & 9.963188 & 3.384850 \\
\hline $\mathrm{Si}$ & 15.596188 & 20.031641 & 1.221741 \\
\hline $\mathrm{Si}$ & $5.45^{\circ}-1$ & 23.2 & 3.385335 \\
\hline & 3.349166 & 23.407995 & 5.54067 \\
\hline
\end{tabular}




\begin{tabular}{|c|c|c|c|}
\hline i & 5.493342 & 11.177725 & .613180 \\
\hline Si & 3.324892 & 11.188890 & 17.765608 \\
\hline $\mathrm{Si}$ & 17.750315 & 23.391003 & 15.588177 \\
\hline $\mathrm{Si}$ & 15.568756 & 23.327162 & 17.718273 \\
\hline & 17.806389 & 11.228216 & 3.318581 \\
\hline $\mathrm{Si}$ & 15.667069 & 11.229429 & 5.48217 \\
\hline$i$ & 3.373684 & 1.282670 & 23.367701 \\
\hline S & 1.241646 & 3.363003 & 23.415279 \\
\hline 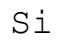 & 3.361304 & 13.405890 & 11.23040 \\
\hline$\Delta \perp$ & 1.258881 & 15.603227 & 11.25588 \\
\hline i & 15.621433 & 1.212516 & 11.21049 \\
\hline i & 13.41025 & 3.284353 & \\
\hline $\mathrm{Si}$ & 15.572641 & 13.435263 & 23.42644 \\
\hline i & 13.485512 & 15.591576 & 23.44877 \\
\hline 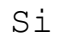 & 3.342127 & 1.216400 & 7.66470 \\
\hline i & 5.481447 & 3.331203 & 7.61421 \\
\hline $\mathrm{Si}$ & 3.297219 & 13.346903 & 19.8915 \\
\hline $\mathrm{Si}$ & 17.775805 & 3.380238 & 19.851767 \\
\hline $\mathrm{Si}$ & 15.650805 & 13.423368 & 7.637760 \\
\hline Si & 17.816099 & 15.570698 & 7.65742 \\
\hline $\mathrm{Si}$ & 3.312755 & 5.444792 & 7.57901 \\
\hline $\mathrm{Si}$ & 1.225382 & 3.318095 & 7.65596 \\
\hline $\mathrm{Si}$ & 3.388734 & 17.699823 & \\
\hline $\mathrm{Si}$ & 1.221498 & 15.555891 & 19.86414 \\
\hline Si & 15.582108 & 5.494798 & 19.93988 \\
\hline $\mathrm{Si}$ & 13.406619 & 3.395046 & 19.92143 \\
\hline $\mathrm{Si}$ & 15.666583 & 17.773132 & 7.63654 \\
\hline $\mathrm{Si}$ & 13.472159 & 15. & 0 \\
\hline $\mathrm{Si}$ & 3.382665 & 5.489701 & 23.40581. \\
\hline $\mathrm{Si}$ & 5.50936 & 3.388491 & 23.406782 \\
\hline $\mathrm{Si}$ & 5.528297 & 15.596915 & 11.13427 \\
\hline Si & 17.878485 & 3.330960 & 11.24181 \\
\hline $\mathrm{Si}$ & 15.649834 & 17.791582 & 23.44295 \\
\hline $\mathrm{Si}$ & 17.734779 & 15.627987 & 23.429115 \\
\hline $\mathrm{Si}$ & 1.643876 & 21.678431 & 23.73109. \\
\hline $\mathrm{Si}$ & 1.616446 & 23.788622 & 21.61385 \\
\hline $\mathrm{Si}$ & 1.674462 & 9.442333 & $11.57582 \varepsilon$ \\
\hline $\mathrm{Si}$ & 1.664509 & 11.605443 & 9.44985 \\
\hline $\mathrm{Si}$ & 13.874875 & 21.669207 & 11.54936 \\
\hline $\mathrm{Si}$ & 13.837978 & 23.857077 & 9.38601 \\
\hline $\mathrm{Si}$ & 13.848660 & 9.430197 & 23.81289 \\
\hline $\mathrm{Si}$ & 13.811761 & 11.631903 & 21.68013 \\
\hline $\mathrm{Si}$ & 17.443241 & 21.701006 & 23.77260 \\
\hline $\mathrm{Si}$ & 17.426249 & 19.569939 & 21.72673 \\
\hline $\mathrm{Si}$ & 17.337160 & 9.465152 & 11.594032 \\
\hline $\mathrm{Si}$ & 17.388866 & 7.244268 & 9.49865 \\
\hline $\mathrm{Si}$ & 5.066109 & 21.681343 & 11.61758 \\
\hline $\mathrm{Si}$ & 5.087713 & 19.548820 & 9.48675 \\
\hline $\mathrm{Si}$ & 5.151556 & 9.468307 & 23.73546 \\
\hline $\mathrm{Si}$ & 5.147429 & 7.289419 & 21.644447 \\
\hline $\mathrm{Si}$ & 17.377459 & 21.733047 & 19.52066 \\
\hline $\mathrm{Si}$ & 17.396391 & 23.908297 & 21.64347 \\
\hline $\mathrm{Si}$ & 17.391050 & 11.680209 & 9.49937 \\
\hline $\mathrm{Si}$ & 5.090869 & 21.603178 & 7.30277 \\
\hline $\mathrm{Si}$ & 5.097180 & 23.772358 & 9.43141 \\
\hline $\mathrm{Si}$ & 5.115386 & 9.470007 & 19.55828 \\
\hline $\mathrm{Si}$ & 5.106890 & 11.631417 & 21.66605 \\
\hline $\mathrm{Si}$ & 1.571538 & 21.599052 & 19.56823 \\
\hline
\end{tabular}




$\begin{array}{lrrr} & & & \\ \mathrm{Si} & 1.604308 & 19.537653 & 21.603422 \\ \mathrm{Si} & 1.612319 & 9.451558 & 7.270728 \\ \mathrm{Si} & 1.594841 & 7.338212 & 9.375821 \\ \mathrm{Si} & 13.879731 & 21.744942 & 7.188437 \\ \mathrm{Si} & 13.864194 & 19.524788 & 9.341594 \\ \mathrm{Si} & 13.853271 & 7.229947 & 21.694937 \\ \mathrm{Si} & 23.769201 & 1.594599 & 21.627697 \\ \mathrm{Si} & 21.670420 & 1.538039 & 23.709002 \\ \mathrm{Si} & 23.846395 & 13.867836 & 9.426312 \\ \mathrm{Si} & 21.712416 & 13.783360 & 11.577527 \\ \mathrm{Si} & 11.553738 & 1.646789 & 9.475832 \\ \mathrm{Si} & 9.418302 & 1.712816 & 11.618308 \\ \mathrm{Si} & 11.677297 & 13.849387 & 21.725281 \\ \mathrm{Si} & 9.455685 & 13.797196 & 23.808769 \\ \mathrm{Si} & 23.808527 & 17.337404 & 21.694452 \\ \mathrm{Si} & 21.681343 & 17.371147 & 19.535469 \\ \mathrm{Si} & 21.683529 & 5.178257 & 7.240142 \\ \mathrm{Si} & 11.642341 & 17.369446 & 9.364898 \\ \mathrm{Si} & 9.437721 & 17.376972 & 7.181397 \\ \mathrm{Si} & 11.608356 & 5.118056 & 21.634493 \\ \mathrm{Si} & 9.488455 & 5.088927 & 19.531828 \\ \mathrm{Si} & 19.531342 & 17.385954 & 21.695423 \\ \mathrm{Si} & 21.715570 & 17.394207 & 23.826733 \\ \mathrm{Si} & 19.558289 & 5.070478 & 9.448402 \\ \mathrm{Si} & 21.740816 & 5.049117 & 11.627048 \\ \mathrm{Si} & 7.254707 & 17.352940 & 9.335282 \\ \mathrm{Si} & 9.438692 & 17.262638 & 11.536018 \\ \mathrm{Si} & 7.300828 & 5.141117 & 21.649544 \\ \mathrm{Si} & 9.416360 & 5.117571 & 23.772600 \\ \mathrm{Si} & 19.548092 & 1.646546 & 21.597111 \\ \mathrm{Si} & 21.669935 & 1.630768 & 19.494446 \\ \mathrm{Si} & 19.551006 & 13.822927 & 9.417088 \\ \mathrm{Si} & 7.293303 & 1.621786 & 9.428983 \\ \mathrm{Si} & 9.428983 & 1.665966 & 7.300343 \\ \mathrm{Si} & 7.286749 & 13.747677 & 21.651972 \\ \mathrm{Si} & 21.701977 & 23.752695 & 1.584888 \\ \mathrm{Si} & 23.822605 & 21.652700 & 1.612562 \\ \mathrm{Si} & 21.703434 & 11.584324 & 13.760541 \\ \mathrm{Si} & 23.838142 & 9.391357 & 13.780932 \\ \mathrm{Si} & 9.461996 & 23.841297 & 13.858369 \\ \mathrm{Si} & 9.466851 & 11.598161 & 1.606979 \\ \mathrm{Si} & 11.615881 & 9.474619 & 1.647517 \\ \mathrm{Si} & 21.713871 & 23.788378 & 17.411442 \\ \mathrm{Si} & 19.546150 & 21.700521 & 17.415569 \\ \mathrm{Si} & 21.758780 & 11.637000 & 4.897400 \\ \mathrm{Si} & 19.610720 & 9.478988 & 5.006636 \\ \mathrm{Si} & 9.359315 & 23.835228 & 5.155439 \\ \mathrm{Si} & 9.453257 & 11.578012 & 17.282059 \\ \mathrm{Si} & 7.275097 & 9.425827 & 17.372602 \\ \mathrm{Si} & 21.669935 & 19.503670 & 17.336920 \\ \mathrm{Si} & 23.831102 & 21.641775 & 17.371147 \\ \mathrm{Si} & 21.704405 & 7.337968 & 5.102278 \\ \mathrm{Si} & 23.783768 & 9.450586 & 5.079217 \\ \mathrm{Si} & 9.489669 & 19.550762 & 4.977021 \\ \mathrm{Si} & 11.581897 & 21.770674 & 5.119027 \\ \mathrm{Si} & 9.445004 & 7.246939 & 17.351969 \\ \mathrm{Si} & 11.612968 & 9.349119 & 17.382555 \\ \mathrm{Si} & 21.677944 & 19.543722 & 1.604308\end{array}$




\begin{tabular}{|c|c|c|c|}
\hline Si & 19.554403 & 21.645903 & 1.648488 \\
\hline Si & 21.718241 & 7.241598 & 13.742579 \\
\hline & 19.544451 & 9.394999 & 13.808848 \\
\hline 1 & 9.454714 & 19.612177 & 13.680193 \\
\hline & 7.260047 & 21.718969 & 13.80229 \\
\hline & 9.427283 & 7.301557 & 1.60989 \\
\hline Si & 7.301072 & 9.434080 & 1.5999 \\
\hline $\mathrm{Si}$ & 11.672441 & 21.742516 & 13.7328 \\
\hline $\mathrm{Si}$ & 11.191804 & 3.417378 & 13.38889 \\
\hline i & 20.064898 & 3.298676 & 13.40637 \\
\hline L & 7.590424 & 1.227324 & 3.36251 \\
\hline 5 & 23.367456 & 15.581865 & 17.67724 \\
\hline i & 5.417120 & 15.513897 & 20.01367 \\
\hline i & 3.421747 & 17.750315 & 11.285261 \\
\hline i & 13.838706 & 9.408349 & 19.540081 \\
\hline $\mathrm{Si}$ & 23.803186 & 5.150099 & 9.409077 \\
\hline L & 15.596673 & 1.279271 & 19.86706 \\
\hline 1 & 15.612207 & 5.478292 & 11.272882 \\
\hline 1 & 17.526018 & 9.552298 & 7226 \\
\hline Al & 9.498651 & 13.718061 & 19.58717 \\
\hline L & 7.255678 & 21.649788 & 5.16029 \\
\hline - & 7.545759 & 17.753471 & 15.44665 \\
\hline 1 & 23.404354 & 5.363716 & 3.41980 \\
\hline 1 & 13.471675 & 19.85 & \\
\hline 1 & 21.778927 & 13.655190 & 7.25009 \\
\hline Al & 16.496775 & 4.547846 & 14.02610 \\
\hline Al & 6.015489 & 18.013937 & 17.99694 \\
\hline 0 & 22.165380 & 0.349797 & 2.86585 \\
\hline O & 24.612017 & 24.602062 & 3.959915 \\
\hline 0 & 24.028212 & 2.102180 & 2.1 \\
\hline O & 22.84 & $2.1 \mathrm{~s}$ & 4.5 \\
\hline 0 & 22.162952 & 12.471560 & 15.065302 \\
\hline 0 & 24.616386 & 12.460395 & 16.08968 \\
\hline 0 & 24.003210 & 14.337063 & 14.32905 \\
\hline 0 & 22.849438 & 14.337063 & 16.7715 \\
\hline O & 9.95 & 0.2 & 15.2 \\
\hline 0 & 12.548755 & 24.620996 & 16.019535 \\
\hline 0 & 11.559078 & 2.119901 & 14.31788 \\
\hline 0 & 10.708011 & 2.105336 & 16.86404 \\
\hline 0 & 9.956955 & 12.482970 & 2.88163 \\
\hline 0 & 12.473745 & 12.4710 & 3.86767 \\
\hline 0 & 11.72 & 14.32 & 2.11504 \\
\hline 0 & 10.774282 & 14.319828 & 4.62285 \\
\hline 0 & 8.930140 & 6.410679 & 2.87775 \\
\hline 0 & 6.419903 & 6.417476 & 3.88612 \\
\hline 0 & 7.080657 & 4.634506 & \\
\hline O & 8.180782 & 4.55 & 4.55270 \\
\hline 0 & 8.933295 & 18.879 & 15.17745 \\
\hline 0 & 6.152154 & 18.634640 & 16.26106 \\
\hline 0 & 7.165376 & 16.861622 & 14.07853 \\
\hline 0 & 7.831471 & 17.016008 & 17.01649 \\
\hline 0 & 21.238817 & 6.323776 & 15.00073 \\
\hline 0 & 18.714745 & 6.404125 & 16.01371 \\
\hline 0 & 19.382296 & 4.553672 & 14.24724 \\
\hline 0 & 20.479507 & 4.543477 & 16.70650 \\
\hline O & 21.225224 & 18.622744 & 2.86634 \\
\hline 0 & 18.704550 & 18.723000 & 3.88417 \\
\hline 0 & 19.297577 & 16.850212 & 2.18034 \\
\hline
\end{tabular}




$$
\begin{aligned}
& 20.477324 \\
& 8.864112 \\
& 16.852640 \\
& 0.331834 \\
& \text { 4. } 554158 \\
& 6.316979 \\
& 7.077745 \\
& 24.686541 \\
& 2.114560 \\
& 2.189326 \\
& 8.180054 \\
& 8.945675 \\
& 6.402183 \\
& \text { 7. } 326560 \\
& 8.093637 \\
& 21.233477 \\
& 18.708920 \\
& 19.376713 \\
& 20.549177 \\
& 21.313826 \\
& 18.719357 \\
& 19.559744 \\
& 20.474653 \\
& 22.253010 \\
& 24.773928 \\
& 24.181627 \\
& 22.763020 \\
& 22.074348 \\
& 24.537008 \\
& 23.935726 \\
& 22.843126 \\
& 9.965208 \\
& 12.548997 \\
& 11.713951 \\
& 10.710196 \\
& 9.884617 \\
& 12.381502 \\
& 11.725117 \\
& 10.621108 \\
& \text { 2. } 946694 \\
& \text { 3. } 963557 \\
& 2.105336 \\
& \text { 4. } 482062 \\
& 2.950820 \\
& \text { 3. } 881994 \\
& \text { 2. } 182772 \\
& 4.635478 \\
& 15.165313 \\
& 16.177807 \\
& 14.344104 \\
& 16.859922 \\
& 15.180120 \\
& 16.103041 \\
& 14.254773 \\
& 16.846329 \\
& \text { 2. } 871200 \\
& \text { 3. } 887334 \\
& 2.122328 \\
& 4.555129 \\
& \text { 2. } 862704 \\
& \text { 4. } 146101 \\
& \text { 2. } 280841 \\
& \text { 4. } 629409 \\
& 15.162157
\end{aligned}
$$




\begin{tabular}{|c|c|c|c|}
\hline 0 & 16.099155 & 6.422088 & 18.698481 \\
\hline O & 14.430279 & 7.169017 & 16.783457 \\
\hline O & 16.938330 & 8.177627 & 16.934931 \\
\hline O & 15.176237 & 21.324991 & 6.324990 \\
\hline O & 16.183390 & 18.795094 & 6.493455 \\
\hline O & 14.327597 & 19.388123 & 4.718740 \\
\hline O & 16.858223 & 20.477324 & 4.621641 \\
\hline 0 & 3.861846 & 8.916060 & 0.267749 \\
\hline & 2.781626 & 6.418447 & 24.615900 \\
\hline & 4.632322 & 7.084299 & 2.100724 \\
\hline O & 2.209231 & 8.269385 & 2.101209 \\
\hline & 3.792906 & 21.232506 & 12.547541 \\
\hline O & 2.880424 & 18.635366 & 12.558221 \\
\hline & 4.549303 & 19.466286 & 14.4 \\
\hline O & 2.109948 & 20.473196 & 14.324198 \\
\hline & 16.025604 & 8.860714 & 12.3 \\
\hline 0 & 15.172111 & 6.312124 & 12.7 \\
\hline & 16.848270 & 7.245968 & 14.4064 \\
\hline O & 14.330024 & 8.261131 & 14.3 \\
\hline & 16.095272 & 21.238089 & 0.2 \\
\hline O & 15.081080 & 18.732708 & 24.6 \\
\hline & 16.863564 & 19.465073 & \\
\hline & 14.418384 & 20.64 & 2.1 \\
\hline & 3.792906 & 22.168049 & 6.4 \\
\hline O & 2.710987 & 24.59 & 6.4 \\
\hline & 4.555371 & 24.024328 & 4.6 \\
\hline O & 2.192724 & 22.83 & 4.5 \\
\hline & 3.801159 & 9.95 & 18.7 \\
\hline 0 & 2.791093 & 12.47 & 18.6 \\
\hline & 4.563868 & 11.72 & 16.8 \\
\hline 0 & 2.10 & 10.68 & 16.8 \\
\hline & 16.102070 & 22.00 & 18.5 \\
\hline 0 & 14.992964 & 24.45 & 18.6 \\
\hline & 16.781759 & 23.932570 & 16.7 \\
\hline ) & 14.406488 & 22.75 & 16.6 \\
\hline & 16.103285 & 9.953799 & 6.3 \\
\hline ) & 15.166284 & 12.48 & 6.4 \\
\hline & 16.916483 & 11.801581 & 4.5 \\
\hline 0 & 14.405033 & 10.786175 & 4.5 \\
\hline & 0.345670 & 2.867073 & 22.1 \\
\hline 0 & 24.609833 & 3.964285 & 24.6 \\
\hline & 2.117473 & 2.114803 & 24.0 \\
\hline 0 & 2.194424 & 4.55 & 22.8 \\
\hline O & 0.421893 & 15.167741 & 9.9 \\
\hline 0 & 24.612986 & 16.167368 & 12.4 \\
\hline 0 & 2.119658 & 14.319344 & 11.801825 \\
\hline 0 & 2.196366 & 16.838320 & 10.7 \\
\hline O & 12.463794 & 2.871200 & 10.0 \\
\hline 0 & 12.546813 & 3.795334 & 12.5 \\
\hline 0 & 14.330267 & 2.028143 & 11.803766 \\
\hline 0 & 14.325412 & 4.473323 & 10.6 \\
\hline 0 & 12.639783 & 15.092489 & 22.153242 \\
\hline 0 & 12.465006 & 16.099642 & 24.604004 \\
\hline O & 14.337549 & 14.326383 & 24.026999 \\
\hline 0 & 14.479798 & 16.776419 & 22.930029 \\
\hline 0 & 6.416262 & 2.870471 & 8.864355 \\
\hline 0 & 6.389075 & 3.955546 & 6.412621 \\
\hline 0 & 36691 & 2.034940 & 7.07628 \\
\hline
\end{tabular}




\begin{tabular}{|c|c|c|c|}
\hline & 4.474294 & 4.480363 & 8.176898 \\
\hline & 6.415534 & 15.099043 & 21.165995 \\
\hline & 6.231047 & 16.188972 & 18.616434 \\
\hline & 4.546632 & 14.328082 & 19.376713 \\
\hline & 4.630622 & 16.857737 & 20.478294 \\
\hline & 18.701880 & 2.948150 & 21.144876 \\
\hline & 18.801163 & 3.977636 & 18.701880 \\
\hline & 16.939785 & 2.193453 & 19.207033 \\
\hline & 16.856525 & 4.621641 & 20.399158 \\
\hline & 18.715958 & 15.166041 & 8.942034 \\
\hline & 18.776888 & 16.033373 & 6.411893 \\
\hline & 16.920853 & 14.329053 & 7.094008 \\
\hline & 16.927893 & 16.856525 & 8.096792 \\
\hline & 0.418980 & 3.884178 & 8.942519 \\
\hline & 24.522926 & 2.629910 & 6.564823 \\
\hline & 2.091985 & 4.543477 & 6.989871 \\
\hline & 2.201706 & 2.189326 & 8.264772 \\
\hline & 0.419222 & 16.085320 & 21.151428 \\
\hline & 24.607647 & 15.079139 & 18.616434 \\
\hline & 2.201949 & 16.777632 & 19.291510 \\
\hline & 2.116017 & 14.325169 & 20.403286 \\
\hline & 12.384658 & 3.799217 & 21.138805 \\
\hline & 12.546083 & 2.871928 & 18.620317 \\
\hline & 14.246278 & 4.721652 & 19.392977 \\
\hline & 14.331965 & 2.268947 & 20.482420 \\
\hline & 12.562834 & 16.098671 & 8.927227 \\
\hline & 12.480057 & 15.239836 & 10679 \\
\hline & 14.416926 & 16.865505 & 7.085027 \\
\hline & 14.413529 & 14.403090 & 8.092909 \\
\hline & 6.410193 & 3.880538 & 22.155184 \\
\hline & 6.411650 & 2.785510 & 24.608131 \\
\hline & 4.632322 & 4.635963 & 24.019472 \\
\hline & 4.533281 & 2.257052 & 22.778070 \\
\hline & 6.321591 & 16.087990 & 9.796015 \\
\hline & 6.503165 & 15.001945 & 12.308193 \\
\hline & 4.709758 & 16.862106 & 11.797212 \\
\hline & 4.542748 & 14.395808 & 10.700244 \\
\hline & 18.621288 & 3.794605 & 9.872479 \\
\hline & 18.893890 & 2.777257 & 12.390969 \\
\hline & 17.012367 & 4.549545 & 11.973931 \\
\hline & 16.847300 & 2.180102 & 10.781077 \\
\hline & 18.635124 & 16.105711 & 22.154213 \\
\hline & 18.713045 & 15.084479 & 24.605219 \\
\hline & 16.928864 & 16.929590 & 24.013405 \\
\hline & 16.774719 & 14.417897 & 22.924932 \\
\hline & 2.870471 & 24.694550 & 22.079933 \\
\hline & 0.430874 & 0.420193 & 20.985634 \\
\hline & 1.009581 & 22.908667 & 22.848225 \\
\hline & 2.200007 & 22.760107 & 20.480722 \\
\hline & 2.953976 & 12.477387 & 9.956226 \\
\hline & 0.518991 & 12.626918 & 8.934023 \\
\hline & 1.094056 & 10.703885 & 10.704127 \\
\hline & 2.184228 & 10.627177 & 8.251422 \\
\hline & 15.161429 & 24.695278 & 9.863012 \\
\hline & 12.564532 & 0.493988 & 8.937907 \\
\hline & 13.325055 & 22.913280 & 10.630818 \\
\hline & 14.329053 & 23.008194 & 8.100919 \\
\hline & 15 & 12. & 22 \\
\hline
\end{tabular}




\begin{tabular}{|c|c|c|c|}
\hline 0 & 12.557250 & 12.566717 & 21.230806 \\
\hline 0 & 13.311219 & 10.698059 & 22.923475 \\
\hline 0 & 14.256472 & 10.714322 & 20.409840 \\
\hline 0 & 16.106197 & 18.703093 & 22.164165 \\
\hline 0 & 18.623714 & 18.616190 & 21.15507 \\
\hline 0 & 18.046465 & 20.386780 & 22.997026 \\
\hline 0 & 16.931532 & 20.563013 & 20.555973 \\
\hline 0 & 16.102070 & 6.413106 & . \\
\hline 0 & 18.631241 & 6.326931 & 1461 \\
\hline 0 & 17.948153 & 8.277881 & 10.62741 \\
\hline 0 & 16.861622 & 8.102860 & 8.16864 \\
\hline 0 & 3.805772 & 18.697023 & 10.032934 \\
\hline 0 & 6.318435 & 18.624685 & 8.941305 \\
\hline 0 & 5.652098 & 20.404985 & 10.78714 \\
\hline 0 & 4.469439 & 20.482906 & 8.34123 \\
\hline 0 & 3.880295 & 6.412135 & 22.165865 \\
\hline O & 6.326204 & 6.324990 & 21.074236 \\
\hline 0 & 5.747740 & 8.248265 & 22.831959 \\
\hline 0 & 4.558041 & 8.256276 & 20.475624 \\
\hline 0 & 16.179993 & 24.852821 & 21.333246 \\
\hline 0 & 18.708433 & 0.419465 & 22.239174 \\
\hline O & 17.878971 & 23.069122 & 20.296234 \\
\hline O & 16.942457 & 22.834631 & 22.766903 \\
\hline 0 & 16.101583 & 12.553123 & \\
\hline 0 & 18.529287 & 12.722802 & 10.039732 \\
\hline 0 & 18.031900 & 10.702186 & 8.412362 \\
\hline 0 & 16.856039 & 10.787632 & 10.790302 \\
\hline O & 3.799703 & 24.607891 & 8.946646 \\
\hline 0 & 6.323047 & 0.428447 & 84 \\
\hline 0 & 5.663749 & 22.831717 & 8.251907 \\
\hline 0 & 4.559012 & 22.910608 & 10.716022 \\
\hline 0 & 3.798004 & 12.474959 & 21.151428 \\
\hline 0 & 6.238572 & 12.647308 & 22.244272 \\
\hline 0 & 5.722737 & 10.707283 & 20.465185 \\
\hline 0 & 4.553915 & 10.696360 & 22 \\
\hline 0 & 2.936498 & 18.806988 & 20.908197 \\
\hline 0 & 0.586232 & 18.460346 & 22.241358 \\
\hline 0 & 0.855922 & 20.390663 & 20.413725 \\
\hline C & 2.195152 & 20.482906 & 22.755251 \\
\hline 0 & 2.796434 & 6.416748 & 38 \\
\hline 0 & 0.417038 & 6.3 & 9 . \\
\hline 0 & 0.942825 & 8.263802 & 8.183696 \\
\hline 0 & 2.191754 & 8.250450 & 10.599504 \\
\hline 0 & 15.161186 & 18.625414 & 8.942277 \\
\hline 0 & 12.571087 & 18.633669 & 9.782663 \\
\hline 0 & 13.390840 & 20.479265 & 433 \\
\hline 0 & 14.323227 & 20.409111 & 10.631789 \\
\hline 0 & 15.171867 & 6.405338 & 21.236391 \\
\hline 0 & 12.641240 & 6.251923 & 22.168293 \\
\hline 0 & 13.306365 & 8.179568 & 20.472225 \\
\hline 0 & 14.327353 & 8.171558 & 22.922747 \\
\hline 0 & 22.170235 & 2.777985 & 24.60303 \\
\hline 0 & 21.074720 & 0.334018 & 0.350040 \\
\hline 0 & 22.923231 & 0.920736 & 22.842884 \\
\hline 0 & 20.559858 & 2.188841 & 22.747000 \\
\hline 0 & 22.167078 & 15.078168 & 12.479571 \\
\hline 0 & 21.239059 & 12.561133 & 12.554823 \\
\hline ? & 22.992657 & 13.247376 & 10.70072 \\
\hline
\end{tabular}




$$
\begin{array}{rrr}
20.484362 & 14.322499 & 10.679609 \\
9.953071 & 3.041121 & 12.394125 \\
8.938149 & 0.593271 & 12.720376 \\
10.620866 & 1.079977 & 10.704370 \\
8.167432 & 2.181315 & 10.698545 \\
9.862770 & 15.092004 & 24.686541 \\
8.869210 & 12.558706 & 0.420922 \\
10.785933 & 13.308791 & 23.001640 \\
8.252150 & 14.310361 & 22.822493 \\
22.162952 & 16.096487 & 18.632938 \\
21.159925 & 18.549192 & 18.548464 \\
22.919834 & 17.949852 & 20.467857 \\
20.479994 & 16.853369 & 20.479994 \\
22.167807 & 3.878595 & 6.401212 \\
21.150217 & 6.402911 & 6.326689 \\
22.915707 & 5.733418 & 8.175927 \\
20.475138 & 4.634021 & 8.195105 \\
9.940449 & 16.093817 & 6.324261 \\
9.020198 & 18.623472 & 6.249253 \\
10.632760 & 17.869261 & 8.188551 \\
8.170102 & 16.865749 & 8.092180 \\
10.022011 & 3.793391 & 18.709646 \\
8.928926 & 6.242941 & 18.524189 \\
10.703156 & 5.740214 & 20.405712 \\
8.262102 & 4.565324 & 20.473682 \\
21.233963 & 16.178535 & 24.772470 \\
22.324619 & 18.627842 & 0.417038 \\
20.477079 & 17.948397 & 22.917891 \\
22.923231 & 16.777632 & 22.917650 \\
21.403156 & 3.800674 & 12.633715 \\
22.245728 & 6.335427 & 12.492437 \\
20.403286 & 5.484603 & 10.790788 \\
22.913036 & 4.555371 & 10.631304 \\
9.017042 & 15.927778 & 12.395582 \\
9.778779 & 18.450396 & 12.642939 \\
8.189279 & 17.786970 & 10.627419 \\
10.783262 & 16.939058 & 10.702913 \\
8.928683 & 3.806015 & 24.607161 \\
9.955013 & 6.326689 & 0.429903 \\
8.9637361240 & 15.407575 & 18.807232 \\
10.709225 & 14.407459 & 20.565199 \\
24.703531 & 22.099594 & 2.884308
\end{array}
$$




\begin{tabular}{|c|c|c|c|}
\hline 0 & 0.497144 & 21.066467 & 0.419222 \\
\hline 0 & 22.997755 & 22.922020 & 1.004483 \\
\hline 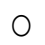 & 22.847980 & 20.490189 & 2.184471 \\
\hline 0 & 24.693577 & 9.878548 & 15.085450 \\
\hline O & 0.507339 & 8.861685 & 12.56089 \\
\hline O & 22.928816 & 10.632275 & 13.230142 \\
\hline 0 & 22.923475 & 8.167432 & 14.321042 \\
\hline 0 & 12.468648 & 22.155426 & 15.07938 \\
\hline 0 & 12.641968 & 21.238333 & 12.54754 \\
\hline 0 & 10.681067 & 22.934399 & 13.22407 \\
\hline 0 & 10.709467 & 20.472225 & 14.16787 \\
\hline 0 & 12.468162 & 9.952585 & 2.9527 \\
\hline 0 & 12.636627 & 8.936451 & 0.503698 \\
\hline 0 & 10.708496 & 10.702671 & $1.0171(\mathrm{C}$ \\
\hline 0 & 10.627419 & 8.264530 & 2.1118 \\
\hline 0 & 18.706007 & 22.168293 & 16.101583 \\
\hline 0 & 18.624929 & 21.153856 & 18.625414 \\
\hline 0 & 20.478294 & 22.911095 & 18.0238 \\
\hline 0 & 20.486305 & 20.484606 & 16.860651 \\
\hline 0 & 18.711588 & 9.946517 & 3.71328 \\
\hline 0 & 18.786598 & 8.934752 & 6.24998 \\
\hline O & 20.487034 & 10.792973 & 5.479020 \\
\hline O & 20.559858 & 8.280551 & 4.464827 \\
\hline 0 & 6.327417 & 22.169020 & \\
\hline 0 & 6.238572 & 20.986605 & 6.410193 \\
\hline 0 & 8.183938 & 23.000669 & 5.820563 \\
\hline 0 & 8.342452 & 20.494072 & 4.478663 \\
\hline O & 6.335427 & 9.874179 & 16.100857 \\
\hline 0 & 6.32 & 8.940578 & 48 \\
\hline 0 & 8.176 & 10.706312 & 17.866833 \\
\hline 0 & 8.187094 & 8.179326 & 16.86866 \\
\hline 0 & 24.854763 & 21.221098 & 16.175863 \\
\hline 0 & 0.503698 & 22.241116 & 18.55307 \\
\hline 0 & 23.006979 & 20.318567 & 17. \\
\hline 0 & 22.9 & 22.835842 & 16. \\
\hline 0 & 24.608374 & 8.853675 & 3.790964 \\
\hline 0 & 0.507096 & 10.034391 & 6.234931 \\
\hline 0 & 22.914494 & 8.263559 & 5.753080 \\
\hline 0 & 22.927359 & 10.623051 & 4.385692 \\
\hline 0 & $12.47^{\prime}$ & 21.408741 & 88 \\
\hline 0 & 12.6 & 22.2 & \\
\hline 0 & 10.864340 & 20.379740 & 5.486059 \\
\hline 0 & 10.687620 & 23.002125 & 4.633050 \\
\hline 0 & 12.472047 & 8.862413 & 16.090418 \\
\hline 0 & 12.628132 & 9.863256 & 18.541424 \\
\hline 0 & 10.699515 & 8.112329 & 363 \\
\hline 0 & 10.629848 & 10.542944 & 16.85652 \\
\hline 0 & 18.704792 & 21.217215 & 2.95737 \\
\hline 0 & 18.608665 & 22.253983 & 0.491075 \\
\hline 0 & 20.403528 & 20.385323 & 1.015164 \\
\hline 0 & 20.573936 & 22.766661 & 2.190540 \\
\hline 0 & 18.731737 & 8.950288 & 15.16118 \\
\hline 0 & 18.528559 & 9.862285 & 12.639783 \\
\hline 0 & 20.473196 & 8.173257 & 13.231599 \\
\hline 0 & 20.479507 & 10.642713 & 14.252831 \\
\hline 0 & 6.497824 & 21.146088 & 15.16871 \\
\hline 0 & 6.24 & 22.172905 & 12.650950 \\
\hline 0 & 8.170344 & 20.478294 & 13.22383 \\
\hline
\end{tabular}




$\begin{array}{rrrr}\mathrm{O} & 8.183453 & 22.922991 & 14.325655 \\ \mathrm{O} & 6.405581 & 8.934752 & 2.868772 \\ \mathrm{O} & 6.327175 & 10.025409 & 0.425291 \\ \mathrm{O} & 8.172771 & 8.180782 & 1.011280 \\ \mathrm{O} & 8.262102 & 10.628148 & 2.114803 \\ \mathrm{O} & 13.317287 & 5.216611 & 8.264530 \\ \mathrm{O} & 12.388784 & 6.659251 & 5.990000 \\ \mathrm{O} & 11.887271 & 7.750637 & 8.510917 \\ \mathrm{O} & 14.490237 & 7.754035 & 7.499152 \\ \mathrm{O} & 9.269741 & 17.800562 & 19.716801 \\ \mathrm{O} & 9.033307 & 19.880653 & 17.534513 \\ \mathrm{O} & 6.917532 & 19.048521 & 19.050705 \\ \mathrm{O} & 8.770656 & 20.639721 & 20.316870 \\ \mathrm{H} & 12.710666 & 22.165380 & 3.134822 \\ \mathrm{H} & 15.872432 & 7.932939 & 7.900168 \\ \mathrm{H} & 25.023954 & 6.154097 & 1.694367 \\ \mathrm{H} & 21.903698 & 16.005943 & 6.547588 \\ \mathrm{H} & 13.674610 & 4.986003 & 9.180168 \\ \mathrm{H} & 9.173857 & 16.290442 & 19.239805 \\ \mathrm{H} & 9.161476 & 19.372343 & 16.059832 \\ \mathrm{H} & 11.888243 & 18.346256 & 19.802975 \\ \mathrm{H} & 11.255889 & 21.314312 & 19.360935 \\ \mathrm{H} & 12.984241 & 20.579762 & 17.572140 \\ \mathrm{H} & 11.348132 & 20.511551 & 16.941971 \\ \mathrm{H} & 12.160846 & 19.004826 & 17.467274 \\ \mathrm{H} & 10.732043 & 18.867918 & 22.003468 \\ \mathrm{H} & 11.034989 & 20.617388 & 21.756109 \\ \mathrm{H} & 12.381502 & 19.481581 & 22.037695 \\ \mathrm{Re} & 13.003174 & 6.871896 & 7.564936 \\ \mathrm{Re} & 8.733758 & 19.377199 & 19.183245 \\ \mathrm{C} & 11.580683 & 19.357292 & 20.079464 \\ \mathrm{C} & 11.517569 & 20.289438 & 19.087360 \\ \mathrm{C} & 12.013499 & 20.068054 & 17.693512 \\ \mathrm{C} & 11.402750 & 19.607565 & 21.541521\end{array}$

\begin{tabular}{lrrr}
605 & & & \\
USY_ReO3_C $4 H 8 M 1$ & \multicolumn{3}{l}{$=-7232.53622802440$} \\
Si & 23.382994 & 3.326349 & 1.243588 \\
Si & 23.428387 & 1.237034 & 3.375869 \\
Si & 23.398529 & 15.571671 & 13.401763 \\
Si & 23.392218 & 13.427494 & 15.574097 \\
Si & 11.247636 & 1.239704 & 15.586963 \\
Si & 11.163646 & 15.565358 & 1.200379 \\
Si & 11.227973 & 13.403705 & 3.365916 \\
Si & 7.632905 & 3.351837 & 1.217857 \\
Si & 7.657908 & 5.500138 & 3.346496 \\
Si & 7.783893 & 15.497632 & 13.281847 \\
Si & 20.003241 & 5.426829 & 15.513411 \\
Si & 19.908569 & 15.644736 & 1.268105 \\
Si & 19.883326 & 17.805176 & 3.350137 \\
Si & 7.626108 & 3.370043 & 5.510577 \\
Si & 7.537748 & 15.543268 & 17.693270 \\
Si & 7.766415 & 13.319230 & 15.462191 \\
Si & 19.917067 & 3.340428 & 17.744732 \\
Si & 19.866089 & 1.201350 & 15.622161 \\
Si & 19.934544 & 15.589875 & 5.434597 \\
Si & 20.010523 & 13.381130 & 3.279984
\end{tabular}




$\begin{array}{lrrr} & & & \\ \mathrm{Si} & 23.434214 & 3.350380 & 5.536308 \\ \mathrm{Si} & 23.371584 & 17.739635 & 15.564387 \\ \mathrm{Si} & 11.236712 & 3.364702 & 17.781145 \\ \mathrm{Si} & 11.267783 & 5.535337 & 15.575554 \\ \mathrm{Si} & 11.251762 & 15.618762 & 5.463241 \\ \mathrm{Si} & 11.182094 & 17.735750 & 3.277071 \\ \mathrm{Si} & 1.308886 & 23.402899 & 3.334116 \\ \mathrm{Si} & 3.379995 & 23.427902 & 1.202564 \\ \mathrm{Si} & 1.256453 & 11.233314 & 15.578953 \\ \mathrm{Si} & 3.411067 & 11.214865 & 13.384771 \\ \mathrm{Si} & 13.456139 & 23.446835 & 15.526519 \\ \mathrm{Si} & 15.607353 & 23.402899 & 13.413415 \\ \mathrm{Si} & 13.425796 & 11.232828 & 3.332660 \\ \mathrm{Si} & 15.609781 & 11.173113 & 1.199165 \\ \mathrm{Si} & 1.360349 & 7.681211 & 3.341884 \\ \mathrm{Si} & 3.381209 & 7.602562 & 5.525627 \\ \mathrm{Si} & 1.308158 & 19.897404 & 15.593274 \\ \mathrm{Si} & 3.424903 & 19.892305 & 17.761238 \\ \mathrm{Si} & 13.431621 & 7.642858 & 15.603712 \\ \mathrm{Si} & 15.584535 & 7.632662 & 17.791340 \\ \mathrm{Si} & 15.552979 & 19.950808 & 5.497468 \\ \mathrm{Si} & 5.526598 & 7.662277 & 3.378539 \\ \mathrm{Si} & 3.406212 & 7.665676 & 1.181445 \\ \mathrm{Si} & 5.527083 & 19.976782 & 15.697412 \\ \mathrm{Si} & 3.443109 & 19.887693 & 13.452740 \\ \mathrm{Si} & 17.878242 & 7.682668 & 15.703966 \\ \mathrm{Si} & 15.625073 & 7.743112 & 13.483084 \\ \mathrm{Si} & 17.723614 & 19.965614 & 3.361546 \\ \mathrm{Si} & 15.612207 & 19.990131 & 1.229023 \\ \mathrm{Si} & 5.500624 & 23.364544 & 3.377325 \\ \mathrm{Si} & 3.409610 & 23.400957 & 5.511062 \\ \mathrm{Si} & 5.515675 & 11.186220 & 15.601527 \\ \mathrm{Si} & 3.364217 & 11.237683 & 17.751772 \\ \mathrm{Si} & 17.723127 & 23.383965 & 15.613421 \\ \mathrm{Si} & 15.541570 & 23.390518 & 17.742304 \\ \mathrm{Si} & 17.821924 & 11.269483 & 3.303287 \\ \mathrm{Si} & 15.662943 & 11.239383 & 5.470767 \\ \mathrm{Si} & 3.358391 & 1.276844 & 23.367456 \\ \mathrm{Si} & 1.242859 & 3.365188 & 23.414793 \\ \mathrm{Si} & 3.396502 & 13.389140 & 11.223846 \\ \mathrm{Si} & 1.288981 & 15.563173 & 11.258316 \\ \mathrm{Si} & 15.613665 & 1.223197 & 11.204912 \\ \mathrm{Si} & 13.361467 & 3.334845 & 11.248849 \\ \mathrm{Si} & 15.562446 & 13.423125 & 23.391973 \\ \mathrm{Si} & 13.446185 & 15.565844 & 23.414064 \\ \mathrm{Si} & 3.359847 & 1.200864 & 7.658879 \\ \mathrm{Si} & 5.517616 & 3.318338 & 7.619796 \\ \mathrm{Si} & 3.300132 & 13.372392 & 19.962702 \\ \mathrm{Si} & 17.774590 & 3.372956 & 19.861477 \\ \mathrm{Si} & 15.643765 & 13.427494 & 7.645285 \\ \mathrm{Si} & 17.822653 & 15.573612 & 7.717866 \\ \mathrm{Si} & 3.339457 & 5.433141 & 7.655723 \\ \mathrm{Si} & 1.241889 & 3.327319 & 7.663491 \\ \mathrm{Si} & 3.429515 & 17.737450 & 19.856136 \\ \mathrm{Si} & 1.262279 & 15.581865 & 19.872158 \\ \mathrm{Si} & 15.565116 & 5.466640 & 19.944983 \\ \mathrm{Si} & 13.393510 & 3.388491 & 19.952991 \\ \mathrm{Si} & 15.660515 & 17.776775 & 7.633390\end{array}$




\begin{tabular}{|c|c|c|c|}
\hline i & 13.466091 & 15.636725 & 7.627565 \\
\hline Si & 3.377568 & 5.510819 & 23.381779 \\
\hline Si & 5.503294 & 3.386307 & 23.346338 \\
\hline & 5.584857 & 15.552979 & 11.140585 \\
\hline & 17.857122 & 3.319551 & 11.254432 \\
\hline$\perp$ & 15.614879 & 17.752985 & 23.436882 \\
\hline & 17.731138 & 15.612451 & 23.426931 \\
\hline & 1.644847 & 21.674305 & 23.725994 \\
\hline & 1.613775 & 23.782797 & 21.613375 \\
\hline & 1.678346 & 9.445974 & 11.58335 \\
\hline & 1.674947 & 11.595976 & 9.45689 \\
\hline & 13.873661 & 21.700277 & 11.55252 \\
\hline & 13.832395 & 23.855135 & 9.38043 \\
\hline 2 & 13.832881 & 9.424128 & 23.7878 \\
\hline & 13.802051 & 11.637243 & 21.65415 \\
\hline & 17.480381 & 21.677217 & 23.77745 \\
\hline L & 17.394449 & 19.536926 & 21.68765 \\
\hline i & 17.427464 & 9.516614 & 11. \\
\hline 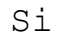 & 17.450768 & 7.327530 & 9.48772 \\
\hline $\mathrm{Si}$ & 5.150827 & 21.645 & 11.64549 \\
\hline S & 5.122911 & 19.542267 & 9.49500 \\
\hline$\perp$ & 5.182627 & 9.435294 & 23.73181 \\
\hline$i$ & 5.152284 & 7.305684 & 21.61410 \\
\hline i & 17.334005 & 21.6 & \\
\hline$i$ & 17.412 & $23 . \varepsilon$ & 21.6 \\
\hline Si & 17.389595 & 11.698415 & 9.49209 \\
\hline $\mathrm{Si}$ & 5.100336 & 21.600752 & 7.31854 \\
\hline$i$ & 5.119027 & 23.744200 & 9.43602 \\
\hline i & 5.076790 & 9.457870 & 19.527 \\
\hline$i$ & 5.025085 & 11.630203 & 21.702948 \\
\hline i & 1.577363 & 21.62 & 19.546392 \\
\hline Si & 1.599939 & 19.533770 & 21.613132 \\
\hline $\mathrm{Si}$ & 1.631253 & 9.422914 & 7.30641 \\
\hline $\mathrm{Si}$ & 1.623485 & 7.276311 & 9.43068 \\
\hline i & 13.875118 & 21.74 & \\
\hline $\mathrm{Si}$ & 13.889 & 19.5 & 9.7 \\
\hline$i$ & 13.810547 & 7.235530 & 21.66119 \\
\hline $\mathrm{Si}$ & 23.772358 & 1.592656 & 21.62696 \\
\hline $\mathrm{Si}$ & 21.672848 & 1.535368 & 23.71240 \\
\hline $\mathrm{Si}$ & 23.862659 & 13.851815 & 9.42509 \\
\hline $\mathrm{Si}$ & 21.733534 & 13.774 & 11.58990 \\
\hline $\mathrm{Si}$ & 11.536 & 1.6 & 9.473 \\
\hline $\mathrm{Si}$ & 9.414903 & 1.703834 & 11.62801 \\
\hline $\mathrm{Si}$ & 11.662731 & 13.824142 & 21.67794 \\
\hline $\mathrm{Si}$ & 9.466608 & 13.762726 & 23.75706 \\
\hline $\mathrm{Si}$ & 23.815809 & 17.347841 & 21.70173 \\
\hline Si & 21.6915 & 17.390324 & 19.55998 \\
\hline $\mathrm{Si}$ & 21.6791 & 5.1 & 7.25179 \\
\hline $\mathrm{Si}$ & 11.674626 & 17.376488 & 9.38698 \\
\hline Si & 9.464423 & 17.367018 & 7.22193 \\
\hline $\mathrm{Si}$ & 11.577042 & 5.157381 & 21.638863 \\
\hline $\mathrm{Si}$ & 9.474619 & 5.111988 & 19.50998 \\
\hline $\mathrm{Si}$ & 19.533770 & 17.385712 & 21.69712 \\
\hline Si & 21.722126 & 17.393963 & 23.82260 \\
\hline Si & 19.581104 & 5.151070 & 9.49258 \\
\hline $\mathrm{Si}$ & 21.729893 & 5.068051 & 11.657147 \\
\hline $\mathrm{Si}$ & 7.283593 & 17.354397 & 9.35858 \\
\hline $\mathrm{Si}$ & 9.490154 & 17.291283 & 11.56806 \\
\hline
\end{tabular}




\begin{tabular}{|c|c|c|c|}
\hline$i$ & 7.293546 & 5.155439 & 21.648573 \\
\hline $\mathrm{S}$ & 9.407864 & 5.116600 & 23.777697 \\
\hline$i$ & 19.552948 & 1.638293 & 21.598082 \\
\hline & 21.680130 & 1.608435 & 19.483278 \\
\hline & 19.554890 & 13.829482 & 9.431168 \\
\hline & 7.307868 & 1.607949 & 9.435537 \\
\hline & 9.425342 & 1.659412 & 7.294759 \\
\hline & 7.246939 & 13.694273 & 21.620901 \\
\hline & 21.703434 & 23.744684 & 1.58707 \\
\hline & 23.837414 & 21.646145 & 1.61741 \\
\hline & 21.715084 & 11.596461 & 13.783360 \\
\hline & 23.851736 & 9.411019 & 13.7 \\
\hline & 9.485299 & 23.799059 & $13.82122 \varepsilon$ \\
\hline & 9.462725 & 11.604958 & 1.65795 \\
\hline & 11.616366 & 9.464423 & 1.64581 \\
\hline & 21.680616 & 23.758520 & 17.38692 \\
\hline$S \perp$ & 19.529644 & 21.6 & \\
\hline i & 21.815 & 11.644 & 4. \\
\hline Si & 19.640821 & 9.536277 & 5.00 \\
\hline $\mathrm{Si}$ & 9.359315 & 23.822849 & 5.16175 \\
\hline & 9.466608 & 11.540873 & 17.29541 \\
\hline $\mathrm{Si}$ & 7.260047 & 9.422186 & 17.369 \\
\hline $\mathrm{Si}$ & 21.6956 & 19.52 & 17. \\
\hline $\mathrm{Si}$ & 23.8 & 21.68 & 17.3 \\
\hline a & 21.7044 & 7.373652 & 5.12169 \\
\hline S & 23.814110 & 9.446703 & 5.13116 \\
\hline $\mathrm{Si}$ & 9.482872 & 19.509737 & 5.0289 \\
\hline $\mathrm{Si}$ & 11.570245 & 21. & \\
\hline $\mathrm{Si}$ & 9.434 & 7.26 & 17. \\
\hline $\mathrm{Si}$ & 11.5765 & 9.3658 & 17.36216 \\
\hline S & 21.697609 & 19.507311 & 1.63465 \\
\hline S & 19.558529 & 21.628426 & 1.65261 \\
\hline $\mathrm{Si}$ & 21.735962 & 7.231403 & 13.784 \\
\hline $\mathrm{Si}$ & 19.5806 & 0 & 13. \\
\hline $\mathrm{Si}$ & 9.4 & 19.5 & 13.7 \\
\hline S & 7.330686 & 21.628910 & 13.79889 \\
\hline S & 9.428254 & 7.297673 & 1.61280 \\
\hline $\mathrm{Si}$ & 7.312723 & 9.422428 & 1.66281 \\
\hline $\mathrm{Si}$ & 11.653992 & 21.72 & 13.691 \\
\hline $\mathrm{Si}$ & 11.2 & 3. & 13. \\
\hline $\mathrm{Si}$ & 20.023 & 3.292364 & 13.41802 \\
\hline $\mathrm{Si}$ & 7.629263 & 1.210089 & 3.36591 \\
\hline S & 23.374741 & 15.606382 & 17.72531 \\
\hline i & 5.457173 & 15. & 19. \\
\hline 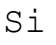 & 3.483 & 17.704 & 11. \\
\hline $\mathrm{S}$ & 13.79 & 9.4 & 19.50 \\
\hline S & 23.792992 & 5.133593 & 9.42218 \\
\hline & 15.580651 & 1.281699 & 19.89691 \\
\hline A & 15.575554 & 5.470524 & 11.20782 \\
\hline 1 & 17.525047 & 9.603274 & 7.20591 \\
\hline & 9.45 & 13.5187 & 19.6420 \\
\hline & 7.254950 & 21.638620 & 5.17825 \\
\hline A & 7.726362 & 17.777988 & $15.45296^{\circ}$ \\
\hline A & 23.372313 & 5.398428 & 3.40839 \\
\hline Al & 13.427980 & 19.835018 & 3.29139 \\
\hline A & 21.793978 & 13.678251 & 7.23650 \\
\hline A] & 16.714762 & 5.188453 & 3.78214 \\
\hline & & 8919 & 18.07 \\
\hline
\end{tabular}




\begin{tabular}{|c|c|c|c|}
\hline 0 & 22.163923 & 0.349069 & 2.866102 \\
\hline 0 & 24.605705 & 24.541864 & 3.955788 \\
\hline 0 & 24.029911 & 2.099510 & 2.117473 \\
\hline 0 & 22.847010 & 2.185442 & 4.557070 \\
\hline $\mathrm{O}$ & 22.163195 & 12.473018 & 15.085692 \\
\hline 0 & 24.610802 & 12.475930 & 16.096729 \\
\hline 0 & 23.941551 & 14.330509 & 14.329296 \\
\hline 0 & 22.846039 & 14.397750 & 16.771807 \\
\hline 0 & 10.024196 & 0.249300 & 15.17623 \\
\hline 0 & 12.558464 & 24.691879 & 16.09284 \\
\hline O & 11.644768 & 2.102180 & 14.25088 \\
\hline 0 & 10.707040 & 2.192239 & 1677032 \\
\hline 0 & 9.943361 & 12.478843 & 2.945480 \\
\hline 0 & 12.481998 & 12.470346 & 3.79776 \\
\hline 0 & 11.719049 & 14.322256 & 2.103394 \\
\hline 0 & 10.774282 & 14.311818 & 4.626010 \\
\hline O & 8.928440 & 6.407765 & 2.879453 \\
\hline O & 6.415291 & 6.409223 & 3.883693 \\
\hline O & 7.077987 & 4.629166 & 2.089800 \\
\hline 0 & 8.181511 & 4.555614 & 4.550274 \\
\hline 0 & 9.006847 & 18.864033 & 15.171381 \\
\hline O & 6.322805 & 18.627356 & 16.349428 \\
\hline O & 7.247910 & 16.687817 & 14. \\
\hline O & 8.005520 & 17.018436 & 9222 \\
\hline 0 & 21.308001 & 6.237601 & 15.008499 \\
\hline 0 & 18.796551 & 6.402425 & 16.025120 \\
\hline 0 & 19.298548 & 4.565567 & 14.247733 \\
\hline O & 20.481207 & 4.467254 & 16.692429 \\
\hline 0 & 21.242216 & 18.547979 & 558 \\
\hline O & 18.719601 & 18.785383 & 3.884907 \\
\hline 0 & 19.283983 & 16.851427 & 2.176703 \\
\hline 0 & 20.398916 & 16.855310 & 4.563139 \\
\hline 0 & 8.930868 & 0.342515 & 3.795334 \\
\hline 0 & 6.40 & 24.6 & 97 \\
\hline O & 7.082600 & 2.1 & 5963 \\
\hline 0 & 8.175927 & 2.190297 & 2.188112 \\
\hline 0 & 9.019470 & 12.467191 & 16.010069 \\
\hline 0 & 6.493698 & 12.393639 & 15.089577 \\
\hline 0 & 7.257134 & 14.338520 & 5536 \\
\hline 0 & 8.25 & 14.2 & 14. \\
\hline $\mathrm{O}$ & 21.147303 & 0.333048 & 16.102554 \\
\hline 0 & 18.632938 & 24.611288 & 15.009470 \\
\hline 0 & 19.297577 & 2.109220 & 16.852640 \\
\hline 0 & 20.475624 & 2.119173 & 14.410858 \\
\hline $\mathrm{O}$ & 21.318924 & 12.466706 & 3.547490 \\
\hline O & 18.707462 & 12.550453 & 2.783326 \\
\hline 0 & 19.559015 & 14.239480 & 4.635235 \\
\hline 0 & 20.480722 & 14.411101 & 2.127426 \\
\hline 0 & 22.257866 & 6.572834 & 3.874226 \\
\hline 0 & 24.775385 & 6.401454 & 2.621171 \\
\hline 0 & 24.110989 & 4.552458 & 4.717040 \\
\hline 0 & 22.759865 & 4.485460 & 2.116017 \\
\hline 0 & 22.085758 & 18.627356 & 16.027788 \\
\hline 0 & 24.600121 & 18.709646 & 15.090062 \\
\hline 0 & 23.934027 & 16.861380 & 16.836863 \\
\hline 0 & 22.862303 & 16.790255 & 14.344104 \\
\hline O & 10.025652 & 6.495640 & 16.00618 \\
\hline 0 & 12.557007 & 6.406309 & 15.01384 \\
\hline
\end{tabular}




\begin{tabular}{|c|c|c|c|}
\hline 0 & 11.726088 & 4.641546 & 16.856039 \\
\hline 0 & 10.771853 & 4.635720 & 14.326383 \\
\hline 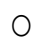 & 9.868596 & 18.529287 & 3.796062 \\
\hline 0 & 12.304794 & 18.706491 & 2.69763 \\
\hline O & 11.801825 & 16.781515 & 4.47113 \\
\hline 0 & 10.611156 & 16.770836 & 2.098782 \\
\hline 0 & 2.950092 & 22.170477 & 0.2568 \\
\hline 0 & 3.969868 & 24.613958 & 24. \\
\hline 0 & 2.098782 & 24.022387 & 3105 \\
\hline 0 & 4.489344 & 22.856234 & 2.1953 \\
\hline 0 & 2.951791 & 9.944818 & 12.4754 \\
\hline 0 & 3.891218 & 12.467920 & $12.4744^{\circ}$ \\
\hline 0 & 2.184957 & 11.732399 & 14.33439 \\
\hline 0 & 4.633778 & 10.704613 & 14.3302 \\
\hline 0 & 15.154389 & 22.159069 & 12.46403 \\
\hline 0 & 16.174408 & 24.615658 & 12.45675 \\
\hline 0 & 14.333908 & 24.014862 & 14.247733 \\
\hline 0 & 16.771807 & 22.846039 & 14.4013 \\
\hline 0 & 15.166041 & 9.859371 & 0.34081 \\
\hline 0 & 16.102798 & 12.397281 & 24.52438 \\
\hline 0 & 14.316431 & 11.714922 & 2.037610 \\
\hline 0 & 16.860165 & 10.709952 & 2.111647 \\
\hline O & 2.868287 & 8.859015 & 6.407765 \\
\hline 0 & 3.895345 & 6.406552 & \\
\hline 0 & 2.193696 & 6.995939 & 4.550759 \\
\hline 0 & 4.555371 & 8.176898 & 4.56605 \\
\hline 0 & 2.865859 & 21.061127 & 18.711832 \\
\hline O & 4.126925 & 18.630512 & 18.624201 \\
\hline 0 & 2.281327 & 19.285440 & 16. \\
\hline 0 & 4.650770 & 20.566654 & 16.929590 \\
\hline 0 & 15.089334 & 8.939849 & 18.61667 \\
\hline 0 & 16.096001 & 6.420389 & 18.711348 \\
\hline 0 & 14.414257 & 7.080657 & 16.775932 \\
\hline 0 & 16.860893 & 8.102618 & 16.857 \\
\hline 0 & 15.174052 & 21.322809 & 90 \\
\hline 0 & 16.177807 & 18.798250 & 6.484716 \\
\hline 0 & 14.256958 & 19.386665 & 4.732819 \\
\hline 0 & 16.772533 & 20.476595 & 4.553430 \\
\hline 0 & 3.862574 & 8.842751 & 0.184487 \\
\hline 0 & 2.779684 & 6.406795 & 24. \\
\hline 0 & 4.638633 & 7.151 & 209 \\
\hline 0 & 2.207775 & 8.266715 & 2.103879 \\
\hline 0 & 3.883450 & 21.157984 & 12.558464 \\
\hline 0 & 3.024130 & 18.615948 & 12.55943 \\
\hline 0 & 4.635478 & 19.383511 & 14.486 \\
\hline 0 & 2.187870 & 20.399887 & 34150 \\
\hline 0 & 16.172224 & 8.851005 & 12.474474 \\
\hline 0 & 15.340818 & 6.241242 & 12.821842 \\
\hline 0 & 16.934931 & 7.071191 & 14.39605 \\
\hline 0 & 14.416442 & 8.254334 & 14.406246 \\
\hline 0 & 16.175137 & 21.229107 & 0.343971 \\
\hline 0 & 15.080594 & 18.721298 & 24.61031 \\
\hline 0 & 16.863077 & 19.380354 & 2.10994 \\
\hline 0 & 14.416926 & 20.569569 & 2.104122 \\
\hline 0 & 3.800674 & 22.168777 & 6.49175 \\
\hline 0 & 2.778956 & 24.612501 & 6.4145 \\
\hline 0 & 4.638633 & 24.018745 & 4.635235 \\
\hline 0 & 2.279870 & 22.841913 & 4.48764 \\
\hline
\end{tabular}




\begin{tabular}{|c|c|c|c|}
\hline & 3.806015 & 9.944818 & 18.629541 \\
\hline 0 & 2.861732 & 12.467920 & 18.697510 \\
\hline 0 & 4.625282 & 11.793571 & 16.847786 \\
\hline 0 & 2.122328 & 10.713594 & 16.850698 \\
\hline 0 & 16.023420 & 22.084059 & 18.623472 \\
\hline 0 & 14.924509 & 24.536036 & 18.635366 \\
\hline 0 & 16.846571 & 23.933784 & 16.861135 \\
\hline 0 & 14.408916 & 22.834871 & 16.68004 \\
\hline O & 16.094301 & 9.961082 & 6.32547 \\
\hline 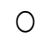 & 15.170167 & 12.487339 & 6.41043 \\
\hline O & 16.921581 & $11.80206^{\circ}$ & \\
\hline 0 & 14.401391 & 10.787632 & 4.541049 \\
\hline 0 & 0.343971 & 2.868287 & 22.16270 \\
\hline $\mathrm{O}$ & 24.608860 & 3.964285 & 24.614927 \\
\hline 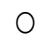 & 2.110676 & 2.115046 & 24.016075 \\
\hline 0 & 2.193210 & 4. & 22.837299 \\
\hline 0 & 0.428447 & 7545 & 9.949187 \\
\hline O & 24.621239 & 16.100613 & 12.476658 \\
\hline 0 & 2.122328 & 14.253317 & 11.79551 \\
\hline O & 2.265548 & 16.768894 & 10.79127 \\
\hline 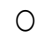 & 12.384415 & 2.939654 & 10.02735 \\
\hline 0 & 12.552880 & 3. & 12 \\
\hline 0 & 14.335608 & 890 & 11.726330 \\
\hline 0 & 14.249433 & 4.557313 & 10.62741 \\
\hline 0 & 12.634929 & 15.077925 & 22.081875 \\
\hline 0 & 12.389755 & 16.094059 & 24.52875 \\
\hline O & 14. & 14. & \\
\hline 0 & 14.410858 & 16.779818 & 22.924688 \\
\hline 0 & 6.483260 & 2.877754 & 8.852946 \\
\hline 0 & 6.396842 & 3.954575 & 6.405338 \\
\hline 0 & 4.646401 & 2.041251 & 7.08308 \\
\hline 0 & 4.5393 & 4.482548 & 8.1 \\
\hline 0 & 6.5 & 15. & 21. \\
\hline 0 & 6.161621 & 16.0 & 18.463745 \\
\hline 0 & 4.548817 & 14.319828 & 19.457548 \\
\hline 0 & 4.643974 & 16.851669 & 20.480236 \\
\hline 0 & 18.704306 & 2.945480 & 21.146818 \\
\hline 0 & 18.7 & 3. & 18.70 \\
\hline 0 & 16.9 & 68 & 19.215288 \\
\hline 0 & 16.857737 & 4.622855 & 20.399403 \\
\hline 0 & 18.706491 & 15.176965 & 9.018985 \\
\hline 0 & 18.802620 & 16.017109 & 6.483988 \\
\hline 0 & 16.933960 & 14.326140 & 7.16294 \\
\hline 0 & 16.9 & 16.8 & 8.102133 \\
\hline 0 & 0.417280 & 3.879567 & 8.950288 \\
\hline 0 & 24.523170 & 2.692296 & 6.564337 \\
\hline 0 & 2.113104 & 4.557070 & 7.007834 \\
\hline 0 & 2.208018 & 2.179374 & 8.256763 \\
\hline 0 & 0.423349 & 16.099886 & 21.152159 \\
\hline 0 & 24.620514 & 15.081324 & 18.63269 \\
\hline 0 & 2.263606 & 16.764767 & 19.28859 \\
\hline 0 & 2.104851 & 14.331238 & 20.470526 \\
\hline 0 & 12.393882 & 3.869614 & 21.151188 \\
\hline 0 & 12.470105 & 2.867558 & 18.693140 \\
\hline 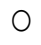 & 14.243607 & 4.699563 & 19.379141 \\
\hline $\mathrm{O}$ & 14.327840 & 2.278171 & 20.554031 \\
\hline 0 & 12.570359 & 16.093575 & 8.92698 \\
\hline & 12 & 15.16 & \\
\hline
\end{tabular}




\begin{tabular}{|c|c|c|c|}
\hline & 14.414742 & 16.861135 & 7.083328 \\
\hline & 14.414257 & 14.401149 & 8.097035 \\
\hline & 6.404367 & 3.871070 & 22.085758 \\
\hline & 6.422574 & 2.790123 & 24.542105 \\
\hline & 4.637905 & 4.643488 & 23.944950 \\
\hline & 4.484732 & 2.272102 & 22.761562 \\
\hline & 6.332029 & 16.089931 & 9.795044 \\
\hline & 6.583272 & 14.999518 & 12.303580 \\
\hline & 4.724080 & 16.775448 & 11.805951 \\
\hline & 4.621641 & 14.322256 & 10.703156 \\
\hline & 18.541910 & 3.958702 & 9.944818 \\
\hline & 18.873743 & 2.776529 & 12.385629 \\
\hline & 16.944885 & 4.540564 & 12.044571 \\
\hline & 16.844387 & 2.194909 & 10.716750 \\
\hline & 18.631241 & 16.107168 & 22.15979 \\
\hline & 18.713045 & 15.083508 & 24.605219 \\
\hline & 16.856039 & 16.856281 & 24.020687 \\
\hline & 16.772533 & 14.405761 & 22.919106 \\
\hline & 2.866587 & 24.693335 & 22.079933 \\
\hline & 0.428932 & 0.421407 & 20.985876 \\
\hline & 1.008124 & 22.911339 & 22.852350 \\
\hline & 2.199521 & 22.772243 & 20.483635 \\
\hline & 2.957617 & 12.473261 & 9.950400 \\
\hline & 0.504426 & 12.578369 & 8.934023 \\
\hline & 1.094056 & 10.699272 & 10.708496 \\
\hline & 2.191025 & 10.617467 & 8.260404 \\
\hline & 15.163371 & 24.693821 & 9.867867 \\
\hline & 12.566717 & 0.509767 & 8.941305 \\
\hline & 13.319230 & 22.929300 & 10.623536 \\
\hline & 14.327840 & 23.010620 & 8.097278 \\
\hline & 15.080594 & 12.562834 & 22.081875 \\
\hline & 12.559678 & 12.561377 & 21.153856 \\
\hline & 13.313404 & 10.709710 & 22.911579 \\
\hline & 14.254288 & 10.701457 & 20.395519 \\
\hline & 16.111052 & 18.630754 & 22.155184 \\
\hline & 18.622259 & 18.608665 & 21.148273 \\
\hline & 18.035055 & 20.392605 & 22.922991 \\
\hline & 16.853853 & 20.475382 & 20.482420 \\
\hline & 16.186789 & 6.496853 & 10.021282 \\
\hline & 18.701151 & 6.422816 & 9.007575 \\
\hline & 18.031416 & 8.346335 & 10.621351 \\
\hline & 16.861866 & 8.169859 & 8.180054 \\
\hline & 3.880780 & 18.626627 & 10.028322 \\
\hline & 6.340526 & 18.616434 & 8.937907 \\
\hline & 5.730505 & 20.392847 & 10.777922 \\
\hline & 4.475022 & 20.480236 & 8.352647 \\
\hline & 3.869128 & 6.483502 & 22.175573 \\
\hline & 6.322562 & 6.325717 & 21.070351 \\
\hline & 5.814495 & 8.272783 & 22.761562 \\
\hline & 4.552944 & 8.190249 & 20.393333 \\
\hline & 16.181934 & 24.771502 & 21.315041 \\
\hline & 18.706247 & 0.416795 & 22.234320 \\
\hline & 17.949368 & 22.928816 & 20.390663 \\
\hline & 16.937601 & 22.845068 & 22.839241 \\
\hline & 16.093817 & 12.546083 & 8.946646 \\
\hline & 18.530500 & 12.718919 & 10.039974 \\
\hline & 18.034328 & 10.707283 & 8.421829 \\
\hline & 16.856525 & 10.791030 & 10.795400 \\
\hline
\end{tabular}




$$
\begin{aligned}
& 3.814025 \\
& 6.323776 \\
& 24.591625 \\
& 0.432573 \\
& 5.723708 \quad 22.830503 \\
& 4.629652 \quad 22.847494 \\
& 3.710615 \quad 12.473018 \\
& \begin{array}{ll}
6.158709 & 12.656775
\end{array} \\
& 5.573933 \quad 10.705098 \\
& \begin{array}{ll}
4.556828 & 10.698059
\end{array} \\
& 2.874598 \quad 18.714987 \\
& 0.503455 \quad 18.536812 \\
& 0.923406 \quad 20.394547 \\
& \begin{array}{ll}
2.196609 & 20.485577
\end{array} \\
& 2.857120 \quad 6.327902 \\
& 0.426262 \quad 6.338826 \\
& 1.020747 \quad 8.176413 \\
& 2.191025 \quad 8.248265 \\
& 15.170167 \\
& 12.633958 \\
& 13.392539 \\
& 14.341189 \\
& 15.092489 \\
& 12.559435 \\
& 13.304908 \\
& 14.324441 \\
& 22.171206 \\
& 21.076178 \\
& 22.922504 \\
& 20.560099 \\
& 22.161495 \\
& 21.239788 \\
& 23.001154 \\
& 20.486305 \\
& 9.962781 \\
& 8.935479 \\
& 10.622322 \\
& 8.173742 \\
& 9.864226 \\
& 8.932810 \\
& 10.785689 \\
& 8.259190 \\
& 22.167078 \\
& 21.227165 \\
& 22.923962 \\
& 20.476839 \\
& 22.147417 \\
& 21.147303 \\
& 22.919106 \\
& 20.485577 \\
& 9.949187 \\
& 9.019227 \\
& 10.700001 \\
& 8.180054 \\
& 9.955013 \\
& 8.929411 \\
& 10.692475 \\
& 8.262102 \\
& 21.232992 \\
& 22.328989
\end{aligned}
$$




$$
\begin{aligned}
& 20.476595 \\
& 17.948639 \\
& 22.923231 \\
& 21.336401 \\
& 22.255924 \\
& 20.390421 \\
& 22.845068 \\
& 9.089867 \\
& 9.866897 \\
& 8.252150 \\
& 10.803654 \\
& 8.929169 \\
& 9.954527 \\
& 8.157722 \\
& 10.622079 \\
& 21.241972 \\
& 22.319281 \\
& 20.402800 \\
& 22.830988 \\
& 21.393932 \\
& 22.343554 \\
& 20.390421 \\
& 23.014505 \\
& 8.852704 \\
& 10.028079 \\
& 8.267200 \\
& 10.628391 \\
& 8.760460 \\
& 10.037062 \\
& 8.005520 \\
& 10.631789 \\
& 24.766401 \\
& 0.496901 \\
& 23.002611 \\
& 22.850409 \\
& 24.686296 \\
& 0.504669 \\
& 22.920319 \\
& 22.924688 \\
& 12.475687 \\
& 12.634929 \\
& 10.697088 \\
& 10.718692 \\
& 12.481028 \\
& 12.570844 \\
& 10.706554 \\
& 10.630818 \\
& 18.707949 \\
& 18.535357 \\
& 20.481449 \\
& 20.483150 \\
& 18.784657 \\
& 18.791939 \\
& 20.564955 \\
& 20.571995 \\
& 6.324747 \\
& 6.236145 \\
& 8.171558 \\
& 16.773991 \\
& \text { 3. } 811355 \\
& 6.322562 \\
& 5.566409 \\
& 4.551973 \\
& 15.931663 \\
& 18.458891 \\
& 17.786242 \\
& 16.935659 \\
& \text { 3. } 803344 \\
& 6.326204 \\
& 5.731476 \\
& \text { 4. } 633293 \\
& 2.863917 \\
& 0.425291 \\
& \text { 1. } 020747 \\
& \text { 2. } 192724 \\
& 15.249060 \\
& 12.644882 \\
& 13.307578 \\
& 14.405761 \\
& \text { 2. } 953976 \\
& 0.590115 \\
& \text { 1. } 007396 \\
& \text { 2. } 197822 \\
& 15.165313 \\
& 12.478115 \\
& 12.983027 \\
& 14.343617 \\
& 22.079205 \\
& 21.064526 \\
& 22.918135 \\
& 20.489218 \\
& 9.934623 \\
& 8.863141 \\
& 10.630575 \\
& 8.178112 \\
& 22.245001 \\
& 21.236147 \\
& 22.933428 \\
& 20.484362 \\
& \text { 9. } 945303 \\
& 8.939607 \\
& 10.707283 \\
& 8.257733 \\
& 22.174360 \\
& 21.146818 \\
& 22.851379 \\
& 20.472954 \\
& 10.031235 \\
& 9.010974 \\
& 10.796371 \\
& 8.330314 \\
& 22.090370 \\
& 20.981993 \\
& 23.007465 \\
& 20.481207
\end{aligned}
$$




\begin{tabular}{|c|c|c|c|}
\hline 0 & 6.327175 & 9.866168 & 00128 \\
\hline 0 & 6.327902 & 8.944219 & 18.619589 \\
\hline 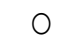 & 8.172044 & 10.695631 & 17.857851 \\
\hline 0 & 8.175200 & 8.178841 & 16.855310 \\
\hline 0 & 24.860102 & 21.232748 & 16.16882 \\
\hline 0 & 0.419222 & 22.253738 & 18.612064 \\
\hline 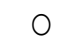 & 22.996784 & 20.380955 & 17.863678 \\
\hline O & 22.862303 & 22.830261 & 16.769379 \\
\hline 0 & 24.691879 & 8.844208 & 3.878838 \\
\hline o & 0.500299 & 10.030993 & 6.31576 \\
\hline $\mathrm{O}$ & 22.915949 & 8.272298 & 5.81328 \\
\hline o & 23.000425 & 10.628877 & 4.390061 \\
\hline 0 & 12.475687 & 21.406069 & 3.709159 \\
\hline 0 & 12.634442 & 22.240145 & 6.24172 \\
\hline 0 & 10.884488 & 20.316383 & 5.48727 \\
\hline o & 10.628148 & 22.920319 & 4.63693 \\
\hline 0 & 12.478843 & 8.849791 & 16.09405 \\
\hline 0 & 12.560891 & 9.871751 & 18.543123 \\
\hline 0 & 10.630818 & 8.176656 & 17.957621 \\
\hline 0 & 10.629362 & 10.536633 & 16.76355 \\
\hline 0 & 18.630512 & 21.244644 & 2.93091 \\
\hline o & 18.701393 & 22.239902 & 0.42189 \\
\hline C & 20.389692 & 20.320024 & 1.095027 \\
\hline 0 & 20.572237 & 22.759378 & 2.192482 \\
\hline 0 & 18.705521 & 8.947860 & 15.16531 \\
\hline 0 & 18.607208 & 9.879762 & 12.64390 \\
\hline 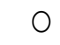 & 20.410082 & 8.090239 & 13.31850 \\
\hline 0 & 20.496500 & 10.634945 & 14.322499 \\
\hline 0 & 6.575261 & 21.075451 & 7012 \\
\hline 0 & 6.320863 & 22.170477 & 12.655320 \\
\hline O & 8.189279 & 20.400373 & 13.153191 \\
\hline 0 & 8.263559 & 22.844097 & 14.325169 \\
\hline 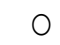 & 6.425972 & 8.940578 & 2.945480 \\
\hline 0 & 6.315765 & 9.952100 & (491 \\
\hline 0 & 8.175442 & 8.183210 & 174 \\
\hline 0 & 8.261374 & 10.630090 & 3500 \\
\hline 0 & 13.151492 & 5.227777 & 8.263074 \\
\hline 0 & 12.461609 & 6.671145 & 5.965969 \\
\hline 0 & 11.811049 & 7.828558 & 8.424014 \\
\hline 0 & 14.486109 & 7.747481 & 7.590424 \\
\hline 0 & 7.424143 & 17. & 19. \\
\hline 0 & 7.581200 & 19.895218 & 18.385096 \\
\hline 0 & 7.669075 & 20.148888 & 21.574051 \\
\hline $\mathrm{H}$ & 12.708481 & 22.176302 & 3.155698 \\
\hline $\mathrm{H}$ & 15.866120 & 7.981731 & 7.923472 \\
\hline $\mathrm{H}$ & 25.047745 & .148756 & 1.711359 \\
\hline $\mathrm{H}$ & 21.950548 & 16.018322 & 6.4 \\
\hline $\mathrm{H}$ & 13.528234 & 5.000810 & 9.167060 \\
\hline $\mathrm{H}$ & 8.983301 & 15.922438 & 19.380840 \\
\hline $\mathrm{H}$ & 10.431523 & 19.100710 & 19.183973 \\
\hline $\mathrm{H}$ & 11.440861 & 18.437044 & 21.453648 \\
\hline $\mathrm{H}$ & 10.468178 & 19.711218 & 22.24791 \\
\hline $\mathrm{H}$ & 11.737011 & 20.129711 & 21.037580 \\
\hline $\mathrm{H}$ & 13.598873 & 20.148159 & 18.591673 \\
\hline $\mathrm{H}$ & 13.268496 & 18.341158 & 18.398689 \\
\hline $\mathrm{H}$ & 11.363182 & 18.862335 & 16.855310 \\
\hline $1+$ & 11.692346 & 20.660597 & 17.070869 \\
\hline & 12.967976 & 6.908551 & 7.56760 \\
\hline
\end{tabular}




\begin{tabular}{|c|c|c|c|}
\hline $\mathrm{Re}$ & 8.119854 & 19.497601 & 20.082376 \\
\hline $\mathrm{C}$ & 9.974433 & 19.297577 & 20.177532 \\
\hline $\mathrm{C}$ & 10.942019 & 19.413368 & 21.305573 \\
\hline $\mathrm{C}$ & 12.985211 & 19.362633 & 18.151089 \\
\hline $\mathrm{C}$ & 11.958881 & 19.638878 & 17.333521 \\
\hline \multicolumn{4}{|c|}{605} \\
\hline US & ReO3_C4H8_M2 & $E=-7232$ & 80643159 \\
\hline $\mathrm{Si}$ & $23 . \overline{3} 8275 \overline{0}$ & 3.326349 & 1.243588 \\
\hline $\mathrm{Si}$ & 23.428387 & 1.236791 & 3.375626 \\
\hline $\mathrm{Si}$ & 23.398771 & 15.571912 & 13.402491 \\
\hline $\mathrm{Si}$ & 23.392460 & 13.427737 & 15.575068 \\
\hline $\mathrm{Si}$ & 11.248607 & 1.240432 & 15.585264 \\
\hline $\mathrm{Si}$ & 11.161461 & 15.577739 & 1.194553 \\
\hline $\mathrm{Si}$ & 11.227973 & 13.408804 & 3.367373 \\
\hline $\mathrm{Si}$ & 7.632662 & 3.352808 & 1.218585 \\
\hline $\mathrm{Si}$ & 7.657665 & 5.501110 & 3.347467 \\
\hline $\mathrm{Si}$ & 7.787292 & 15.494720 & 13.278206 \\
\hline $\mathrm{Si}$ & 19.964643 & 5.439452 & 15.53 \\
\hline $\mathrm{Si}$ & 19.908813 & 15.644736 & 1.26 \\
\hline $\mathrm{Si}$ & 19.883566 & 17.804932 & 3.349652 \\
\hline $\mathrm{Si}$ & 7.626108 & 3.370528 & 5.5 \\
\hline $\mathrm{Si}$ & 7.525368 & 15.540599 & 17.702251 \\
\hline $\mathrm{Si}$ & 7.766415 & 13.322386 & 15.4 \\
\hline $\mathrm{Si}$ & 19.916580 & 3.339457 & 17.74 \\
\hline $\mathrm{Si}$ & 19.870459 & 1.218828 & 15.6 \\
\hline $\mathrm{Si}$ & 19.935028 & 15.589634 & 5.43 \\
\hline $\mathrm{Si}$ & 20.010765 & 13.381372 & 3.2 \\
\hline $\mathrm{Si}$ & 23.436155 & 3.349895 & 5.535094 \\
\hline $\mathrm{Si}$ & 23.372313 & 17.739635 & 15.5 \\
\hline $\mathrm{Si}$ & 11.237440 & 3.366401 & 17.780174 \\
\hline $\mathrm{Si}$ & 11.267783 & 5.536793 & 15.5 \\
\hline $\mathrm{Si}$ & 11.238654 & 15.649834 & 5.45 \\
\hline $\mathrm{Si}$ & 11.185493 & 17.781630 & 3.2 \\
\hline $\mathrm{Si}$ & 1.308401 & 23.402655 & 3.33 \\
\hline $\mathrm{Si}$ & 3.378296 & 23.427416 & 1.2 \\
\hline $\mathrm{Si}$ & 1.256696 & 11.232828 & $15.5^{\circ}$ \\
\hline $\mathrm{Si}$ & 3.411552 & 11.214865 & 13.3 \\
\hline $\mathrm{Si}$ & 13.456139 & 23.445621 & 15.52 \\
\hline $\mathrm{Si}$ & 15.608324 & 23.403141 & 13.4 \\
\hline $\mathrm{Si}$ & 13.425309 & 11.233314 & 3.3 \\
\hline $\mathrm{Si}$ & 15.610024 & 11.173113 & 1.1 \\
\hline $\mathrm{Si}$ & 1.360591 & 7.681697 & 3.3 \\
\hline $\mathrm{Si}$ & 3.381209 & 7.602804 & 5.5 \\
\hline $\mathrm{Si}$ & 1.308401 & 19.897646 & 15.593760 \\
\hline $\mathrm{Si}$ & 3.425389 & 19.886723 & 17.7 \\
\hline $\mathrm{Si}$ & 13.432107 & 7.643343 & 15.6 \\
\hline $\mathrm{Si}$ & 15.583079 & 7.634362 & 17.7 \\
\hline $\mathrm{Si}$ & 15.557590 & 19.952021 & 0867 \\
\hline $\mathrm{Si}$ & 5.526113 & 7.662520 & 3.3 \\
\hline $\mathrm{Si}$ & 3.406455 & 7.666404 & 1.181202 \\
\hline $\mathrm{Si}$ & 5.526841 & 19.977995 & 15.6 \\
\hline $\mathrm{Si}$ & 3.441895 & 19.887936 & 13.452740 \\
\hline $\mathrm{Si}$ & 17.878000 & 7.686309 & 15.715860 \\
\hline $\mathrm{Si}$ & 15.627258 & 7.739471 & 13.486239 \\
\hline $\mathrm{Si}$ & 17.724098 & 19.965858 & 1061 \\
\hline $\mathrm{Si}$ & 15.608810 & 20.004698 & 1.2 \\
\hline $\mathrm{Si}$ & 5.498925 & 23.365515 & 3.37781 \\
\hline
\end{tabular}




\begin{tabular}{|c|c|c|c|}
\hline Si & 3.409610 & 23.400713 & 11548 \\
\hline i & 5.516160 & 11.188163 & 15.601770 \\
\hline S & 3.363731 & 11.239625 & 17.752743 \\
\hline & 17.727253 & 23.387848 & 15.612451 \\
\hline & 15.543511 & 23.390276 & 17.74181 \\
\hline & 17.821924 & 11.269726 & 3.30328 \\
\hline i & 15.662700 & 11.239868 & $5 \quad 170$ \\
\hline i & 3.357177 & 1.276844 & 23.36745 \\
\hline & 1.242374 & 3.365673 & 23.41454 \\
\hline S & 3.396987 & 13.389627 & 11.22433 \\
\hline & 1.288981 & 15.563173 & 11.25880 \\
\hline i & 15.613908 & 1.222954 & 11.20491 \\
\hline i & 13.361224 & 3.333388 & 11. \\
\hline 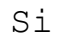 & 15.563416 & 13.422640 & 23.39124 \\
\hline & 13.448370 & 15.564387 & 23.41236 \\
\hline & 3.360090 & 1.200379 & 7.65960 \\
\hline & 5.517131 & 3.318338 & 7.62003 \\
\hline i & 3.298676 & 13.373119 & 19. \\
\hline $\mathrm{Si}$ & 17.774834 & 3.373441 & 19.86196 \\
\hline S & 15.642065 & 13.429679 & $7.64455^{\circ}$ \\
\hline $\mathrm{Si}$ & 17.823868 & 15.573369 & 7.71810 \\
\hline 1 & 3.339214 & 5.433626 & 7.65596 \\
\hline$i$ & 1.242374 & 3.327077 & 7.66324 \\
\hline i & 3.438740 & 17.736237 & 19. \\
\hline i & 1.266649 & 15.584050 & 19.871674 \\
\hline $\mathrm{Si}$ & 15.565844 & 5.468096 & 19.94449 \\
\hline 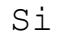 & 13.394724 & 3.389948 & 19.95274 \\
\hline 1 & 15.665128 & 17.778717 & 7.63581 \\
\hline i & 13.468760 & 15.648378 & 5836 \\
\hline $\mathrm{Si}$ & 3.377082 & 5.512033 & 23.38153 \\
\hline Si & 5.502323 & 3.387763 & 23.34682 \\
\hline $\mathrm{Si}$ & 5.586556 & 15.553222 & 11.13912 \\
\hline Si & 17.854939 & 3.322222 & 11.25151 \\
\hline$i$ & 15.614392 & 17.756384 & 23.4417 \\
\hline$i$ & 17.731138 & 15. & 23. \\
\hline $\mathrm{Si}$ & 1.644604 & 21.672119 & 23.72429 \\
\hline $\mathrm{Si}$ & 1.613290 & 23.781096 & 21.61264 \\
\hline Si & 1.678103 & 9.446460 & 11.58456 \\
\hline $\mathrm{Si}$ & 1.675190 & 11.596219 & 9.45738 \\
\hline $\mathrm{Si}$ & 13.875 & 21.701248 & 11.5517 \\
\hline $\mathrm{Si}$ & 13.8 & $23 . \varepsilon$ & 9. \\
\hline $\mathrm{Si}$ & 13.833122 & 9.424128 & 23.78837 \\
\hline $\mathrm{Si}$ & 13.801323 & 11.637486 & 21.65439 \\
\hline $\mathrm{Si}$ & 17.483780 & 21.678188 & 23.77502 \\
\hline $\mathrm{Si}$ & 17.394691 & 19.536196 & 21.68717 \\
\hline $\mathrm{Si}$ & 17.416540 & 9.518312 & 11.586 \\
\hline $\mathrm{Si}$ & 17.452709 & 7.327530 & 9.47898 \\
\hline $\mathrm{Si}$ & 5.153255 & 21.643961 & 11.64743 \\
\hline Si & 5.122426 & 19.541780 & 9.49500 \\
\hline $\mathrm{Si}$ & 5.182384 & 9.436750 & 23.73060 \\
\hline $\mathrm{Si}$ & 5.151798 & 7.307625 & 21.61337 \\
\hline $\mathrm{Si}$ & 17.334248 & 21.698822 & 19.51969 \\
\hline $\mathrm{Si}$ & 17.413628 & 23.852463 & 21.66265 \\
\hline $\mathrm{Si}$ & 17.388624 & 11.699629 & 9.49015 \\
\hline $\mathrm{Si}$ & 5.102520 & 21.599781 & 7.31709 \\
\hline $\mathrm{Si}$ & 5.119513 & 23.742498 & 9.43626 \\
\hline $\mathrm{Si}$ & 5.076547 & 9.459811 & 19.52721 \\
\hline Si & 5.024114 & 11.632145 & 21.70246 \\
\hline
\end{tabular}




\begin{tabular}{|c|c|c|c|}
\hline$i$ & 1.578334 & 21.622356 & 19.544935 \\
\hline S & 1.603580 & 19.529886 & 21.610704 \\
\hline & 1.631253 & 9.423400 & 7.306897 \\
\hline & 1.623485 & 7.277040 & 9.431168 \\
\hline & 13.875846 & 21.743000 & 7.184796 \\
\hline & 13.891140 & 19.549549 & 93496 \\
\hline & 13.810790 & 7.236258 & 21.660954 \\
\hline & 23.772358 & 1.592414 & 21.62 \\
\hline & 21.673090 & 1.535126 & 23.712641 \\
\hline & 23.862902 & 13.852057 & 9.42534 \\
\hline & 21.734020 & 13.774864 & 11.5903 \\
\hline & 11.5 & 1.652372 & \\
\hline & 9.414903 & 1.703106 & 11.628018 \\
\hline & 11.655691 & 13.816617 & 21.6968 \\
\hline & 9.458840 & 13.764183 & 23.76726 \\
\hline & 23.818235 & 17.347357 & 21.70076 \\
\hline$S \perp$ & 21.692995 & 17.390079 & \\
\hline 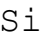 & 21.681 & 5.1 & 7. \\
\hline S & 11.670 & 17.3944 & 9.390. \\
\hline $\mathrm{Si}$ & 9.462725 & 17.391537 & 7.20591 \\
\hline & 11.577285 & 5.157866 & 21.638 \\
\hline & 9.474376 & 5.113202 & 19. \\
\hline $\mathrm{Si}$ & 19.534985 & 17. & \\
\hline 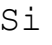 & 21.72 & 17.3 & 23. \\
\hline 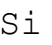 & 19.564 & 5.1551 & 9.457 \\
\hline S & 21.720911 & 5.099365 & 11.63263 \\
\hline S & 7.284564 & 17.355610 & 9.3566 \\
\hline $\mathrm{Si}$ & 9.4 & 17. & \\
\hline $\mathrm{Si}$ & 7.2 & 5. & 21 . \\
\hline $\mathrm{Si}$ & 9.40 & 5.1 & 23.77794. \\
\hline S & 19.553432 & 1.638293 & 21.59832 \\
\hline $\mathrm{Si}$ & 21.6801 & 1.608192 & 19.48303 \\
\hline $\mathrm{Si}$ & 19.555 & 13.8289 & 9.43165 \\
\hline $\mathrm{Si}$ & 7.30 & 7 & \\
\hline & 9.42 & 1.659 & 7.29524 \\
\hline S & 7.243298 & 13.697670 & 21.61968 \\
\hline S & 21.703676 & 23.744442 & 1.58683 \\
\hline $\mathrm{Si}$ & 23.837656 & 21.645903 & 1.61693 \\
\hline $\mathrm{Si}$ & 21.714 & 11.5967 & 13.783 \\
\hline $\mathrm{Si}$ & 23.849 & 9. & 13. \\
\hline S & 9.48 & 23.795176 & 13.82074 \\
\hline S & 9.462725 & 11.604230 & 1.66062 \\
\hline $\mathrm{Si}$ & 11.61636 & 9.464666 & 1.64581 \\
\hline $\mathrm{Si}$ & 21.679 & 23.7 & 17.3823 \\
\hline $\mathrm{Si}$ & 19.530 & 21.6 & 17.398 \\
\hline $\mathrm{Si}$ & 21.815 & 11.644768 & 4.88235 \\
\hline $\mathrm{Si}$ & 19.640821 & 9.536519 & 5.00833 \\
\hline S & 9.358829 & 23.822363 & 5.16223 \\
\hline $\mathrm{Si}$ & 9.465880 & 11.543057 & 17.29492 \\
\hline $\mathrm{Si}$ & 7.259319 & 9.423885 & 17.36944 \\
\hline $\mathrm{Si}$ & 21.6 & 19.5 & 4298 \\
\hline $\mathrm{Si}$ & 23.835957 & 21.681343 & 17.35099 \\
\hline $\mathrm{Si}$ & 21.704889 & 7.374381 & 5.12096 \\
\hline $\mathrm{Si}$ & 23.814352 & 9.446945 & 5.13116 \\
\hline $\mathrm{Si}$ & 9.482387 & 19.528187 & 4.97920 \\
\hline $\mathrm{Si}$ & 11.570003 & 21.726980 & 5.13019 \\
\hline $\mathrm{Si}$ & 9.433595 & 7.262960 & 17.32162 \\
\hline & & 9.36708 & 17.361 \\
\hline
\end{tabular}




\begin{tabular}{|c|c|c|c|}
\hline Si & 21.698093 & 19.506824 & 1.634166 \\
\hline Si & 19.559502 & 21.628426 & 1.652129 \\
\hline & 21.720669 & 7.237957 & 13.783360 \\
\hline 1 & 19.562414 & 9.415874 & 13.829724 \\
\hline & 9.491854 & 19.508282 & 13.784574 \\
\hline & 7.349134 & 21.616287 & 13.79428 \\
\hline 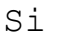 & 9.428012 & 7.298401 & 1.613047 \\
\hline $\mathrm{Si}$ & 7.312238 & 9.422428 & 1.662567 \\
\hline i & 11.658119 & 21.707802 & 13.69621 \\
\hline i & 11.212438 & 3.404027 & 13.37603 \\
\hline 1 & 19.998629 & 3.348681 & 13.41997 \\
\hline$\perp$ & 7.628778 & 1.210574 & 3.36688 \\
\hline $\mathrm{Si}$ & 23.376925 & 15.606139 & 17.726526 \\
\hline Si & 5.458629 & 15.549823 & 19.947166 \\
\hline L & 3.483405 & 17.703707 & 11.29181 \\
\hline i & 13.796226 & 9.427283 & 19.50828 \\
\hline L & 23.793718 & 5.133349 & 9.42097 \\
\hline 1 & 15.582108 & 1.281942 & $19.89594^{\circ}$ \\
\hline 1 & 15.578223 & 5.474650 & 11.2 \\
\hline Al & 17.524563 & 9.604488 & 7.204944 \\
\hline L & 9.447674 & 13.516825 & 19.64324 \\
\hline - & 7.259319 & 21.632309 & 5.16927 \\
\hline Al & 7.716410 & 17.753714 & $15.45563^{\circ}$ \\
\hline 1 & 23.372555 & 913 & 3.40815 \\
\hline 1 & 13.455168 & 19.860506 & 3.28920 \\
\hline 1 & 21.794464 & 13.678008 & 7.23722 \\
\hline Al & 16.738064 & 5.182627 & 13.79064 \\
\hline 1 & 6.959043 & 18.320284 & 18.04185 \\
\hline O & 22.163923 & 0.349069 & 2.866102 \\
\hline 0 & 24.605705 & 24.541864 & 3.955788 \\
\hline 0 & 24.029911 & 267 & 2.11747 \\
\hline 0 & 22.847254 & 2.185199 & 4.55682 \\
\hline 0 & 22.163195 & 12.473018 & 15.085692 \\
\hline 0 & 24.610802 & 12.475687 & 16.096487 \\
\hline 0 & 23.941309 & 14.330267 & 14.329296 \\
\hline O & 22.846 & 14.3 & $16.77180^{\circ}$ \\
\hline 0 & 10.024681 & 0.249057 & 15.176237 \\
\hline 0 & 12.558221 & 24.691393 & 16.09333 \\
\hline 0 & 11.644768 & 2.102666 & 14.25040 \\
\hline 0 & 10.707283 & 2.191511 & $16.77981 \varepsilon$ \\
\hline 0 & 9.944575 & 12.480542 & 2.94693 \\
\hline 0 & 12.481028 & 12.4 & 3.79654 \\
\hline 0 & 11.714679 & 14.332694 & 2.10436 \\
\hline 0 & 10.782048 & 14.322985 & 4.62795 \\
\hline 0 & 8.928440 & 6.407523 & 2.879453 \\
\hline 0 & 6.415049 & 6.409465 & 3.88369 \\
\hline 0 & 7.077987 & 4.629409 & 2.09028 \\
\hline 0 & 8.181511 & 4.555371 & 4.55003 \\
\hline 0 & 9.019712 & 18.800676 & 15.163371 \\
\hline 0 & 6.326689 & 18.633425 & 16.34166 \\
\hline 0 & 7.243541 & 16.681505 & 14.24117 \\
\hline 0 & 8.003335 & 17.015522 & 17.11820 \\
\hline 0 & 21.249498 & 6.248039 & 14.99854 \\
\hline 0 & 18.786112 & 6.410679 & 16.10231 \\
\hline 0 & 19.224512 & 4.627467 & 14.24214 \\
\hline O & 20.478050 & 4.466041 & 16.68805 \\
\hline 0 & 21.242216 & 18.548222 & 2.86755 \\
\hline 0 & 18.719357 & 18.785383 & 3.88417 \\
\hline
\end{tabular}




$\begin{array}{rrr}19.283741 & 16.851427 & 2.176461 \\ 20.398916 & 16.855553 & 4.562896 \\ 8.930868 & 0.342515 & 3.795091 \\ 6.401698 & 24.609589 & 2.782355 \\ 7.082600 & 2.106550 & 4.636206 \\ 8.175685 & 2.190054 & 2.187870 \\ 9.019712 & 12.466463 & 16.009583 \\ 6.494426 & 12.393639 & 15.089334 \\ 7.260047 & 14.338763 & 16.701895 \\ 8.258946 & 14.240937 & 14.234867 \\ 21.145603 & 0.337902 & 16.096972 \\ 18.638523 & 24.616144 & 15.007528 \\ 19.296850 & 2.109463 & 16.853611 \\ 20.468342 & 2.189569 & 14.424452 \\ 21.318680 & 12.466706 & 3.547490 \\ 18.707462 & 12.550453 & 2.783326 \\ 19.559015 & 14.239480 & 4.634992 \\ 20.480722 & 14.411345 & 2.127183 \\ 22.257866 & 6.572834 & 3.874226 \\ 24.775385 & 6.401454 & 2.620929 \\ 24.111717 & 4.552701 & 4.716555 \\ 22.759865 & 4.485460 & 2.116017 \\ 22.085758 & 18.627356 & 16.027548 \\ 24.599878 & 18.710133 & 15.089819 \\ 23.934027 & 16.861380 & 16.836863 \\ 22.862303 & 16.790499 & 14.344104 \\ 10.025409 & 6.495640 & 16.006670 \\ 12.557250 & 6.406309 & 15.013840 \\ 11.726088 & 4.641789 & 16.855795 \\ 10.770883 & 4.635720 & 14.326869 \\ 9.877819 & 18.608665 & 3.705275 \\ 12.372520 & 18.700422 & 2.690839 \\ 11.725117 & 16.848757 & 4.470410 \\ 10.617710 & 16.840017 & 2.025715 \\ 2.949849 & 22.170235 & 0.256340 \\ 3.969868 & 24.613716 & 24.528997 \\ 2.098539 & 24.022629 & 2.031298 \\ 4.489101 & 22.856962 & 2.194667 \\ 2.951791 & 9.944818 & 12.475445 \\ 3.891218 & 12.467677 & 12.474232 \\ 2.184714 & 11.731914 & 14.334394 \\ 4.634021 & 10.704613 & 14.330509 \\ 15.156089 & 22.159554 & 12.464035 \\ 16.174892 & 24.616144 & 12.456753 \\ 14.334150 & 24.015104 & 14.247249 \\ 16.773262 & 22.847494 & 14.401876 \\ 15.166041 & 9.859371 & 0.341058 \\ 16.102798 & 12.397281 & 24.524385 \\ 14.315945 & 11.715164 & 2.037610 \\ 16.860165 & 10.709952 & 2.111890 \\ 2.868044 & 8.859015 & 6.407765 \\ 3.895345 & 6.406552 & 6.478890 \\ 2.193696 & 6.995939 & 4.551002 \\ 4.555371 & 8.176898 & 4.566052 \\ 2.866345 & 21.063068 & 18.710133 \\ 4.116487 & 18.633183 & 18.622988 \\ 2.280599 & 19.287868 & 16.779573 \\ 4.653198 & 20.565926 & 16.929348\end{array}$




\begin{tabular}{|c|c|c|c|}
\hline 0 & 15.088363 & 8.940334 & 18.617889 \\
\hline 0 & 16.096001 & 6.421360 & 18.710861 \\
\hline 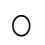 & 14.413771 & 7.080657 & 16.776419 \\
\hline 0 & 16.856039 & 8.112814 & 16.86089 \\
\hline O & 15.174294 & 21.323780 & 6.32377 \\
\hline O & 16.179749 & 18.798977 & 6.485445 \\
\hline 0 & 14.262783 & 19.389336 & 4. \\
\hline 0 & 16.773504 & 20.477566 & 4.55343 \\
\hline 0 & 3.863060 & 8.842751 & 0.18424 \\
\hline 0 & 2.779684 & 6.406795 & 24.62027 \\
\hline 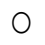 & 4.638391 & 7.151297 & 2.1009 \\
\hline 0 & 2.208018 & 8.266715 & 2.103879 \\
\hline 0 & 3.883936 & 21.158468 & 12.55846 \\
\hline 0 & 3.023887 & 18.615948 & 12.55894 \\
\hline 0 & 4.634992 & 19.383753 & 14.4846 \\
\hline 0 & 2.187384 & 20.400373 & 14.33342 \\
\hline O & 16.168581 & 8.847606 & 12.47374 \\
\hline 0 & 15.343003 & 6.240757 & 12.8247 \\
\hline 0 & 16.940271 & 7.070220 & 14.401876 \\
\hline 0 & 14.417413 & 8.253849 & 14.406732 \\
\hline 0 & 16.178049 & 21.232748 & 0.340573 \\
\hline O & 15.077925 & 18.730038 & 24.613716 \\
\hline O & 16.858709 & 19.382540 & 2.110191 \\
\hline 0 & 14.425423 & 20.632681 & \\
\hline 0 & 3.801645 & 22.168535 & 6.491513 \\
\hline 0 & 2.778956 & 24.612261 & 6.41480 \\
\hline 0 & 4.638148 & 24.019472 & 4.635235 \\
\hline O & 2.279870 & 22.841913 & 4.487645 \\
\hline 0 & 3.805772 & 75 & 18. \\
\hline 0 & 2.860276 & 12.466949 & 18.698238 \\
\hline 0 & 4.625040 & 11.793086 & 16.84778 \\
\hline 0 & 2.122328 & 10.713108 & 16.850698 \\
\hline 0 & 16.024633 & 22.085274 & 18.62323 \\
\hline 0 & 14.924266 & 24.536036 & 18.635124 \\
\hline 0 & 16.848028 & 23.934755 & 16. \\
\hline 0 & 14.409401 & 22.835602 & 16.679804 \\
\hline 0 & 16.093817 & 9.962052 & 6.325232 \\
\hline 0 & 15.169683 & 12.488068 & 6.410193 \\
\hline 0 & 16.921095 & 11.802067 & 4.557313 \\
\hline 0 & 14.400906 & 8117 & 49 \\
\hline 0 & 0.343971 & 2.8 & 22 \\
\hline 0 & 24.608860 & 3.964285 & 24.614927 \\
\hline 0 & 2.110676 & 2.115288 & 24.016075 \\
\hline 0 & 2.193210 & 4.554886 & 22.837299 \\
\hline 0 & 0.428689 & 15.157788 & 9.949187 \\
\hline 0 & 24.620996 & 16.1 & 6416 \\
\hline 0 & 2.122328 & 14.253317 & 11.795513 \\
\hline 0 & 2.265306 & 16.768894 & 10.791030 \\
\hline 0 & 12.384901 & 2.939411 & 10.028322 \\
\hline 0 & 12.552638 & 3.874712 & 12.552638 \\
\hline 0 & 14.336092 & 2.111890 & 11.726330 \\
\hline 0 & 14.252103 & 4.557798 & 10.631546 \\
\hline 0 & 12.632744 & 15.076711 & 22.082361 \\
\hline 0 & 12.392668 & 16.093575 & 24.525599 \\
\hline 0 & 14.332936 & 14.322256 & 24.015589 \\
\hline 0 & 14.411345 & 16.781515 & 22.925417 \\
\hline 0 & 6.483260 & 2.877754 & 8.852946 \\
\hline 0 & 6.396599 & 3.954575 & 6.40558 \\
\hline
\end{tabular}




\begin{tabular}{|c|c|c|c|}
\hline 0 & 4.646644 & 2.041008 & 7.083086 \\
\hline 0 & 4.539107 & 4.482548 & 8.186608 \\
\hline 0 & 6.502923 & 15.086178 & 21.074965 \\
\hline 0 & 6.168904 & 16.016138 & 18.48340 \\
\hline $\mathrm{O}$ & 4.544691 & 14.312546 & 19.46264 \\
\hline 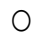 & 4.641061 & 16.843416 & 20.480965 \\
\hline 0 & 18.704550 & 2.945480 & 21.1468 \\
\hline 0 & 18.797035 & 3.976422 & 18.7 \\
\hline 0 & 16.932989 & 2.191754 & $19.2152 \varepsilon$ \\
\hline 0 & 16.857496 & 4.622855 & 20.3991 \\
\hline 0 & 18.706734 & 15.176478 & 9.0192 \\
\hline 0 & 18.802862 & 16.017109 & 6.4837 \\
\hline 0 & 16.933474 & 14.326626 & 7.16294 \\
\hline 0 & 16.925220 & 16.862350 & 8.1026 \\
\hline 0 & 0.417280 & 3.879081 & 8.9500 \\
\hline 0 & 24.523899 & 2.692053 & 6.5643 \\
\hline O & 2.113104 & 4.556828 & 7.007834 \\
\hline 0 & 2.208503 & 2.179131 & \\
\hline 0 & 0.423106 & 16.099400 & 21.15191 \\
\hline 0 & 24.620996 & 15.081567 & 18.63342 \\
\hline 0 & 2.263849 & 16.765982 & 19.28956 \\
\hline O & 2.102666 & 14.330267 & 20.47149 \\
\hline O & 12.393639 & 3.869128 & 21.151428 \\
\hline 0 & 12.469619 & 2.867316 & 18.693869 \\
\hline 0 & 14.243850 & 4.700048 & 19.379383 \\
\hline 0 & 14.327353 & 2.277686 & 20.55354 \\
\hline 0 & 12.566717 & 16.100128 & 8.92237 \\
\hline 0 & 12.483941 & 15.237894 & 6.40922 \\
\hline 0 & 14.420325 & 16.866234 & \\
\hline 0 & 14.410374 & 14.404305 & 8.093394 \\
\hline 0 & 6.404610 & 3.870585 & 22.086000 \\
\hline 0 & 6.423059 & 2.789880 & 24.541864 \\
\hline 0 & 4.637662 & 4.643731 & 23.9449 \\
\hline 0 & 4.485703 & 2.271132 & 22.761 \\
\hline 0 & 6.332272 & 30660 & \\
\hline 0 & 6.584000 & 14.998789 & 12.30139 \\
\hline 0 & 4.724322 & 16.775204 & 11.80522 \\
\hline 0 & 4.621884 & 14.322742 & 10.70291 \\
\hline 0 & 18.541182 & 3.961372 & 9.942390 \\
\hline 0 & 18.873 & 2.781626 & 12 \\
\hline 0 & 16.9 & 806 & 12 . \\
\hline 0 & 16.845358 & 2.194424 & 10.71626 \\
\hline 0 & 18.630997 & 16.107410 & 22.160040 \\
\hline 0 & 18.712803 & 15.083508 & 24.60521 \\
\hline 0 & 16.855310 & 16.857010 & 24.0216 \\
\hline 0 & 16.772778 & 14.406004 & 22.919348 \\
\hline 0 & 2.866587 & 24.693094 & 22.07968 \\
\hline 0 & 0.429175 & 0.421650 & 20.98587 \\
\hline 0 & 1.008124 & 22.911579 & 22.85283 \\
\hline 0 & 2.199521 & 22.772001 & 20.483150 \\
\hline 0 & 2.957617 & 12.473261 & 9.950644 \\
\hline 0 & 0.504426 & 12.578369 & 8.93378 \\
\hline 0 & 1.093813 & 10.699272 & 10.70849 \\
\hline 0 & 2.190783 & 10.617467 & 8.26040 \\
\hline 0 & 15.163614 & 24.694063 & 9.86762 \\
\hline 0 & 12.566717 & 0.510009 & 8.941063 \\
\hline 0 & 13.319715 & 22.929787 & 10.623051 \\
\hline ? & 14.328082 & 23.011106 & 8.09703 \\
\hline
\end{tabular}




\begin{tabular}{|c|c|c|c|}
\hline & 15.080353 & 12.562834 & 22.081631 \\
\hline O & 12.557250 & 12.561863 & 21.15701 \\
\hline & 13.313404 & 10.709467 & 22.91206 \\
\hline 0 & 14.253559 & 10.701942 & 20.396004 \\
\hline 0 & 16.110081 & 18.631483 & 57368 \\
\hline 0 & 18.621773 & 18.607937 & 21.1 \\
\hline 0 & 18.035786 & 20.392363 & 22.9227 \\
\hline 0 & 16.854340 & 20.475624 & 20.4831 \\
\hline & 16.190672 & 6.496368 & 10.0215 \\
\hline & 18.689014 & 6.423544 & 8.9430 \\
\hline 0 & 18. & 4637 & 10.6218 \\
\hline 0 & 16.858709 & 8.173257 & 8.17884 \\
\hline 0 & 3.880538 & 18.626627 & 10.02832 \\
\hline 0 & 6.339797 & 18.617161 & 8.93693 \\
\hline 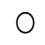 & 5.730748 & 20.392363 & 10.77767 \\
\hline O & 4.475750 & 20.480478 & \\
\hline u & 3.869371 & 6.483260 & 22. \\
\hline 0 & 6.322562 & 6.325717 & 21.0703 \\
\hline O & 5.814737 & 8.272541 & 22.76180 \\
\hline O & 4.552944 & 8.190735 & 20.39284 \\
\hline O & 16.181690 & 24.771502 & 21.314796 \\
\hline 0 & 18. & 88 & 22. \\
\hline 0 & 17.949 & 22.9 & 20 \\
\hline o & 16.937843 & 22.845797 & 22.83851 \\
\hline $\mathrm{O}$ & 16.093088 & 12.547784 & 8.94518 \\
\hline 0 & 18.530016 & 12.718676 & 10.03973 \\
\hline O & 18. & 10. & \\
\hline 0 & 16.852882 & 10. & 10. \\
\hline 0 & 3.814996 & 24.590654 & 8.94737 \\
\hline 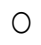 & 6.323776 & 0.432331 & 9.95841 \\
\hline 0 & 5.723950 & 22.830017 & 8.24680 \\
\hline O & 4.6 & 22.847 & 10. \\
\hline 0 & & 12. & 21. \\
\hline 0 & 6.1 & 12.6 & 22.2 \\
\hline 0 & 5.573933 & 10.704856 & 20.47246 \\
\hline 0 & 4.557070 & 10.698059 & 22.92808 \\
\hline 0 & 2.875812 & 18.713774 & 20.99558 \\
\hline 0 & 5 & 18. & 22 \\
\hline 0 & 0.9 & 20 . & 20. \\
\hline 0 & 2.196609 & 20.485092 & 22.758408 \\
\hline 0 & 2.856878 & 6.327902 & 8.934994 \\
\hline 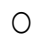 & 0.426262 & 6.338826 & 10.029293 \\
\hline 0 & 1.02 & 8.1 & 8.18976 \\
\hline 0 & 3 & & 10. \\
\hline 0 & 15.171138 & 18.6 & 305 \\
\hline 0 & 12.635900 & 18.626871 & 9.789946 \\
\hline 0 & 13.393267 & 20.481449 & 8.099220 \\
\hline 0 & 14.342890 & 20.471254 & 10.615767 \\
\hline 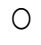 & 15.092489 & 486 & 21.241001 \\
\hline 0 & 12.5 & 942 & 22. \\
\hline 0 & 13.304665 & 8.164276 & 20.40280 \\
\hline 0 & 14.324441 & 8.190007 & 22.858419 \\
\hline 0 & 22.171206 & 2.777985 & 24.604977 \\
\hline 0 & 21.076178 & 0.332319 & 0.353438 \\
\hline 0 & 22.922504 & 0.921706 & 22.842154 \\
\hline O & 20.560343 & 2.187141 & 22.747726 \\
\hline 0 & 22.161737 & 15.081808 & 12.47860 \\
\hline & & 12.55 & \\
\hline
\end{tabular}




\begin{tabular}{|c|c|c|}
\hline 01154 & 240337 & 400 \\
\hline 20.486547 & 14.258171 & 10.699030 \\
\hline 9.962296 & 3.043549 & 395339 \\
\hline 8.937179 & 0.590358 & \\
\hline 10.622564 & 1.081676 & 0.70 \\
\hline 8.173742 & 2.181801 & 10.702 \\
\hline 9.8610 & 15.090304 & 24.609 \\
\hline 8.92941 & 12. & 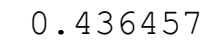 \\
\hline 10.799769 & 13.238881 & 22 . \\
\hline 8.261374 & 14.237782 & 22.7649 \\
\hline 22.166836 & 16.090660 & 18.697 \\
\hline 21.227165 & 18.618618 & \\
\hline 22.923717 & 17.954950 & 20.479 \\
\hline 20.477079 & 16.855553 & 20.480 \\
\hline 22.150330 & 3.877867 & \\
\hline 21.147789 & 6.402183 & \\
\hline 22.918861 & 5.732932 & 8.1810 \\
\hline 20.4 & 963 & \\
\hline 9.943847 & 16.100370 & 6.3308 \\
\hline 9.017771 & 18.618376 & 6.2545 \\
\hline 10.692233 & 17.933104 & 8.16524 \\
\hline 8.186123 & 16.870361 & 8.0941 \\
\hline 9.955013 & 3.787080 & 18 \\
\hline 8.929411 & 6.234688 & 18.46228 \\
\hline 10.692475 & 5.750653 & 20.38993 \\
\hline 8.262102 & 4.631836 & $20.4690^{\circ}$ \\
\hline 21.233234 & 16.180964 & 24.77198 \\
\hline 22.328989 & 18.626143 & 0.418 \\
\hline 20 . & 17. & 22 \\
\hline 22.923475 & 16.773991 & 22.91765 \\
\hline 21.315283 & 3.877139 & 12.64585 \\
\hline 22.252039 & 6.335670 & 12.54414 \\
\hline 20.396975 & 5.579274 & 10.7987 \\
\hline 22.8 & 4.553672 & 10.62 \\
\hline 9.0 & 15.93 & 12. \\
\hline 9.877577 & 18.449665 & 12.62279 \\
\hline 8.248752 & 17.786484 & 10.62086 \\
\hline 10.786418 & 16.932261 & 10.63979 \\
\hline 8.929169 & 3.803102 & 24.6081 \\
\hline .7 & 6 & \\
\hline 8.157722 & 5.7 & 22 . \\
\hline 10.622079 & 4.632807 & 22.83681 \\
\hline 21.242216 & 2.864645 & 18.54263 \\
\hline 22.317823 & 0.425534 & 18.5453 \\
\hline 20.402800 & 1.021232 & 20.313 \\
\hline 22.8 & 2.192724 & 20.46 \\
\hline 21.394176 & 15.249060 & 6.24658 \\
\hline 22.343554 & 12.644882 & 6.00043 \\
\hline 20.390421 & 13.307336 & 8.16476 \\
\hline 23.014505 & 14.405761 & 8.1953 \\
\hline 8.852946 & 2.9 & 6.49297 \\
\hline 10.027594 & 0.589873 & 6.24512 \\
\hline 8.267200 & 1.007396 & 8.25724 \\
\hline 10.628633 & 2.197822 & 8.25797 \\
\hline 8.767742 & 15.157545 & 18.78854 \\
\hline 10.037547 & 12.477387 & 18.4494 \\
\hline & 12. & 20.397 \\
\hline 10.629604 & 14.337549 & 20.5676 \\
\hline
\end{tabular}




\begin{tabular}{|c|c|c|c|}
\hline 0 & 24.766159 & 22.079205 & 2.866345 \\
\hline 0 & 0.497144 & 21.064526 & 0.416795 \\
\hline - & 23.002853 & 22.918135 & 1.012008 \\
\hline 0 & 22.850166 & 20.489218 & 2.19078 \\
\hline $\mathrm{O}$ & 24.685568 & 9.933652 & 15.08423 \\
\hline O & 0.503941 & 8.862899 & 12.567445 \\
\hline 0 & 22.919592 & 10.629848 & 13.230628 \\
\hline 0 & 22.920076 & 8.175442 & 14.32000 \\
\hline 0 & 12.475930 & 22.244028 & 15.00315 \\
\hline 0 & 12.637841 & 21.237362 & 12.48952 \\
\hline 0 & 10.697816 & 22.921776 & 13.14566 \\
\hline 0 & 10.789816 & 20.413967 & 14.160830 \\
\hline 0 & 12.481028 & 5546 & 43781 \\
\hline 0 & 12.570601 & 8.939607 & 0.42966 \\
\hline 0 & 10.706312 & 10.707525 & 1.08895 \\
\hline 0 & 10.631061 & 8.257733 & 2.115531 \\
\hline 0 & 18.708191 & 22.175331 & 16.085077 \\
\hline 0 & 18.535841 & 21.147303 & \\
\hline 0 & 20.481207 & 22.8 & 18.028503 \\
\hline 0 & 20.483150 & 20.473196 & 16.857496 \\
\hline 0 & 18.784412 & 10.031235 & 3.705518 \\
\hline O & 18.791939 & 9.010974 & 6.243184 \\
\hline 0 & 20.564955 & 10.796614 & 5.488972 \\
\hline 0 & 20.571751 & 30557 & 352 \\
\hline 0 & 6.323047 & 22.091827 & 3.792663 \\
\hline 0 & 6.239058 & 20.981993 & 6.412378 \\
\hline 0 & 8.171801 & 23.004309 & 5.818379 \\
\hline O & 8.351191 & 20.490675 & 4.481091 \\
\hline 0 & 6.326931 & 9.866411 & 86 \\
\hline 0 & 6.328146 & 8.9 & 18.619831 \\
\hline 0 & 8.172529 & 10.695631 & 17.858093 \\
\hline 0 & 8.174714 & 8.179083 & 16.855553 \\
\hline 0 & 24.859375 & 21.233477 & 16.168097 \\
\hline 0 & 0.418980 & 22.254223 & 18.612 \\
\hline 0 & 22.996542 & 20 & 578 \\
\hline 0 & 22.861090 & 22.831232 & 16.768894 \\
\hline 0 & 24.691879 & 8.844208 & 3.878838 \\
\hline 0 & 0.500299 & 10.031235 & 6.316008 \\
\hline 0 & 22.915949 & 8.272541 & 5.813281 \\
\hline 0 & 23.000425 & 10.62 & 61 \\
\hline 0 & 12.47 & 21.4 & 402 \\
\hline 0 & 12.634686 & 22.240145 & 6.241971 \\
\hline 0 & 10.879390 & 20.318083 & 5.484603 \\
\hline 0 & 10.628633 & 22.920805 & 4.636691 \\
\hline 0 & 12.478843 & 8.8497 & 16.094301 \\
\hline 0 & 12.560649 & 9.8 & 18. \\
\hline 0 & 10.631304 & 8.176656 & 17.957863 \\
\hline 0 & 10.629119 & 10.536875 & 16.763554 \\
\hline 0 & 18.630270 & 21.244644 & 2.929701 \\
\hline 0 & 18.702364 & 22.240631 & 0.421650 \\
\hline 0 & 20.389935 & 20.320024 & 1.095027 \\
\hline 0 & 20.572481 & 22.759621 & 2.192724 \\
\hline 0 & 18.706007 & 8.944704 & 15.163371 \\
\hline 0 & 18.559629 & 9.937293 & 12.660903 \\
\hline 0 & 20.399158 & 8.101404 & 13.312190 \\
\hline 0 & 20.493832 & 10.635430 & 14.320071 \\
\hline 0 & 6.57 & 21.074720 & 15.161672 \\
\hline 0 & 6.327902 & 22.163439 & 12.65726 \\
\hline
\end{tabular}




\begin{tabular}{|c|c|c|c|}
\hline 0 & 8.255548 & 20.410812 & 61446 \\
\hline 0 & 8.263559 & 22.845068 & 14.326383 \\
\hline 0 & 6.425972 & 8.940578 & 2.945723 \\
\hline 0 & 6.315765 & 9.952100 & 0.49180 \\
\hline 0 & 8.175442 & 8.183210 & 1.023174 \\
\hline 0 & 8.261617 & 10.629119 & 2.184714 \\
\hline 0 & 13.149064 & 5.238944 & 8.33128 \\
\hline 0 & 12.467191 & 6.659736 & 5.5 \\
\hline 0 & 11.810321 & 7.830986 & 8.42474 \\
\hline 0 & 14.484653 & 7.745782 & 7.59285 \\
\hline 0 & 7.162220 & 18.041367 & 19.80588 \\
\hline 0 & 7.847978 & 19.823608 & 18.37077 \\
\hline 0 & 7.671502 & 19.718498 & 21.8328 \\
\hline $\mathrm{H}$ & 12.716006 & 22.184071 & 3.16686 \\
\hline $\mathrm{H}$ & 15.860780 & 7.987314 & 7.92444 \\
\hline $\mathrm{H}$ & 25.047018 & 6.149970 & 1.71087 \\
\hline $\mathrm{H}$ & 21.950064 & 16.018322 & 6.47816 \\
\hline $\mathrm{H}$ & 13.550081 & 501600 & \\
\hline $\mathrm{H}$ & 9.143514 & 15.921710 & 19.27111 \\
\hline $\mathrm{H}$ & 10.583240 & 19.153145 & 19.39127 \\
\hline $\mathrm{H}$ & 10.938863 & 17.744246 & 21.46141 \\
\hline $\mathrm{H}$ & 10.439534 & 19.100954 & 22.50279 \\
\hline $\mathrm{H}$ & 11.951114 & 19.196110 & 21.54467 \\
\hline $\mathrm{H}$ & 10.289517 & 21. & 21. \\
\hline $\mathrm{H}$ & 10.58 & 21. & 19. \\
\hline $\mathrm{H}$ & 8.353132 & 22.077021 & 19.15265 \\
\hline $\mathrm{H}$ & 8.087568 & 22.194752 & 20.96742 \\
\hline $\mathrm{Re}$ & 12.969919 & 6.899812 & 7.58508 \\
\hline $\mathrm{Re}$ & 8.173257 & 19.5 & 20 \\
\hline $\mathrm{C}$ & 10.1 & 19. & 20 \\
\hline $\mathrm{C}$ & 10.9 & 18.841 & 21.54613 \\
\hline $\mathrm{C}$ & 9.938507 & 21.085888 & 20.38605 \\
\hline $\mathrm{C}$ & 8.50 & 21.6310 & 20.13432 \\
\hline \multicolumn{4}{|c|}{605} \\
\hline \multicolumn{2}{|c|}{ USY } & \multicolumn{2}{|c|}{$E=-7232.52800905503$} \\
\hline $\mathrm{Si}$ & $23 . \overline{3} 8299 \overline{4}$ & 3.326349 & 1.24358 \\
\hline $\mathrm{Si}$ & 23.428873 & 1.237034 & 3.37611 \\
\hline $\mathrm{Si}$ & 23.399015 & 15.572641 & 13.40249 \\
\hline $\mathrm{Si}$ & 23.391733 & 13.427494 & 15.57409 \\
\hline $\mathrm{Si}$ & 11.2 & 1 . & 15 \\
\hline $\mathrm{Si}$ & 11.160732 & 15.579924 & 1.19673 \\
\hline $\mathrm{Si}$ & 11.228216 & 13.409045 & 3.36761 \\
\hline $\mathrm{Si}$ & 7.632420 & 3.352079 & 1.21809 \\
\hline $\mathrm{Si}$ & 7.657665 & 5.500138 & 3.34722 \\
\hline $\mathrm{Si}$ & 7.786321 & 15.491321 & 13.27480 \\
\hline $\mathrm{Si}$ & 20. & 5. & 15 \\
\hline $\mathrm{Si}$ & 19.909784 & 15.644736 & 1.26834 \\
\hline $\mathrm{Si}$ & 19.884296 & 17.804447 & 3.34989 \\
\hline $\mathrm{Si}$ & 7.625865 & 3.370286 & 5.51106 \\
\hline $\mathrm{Si}$ & 7.597707 & 15.533073 & 17.72312 \\
\hline $\mathrm{Si}$ & 7.75 & 13.330396 & 15.48525 \\
\hline $\mathrm{Si}$ & 19.917553 & 3.340670 & 17.74400 \\
\hline Si & 19.867060 & 1.201107 & 15.62240 \\
\hline $\mathrm{Si}$ & 19.935514 & 15.589391 & 5.43508 \\
\hline $\mathrm{Si}$ & 20.010765 & 13.380888 & 3.28046 \\
\hline $\mathrm{Si}$ & 23.434456 & 3.350380 & 5.53630 \\
\hline $\mathrm{Si}$ & 23.374010 & 17.741577 & 15.56341 \\
\hline
\end{tabular}




\begin{tabular}{|c|c|c|c|}
\hline i & 11.236955 & 3.365673 & 17.780174 \\
\hline $\mathrm{Si}$ & 11.267783 & 5.536308 & 15.575068 \\
\hline $\mathrm{Si}$ & 11.238411 & 15.650320 & 5.452075 \\
\hline $\mathrm{Si}$ & 11.185249 & 17.783087 & 3.229978 \\
\hline $\mathrm{SI}$ & 1.309372 & 23.402899 & 3.334602 \\
\hline $\mathrm{Si}$ & 3.379267 & 23.427902 & 1.202321 \\
\hline$i$ & 1.256453 & 11.233556 & 15.578710 \\
\hline S & 3.411795 & 11.215350 & 13.384771 \\
\hline S & 13.455411 & 23.446350 & 15.525791 \\
\hline S & 15.607840 & 23.403141 & 13.41365 \\
\hline i & 13.425309 & 11.233314 & 3.332660 \\
\hline i & 15.610024 & 11.172384 & 1.200136 \\
\hline i & 1.360349 & 7.681211 & 3.341884 \\
\hline 2 & 3.381209 & 7.602562 & 5.525870 \\
\hline 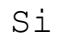 & 1.312770 & 19.897646 & 15.591089 \\
\hline i & 3.429273 & 19.895704 & 17.751772 \\
\hline $\mathrm{Si}$ & 13.431621 & 7.643343 & 15.602741 \\
\hline $\mathrm{Si}$ & 15.585021 & 7.632905 & 17.789154 \\
\hline $\mathrm{Si}$ & 15.557834 & 19.952507 & 5.502080 \\
\hline Si & 5.526113 & 7.662277 & 3.378782 \\
\hline Si & 3.405969 & 7.665676 & 1.18144 \\
\hline i & 5.553543 & 19.953722 & 15.68090 \\
\hline $\mathrm{Si}$ & 3.459373 & 19.884296 & 13 \\
\hline $\mathrm{Si}$ & 17.869987 & 7.680241 & 15.68478 \\
\hline Si & 15.607840 & 7.748209 & 13.45322 \\
\hline $\mathrm{Si}$ & 17.725311 & 19.966101 & 3.36251 \\
\hline $\mathrm{Si}$ & 15.610995 & 20.005669 & 1.23484 \\
\hline i & 5.49 & 23. & 3. \\
\hline $\mathrm{Si}$ & 3.410096 & 23.400713 & 5.511791 \\
\hline $\mathrm{Si}$ & 5.516888 & 11.188405 & 15.60128 \\
\hline $\mathrm{Si}$ & 3.364460 & 11.237926 & 17.751043 \\
\hline Si & 17.724098 & 23.383965 & 15.613421 \\
\hline i & 15.542541 & 23.390762 & 17.74133 \\
\hline $\mathrm{Si}$ & 17.822168 & 11.269483 & 3.30377 \\
\hline $\mathrm{Si}$ & 15.662943 & 11.239625 & 5.47125 \\
\hline $\mathrm{Si}$ & 3.357420 & 1.276116 & 23.366730 \\
\hline $\mathrm{Si}$ & 1.242617 & 3.364702 & 23.41454 \\
\hline $\mathrm{Si}$ & 3.396987 & 13.390354 & 11.22384 \\
\hline $\mathrm{Si}$ & 1.289709 & 15.563902 & 11.258801 \\
\hline $\mathrm{Si}$ & 15.613908 & 1.223197 & 11.20491 \\
\hline $\mathrm{Si}$ & 13.360010 & 3.333388 & 11.24909 \\
\hline $\mathrm{Si}$ & 15.564873 & 13.421911 & 23.39246 \\
\hline $\mathrm{Si}$ & 13.451526 & 15.566330 & 23.41673 \\
\hline $\mathrm{Si}$ & 3.360333 & 1.199893 & 7.65985 \\
\hline $\mathrm{Si}$ & 5.517131 & 3.318095 & 7.620039 \\
\hline $\mathrm{Si}$ & 3.304015 & 13.377732 & 19.95906 \\
\hline $\mathrm{Si}$ & 17.775805 & 3.373684 & 19.86123 \\
\hline $\mathrm{Si}$ & 15.642065 & 13.429922 & $7.64455^{\circ}$ \\
\hline $\mathrm{Si}$ & 17.823868 & 15.573126 & 7.71810 \\
\hline $\mathrm{Si}$ & 3.339214 & 5.433141 & 7.65596 \\
\hline $\mathrm{Si}$ & 1.242374 & 3.326834 & 7.66373 \\
\hline $\mathrm{Si}$ & 3.411067 & 17.755898 & 19.87070 \\
\hline $\mathrm{Si}$ & 1.261794 & 15.583323 & 19.87361 \\
\hline $\mathrm{Si}$ & 15.565844 & 5.467611 & 19.94425 \\
\hline $\mathrm{Si}$ & 13.393995 & 3.389705 & 19.952507 \\
\hline $\mathrm{Si}$ & 15.665128 & 17.778473 & 7.636303 \\
\hline $\mathrm{Si}$ & 13.468276 & 15.648378 & 7.62732 \\
\hline $\mathrm{Si}$ & 3.377325 & 5.510819 & 23.38177 \\
\hline
\end{tabular}




\begin{tabular}{|c|c|c|c|}
\hline $8 i$ & 5.502323 & 3.386549 & 23.346338 \\
\hline $\mathrm{Si}$ & 5.586314 & 15.553465 & 11.138885 \\
\hline $\mathrm{Si}$ & 17.858093 & 3.318823 & 11.254432 \\
\hline $\mathrm{Si}$ & 15.621433 & 17.753956 & 23.446835 \\
\hline SI & 17.733809 & 15.610995 & 23.4264 \\
\hline $\mathrm{Si}$ & 1.643876 & 21.676489 & 23.72647 \\
\hline i & 1.614018 & 23.780611 & 21.611433 \\
\hline S & 1.678589 & 9.446460 & 11.583 \\
\hline L & 1.675433 & 11.596461 & $9.4573 \xi$ \\
\hline 1 & 13.875603 & 21.701492 & 11.5517 \\
\hline i & 13.832395 & 23.855862 & $9.3806^{\circ}$ \\
\hline i & 13.834823 & 9.419030 & \\
\hline $\mathrm{Si}$ & 13.810306 & 11.629475 & 21.66 \\
\hline 2 & 17.484751 & 21.679159 & 23.77 \\
\hline i & 17.396635 & 19.536682 & 21.68644 \\
\hline i & 17.395906 & 9.509332 & 11.57777 \\
\hline $\mathrm{Si}$ & 17.450768 & 7.326317 & \\
\hline $\mathrm{Si}$ & 5.153983 & 21.644203 & 11.649623 \\
\hline $\mathrm{Si}$ & 5.122183 & 19.542509 & 9.4962 \\
\hline Si & 5.182141 & 9.435537 & 23.73133 \\
\hline $\mathrm{Si}$ & 5.151798 & 7.306169 & 21.61361 \\
\hline $\mathrm{Si}$ & 17.334734 & 21.699551 & 19.5182 \\
\hline $\mathrm{Si}$ & 17.414354 & 23.853920 & \\
\hline $\mathrm{Si}$ & 17.3895 & 11.7 & 9. \\
\hline Si & 5.102520 & 21.599539 & 7.31757 \\
\hline $\mathrm{Si}$ & 5.119513 & 23.741772 & 9.43699 \\
\hline $\mathrm{Si}$ & 5.077033 & 9.457870 & 19.52648 \\
\hline $\mathrm{Si}$ & 5.0 & 11.6 & \\
\hline $\mathrm{Si}$ & 1.583918 & 21.621628 & 19. \\
\hline $\mathrm{Si}$ & 1.59921 & 19.535469 & 21.620171 \\
\hline $\mathrm{Si}$ & 1.63125 & 9.423157 & 7.30689 \\
\hline Si & 1.623728 & 7.276554 & 9.43092 \\
\hline $\mathrm{Si}$ & 13.875603 & 21.743486 & 7.1855 \\
\hline $\mathrm{Si}$ & 13.890897 & 19.549549 & \\
\hline $\mathrm{Si}$ & 13.8 & 287 & 21.6 \\
\hline $\mathrm{Si}$ & 23.773087 & 1.591928 & 21.626484 \\
\hline $\mathrm{Si}$ & 21.673574 & 1.535368 & 23.712400 \\
\hline $\mathrm{Si}$ & 23.863388 & 13.852300 & 9.42558 \\
\hline $\mathrm{Si}$ & 21.734262 & 13.775107 & 11.59039 \\
\hline $\mathrm{Si}$ & 11.536504 & 1.651644 & \\
\hline $\mathrm{Si}$ & 9.414417 & 1.703349 & 11.62 \\
\hline $\mathrm{Si}$ & 11.672684 & 13.829967 & 21.71265 \\
\hline $\mathrm{Si}$ & 9.452286 & 13.777534 & 23.77745 \\
\hline $\mathrm{Si}$ & 23.817022 & 17.348328 & 21.70149 \\
\hline $\mathrm{Si}$ & 21.693239 & 17.390566 & 19.55852 \\
\hline $\mathrm{Si}$ & 21.679401 & 5.179714 & 7.25203 \\
\hline $\mathrm{Si}$ & 11.669043 & 17.394449 & 9.35761 \\
\hline $\mathrm{Si}$ & 9.462239 & 17.391294 & 7.20664 \\
\hline $\mathrm{Si}$ & 11.576556 & 5.157624 & 21.63862 \\
\hline $\mathrm{Si}$ & 9.474133 & 5.112716 & 19.50949 \\
\hline $\mathrm{Si}$ & 19.536682 & 17.384741 & 21.69639 \\
\hline $\mathrm{Si}$ & 21.723581 & 17.393963 & 23.82187 \\
\hline $\mathrm{Si}$ & 19.581835 & 5.150585 & 9.49282 \\
\hline $\mathrm{Si}$ & 21.730135 & 5.068536 & 11.65787 \\
\hline $\mathrm{Si}$ & 7.284079 & 17.356339 & 9.357130 \\
\hline $\mathrm{Si}$ & 9.495009 & 17.285456 & 11.555437 \\
\hline $\mathrm{Si}$ & 7.292818 & 5.155682 & 21.64857 \\
\hline $\mathrm{Si}$ & 9.407378 & 5.116843 & 23.77769 \\
\hline
\end{tabular}




\begin{tabular}{|c|c|c|c|}
\hline $\mathrm{Si}$ & 19.554403 & 1.638778 & 839 \\
\hline $\mathrm{Si}$ & 21.680857 & 1.608192 & 19.482794 \\
\hline $\mathrm{Si}$ & 19.556103 & 13.829482 & 9.431410 \\
\hline$S$ & 7.307140 & 1.607707 & 9.43578 \\
\hline & 9.424856 & 1.659412 & 7.295002 \\
\hline$i$ & 7.250580 & 13.690874 & 21.62284 \\
\hline S & 21.704405 & 23.745413 & 15868 \\
\hline S & 23.838142 & 21.646389 & $1.01 / 41$ \\
\hline S & 21.713387 & 11.595490 & 13.7828 \\
\hline i & 23.851492 & 9.411262 & 13.78942 \\
\hline 1 & 9.486756 & 23.796391 & 13.8209 \\
\hline$i$ & 9.462967 & 11.604472 & 5603 \\
\hline$i$ & 11. & 4423 & 1.64654 \\
\hline i & 21.682800 & 23.757065 & 17.386438 \\
\hline S & 19.531586 & 21.671633 & 17.3988 \\
\hline i & 21.816069 & 11.644282 & 4.88259 \\
\hline$i$ & 19.641064 & 9.536034 & $5.0085^{\circ}$ \\
\hline $\mathrm{Si}$ & 9.358101 & 23. & $2 \pi$ \\
\hline $\mathrm{Si}$ & 9.466366 & 11.545241 & 17.298565 \\
\hline $\mathrm{Si}$ & 7.260533 & 9.424128 & 17.36896 \\
\hline i & 21.697851 & 19.522360 & 17.34226 \\
\hline$i$ & 23.840326 & 21.679646 & 17.3490 \\
\hline $\mathrm{Si}$ & 21.704647 & 7.373410 & 5.1216 \\
\hline $\mathrm{Si}$ & 23.814352 & 9. & \\
\hline $\mathrm{Si}$ & 9.481416 & 19.5 & 4.97993 \\
\hline $\mathrm{Si}$ & 11.568789 & 21.727467 & 5.13116 \\
\hline $\mathrm{Si}$ & 9.433838 & 7.262475 & 17.32186 \\
\hline $\mathrm{Si}$ & 11.575342 & 9.367568 & 17.3614 \\
\hline $\mathrm{Si}$ & 21.65 & 19.507311 & 7621 \\
\hline $\mathrm{Si}$ & 19.560957 & 21.628668 & 1.65237 \\
\hline $\mathrm{Si}$ & 21.732077 & 7.234559 & 13.78336 \\
\hline $\mathrm{Si}$ & 19.564842 & 9.403737 & 13.83288 \\
\hline Si & 9.499621 & 19.505854 & 13.79549 \\
\hline $\mathrm{Si}$ & 7.343552 & 21.623327 & 13.8069 \\
\hline $\mathrm{Si}$ & 769 & 73 & 16130 \\
\hline $\mathrm{Si}$ & 7.312238 & 9.422186 & 1.66281 \\
\hline $\mathrm{Si}$ & 11.659090 & 21.709503 & $13.69572 \xi$ \\
\hline Si & 11.212680 & 3.403299 & 13.37603 \\
\hline $\mathrm{Si}$ & 20.023632 & 3.292364 & 13.4182 \\
\hline $\mathrm{Si}$ & 7.6 & 32 & 366 \\
\hline $\mathrm{Si}$ & 23.3 & 15. & 17. \\
\hline $\mathrm{Si}$ & 5.457658 & 15.563416 & 19.97702 \\
\hline $\mathrm{Si}$ & 3.483648 & 17.704678 & 11.29302 \\
\hline $\mathrm{Si}$ & 13.797440 & 9.427283 & 19.50779 \\
\hline $\mathrm{Si}$ & 23.793234 & 5.133593 & 9.4224 \\
\hline $\mathrm{Al}$ & 15.581865 & 1.2 & $19.8957 c$ \\
\hline Al & 15.576768 & 5.468582 & 11.20491 \\
\hline Al & 17.524803 & 9.602789 & 7.20615 \\
\hline Al & 9.477531 & 13.529691 & 19.65538 \\
\hline Al & 7.258348 & 21.632795 & 5.16927 \\
\hline Al & 7.728304 & 17.741091 & 15.43184 \\
\hline Al & 23.372313 & 8428 & 3.40839 \\
\hline $\mathrm{Al}$ & 13.454440 & 19.862206 & 3.29212 \\
\hline Al & 21.794949 & 13.677523 & 7.23747 \\
\hline Al & 16.713791 & 5.196949 & 13.77389 \\
\hline $\mathrm{Al}$ & 6.942536 & 18.178762 & 18.10132 \\
\hline 0 & 22.1 & 0.349311 & 2.86634 \\
\hline 0 & 24.605705 & 24.541864 & 3.95578 \\
\hline
\end{tabular}




$$
\begin{aligned}
& 24.029911 \\
& 2.099510 \\
& 2.185685 \\
& \begin{array}{l}
22.847010 \\
22.162466
\end{array} \\
& 12.472290 \\
& 24.610559 \\
& 12.475687 \\
& 14.330267 \\
& 23.941067 \\
& 22.846039 \\
& 10.025167 \\
& 12.558464 \\
& 11.645253 \\
& 10.707283 \\
& 9.944575 \\
& 12.481028 \\
& 11.714437 \\
& 10.782291 \\
& 8.928440 \\
& 6.415291 \\
& 7.077987 \\
& 8.181511 \\
& 9.027723 \\
& 6.402911 \\
& 7.235530 \\
& 8.008433 \\
& 21.305088 \\
& 18.791452 \\
& 19.298548 \\
& 20.480722 \\
& 21.242216 \\
& 18.719357 \\
& 19.283741 \\
& 20.398916 \\
& 8.930868 \\
& 6.401454 \\
& 7.082600 \\
& 8.175927 \\
& 9.018499 \\
& 6.494426 \\
& 7.250580 \\
& 8.254334 \\
& 21.147545 \\
& 18.633183 \\
& 19.297577 \\
& 20.475866 \\
& 21.318924 \\
& 18.707462 \\
& 19.559015 \\
& 20.480722 \\
& 22.257866 \\
& 24.775385 \\
& 24.110989 \\
& 22.759865 \\
& 22.086000 \\
& 24.600121 \\
& 23.933784 \\
& 22.862061 \\
& 10.025652 \\
& \text { O } 12.557250 \\
& \text { o } 10.771853
\end{aligned}
$$$$
\text { O } 11.726330
$$ 


\begin{tabular}{|c|c|c|c|}
\hline & 9.877819 & 18.609636 & 3.706003 \\
\hline O & 12.373249 & 18.701393 & 2.690111 \\
\hline 0 & 11.725359 & 16.848999 & 4.470653 \\
\hline $\mathrm{O}$ & 10.618681 & 16.843174 & 2.026201 \\
\hline 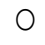 & 2.949849 & 22.170961 & 0.257311 \\
\hline 0 & 3.970111 & 24.614202 & 24.529238 \\
\hline 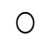 & 2.098539 & 24.022629 & 2.0315 \\
\hline 0 & 4.488859 & 22.856962 & 2.1951 \\
\hline 0 & 2.951791 & 9.944818 & 12.47568 \\
\hline 0 & 3.891218 & 12.467920 & 12.473989 \\
\hline U & 2.184957 & 11.732157 & 14.3343 \\
\hline 0 & 4.634264 & 10.705098 & 14.330752 \\
\hline 0 & 15.156089 & 22.159554 & 12.4637 \\
\hline 0 & 16.174652 & 24.616144 & 12.45699 \\
\hline 0 & 14.333908 & 24.015104 & 14.2474 \\
\hline 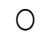 & 16.772049 & 22.846283 & 14.40163 \\
\hline $\mathrm{O}$ & 15.167498 & 9858400 & \\
\hline 0 & 16.103041 & 12.396794 & 24.525112 \\
\hline 0 & 14.315945 & 11.715164 & 2.03761 \\
\hline 0 & 16.860409 & 10.709952 & 2.112133 \\
\hline O & 2.868044 & 8.859258 & 6.40776 \\
\hline $\mathrm{O}$ & 3.895345 & 6.2 & \\
\hline 0 & 2.193696 & 5939 & 4.551002 \\
\hline 0 & 4.555371 & 8.176898 & 4.566295 \\
\hline 0 & 2.868772 & 21.066467 & 18.706007 \\
\hline O & 4.036866 & 18.625656 & 18.62225 \\
\hline 0 & 2.275744 & 19.290052 & 16.77641 \\
\hline 0 & 4.630865 & 20. & 16. \\
\hline $\mathrm{O}$ & 15.089819 & 8.939849 & 18.616190 \\
\hline 0 & 16.096001 & 6.421360 & 18.710861 \\
\hline 0 & 14.413771 & 7.080657 & 16.775690 \\
\hline 0 & 16.859922 & 8.098492 & 16.850212 \\
\hline 0 & 15.172 & 21. & 19 \\
\hline $\mathrm{O}$ & 16.179993 & 8735 & 6.485687 \\
\hline 0 & 14.263754 & 19.389336 & 4.733304 \\
\hline 0 & 16.773504 & 20.477566 & 4.553430 \\
\hline 0 & 3.862574 & 8.842994 & 0.18448 \\
\hline 0 & 2.779684 & 6.406795 & 24.62027 \\
\hline 0 & 4.6 & 97 & 66 \\
\hline 0 & 2.207775 & 8.266715 & 2.103879 \\
\hline 0 & 3.882965 & 21.160410 & 12.556279 \\
\hline 0 & 3.022916 & 18.615704 & 12.559192 \\
\hline 0 & 4.711943 & 19.380112 & 14.416442 \\
\hline 0 & 2.198793 & 20.396004 & 14.332694 \\
\hline 0 & 16.095030 & 8.846878 & 12.398979 \\
\hline 0 & 15.334507 & 6.236387 & 12.816504 \\
\hline 0 & 16.945856 & 7.084056 & 14.338277 \\
\hline 0 & 14.417897 & 8.253364 & 14.404547 \\
\hline 0 & 16.177807 & 21.232506 & 0.339844 \\
\hline 0 & 15.077197 & 18.732708 & 24.612261 \\
\hline 0 & 16.859194 & 19.382296 & 2.110676 \\
\hline 0 & 14.424211 & 20.631952 & 2.101209 \\
\hline 0 & 3.801645 & 22.168535 & 6.491756 \\
\hline 0 & 2.778956 & 24.612261 & 6.414805 \\
\hline 0 & 4.638148 & 24.019472 & 4.635235 \\
\hline $\mathrm{O}$ & 2.279870 & 22.841913 & 4.488131 \\
\hline 0 & 3.805772 & 9.945061 & 18.62954 \\
\hline & & & \\
\hline
\end{tabular}




\begin{tabular}{|c|c|c|c|}
\hline 0 & 4.625282 & 11.793571 & 16.848028 \\
\hline 0 & 2.121843 & 10.713594 & 16.850941 \\
\hline 0 & 16.023663 & 22.085030 & 18.623959 \\
\hline 0 & 14.924266 & 24.535793 & 18.635851 \\
\hline O & 16.847057 & 23.934027 & 16.86089 \\
\hline 0 & 14.409160 & 22.835358 & 16.680048 \\
\hline 0 & 16.094545 & 9.961567 & 25232 \\
\hline 0 & 15.169683 & 12.488068 & 6. \\
\hline 0 & 16.921095 & 11.802067 & 4.55731 \\
\hline 0 & 14.400906 & 10.788117 & 4.54104 \\
\hline 0 & 0.343728 & 2.868287 & 22.16270 \\
\hline 0 & 24.608860 & 3.964285 & 24.614927 \\
\hline 0 & 2.110676 & 2.115046 & 24.01631 \\
\hline 0 & 2.192967 & 4.554886 & 22.837543 \\
\hline 0 & 0.428447 & 15.157788 & 9.94943 \\
\hline 0 & 24.621239 & 16.100613 & 12.47641 \\
\hline O & 2.121843 & 14.253317 & 11.795513 \\
\hline 0 & 2.265791 & 16.768894 & \\
\hline 0 & 12.383930 & 2.939654 & 10.027108 \\
\hline 0 & 12.552638 & 3.874712 & 12.55239 \\
\hline 0 & 14.335850 & 2.112133 & 11.726088 \\
\hline 0 & 14.249675 & 4.555857 & 10.62717 \\
\hline O & 12.643668 & 15.087392 & 22.084787 \\
\hline 0 & 12.392911 & 16.094788 & 814 \\
\hline 0 & 14.334394 & 14.322499 & 24.016560 \\
\hline 0 & 14.413286 & 16.785885 & 22.92735 \\
\hline r & 6.483502 & 2.877754 & 8.852946 \\
\hline 0 & 6.396599 & 3.954332 & 6.405581 \\
\hline 0 & 4.646401 & 2.041251 & 86 \\
\hline 0 & 4.539107 & 4.482548 & 8.186608 \\
\hline 0 & 6.505350 & 15.084722 & 21.084917 \\
\hline 0 & 6.319649 & 16.180233 & 18.616674 \\
\hline 0 & 4.557556 & 14.330752 & 19.45075 \\
\hline 0 & 4.641546 & 16.860651 & 20.48 \\
\hline 0 & 18.704306 & 80 & 21. \\
\hline 0 & 18.797279 & 3.975937 & 18.703821 \\
\hline 0 & 16.932989 & 2.191754 & 19.215288 \\
\hline 0 & 16.857496 & 4.622612 & 20.399158 \\
\hline 0 & 18.706734 & 15.176722 & 9.019227 \\
\hline 0 & 18.802620 & 16.017 & 502 \\
\hline 0 & 16.933474 & 14.3 & 7 . \\
\hline 0 & 16.925220 & 16.862350 & 8.102618 \\
\hline 0 & 0.417280 & 3.879567 & 8.950045 \\
\hline 0 & 24.523170 & 2.692296 & 6.564337 \\
\hline 0 & 2.112861 & 4.556828 & 7.007 \\
\hline 0 & 2.208260 & 2.1 & 763 \\
\hline 0 & 0.421893 & 16.098671 & 21.153856 \\
\hline 0 & 24.620270 & 15.080837 & 18.63342 \\
\hline 0 & 2.259965 & 16.763554 & 19.298063 \\
\hline 0 & 2.106550 & 14.330752 & 20.470041 \\
\hline 0 & 12.393882 & 3.869371 & 21.151428 \\
\hline 0 & 12.469862 & 2.867558 & 18.693626 \\
\hline 0 & 14.243850 & 4.699805 & 19.379141 \\
\hline 0 & 14.327353 & 2.277928 & 20.553787 \\
\hline 0 & 12.566231 & 16.100128 & 8.922857 \\
\hline 0 & 12.484183 & 15.238137 & 6.409465 \\
\hline 0 & 14.420325 & 16.866234 & 7.084056 \\
\hline ? & 14.410131 & 14.404305 & 8.09339 \\
\hline
\end{tabular}




\begin{tabular}{|c|c|c|c|}
\hline 0 & 6.404367 & 3.870827 & 22.086000 \\
\hline 0 & 6.422574 & 2.790123 & 24.541864 \\
\hline 0 & 4.637905 & 4.643731 & 23.944950 \\
\hline 0 & 4.484732 & 2.271860 & 22.76180 \\
\hline O & 6.332272 & 16.090660 & 9.79164 \\
\hline 0 & 6.584485 & 14.994905 & 12.298483 \\
\hline 0 & 4.724565 & 16.774719 & 11.805223 \\
\hline 0 & 4.621641 & 14.322499 & 10.702671 \\
\hline 0 & 18.541910 & 3.958702 & 9.94457 \\
\hline 0 & 18.874229 & 2.776286 & 12.38562 \\
\hline 0 & 16.944885 & 4.540321 & 12.0433 \\
\hline 0 & 16.844387 & 2.194909 & 10.716507 \\
\hline 0 & 18.630997 & 16.107653 & 22.15955 \\
\hline 0 & 18.713289 & 15.083265 & 24.60594 \\
\hline 0 & 16.857737 & 16.856039 & 2262 \\
\hline 0 & 16.773020 & 14.406732 & 22.91959 \\
\hline O & 2.866345 & 24.693335 & 22.079447 \\
\hline 0 & 0.429418 & 0.421650 & 20 . \\
\hline 0 & 1.007639 & 22.913280 & 22 . \\
\hline 0 & 2.199036 & 22.771273 & 20.483391 \\
\hline 0 & 2.957617 & 12.473261 & 9.950644 \\
\hline O & 0.504426 & 12.578369 & 8.933781 \\
\hline O & 1.093813 & 10.699272 & 10.708496 \\
\hline 0 & 2.190783 & 10. & \\
\hline 0 & 15.163614 & 24.693821 & 9.867625 \\
\hline 0 & 12.566717 & 0.510009 & 8.941063 \\
\hline 0 & 13.319715 & 22.929787 & 10.623051 \\
\hline 0 & 14.328325 & 23.011106 & 8.097278 \\
\hline 0 & 15.085450 & 12.562104 & 22 . \\
\hline 0 & 12.558706 & 12.556522 & 21.223040 \\
\hline 0 & 13.315831 & 10.704127 & 22.91862 \\
\hline 0 & 14.252346 & 10.703156 & 20.396975 \\
\hline 0 & 16.108381 & 18.631725 & 22.158339 \\
\hline 0 & 18.621044 & 18.606480 & 21.14 \\
\hline 0 & 18.035542 & 20 . & 91 \\
\hline 0 & 16.853369 & 20.475624 & 20.483391 \\
\hline 0 & 16.187031 & 6.496368 & 10.020311 \\
\hline 0 & 18.701637 & 6.422331 & 9.007333 \\
\hline 0 & 18.030445 & 8.345850 & 10.620623 \\
\hline 0 & 16.861622 & 8.1 & 68 \\
\hline 0 & 3.880538 & 18.6 & 10. \\
\hline 0 & 6.339312 & 18.617405 & 8.936936 \\
\hline 0 & 5.731233 & 20.392847 & 10.777679 \\
\hline 0 & 4.475750 & 20.480236 & 8.351918 \\
\hline 0 & 3.869128 & 6.483260 & 22.175573 \\
\hline 0 & 6.322562 & 17 & 21. \\
\hline 0 & 5.814495 & 8.272783 & 22.761562 \\
\hline 0 & 4.552944 & 8.190735 & 20.393091 \\
\hline 0 & 16.181204 & 24.770771 & 21.314796 \\
\hline 0 & 18.706247 & 0.417280 & 22.234076 \\
\hline 0 & 17.949368 & 22.928329 & 20.390663 \\
\hline 0 & 16.937359 & 22.845552 & 22.838270 \\
\hline 0 & 16.093330 & 12.548025 & 8.944947 \\
\hline 0 & 18.530743 & 12.719405 & 10.039489 \\
\hline 0 & 18.034328 & 10.707525 & 8.421102 \\
\hline 0 & 16.853611 & 10.795885 & 10.790302 \\
\hline 0 & 3.815239 & 24.590654 & 8.947618 \\
\hline 0 & 6.323776 & 0.432331 & 9.95841 \\
\hline
\end{tabular}




\begin{tabular}{|c|c|c|c|}
\hline 0 & 5.724193 & 22.830261 & 8.246809 \\
\hline 0 & 4.631593 & 22.846767 & 10.710681 \\
\hline 0 & 3.712557 & 12.475203 & 21.236633 \\
\hline 0 & 6.160165 & 12.654591 & 22.247427 \\
\hline O & 5.574176 & 10.704613 & 20.471741 \\
\hline 0 & 4.556585 & 10.698301 & 22.928087 \\
\hline 0 & 2.884308 & 18.701880 & 21.059185 \\
\hline 0 & 0.499814 & 18.537785 & 22.25373 \\
\hline 0 & 0.926804 & 20.392605 & 20.39891 \\
\hline 0 & 2.195637 & 20.489462 & 22.762533 \\
\hline 0 & 2.856878 & 6.327902 & 8.9349 \\
\hline 0 & 0.426505 & 6.339068 & 10.029536 \\
\hline 0 & 00504 & 8.176413 & 8.18976 \\
\hline 0 & 2.191025 & 8.248508 & 10.61504 \\
\hline 0 & 15.170897 & 18.630997 & 8.94130 \\
\hline 0 & 12.635900 & 18.626871 & 9.7899 \\
\hline 0 & 13.393267 & 20.481207 & 8.098977 \\
\hline 0 & 14.343133 & 20.471254 & 10.615767 \\
\hline 0 & 15.092731 & 6.333243 & 21.240761 \\
\hline 0 & 12.559435 & 6.334942 & 22.169991 \\
\hline 0 & 13.305635 & 8.164519 & 20.402557 \\
\hline 0 & 14.325412 & 8.187822 & 22.858662 \\
\hline O & 22.171206 & 2.778228 & 24.604977 \\
\hline 0 & 21.076178 & 0.332319 & 438 \\
\hline 0 & 22.922504 & 0.921706 & 22.842154 \\
\hline 0 & 20.560099 & 2.187141 & 22.747971 \\
\hline 0 & 22.161737 & 15.081808 & 12.478601 \\
\hline 0 & 21.239546 & 12.552153 & 12.565260 \\
\hline 0 & 23.001154 & 13.240337 & 10. \\
\hline 0 & 20.486790 & 14.258414 & 10.699272 \\
\hline 0 & 9.962781 & 3.043792 & 12.395096 \\
\hline 0 & 8.937179 & 0.590601 & 12.722075 \\
\hline 0 & 10.622322 & 1.081919 & 10.706312 \\
\hline 0 & 8.173742 & 2.181801 & 10.702428 \\
\hline 0 & 9.856 & 15.0 & 24 . \\
\hline 0 & 8.930383 & 12.550453 & 0.435001 \\
\hline 0 & 10.800983 & 13.298596 & 22.998968 \\
\hline 0 & 8.259432 & 14.239237 & 22.766903 \\
\hline 0 & 22.167078 & 16.090418 & 18.697268 \\
\hline 0 & 21.226 & 18.618618 & 18. \\
\hline 0 & 22.923 & 17.9 & 20 . \\
\hline 0 & 20.477324 & 16.855553 & 20.480478 \\
\hline 0 & 22.147417 & 3.878353 & 6.409465 \\
\hline 0 & 21.147303 & 6.401698 & 6.328874 \\
\hline 0 & 22.919106 & 5.732932 & 8.181753 \\
\hline 0 & 20.485819 & 4.6 & 89 \\
\hline 0 & 9.943847 & 16.100370 & 6.330815 \\
\hline 0 & 9.017771 & 18.618132 & 6.254836 \\
\hline 0 & 10.692233 & 17.932617 & 8.165490 \\
\hline 0 & 8.185881 & 16.870119 & 8.094608 \\
\hline 0 & 9.955256 & 3.787323 & 18.700666 \\
\hline 0 & 8.929654 & 6.234688 & 18.462532 \\
\hline 0 & 10.692233 & 5.750896 & 20.389692 \\
\hline 0 & 8.262102 & 4.632079 & 20.468828 \\
\hline 0 & 21.232992 & 16.180964 & 24.771986 \\
\hline 0 & 22.328747 & 18.626385 & 0.41800 \\
\hline 0 & 20.476109 & 17.948639 & 22.920563 \\
\hline O & 22.922991 & 16.773748 & 22.91789 \\
\hline
\end{tabular}




$$
\begin{array}{rrr}
21.336645 & 3.811355 & 12.636627 \\
22.254709 & 6.323290 & 12.548997 \\
20.390421 & 5.566409 & 10.858999 \\
22.845068 & 4.552458 & 10.621837 \\
9.094721 & 15.929478 & 12.393397 \\
9.874421 & 18.451366 & 12.625219 \\
8.249237 & 17.786242 & 10.621108 \\
10.786418 & 16.932747 & 10.641499 \\
8.929169 & 3.803102 & 24.608374 \\
9.954527 & 6.326204 & 0.429903 \\
8.157722 & 5.731476 & 22.912310 \\
10.622079 & 4.633050 & 22.836813 \\
21.242216 & 2.863917 & 18.542639 \\
22.319281 & 0.425291 & 18.546522 \\
20.402800 & 1.020990 & 20.313471 \\
22.830746 & 2.192724 & 20.462030 \\
21.394661 & 15.249060 & 6.246583 \\
22.343554 & 12.644882 & 6.000196 \\
20.390421 & 13.307336 & 8.164519 \\
23.014505 & 14.405761 & 8.195348 \\
8.852946 & 2.953733 & 6.492727 \\
10.027594 & 0.589873 & 6.245126 \\
8.267200 & 1.007396 & 8.257005 \\
10.628391 & 2.197822 & 8.257733 \\
8.845907 & 15.169439 & 18.791939 \\
10.036819 & 12.477872 & 18.452822 \\
8.013288 & 12.989338 & 20.395033 \\
10.688834 & 14.335364 & 20.554518 \\
24.766159 & 22.079447 & 2.866830 \\
0.496416 & 21.065012 & 0.417038 \\
23.002611 & 22.918135 & 1.012251 \\
22.850166 & 20.489462 & 2.191025 \\
24.686296 & 9.934380 & 15.084479 \\
0.504426 & 8.863384 & 12.566960 \\
22.919592 & 10.630575 & 13.230385 \\
22.923717 & 8.178355 & 14.327353 \\
12.476416 & 22.244514 & 15.003159 \\
12.638084 & 21.237362 & 12.488795 \\
10.698059 & 22.922260 & 13.145667 \\
10.794671 & 20.415180 & 14.162286 \\
12.481028 & 9.945546 & 2.944023 \\
12.570359 & 8.938149 & 0.431117 \\
10.706312 & 10.707768 & 1.088716 \\
10.630333 & 8.257733 & 2.115531 \\
18.707949 & 22.174118 & 16.086290 \\
18.535599 & 21.146818 & 18.551134 \\
20.481449 & 22.851624 & 18.030201 \\
20.483391 & 20.472469 & 16.857496 \\
18.784657 & 10.030993 & 3.705518 \\
18.791939 & 9.010974 & 6.243184 \\
20.564955 & 10.796371 & 5.488972 \\
20.571995 & 8.330314 & 4.472595 \\
6.323047 & 22.092072 & 3.792663 \\
6.239058 & 20.981993 & 6.412621 \\
8.171801 & 23.004309 & 5.818379 \\
8.351191 & 20.491159 & 4.481576 \\
6.326931 & 9.866653 & 16.099642 \\
6.328388 & 8.944704 & 18.619347
\end{array}
$$




\begin{tabular}{|c|c|c|c|}
\hline & 8.172529 & 10.695631 & 17.858093 \\
\hline 0 & 8.174956 & 8.179083 & 16.855553 \\
\hline 0 & 24.857918 & 21.233234 & 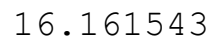 \\
\hline 0 & 0.419708 & 22.252768 & 18.6125 \\
\hline & 22.997271 & 20.381681 & 17.86367 \\
\hline 0 & 22.863760 & 22.829775 & 167693 \\
\hline$c$ & 24.691879 & 8.844208 & 3.8788 \\
\hline 0 & 0.500299 & 10.030993 & 315 \\
\hline 0 & 22.915949 & 8.272298 & 5.813 \\
\hline 0 & 23.000669 & 10.628877 & 4.3900 \\
\hline$c$ & 12.475203 & 21.408741 & 3.7096 \\
\hline 0 & 12.634929 & 22.240145 & \\
\hline O & 10.879633 & 20.318083 & 5.4848 \\
\hline 0 & 10.628391 & 22.921047 & 4.6366 \\
\hline 0 & 12.479086 & 8.850034 & 16.0940 \\
\hline 0 & 12.561133 & 9.871751 & 18.5428 \\
\hline 0 & 10.631304 & 8.176898 & 17.9576 \\
\hline 0 & 10.628148 & 10.537603 & 16.7640 \\
\hline 0 & 18.630270 & 21.244644 & 2.9292 \\
\hline 0 & 18.702608 & 22.240631 & 0.4218 \\
\hline 0 & 20.389935 & 20.319782 & 1.0950 \\
\hline 0 & 20.572481 & 22.759865 & 2.19272 \\
\hline 0 & 18.705036 & 8. & 15. \\
\hline 0 & 18.546038 & 9.869809 & 12.6613 \\
\hline 0 & 20.405228 & 8.093637 & 13.3163 \\
\hline 0 & 20.490189 & 10.633245 & 14.3152 \\
\hline 0 & 6.581572 & 21.081032 & 15.1687 \\
\hline 0 & 6.330087 & 22.162708 & 12.65 \\
\hline 0 & 8.254578 & 20. & 13. \\
\hline 0 & 8.264287 & 22.849922 & 14.3275 \\
\hline 0 & 6.425729 & 8.940578 & 2.9454 \\
\hline 0 & 6.315765 & 9.952100 & 0.4915 \\
\hline 0 & 8.175442 & 8.183210 & 1.0229 \\
\hline 0 & 8.261374 & 10.6 & 8 \\
\hline 0 & 13.233784 & 5.219282 & 8.2596 \\
\hline 0 & 12.467434 & 6.662406 & 5.9608 \\
\hline 0 & 11.806194 & 7.754035 & 8.4300 \\
\hline 0 & 14.488052 & 7.753793 & 7.5884 \\
\hline 0 & 8.270599 & 18.026802 & 19.388123 \\
\hline 0 & 6.580116 & 19.721655 & 62 \\
\hline 0 & 7.582899 & 19.4 & 21.9204 \\
\hline $\mathrm{H}$ & 12.712851 & 22.185770 & 3.1675 \\
\hline $\mathrm{H}$ & 15.863208 & 7.983673 & 7.9234 \\
\hline $\mathrm{H}$ & 25.047745 & 6.148513 & 1.7116 \\
\hline $\mathrm{H}$ & 21.949335 & 16.018564 & 6.480590 \\
\hline $\mathrm{H}$ & 13.582608 & 4.993285 & 9.1777 \\
\hline $\mathrm{H}$ & 9.142057 & 15.978513 & 19.27791 \\
\hline $\mathrm{H}$ & 10.677424 & 18.555988 & 21.64857 \\
\hline $\mathrm{H}$ & 12.173469 & 19.631353 & 23.2875 \\
\hline $\mathrm{H}$ & 12.778150 & 20.800903 & 22.036482 \\
\hline $\mathrm{H}$ & 13.280390 & 19.106779 & 22.02920 \\
\hline $\mathrm{H}$ & 11.802795 & 20.724924 & 19.75005 \\
\hline $\mathrm{H}$ & 10.963137 & 19.220385 & 19.2584 \\
\hline $\mathrm{H}$ & 9.520255 & 21.166237 & 18.9623 \\
\hline $\mathrm{H}$ & 9.604488 & 21.587887 & 20.69263 \\
\hline $\mathrm{Re}$ & 12.987638 & 6.883791 & 7.560810 \\
\hline $\mathrm{Re}$ & 7.914976 & 19.622858 & 20.2722 \\
\hline C & 11.318760 & 19.382296 & 21.3313 \\
\hline
\end{tabular}




$\begin{array}{rrrr}\text { C } & 12.415243 & 19.781128 & 22.223152 \\ \mathrm{C} & 11.027223 & 20.000813 & 20.037710 \\ \mathrm{C} & 9.609586 & 20.745800 & 19.980907\end{array}$

596

USY_ReO3_C4H8_M4 E $=-7211.89800005573$

$\mathrm{Si}-23 . \overline{3} 8250 \overline{7}$

$3.326106 \quad 1.243588$

Si 23.428143

$1.237034 \quad 3.375869$

Si 23.398529

15.571184

13.401278

Si 23.391973

13.427009

15.573612

Si 11.248607

1.239946

15.579194

15.587449

11.163160

13.409775

3.351837

5.500138

15.498846

5.426829

1.192611

7.633390

7.658150

7.787048

20.002028

19.907356

15.644493

19.882839

17.805418

3.370043

7.626108

7. 531195

7.761318

15.550309

3. 366401

1. 218099

3. 346739

13.281118

15.513167

1.267377

3. 349895

5.510577

13.328940

17.721672

19.916580

3. 340670

1. 201107

19.865602

15.590118

20.010279

13.381372

3. 350380

23.434214

23.370371

17.739391

3.364460

5.535094

11.267783

11.238896

15.650561

11.185735

17.781872

1. 308644

3. 379752

23.402899

23.428387

1.255968

3. 411309

11.233070

11.214865

13.458323

23.448776

15.607840

23.403870

13.425309

11.233799

11.173841

7.681211

7.602562

1. 360106

3. 381452

1. 306702

19.897404

3. 424661

19.897161

7. 642614

7. 632420

15.585021

15.556620

5.526841

3. 405969

19.952265

7. 662277

7.665433

5.523200

19.985521

3.441167

19.888180

7.679270

7.743597

15.481126

17.744732

15.622161

5.433869

3.279499

5.536308

15.564145

17.781385

15.575554

5.450133

3.226094

3. 334602

1.203292

15.578223

13.384286

15.529431

13.414386

3. 332174

1. 198437

3. 342127

5.525627

15.592546

17.756384

15.603227

17.789883

5.499410

3.378539

1.181930

15.688915

13.449584

15.688674

15.618278

17.722399

19.966101

13.470218

3. 359847

1. 230237

3. 377568

5.511062

23.400957

11.187192

15.601042 


\begin{tabular}{|c|c|c|c|}
\hline $\mathrm{Si}$ & .364460 & 11.235498 & 3 \\
\hline , & 17.722399 & 23.384207 & 15.614635 \\
\hline$\perp$ & 15.541327 & 23.389305 & 17.744732 \\
\hline$S$ & 17.821440 & 11.269726 & 3.30280 \\
\hline & 15.662700 & 11.239625 & 5.47052 \\
\hline$i$ & 3.358391 & 1.277572 & 23.36794 \\
\hline 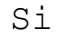 & 1.242374 & 3.365430 & 23.4147 \\
\hline i & 3.396987 & 13.389383 & 11.22336 \\
\hline L & 1.288738 & 15.562931 & 11.25807 \\
\hline i & 15.613908 & 1.222954 & 11.2051 \\
\hline I & 13.360253 & 3.333631 & 11.2493 \\
\hline$i$ & 15.561717 & 13.424338 & 23.3917 \\
\hline i & 13.447642 & 15.568028 & 23.41042 \\
\hline i & 3.359847 & 1.200864 & 7.65912 \\
\hline 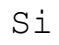 & 5.517616 & 3.318338 & 7.6195 \\
\hline i & 3.294791 & 13.366565 & 19.9629 \\
\hline$i$ & 17.774347 & 3.372713 & 19.86147 \\
\hline$i$ & 15.641824 & 13.429436 & \\
\hline $\mathrm{Si}$ & 17.823381 & 15.573612 & 7.71786 \\
\hline $\mathrm{Si}$ & 3.339457 & 5.433141 & 7.65572. \\
\hline i & 1.241889 & 3.327319 & 7.66349 \\
\hline $\mathrm{Si}$ & 3.422476 & 17.736237 & 19.85832 \\
\hline $\mathrm{Si}$ & 1.258152 & 15.579680 & 19.8724 \\
\hline $\mathrm{Si}$ & 15.565116 & 5.466882 & 19 \\
\hline $\mathrm{Si}$ & 13.394238 & 3.388491 & 19.95299 \\
\hline $\mathrm{Si}$ & 15.665128 & 17.779203 & 7.63508 \\
\hline Si & 13.468276 & 15.648378 & 7.62659 \\
\hline $\mathrm{Si}$ & 3.377568 & 5.510819 & 23.38202 \\
\hline $\mathrm{Si}$ & 5.503537 & 5792 & \\
\hline $\mathrm{Si}$ & 5.587285 & 15.553465 & 11.13937 \\
\hline $\mathrm{Si}$ & 17.857609 & 3.318823 & 11.25418 \\
\hline $\mathrm{Si}$ & 15.611480 & 17.757597 & 23.44028 \\
\hline Si & 17.728468 & 15.614392 & 23.42741 \\
\hline$i$ & 1.644119 & 21.675276 & 23.7274 \\
\hline $\mathrm{Si}$ & 1.613533 & 23.783768 & 21 . \\
\hline $\mathrm{Si}$ & 1.678103 & 9.445974 & 11.583110 \\
\hline $\mathrm{Si}$ & 1.674947 & 11.595976 & 9.45665 \\
\hline $\mathrm{Si}$ & 13.878758 & 21.702219 & 11.54961 \\
\hline $\mathrm{Si}$ & 13.832881 & 23.855862 & 9.37970 \\
\hline $\mathrm{Si}$ & 13.833122 & 9.424613 & 23.787 \\
\hline $\mathrm{Si}$ & 13.8 & 11.6 & 21 . \\
\hline $\mathrm{Si}$ & 17.482323 & 21.677944 & 23.77478 \\
\hline $\mathrm{Si}$ & 17.392508 & 19.536926 & 21.68765 \\
\hline $\mathrm{Si}$ & 17.426493 & 9.517342 & 11.58942 \\
\hline $\mathrm{Si}$ & 17.450523 & 7.328259 & 9.48699 \\
\hline $\mathrm{Si}$ & 5.153012 & 21.644932 & 11.64549 \\
\hline $\mathrm{Si}$ & 5.122911 & 19.542267 & 9.49403 \\
\hline $\mathrm{Si}$ & 5.182870 & 9.434566 & 23.73279 \\
\hline $\mathrm{Si}$ & 5.152284 & 7.305198 & 21.61434 \\
\hline $\mathrm{Si}$ & 17.332306 & 21.698822 & 19.52090 \\
\hline $\mathrm{Si}$ & 17.412657 & 23.851978 & 21.66289 \\
\hline $\mathrm{Si}$ & 17.389109 & 11.698900 & 9.49136 \\
\hline $\mathrm{Si}$ & 5.103006 & 21.600266 & 7.31612 \\
\hline $\mathrm{Si}$ & 5.119513 & 23.743713 & 9.43529 \\
\hline $\mathrm{Si}$ & 5.077518 & 9.456899 & 19.52721 \\
\hline $\mathrm{Si}$ & 5.025570 & 11.627775 & 21.70343 \\
\hline $\mathrm{Si}$ & 1.576150 & 21.625998 & 19.54663 \\
\hline $\mathrm{Si}$ & 1.597026 & 19.535711 & 21.61483 \\
\hline
\end{tabular}




\begin{tabular}{|c|c|c|c|}
\hline Si & 1.631253 & 9.422914 & 7.306411 \\
\hline i & 1.623485 & 7.276068 & 9.430439 \\
\hline S & 13.875603 & 21.743244 & 7.183582 \\
\hline & 13.891382 & 19.549549 & 9.348634 \\
\hline & 13.810790 & 7.235773 & 1.661196 \\
\hline & 23.771387 & 1.592899 & 21.626968 \\
\hline i & 21.672119 & 1.535368 & 23.712 \\
\hline & 23.862173 & 13.851815 & 9.42485 \\
\hline & 21.733047 & 13.774378 & 11.58966 \\
\hline & 11.536989 & 1.651886 & 9.47316 \\
\hline & 9.414903 & 1.703591 & 11.6280 \\
\hline i & 11.664673 & 13.825356 & 21.677 \\
\hline i & 9.470007 & 13.762241 & 23. \\
\hline S & 23.813381 & 17.348085 & 21.702219 \\
\hline & 21.689596 & 17.390566 & 19.560472 \\
\hline & 21.678915 & 5.179714 & 7.25179 \\
\hline & 11.670013 & 17.394207 & 9.35761 \\
\hline i & 9.463452 & 17.391537 & \\
\hline $\mathrm{Si}$ & 11.577527 & 381 & 21.63862 \\
\hline S & 9.475104 & 5.111988 & 19.509981 \\
\hline $\mathrm{Si}$ & 19.531342 & 17.386196 & 21.69736 \\
\hline L & 21.720425 & 17.394207 & 23.82309 \\
\hline 1 & 19.580864 & 5.151313 & 9.4925 \\
\hline i & 21.729650 & 3051 & 11.6 \\
\hline i & 7.286507 & 17.3 & 9.35664 \\
\hline $\mathrm{Si}$ & 9.496709 & 17.289583 & 11.55738 \\
\hline 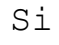 & 7.294032 & 5.155439 & 21.64857 \\
\hline$i$ & 9.408349 & 5.116600 & 23.7774 \\
\hline$i$ & 19.552217 & 1.6 & 21 \\
\hline $\mathrm{Si}$ & 21.679401 & 1.608435 & 19.48327 \\
\hline $\mathrm{Si}$ & 19.554646 & 13.829239 & 9.430924 \\
\hline $\mathrm{Si}$ & 7.308111 & 1.607949 & 9.435537 \\
\hline Si & 9.425342 & 1.659169 & 7.29451 \\
\hline$i$ & 7.251794 & 13.689174 & 21.6259 \\
\hline $\mathrm{Si}$ & 21.702705 & 23.7 & 1. \\
\hline $\mathrm{Si}$ & 23.836685 & 21.646389 & 1.617902 \\
\hline $\mathrm{Si}$ & 21.714357 & 11.595976 & 13.78311 \\
\hline Si & 23.851250 & 9.410776 & 13.78894 \\
\hline $\mathrm{Si}$ & 9.487484 & 23.799303 & 13.82171 \\
\hline $\mathrm{Si}$ & 9.463 & 11.6 & 1.658 \\
\hline $\mathrm{Si}$ & 11.6 & 9. & 1 . \\
\hline Si & 21.679401 & 23.759007 & 17.386925 \\
\hline $\mathrm{Si}$ & 19.527700 & 21.672119 & 17.39979 \\
\hline $\mathrm{Si}$ & 21.815338 & 11.644768 & 4.88210 \\
\hline $\mathrm{Si}$ & 19.640335 & 9.5 & 5.008 \\
\hline $\mathrm{Si}$ & 9.359072 & $23 . \varepsilon$ & \\
\hline $\mathrm{Si}$ & 9.466851 & 11.541600 & 17.29541 \\
\hline $\mathrm{Si}$ & 7.261018 & 9.422428 & 17.36896 \\
\hline Si & 21.694210 & 19.522604 & 17.34298 \\
\hline $\mathrm{Si}$ & 23.834015 & 21.680857 & 17.35075 \\
\hline $\mathrm{Si}$ & 21.704163 & 7.373652 & 5.12145 \\
\hline $\mathrm{Si}$ & 23.813866 & 9.446703 & 5.13116 \\
\hline $\mathrm{Si}$ & 9.483600 & 19.528429 & 4.97799 \\
\hline $\mathrm{Si}$ & 11.570731 & 21.726980 & 5.12849 \\
\hline $\mathrm{Si}$ & 9.434566 & 7.261018 & 17.32211 \\
\hline $\mathrm{Si}$ & 11.576799 & 9.366111 & 17.361921 \\
\hline $\mathrm{Si}$ & 21.696878 & 19.507311 & 1.63465 \\
\hline $\mathrm{Si}$ & 19.557802 & 21.628910 & 1.65188 \\
\hline
\end{tabular}




\begin{tabular}{|c|c|c|c|}
\hline $\mathrm{Si}$ & 21.735233 & 7.231161 & 13.784331 \\
\hline $\mathrm{Si}$ & 19.579407 & 9.401310 & 13.840405 \\
\hline $\mathrm{Si}$ & 9.499136 & 19.537653 & $13.7794^{\circ}$ \\
\hline$i$ & 7.349620 & 21.622356 & 13.791128 \\
\hline 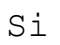 & 9.428740 & 7.297673 & 1.61304 \\
\hline 1 & 7.313451 & 9.422671 & 1.6632 \\
\hline $\mathrm{Si}$ & 11.669286 & 21.731592 & 13.6901 \\
\hline $\mathrm{Si}$ & 11.213165 & 3.402813 & 13.3765 \\
\hline i & 20.023146 & 3.292121 & 13.41827 \\
\hline i & 7.629263 & 1.210089 & 3.36615 \\
\hline$i$ & 23.372797 & 15.606626 & 17.72434 \\
\hline$i$ & 5.442608 & 15.544239 & 19.9770 \\
\hline $\mathrm{Si}$ & 3.483405 & 17.703222 & 11.2903 \\
\hline $\mathrm{Si}$ & 13.798167 & 9.427041 & 19.5082 \\
\hline i & 23.792747 & 5.133593 & 9.422 \\
\hline 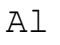 & 15.580408 & 1.280485 & 19.89716 \\
\hline 1 & 15.576768 & 5.469795 & 11.20636 \\
\hline 1 & 17.524563 & 9.603031 & 7.205914 \\
\hline 1 & 9.453985 & 13.517068 & 19.643 \\
\hline Al & 7.260290 & 21.632309 & 5.16879 \\
\hline 1 & 7.725634 & 17.780415 & 15.45078 \\
\hline Al & 23.371826 & 5.398428 & 3.40863 \\
\hline Al & 13.455168 & 19.860992 & 3.28702 \\
\hline Al & 21.793736 & 13.678493 & \\
\hline Al & 16.715733 & 5.191366 & 13.77680 \\
\hline Al & 6.748096 & 18.186287 & 18.08409 \\
\hline 0 & 22.164165 & 0.349069 & 2.86610 \\
\hline 0 & 24.605705 & 24.542105 & 3.95578 \\
\hline 0 & 24.029911 & 2.099510 & 2.117473 \\
\hline 0 & 22.847010 & 2.185685 & $4.55^{7}-x$ \\
\hline 0 & 22.1 & 12.4 & 15. \\
\hline 0 & 24.610802 & 12.475930 & 16.09672 \\
\hline 0 & 23.941551 & 14.330509 & 14.32929 \\
\hline 0 & 22.846039 & 14.397508 & 16.77180 \\
\hline 0 & 10.024681 & 0.249786 & 15.17 \\
\hline 0 & 12.558949 & 24.693094 & 16. \\
\hline 0 & 11.644768 & 2.102180 & 14.25064 \\
\hline 0 & 10.706798 & 2.192482 & 16.77933 \\
\hline 0 & 9.944575 & 12.480542 & 2.94645 \\
\hline 0 & 12.480785 & 12.472047 & 3.79654 \\
\hline 0 & 11.714679 & 14.332694 & 2.10387 \\
\hline 0 & 10.7820 & 14.322742 & 4.627 \\
\hline 0 & 8.928440 & 6.407765 & 2.87945 \\
\hline 0 & 6.415291 & 6.409223 & 3.88369 \\
\hline 0 & 7.077987 & 4.629166 & 2.08980 \\
\hline 0 & 8.181511 & 4.555614 & 4550 \\
\hline 0 & 9.009517 & 18.866461 & 15.17283 \\
\hline 0 & 6.319649 & 18.635 & 16.344 \\
\hline 0 & 7.248153 & 16.688301 & 14.24579 \\
\hline 0 & 7.933667 & 17.023291 & 17.11068 \\
\hline 0 & 21.307030 & 6.237601 & 15.00874 \\
\hline 0 & 18.793636 & 6.402911 & 16.02172 \\
\hline 0 & 19.298307 & 4.565081 & 14.24724 \\
\hline 0 & 20.480722 & 4.467497 & 16.69242 \\
\hline 0 & 21.242216 & 18.547979 & 2.86780 \\
\hline 0 & 18.719114 & 18.785870 & 3.88417 \\
\hline 0 & 19.283983 & 16.851427 & 2.17670 \\
\hline 0 & 20.398916 & 16.855553 & 4.56289 \\
\hline
\end{tabular}




$$
\begin{aligned}
& \begin{array}{lll}
8.930868 & 0.342515 & 3.795576
\end{array} \\
& \begin{array}{lll}
6.401454 & 24.609833 & 2.782355
\end{array} \\
& \begin{array}{lll}
7.082600 & 2.106307 & 4.635963
\end{array} \\
& \begin{array}{lll}
8.175685 & 2.190297 & 2.188112
\end{array} \\
& \begin{array}{lll}
9.018013 & 12.468648 & 16.011040
\end{array} \\
& \begin{array}{lll}
6.494426 & 12.397524 & 15.092975
\end{array} \\
& \begin{array}{lll}
7.254464 & 14.324684 & 16.762096
\end{array} \\
& \begin{array}{lll}
8.254578 & 14.244093 & 14.245548
\end{array} \\
& \begin{array}{lll}
21.147303 & 0.333048 & 16.102312
\end{array} \\
& \begin{array}{lll}
18.632696 & 24.611044 & 15.009470
\end{array} \\
& \begin{array}{lll}
19.297577 & 2.109220 & 16.852398
\end{array} \\
& 20.475866 \quad 2.118930 \quad 14.410615 \\
& \begin{array}{lll}
21.318924 & 12.466706 & 3.547490
\end{array} \\
& \begin{array}{lll}
18.707462 & 12.550453 & 2.783326
\end{array} \\
& \begin{array}{lll}
19.559015 & 14.239480 & 4.634992
\end{array} \\
& 20.480722 \quad 14.411345 \quad 2.127183 \\
& \begin{array}{lll}
22.257866 & 6.572834 & 3.874469
\end{array} \\
& \begin{array}{lll}
24.775141 & 6.401454 & 2.621171
\end{array} \\
& \begin{array}{lll}
24.110989 & 4.552458 & 4.717040
\end{array} \\
& \begin{array}{lll}
22.759865 & 4.485460 & 2.116017
\end{array} \\
& \begin{array}{lll}
22.085758 & 18.627356 & 16.027788
\end{array} \\
& \begin{array}{lll}
24.600121 & 18.709404 & 15.090062
\end{array} \\
& \begin{array}{lll}
23.934027 & 16.861380 & 16.836618
\end{array} \\
& \begin{array}{lll}
22.862303 & 16.790012 & 14.344104
\end{array} \\
& \begin{array}{lll}
10.025652 & 6.495640 & 16.006186
\end{array} \\
& \begin{array}{lll}
12.557007 & 6.406309 & 15.013597
\end{array} \\
& \begin{array}{lll}
11.726088 & 4.641546 & 16.856039
\end{array} \\
& \begin{array}{lll}
10.771611 & 4.635720 & 14.326383
\end{array} \\
& \begin{array}{lll}
9.877577 & 18.607695 & 3.705760
\end{array} \\
& \begin{array}{lll}
12.373249 & 18.699696 & 2.691325
\end{array} \\
& \begin{array}{lll}
11.724874 & 16.849241 & 4.470410
\end{array} \\
& \begin{array}{lll}
10.616738 & 16.837349 & 2.026929
\end{array} \\
& \begin{array}{lll}
2.950335 & 22.170477 & 0.256582
\end{array} \\
& \begin{array}{lll}
3.969868 & 24.613958 & 24.528997
\end{array} \\
& \begin{array}{lll}
2.098539 & 24.022387 & 2.031298
\end{array} \\
& \begin{array}{lll}
4.488859 & 22.856720 & 2.195152
\end{array} \\
& \begin{array}{lll}
2.951791 & 9.944818 & 12.475445
\end{array} \\
& \begin{array}{lll}
3.891461 & 12.468162 & 12.474232
\end{array} \\
& \begin{array}{lll}
2.185199 & 11.732399 & 14.334394
\end{array} \\
& \begin{array}{lll}
4.634264 & 10.705098 & 14.330752
\end{array} \\
& \begin{array}{lll}
15.156817 & 22.160040 & 12.463794
\end{array} \\
& \begin{array}{lll}
16.174652 & 24.616144 & 12.456753
\end{array} \\
& \begin{array}{lll}
14.334150 & 24.015589 & 14.247249
\end{array} \\
& \begin{array}{lll}
16.772291 & 22.846283 & 14.401391
\end{array} \\
& \begin{array}{lll}
15.166041 & 9.859371 & 0.341058
\end{array} \\
& \begin{array}{lll}
16.102798 & 12.397524 & 24.524143
\end{array} \\
& \begin{array}{lll}
14.316188 & 11.715164 & 2.037610
\end{array} \\
& \begin{array}{lll}
16.860165 & 10.709952 & 2.111647
\end{array} \\
& \begin{array}{lll}
2.868287 & 8.859015 & 6.407765
\end{array} \\
& \begin{array}{lll}
3.895345 & 6.406552 & 6.478890
\end{array} \\
& \begin{array}{lll}
2.193938 & 6.995939 & 4.550759
\end{array} \\
& \begin{array}{lll}
4.555371 & 8.176898 & 4.566052
\end{array} \\
& \begin{array}{lll}
2.866102 & 21.059914 & 18.712803
\end{array} \\
& \begin{array}{lll}
4.132265 & 18.627842 & 18.623472
\end{array} \\
& \begin{array}{lll}
2.281327 & 19.283499 & 16.782001
\end{array} \\
& \begin{array}{lll}
4.645673 & 20.569569 & 16.921824
\end{array} \\
& \begin{array}{lll}
15.090062 & 8.939607 & 18.615704
\end{array} \\
& \begin{array}{llll}
0 & 16.096243 & 6.420874 & 18.711103
\end{array}
\end{aligned}
$$




$$
\begin{array}{rrr}
14.413771 & 7.080657 & 16.775690 \\
16.860409 & 8.098735 & 16.851185 \\
15.174537 & 21.323536 & 6.324019 \\
16.179506 & 18.798977 & 6.485202 \\
14.261812 & 19.389578 & 4.733061 \\
16.773748 & 20.477566 & 4.553672 \\
3.862331 & 8.842751 & 0.184487 \\
2.779684 & 6.406795 & 24.620270 \\
4.638633 & 7.151297 & 2.101209 \\
2.207775 & 8.266715 & 2.103879 \\
3.883936 & 21.158712 & 12.558221 \\
3.023401 & 18.615219 & 12.558949 \\
4.634264 & 19.383511 & 14.483439 \\
2.187870 & 20.399887 & 14.334394 \\
16.168097 & 8.854646 & 12.470105 \\
15.336206 & 6.239300 & 12.817474 \\
16.948282 & 7.075317 & 14.349444 \\
14.417897 & 8.253120 & 14.405275 \\
16.178535 & 21.232748 & 0.341058 \\
15.078652 & 18.729067 & 24.614685 \\
16.858709 & 19.382540 & 2.109948 \\
14.426637 & 20.633167 & 2.102666 \\
3.801402 & 22.168535 & 6.491513 \\
2.778956 & 24.612261 & 6.414805 \\
4.638148 & 24.019472 & 4.635235 \\
2.279870 & 22.841913 & 4.487645 \\
3.806015 & 9.944575 & 18.629784 \\
2.861004 & 12.467191 & 18.695568 \\
4.625040 & 11.793571 & 16.848028 \\
2.121600 & 10.713108 & 16.850456 \\
16.025604 & 22.085030 & 18.622988 \\
14.924509 & 24.537008 & 18.635124 \\
16.846571 & 23.934027 & 16.861135 \\
14.410615 & 22.835602 & 16.680048 \\
16.094301 & 9.961810 & 6.325232 \\
15.169683 & 12.487824 & 6.410436 \\
16.921337 & 11.802067 & 4.557313 \\
14.400663 & 10.788117 & 4.541049 \\
0.343971 & 2.868287 & 22.162466 \\
24.608860 & 3.964285 & 24.614927 \\
2.110676 & 2.115046 & 24.016075 \\
2.193210 & 4.554886 & 22.837299 \\
0.428447 & 15.157302 & 9.949187 \\
24.621239 & 16.100613 & 12.476658 \\
2.122571 & 14.253559 & 11.795513 \\
2.265306 & 16.768650 & 10.791030 \\
12.384171 & 2.939897 & 10.027108 \\
12.552638 & 3.874712 & 12.552395 \\
14.335608 & 2.112133 & 11.726088 \\
14.249918 & 4.556342 & 10.627663 \\
12.635900 & 15.075983 & 22.081631 \\
12.393882 & 16.092604 & 24.526327 \\
14.332209 & 14.322742 & 24.015589 \\
14.413043 & 16.780302 & 22.925659 \\
& 2.877511 & 8.853189 \\
\hline & 3.954575 & 6.405338 \\
\hline & 4.041251 & 7.083086 \\
\hline
\end{array}
$$




\begin{tabular}{|c|c|c|c|}
\hline & 6.504622 & 15.084964 & 21.077148 \\
\hline $\mathrm{O}$ & 6.169632 & 16.107168 & 18.52710 \\
\hline & 4.545176 & 14.317159 & 45900 \\
\hline 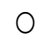 & 4.642517 & 16.856281 & 20.484362 \\
\hline 0 & 18.704550 & 2.945723 & 21.146818 \\
\hline 0 & 18.797035 & 3.975937 & 18.7040 \\
\hline O & 16.932503 & 2.191268 & $19.2152 \varepsilon$ \\
\hline 0 & 16.857737 & 4.623097 & 20.3991 \\
\hline 0 & 18.706734 & 15.176722 & 9.0189 \\
\hline & 18.802620 & 16.017109 & 6.4837 \\
\hline O & 16.933474 & 14.326383 & 7. \\
\hline 0 & 16.924978 & 16.862350 & 8.1026 \\
\hline 0 & 0.417280 & 3.879567 & 8.95028 \\
\hline 0 & 24.523170 & 2.692296 & 6.56433 \\
\hline 0 & 2.113104 & 4.556828 & 7.00783 \\
\hline O & 2.208018 & & \\
\hline O & 0.423349 & 16.1 & 21. \\
\hline O & 24.619783 & 15.081324 & 18.631 \\
\hline O & 2.263121 & 16.762825 & 19.28762 \\
\hline O & 2.104608 & 14.331238 & 20.47077 \\
\hline O & 12.393882 & 3.869371 & 21.15 \\
\hline 0 & 12.4 & & 18. \\
\hline 0 & 14.24 & 4.6 & 19. \\
\hline 0 & 14.328082 & 2.278414 & 20.55403 \\
\hline $\mathrm{O}$ & 12.566231 & 16.099886 & 8.92310 \\
\hline 0 & 12.483698 & 15.238137 & 6.40946 \\
\hline 0 & 14. & 16. & \\
\hline 0 & 14.410 & 14. & 8 . \\
\hline 0 & 6.404367 & 3.871070 & 22.08575 \\
\hline 0 & 6.422574 & 2.790123 & 24.54234 \\
\hline O & 4.637905 & 4.643488 & 23.94495 \\
\hline O & 4.482 & 2.2 & 22. \\
\hline 0 & 6.3 & 16. & \\
\hline 0 & 6.58 & 15. & 12. \\
\hline 0 & 4.724322 & 16.775 & 11.80570 \\
\hline$\Omega$ & 4.622612 & 14.322742 & 10.70315 \\
\hline 0 & 18.542395 & 3.958459 & 9.94457 \\
\hline O & 18.87 & & 12 \\
\hline 0 & 16.9 & 4 & 12. \\
\hline 0 & 16.844387 & 909 & 10.71650 \\
\hline 0 & 18.631241 & 16.106924 & 22.160280 \\
\hline 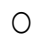 & 18.712803 & 15.083508 & 24.60473 \\
\hline 0 & 16.8 & 16.8 & 24. \\
\hline 0 & 16. & 14. & \\
\hline 0 & 2.866 & 24.6 & 22.079933 \\
\hline 0 & 0.428932 & 0.421650 & 20.98587 \\
\hline 0 & 1.008124 & 22.911095 & 22.85210 \\
\hline 0 & 2.199521 & 22.772243 & 20.48363 \\
\hline O & 2.957617 & 12.4 & 9.950400 \\
\hline 0 & 0.5 & 12. & 8 . \\
\hline 0 & 1.09 & 10.6 & 10.7084 \\
\hline 0 & 2.191025 & 10.617467 & 8.26040 \\
\hline 0 & 15.163614 & 24.694063 & 9.86762 \\
\hline 0 & 12.566717 & 0.510009 & 8.94106 \\
\hline 0 & 13.319958 & 22.930029 & 10.62256 \\
\hline O & 14.328082 & 23.010862 & 8.09703 \\
\hline 0 & 15.080837 & 12.562591 & 22.08187 \\
\hline & & 12 & \\
\hline
\end{tabular}




\begin{tabular}{|c|c|c|c|}
\hline & 13.313404 & 10.709710 & 22.911339 \\
\hline & 14.254530 & 10.701215 & 20.395519 \\
\hline & 16.111780 & 18.630754 & 22.157612 \\
\hline & 18.622501 & 18.609392 & 21.148516 \\
\hline & 18.036026 & 20.392363 & 22.922747 \\
\hline & 16.854824 & 20.475624 & 20.483150 \\
\hline & 16.187031 & 6.496853 & 10.021040 \\
\hline & 18.701151 & 6.423059 & 9.007575 \\
\hline & 18.031172 & 8.346093 & 10.621593 \\
\hline & 16.861622 & 8.170102 & 8.179811 \\
\hline & 3.880780 & 18.626627 & 10.028079 \\
\hline & 6.340282 & 18.616674 & 8.937422 \\
\hline & 5.730505 & 20.392363 & 10.777922 \\
\hline & 4.475750 & 20.480478 & 8.351676 \\
\hline & 3.868886 & 6.483502 & 22.175573 \\
\hline & 6.322805 & 6.325475 & 21.070351 \\
\hline & 5.814495 & 8.272783 & 22.761320 \\
\hline & 4.552944 & 8.190249 & 20.393576 \\
\hline & 16.182419 & 24.772226 & 21.315283 \\
\hline & 18.706007 & 0.416795 & 22.234320 \\
\hline & 17.949852 & 22.928816 & 20.390421 \\
\hline & 16.938087 & 22.846039 & 22.838757 \\
\hline & 16.093088 & 12.547297 & 8.945918 \\
\hline & 18.530258 & 12.718919 & 10.039974 \\
\hline & 18.034084 & 10.707525 & 8.421829 \\
\hline & 16.856525 & 10.792001 & 10.794671 \\
\hline & 3.814268 & 24.591139 & 8.947132 \\
\hline & 6.323776 & 0.432331 & 9.958411 \\
\hline & 5.723950 & 22.830017 & 8.247052 \\
\hline & 4.630380 & 22.847494 & 10.709710 \\
\hline & 3.710858 & 12.472531 & 21.239788 \\
\hline & 6.158223 & 12.655562 & 22.248156 \\
\hline & 5.573690 & 10.705098 & 20.471498 \\
\hline & 4.556828 & 10.697574 & 22.928572 \\
\hline & 2.873870 & 18.716688 & 20.995344 \\
\hline & 0.503455 & 18.536083 & 22.252039 \\
\hline & 0.923163 & 20.394547 & 20.398432 \\
\hline & 2.196851 & 20.486063 & 22.759378 \\
\hline & 2.857120 & 6.327902 & 8.934994 \\
\hline & 0.426262 & 6.338826 & 10.029536 \\
\hline & 1.020747 & 8.176413 & 8.189764 \\
\hline & 2.191025 & 8.248265 & 10.614797 \\
\hline & 15.171138 & 18.630754 & 8.941305 \\
\hline & 12.635900 & 18.626871 & 9.790189 \\
\hline ) & 13.393024 & 20.481449 & 8.098977 \\
\hline & 14.343861 & 20.470526 & 10.615525 \\
\hline & 15.092731 & 6.333486 & 21.240761 \\
\hline 0 & 12.559435 & 6.335185 & 22.170235 \\
\hline & 13.305394 & 8.164276 & 20.402315 \\
\hline 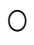 & 14.324441 & 8.190007 & 22.858175 \\
\hline 0 & 22.171206 & 2.777985 & 24.604733 \\
\hline 0 & 21.076178 & 0.332319 & 0.353438 \\
\hline 0 & 22.922504 & 0.921706 & 22.842154 \\
\hline 0 & 20.560099 & 2.187141 & 22.747484 \\
\hline o & 22.161495 & 15.081324 & 12.478358 \\
\hline 0 & 21.239788 & 12.552153 & 12.565746 \\
\hline 0 & 23.001396 & 13.240337 & 10.703642 \\
\hline 0 & 20.486305 & 14.258414 & 10.698787 \\
\hline
\end{tabular}




$\begin{array}{rrr}9.962781 & 3.043792 & 12.395339 \\ 8.935966 & 0.591087 & 12.722075 \\ 10.622322 & 1.081919 & 10.706312 \\ 8.173742 & 2.181801 & 10.702671 \\ 9.863498 & 15.086421 & 24.607891 \\ 8.933052 & 12.550453 & 0.431845 \\ 10.786175 & 13.234755 & 22.939011 \\ 8.260404 & 14.238509 & 22.766418 \\ 22.167078 & 16.091146 & 18.697996 \\ 21.227409 & 18.618618 & 18.610363 \\ 22.923962 & 17.954708 & 20.479023 \\ 20.476595 & 16.855553 & 20.481693 \\ 22.147417 & 3.878353 & 6.409465 \\ 21.147303 & 6.401698 & 6.328874 \\ 22.919106 & 5.733175 & 8.181753 \\ 20.485577 & 4.640575 & 8.262831 \\ 9.944090 & 16.100128 & 6.330815 \\ 9.017771 & 18.618618 & 6.254593 \\ 10.691504 & 17.931889 & 8.166703 \\ 8.186366 & 16.870361 & 8.094365 \\ 9.955013 & 3.787566 & 18.700422 \\ 8.929411 & 6.234446 & 18.462774 \\ 10.692233 & 5.751381 & 20.389935 \\ 8.262102 & 4.632322 & 20.468584 \\ 21.232992 & 16.180477 & 24.772226 \\ 22.328989 & 18.626385 & 0.418009 \\ 20.476595 & 17.948639 & 22.919592 \\ 22.922991 & 16.773991 & 22.918861 \\ 21.336401 & 3.811355 & 12.636627 \\ 22.255680 & 6.322562 & 12.548268 \\ 20.390421 & 5.566651 & 10.858999 \\ 22.845068 & 4.552216 & 10.621837 \\ 9.093265 & 15.932147 & 12.394609 \\ 9.873693 & 18.453308 & 12.634442 \\ 8.249965 & 17.786726 & 10.622322 \\ 10.787146 & 16.933960 & 10.645140 \\ 8.929169 & 3.803344 & 24.608131 \\ 9.954285 & 6.326204 & 0.429903 \\ 8.005034 & 12.983513 & 20.396246 \\ 10.631061 & 14.342403 & 20.558643 \\ 24.766401 & 22.079205 & 2.866345 \\ 0.496658 & 21.064526 & 0.416795\end{array}$




$\begin{array}{rrr}23.002611 & 22.918377 & 1.012008 \\ 22.850409 & 20.488976 & 2.190783 \\ 24.686054 & 9.934380 & 15.084236 \\ 0.504669 & 8.863141 & 12.566960 \\ 22.920319 & 10.630818 & 13.230385 \\ 22.924688 & 8.178112 & 14.327597 \\ 12.479086 & 22.248156 & 15.003645 \\ 12.639298 & 21.238575 & 12.486854 \\ 10.697574 & 22.930271 & 13.141297 \\ 10.777679 & 20.464458 & 14.161072 \\ 12.481028 & 9.945546 & 2.944023 \\ 12.570844 & 8.939607 & 0.429418 \\ 10.706554 & 10.707525 & 1.088716 \\ 10.630818 & 8.257976 & 2.115288 \\ 18.708191 & 22.174603 & 16.085564 \\ 18.535357 & 21.146574 & 18.551620 \\ 20.481449 & 22.851137 & 18.030445 \\ 20.482906 & 20.473440 & 16.857252 \\ 18.784412 & 10.031235 & 3.705518 \\ 18.791939 & 9.010974 & 6.243184 \\ 20.564955 & 10.796371 & 5.488972 \\ 20.571751 & 8.330557 & 4.472352 \\ 6.322805 & 22.091585 & 3.792906 \\ 6.239300 & 20.981993 & 6.412378 \\ 8.171801 & 23.004553 & 5.818379 \\ 8.351191 & 20.490189 & 4.481576 \\ 6.327175 & 9.866653 & 16.099886 \\ 6.328146 & 8.944462 & 18.619102 \\ 8.172286 & 10.695874 & 17.857851 \\ 8.175200 & 8.178841 & 16.855310 \\ 24.860588 & 21.232506 & 16.169552 \\ 0.419465 & 22.253738 & 18.611336 \\ 22.997026 & 20.380468 & 17.863678 \\ 22.862061 & 22.830746 & 16.769379 \\ 24.691879 & 8.844208 & 3.878838 \\ 0.500057 & 10.030993 & 6.315765 \\ 22.915949 & 8.272298 & 5.813281 \\ 23.000425 & 10.629119 & 4.390061 \\ 12.475203 & 21.408012 & 3.709159 \\ 12.634201 & 22.239660 & 6.242213 \\ 10.879633 & 20.318567 & 5.485088 \\ 10.628391 & 22.920805 & 4.636691 \\ 12.479086 & 8.849791 & 16.093817 \\ 12.561133 & 9.871509 & 18.543123 \\ 10.631061 & 8.176898 & 17.957621 \\ 10.629119 & 10.536633 & 16.763309 \\ 18.630754 & 21.244400 & 2.930430 \\ 18.702364 & 22.240631 & 0.421407 \\ 20.389692 & 20.320267 & 1.095027 \\ 20.572237 & 22.759378 & 2.192724 \\ 18.704792 & 8.947860 & 15.164342 \\ 18.605509 & 9.880004 & 12.643425 \\ 20.409840 & 8.090482 & 13.318259 \\ 20.496016 & 10.634702 & 14.322256 \\ 6.325717 & 22.164894 & 12.656533 \\ 8.251664 & 20.417364 & 13.154891 \\ 8.264287 & 22.846523 & 14.326140\end{array}$




\begin{tabular}{|c|c|c|c|}
\hline O & 6.425972 & 8.940820 & 5480 \\
\hline 0 & 6.316008 & 9.952100 & 0.492046 \\
\hline 0 & 8.175442 & 8.183210 & 1.023174 \\
\hline 0 & 8.261617 & 10.630575 & 2.183743 \\
\hline 0 & 13.233541 & 5.220010 & 8.259675 \\
\hline 0 & 12.467434 & 6.661678 & 5.9603 \\
\hline 0 & 11.806194 & 7.754278 & 8.43032 \\
\hline 0 & 14.488294 & 7.753307 & 7.58848 \\
\hline 0 & 7.589696 & 17.863192 & 19.724569 \\
\hline 0 & 7.069977 & 20.045721 & 18.53899 \\
\hline 0 & 7.332142 & 20.069511 & 21.66435 \\
\hline $\mathrm{H}$ & 12.683477 & 22.176788 & 3.14331 \\
\hline $\mathrm{H}$ & 15.863451 & 7.983430 & 7.923 \\
\hline $\mathrm{H}$ & 25.047018 & 6.148999 & 1.71135 \\
\hline $\mathrm{H}$ & 21.949093 & 16.018808 & 6. \\
\hline $\mathrm{H}$ & 13.583581 & 4.993527 & 9.17725 \\
\hline $\mathrm{H}$ & 9.052484 & 15.934332 & 19.34612 \\
\hline $\mathrm{H}$ & 10.224218 & 19. & \\
\hline $\mathrm{H}$ & 10.262815 & 20.2 & 20 \\
\hline $\mathrm{Re}$ & 12.987153 & 6.8 & 7.56008 \\
\hline$R e$ & 7.843608 & 19.64 & 20.11636 \\
\hline $\mathrm{C}$ & 9.681438 & 19.97 & 20.07120 \\
\hline \multicolumn{4}{|c|}{608} \\
\hline USY & $\mathrm{ReO} 3 \_\mathrm{C} 4 \mathrm{H} 8$ _M & $E=-7239$ & 406549707 \\
\hline Si & $23 . \overline{3} 8275 \overline{0}$ & 3.326106 & 1.24334 \\
\hline$S$ & 23.428387 & 1.237034 & 3.37586 \\
\hline Si & 23.398529 & 15.571427 & 13.40176 \\
\hline Si & 23.392460 & 13.427252 & 15 \\
\hline $\mathrm{Si}$ & 11.248849 & 1.24 & 15 \\
\hline $\mathrm{Si}$ & 11.163403 & 15.567058 & 1.19989 \\
\hline $\mathrm{Si}$ & 11.228216 & 13.404434 & 3.36518 \\
\hline Si & 7.633148 & 3.351837 & 1.21785 \\
\hline$i$ & 7.657908 & 5.500 & 3.3464 \\
\hline $\mathrm{Si}$ & 7.7 & 15.4 & 13. \\
\hline $\mathrm{Si}$ & 20.003483 & 5.426 & 15. \\
\hline $\mathrm{Si}$ & 19.908083 & 15.644736 & 1.26786 \\
\hline $\mathrm{Si}$ & 19.883081 & 17.805418 & 3.34989 \\
\hline $\mathrm{Si}$ & 7.626108 & 3.370043 & 5.51057 \\
\hline $\mathrm{Si}$ & 7.613485 & 15.534 & 17.69642 \\
\hline $\mathrm{Si}$ & 7 . & 13. & 15 \\
\hline Si & 19.916822 & 3.340428 & 17.74 \\
\hline $\mathrm{Si}$ & 19.866089 & 1.201107 & 15.62240 \\
\hline Si & 19.934544 & 15.590118 & 5.43411 \\
\hline $\mathrm{Si}$ & 20.010523 & 13.381130 & 3.27974 \\
\hline $\mathrm{Si}$ & 23.434214 & & .53606 \\
\hline $\mathrm{Si}$ & 23.3 & 17.7 & 15.56390 \\
\hline Si & 11.236955 & 3.364945 & 17.78163 \\
\hline Si & 11.267541 & 5.535580 & 15.57579 \\
\hline $\mathrm{Si}$ & 11.251762 & 15.618762 & 5.46251 \\
\hline $\mathrm{Si}$ & 11.182337 & 17.735992 & 3.27634 \\
\hline $\mathrm{Si}$ & 1.308886 & 23.402899 & 3.3 \\
\hline $\mathrm{Si}$ & 3.379995 & 23.427902 & 1.20304 \\
\hline Si & 1.256696 & 11.233314 & 15.57895 \\
\hline $\mathrm{Si}$ & 3.411552 & 11.214622 & 13.38477 \\
\hline $\mathrm{Si}$ & 13.458566 & 23.449505 & 15.52943 \\
\hline $\mathrm{Si}$ & 15.608081 & 23.403870 & 13.41414 \\
\hline Si & 13.425309 & 11.233070 & 3.33266 \\
\hline
\end{tabular}




\begin{tabular}{|c|c|c|c|}
\hline$i$ & 15.609781 & 11.173355 & 1.199165 \\
\hline & 1.360591 & 7.681454 & 3.341641 \\
\hline & 3.381452 & 7.602562 & 5.525384 \\
\hline & 1.307673 & 19.896191 & 15.594488 \\
\hline & 3.434370 & 19.898617 & 17.743761 \\
\hline & 13.430893 & 7.642858 & 15.6041 \\
\hline & 15.584293 & 7.632420 & 17.791582 \\
\hline & 15.552735 & 19.950808 & 5.4967 \\
\hline & 5.526598 & 7.662035 & 3.37853 \\
\hline & 3.406455 & 7.666161 & 1.180 \\
\hline & 5.522228 & 19.961731 & 15.6539 \\
\hline & 3.421990 & 19.885267 & 13.431 \\
\hline & 17.878485 & 7.682668 & 15.70 \\
\hline & 15.625073 & 7.742869 & 13.483 \\
\hline & 17.723127 & 19.965614 & 3.36081 \\
\hline & 15.611237 & 19.990131 & 1.2280 \\
\hline L & 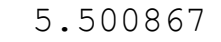 & 23. & \\
\hline 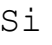 & 3.4098 & 23. & 5.5 \\
\hline & 5.516403 & 11.1 & 15.601 \\
\hline & 3.364945 & 11.236955 & 17.751 \\
\hline & 17.722885 & 23.384207 & 15.614 \\
\hline $\mathrm{Si}$ & 15.541813 & 23.389547 & 17.744 \\
\hline $\mathrm{Si}$ & 17.821682 & 11. & \\
\hline 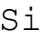 & 15.6 & 11.2 & 5.4 \\
\hline 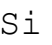 & 3.35814 & 1.27 & 23.367 \\
\hline $\mathrm{S}$ & 1.242617 & 3.365188 & 23.4145 \\
\hline SI & 3.396016 & 13.389383 & 11.2240 \\
\hline $\mathrm{Si}$ & 2 & 15. & \\
\hline $\mathrm{Si}$ & 15.6 & 1.2 & 11.26 \\
\hline & 13.36025 & 3.332417 & 11.25 \\
\hline$C_{1}$ & 15.561960 & 13.424096 & 23.39294 \\
\hline S & 13.446915 & 15.568514 & 23.41697 \\
\hline S & 3.359847 & 107 & $7.658 \varepsilon$ \\
\hline $\mathrm{Si}$ & 5.5 & & \\
\hline & 3.3 & 13.3 & $19.96221^{\circ}$ \\
\hline S & 17.774347 & 3.372470 & 19.86171 \\
\hline S & 15.643522 & 13.427494 & 7.64479 \\
\hline $\mathrm{Si}$ & 17.822653 & 15.573853 & 7.71762 \\
\hline Si & 3.3394 & 41 & 7.655 \\
\hline S & 1.2 & 3. & 7 . \\
\hline & 3.424903 & 17.7 & 19.852 \\
\hline & 1.260580 & 15.580408 & 19.87240 \\
\hline S & 15.565116 & 5.466397 & 19.94522 \\
\hline $\mathrm{Si}$ & 13.3 & 3.3 & 19.9532 \\
\hline $\mathrm{Si}$ & 15.660515 & 17. & \\
\hline $\mathrm{Si}$ & 13.465848 & 15.6 & 7.62707 \\
\hline $\mathrm{Si}$ & 3.377568 & 819 & 23.38177 \\
\hline S & 5.503294 & 3.386549 & 23.34633 \\
\hline $\mathrm{Si}$ & 5.581459 & 15.556134 & $11.14349^{\circ}$ \\
\hline $\mathrm{Si}$ & 17.857609 & 9066 & 11.25443 \\
\hline $\mathrm{Si}$ & 15.6 & 17. & 23.43615 \\
\hline Si & 17.730167 & 15.613180 & 23.42717 \\
\hline S & 1.644361 & 21.674545 & 23.72647 \\
\hline$S$ & 1.614018 & 23.782310 & 21.61264 \\
\hline $\mathrm{Si}$ & 1.678346 & 9.445974 & 11.58335 \\
\hline $\mathrm{Si}$ & 1.675190 & 11.595734 & 9.45689 \\
\hline S & 3.878758 & 21.702219 & 11.54888 \\
\hline & & & 9.3792 \\
\hline
\end{tabular}




\begin{tabular}{|c|c|c|c|}
\hline$i$ & 3.833365 & 9.422914 & 349 \\
\hline & 13.805449 & 11.633844 & 21.656826 \\
\hline & 17.479895 & 21.676973 & 212 \\
\hline & 7.393721 & 19.537411 & 21.687654 \\
\hline & 17.427464 & 9.516371 & 11.590393 \\
\hline & 17.450523 & 7.327773 & 9.487242 \\
\hline & 5.150342 & 21.645174 & 11.644768 \\
\hline & 5.122183 & 19.541780 & 9.495 \\
\hline & 5.183113 & 9.436508 & 23.7313 \\
\hline & 5.152284 & 7.305926 & 21.61410 \\
\hline & 17.333035 & 21.699306 & \\
\hline & 17.412657 & 23.851978 & 21.663 \\
\hline & 17.389595 & 11.698415 & 9.491 \\
\hline & 5.100336 & 21.600752 & 7.318 \\
\hline & 5.119270 & 23.744442 & 9.43553 \\
\hline & 5.077518 & 9.457384 & 19.52721 \\
\hline S & 5.032853 & 11.6 & \\
\hline Si & 1.581247 & 21.623 & 19. \\
\hline & 1.598968 & 19.534740 & 21.6 \\
\hline & 1.631253 & 9.422914 & 7.30641 \\
\hline & 1.623485 & 7.276311 & 9.43068 \\
\hline L & 13.875118 & 21.743000 & 71038 \\
\hline S & 13.8896 & 19.5 & \\
\hline S & 13.8 & 7.2 & 21. \\
\hline $\mathrm{Si}$ & 23.772 & 1.592656 & 21.62696 \\
\hline $\mathrm{Si}$ & 21.672604 & 1.535368 & 23.71240 \\
\hline S & 23.862173 & 13.851086 & 9.42437. \\
\hline i & 21. & 13. & \\
\hline L & 11.53 & 129 & 316 \\
\hline & 9.4151 & 1.704077 & 11.62753 \\
\hline $\mathrm{Si}$ & 11.668799 & 13.807634 & 21.708773 \\
\hline $\mathrm{Si}$ & 9.461754 & 13.765640 & 23.76871 \\
\hline L & 23.814838 & 17.348085 & $21.70197^{\circ}$ \\
\hline 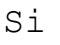 & 21.6 & 17.3 & 19. \\
\hline & 21.67 & 5.1 & 7.2 \\
\hline S & 11.674383 & 17.376728 & 9.38650 \\
\hline $\mathrm{Si}$ & 9.464181 & 17.367018 & 7.22120 \\
\hline $\mathrm{Si}$ & 11.577 & 5.157138 & 21.63862 \\
\hline 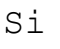 & 9.4746 & 5.1 & 19.509 \\
\hline $\mathrm{Si}$ & 19.5 & 17. & 21. \\
\hline & 21.721395 & 17.394207 & 23.823092 \\
\hline & 19.581104 & 5.151313 & 9.492582 \\
\hline $\mathrm{Si}$ & 21.72989 & 5.068051 & $11.65714^{\circ}$ \\
\hline i & 7.283108 & 17.3 & 9.35882 \\
\hline $\mathrm{Si}$ & 9.4 & 17.2 & 11.56927 \\
\hline $\mathrm{Si}$ & 7.2 & 5.1 & 21.64857 \\
\hline $\mathrm{Si}$ & 9.407864 & 5.116600 & 23.77769 \\
\hline SI & 19.552462 & 1.638050 & 21.59832 \\
\hline $\mathrm{Si}$ & 21.679886 & 1.608435 & 19.48352 \\
\hline $\mathrm{Si}$ & 19.554890 & 13.829482 & 9.43068 \\
\hline Si & & 1. & \\
\hline $\mathrm{Si}$ & 9.425342 & 1.659412 & $7.29451^{\circ}$ \\
\hline $\mathrm{SI}_{\mathrm{I}}$ & 7.263445 & 13.705195 & 21.60075 \\
\hline$S$ & 21.702948 & 23.744442 & 1.58707 \\
\hline Si & 23.837172 & 21.646389 & 1.61765 \\
\hline$S$ & 21.715084 & 11.596219 & 13.78311 \\
\hline S & 23.851978 & 9.411019 & 13.78918 \\
\hline & 9.48 & 23.80197 & 13.821 \\
\hline
\end{tabular}




\begin{tabular}{|c|c|c|c|}
\hline Si & 9.462725 & 11.604230 & 1.659897 \\
\hline Si & 11.616123 & 9.464666 & 1.646061 \\
\hline & 21.680616 & 23.758520 & 17.386925 \\
\hline & 19.528915 & 21.672119 & 17.399790 \\
\hline & 21.815582 & 11.644526 & 4.882107 \\
\hline & 19.640577 & 9.536277 & 5.008335 \\
\hline & 9.359557 & 23.822605 & 5.161751 \\
\hline & 9.467337 & 11.546213 & 17.3039 \\
\hline Si & 7.261504 & 9.423642 & 17.36944 \\
\hline & 21.695181 & 19.522604 & 17.342987 \\
\hline & 23.835714 & 21.680616 & 17.351240 \\
\hline & 21.704405 & 7.373652 & 5.1214 \\
\hline $\mathrm{Si}$ & 23.814110 & 9.446703 & 51309 \\
\hline i & 9.483114 & 19.509495 & 5.028483 \\
\hline i & 11.570488 & 21.726009 & 5.127 \\
\hline L & 9.434080 & 7.261504 & 17.322111 \\
\hline i & 11.574615 & 9.367325 & 17.36240 \\
\hline Si & 21.697123 & 19.507553 & 1.634652 \\
\hline Si & 19.557802 & 21.628668 & 1.652372 \\
\hline $\mathrm{Si}$ & 21.735962 & 7.2 & 13.78408 \\
\hline $\mathrm{Si}$ & 19.580620 & 9.401310 & $13.84137^{\circ}$ \\
\hline Si & 9.489669 & 19.556831 & 13.775592 \\
\hline $\mathrm{Si}$ & 7.339668 & 21.641533 & 13.79768 \\
\hline $\mathrm{Si}$ & 9.428497 & 673 & \\
\hline $\mathrm{Si}$ & 7.312965 & 9.4 & 1.66305 \\
\hline Si & 11.669286 & 21.734020 & 13.68966 \\
\hline $\mathrm{Si}$ & 11.212680 & 3.403056 & 13.376761 \\
\hline $\mathrm{Si}$ & 20.023146 & 3.292 & 13.41827 \\
\hline $\mathrm{Si}$ & 7.629507 & 1.210089 & 3.365916 \\
\hline $\mathrm{Si}$ & 23.374010 & 15.606626 & 17.724 \\
\hline $\mathrm{Si}$ & 5.46 & 15.5 & 19.9 \\
\hline $\mathrm{Si}$ & 3.471268 & 17.707106 & 11.29521 \\
\hline Si & 13.796953 & 9.426312 & 19.50852 \\
\hline $\mathrm{Si}$ & 23.792992 & 5.133593 & 9.42218 \\
\hline Al & 15.580651 & 9971 & 19. \\
\hline Al & 15.5 & 5.4 & 11.2 \\
\hline Al & 17.524563 & 9.603031 & 7.205672 \\
\hline Al & 9.478260 & 13.594019 & 19.605865 \\
\hline Al & 7.255435 & 21.638376 & 5.17825 \\
\hline Al & 7.727333 & 17.779203 & 15.44932 \\
\hline 1 & 23.372313 & 5.398428 & 3.40839 \\
\hline Al & 13.427980 & 19.83 & 3.29 \\
\hline Al & 21.793736 & 13.678251 & 7.23601 \\
\hline Al & 16.716703 & 5.188210 & 13.78336 \\
\hline Al & 6.781837 & 18.142107 & 18.082150 \\
\hline 0 & 22.163923 & 0.349069 & 2.8661 \\
\hline 0 & 24.605705 & 24.5 & 3.95578 \\
\hline 0 & 24.0 & 2. & 2.11747 \\
\hline 0 & 22.847010 & 2.185442 & 4.557070 \\
\hline 0 & 22.163195 & 12.473018 & 15.085692 \\
\hline O & 24.610802 & 12.475930 & 16.09672 \\
\hline O & 23.941551 & 14.330509 & 14.32929 \\
\hline 0 & 22.846039 & 14.397508 & $16.77180^{\circ}$ \\
\hline 0 & 10.025895 & 0.250271 & 15.17672 \\
\hline 0 & 12.558949 & 24.693094 & 16.09357 \\
\hline O & 11.645011 & 2.102423 & 14.25064 \\
\hline 0 & 10.707040 & 2.192482 & 16.779573 \\
\hline 0 & 9.943604 & 12.478843 & 2.94620 \\
\hline
\end{tabular}




$\begin{array}{rrr}12.481998 & 12.470590 & 3.797761 \\ 11.718805 & 14.322742 & 2.103151 \\ 10.774282 & 14.311575 & 4.626253 \\ 8.928440 & 6.407765 & 2.879453 \\ 6.415291 & 6.409223 & 3.883693 \\ 7.077987 & 4.629166 & 2.089800 \\ 8.181511 & 4.555614 & 4.550274 \\ 9.009760 & 18.870831 & 15.168225 \\ 6.322320 & 18.624443 & 16.332678 \\ 7.249124 & 16.688059 & 14.244821 \\ 7.999208 & 17.027903 & 17.100485 \\ 21.308001 & 6.237601 & 15.008499 \\ 18.796551 & 6.402183 & 16.025120 \\ 19.298307 & 4.565567 & 14.247733 \\ 20.481207 & 4.467254 & 16.692671 \\ 21.242216 & 18.547979 & 2.867801 \\ 18.719601 & 18.785627 & 3.884907 \\ 19.283983 & 16.851427 & 2.176703 \\ 20.398916 & 16.855553 & 4.562896 \\ 8.931110 & 0.342757 & 3.795334 \\ 6.401454 & 24.609589 & 2.782597 \\ 7.082600 & 2.106307 & 4.635963 \\ 8.175927 & 2.190297 & 2.188355 \\ 9.018742 & 12.470105 & 16.012011 \\ 6.493455 & 12.393397 & 15.089577 \\ 7.242084 & 14.348958 & 16.700924 \\ 8.258219 & 14.241179 & 14.238752 \\ 21.147303 & 0.332805 & 16.102554 \\ 18.632938 & 24.611288 & 15.009470 \\ 19.297577 & 2.109463 & 16.852398 \\ 20.475624 & 2.118930 & 14.410858 \\ 21.318924 & 12.466706 & 3.547490 \\ 18.707462 & 12.550453 & 2.783326 \\ 19.559015 & 14.239480 & 4.635235 \\ 20.480722 & 14.411345 & 2.127426 \\ 22.257866 & 6.572834 & 3.874226 \\ 24.775385 & 6.401454 & 2.620929 \\ 24.110989 & 4.552458 & 4.717040 \\ 22.759865 & 4.485460 & 2.116017 \\ 22.085758 & 18.627356 & 16.027788 \\ 24.599634 & 18.709404 & 15.089819 \\ 23.934027 & 16.861380 & 16.836618 \\ 22.861574 & 16.790012 & 14.344104 \\ 10.025409 & 6.495883 & 16.006428 \\ 12.557007 & 6.406309 & 15.013840 \\ 11.726088 & 4.641546 & 16.856039 \\ 10.771611 & 4.635963 & 14.326383 \\ 9.868596 & 18.529287 & 3.796304 \\ 12.305037 & 18.706491 & 2.698122 \\ 11.801825 & 16.781759 & 4.471381 \\ 10.610912 & 16.771078 & 2.099996 \\ 2.950092 & 22.170477 & 0.256825 \\ 3.969868 & 24.613958 & 24.528997 \\ 2.098782 & 24.022387 & 2.031056 \\ 4.489587 & 22.856478 & 2.195395 \\ 2.951791 & 9.944818 & 12.475445 \\ 3.891218 & 12.467920 & 12.474232 \\ 2.184957 & 11.732399 & 14.334394\end{array}$




\begin{tabular}{|c|c|c|c|}
\hline 0 & 4.633778 & 10.704613 & 14.330509 \\
\hline 0 & 15.156574 & 22.159796 & 12.463794 \\
\hline 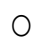 & 16.174892 & 24.615900 & 12.456753 \\
\hline 0 & 14.334150 & 24.015833 & 14.24724 \\
\hline O & 16.772291 & 22.846039 & 14.40139 \\
\hline 0 & 15.166527 & 9.859129 & 0.341058 \\
\hline 0 & 16.102798 & 12.397281 & 24.524385 \\
\hline 0 & 14.316431 & 11.714922 & 2.03785 \\
\hline 0 & 16.860409 & 10.709952 & 2.111647 \\
\hline 0 & 2.868287 & 8.859015 & 6.40776 \\
\hline 0 & 3.895345 & 6.406552 & 6.4788 \\
\hline 0 & 2.193938 & 6.995939 & 4.5507 \\
\hline 0 & 4.555371 & 8.1 & \\
\hline 0 & 2.870714 & 21.057487 & 18.70843 \\
\hline 0 & 4.128138 & 18.629784 & 18.61934 \\
\hline 0 & 2.279142 & 19.280828 & 16.782001 \\
\hline O & 4.629166 & 20.565199 & 16.860651 \\
\hline 0 & 15.089334 & 8 . & \\
\hline 0 & 16.096001 & 6.420146 & 18.711348 \\
\hline 0 & 14.414014 & 7.080901 & 16.775932 \\
\hline 0 & 16.860893 & 8.102860 & 16.858223 \\
\hline O & 15.174052 & 21.322565 & 6.32353 \\
\hline O & 16.177565 & 18.798492 & 6.48447 \\
\hline 0 & 14.256472 & 19. & \\
\hline 0 & 16.772533 & 20.476 & 4.553430 \\
\hline 0 & 3.862817 & 8.843479 & 0.183759 \\
\hline 0 & 2.779927 & 6.406795 & 24.620028 \\
\hline 0 & 4.638391 & 7.151539 & 2.100966 \\
\hline 0 & 2.208018 & 8.266715 & 37 \\
\hline 0 & 3.880052 & 21.158955 & 12.553852 \\
\hline 0 & 2.954219 & 18.615948 & 12.55190 \\
\hline 0 & 4.647857 & 19.378654 & 14.419598 \\
\hline 0 & 2.187870 & 20.401344 & 14.336578 \\
\hline 0 & 16.172224 & 8.851005 & 12.474474 \\
\hline 0 & 15.340818 & 42 & 12. \\
\hline 0 & 16.935175 & 7.071434 & 14.396295 \\
\hline 0 & 14.416442 & 8.254334 & 14.406488 \\
\hline 0 & 16.175379 & 21.229107 & 0.344214 \\
\hline 0 & 15.081080 & 18.721058 & 24.610802 \\
\hline 0 & 16.862837 & 19. & 48 \\
\hline 0 & 14.417170 & 20 & 2 . \\
\hline 0 & 3.800674 & 22.168777 & 6.491756 \\
\hline 0 & 2.778956 & 24.612501 & 6.414563 \\
\hline 0 & 4.638633 & 24.01898 & 4.635235 \\
\hline 0 & 2.279870 & 22.841913 & 4.487645 \\
\hline 0 & 3.806015 & 9.944818 & 18. \\
\hline 0 & 2.862461 & 12.46 & 18.697510 \\
\hline 0 & 4.625040 & 11.793328 & 16.847786 \\
\hline 0 & 2.122086 & 10.713594 & 16.850941 \\
\hline 0 & 16.025362 & 22.084787 & 18.623230 \\
\hline 0 & 14.924509 & 24.537008 & 18.635124 \\
\hline 0 & 16.846571 & 23.934027 & 16.86113 \\
\hline 0 & 14.410615 & 22.835602 & 16.680048 \\
\hline 0 & 16.094301 & 9.961325 & 6.325475 \\
\hline 0 & 15.170167 & 12.487339 & 6.410436 \\
\hline 0 & 16.921581 & 11.802067 & 4.55755 \\
\hline 0 & 14.401391 & 10.787874 & 4.541049 \\
\hline 0 & 0.343971 & 2.868287 & 22.16246 \\
\hline
\end{tabular}




$$
\begin{aligned}
& 24.608860 \\
& \text { 3. } 964285 \\
& \text { 2. } 115046 \\
& 4.554886 \\
& 2.193210 \\
& 0.427961 \\
& 24.620996 \\
& 2.121600 \\
& 2.263364 \\
& 12.384658 \\
& 12.552638 \\
& 14.336092 \\
& 14.251132 \\
& 12.639055 \\
& 12.389998 \\
& 14.332694 \\
& 14.412072 \\
& 6.483260 \\
& 6.396842 \\
& \text { 4. } 646644 \\
& \text { 4. } 539107 \\
& 6.498796 \\
& 6.250709 \\
& \text { 4. } 550274 \\
& \text { 4. } 649557 \\
& 18.704550 \\
& 18.796793 \\
& 16.932503 \\
& 16.857737 \\
& 18.706491 \\
& 18.802620 \\
& 16.933960 \\
& 16.922066 \\
& 0.417280 \\
& 24.523170 \\
& \text { 2. } 113104 \\
& \text { 2. } 208018 \\
& 0.423349 \\
& 24.620028 \\
& \text { 2. } 263606 \\
& \text { 2. } 106064 \\
& 12.393882 \\
& 12.470105 \\
& 14.243607 \\
& 14.327840 \\
& 12.570359 \\
& 12.484669 \\
& 14.414500 \\
& 14.414014 \\
& 6.404367 \\
& 6.422574 \\
& \text { 4. } 637905 \\
& \text { 4. } 484732 \\
& 6.332272 \\
& 6.582301 \\
& \text { 4. } 719468 \\
& \text { 4. } 621155 \\
& 18.542152 \\
& 18.873987 \\
& 15.156574 \\
& 16.100613 \\
& 14.252831 \\
& 16.765495 \\
& \text { 2. } 939168 \\
& \text { 3. } 874712 \\
& 2.111647 \\
& \text { 4. } 556828 \\
& 15.072827 \\
& 16.093817 \\
& 14.322985 \\
& 16.779089 \\
& 2.877754 \\
& \text { 3. } 954575 \\
& \text { 2. } 041251 \\
& \text { 4. } 482548 \\
& 15.092975 \\
& 16.101828 \\
& 14.323956 \\
& 16.856766 \\
& \text { 2. } 945480 \\
& \text { 3. } 975937 \\
& \text { 2. } 191268 \\
& \text { 4. } 622855 \\
& 15.176965 \\
& 16.017109 \\
& 14.326140 \\
& 16.861866 \\
& 3.879567 \\
& \text { 2. } 692296 \\
& 4.557070 \\
& \text { 2. } 179374 \\
& 16.100128 \\
& 15.081324 \\
& 16.763067 \\
& 14.331238 \\
& 3.869614 \\
& 2.867558 \\
& 4.699320 \\
& \text { 2. } 278171 \\
& 16.093817 \\
& 15.167741 \\
& 16.861380 \\
& 14.401149 \\
& 3.871070 \\
& 2.790123 \\
& \text { 4. } 643488 \\
& \text { 2. } 272102 \\
& 16.090660 \\
& 15.000246 \\
& 16.780788 \\
& 14.323470 \\
& \text { 3. } 958702 \\
& \text { 2. } 776286 \\
& \text { 4. } 540321
\end{aligned}
$$




\begin{tabular}{|c|c|c|c|}
\hline O & 16.844387 & 2.194909 & 10.716750 \\
\hline 0 & 18.631241 & 16.106924 & 22.159796 \\
\hline O & 18.713045 & 15.083508 & 24.604977 \\
\hline 0 & 16.855795 & 16.856525 & 24.020929 \\
\hline O & 16.772778 & 14.405518 & 22.919106 \\
\hline 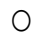 & 2.866587 & 24.693094 & 22.079689 \\
\hline O & 0.429175 & 0.421407 & 20.985876 \\
\hline 0 & 1.008367 & 22.911095 & 51864 \\
\hline 0 & 2.200007 & 22.771030 & 20.482906 \\
\hline O & 2.957617 & 12.473261 & 9.950400 \\
\hline 0 & 0.504426 & 12.577883 & 8.933781 \\
\hline 0 & 1.094056 & 10.699272 & 10.708496 \\
\hline 0 & 2.191025 & 10.617467 & 8.260404 \\
\hline 0 & 15.163614 & 24.693821 & 9.867625 \\
\hline 0 & 12.566717 & 0.510009 & 8.941063 \\
\hline 0 & 13.319958 & 22.930029 & 10.622564 \\
\hline O & 14.328082 & 23.010378 & 8.096792 \\
\hline 0 & 15.082051 & 12.562591 & 22.082361 \\
\hline 0 & 12.563075 & 12.556279 & 21.158712 \\
\hline 0 & 13.314132 & 10.708254 & 22.913521 \\
\hline 0 & 14.255501 & 10.700972 & 20.395275 \\
\hline 0 & 16.112022 & 18.630270 & 22.155184 \\
\hline O & 18.622501 & 18.609152 & 21.14827 \\
\hline 0 & 18.035299 & 20.392605 & 22.923231 \\
\hline 0 & 16.854582 & 20.475624 & 20.482906 \\
\hline 0 & 16.186789 & 6.496611 & 10.021525 \\
\hline 0 & 18.701151 & 6.423059 & 9.007575 \\
\hline 0 & 18.031416 & 8.346335 & 10.621351 \\
\hline 0 & 16.861380 & 8.169859 & 8.180054 \\
\hline 0 & 3.880052 & 18.626627 & 10.031478 \\
\hline 0 & 6.339797 & 18.616190 & 8.938149 \\
\hline 0 & 5.730505 & 20.392605 & 10.778165 \\
\hline 0 & 4.475022 & 20.479994 & 8.35288 \\
\hline 0 & 3.869128 & 6.483502 & 22.17557 \\
\hline 0 & 6.322562 & 6.3 & 21.070351 \\
\hline 0 & 5.814495 & 8.273026 & 22.761320 \\
\hline 0 & 4.552944 & 8.190493 & 20.393333 \\
\hline 0 & 16.182419 & 24.771744 & 21.315283 \\
\hline 0 & 18.706007 & 0.416795 & 22.234562 \\
\hline 0 & 17.949852 & 22.928816 & 20.39 \\
\hline 0 & 16.937843 & 22.845312 & 22.839485 \\
\hline 0 & 16.093817 & 12.546327 & 8.946646 \\
\hline 0 & 18.530500 & 12.718919 & 10.040217 \\
\hline 0 & 18.034328 & 10.70704 & 8.421829 \\
\hline 0 & 16.856525 & 10.79127 & 10.795643 \\
\hline 0 & 3.814025 & 24.591625 & 8.947132 \\
\hline 0 & 6.323776 & 0.432573 & 9.95865 \\
\hline 0 & 5.723465 & 22.830261 & 8.24753 \\
\hline 0 & 4.629894 & 22.847980 & 10.708982 \\
\hline 0 & 3.713528 & 12.473745 & 21.239304 \\
\hline 0 & 6.177157 & 12.659447 & 22.230192 \\
\hline 0 & 5.574904 & 10.704856 & 20.47077 \\
\hline 0 & 4.559498 & 10.700729 & 22.92711 \\
\hline 0 & 2.875326 & 18.714987 & 20.995344 \\
\hline 0 & 0.503698 & 18.536570 & 22.251797 \\
\hline 0 & 0.924377 & 20.395033 & 20.397945 \\
\hline 0 & 2.196851 & 20.2 & 22.758894 \\
\hline 0 & 2.857120 & 6.327902 & 8.93499 \\
\hline
\end{tabular}




$$
\begin{aligned}
& 0.426262 \\
& 6.338826 \\
& 8.176413 \\
& 8.248265 \\
& 2.191025 \\
& 15.170167 \\
& 12.634201 \\
& 13.392539 \\
& 14.343133 \\
& 15.092489 \\
& 12.559435 \\
& 13.304908 \\
& 14.324684 \\
& 22.171206 \\
& 21.076178 \\
& 22.922504 \\
& 20.560099 \\
& 22.161253 \\
& 21.239788 \\
& 23.001154 \\
& 20.486305 \\
& 9.963024 \\
& 8.935966 \\
& 10.622564 \\
& 8.173742 \\
& 9.861556 \\
& 8.928926 \\
& 10.800983 \\
& 8.263802 \\
& 22.167078 \\
& 21.227409 \\
& 22.923962 \\
& 20.476839 \\
& 22.147417 \\
& 21.147303 \\
& 22.919106 \\
& 20.485577 \\
& 9.949187 \\
& 9.018985 \\
& 10.699759 \\
& 8.180054 \\
& 9.955013 \\
& 8.929411 \\
& 10.692233 \\
& 8.262102 \\
& 21.232992 \\
& 22.328989 \\
& 20.476595 \\
& 22.923231 \\
& 21.336401 \\
& 22.255680 \\
& 20.390179 \\
& 22.845068 \\
& 9.090837 \\
& 9.866653 \\
& 8.252150 \\
& 10.803411 \\
& 8.929169 \\
& 9.954527 \\
& 8.157722
\end{aligned}
$$




$$
\begin{aligned}
& 10.622079 \quad 4.633293 \\
& \begin{array}{ll}
21.242216 & 2.863917
\end{array} \\
& \begin{array}{ll}
22.319281 & 0.425291
\end{array} \\
& 20.402800 \quad 1.020990 \\
& 22.831232 \quad 2.192724 \\
& 21.393932 \quad 15.249060 \\
& 22.343554 \quad 12.644639 \\
& 20.390421 \quad 13.307336 \\
& 23.014261 \quad 14.405518 \\
& 8.852704 \\
& 10.027837 \\
& 2.953733 \\
& 0.590115 \\
& 1.007396 \\
& 2.197822 \\
& 10.628391 \\
& 8.932810 \\
& 10.043615 \\
& 8.002364 \\
& 10.693204 \\
& 24.766401 \\
& 0.496901 \\
& 23.002611 \\
& 22.850409 \\
& 24.686296 \\
& 0.504669 \\
& 22.920319 \\
& 22.924688 \\
& 12.479329 \\
& 12.639542 \\
& 10.697330 \\
& 10.780107 \\
& 12.481028 \\
& 12.570601 \\
& 10.706312 \\
& 10.630818 \\
& 18.708191 \\
& 18.535357 \\
& 20.481693 \\
& 20.482906 \\
& 18.784657 \\
& 18.791939 \\
& 20.564955 \\
& 20.571995 \\
& 6.324747 \\
& 6.236145 \\
& 8.171558 \\
& 8.355074 \\
& 6.327175 \\
& 6.328388 \\
& 8.172286 \\
& 8.175200 \\
& 24.860344 \\
& 0.420436 \\
& 22.996784 \\
& 22.862545 \\
& 24.691879 \\
& 0.500057 \\
& 22.915949 \\
& 23.000425 \\
& 15.164827 \\
& 12.479086 \\
& 13.069930 \\
& 14.331481 \\
& 22.079205 \\
& 21.064526 \\
& 22.918135 \\
& 20.489218 \\
& 9.934623 \\
& 8.863141 \\
& 10.630575 \\
& 8.178355 \\
& 22.248398 \\
& 21.238817 \\
& 22.931244 \\
& 20.465916 \\
& 9.945303 \\
& 8.939121 \\
& 10.707283 \\
& 8.257733 \\
& 22.174360 \\
& 21.146818 \\
& 22.851379 \\
& 20.473196 \\
& 10.031235 \\
& 9.010974 \\
& 10.796371 \\
& 8.330314 \\
& 22.090370 \\
& 20.981993 \\
& 23.007465 \\
& 20.481207 \\
& 9.866168 \\
& 8.944219 \\
& 10.696360 \\
& 8.179083 \\
& 21.232264 \\
& 22.253252 \\
& 20.380711 \\
& 22.830503 \\
& 8.844208 \\
& 10.030993 \\
& 8.272298 \\
& 10.628877 \\
& 22.836813 \\
& 18.542639 \\
& 18.546764 \\
& 20.313471 \\
& 20.461546 \\
& 6.246339 \\
& 6.000196 \\
& 8.164762 \\
& 8.194619 \\
& 6.492970 \\
& 6.245126 \\
& 8.257005 \\
& 8.257733 \\
& 18.631725 \\
& 18.452579 \\
& 20.321238 \\
& 20.554758 \\
& 2.866345 \\
& 0.416552 \\
& 1.012008 \\
& 2.190783 \\
& 15.084479 \\
& 12.566960 \\
& 13.230385 \\
& 14.327597 \\
& 15.003886 \\
& 12.486854 \\
& 13.141539 \\
& 14.162286 \\
& 2.943781 \\
& 0.429903 \\
& 1.088473 \\
& 2.115531 \\
& 16.085806 \\
& 18.551376 \\
& 18.030445 \\
& 16.857252 \\
& 3.705518 \\
& 6.243427 \\
& 5.488972 \\
& 4.472595 \\
& 3.793634 \\
& 6.415291 \\
& 5.819350 \\
& 4.547846 \\
& 16.100128 \\
& 18.619102 \\
& 17.858337 \\
& 16.855310 \\
& 16.169067 \\
& 18.611820 \\
& 17.863920 \\
& 16.769621 \\
& 3.878838 \\
& 6.315765 \\
& 5.813281 \\
& 4.390061 \\
& 3.709159
\end{aligned}
$$




\begin{tabular}{|c|c|c|c|}
\hline O & 12.634201 & 22.239902 & 6.241727 \\
\hline 0 & 10.884245 & 20.316383 & 5.487516 \\
\hline 0 & 10.628391 & 22.920319 & 4.636934 \\
\hline 0 & 12.478601 & 8.850276 & 16.094301 \\
\hline 0 & 12.561133 & 9.871994 & 18.542639 \\
\hline 0 & 10.631061 & 8.176656 & 17.957621 \\
\hline 0 & 10.627905 & 10.538332 & 16.763796 \\
\hline 0 & 18.630512 & 21.244644 & 2.931158 \\
\hline 0 & 18.701151 & 22.239902 & 0.421893 \\
\hline 0 & 20.389692 & 20.320267 & $1.09527 c$ \\
\hline 0 & 20.572237 & 22.759378 & 2.192482 \\
\hline 0 & 18.705521 & 8.948103 & 15.165313 \\
\hline 0 & 18.606966 & 9.879762 & 12.643909 \\
\hline 0 & 20.410082 & 8.090239 & 13.318501 \\
\hline 0 & 20.496258 & 10.634945 & 14.32249 \\
\hline 0 & 6.579387 & 21.071323 & 15.15754 \\
\hline 0 & 6.321591 & 22.168777 & 12.65483 \\
\hline 0 & 8.265744 & 20.468584 & 13.15100 \\
\hline 0 & 8.263074 & 22.855991 & 14.330267 \\
\hline 0 & 6.425729 & 8.940 & 2.94548 \\
\hline 0 & 6.316008 & 9.952100 & 0.49204 \\
\hline 0 & 8.175200 & 8.182967 & 1.02293 \\
\hline 0 & 8.261374 & 10.629119 & 2.18447 \\
\hline 0 & 13.149064 & 5.238944 & \\
\hline 0 & 12.467434 & 6.6 & 5 . \\
\hline 0 & 11.810563 & 7.830986 & 8.42498 \\
\hline 0 & 14.485381 & 7.745539 & 7.59285 \\
\hline 0 & 7.674657 & 17.870958 & 19.7286 \\
\hline 0 & 6.907580 & 19.975811 & 18.54676 \\
\hline 0 & 7.329230 & 20.149374 & 21.66022 \\
\hline $\mathrm{H}$ & 12.65 & 22.1 & 3.14768 \\
\hline $\mathrm{H}$ & 15.864179 & 7.981731 & 7.92420 \\
\hline $\mathrm{H}$ & 25.047987 & 6.148271 & 1.71160 \\
\hline $\mathrm{H}$ & 21.950064 & 16.018564 & 6.476949 \\
\hline $\mathrm{H}$ & 13.548383 & 5.016103 & $9.22968 \varepsilon$ \\
\hline $\mathrm{H}$ & 9.7 & 60283 & 18 \\
\hline $\mathrm{H}$ & 10.061821 & 20.081646 & $18.96865^{\circ}$ \\
\hline $\mathrm{H}$ & 10.114740 & 20.676374 & 20.73269 \\
\hline $\mathrm{H}$ & 12.008644 & 15.173323 & 18.09477 \\
\hline $\mathrm{H}$ & 10.634945 & 17.874601 & 17.66875 \\
\hline $\mathrm{H}$ & 10.679124 & 17.645935 & 20.25618 \\
\hline $\mathrm{H}$ & 12.2 & 18.282171 & 19.66169 \\
\hline $\mathrm{H}$ & 12.079769 & 16.565956 & 20.11587 \\
\hline $\mathrm{H}$ & 12.091178 & 15.548609 & 15.54739 \\
\hline $\mathrm{H}$ & 10.609699 & 16.517166 & 15.64473 \\
\hline $\mathrm{H}$ & 10.589794 & 14.752160 & 15.98191 \\
\hline $\operatorname{Re}$ & 12.970160 & 6.899812 & 7.58484 \\
\hline $\operatorname{Re}$ & 7.771513 & 19.651745 & 20.110 \\
\hline $\mathrm{C}$ & 9.545258 & 20.207390 & 19.92653 \\
\hline $\mathrm{C}$ & 11.465622 & 15.955209 & 17.55757 \\
\hline C & 11.162189 & 17.094173 & 18.22488 \\
\hline C & 11.557137 & 17.401976 & 19.63790 \\
\hline C & 11.155635 & 15.687460 & 16.11396 \\
\hline \multicolumn{4}{|c|}{608} \\
\hline \multirow{3}{*}{$\begin{array}{l}\mathrm{US} \\
\mathrm{Si} \\
\mathrm{Si}\end{array}$} & Ne & $E=-7239$ & 49244750 \\
\hline & 23.382750 & 3.326106 & 1.243 \\
\hline & 23.428387 & 1.236791 & 3.37562 \\
\hline
\end{tabular}




\begin{tabular}{|c|c|c|c|}
\hline & 23.397800 & 15.571184 & 13.40 \\
\hline & 23.391973 & 13.427494 & 15.574340 \\
\hline & 11.249334 & 1.240189 & 15.586478 \\
\hline & 11.162432 & 15.577739 & 1.193339 \\
\hline & 11.227973 & 13.409045 & 3.367130 \\
\hline & 7.632662 & 3.352565 & 1.218099 \\
\hline & 7.657908 & 5.500624 & 3.346982 \\
\hline & 7.754278 & 15.485981 & 13.238 \\
\hline & 20.005911 & 5.431199 & 15.510 \\
\hline & 9.908327 & 15.644736 & 1.2673 \\
\hline & 19.883326 & 17.805176 & 3.34 \\
\hline & 7.62610 & 3.370528 & \\
\hline & 7.551342 & 15.536957 & 17.108321 \\
\hline & 7.750637 & 13.323600 & 15.48185 \\
\hline & 19.917553 & 3.341641 & 17.74327 \\
\hline & 19.868275 & 1.204991 & 15.61779 \\
\hline & 19.9 & 15. & \\
\hline Si & 20.010 & 13.3 & 3.2 \\
\hline & 23.436155 & 3.349895 & 5.53 \\
\hline & 23.368671 & 17.737450 & 15.56390 \\
\hline & 11.237440 & 3.365188 & 17.780 \\
\hline & 11.267783 & 5.535822 & 15.5750 \\
\hline $\mathrm{Si}$ & 11.238896 & 15. & \\
\hline $\mathrm{Si}$ & 11.1 & 17.7 & 3.2 \\
\hline 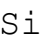 & 1.30840 & 23.4026 & 3.33435 \\
\hline $\mathrm{Si}$ & 3.378539 & 23.427658 & 1.202321 \\
\hline S & 1.255968 & 11.233070 & 15.57943 \\
\hline $\mathrm{Si}$ & 3.4 .3 & 11.2 & \\
\hline $\mathrm{Si}$ & 13.4 & 23. & $15.52627^{\circ}$ \\
\hline & 15.6073 & 23.4 & 13.41414 \\
\hline$C_{1}$ & 13.42536 & 11.2335 & 3.33217 \\
\hline $\mathrm{Si}$ & 15.610024 & 11.173355 & 1.19868 \\
\hline S & 1.360349 & 7.681 & 3.34188 \\
\hline $\mathrm{Si}$ & 3.3812 & 9 & \\
\hline & 1.3 & $19 . \varepsilon$ & 15.5 \\
\hline & 3.432185 & 19.860748 & 17.73138 \\
\hline S & 13.43137 & 7.643100 & 15.60395 \\
\hline S & 15.584778 & 7.6329 & 17.79085 \\
\hline i & 15.557105 & 19.9 & 0 \\
\hline 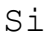 & 5.5 & 7 . & 3 . \\
\hline S & 3.406212 & 7.665 & 1.18144 \\
\hline & 5.520772 & 19.966585 & 15.64158 \\
\hline S & 3.41810 & 19.886723 & 13.42919 \\
\hline i & 17.8789 & 7.6 & 15. \\
\hline $\mathrm{Si}$ & 15.6236 & 7. & 17 \\
\hline $\mathrm{S}$ & 17.7236 & 19. & 3.36057 \\
\hline $\mathrm{Si}$ & 15.608324 & 20.0 & 1.23120 \\
\hline S & 5.498925 & 23.365759 & 3.37756 \\
\hline $\mathrm{Si}$ & 3.409610 & 23.400957 & 5.51130 \\
\hline $\mathrm{Si}$ & 5.513732 & 11.184278 & 15.60298 \\
\hline $\mathrm{Si}$ & & 11. & 17. \\
\hline $\mathrm{Si}$ & 17.723856 & 23.384935 & 15.61366 \\
\hline$S$ & 15.541570 & 23.389547 & 17.74279 \\
\hline & 17.821924 & 11.269726 & 3.30280 \\
\hline $\mathrm{Si}$ & 15.662943 & 11.240110 & 5.47028 \\
\hline$S$ & 3.356934 & 1.276116 & 23.36648 \\
\hline S: & 1.242131 & 3.365188 & 23.41430 \\
\hline & 3.37 & 13.381372 & \\
\hline
\end{tabular}




\begin{tabular}{|c|c|c|c|}
\hline $\mathrm{Si}$ & 1.284612 & 15.562202 & 11.25 \\
\hline . & 15.613665 & 1.223683 & 11.205398 \\
\hline $\mathrm{Si}$ & 13.362438 & 3.337029 & 11.247393 \\
\hline$S$ & 15.562688 & 13.423368 & 23.3914 \\
\hline & 13.448370 & 15.566330 & 23.41066 \\
\hline$i$ & 3.360090 & 1.200622 & 7.65912 \\
\hline i & 5.517131 & 3.318338 & 76200 \\
\hline i & 3.300860 & 13.374333 & 19.96221 \\
\hline & 17.775074 & 3.372956 & 19.86123 \\
\hline i & 15.642065 & 13.429922 & 7.644 \\
\hline 1 & 17.823624 & 15.573612 & $7.717 \xi$ \\
\hline$i$ & 3.339457 & 5.433383 & 7.6559 \\
\hline $\mathrm{Si}$ & 1.242131 & 3.327077 & 7.66324 \\
\hline i & 3.433157 & 17.740849 & 19.85273 \\
\hline 2 & 1.263736 & 15.583564 & 19.8719 \\
\hline i & 15.565600 & 5.467125 & 19.9447 \\
\hline$i$ & 13.394481 & 3.388977 & 19.9527 \\
\hline $\mathrm{Si}$ & 15.665128 & 17.779203 & \\
\hline $\mathrm{Si}$ & 13.468760 & 15.648620 & 7.62683 \\
\hline $\mathrm{Si}$ & 3.377082 & 5.511305 & 23.38177 \\
\hline $\mathrm{Si}$ & 5.502323 & 3.386792 & 23.34633 \\
\hline $\mathrm{Si}$ & 5.556456 & 15.553465 & 11.11218 \\
\hline $\mathrm{Si}$ & 17.857851 & 3.320765 & 25370 \\
\hline $\mathrm{Si}$ & 15.613180 & 17.757355 & $23 \quad 100$ \\
\hline $\mathrm{Si}$ & 17.730167 & 15.613908 & 23.42693 \\
\hline $\mathrm{Si}$ & 1.644361 & 21.673090 & 23.72477 \\
\hline $\mathrm{Si}$ & 1.614989 & 23.777941 & 21.60827 \\
\hline $\mathrm{Si}$ & 1.677860 & 9.445489 & 11.58359 \\
\hline $\mathrm{Si}$ & 1.671792 & 11.593549 & \\
\hline $\mathrm{Si}$ & 13.874875 & 21.701492 & $11.55276^{\circ}$ \\
\hline Si & 13.832881 & 23.855619 & 9.380915 \\
\hline $\mathrm{Si}$ & 13.833122 & 9.424371 & 23.78765 \\
\hline Si & 13.802294 & 11.637486 & 21.65367 \\
\hline $\mathrm{Si}$ & 17.483294 & 21.678188 & 23.7745 \\
\hline $\mathrm{Si}$ & 17.3 & 19 & 21.6871 \\
\hline $\mathrm{Si}$ & 17.417025 & 9.518798 & 11.586509 \\
\hline $\mathrm{Si}$ & 17.453436 & 7.327045 & 9.47923 \\
\hline $\mathrm{Si}$ & 5.151070 & 21.645418 & 11.64549 \\
\hline $\mathrm{Si}$ & 5.118299 & 19.544209 & 9.49598 \\
\hline $\mathrm{Si}$ & 5.1823 & 9.435780 & 23.7315 \\
\hline $\mathrm{Si}$ & 5.1 & 7 . & 21.61385 \\
\hline $\mathrm{Si}$ & 17.333035 & 21.699064 & 19.51993 \\
\hline $\mathrm{Si}$ & 17.413141 & 23.852463 & 21.66265 \\
\hline $\mathrm{Si}$ & 17.388624 & 11.699629 & 9.49015 \\
\hline $\mathrm{Si}$ & 5.102520 & 21.600266 & 7.3168 \\
\hline $\mathrm{Si}$ & 5.119513 & 23.743471 & 9.435 \\
\hline $\mathrm{Si}$ & 5.077033 & 9.458112 & 19.52721 \\
\hline $\mathrm{Si}$ & 5.025085 & 11.630203 & 21.70270 \\
\hline $\mathrm{Si}$ & 1.586102 & 21.610947 & 19.53862 \\
\hline $\mathrm{Si}$ & 1.601153 & 19.532070 & 21.61216 \\
\hline $\mathrm{Si}$ & 1.631010 & 9.422428 & 7.30616 \\
\hline $\mathrm{Si}$ & 1.623485 & 7.276311 & 9.43068 \\
\hline $\mathrm{Si}$ & 13.875846 & 21.743000 & 7.18503 \\
\hline $\mathrm{Si}$ & 13.890654 & 19.549549 & 9.35009 \\
\hline $\mathrm{Si}$ & 13.810790 & 7.236015 & 21.66070 \\
\hline $\mathrm{Si}$ & 23.772600 & 1.591443 & 21.62648 \\
\hline $\mathrm{Si}$ & 21.672848 & 1.535126 & 23.71215 \\
\hline $\mathrm{Si}$ & 23.861689 & 13.851572 & 9.42461 \\
\hline
\end{tabular}




\begin{tabular}{|c|c|c|c|}
\hline i & 1.733534 & 13.774621 & .58990 \\
\hline $\mathrm{S}$ & 11.537475 & 1.651886 & 9.47364 \\
\hline & 9.415389 & 1.703834 & 11.62801 \\
\hline $\mathrm{Si}$ & 11.662973 & 13.823656 & 21.677460 \\
\hline & 9.467822 & 13.761514 & 23.75706 \\
\hline Si & 23.816294 & 17.348085 & 217014 \\
\hline i & 21.691538 & 17.390324 & 19.56023 \\
\hline 1 & 21.681343 & 5.179228 & 7.247 \\
\hline 1 & 11.668314 & 17.394691 & 9.354 \\
\hline & 9.462725 & 17.392265 & 7.204 \\
\hline & 11.577527 & 5.157624 & 21.6386 \\
\hline$i$ & 9.474861 & 5.112473 & 19.509 \\
\hline i & 19.533527 & 17.385712 & 21.696 \\
\hline & 21.722126 & 17.393963 & 23.822 \\
\hline & 19.563385 & 5.153255 & 9.45689 \\
\hline & 21.722853 & 5.108832 & 11.6280 \\
\hline i & & & \\
\hline 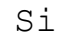 & 9.4 & 17.2 & 11.529 \\
\hline S & 7.293303 & 5.155925 & 21.64833 \\
\hline Si & 9.407864 & 5.117085 & 23.77745 \\
\hline & 19.552948 & 1.638293 & 21.59783 \\
\hline & 21.680130 & 1.607949 & 19.4825 \\
\hline $\mathrm{Si}$ & 19.5 & 13. & \\
\hline i & 7.3 & 1. & 9. \\
\hline 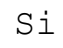 & 9.425342 & 1.659412 & 7.29524 \\
\hline 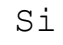 & 7.249124 & 13.691602 & 21.62259 \\
\hline i & 21.703434 & 23.744684 & 1.58683 \\
\hline 2 & 23. & 21 & \\
\hline & 21.7 & 11 & 13.78336 \\
\hline & 23.8 & 9.2 & 13.789429 \\
\hline $\mathrm{Si}$ & 9.48918 & 23.798332 & 13.82147 \\
\hline $\mathrm{Si}$ & 9.463210 & 11.605443 & 1.65868 \\
\hline & 11.616609 & 4666 & 1.6455 \\
\hline $\mathrm{Si}$ & 21.6 & 23. & 3474 \\
\hline 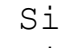 & 19.5 & 21.6 & 17.39930 \\
\hline 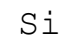 & 21.815582 & 11.644768 & 4.88210 \\
\hline S & 19.640821 & 9.536519 & 5.00809 \\
\hline S & 9.35 & 23.822363 & 5.1619 \\
\hline $\mathrm{Si}$ & 9.4 & 11.5 & $17.293^{\circ}$ \\
\hline S & 7. & 9 . & 17.36944 \\
\hline$S$ & 21.695181 & 19.5 & 17.34323 \\
\hline $\mathrm{Si}$ & 23.839113 & 21.677702 & 17.35124 \\
\hline $\mathrm{S}-\mathrm{t}+\mathrm{C}$ & 21.704647 & 7.374137 & 5.12096 \\
\hline $\mathrm{Si}$ & 23.8 & 9.4 & \\
\hline $\mathrm{Si}$ & & 19. & \\
\hline $\mathrm{Si}$ & $11.5^{-}$ & 21. & 5.1297 \\
\hline $\mathrm{Si}$ & 9.4 & 1746 & 17.3218 \\
\hline $\mathrm{Si}$ & 11.575828 & 9.367083 & 17.36192 \\
\hline $\mathrm{Si}$ & 21.697609 & 19.507069 & 1.6341 \\
\hline $\mathrm{Si}$ & 19.559015 & 21.628668 & 1.65188 \\
\hline $\mathrm{Si}$ & 21. & & 13.7668 \\
\hline $\mathrm{Si}$ & 19.56 & 9.416360 & 13.82996 \\
\hline $\mathrm{Si}$ & 9.480687 & 19.522846 & 13.73651 \\
\hline $\mathrm{Si}$ & 7.342824 & 21.640078 & 13.79501 \\
\hline $\mathrm{Si}$ & 9.428254 & 7.297915 & 1.613 \\
\hline $\mathrm{Si}$ & 7.312723 & 9.422671 & 1.66281 \\
\hline $\mathrm{Si}$ & 11.656177 & 21.704647 & 13.69767 \\
\hline & 11.2136 & 3.40329 & 13.3 \\
\hline
\end{tabular}




\begin{tabular}{|c|c|c|c|}
\hline Si & 20.027031 & 3.307900 & 13.413901 \\
\hline $\mathrm{Si}$ & 7.628778 & 1.210574 & 3.366401 \\
\hline $\mathrm{Si}$ & 23.375225 & 15.606382 & 17.725555 \\
\hline & 5.460814 & 15.554677 & 19.961245 \\
\hline & 3.470054 & 17.707106 & 11.295941 \\
\hline & 13.797440 & 9.427041 & 19.5077 \\
\hline 5 & 23.793718 & 5.133349 & 9.4207 \\
\hline 1 & 15.581138 & 1.280971 & 19.89619 \\
\hline & 15.574826 & 5.471737 & 11.20685 \\
\hline & 17.525047 & 9.604974 & 7.20470 \\
\hline & 9.451558 & 13.524350 & 19.63912 \\
\hline & 7.259319 & 21.632551 & 5.1692 \\
\hline Al & 7.676114 & 17.725798 & 15.37917 \\
\hline 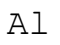 & 23.372313 & 5.398428 & 3.40839 \\
\hline & 13.455411 & 19.860506 & 3.28823 \\
\hline 1 & 21.793736 & 13.678008 & 7.23625 \\
\hline & 16.715733 & 5.187239 & 13.78311 \\
\hline & 6.910978 & 18.216873 & 18.01296 \\
\hline 0 & 22.163923 & 0.349069 & 2.866102 \\
\hline 0 & 24.605705 & 24.541864 & 3.95578 \\
\hline 0 & 24.029911 & 2.099510 & 2.11747 \\
\hline 0 & 22.847254 & 2.185442 & 4.55682 \\
\hline 0 & 22.163195 & 12.472775 & 15.085692 \\
\hline 0 & 24.610802 & 12.4 & \\
\hline 0 & 23.9 & 14.3 & 14.32929 \\
\hline O & 22.846039 & 14.397750 & 16.77204 \\
\hline 0 & 10.025895 & 0.250271 & 15.176237 \\
\hline 0 & 12.558949 & 24.691635 & 16.09308 \\
\hline 0 & 11.645253 & 2.102666 & 14 \\
\hline 0 & 10.707283 & 1996 & 16.779573 \\
\hline 0 & 9.944575 & 12.480542 & 2.94645 \\
\hline 0 & 12.480785 & 12.472047 & 3.79654 \\
\hline 0 & 11.714679 & 14.332694 & 2.104122 \\
\hline 0 & 10.782048 & 14.322985 & 4.62795 \\
\hline 0 & 8.928440 & 6.407765 & 2870 \\
\hline 0 & 6.4 & 6.4 & 369 \\
\hline 0 & 7.077987 & 4.629166 & 2.090043 \\
\hline 0 & 8.181511 & 4.555614 & 4.55027 \\
\hline 0 & 8.950773 & 18.799463 & 15.08520 \\
\hline 0 & 6.325717 & 18.620317 & 16.28315 \\
\hline 0 & 7.1 & 16.6 & 14.15937 \\
\hline 0 & 7.993868 & 16.9 & 17.02790 \\
\hline 0 & 21.311642 & 6.239058 & 14.99417 \\
\hline 0 & 18.798006 & 6.404367 & 16.02512 \\
\hline 0 & 19.293938 & 4.570422 & 14.24967 \\
\hline 0 & 20.481693 & 4.469196 & 16.69097 \\
\hline 0 & 21.242216 & 18.548222 & 2.86755 \\
\hline 0 & 18.719114 & 18.785627 & 3.88417 \\
\hline 0 & 19.283741 & 16.851427 & 2.17646 \\
\hline 0 & 20.398916 & 16.855553 & 4.56289 \\
\hline 0 & 8.930868 & 0.342515 & 3.79533 \\
\hline 0 & 6.401698 & 24.609589 & 2.78235 \\
\hline 0 & 7.082600 & 2.106550 & 4.63620 \\
\hline 0 & 8.175685 & 2.190054 & 2.18787 \\
\hline 0 & 9.017042 & 12.470590 & 16.00764 \\
\hline 0 & 6.489814 & 12.391212 & 15.09200 \\
\hline 0 & 7.253979 & 14.312546 & 16.75894 \\
\hline 0 & 8.184667 & 14.247733 & 14.23729 \\
\hline
\end{tabular}




\begin{tabular}{|c|c|c|c|}
\hline 0 & 21.147789 & 0.333776 & 101099 \\
\hline 0 & 18.634153 & 24.612261 & 15.008986 \\
\hline 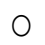 & 19.298307 & 2.109948 & 16.851185 \\
\hline 0 & 20.476595 & 2.128882 & 14.40600 \\
\hline 0 & 21.318680 & 12.466706 & 3.54749 \\
\hline 0 & 18.707462 & 12.550453 & 2.783326 \\
\hline 0 & 19.559015 & 14.239480 & 4.634 \\
\hline 0 & 20.480722 & 14.411345 & 2.12718 \\
\hline 0 & 22.257866 & 6.572834 & 3.87398 \\
\hline 0 & 24.775385 & 6.401454 & 2.62092 \\
\hline 0 & 24.111717 & 4.552701 & 4.7165 \\
\hline 0 & 22.759865 & 4.485218 & 2.1160 \\
\hline 0 & 22.085030 & 18.627113 & 16.028032 \\
\hline 0 & 24.594782 & 18.707462 & 15.0873 \\
\hline 0 & 23.933540 & 16.860893 & 16.8361 \\
\hline 0 & 22.858419 & 16.788313 & 14.34386 \\
\hline O & 10.025409 & 6.495640 & 16.006428 \\
\hline 0 & 12.557250 & 6.406309 & \\
\hline 0 & 11.726088 & 4.641789 & 16.85603 \\
\hline 0 & 10.771853 & 4.635720 & 14.32638 \\
\hline 0 & 9.877577 & 18.607937 & 3.70527 \\
\hline O & 12.372035 & 18.700180 & 2.69035 \\
\hline O & 11.724874 & 16.848757 & 4.470410 \\
\hline 0 & 10.617224 & 16.839291 & \\
\hline 0 & 2.949849 & 22.170235 & 0.256340 \\
\hline 0 & 3.969868 & 24.613716 & 24.52875 \\
\hline 0 & 2.098539 & 24.022629 & 2.03129 \\
\hline O & 4.488859 & 22.856962 & 2.194667 \\
\hline 0 & 2.951549 & 9.944575 & 12.47 \\
\hline 0 & 3.889519 & 12.467434 & 12.47471 \\
\hline 0 & 2.184714 & 11.732157 & 14.33487 \\
\hline 0 & 4.633535 & 10.704127 & 14.330509 \\
\hline 0 & 15.155603 & 22.159554 & 12.46379 \\
\hline 0 & 16.174408 & 24.616144 & 12.456 \\
\hline 0 & 14.333423 & 24.014618 & 14. \\
\hline 0 & 16.772291 & 22.846523 & 14.401391 \\
\hline 0 & 15.166041 & 9.859371 & 0.341058 \\
\hline 0 & 16.102798 & 12.397524 & 24.52438 \\
\hline 0 & 14.316188 & 11.715164 & 2.037610 \\
\hline 0 & 16.860165 & 10.709952 & 2 . \\
\hline 0 & 2.868287 & 015 & 6 . \\
\hline 0 & 3.895587 & 6.406552 & 6.478890 \\
\hline 0 & 2.193696 & 6.995939 & 4.550759 \\
\hline 0 & 4.555371 & 8.176898 & 4.566052 \\
\hline 0 & 2.860276 & 20.997286 & 18.7123 \\
\hline 0 & 4.13 & 18.625656 & 18.62 \\
\hline 0 & 2.279142 & 19.220869 & 16.77350 \\
\hline 0 & 4.629894 & 20.557186 & 16.86186 \\
\hline 0 & 15.089334 & 8.939849 & 18.61667 \\
\hline 0 & 16.096001 & 6.420389 & 18.711103 \\
\hline 0 & 14.414014 & 7.080657 & 16.776175 \\
\hline 0 & 16.861135 & 8.102375 & 16.85798 \\
\hline 0 & 15.174294 & 21.323536 & 6.32377 \\
\hline 0 & 16.179749 & 18.798977 & 6.48544 \\
\hline 0 & 14.262298 & 19.389336 & 4.73306 \\
\hline 0 & 16.773504 & 20.477566 & 4.55343 \\
\hline 0 & 3.862817 & 8.842751 & 0.184487 \\
\hline 0 & 2.779684 & 6.406795 & 24.62027 \\
\hline
\end{tabular}




\begin{tabular}{|c|c|c|c|}
\hline 0 & 4.638633 & 7.151297 & 2.100966 \\
\hline 0 & 2.208018 & 8.266715 & 2.103879 \\
\hline 0 & 3.879324 & 21.159683 & 12.55312 \\
\hline 0 & 2.953005 & 18.615948 & 12.551424 \\
\hline 0 & 4.643974 & 19.380112 & 14.41498 \\
\hline 0 & 2.187141 & 20.400131 & 340219 \\
\hline 0 & 16.168097 & 8.848820 & 12.47277 \\
\hline 0 & 15.340575 & 6.240514 & 12.82160 \\
\hline 0 & 16.934931 & 7.071191 & 14.3955 \\
\hline 0 & 14.416199 & 8.254092 & 14.40648 \\
\hline O & 16.178535 & 21.232748 & 0.3408 \\
\hline 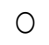 & 15.078168 & 18.729553 & 24.6142 \\
\hline O & 16.858709 & 19.382540 & 2.110191 \\
\hline 0 & 14.425666 & 20.632923 & 2.10242 \\
\hline 0 & 3.801402 & 22.168777 & 6.49151 \\
\hline 0 & 2.778956 & 24.612501 & 6.41480 \\
\hline 0 & 4.638148 & 24.019472 & 4.635235 \\
\hline 0 & 2.279870 & 22.841913 & 4.487645 \\
\hline 0 & 3.805772 & 9.944575 & 18.62978 \\
\hline 0 & 2.862218 & 12.468648 & 18.69799 \\
\hline 0 & 4.624069 & 11.792843 & 16.84851 \\
\hline O & 2.121843 & 10.713351 & 16.85094 \\
\hline 0 & 16.024876 & 22.085274 & 18. \\
\hline O & 14.924266 & 24.536278 & 18.635124 \\
\hline 0 & 16.846815 & 23.934269 & 16.86089 \\
\hline 0 & 14.409401 & 22.835358 & 16.679319 \\
\hline 0 & 16.093817 & 9.962052 & 6.32499 \\
\hline O & 15.169683 & 12.488068 & \\
\hline 0 & 1337 & 11.802067 & \\
\hline 0 & 14.400663 & 10.788117 & 4.541049 \\
\hline 0 & 0.343971 & 2.868044 & 22.162224 \\
\hline 0 & 24.608860 & 3.964285 & 24.614927 \\
\hline 0 & 2.110676 & 2.115046 & 24.015833 \\
\hline 0 & 2.193210 & 643 & 22 . \\
\hline 0 & 0.427233 & 15.15 & 9.947973 \\
\hline 0 & 24.619541 & 16.101583 & 12.477144 \\
\hline 0 & 2.109705 & 14.250161 & 11.800367 \\
\hline 0 & 2.262393 & 16.764767 & 10.788845 \\
\hline 0 & 12.384415 & 2.940625 & 10.026381 \\
\hline 0 & 12.553123 & 3.87 & 909 \\
\hline $\mathrm{O}$ & 14.335364 & 2.112618 & 11.725602 \\
\hline 0 & 14.249675 & 4.558284 & 10.625235 \\
\hline 0 & 12.635172 & 15.077438 & 22.081146 \\
\hline 0 & 12.393154 & 16.093330 & 24.525841 \\
\hline $\mathrm{O}$ & 14.332452 & 14.322499 & 24.015589 \\
\hline $\mathrm{O}$ & 14.412072 & 16.781031 & 22.925417 \\
\hline 0 & 6.483260 & 2.877754 & 8.85294 \\
\hline 0 & 6.396842 & 3.954575 & 6.405581 \\
\hline 0 & 4.646644 & 2.041251 & 7.083086 \\
\hline 0 & 4.539350 & 4.482548 & 8.186366 \\
\hline 0 & 6.506320 & 15.085207 & 21.077877 \\
\hline 0 & 6.229105 & 16.100613 & 18.533171 \\
\hline 0 & 4.550517 & 14.322985 & 19.458277 \\
\hline 0 & 4.646644 & 16.853369 & 20.48120 \\
\hline 0 & 18.704550 & 2.945480 & 21.146818 \\
\hline $\mathrm{O}$ & 18.797279 & 3.975937 & 18.703335 \\
\hline $\mathrm{O}$ & 16.932747 & 2.191511 & 19.21528 \\
\hline ( & 16.857737 & 4.622855 & 20.39915 \\
\hline
\end{tabular}




\begin{tabular}{|c|c|c|c|}
\hline 0 & 06734 & 15.176722 & 9.018985 \\
\hline 0 & 18.802862 & 16.017109 & 6.483746 \\
\hline 0 & 16.933474 & 14.326626 & 7.162949 \\
\hline 0 & 16.924978 & 16.862350 & 8.102618 \\
\hline 0 & 0.417523 & 3.879081 & 8.94955 \\
\hline O & 24.523899 & 2.692053 & 6.564095 \\
\hline 0 & 2.113104 & 4.556828 & 7.007591 \\
\hline 0 & 2.208260 & 2.179374 & 8.256763 \\
\hline 0 & 0.423349 & 16.099886 & 21.15215 \\
\hline 0 & 24.620756 & 15.081567 & 18.63293 \\
\hline O & 2.265548 & 16.765009 & 19.28981 \\
\hline O & 2.105093 & 14.331238 & 20.470526 \\
\hline 0 & 12.393882 & 3.869371 & 21.151188 \\
\hline 0 & 12.469862 & 2.867558 & 18.69362 \\
\hline 0 & 14.243607 & 4.699805 & 19.37938 \\
\hline 0 & 14.327840 & 2.277928 & 20.55403 \\
\hline 0 & 12.566231 & 16.100370 & 8.92261 \\
\hline 0 & 12.483941 & 15.238137 & 09465 \\
\hline 0 & 14.420325 & 16.866234 & 7.08405 \\
\hline 0 & 14.410131 & 14.404305 & 8.09339 \\
\hline ? & 6.404367 & 3.870827 & 22.08600 \\
\hline 0 & 6.422816 & 2.789880 & 24.54186 \\
\hline 0 & 4.637662 & 4.643731 & 23.944950 \\
\hline 0 & 4.484732 & 2.271374 & 22.761562 \\
\hline 0 & 6.319649 & 16.087748 & 9.777566 \\
\hline 0 & 6.589582 & 14.981797 & 12.22711 \\
\hline 0 & 4.719468 & 16.780544 & 11.80546 \\
\hline O & 4.564110 & 14.338277 & 10.696116 \\
\hline 0 & 18.539967 & 886 & 2390 \\
\hline 0 & 18.877384 & 2.778956 & 12.384901 \\
\hline 0 & 16.945370 & 4.540806 & 12.04432 \\
\hline 0 & 16.845358 & 2.194909 & 10.716992 \\
\hline 0 & 18.630997 & 16.107168 & 22.16004 \\
\hline 0 & 18.712803 & 15.083508 & 24.60497 \\
\hline 0 & 16.855068 & 16.857252 & 1416 \\
\hline 0 & 16.772778 & 14.406004 & 22.919348 \\
\hline 0 & 2.866345 & 24.692122 & 22.078476 \\
\hline 0 & 0.430146 & 0.420679 & 20.985390 \\
\hline 0 & 1.008852 & 22.911095 & 22.850893 \\
\hline 0 & 2.201221 & 22.765690 & 20.478294 \\
\hline 0 & 2.9 & 12.4 & 19673 \\
\hline 0 & 0.503212 & 12.577883 & 8.93329 \\
\hline 0 & 1.093085 & 10.698545 & 10.708739 \\
\hline 0 & 2.190783 & 10.616738 & 8.25967 \\
\hline 0 & 15.163857 & 24.694063 & 9.86786 \\
\hline 0 & 12.566960 & 0.510 & 63 \\
\hline 0 & 13.319472 & 22.929543 & 10.62305 \\
\hline 0 & 14.328082 & 23.011106 & 8.09727 \\
\hline 0 & 15.080837 & 12.562834 & 22.081875 \\
\hline 0 & 12.559678 & 12.561133 & 21.153856 \\
\hline 0 & 13.313404 & 10.709710 & 22.911579 \\
\hline 0 & 14.254288 & 10.701215 & 20.39551 \\
\hline 0 & 16.110807 & 18.631241 & 22.15736 \\
\hline 0 & 18.622015 & 18.608665 & 21.148516 \\
\hline 0 & 18.035786 & 20.392363 & 22.922747 \\
\hline 0 & 16.854582 & 20.475624 & 20.482906 \\
\hline 0 & 16.190914 & 6.496853 & 10.020797 \\
\hline ? & 18.689257 & 6.423059 & 8.94300 \\
\hline
\end{tabular}




\begin{tabular}{|c|c|c|}
\hline 2871 & 8.344879 & 621593 \\
\hline 6.858952 & 8.173257 & 8.178597 \\
\hline 3.878838 & 18.626871 & 10.03172 \\
\hline 6.331786 & 18.620317 & 8.93159 \\
\hline 5.730019 & 20.392847 & 10.777 \\
\hline 4.474051 & 20.481449 & 8.351 \\
\hline 3.869128 & 6.483260 & 22.175 \\
\hline 6.322 & 6.325717 & \\
\hline 5.8144 & 8.272783 & 22.761 \\
\hline 4.552944 & 8.190493 & 20.393 \\
\hline 16.181934 & 24.771744 & 21.315 \\
\hline 18. & 0.417038 & \\
\hline 17.949610 & 22.928816 & 20.3 \\
\hline 16.937843 & 22.846039 & 22.838 \\
\hline 16.09308 & 12.547784 & 8.945 \\
\hline 3.53001 & 12.718676 & 10.039 \\
\hline .034328 & 10.707525 & 8.421 \\
\hline 16. & 10.792486 & 10. \\
\hline 3.814511 & 24.591139 & 8.9468 \\
\hline 6.323776 & 0.432331 & 9.958 \\
\hline 5.724193 & 22.830017 & 8.2468 \\
\hline 4.629894 & 22.847738 & 10.708 \\
\hline 0858 & 12.473261 & 21 . \\
\hline 6.15 & 12.655562 & 22.24839 \\
\hline 5.57 & 10.704856 & 20. \\
\hline 4.556828 & 10.698059 & 22.9280 \\
\hline 2.875326 & 18.714502 & 20.9955 \\
\hline 0.503698 & 18.536570 & 22.25252 \\
\hline 0.9 & 20 . & \\
\hline 2.19 & 20.485092 & 22.75889 \\
\hline 2.857120 & 6.327902 & 8.9349 \\
\hline 0.426505 & 6.338826 & 10.02905 \\
\hline 1.0205 & 8.176170 & 8.1895 \\
\hline $2 \cdot$ & 8.248 & 10 . \\
\hline 15.1 & 18.6 & 8 . \\
\hline 12.635172 & 18.626143 & 9.79018 \\
\hline 13.393024 & 20.481207 & 8.09922 \\
\hline 14.34240 & 20.471254 & 10.6160 \\
\hline 15.0924 & 6.333243 & 21.241 \\
\hline 12.5 & 6 . & 17 \\
\hline 13.3 & 8.164276 & 20 . \\
\hline 14.324441 & 8.189764 & 22.85817 \\
\hline 22.171206 & 2.777985 & 24.60473 \\
\hline 21.0761 & 0.332319 & 0.3531 \\
\hline 22.922 & 0.921706 & 22.842 \\
\hline 20 & 2. & 22 \\
\hline 22.1612 & 15.081324 & 12.47835 \\
\hline 21.2397 & 12.552395 & 12.56550 \\
\hline 23.00091 & 13.240337 & 10.7034 \\
\hline 20.48630 & 14.258414 & 10.69878 \\
\hline 9.96 & 3.044035 & 12.39533 \\
\hline 8.9374 & 0.590844 & 12.72183 \\
\hline 10.622564 & 1.081919 & 10.70631 \\
\hline 8.173742 & 2.181801 & 10.70267 \\
\hline 9.863498 & 15.086663 & 24.6074 \\
\hline .933052 & 12.550696 & 0.432 \\
\hline 10. & 13.234998 & 93998 \\
\hline 8.2604 & 14.238994 & 22.76593 \\
\hline
\end{tabular}




\begin{tabular}{rrr}
22.167078 & 16.090902 & 18.697510 \\
21.227165 & 18.618618 & 18.610607 \\
22.923717 & 17.954708 & 20.479507 \\
20.476839 & 16.855553 & 20.481207 \\
22.150572 & 3.877867 & 6.408252 \\
21.147545 & 6.402183 & 6.327902 \\
22.918861 & 5.732932 & 8.180782 \\
20.486790 & 4.635720 & 8.251664 \\
9.943847 & 16.100370 & 6.330815 \\
9.017285 & 18.618618 & 6.253379 \\
10.691504 & 17.934317 & 8.163791 \\
8.185881 & 16.870604 & 8.094123 \\
9.955013 & 3.787323 & 18.700666 \\
8.929411 & 6.234446 & 18.462532 \\
10.692233 & 5.751138 & 20.389935 \\
8.262102 & 4.632079 & 20.468828 \\
21.233234 & 16.180719 & 24.771986 \\
22.328989 & 18.626385 & 0.418009 \\
20.476353 & 17.948639 & 22.920076 \\
22.923231 & 16.773991 & 22.918135 \\
21.326935 & 3.880295 & 12.642211 \\
22.248398 & 6.398784 & 12.476658 \\
20.394547 & 5.576118 & 10.796856 \\
22.846283 & 4.553187 & 10.621108 \\
9.088653 & 15.931904 & 12.394853 \\
9.868353 & 18.468843 & 12.564046 \\
8.191949 & 17.781145 & 10.630575 \\
10.775252 & 16.930805 & 10.632275 \\
8.929169 & 3.803102 & 24.608131 \\
9.954527 & 6.326204 & 0.429903 \\
8.157722 & 5.731476 & 22.912550 \\
10.622079 & 4.633050 & 22.836573 \\
21.241972 & 2.864160 & 18.542395 \\
22.319523 & 0.425048 & 18.546038 \\
20.403044 & 1.020990 & 20.313227 \\
22.831232 & 2.192239 & 20.461302 \\
21.393932 & 15.249060 & 6.246339 \\
22.343554 & 12.644882 & 6.000196 \\
20.390179 & 13.307336 & 8.164762 \\
22.920076 & 10.631061 & 13.230385 \\
23.014019 & 14.405761 & 8.194134 \\
12.475203 & 22.242573 & 15.002674 \\
12.637114 & 21.236876 & 12.490009 \\
10.027594 & 2.953733 & 6.492970 \\
8.267200 & 0.589873 & 6.245126 \\
10.628633 & 2.1907396 & 8.257005 \\
8.770656 & 15.168712 & 18.7973977 \\
10.036575 & 12.478601 & 18.447968 \\
8.006491 & 12.983270 & 20.396732 \\
10.632031 & 14.342890 & 20.559372 \\
24.766159 & 22.079205 & 2.866345 \\
0.497144 & 21.064770 & 0.416552 \\
23.002611 & 22.918135 & 1.012008 \\
22.850409 & 20.489218 & 2.190783 \\
0.686296 & 9.934380 & 15.084236 \\
\hline & &
\end{tabular}




\begin{tabular}{|c|c|c|c|}
\hline D & 10.697574 & 22.920805 & 13.146395 \\
\hline 0 & 10.786418 & 20.405956 & 14.157916 \\
\hline 0 & 12.481028 & 9.945546 & 2.944023 \\
\hline 0 & 12.570844 & 8.939607 & 0.429660 \\
\hline 0 & 10.706554 & 10.707525 & 1.088716 \\
\hline 0 & 10.630818 & 8.257976 & 2.115531 \\
\hline 0 & 18.708191 & 22.174847 & 16.085564 \\
\hline 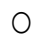 & 18.535599 & 21.147058 & 18.55137 \\
\hline 0 & 20.482420 & 22.851864 & 18.02923 \\
\hline 0 & 20.482906 & 20.473196 & 16.85725 \\
\hline 0 & 18.784412 & 10.031235 & 3.705518 \\
\hline 0 & 18.791939 & 9.010974 & 6.242941 \\
\hline 0 & 20.564955 & 10.796371 & 5.48897 \\
\hline 0 & 20.571751 & 8.330557 & 4.47235 \\
\hline ? & 6.323047 & 22.091827 & 3.792421 \\
\hline O & 6.239058 & 20.981993 & 6.412378 \\
\hline 0 & 8.171801 & 23.004309 & 5.818136 \\
\hline 0 & 8.350947 & 20.490675 & 4.480606 \\
\hline 0 & 6.326689 & 9.865197 & 16.10012 \\
\hline 0 & 6.328146 & 8.944219 & 18.619589 \\
\hline 0 & 8.172286 & 10.696116 & 17.857122 \\
\hline O & 8.175200 & 8.178841 & 16.855553 \\
\hline 0 & 24.865444 & 21.223040 & 16. \\
\hline 0 & 0.424320 & 22.251554 & 18.611578 \\
\hline 0 & 22.996542 & 20.380711 & 17.863920 \\
\hline 0 & 22.867401 & 22.830017 & 16.769379 \\
\hline 0 & 24.691879 & 8.844208 & 3.878838 \\
\hline 0 & 0.500057 & 10.031235 & $\$ 765$ \\
\hline 0 & 22.915949 & 8.272541 & 3281 \\
\hline 0 & 23.000425 & 10.629119 & 4.390061 \\
\hline 0 & 12.475445 & 21.408255 & 3.709159 \\
\hline 0 & 12.634686 & 22.239902 & 6.242213 \\
\hline 0 & 10.879633 & 20.318325 & 5.484603 \\
\hline 0 & 10.628633 & 22.92 & 91 \\
\hline 0 & 12.478601 & 8.850034 & 16.094301 \\
\hline 0 & 12.560891 & 9.871751 & 18.542881 \\
\hline 0 & 10.631061 & 8.176898 & 17.957621 \\
\hline 0 & 10.628391 & 10.537360 & 16.763309 \\
\hline 0 & 18.630512 & 21.244644 & 2.929944 \\
\hline 0 & 18.702364 & 22.240631 & 0.421407 \\
\hline 0 & 20.389935 & 20.320024 & 1.095027 \\
\hline 0 & 20.572481 & 22.759378 & 2.192724 \\
\hline 0 & 18.702850 & 8.951015 & 15.162157 \\
\hline 0 & 18.560600 & 9.937778 & 12.659931 \\
\hline 0 & 20.397945 & 8.099948 & 13.316074 \\
\hline 0 & 20.494316 & 10.635673 & 14.319828 \\
\hline 0 & 6.579387 & 21.075691 & 15.155117 \\
\hline 0 & 6.321591 & 22.169264 & 12.654591 \\
\hline 0 & 8.267443 & 20.471983 & 13.145667 \\
\hline 0 & 8.262831 & 22.856234 & 14.330752 \\
\hline 0 & 6.426215 & 8.940578 & 2.945480 \\
\hline 0 & 6.315765 & 9.952100 & 0.491803 \\
\hline 0 & 8.175442 & 8.183210 & 1.023174 \\
\hline 0 & 8.261617 & 10.630333 & 2.183743 \\
\hline 0 & 13.234511 & 5.212970 & 8.183453 \\
\hline 0 & 12.471317 & 6.674301 & 5.905282 \\
\hline 0 & 11.809592 & 7.746995 & 8.41746 \\
\hline , & 14.483196 & 7.752336 & 7.58338 \\
\hline
\end{tabular}




\begin{tabular}{|c|c|c|c|}
\hline O & 7.674171 & 17.866104 & 39122 \\
\hline 0 & 7.247182 & 19.980907 & 18.462048 \\
\hline 0 & 7.079444 & 19.809772 & 21.579391 \\
\hline $\mathrm{H}$ & 12.712364 & 22.182856 & 3.163466 \\
\hline $\mathrm{H}$ & 15.863692 & 7.988042 & 7.922501 \\
\hline $\mathrm{H}$ & 25.047018 & 6.149241 & 1.711117 \\
\hline $\mathrm{H}$ & 21.949093 & 16.018564 & 6479619 \\
\hline $\mathrm{H}$ & 13.559792 & 4.989644 & 9.106616 \\
\hline $\mathrm{H}$ & 8.965823 & 15.933118 & 19.393221 \\
\hline $\mathrm{H}$ & 9.944332 & 21.233963 & 19.50488 \\
\hline $\mathrm{H}$ & 9.061951 & 21.850294 & 20.97616 \\
\hline $\mathrm{H}$ & 11.479458 & 20.192097 & 21.04680 \\
\hline $\mathrm{H}$ & 9.590894 & 17.952036 & 21.37062 \\
\hline $\mathrm{H}$ & 10.950757 & 19.092215 & 18.8079 \\
\hline $\mathrm{H}$ & 10.148239 & 17.519463 & 18.87034 \\
\hline $\mathrm{H}$ & 11.594034 & 17.811487 & 19.85346 \\
\hline $\mathrm{H}$ & 10.574986 & 21.127882 & 23.18734 \\
\hline $\mathrm{H}$ & 10.847347 & 19.378899 & 23 \\
\hline $\mathrm{H}$ & 9.197888 & 20.006882 & 23.1 \\
\hline $\mathrm{Re}$ & 12.980114 & 6.890588 & 7.5 \\
\hline $\mathrm{Re}$ & 7.919345 & 19.667767 & 20.12558 \\
\hline $\mathrm{C}$ & 9.504720 & 20.968399 & 20.47926 \\
\hline $\mathrm{C}$ & 10.423270 & 20.040138 & 21.31139 \\
\hline C & 9.8 & 18. & 20. \\
\hline $\mathrm{C}$ & 10.6 & 18.2 & 19. \\
\hline $\mathrm{C}$ & 10.2 & 20.1 & 22.8 \\
\hline \multicolumn{4}{|c|}{608} \\
\hline \multicolumn{2}{|c|}{ USY } & \multicolumn{2}{|c|}{$E=-7239.47026519494$} \\
\hline $\mathrm{Si}$ & 23.382994 & 3.326349 & 1.24358 \\
\hline $\mathrm{Si}$ & 23.428629 & 1.236548 & 3.37586 \\
\hline $\mathrm{Si}$ & 23.398045 & 15.571184 & 13.40249 \\
\hline $\mathrm{Si}$ & 23.392460 & 13.428223 & 15.57482 \\
\hline $\mathrm{Si}$ & 11.248849 & 1.244801 & 15.58186 \\
\hline $\mathrm{Si}$ & 11.1 & 15.577982 & 1. \\
\hline $\mathrm{Si}$ & 11.227730 & 13.410016 & 3.365 \\
\hline $\mathrm{Si}$ & 7.632420 & 3.352322 & 1.21882 \\
\hline $\mathrm{Si}$ & 7.657665 & 5.501110 & 3.34698 \\
\hline $\mathrm{Si}$ & 7.722721 & 15.465346 & 13.24155 \\
\hline $\mathrm{Si}$ & 20.0 & 5.427557 & 15.513 \\
\hline $\mathrm{Si}$ & 19. & 15. & 1. \\
\hline $\mathrm{Si}$ & 19.883566 & 17.805176 & 3.349 \\
\hline $\mathrm{Si}$ & 7.625865 & 3.370528 & 5.51009 \\
\hline Si & 7.537263 & 15.577496 & 17.71608 \\
\hline Si & 7.745539 & 13.333308 & 15.49763 \\
\hline $\mathrm{Si}$ & 19.918524 & 3.339942 & 17.74473 \\
\hline $\mathrm{Si}$ & 19.8 & 1.19 & 15.624 \\
\hline Si & 19.935028 & 15.589875 & 5.4338 \\
\hline Si & 20.010765 & 13.381372 & 3.27925 \\
\hline $\mathrm{Si}$ & 23.434456 & 3.350380 & 5.53582 \\
\hline $\mathrm{Si}$ & 23.370127 & 17.736237 & 15.56390 \\
\hline Si & 11.237440 & 3.370771 & 17.78090 \\
\hline $\mathrm{Si}$ & 11.267541 & 5.539706 & 15.57434 \\
\hline $\mathrm{Si}$ & 11.238411 & 15.650320 & 5.44940 \\
\hline $\mathrm{Si}$ & 11.185007 & 17.780415 & 3.22512 \\
\hline $\mathrm{Si}$ & 1.308644 & 23.402655 & 3.33363 \\
\hline $\mathrm{Si}$ & 3.378296 & 23.426445 & 1.20159 \\
\hline $\mathrm{Si}$ & 1.255239 & 11.233070 & 15.57895 \\
\hline
\end{tabular}




\begin{tabular}{|c|c|c|c|}
\hline$i$ & 3.411309 & 11.213651 & 198 \\
\hline $\mathrm{Si}$ & 13.458809 & 23.446592 & .521664 \\
\hline Si & 5.610509 & 23.402655 & 13.410746 \\
\hline Si & 13.424582 & 11.234042 & 3.332417 \\
\hline Si & 15.609781 & 11.174084 & 1.198922 \\
\hline $\mathrm{Si}$ & 1.360591 & 7.681697 & 2016 \\
\hline i & 3.381452 & 7.602562 & 225142 \\
\hline i & 1.316169 & 19.876528 & 15.59 \\
\hline i & 3.433157 & 19.850796 & 17.738 \\
\hline & 13.431864 & 7.644799 & 15.604 \\
\hline i & 15.584293 & 7.634119 & 17.7913 \\
\hline i & 15.556862 & 19.951778 & 5.499 \\
\hline 2 & 5.526841 & 7.663005 & 3.377 \\
\hline & 3.406697 & 7.666890 & 1.180 \\
\hline & 5.517616 & 19.966101 & 15.64206 \\
\hline & 3.417864 & 19.885994 & 13.4289 \\
\hline i & 17.879456 & & \\
\hline i & 15.6 & 7.7 & 13.483 \\
\hline S & 17.723614 & 19.965858 & 3.360 \\
\hline Si & 15.607353 & 20.003969 & 1.23169 \\
\hline & 5.498682 & 23.365030 & 3.3768 \\
\hline & 3.409853 & 23.400713 & 5.5105 \\
\hline Si & 5.5 & 11. & \\
\hline i & 3.3 & 11. & 17. \\
\hline 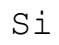 & 17.727253 & 23.383720 & 15.61293 \\
\hline 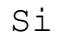 & 15.547395 & 23.391003 & 17.74279 \\
\hline i & 17.821682 & 11.269726 & 3.30280 \\
\hline $\mathrm{Si}$ & 15. & 11. & \\
\hline & 3.3 & 1.2 & 23.366730 \\
\hline & 1.242617 & 3.3 & 23.414549 \\
\hline $\mathrm{S}$ & 3.372470 & 13.379674 & 11.22942 \\
\hline $\mathrm{Si}$ & 1.284855 & 15.562202 & 11.25904 \\
\hline & 15.6 & 1.2241 & 11.2032 \\
\hline $\mathrm{Si}$ & 13. & & 0 \\
\hline $\mathrm{Si}$ & 15.5 & 13. & 23.39197 \\
\hline Si & 13.445701 & 15.566330 & 23.41042 \\
\hline$\sigma_{1}$ & 3.359605 & 1.200622 & 7.65863 \\
\hline$\perp$ & 5.516645 & 3.318823 & 7.6193 \\
\hline $\mathrm{Si}$ & & 13.3 & 1 \\
\hline $\mathrm{S}$ & 17. & 3. & 19.86147 \\
\hline SI & 15.6 & 13.42 & 7.64479 \\
\hline $\mathrm{Si}$ & 17.823868 & 15.573126 & 7.71762 \\
\hline Si & 3.339214 & 5.433869 & 7.65572 \\
\hline $\mathrm{Si}$ & 1.2 & 3. & 62 \\
\hline $\mathrm{Si}$ & & 17. & $9.82870^{\circ}$ \\
\hline $\mathrm{Si}$ & 1.2 & 15.58 & 19.86196 \\
\hline Si & 15.566814 & 5.469310 & 19.94546 \\
\hline $\mathrm{Si}$ & 13.395452 & 3.392618 & 19.95396 \\
\hline $\mathrm{Si}$ & 15.665128 & 17.778473 & 7.6348 \\
\hline $\mathrm{Si}$ & 13.467546 & 15.648135 & 7.62659 \\
\hline $\mathrm{Si}$ & 3.3 & & 23.3815 \\
\hline $\mathrm{Si}$ & 5.501352 & 3.386792 & 23.34706 \\
\hline Si & 5.557426 & 15.541813 & 11.11558 \\
\hline Si & 17.858580 & 3.320037 & 11.25443 \\
\hline $\mathrm{Si}$ & 1723 & 5655 & 23.44198 \\
\hline $\mathrm{Si}$ & 17.729925 & 15.613421 & 23.42717 \\
\hline $\mathrm{Si}$ & 1.645332 & 21.670420 & 23.7228 \\
\hline & 1.61 & 23.77648 & 21.68 \\
\hline
\end{tabular}




\begin{tabular}{|c|c|c|c|}
\hline $\mathrm{Si}$ & 1.678589 & 9.445974 & 11.584081 \\
\hline & 1.671549 & 11.593306 & 9.455441 \\
\hline & 13.876089 & 21.700764 & 11.54815 \\
\hline & 13.832638 & 23.855375 & 9.3787 \\
\hline & 13.832638 & 9.425827 & 23.78862 \\
\hline & 13.800838 & 11.639428 & 21.6541 \\
\hline & 17.483294 & 21.677460 & 23.776 \\
\hline & 17.394449 & 19.534498 & 21.6893 \\
\hline & 17.427948 & 9.517342 & 11.5901 \\
\hline & 17.450768 & 7.328501 & 9.4869 \\
\hline & 5.151556 & 21.643717 & 11.6457 \\
\hline & 5.117571 & 19.543238 & 9.4952 \\
\hline S & 5.182384 & 9.438207 & 23.730 \\
\hline & 5.151313 & 7.308596 & 21.613 \\
\hline & 17.336433 & 21.697609 & 19.5218 \\
\hline & 17.414354 & 23.852463 & 21.6645 \\
\hline S & 17.389109 & 11.699386 & 9.4913 \\
\hline S & 5.102278 & 21.599781 & \\
\hline $\mathrm{Si}$ & 5.119027 & 23.742985 & 9.4350 \\
\hline S & 5.075819 & 9.461025 & 19.5272 \\
\hline S & 5.023871 & 11.634330 & 21.7012 \\
\hline S & 1.590715 & 21.607307 & 19.5415 \\
\hline \pm & 1.606493 & 19.525517 & 21.609 \\
\hline$i$ & 1.631010 & 9.422428 & \\
\hline i & 1.623485 & 7.276796 & 9.4306 \\
\hline $\mathrm{Si}$ & 13.875118 & 21.742029 & 7.18285 \\
\hline Si & 13.888712 & 19.546392 & 9.34814 \\
\hline i & 13.811033 & 7.238200 & 21.6616 \\
\hline i & 23.773813 & 1.590957 & 21.62 \\
\hline $\mathrm{Si}$ & 21.673819 & 1.534883 & 23.71288 \\
\hline i & 23.861931 & 13.851815 & 9.4246 \\
\hline $\mathrm{Si}$ & 21.733778 & 13.774621 & 11.59015 \\
\hline $\mathrm{Si}$ & 11.537231 & 1.652372 & 9.47170 \\
\hline i & 9.414660 & 1.704320 & 11.624 \\
\hline $\mathrm{Si}$ & 11.659818 & 13.824627 & 21. \\
\hline $\mathrm{Si}$ & 9.464666 & 13.763697 & 23.75415 \\
\hline $\mathrm{Si}$ & 23.819206 & 17.347115 & 21.70076 \\
\hline $\mathrm{Si}$ & 21.693239 & 17.389837 & 19.56071 \\
\hline $\mathrm{Si}$ & 21.679401 & 5.179714 & 7.2513 \\
\hline $\mathrm{Si}$ & 11.664187 & 17.391537 & 9 . \\
\hline i & 9.461 & 17.3 & 7 . \\
\hline $\mathrm{Si}$ & 11.577042 & 5.158838 & 21.64056 \\
\hline $\mathrm{Si}$ & 9.473163 & 5.114901 & 19.51095 \\
\hline $\mathrm{Si}$ & 19.534740 & 17.384983 & 21.69712 \\
\hline $\mathrm{Si}$ & 21.723339 & 17.393721 & 23.82115 \\
\hline $\mathrm{Si}$ & 19.581835 & 5.151798 & \\
\hline $\mathrm{Si}$ & 21.730379 & 5.068294 & 11.6569 \\
\hline $\mathrm{Si}$ & 7.262231 & 17.350269 & 9.34110 \\
\hline $\mathrm{Si}$ & 9.457141 & 17.272591 & 11.52776 \\
\hline $\mathrm{Si}$ & 7.291604 & 5.157138 & 21.64954 \\
\hline $\mathrm{Si}$ & 9.407378 & 5.117571 & 23.77915 \\
\hline $\mathrm{Si}$ & 19.555132 & 1.638293 & 21.59808 \\
\hline $\mathrm{Si}$ & 21.681829 & 1.606250 & 19.48230 \\
\hline $\mathrm{Si}$ & 19.555616 & 13.829239 & 9.43116 \\
\hline $\mathrm{Si}$ & 7.306169 & 1.607707 & 9.43383 \\
\hline $\mathrm{Si}$ & 9.424613 & 1.659169 & 7.29354 \\
\hline $\mathrm{Si}$ & 7.243298 & 13.698400 & 21.61871 \\
\hline $\mathrm{Si}$ & 21.703918 & 23.744200 & 1.58707 \\
\hline
\end{tabular}




\begin{tabular}{|c|c|c|c|}
\hline & 23.83814 & 21.645418 & 1.616446 \\
\hline & 21.716055 & 11.597432 & 13.783603 \\
\hline & 23.851978 & 9.411505 & 18894 \\
\hline & 9.485785 & 23.797117 & 13.818316 \\
\hline & 9.462725 & 11.607628 & 1.6574 \\
\hline & 11.616123 & 9.465880 & 160150 \\
\hline & 21.701977 & 23.750996 & 17.380371 \\
\hline & 19.534985 & 21.672119 & 17.3 \\
\hline & 21.815582 & 11.644768 & 4.8821 \\
\hline & 19.640821 & 9.536519 & 5.008 \\
\hline & 9.358586 & 23.821392 & 5.1612 \\
\hline & 9.464423 & 11.547426 & 17.294 \\
\hline & 7.258590 & 9.425342 & 17.369 \\
\hline & 21.697851 & 19.520418 & 17.34323 \\
\hline & 23.853678 & 21.671391 & 17.35512 \\
\hline & 21.704647 & 7.373895 & 5.121 \\
\hline$S \perp$ & 23.814110 & & \\
\hline i & 9.4831 & 19.5 & 4.9 \\
\hline Si & 11.570003 & 21.726252 & 5.128 \\
\hline $\mathrm{Si}$ & 9.432867 & 7.264902 & 17.32235 \\
\hline S & 11.574615 & 9.369510 & 17.36216 \\
\hline 1 & 21.698336 & 19.506824 & 1.63392 \\
\hline $\mathrm{Si}$ & 19.559502 & 21. & \\
\hline $\mathrm{Si}$ & 21.7 & 7.2 & 13.78384 \\
\hline $\mathrm{Si}$ & 19.581591 & 9.4022 & 13.841 \\
\hline $\mathrm{Si}$ & 9.500108 & 19.486677 & 13.75374 \\
\hline- & 7.341367 & 21.623814 & 13.80253 \\
\hline 2 & 9.4 & & \\
\hline i & 7.3129 & 9.4 & 1.6 \\
\hline $\mathrm{Si}$ & 11.656420 & 21.703918 & 13.69305 \\
\hline $\mathrm{Si}$ & 11.212438 & 3.406697 & 13.37360 \\
\hline $\mathrm{Si}$ & 20.023632 & 3.292 & 13.41851 \\
\hline $\mathrm{Si}$ & 7.6285 & 1.2 & 3.3661 \\
\hline 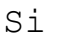 & 23.374254 & 15.6 & 17. \\
\hline & 5.4 & 15.5 & 19.92 \\
\hline $\mathrm{Si}$ & 3.472724 & 17.704193 & 11.29667 \\
\hline $\mathrm{Si}$ & 13.796710 & 9.428497 & 19.50876 \\
\hline $\mathrm{Si}$ & 23.793478 & 5.133835 & 9.42218 \\
\hline 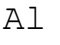 & 15.584535 & 1.2 & 19.897 \\
\hline & 15.5 & 5. & 11. \\
\hline & 17.524803 & 9.6 & 7.20518 \\
\hline & 9.444275 & 13.530905 & 19.63548 \\
\hline Al & 7.259319 & 21.631823 & 5.16830 \\
\hline 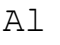 & $7.6^{\circ}$ & 17.7 & 15. \\
\hline 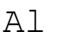 & 23.3 & & \\
\hline Al & 13.45 & 19.8 & 3.28702 \\
\hline Al & 21.793978 & 13.678251 & 7.23625 \\
\hline Al & 16.718645 & 5.190395 & 13.78336 \\
\hline Al & 7.119255 & 18.445782 & 17.9432 \\
\hline 0 & 22.1 & 0.3 & 6610 \\
\hline O & 24.6 & 24.5 & \\
\hline 0 & 24.029911 & 2.0 & 2.11747 \\
\hline 0 & 22.847010 & 2.185442 & 4.55707 \\
\hline 0 & 22.163195 & 12.473018 & 15.08569 \\
\hline O & 24.610559 & 12.475930 & 16.09648 \\
\hline 0 & 23.941309 & 14.330024 & 14.32905 \\
\hline 0 & 22.844339 & 14.397508 & 16.77059 \\
\hline 0 & 10.0256 & 0 & 15.1774 \\
\hline
\end{tabular}




$\begin{array}{rrr}12.556522 & 24.689451 & 16.094301 \\ 11.644768 & 2.103394 & 14.249190 \\ 10.708254 & 2.190054 & 16.781759 \\ 9.944575 & 12.480300 & 2.946451 \\ 12.480542 & 12.472290 & 3.796547 \\ 11.714679 & 14.332936 & 2.103879 \\ 10.782048 & 14.322742 & 4.627953 \\ 8.928683 & 6.407523 & 2.879210 \\ 6.415049 & 6.409465 & 3.883693 \\ 7.077987 & 4.629409 & 2.090043 \\ 8.181511 & 4.555614 & 4.550031 \\ 9.023354 & 18.714016 & 15.092731 \\ 6.327417 & 18.625414 & 16.266167 \\ 7.147413 & 16.674223 & 14.154276 \\ 8.004306 & 17.011639 & 17.030573 \\ 21.308001 & 6.237601 & 15.008256 \\ 18.796309 & 6.402183 & 16.025120 \\ 19.298307 & 4.565567 & 14.247733 \\ 20.481207 & 4.467254 & 16.692429 \\ 21.242216 & 18.548222 & 2.867558 \\ 18.719114 & 18.785627 & 3.884178 \\ 19.283741 & 16.851427 & 2.176461 \\ 20.398916 & 16.855553 & 4.562896 \\ 8.930868 & 0.342757 & 3.795334 \\ 6.401454 & 24.609589 & 2.782355 \\ 7.082600 & 2.106307 & 4.636206 \\ 8.175927 & 2.190054 & 2.188112 \\ 9.015100 & 12.471804 & 16.008612 \\ 6.489572 & 12.393397 & 15.096616 \\ 7.257377 & 14.331238 & 16.766708 \\ 8.182724 & 14.245305 & 14.241422 \\ 21.151188 & 0.330135 & 16.102798 \\ 18.634153 & 24.611530 & 15.009956 \\ 19.298063 & 2.108734 & 16.852156 \\ 20.475866 & 2.118444 & 14.411101 \\ 21.318680 & 12.466706 & 3.547490 \\ 18.707462 & 12.550453 & 2.783326 \\ 19.559015 & 14.239480 & 4.634992 \\ 20.480722 & 14.411345 & 2.127183 \\ 22.257866 & 6.572590 & 3.874469 \\ 24.775385 & 6.401454 & 2.620929 \\ 24.110989 & 4.552701 & 4.717040 \\ 22.759865 & 4.485460 & 2.116017 \\ 22.085516 & 18.627113 & 16.027548 \\ 24.595024 & 18.706491 & 15.086421 \\ 23.932814 & 16.860409 & 16.836134 \\ 22.857691 & 16.788313 & 14.343374 \\ 10.024923 & 6.495640 & 16.007156 \\ 12.557735 & 6.406552 & 15.013840 \\ 11.726330 & 4.641789 & 16.854824 \\ 10.771853 & 4.635478 & 14.327111 \\ 2.9493606 & 18.607208 & 3.705760 \\ 3.969625 & 24.699451 & 2.691325 \\ 2.098539 & 24.022629 & 2.031056\end{array}$




\begin{tabular}{|c|c|c|c|}
\hline 0 & 4.487888 & 22.856720 & 2.194909 \\
\hline 0 & 2.951791 & 9.944575 & 12.475445 \\
\hline 0 & 3.889276 & 12.466949 & 12.474959 \\
\hline 0 & 2.184228 & 11.732157 & 14.33439 \\
\hline O & 4.634021 & 10.704613 & 14.33172 \\
\hline 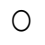 & 15.154389 & 22.158825 & 12.464278 \\
\hline 0 & 16.174408 & 24.616144 & 12.456268 \\
\hline 0 & 14.334150 & 24.014618 & 14.24651 \\
\hline 0 & 16.771807 & 22.846283 & 14.40236 \\
\hline 0 & 15.166284 & 9.859371 & 0.34105 \\
\hline 0 & 16.102798 & 12.397524 & 24.524143 \\
\hline 0 & 14.316188 & 11.715164 & 2.0378 \\
\hline 0 & 16.860165 & 10.709952 & 11647 \\
\hline 0 & 2.868287 & 8.858772 & 6.40752 \\
\hline 0 & 3.895345 & 6.406552 & 6.4786 \\
\hline 0 & 2.193938 & 6.995939 & 4.550759 \\
\hline 0 & 4.555614 & 8.176898 & 4.565809 \\
\hline 0 & 2.861004 & 20.998257 & 18. \\
\hline 0 & 4.119885 & 18.622744 & 18.623959 \\
\hline 0 & 2.279628 & 19.220142 & 16.773262 \\
\hline 0 & 4.629894 & 20.555244 & 16.86550 \\
\hline 0 & 15.089090 & 8.940092 & 18.61691 \\
\hline O & 16.096001 & 6.420631 & 18.710861 \\
\hline 0 & 14.414014 & 7.0 & 16. \\
\hline 0 & 16.860893 & 8.102618 & 16.857981 \\
\hline 0 & 15.174537 & 21.323294 & 6.32401 \\
\hline 0 & 16.179506 & 18.798977 & 6.48544 \\
\hline 0 & 14.261812 & 19.389578 & 4.732819 \\
\hline 0 & 16.773748 & 20.477566 & 30 \\
\hline 0 & 3.863302 & 8.842994 & 0.183759 \\
\hline 0 & 2.779684 & 6.406795 & 24.620270 \\
\hline 0 & 4.638633 & 7.151297 & 2.100966 \\
\hline 0 & 2.208018 & 8.266715 & 2.103637 \\
\hline 0 & 3.880052 & 21.160168 & 12.553123 \\
\hline 0 & 2.953976 & 18.6 & 12. \\
\hline 0 & 4.643246 & 19.381569 & 14.413529 \\
\hline 0 & 2.187141 & 20.399887 & 14.338520 \\
\hline 0 & 16.172466 & 8.851005 & 12.474232 \\
\hline 0 & 15.340818 & 6.241485 & 12.822572 \\
\hline 0 & 16.935175 & 7.07 & 14 \\
\hline 0 & 14.416442 & 8.2 & 14. \\
\hline 0 & 16.178291 & 21.232748 & 0.340815 \\
\hline 0 & 15.078652 & 18.729553 & 24.614202 \\
\hline 0 & 16.858709 & 19.382540 & 2.109948 \\
\hline 0 & 14.426152 & 20.633410 & 2.102 \\
\hline 0 & 3.801402 & 22.16 & $\$ 13$ \\
\hline 0 & 2.778956 & 24.612261 & 6.414563 \\
\hline 0 & 4.638391 & 24.019472 & 4.63523 \\
\hline 0 & 2.279628 & 22.841913 & 4.487645 \\
\hline 0 & 3.804315 & 9.947245 & 18.630026 \\
\hline 0 & 2.792307 & 12.457239 & 18.696053 \\
\hline 0 & 4.615329 & 11.793328 & 16.852156 \\
\hline 0 & 2.118444 & 10.708739 & 16.847786 \\
\hline 0 & 16.023663 & 22.084545 & 18.623714 \\
\hline 0 & 14.922324 & 24.534822 & 18.635611 \\
\hline 0 & 16.846815 & 23.934269 & 16.859922 \\
\hline 0 & 14.406732 & 22.835115 & 16.681747 \\
\hline 0 & 16.094059 & 9.961567 & 6.32547 \\
\hline
\end{tabular}




$$
\begin{array}{rrr}
15.169683 & 12.487824 & 6.410436 \\
16.921337 & 11.802067 & 4.557313 \\
14.400663 & 10.788117 & 4.541049 \\
0.343971 & 2.868287 & 22.162466 \\
24.608860 & 3.964285 & 24.614927 \\
2.110676 & 2.115046 & 24.015833 \\
2.193210 & 4.554886 & 22.837299 \\
0.427233 & 15.157302 & 9.947973 \\
24.619297 & 16.101583 & 12.476901 \\
2.108977 & 14.249918 & 11.800611 \\
2.263364 & 16.764280 & 10.789088 \\
12.384415 & 2.939168 & 10.028322 \\
12.553123 & 3.874469 & 12.552395 \\
14.335850 & 2.111647 & 11.726816 \\
14.251617 & 4.557070 & 10.631546 \\
12.635656 & 15.075497 & 22.080660 \\
12.393882 & 16.092604 & 24.526569 \\
14.332452 & 14.322985 & 24.015589 \\
14.412557 & 16.780302 & 22.925175 \\
6.483017 & 2.877996 & 8.852946 \\
6.396842 & 3.954575 & 6.405581 \\
4.646644 & 2.041008 & 7.083086 \\
4.539107 & 4.482790 & 8.186608 \\
6.491756 & 15.082294 & 21.071566 \\
6.234202 & 16.097944 & 18.555017 \\
4.559983 & 14.255986 & 19.390306 \\
4.561925 & 16.767923 & 20.473682 \\
18.704550 & 2.944994 & 21.146818 \\
18.797279 & 3.975694 & 18.703335 \\
16.932989 & 2.191996 & 19.215044 \\
16.857252 & 4.622369 & 20.399158 \\
18.706734 & 15.176722 & 9.019227 \\
18.802862 & 16.017109 & 6.483746 \\
16.933233 & 14.326383 & 7.163191 \\
16.924978 & 16.862350 & 8.102618 \\
0.417280 & 3.879324 & 8.950288 \\
24.523170 & 2.692296 & 6.564337 \\
2.113104 & 4.556828 & 7.007834 \\
2.208018 & 2.179374 & 8.256763 \\
0.424320 & 16.098671 & 21.150944 \\
24.613472 & 15.073312 & 18.630754 \\
2.204619 & 16.781759 & 19.225969 \\
2.110676 & 14.339248 & 20.462273 \\
12.393397 & 3.869128 & 21.151188 \\
12.468891 & 2.866102 & 18.694111 \\
14.244093 & 4.700048 & 19.379141 \\
14.326140 & 2.276715 & 20.552090 \\
12.565989 & 16.100370 & 8.923828 \\
12.483212 & 15.238379 & 6.409465 \\
14.420082 & 16.866234 & 7.084542 \\
14.410131 & 14.404305 & 8.093637 \\
6.404367 & 3.870342 & 22.086000 \\
6.422816 & 2.790123 & 24.541620 \\
4.637662 & 4.643731 & 23.944950 \\
6.520377 & 16.0873077 & 22.761562 \\
4.723109 & 14.920382 & 12.225174 \\
& & \\
14 & & \\
14 & .776661 & 11.805466
\end{array}
$$




\begin{tabular}{|c|c|c|c|}
\hline 0 & 4.559255 & 14.336578 & 10.692475 \\
\hline 0 & 18.542152 & 3.958702 & 9.944575 \\
\hline O & 18.873987 & 2.776286 & 12.385872 \\
\hline 0 & 16.945370 & 4.540321 & 12.04505 \\
\hline 0 & 16.844872 & 2.195152 & 10.716750 \\
\hline 0 & 18.630997 & 16.107168 & 22.160040 \\
\hline 0 & 18.712803 & 15.083508 & 24.604977 \\
\hline 0 & 16.855310 & 16.857737 & 24.02141 \\
\hline 0 & 16.772778 & 14.405761 & 22.91934 \\
\hline 0 & 2.866102 & 24.692122 & 22.07847 \\
\hline O & 0.430389 & 0.420436 & 20.98539 \\
\hline 0 & 1.009095 & 22.910852 & 22.85113 \\
\hline 0 & 2.202677 & 22.766418 & 20.478781 \\
\hline 0 & 2.953248 & 12.469134 & 9.94918 \\
\hline 0 & 0.502970 & 12.577641 & 8.93329 \\
\hline o & 1.092842 & 10.698301 & 10.70873 \\
\hline 0 & 2.190540 & 10.616496 & 8.25967 \\
\hline 0 & 15.163371 & 24.693821 & \\
\hline 0 & 12.566717 & 0.510009 & 41305 \\
\hline 0 & 13.319472 & 22.930029 & 10.62305 \\
\hline 0 & 14.328082 & 23.010378 & 8.09703 \\
\hline O & 15.081080 & 12.562591 & 22.08187 \\
\hline O & 12.559435 & 12.561377 & 21.153856 \\
\hline 0 & 13.313404 & 10.709710 & 22 . \\
\hline 0 & 14.254530 & 10.701700 & 20.396004 \\
\hline 0 & 16.110567 & 18.630997 & 22.15736 \\
\hline 0 & 18.622259 & 18.608179 & 21.148273 \\
\hline O & 18.035786 & 20.392847 & 22.92250 \\
\hline 0 & 16.854097 & 20.475138 & 20. \\
\hline 0 & 16.186789 & 6.496611 & 21282 \\
\hline 0 & 18.700909 & 6.422816 & 9.007575 \\
\hline 0 & 18.031172 & 8.346335 & 10.621351 \\
\hline 0 & 16.861380 & 8.169859 & 8.179811 \\
\hline 0 & 3.879081 & 18.626143 & 10.032449 \\
\hline 0 & 6.331058 & 18.619831 & \\
\hline 0 & 5.730748 & 20.392363 & 10.777436 \\
\hline 0 & 4.473809 & 20.481693 & 8.351676 \\
\hline 0 & 3.869371 & 6.483260 & 22.175089 \\
\hline 0 & 6.322320 & 6.325717 & 21.070351 \\
\hline 0 & 5.814495 & 8.27 & 22. \\
\hline 0 & 4.552701 & 8.1 & 20 . \\
\hline 0 & 16.181448 & 24.770529 & 21.314554 \\
\hline 0 & 18.706247 & 0.417523 & 22.233833 \\
\hline 0 & 17.949123 & 22.929300 & 20.390179 \\
\hline 0 & 16.937843 & 22.845552 & 22.838757 \\
\hline 0 & 16.093088 & 12.54 & 18 \\
\hline 0 & 18.530258 & 12.718919 & 10.039974 \\
\hline 0 & 18.033844 & 10.707283 & 8.421829 \\
\hline 0 & 16.856525 & 10.791273 & 10.795156 \\
\hline 0 & 3.814511 & 24.591381 & 8.947374 \\
\hline 0 & 6.323776 & 0.432331 & 9.958411 \\
\hline 0 & 5.723950 & 22.829775 & 809 \\
\hline 0 & 4.631108 & 22.847738 & 10.709952 \\
\hline 0 & 3.708673 & 12.473989 & 21.233234 \\
\hline 0 & 6.158951 & 12.654834 & 22.248398 \\
\hline 0 & 5.573448 & 10.704856 & 20.472469 \\
\hline 0 & 342 & 10.699030 & 22.926874 \\
\hline 0 & 2.873870 & 18.705036 & 20.9880 \\
\hline
\end{tabular}




\begin{tabular}{|c|c|c|c|}
\hline 0 & 0.503455 & 18.536812 & 22.252768 \\
\hline 0 & 0.921949 & 20.393091 & 20.402073 \\
\hline 0 & 2.197094 & 20.483391 & 22.757193 \\
\hline 0 & 2.856878 & 6.327902 & 8.93499 \\
\hline O & 0.426262 & 6.338826 & 10.02953 \\
\hline 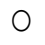 & 1.020504 & 8.176170 & 8.189522 \\
\hline 0 & 2.191025 & 8.248265 & 10.615040 \\
\hline 0 & 15.170653 & 18.630754 & 8 \\
\hline 0 & 12.632987 & 18.624685 & 9. \\
\hline 0 & 13.392782 & 20.481207 & 8.09970 \\
\hline 0 & 14.342162 & 20.469555 & 10.61552 \\
\hline 0 & 15.092004 & 6.333243 & 21.241245 \\
\hline 0 & 12.560163 & 6.335185 & 22.170235 \\
\hline 0 & 13.305151 & 8.164034 & 20.40207 \\
\hline 0 & 14.324441 & 8.190249 & 22.85914 \\
\hline 0 & 22.171206 & 2.778228 & 24.60497 \\
\hline 0 & 21.076178 & 0.332319 & 0.353438 \\
\hline 0 & 22.922747 & 0.921706 & 22.841913 \\
\hline 0 & 20.560343 & 2.187141 & 22.747971 \\
\hline 0 & 22.161011 & 15.081080 & 12.47835 \\
\hline 0 & 21.239546 & 12.551909 & 12.565503 \\
\hline 0 & 23.000910 & 13.240337 & 10.70340 \\
\hline O & 20.486305 & 14.258171 & 10.699030 \\
\hline 0 & 9.962052 & 3.043306 & 12.395582 \\
\hline 0 & 8.937664 & 0.591087 & 12.722075 \\
\hline 0 & 10.622808 & 1.081676 & 10.706554 \\
\hline 0 & 8.173015 & 2.181801 & 10.702186 \\
\hline 0 & 9.863012 & 15.085450 & 24.607405 \\
\hline 0 & 8.933052 & 12.550939 & \\
\hline 0 & 10.785689 & 13.234998 & 22.939739 \\
\hline 0 & 8.258219 & 14.238752 & 22.763990 \\
\hline 0 & 22.165865 & 16.091146 & 18.696781 \\
\hline 0 & 21.227409 & 18.619102 & 18.61109 \\
\hline 0 & 22.923962 & 17.954708 & 20.4 \\
\hline 0 & 20.476839 & 16.855795 & 20. \\
\hline 0 & 22.147657 & 3.878353 & 6.409465 \\
\hline 0 & 21.147303 & 6.401698 & 6.328874 \\
\hline 0 & 22.919106 & 5.733175 & 8.181753 \\
\hline 0 & 20.485819 & 4.640575 & 8.262589 \\
\hline 0 & 9.943847 & 16.1 & 58 \\
\hline 0 & 9.017528 & 18.6 & 108 \\
\hline 0 & 10.690534 & 17.932859 & 8.165247 \\
\hline 0 & 8.184909 & 16.869875 & 8.093880 \\
\hline 0 & 9.954527 & 3.787080 & 18.700909 \\
\hline 0 & 8.929654 & 6.234446 & 18.462048 \\
\hline 0 & 10.693204 & 5.7 & 20. \\
\hline 0 & 8.261860 & 4.631593 & 20.469313 \\
\hline 0 & 21.233234 & 16.180964 & 24.77198 \\
\hline 0 & 22.328989 & 18.626143 & 0.417766 \\
\hline 0 & 20.476353 & 17.948881 & 22.920319 \\
\hline 0 & 22.923475 & 16.773748 & 22.917406 \\
\hline 0 & 21.336401 & 3.811355 & 12.636627 \\
\hline 0 & 22.255680 & 6.322562 & 12.548268 \\
\hline 0 & 20.390179 & 5.566409 & 10.859241 \\
\hline 0 & 22.845068 & 4.551973 & 10.621837 \\
\hline 0 & 9.031851 & 15.936032 & 12.385872 \\
\hline 0 & 9.872479 & 18.462774 & 12.557978 \\
\hline 0 & 8.188793 & 17.784784 & 10.62693 \\
\hline
\end{tabular}




$$
\begin{aligned}
& 10.768941 \\
& 8.929169 \\
& 16.924736 \\
& 3.802859 \\
& 9.954527 \quad 6.326204 \\
& 8.158208 \quad 5.731476 \\
& 10.621837 \\
& 4.632807 \\
& 2.863432 \\
& 21.242458 \\
& 0.424563 \\
& 22.321707 \\
& 1.020747 \\
& 22.831232 \\
& 21.393690 \\
& 2.191996 \\
& 15.249060 \\
& 12.644639 \\
& 22.343554 \\
& 20.390421 \\
& 23.014019 \\
& 8.852704 \\
& 10.027594 \\
& 8.267443 \\
& 10.628148 \\
& 8.773568 \\
& 10.036819 \\
& 8.005520 \\
& 10.632275 \\
& 24.765915 \\
& 0.497144 \\
& 23.002853 \\
& 22.850166 \\
& 24.685568 \\
& 0.504426 \\
& 22.920076 \\
& 22.924688 \\
& 12.473989 \\
& 12.638084 \\
& 10.697574 \\
& 10.788117 \\
& 12.481028 \\
& 12.570601 \\
& 10.706312 \\
& 10.631304 \\
& 18.708191 \\
& 18.536327 \\
& 20.489946 \\
& 20.483877 \\
& 18.784412 \\
& 18.791939 \\
& 20.564955 \\
& 20.571751 \\
& 6.322805 \\
& 6.239058 \\
& 8.171558 \\
& 8.351433 \\
& 6.325717 \\
& 6.327417 \\
& 8.172771 \\
& 8.174228 \\
& 24.867872 \\
& 0.427718 \\
& 22.995813 \\
& 22.911823 \\
& 24.691879
\end{aligned}
$$




\begin{tabular}{|c|c|c|c|}
\hline 0 & 0.500057 & 10.030993 & 6.315765 \\
\hline 0 & 22.915949 & 8.272298 & 5.813281 \\
\hline 0 & 23.000425 & 10.628877 & 4.390061 \\
\hline O & 12.475445 & 21.408255 & 3.7094 \\
\hline 0 & 12.634201 & 22.239660 & 6.2419 \\
\hline 0 & 10.879390 & 20.318325 & 5.4853 \\
\hline O & 10.628633 & 22.920805 & 4.636691 \\
\hline 0 & 12.478358 & 8.850276 & 16.0945 \\
\hline o & 12.560891 & 9.871509 & $18.5428 \varepsilon$ \\
\hline 0 & 10.631546 & 8.176656 & 17.95762 \\
\hline 0 & 10.627663 & 10.538089 & 16.7633 \\
\hline$c$ & 18.630512 & 21.244644 & 2.9299 \\
\hline 0 & 18.702608 & 22.240873 & 0.4216 \\
\hline 0 & 20.389935 & 20.320024 & 1.095027 \\
\hline c & 20.572237 & 22.759621 & 2.1927 \\
\hline 0 & 18.705521 & 8.948345 & $15.1650^{\circ}$ \\
\hline 0 & 18.606966 & 9.879519 & 12.64390 \\
\hline 0 & 20.409597 & 8.090239 & 13.318501 \\
\hline $\mathrm{O}$ & 20.496744 & 10. & \\
\hline 0 & 6.574290 & 1032 & 15. \\
\hline 0 & 6.328631 & 22.163195 & 12.65580 \\
\hline 0 & 8.254334 & 20.410568 & 13.21557 \\
\hline 0 & 8.264772 & 22.848709 & 14.32856 \\
\hline 0 & 6.426942 & 0578 & \\
\hline 0 & 6.315765 & 9. & 0 . \\
\hline 0 & 8.175200 & 3210 & 1.02317 \\
\hline 0 & 8.262102 & 10.630333 & 2.18374 \\
\hline 0 & 13.148823 & 5.238944 & 8.3305 \\
\hline 0 & 12.467191 & 6.659979 & 5.98053 \\
\hline 0 & 11.810321 & & \\
\hline 0 & 14.485138 & 7 . & 7. \\
\hline 0 & 7.835841 & 18.286299 & 19.556103 \\
\hline ? & 7.669560 & 20.146461 & 18.024860 \\
\hline 0 & 8.433238 & 20.477566 & 21.240274 \\
\hline $\mathrm{H}$ & 12.687847 & 22.177759 & 3.146716 \\
\hline $\mathrm{H}$ & 15.864906 & 7 . & 957 \\
\hline $\mathrm{H}$ & 25.047501 & 484 & 1.7 \\
\hline $\mathrm{H}$ & 21.950548 & 16.018080 & 6.477191 \\
\hline$H$ & 13.549595 & 5.017802 & 9.228231 \\
\hline $\mathrm{H}$ & 9.032336 & 15.9 & 19.367002 \\
\hline $\mathrm{H}$ & 7.808653 & 22.4 & 18.519819 \\
\hline $\mathrm{H}$ & 8.56 & $22 . \varepsilon$ & 20. \\
\hline $\mathrm{H}$ & 9.997251 & 21.791552 & 17. \\
\hline $\mathrm{H}$ & 11.123349 & 19.762436 & 20.252783 \\
\hline $\mathrm{H}$ & 10.488569 & 19.103622 & 17.248074 \\
\hline $\mathrm{H}$ & 11.503733 & 18.106667 & 18. \\
\hline $\mathrm{H}$ & 12.038016 & 19.7 & 17.897661 \\
\hline $\mathrm{H}$ & 11.427510 & 23.5 & 18.878 \\
\hline $\mathrm{H}$ & 12.072244 & 21.964870 & 19.084206 \\
\hline 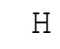 & 11.112426 & 22.727335 & 20.400373 \\
\hline $\operatorname{Re}$ & 12.969919 & 6.900540 & 7.584598 \\
\hline $\operatorname{Re}$ & 8.643699 & 20.033098 & 19.623100 \\
\hline C & 8.661177 & 22.357391 & 19. \\
\hline 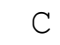 & 9.934865 & 22.118530 & 18.683187 \\
\hline C & 10.481287 & 19.638151 & 19.37088 \\
\hline C & 11.140100 & 19.131296 & 18.130939 \\
\hline & 11.204670 & 22.596012 & 19.3152 \\
\hline
\end{tabular}




\begin{tabular}{|c|c|c|c|}
\hline USY & C4H8_M8 & $E=-721 \varepsilon$ & \\
\hline $\mathrm{Si}$ & $23 . \overline{3} 8275 \overline{0}$ & 3.326106 & 1.243588 \\
\hline $\mathrm{Si}$ & 23.428387 & 1.237034 & 3.375869 \\
\hline & 23.397558 & 15.570213 & 13.401035 \\
\hline 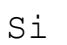 & 23.391973 & 13.426281 & 15.572883 \\
\hline & 11.248849 & 1.240189 & 15.587690 \\
\hline & 11.162432 & 15.579194 & 1.192611 \\
\hline & 11.227973 & 13.409775 & 3.366644 \\
\hline II & 7.633148 & 3.351594 & 1.217857 \\
\hline 然 & 7.657908 & 5.499896 & 3.346496 \\
\hline & 7.724663 & 15.497146 & 13.270680 \\
\hline i & 20.003241 & 5.426829 & 15.513897 \\
\hline & 19.907598 & 15.644493 & 1.26762 \\
\hline $\mathrm{Si}$ & 19.882839 & 17.805662 & 3.34989 \\
\hline Si & 7.626351 & 3.370043 & 5.510334 \\
\hline Si & 7.494782 & 15.546424 & 17.727497 \\
\hline Si & 7.749423 & 13.326269 & 15.48719 \\
\hline $\mathrm{Si}$ & 19.916822 & 3.340428 & 17.7 \\
\hline $\mathrm{Si}$ & 19.866089 & 1.201107 & 15.62240 \\
\hline Si & 19.934301 & 15.590118 & 5.433869 \\
\hline $\mathrm{Si}$ & 20.010279 & 13.381130 & 3.279741 \\
\hline $\mathrm{Si}$ & 23.434214 & 3.350380 & 36065 \\
\hline Si & 23.366730 & 17.7 & 15. \\
\hline Si & 11.236955 & 3.3 & 17. \\
\hline $\mathrm{Si}$ & 11.267783 & 5.535580 & 15.575797 \\
\hline Si & 11.238896 & 15.650805 & 5.45013 \\
\hline $\mathrm{Si}$ & 11.185493 & 17.781872 & 3.2 \\
\hline Si & 1.308886 & 23.4 & 3.33 \\
\hline $\mathrm{Si}$ & 3.379267 & 23.4 & $1.20207 \varepsilon$ \\
\hline $\mathrm{Si}$ & 1.254511 & 11.2 & 15.5 \\
\hline Si & 3.411067 & 11.213165 & 13.38598 \\
\hline $\mathrm{Si}$ & 13.454925 & 23.445137 & 15.527004 \\
\hline $\mathrm{Si}$ & 15.606867 & 23.4 & 13.413901 \\
\hline Si & 13.4 & 11.2 & 3. \\
\hline $\mathrm{Si}$ & 15.609781 & 11.173598 & 1.19892 \\
\hline Si & 1.360349 & 7.681211 & 3.341884 \\
\hline $\mathrm{Si}$ & 3.381695 & 7.602319 & 5.525384 \\
\hline $\mathrm{Si}$ & 1.308644 & 19.881382 & 15.59643 \\
\hline Si & 3.439 & 19.8 & 17. \\
\hline $\mathrm{Si}$ & 13.4 & 7.6 & 15. \\
\hline $\mathrm{Si}$ & 15.584293 & 7.632662 & 17.79182 \\
\hline Si & 15.556134 & 19.952265 & 5.499653 \\
\hline $\mathrm{Si}$ & 5.527083 & 7.662277 & 3.378539 \\
\hline Si & 3.406212 & 7.665433 & 1.18168 \\
\hline $\mathrm{Si}$ & 5.50 & 19.9 & 15.62 \\
\hline $\mathrm{Si}$ & 0 & 19.8 & 13. \\
\hline Si & 17.878242 & 7.682668 & $15.70420 s$ \\
\hline $\mathrm{Si}$ & 15.625073 & 7.743354 & 13.483327 \\
\hline $\mathrm{Si}$ & 17.722399 & 6101 & 3.36009 \\
\hline Si & 15.606867 & 20.0 & 1.23072 \\
\hline $\mathrm{Si}$ & 5.499896 & 23.3 & 3.37683 \\
\hline $\mathrm{Si}$ & 3.410096 & 23.401199 & $5 \quad 51057$ \\
\hline Si & 0819 & 11.186220 & 15.60662 \\
\hline $\mathrm{Si}$ & 3.350623 & 11.237926 & 17.75395 \\
\hline Si & 17.722157 & 23.384207 & 15.61415 \\
\hline Si & 15.5 & 23.3 & 17.7 \\
\hline Si & 17.821440 & 11.269726 & 3.30304 \\
\hline
\end{tabular}




\begin{tabular}{|c|c|c|c|}
\hline i & 15.662457 & 11.239625 & 5.470524 \\
\hline $\mathrm{Si}$ & 3.358148 & 1.276358 & 23.366730 \\
\hline $\mathrm{Si}$ & 1.242859 & 3.365188 & 23.414549 \\
\hline$S=$ & 3.374412 & 13.381130 & 11.228702 \\
\hline SI & 1.284369 & 15.561232 & 11.258316 \\
\hline $\mathrm{Si}$ & 15.613908 & 1.222469 & 11.204912 \\
\hline$i$ & 13.360497 & 3.332417 & 11.250305 \\
\hline L & 15.561960 & 13.423853 & 23.391973 \\
\hline S & 13.447642 & 15.567543 & 23.4111 \\
\hline S & 3.359847 & 1.200864 & 7.6586 \\
\hline i & 5.517616 & 3.318338 & 7.61955 \\
\hline i & 3.269546 & 13.341805 & 19.961 \\
\hline i & 17.774103 & 3.372470 & 19.86171 \\
\hline 2 & 15.641581 & 13.429436 & 7.645042 \\
\hline i & 17.822897 & 15.573853 & 7.71786 \\
\hline i & 3.339700 & 5.433141 & 7.65572 \\
\hline $\mathrm{Si}$ & 1.241889 & 3.327319 & \\
\hline $\mathrm{Si}$ & 3.410338 & 17.696669 & 19.803703 \\
\hline $\mathrm{Si}$ & 1.248200 & 15.577982 & 19.862206 \\
\hline Si & 15.564873 & 5.466397 & 19.94522 \\
\hline Si & 13.393753 & 3.388248 & 19.95323 \\
\hline i & 15.664398 & 17.779444 & 7.63533 \\
\hline $\mathrm{Si}$ & 13.467546 & 15.648863 & \\
\hline $\mathrm{Si}$ & 3.377810 & 5.510819 & 23.38177 \\
\hline Si & 5.503294 & 3.386307 & 23.34633 \\
\hline $\mathrm{Si}$ & 5.555242 & 15.554193 & 11.112911 \\
\hline $\mathrm{Si}$ & 17.857367 & 3.319066 & 11.25443 \\
\hline $\mathrm{Si}$ & 15.611480 & 17. & 23.44101 \\
\hline $\mathrm{Si}$ & 17.728710 & 15.614151 & 23.42741 \\
\hline $\mathrm{Si}$ & 1.64630 & 21.669449 & 23.723082 \\
\hline $\mathrm{Si}$ & 1.615475 & 23.778667 & 21.60876 \\
\hline Si & 1.678103 & 9.445246 & 11.58335 \\
\hline $\mathrm{Si}$ & 1.672034 & 11.593063 & 9.45519 \\
\hline $\mathrm{Si}$ & 13.874632 & 21.701006 & 11. \\
\hline $\mathrm{Si}$ & 13.8323 & 23.855135 & 994 \\
\hline Si & 13.832881 & 9.424371 & 23.787893 \\
\hline $\mathrm{Si}$ & 13.802051 & 11.637971 & 21.654158 \\
\hline $\mathrm{Si}$ & 17.482323 & 21.678188 & 23.77527 \\
\hline $\mathrm{Si}$ & 17.392750 & 19.537169 & 21.688141 \\
\hline $\mathrm{Si}$ & 17.427219 & 9.516856 & 11.59039 \\
\hline $\mathrm{Si}$ & 17.450523 & 7.328016 & 9.487242 \\
\hline $\mathrm{Si}$ & 5.153983 & 21.645418 & 11.644282 \\
\hline $\mathrm{Si}$ & 5.118542 & 19.543964 & 9.494281 \\
\hline $\mathrm{Si}$ & 5.183113 & 9.434566 & 23.73254 \\
\hline $\mathrm{Si}$ & 5.152284 & 7.305684 & 21.613859 \\
\hline $\mathrm{Si}$ & 17.332550 & 21.699306 & $19.52114^{\circ}$ \\
\hline $\mathrm{Si}$ & 17.412657 & 23.852463 & 21.663137 \\
\hline $\mathrm{Si}$ & 17.388866 & 11.698900 & 9.491611 \\
\hline $\mathrm{Si}$ & 5.102520 & 21.600510 & 7.31563 \\
\hline $\mathrm{Si}$ & 5.119756 & 23.744442 & 9.43456 \\
\hline $\mathrm{Si}$ & 5.076790 & 9.457870 & 19.52697 \\
\hline $\mathrm{Si}$ & 5.023628 & 11.628018 & 21.70343 \\
\hline $\mathrm{Si}$ & 1.586345 & 21.610462 & 19.53862 \\
\hline $\mathrm{Si}$ & 1.608192 & 19.526974 & 21.59929 \\
\hline $\mathrm{Si}$ & 1.631010 & 9.422186 & 7.30568 \\
\hline $\mathrm{Si}$ & 1.623485 & 7.276068 & 9.430439 \\
\hline $\mathrm{Si}$ & 13.875361 & 21.742758 & 7.18406 \\
\hline $\mathrm{Si}$ & 13.888712 & 19.547850 & 9.34984 \\
\hline
\end{tabular}




\begin{tabular}{|c|c|c|c|}
\hline $\mathrm{Si}$ & 13.810547 & 7.235530 & 21.661196 \\
\hline$i$ & 23.772842 & 1.591928 & 21.626968 \\
\hline & 21.672604 & 1.535368 & 23.712400 \\
\hline $\mathrm{Si}$ & 23.861446 & 13.851086 & 9.423885 \\
\hline & 21.732807 & 13.773893 & 11.58917 \\
\hline & 11.537231 & 1.652129 & 9.47291 \\
\hline 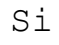 & 9.415389 & 1.703834 & 11.627775 \\
\hline & 11.663217 & 13.825112 & 21.67746 \\
\hline i & 9.468065 & 13.762726 & 23.75779 \\
\hline & 23.815081 & 17.348085 & 21.70149 \\
\hline & 21.689840 & 17.390566 & 19.560957 \\
\hline & 21.678915 & 5.179714 & 7.25179 \\
\hline $\mathrm{Si}$ & 11.663945 & 17.391779 & 9.35980 \\
\hline$i$ & 9.462725 & 17.392021 & 7.203730 \\
\hline S & 11.577285 & 5.157138 & 21.638863 \\
\hline S & 9.474861 & 5.111988 & 19.50998 \\
\hline$\perp$ & 19.531586 & 17.386196 & 21.69760 \\
\hline$i$ & 21.720669 & 17.394207 & 23.82309 \\
\hline$i$ & 19.580864 & 5.151313 & \\
\hline $\mathrm{Si}$ & 21.729893 & 5.068051 & $11.65714^{\circ}$ \\
\hline i & 7.264174 & 17.351727 & $9.340 \varepsilon$ \\
\hline L & 9.457870 & 17.275261 & 11.530678 \\
\hline $\mathrm{Si}$ & 7.293788 & 5.155439 & 21.64857 \\
\hline $\mathrm{Si}$ & 9.408106 & 5. & 23. \\
\hline $\mathrm{Si}$ & 19.552462 & 1.6380 & 21.59832 \\
\hline $\mathrm{Si}$ & 21.679886 & 1.608192 & 19.48352 \\
\hline $\mathrm{Si}$ & 19.554646 & 13.829239 & 9.43068 \\
\hline Si & 7.308111 & 1.608192 & 9.4352 \\
\hline $\mathrm{Si}$ & 9.425584 & 1.659412 & 7.29427 \\
\hline$i$ & 7.247910 & 13.691602 & 21.623814 \\
\hline $\mathrm{Si}$ & 21.703192 & 23.74 & 1.58707 \\
\hline Si & 23.837656 & 21.646145 & 1.61668 \\
\hline $\mathrm{Si}$ & 21.714600 & 11.595734 & 13.78287 \\
\hline$i$ & 23.851007 & 9.410534 & 13.78845 \\
\hline$i$ & 9.489427 & 23.7 & $13.82147]$ \\
\hline $\mathrm{Si}$ & 9.4 & 11.6 & 1.65892 \\
\hline $\mathrm{Si}$ & 11.616609 & 9.464666 & 1.645818 \\
\hline $\mathrm{Si}$ & 21.682072 & 23.758036 & 17.38619 \\
\hline $\mathrm{Si}$ & 19.527945 & 21.672361 & 17.39979 \\
\hline $\mathrm{Si}$ & 21.815338 & 11.644526 & $4.88210^{\circ}$ \\
\hline $\mathrm{Si}$ & 19.640335 & 9.536277 & 5.00833 \\
\hline $\mathrm{Si}$ & 9.359072 & 23.82 & 5.1615 \\
\hline $\mathrm{Si}$ & 9.465637 & 11.543543 & 17.29273 \\
\hline Si & 7.260533 & 9.422671 & 17.36920 \\
\hline $\mathrm{Si}$ & 21.693724 & 19.522120 & 17.343231 \\
\hline $\mathrm{Si}$ & 23.837172 & 21.677460 & 17.35075 \\
\hline Si & 21.704163 & 7.373652 & 5.12145 \\
\hline $\mathrm{Si}$ & 23.813866 & 9.446703 & 5.130 \\
\hline $\mathrm{Si}$ & 9.483843 & 19.528671 & 4.97750 \\
\hline Si & 11.570731 & 21.726980 & 5.12825 \\
\hline $\mathrm{Si}$ & 9.434323 & 7.261261 & 17.32211 \\
\hline $\mathrm{Si}$ & 11.576071 & 9.366597 & 17.36192 \\
\hline $\mathrm{Si}$ & 21.697123 & 19.507553 & 1.63465 \\
\hline Si & 19.558044 & 21.628910 & 1.65212 \\
\hline Si & 21.735476 & 7.231161 & 13.78408 \\
\hline $\mathrm{Si}$ & 19.580133 & 9.401067 & 13.841377 \\
\hline $\mathrm{Si}$ & 9.501806 & 19.485220 & 13.76127 \\
\hline $\mathrm{Si}$ & 7.349134 & 21.626968 & 13.79986 \\
\hline
\end{tabular}




\begin{tabular}{|c|c|c|c|}
\hline $\mathrm{Si}$ & 9.428497 & 7.297673 & 1.613047 \\
\hline $\mathrm{Si}$ & 7.313451 & 9.422671 & 1.663296 \\
\hline$\therefore$ & 11.656662 & 21.704163 & 13.697670 \\
\hline$i$ & 11.212923 & 3.403056 & 13.37676 \\
\hline L & 20.023146 & 3.292364 & 13.41827 \\
\hline$\perp$ & 7.629263 & 1.209846 & 3.36567 \\
\hline & 23.369642 & 15.605168 & 17.72215 \\
\hline $\mathrm{Si}$ & 5.420761 & 15.494963 & 19.9583 \\
\hline i & 3.469326 & 17.706379 & 11.29327 \\
\hline i & 13.797196 & 9.426798 & 19.50852 \\
\hline 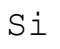 & 23.792992 & 5.133593 & 9.42218 \\
\hline 1 & 15.580167 & 1.280728 & 19.89716 \\
\hline 1 & 15.578466 & 5.471252 & 11.2097 \\
\hline 1 & 17.524319 & 9.603274 & 7.20567 \\
\hline & 9.450586 & 13.519737 & 19.64057 \\
\hline 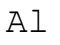 & 7.260290 & 21.632551 & 5.16854 \\
\hline 1 & 7.660578 & 17.744976 & 15.43985 \\
\hline 1 & 23.372068 & 5.398428 & 3.40839 \\
\hline Al & 13.454440 & 19.861477 & 3.287023 \\
\hline Al & 21.793249 & 13.678251 & 7.23577 \\
\hline $\mathrm{L}$ & 16.715488 & 5.188453 & 13.78336 \\
\hline & 6.617741 & 18.207647 & 18.05496 \\
\hline 0 & 22.164165 & 0.349069 & 2.86610 \\
\hline 0 & 24.605705 & 24.541864 & \\
\hline 0 & 24.029911 & 2.099510 & 2.11747 \\
\hline 0 & 22.847010 & 2.185442 & 4.55707 \\
\hline 0 & 22.162952 & 12.472775 & 15.08520 \\
\hline 0 & 24.610559 & 12.475930 & 16.09648 \\
\hline 0 & 23.941551 & 14.330267 & 14.32929 \\
\hline 0 & 22.844097 & 14.396536 & 16. \\
\hline 0 & 10.025409 & 0.2 & 15. \\
\hline 0 & 12.558706 & 24.691635 & 16.09284 \\
\hline 0 & 11.644768 & 2.102180 & 14.25064 \\
\hline 0 & 10.707040 & 2.192482 & 16.77933 \\
\hline 0 & 9.944575 & 12.480542 & 2016 \\
\hline 0 & 12.480785 & 12.4 & 3. \\
\hline 0 & 11.714437 & 14.332694 & 2.10387 \\
\hline 0 & 10.782048 & 14.322985 & 4.62795 \\
\hline 0 & 8.928440 & 6.407765 & 2.87945 \\
\hline 0 & 6.415291 & 6.409223 & 3.88369 \\
\hline 0 & 7.077987 & 4.629166 & 2.08955 \\
\hline 0 & 8.181511 & 4.5 & 4.55003 \\
\hline 0 & 9.018742 & 18.713289 & 15.10122 \\
\hline 0 & 6.247311 & 18.623230 & 16.27660 \\
\hline 0 & 7.157851 & 16.673008 & 14.23365 \\
\hline 0 & 7.849920 & 17.026203 & 17.1121 \\
\hline 0 & 21.307758 & 6.2 & 15.00849 \\
\hline 0 & 18.796551 & 6.402425 & 16.02512 \\
\hline 0 & 19.298307 & 4.565567 & 14.24773 \\
\hline 0 & 20.481207 & 4.467011 & 16.69267 \\
\hline 0 & 21.242216 & 18.548222 & 2.86755 \\
\hline 0 & 18.719114 & 18.785870 & 3.88417 \\
\hline 0 & 19.284227 & 16.851427 & 2.17670 \\
\hline 0 & 20.398916 & 16.855553 & 4.56289 \\
\hline 0 & 8.930868 & 0.342515 & 3.79557 \\
\hline 0 & 6.401212 & 24.609833 & 2.78235 \\
\hline 0 & 7.082600 & 2.106307 & 4.63596 \\
\hline 0 & 8.175685 & 2.190297 & 2.18835 \\
\hline
\end{tabular}




$$
\begin{aligned}
& 9.016557 \quad 12.470590 \\
& 6.488843 \quad 12.391697 \\
& 7.256649 \quad 14.318616 \\
& 8.181996 \quad 14.246035 \\
& \begin{array}{ll}
21.148031 & 0.332562
\end{array} \\
& 18.632938 \quad 24.611044 \\
& \begin{array}{ll}
19.297821 & 2.109220
\end{array} \\
& 20.475866 \quad 2.118930 \\
& 21.318680 \quad 12.466706 \\
& 18.707462 \quad 12.550453 \\
& 19.559015 \quad 14.239480 \\
& 20.480722 \quad 14.411101 \\
& 22.257866 \quad 6.572834 \\
& 24.775385 \quad 6.401454 \\
& 24.110989 \quad 4.552458 \\
& 22.759865 \quad 4.485460 \\
& 22.085030 \quad 18.627356 \\
& \begin{array}{ll}
24.595024 & 18.706247
\end{array} \\
& 23.933056 \quad 16.860409 \\
& 22.858175 \quad 16.787827 \\
& 10.025409 \quad 6.495883 \\
& 12.557007 \quad 6.406309 \\
& 11.725844 \quad 4.641546 \\
& \begin{array}{ll}
10.771611 & 4.635963
\end{array} \\
& 9.877577 \quad 18.607208 \\
& 12.373491 \quad 18.699451 \\
& 11.724874 \quad 16.849241 \\
& 10.616738 \quad 16.836863 \\
& 2.949606 \quad 22.169264 \\
& 3.969382 \quad 24.613472 \\
& 2.098539 \quad 24.022142 \\
& 4.487888 \quad 22.855991 \\
& 2.951549 \quad 9.944575 \\
& 3.889519 \quad 12.467434 \\
& \begin{array}{ll}
2.184471 & 11.732399
\end{array} \\
& 4.633293 \quad 10.704613 \\
& \begin{array}{ll}
15.155117 & 22.159309
\end{array} \\
& 16.174652 \quad 24.615658 \\
& 14.333179 \quad 24.014618 \\
& 16.772049 \quad 22.846039 \\
& \begin{array}{ll}
15.166041 & 9.859371
\end{array} \\
& 16.102798 \quad 12.397524 \\
& 14.316188 \quad 11.715164 \\
& 16.860165 \quad 10.709952 \\
& 2.868287 \quad 8.858772 \\
& 3.895345 \quad 6.406552 \\
& 2.193696 \quad 6.995939 \\
& \begin{array}{ll}
4.555614 & 8.176898
\end{array} \\
& 2.866102 \quad 20.982721 \\
& 4.207031 \quad 18.554775 \\
& 2.280356 \quad 19.218443 \\
& 4.627710 \quad 20.565683 \\
& 15.089334 \quad 8.940092 \\
& 16.096001 \quad 6.420146 \\
& \begin{array}{ll}
14.414014 & 7.080901
\end{array} \\
& \begin{array}{ll}
16.860651 & 8.102618
\end{array} \\
& 15.174537 \quad 21.323536 \\
& \begin{array}{lll}
0 & 16.179506 & 18.798977
\end{array}
\end{aligned}
$$




$$
\begin{array}{rrr}
14.261569 & 19.389578 & 4.732819 \\
16.773748 & 20.477324 & 4.553672 \\
3.862574 & 8.842751 & 0.184487 \\
2.779684 & 6.406552 & 24.620028 \\
4.638633 & 7.151297 & 2.101209 \\
2.207775 & 8.266715 & 2.103879 \\
3.879567 & 21.162109 & 12.551667 \\
2.950820 & 18.615463 & 12.549726 \\
4.635963 & 19.383753 & 14.407702 \\
2.186413 & 20.400616 & 14.340948 \\
16.172224 & 8.850761 & 12.474474 \\
15.340818 & 6.241242 & 12.822329 \\
16.934931 & 7.071434 & 14.396295 \\
14.416442 & 8.254334 & 14.406246 \\
16.178535 & 21.232748 & 0.341058 \\
15.078652 & 18.729067 & 24.614685 \\
16.858709 & 19.382540 & 2.109705 \\
14.426637 & 20.633167 & 2.102909 \\
3.801402 & 22.168777 & 6.491513 \\
2.778956 & 24.612261 & 6.414805 \\
4.638148 & 24.019472 & 4.634992 \\
2.279628 & 22.841913 & 4.487645 \\
3.804558 & 9.947245 & 18.630512 \\
2.791093 & 12.456268 & 18.695568 \\
4.615815 & 11.793814 & 16.852156 \\
2.117959 & 10.708739 & 16.847542 \\
16.025120 & 22.084787 & 18.622744 \\
14.924509 & 24.536764 & 18.634880 \\
16.846329 & 23.934027 & 16.861135 \\
14.409401 & 22.834631 & 16.679077 \\
16.094059 & 9.961567 & 6.325475 \\
15.169683 & 12.487824 & 6.410436 \\
16.921337 & 11.802067 & 4.557313 \\
14.400663 & 10.788117 & 4.541049 \\
0.343971 & 2.868044 & 22.162466 \\
24.608860 & 3.964285 & 24.614927 \\
2.110676 & 2.114803 & 24.015833 \\
2.193210 & 4.554886 & 22.837299 \\
0.426990 & 15.156817 & 9.947973 \\
24.619541 & 16.101583 & 12.476901 \\
2.109705 & 14.250161 & 11.800367 \\
2.261664 & 16.764280 & 10.788360 \\
12.384415 & 2.939168 & 10.028079 \\
12.552638 & 3.874712 & 12.552638 \\
14.336092 & 2.111647 & 11.726573 \\
14.251132 & 4.556828 & 10.631061 \\
12.635656 & 15.076468 & 22.081146 \\
12.393639 & 16.092604 & 24.526327 \\
14.332209 & 14.322742 & 24.015347 \\
14.413043 & 16.780060 & 22.925417 \\
6.483260 & 2.877754 & 8.853189 \\
6.396842 & 3.954575 & 6.405338 \\
4.646644 & 2.041251 & 7.083086 \\
4.56149670 & 15.482548 & 8.186366 \\
\hline & 16.025362 & 18.480253 \\
14.5390 & 16.770350 & 20.478294
\end{array}
$$




\begin{tabular}{|c|c|c|c|}
\hline 0 & 18.704550 & 2.945723 & 21.146818 \\
\hline 0 & 18.797035 & 3.975694 & 18.704063 \\
\hline 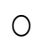 & 16.932503 & 2.191268 & 19.215288 \\
\hline 0 & 16.857737 & 4.622855 & 20.39940 \\
\hline O & 18.706491 & 15.176722 & 9.01898 \\
\hline O & 18.802620 & 16.017109 & 6.483746 \\
\hline 0 & 16.933474 & 14.326383 & 7.1631 \\
\hline 0 & 16.924494 & 16.862593 & 8.1028 \\
\hline 0 & 0.417280 & 3.879324 & $8.9502 \xi$ \\
\hline 0 & 24.523170 & 2.692296 & 6.56433 \\
\hline 0 & 2.113104 & 4.556828 & 7.0078 \\
\hline 0 & 2.208018 & 2.179374 & 8.2567 \\
\hline 0 & 0.425048 & 16.100613 & 21.150702 \\
\hline 0 & 24.612017 & 15.072827 & 18.62856 \\
\hline 0 & 2.204376 & 16.775690 & 19.21723 \\
\hline 0 & 2.104122 & 14.338520 & 20.46737 \\
\hline 0 & 12.393882 & 3.869614 & 21.151188 \\
\hline 0 & 12.470105 & 2.867558 & 18.6931 \\
\hline 0 & 14.243363 & 320 & 19.379141 \\
\hline 0 & 14.328082 & 2.278414 & 20.55403 \\
\hline ○ & 12.565503 & 16.100370 & 8.92407 \\
\hline O & 12.483455 & 15.238379 & 6.40970 \\
\hline O & 14.420082 & 16.865992 & 7.084542 \\
\hline 0 & 14.410131 & 14.2 & 637 \\
\hline 0 & 6.404125 & 3.871070 & 22.085758 \\
\hline 0 & 6.422331 & 2.790123 & 24.54210 \\
\hline 0 & 4.637905 & 4.643246 & 23.944950 \\
\hline O & 4.484247 & 2.271860 & 22.761562 \\
\hline 0 & 6.319406 & 16.087263 & 51 \\
\hline 0 & 6.579387 & 14.989079 & 12.237554 \\
\hline 0 & 4.718254 & 16.782001 & 11.80546 \\
\hline 0 & 4.563868 & 14.338520 & 10.695631 \\
\hline 0 & 18.541910 & 3.958702 & 9.94481 \\
\hline 0 & 18.873987 & 2.776286 & 12.385 \\
\hline 0 & 16.945370 & 321 & 12. \\
\hline 0 & 16.844387 & 2.194909 & 10.716750 \\
\hline 0 & 18.631241 & 16.106924 & 22.160280 \\
\hline 0 & 18.712803 & 15.083508 & 24.604733 \\
\hline 0 & 16.854824 & 16.857496 & 24.021416 \\
\hline 0 & 16.772778 & 14. & 22.919 \\
\hline 0 & 2.866102 & 24.6 & 22 . \\
\hline 0 & 0.430146 & 0.420193 & 20.985390 \\
\hline 0 & 1.009823 & 22.907454 & 22.849195 \\
\hline 0 & 2.202192 & 22.76471 & 20.476839 \\
\hline 0 & 2.953733 & 12.469619 & 9.94943 \\
\hline 0 & 0.503212 & 12.57 & 295 \\
\hline 0 & 1.093085 & 10.698545 & 10.708739 \\
\hline 0 & 2.190783 & 10.616496 & 8.25967 \\
\hline 0 & 15.163371 & 24.693821 & 9.86786 \\
\hline 0 & 12.566717 & 0.509767 & 8.941063 \\
\hline 0 & 13.319472 & 22.929543 & 10.623293 \\
\hline 0 & 14.328082 & 23.010620 & 8.09703 \\
\hline 0 & 15.080837 & 12.562834 & 22.08187 \\
\hline 0 & 12.559192 & 12.561377 & 21.15361 \\
\hline 0 & 13.313404 & 10.709710 & 22.911579 \\
\hline 0 & 14.254288 & 10.701457 & 20.395519 \\
\hline 0 & 16.111538 & 18.630754 & 22.157125 \\
\hline ? & 18.622259 & 18.609152 & 21.14851 \\
\hline
\end{tabular}




\begin{tabular}{|c|c|c|c|}
\hline 0 & 18.036026 & 20.392363 & 22.922747 \\
\hline 0 & 16.854582 & 20.475382 & 20.482906 \\
\hline 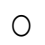 & 16.186789 & 6.496611 & 10.021525 \\
\hline 0 & 18.701151 & 6.423059 & 9.00757 \\
\hline O & 18.031172 & 8.346335 & 10.621351 \\
\hline O & 16.861380 & 8.169859 & 8.180054 \\
\hline 0 & 3.878595 & 18.627356 & 10.031235 \\
\hline 0 & 6.331058 & 18.620317 & 8 \\
\hline 0 & 5.730262 & 20.392120 & 10.77767 \\
\hline 0 & 4.473809 & 20.481936 & 8.35143 \\
\hline 0 & 3.869128 & 6.483502 & 22.175573 \\
\hline 0 & 6.322562 & 6.325475 & 21.070351 \\
\hline 0 & 5.814495 & 8.2 & 22.7613 \\
\hline 0 & 4.552701 & 8.190735 & 20.393091 \\
\hline 0 & 16.182419 & 24.771986 & 21.31504 \\
\hline 0 & 18.706247 & 0.416795 & 22.234320 \\
\hline O & 17.949852 & 22.928816 & 20.390421 \\
\hline 0 & 16.938087 & 22.845797 & 22.8387 \\
\hline 0 & 16.093088 & 12.547297 & 8.945918 \\
\hline 0 & 18.530258 & 12.718919 & 10.039974 \\
\hline 0 & 18.033844 & 10.707283 & 8.421829 \\
\hline O & 16.856525 & 10.791273 & 10.795156 \\
\hline 0 & 3.814268 & 24.591139 & 8.947132 \\
\hline 0 & 6.324019 & 0.432573 & \\
\hline 0 & 5.723950 & 22.830017 & 8.247052 \\
\hline 0 & 4.631108 & 22.847980 & 10.709467 \\
\hline 0 & 3.704547 & 12.466220 & 21.242216 \\
\hline O & 6.157009 & 12.655320 & 22.247669 \\
\hline 0 & 5.573448 & 10.705341 & 20.471741 \\
\hline 0 & 4.557070 & 10.697088 & 22.929058 \\
\hline 0 & 2.871442 & 18.720085 & 20.914022 \\
\hline 0 & 0.510009 & 18.535357 & 22.249126 \\
\hline 0 & 0.920007 & 20.395519 & 20.399158 \\
\hline 0 & 2.199764 & 20.478781 & 22 . \\
\hline 0 & 2.857120 & 7660 & \\
\hline 0 & 0.426262 & 6.338826 & 10.029536 \\
\hline 0 & 1.020504 & 8.176170 & 8.189764 \\
\hline 0 & 2.191025 & 8.248023 & 10.614797 \\
\hline 0 & 15.170167 & 18.630997 & 8.941792 \\
\hline 0 & 12.632987 & 18.624443 & 30 \\
\hline 0 & 13.392782 & 20. & 8 . \\
\hline 0 & 14.341676 & 20.470526 & 10.615767 \\
\hline 0 & 15.092489 & 6.333486 & 21.241001 \\
\hline 0 & 12.559435 & 6.335185 & 22.170235 \\
\hline 0 & 13.304908 & 8.164034 & 20.402557 \\
\hline 0 & 14.324441 & 8.190007 & 22. \\
\hline 0 & 22.171206 & 2.777985 & 24.604733 \\
\hline 0 & 21.076422 & 0.332319 & 0.353438 \\
\hline 0 & 22.922747 & 0.921706 & 22.842154 \\
\hline 0 & 20.560343 & 2.187141 & 22.747484 \\
\hline 0 & 22.160767 & 15.080837 & 12.478115 \\
\hline 0 & 21.239546 & 12.552153 & 12.565503 \\
\hline 0 & 23.001154 & 13.240337 & 10.703400 \\
\hline 0 & 20.486063 & 14.258414 & 10.698787 \\
\hline 0 & 9.962781 & 3.043792 & 12.395339 \\
\hline 0 & 8.937422 & 0.590358 & 12.722075 \\
\hline 0 & 10.622564 & 1.081676 & 10.706312 \\
\hline 0 & 8.173742 & 2.181801 & 10.70267 \\
\hline
\end{tabular}




\begin{tabular}{|c|c|c|}
\hline 63256 & 15.086178 & 24.607647 \\
\hline 8.932810 & 12.550696 & 0.431602 \\
\hline 10.785447 & 13.234998 & 22.93828 \\
\hline 8.259190 & 14.238994 & 22.76496 \\
\hline 22.166107 & 16.091631 & 18.697 \\
\hline 21.227165 & 18.619102 & 18.611 \\
\hline 22.923962 & 17.954950 & 20. \\
\hline 20.4 & 16. & 20.48 \\
\hline 22.147417 & 3.878353 & 6.409 \\
\hline 21.147303 & 6.401698 & 6.328 \\
\hline 22.919106 & 5.733175 & 8.181 \\
\hline 20.485577 & 4.640575 & \\
\hline 9.943847 & 16.100128 & 6 . \\
\hline 9.017528 & 18.618860 & 6.253 \\
\hline 10.690776 & 17.932617 & 8.165 \\
\hline 8.185394 & 16.870119 & 8.093 \\
\hline 9.955013 & 3.787566 & 18.700 \\
\hline 8.929411 & & \\
\hline 10.692475 & 5.751381 & 20.3899 \\
\hline 8.261860 & 4.632322 & 20.468 \\
\hline 21.232992 & 16.180477 & 24.771 \\
\hline 22.328989 & 18.626385 & 0.418 \\
\hline 20.476595 & 17.948639 & 22.919 \\
\hline 22.923717 & 16.774477 & 22 . \\
\hline 21.336401 & 3.811112 & 12. \\
\hline 22.255680 & 6.322562 & 12.5480 \\
\hline 20.390179 & 5.566409 & 10.8589 \\
\hline 22.845068 & 4.551973 & 10.621 \\
\hline 9.0 & 15. & \\
\hline 9.871751 & 18.463989 & 12.56064 \\
\hline 8.188793 & 17.784784 & 10.62717 \\
\hline 10.767241 & 16.923523 & 10.63640 \\
\hline 8.929169 & 3.803344 & 24.6083 \\
\hline 9.5 & 6.3 & \\
\hline 8.1 & 5.7 & 22. \\
\hline 10.622079 & 4.633293 & 22.83657 \\
\hline 21.242216 & 2.863917 & 18.54263 \\
\hline 22.320009 & 0.424805 & 18.5465 \\
\hline 20.403044 & 1.0209 & 20.3132 \\
\hline $22 . \varepsilon$ & 2 . & 20 . \\
\hline 21.393690 & 15.2 & 6 . \\
\hline 22.343554 & 12.644639 & 6.00019 \\
\hline 20.390421 & 13.307336 & 8.16476 \\
\hline 23.014019 & 14.405761 & 8.1941. \\
\hline 8.8527 & 2.953 & 6.4 \\
\hline 10.0 & 0. & \\
\hline 8.267443 & 1.0 & 8 . \\
\hline 10.628391 & 2.197822 & 8.25797 \\
\hline 8.755605 & 15.17575 & 18.79242 \\
\hline 10.036819 & 12.478601 & 18.4472 \\
\hline 8.005 & 12. & 3964 \\
\hline 1061 & 14.34410 & 20.5564 \\
\hline 24.766401 & 22.07896 & 2.86585 \\
\hline 0.497872 & 21.06379 & 0.4153 \\
\hline 23.002853 & 22.91813 & 1.01176 \\
\hline 22.850653 & 20.48921 & 2.1905 \\
\hline 24 . & & \\
\hline 0.502 & 8.862656 & 12.56671 \\
\hline
\end{tabular}




\begin{tabular}{|c|c|c|c|}
\hline 0 & 22.920319 & 10.630575 & 13.230142 \\
\hline 0 & 22.924446 & 8.177870 & 14.327597 \\
\hline O & 12.475203 & 22.241844 & 15.002915 \\
\hline 0 & 12.636870 & 21.236391 & 12.49025 \\
\hline 0 & 10.697088 & 22.919348 & 13.1463 \\
\hline 0 & 10.787874 & 20.403528 & 14.160587 \\
\hline 0 & 12.481028 & 9.945546 & 2.943 \\
\hline 0 & 12.570844 & 8.939607 & 0 \\
\hline 0 & 10.706554 & 10.707525 & 1.08871 \\
\hline 0 & 10.630818 & 8.257976 & 2.1152 \\
\hline 0 & 18.708191 & 22.174603 & 16.0855 \\
\hline 0 & 18.535357 & 21.146818 & 51620 \\
\hline 0 & 32664 & 22.851137 & \\
\hline 0 & 20.482906 & 20.473440 & 16.85725 \\
\hline 0 & 18.784412 & 10.031235 & 0551 \\
\hline 0 & 18.791939 & 9.010974 & 6.243427 \\
\hline 0 & 20.564955 & 10.796371 & 5.488972 \\
\hline 0 & 20.571751 & 8.330557 & \\
\hline 0 & 6.322562 & 22.091585 & 2906 \\
\hline 0 & 6.239058 & 20.981993 & 6.412378 \\
\hline 0 & 8.171801 & 23.004553 & 5.81837 \\
\hline 0 & 8.351191 & 20.490433 & 4.481576 \\
\hline 0 & 6.325717 & 9.866411 & 16.100857 \\
\hline 0 & 6.327902 & 704 & 18. \\
\hline 0 & 8.172286 & 10.696116 & 17.857367 \\
\hline 0 & 8.174956 & 8.178841 & 16.855553 \\
\hline 0 & 24.865927 & 21.222069 & 16.172224 \\
\hline O & 0.427961 & 22.250340 & 18.608421 \\
\hline 0 & 22.996784 & 20.380224 & 17. \\
\hline 0 & 22.867643 & 22.830017 & 16.769621 \\
\hline 0 & 24.691879 & 8.844208 & 3.878838 \\
\hline 0 & 0.500057 & 10.030993 & 6.315765 \\
\hline 0 & 22.915949 & 8.272298 & 5.813281 \\
\hline 0 & 23.000425 & 10.629119 & \\
\hline 0 & 12.475203 & 21.408255 & \\
\hline 0 & 12.634201 & 22.239416 & 6.242213 \\
\hline 0 & 10.879633 & 20.318325 & 5.485331 \\
\hline 0 & 10.628633 & 22.920805 & 4.636691 \\
\hline 0 & 12.478601 & 8.850034 & 16.094301 \\
\hline 0 & 12.560891 & 9.871751 & 18 \\
\hline 0 & 10.6 & 8.1 & 17. \\
\hline 0 & 10.628633 & 10.537360 & 16.763309 \\
\hline 0 & 18.630754 & 21.244400 & 2.930430 \\
\hline 0 & 18.702364 & 22.240631 & 0.421407 \\
\hline 0 & 20.389935 & 20.320267 & 1.095027 \\
\hline 0 & 20.572481 & 22.759378 & 482 \\
\hline 0 & 18.705521 & 8.947860 & 15.165313 \\
\hline 0 & 18.606966 & 9.879762 & 12.643668 \\
\hline 0 & 20.409840 & 8.090239 & 13.318501 \\
\hline 0 & 20.496258 & 10.634702 & 14.322499 \\
\hline 0 & 6.576231 & 21.078363 & 15.160944 \\
\hline 0 & 6.328146 & 22.162224 & 12.657262 \\
\hline 0 & 8.253849 & 20.407169 & 13.218733 \\
\hline 0 & 8.264287 & 22.850166 & 14.327353 \\
\hline 0 & 6.426215 & 8.940820 & 2.945480 \\
\hline 0 & 6.316008 & 9.952100 & 0.492046 \\
\hline 0 & 8.175442 & 8.183210 & 1.023174 \\
\hline 0 & 8.261617 & 10.630575 & 2.18374 \\
\hline
\end{tabular}




\begin{tabular}{|c|c|c|c|}
\hline O & 13.148823 & 5.238944 & 8.331042 \\
\hline 0 & 12.467434 & 6.659979 & 5.980533 \\
\hline 0 & 11.810563 & 7.830986 & 8.424743 \\
\hline 0 & 14.485381 & 7.745539 & 7.592852 \\
\hline 0 & 7.336027 & 17.856638 & 19.728453 \\
\hline 0 & 7.325346 & 19.977753 & 18.37077 \\
\hline 0 & 8.082956 & 20.151558 & 21.486418 \\
\hline $\mathrm{H}$ & 12.679837 & 22.176060 & 3.14089 \\
\hline $\mathrm{H}$ & 15.864179 & 7.981731 & 7.92420 \\
\hline $\mathrm{H}$ & 25.047745 & 6.148513 & 1.71160 \\
\hline $\mathrm{H}$ & 21.948122 & 16.019051 & 6.48010 \\
\hline $\mathrm{H}$ & 13.548383 & 5.016103 & 9.22968 \\
\hline $\mathrm{H}$ & 9.005148 & 15.930449 & 19.370 \\
\hline $\mathrm{H}$ & 10.611641 & 19.407057 & 20.49698 \\
\hline $\mathrm{H}$ & 10.024196 & 18.8 & 17.46120 \\
\hline $\mathrm{H}$ & 11.098104 & 17.842802 & 18.46957 \\
\hline $\mathrm{H}$ & 11.514413 & 19.535225 & 18.17536 \\
\hline $\mathrm{Re}$ & 12.970160 & 6.899812 & \\
\hline $\mathrm{Re}$ & 8.1 & 19.5 & 19. \\
\hline $\mathrm{C}$ & 9.972 & 19.2 & 19.6204 \\
\hline C & 10.66 & 18.8 & 18.35 \\
\hline \multicolumn{4}{|c|}{605} \\
\hline US : & $\mathrm{ReO} 3$ C & $E=-7232$ & 336261187 \\
\hline $\mathrm{Si}$ & $23 . \overline{3} 8275 \overline{0}$ & 3.326106 & 1.2 \\
\hline $\mathrm{Si}$ & 23.428387 & 1.237034 & 3.37586 \\
\hline $\mathrm{Si}$ & 23.398529 & 15.571912 & 13.40200 \\
\hline $\mathrm{Si}$ & 23.391247 & 13.427252 & 15.57361 \\
\hline $\mathrm{Si}$ & 11.248607 & 1.240432 & 15.58696 \\
\hline $\mathrm{Si}$ & 11.1 & 15.5 & 1.19503 \\
\hline $\mathrm{Si}$ & 11.228702 & 13.410502 & 3.36640 \\
\hline $\mathrm{Si}$ & 7.633148 & 3.351837 & 1.21809 \\
\hline $\mathrm{Si}$ & 7.657908 & 5.500138 & 3.34673 \\
\hline $\mathrm{Si}$ & 6806 & 15.489864 & 13.27140 \\
\hline $\mathrm{Si}$ & 20 & 5.4 & 15. \\
\hline $\mathrm{Si}$ & 19.9 & 15.644736 & 1.26762 \\
\hline $\mathrm{Si}$ & 19.883081 & 17.805418 & 3.34965 \\
\hline $\mathrm{Si}$ & 7.626108 & 3.370043 & 5.51057 \\
\hline $\mathrm{Si}$ & 7.609359 & 15.523363 & $17.72215^{\circ}$ \\
\hline $\mathrm{Si}$ & 7.76 & 13.325298 & 15.481 \\
\hline $\mathrm{Si}$ & 19. & 3. & 17 \\
\hline $\mathrm{Si}$ & 19.866333 & 1.201107 & 15.62240 \\
\hline $\mathrm{Si}$ & 19.934544 & 15.590118 & 5.43411 \\
\hline Si & 20.010523 & 13.381372 & 3.27974 \\
\hline Si & 23.434214 & 3.350380 & 5.53630 \\
\hline $\mathrm{Si}$ & 23.372068 & 17.740849 & 15.56365 \\
\hline $\mathrm{Si}$ & 11.2 & 3.3 & 17.781 \\
\hline Si & 11.268026 & 5.535580 & 15.57531 \\
\hline Si & 11.238896 & 15.650805 & 5.45013 \\
\hline $\mathrm{Si}$ & 11.186220 & 17.783813 & 3.22730 \\
\hline $\mathrm{Si}$ & 1.308644 & 23.402899 & 3.33460 \\
\hline $\mathrm{Si}$ & $3.3^{\prime}-1$ & 23.428629 & 1.20329 \\
\hline $\mathrm{Si}$ & 1.255239 & 11.233070 & 15.57773 \\
\hline $\mathrm{Si}$ & 3.411067 & 11.215107 & 13.38404 \\
\hline $\mathrm{Si}$ & 13.456866 & 23.447321 & 15.52603 \\
\hline $\mathrm{Si}$ & 15.608081 & 23.403141 & 13.41365 \\
\hline $\mathrm{Si}$ & 13.425309 & 11.234042 & 3.33241 \\
\hline Si & 15.610266 & 11.173598 & 1.19916 \\
\hline
\end{tabular}




\begin{tabular}{|c|c|c|c|}
\hline $\mathrm{Si}$ & 1.360106 & 7.681211 & 3.342127 \\
\hline & 3.381209 & 7.602562 & 5.525627 \\
\hline & 1.309372 & 19.898132 & 15.589634 \\
\hline & 3.423932 & 19.896431 & 17.750072 \\
\hline & 13.432107 & 7.642858 & 15.602741 \\
\hline & 15.585021 & 7.632662 & 17.789640 \\
\hline & 15.556862 & 19.952265 & 5.499896 \\
\hline & 5.526598 & 7.662035 & 3.378782 \\
\hline & 3.405969 & 7.665433 & 1.181930 \\
\hline & 5.550145 & 19.958818 & 15.67823 \\
\hline & 3.457431 & 19.884537 & 13.44958 \\
\hline & 17.869747 & 7.679997 & 15.68503 \\
\hline & 15.607840 & 7.747967 & 13.453226 \\
\hline S & 17.722885 & 19.966101 & 3.36033 \\
\hline & 15.607353 & 20.005182 & 1.23120 \\
\hline & 5.499896 & 23.365515 & 3.37781 \\
\hline S & 3.409853 & 23.400713 & 5.51130 \\
\hline i & 5.516160 & 11.186220 & 15.60079 \\
\hline $\mathrm{Si}$ & 3.364460 & 11.234528 & 17.74813 \\
\hline $\mathrm{Si}$ & 17.723370 & 23.383720 & 15.61390 \\
\hline S & 15.542297 & 23.389790 & 17.74351 \\
\hline & 17.821682 & 11.269726 & 3.30304 \\
\hline 1 & 15.662943 & 11.239625 & 5.47052 \\
\hline i & 3.357662 & 1.276844 & 23.367 \\
\hline i & 1.242374 & 3.364945 & 23.41454 \\
\hline , & 3.396259 & 13.389627 & 11.22336 \\
\hline $\mathrm{Si}$ & 1.288738 & 15.563416 & 11.258316 \\
\hline$\perp$ & 15.613908 & 1.222954 & 11.20491 \\
\hline i & 13.360253 & 3.333388 & 11.249092 \\
\hline$i$ & 15.563173 & 13.425067 & 23.39294 \\
\hline$i$ & 13.451041 & 15.571671 & 23.41552 \\
\hline . & 3.360090 & 1.200379 & 7.65936 \\
\hline$i$ & 5.517374 & 3.318095 & 7.61979 \\
\hline$i$ & 3.307657 & 13.378460 & 19.94668 \\
\hline i & 17.774834 & 3.372713 & 19.861 \\
\hline i & 15.641824 & 13.429922 & 7.64407 \\
\hline $\mathrm{Si}$ & 17.823381 & 15.573612 & 7.71786 \\
\hline $\mathrm{Si}$ & 3.339457 & 5.432898 & 7.65572 \\
\hline $\mathrm{Si}$ & 1.241889 & 3.327077 & 7.66349 \\
\hline $\mathrm{Si}$ & 3.404998 & 17.754442 & 19.87070 \\
\hline$i$ & 1.258152 & 15.581379 & 19.87385 \\
\hline $\mathrm{Si}$ & 15.565600 & 5.467125 & 19.94 \\
\hline $\mathrm{Si}$ & 13.394238 & 3.388977 & 19.95299 \\
\hline $\mathrm{Si}$ & 15.665128 & 17.779203 & 7.63508 \\
\hline $\mathrm{Si}$ & 13.468519 & 15.648378 & 7.62610 \\
\hline $\mathrm{Si}$ & 3.377325 & 5.510577 & 23.38202 \\
\hline $\mathrm{Si}$ & 5.503051 & 3.386307 & 23.34658 \\
\hline Si & 5.585586 & 15.553222 & 11.13718 \\
\hline $\mathrm{Si}$ & 17.858093 & 3.318823 & 11.25443 \\
\hline $\mathrm{Si}$ & 15.613180 & 17.758812 & 23.44173 \\
\hline $\mathrm{Si}$ & 17.729681 & 15.614635 & 23.42741 \\
\hline $\mathrm{Si}$ & 1.643148 & 21.677460 & 23.72817 \\
\hline $\mathrm{Si}$ & 1.613533 & 23.782310 & 21.61240 \\
\hline $\mathrm{Si}$ & 1.678103 & 9.445974 & 11.58311 \\
\hline $\mathrm{Si}$ & 1.674947 & 11.595976 & 9.45665 \\
\hline $\mathrm{Si}$ & 13.876575 & 21.701492 & 11.55082 \\
\hline $\mathrm{Si}$ & 13.832638 & 23.855375 & 9.37994 \\
\hline $\mathrm{Si}$ & 13.835064 & 9.419516 & 23.79202 \\
\hline
\end{tabular}




\begin{tabular}{|c|c|c|c|}
\hline i & .810790 & 30932 & 21.667021 \\
\hline $\mathrm{Si}$ & 17.482809 & 21.678188 & 23.775270 \\
\hline 1 & 17.393478 & 19.536682 & 21.68789 \\
\hline $\mathrm{Si}$ & 17.395906 & 9.509332 & 11.577527 \\
\hline Si & 17.450768 & 7.326560 & 9.485299 \\
\hline $\mathrm{Si}$ & 5.153983 & 21.644688 & 11.647923 \\
\hline i & 5.122183 & 19.542751 & 9.495009 \\
\hline i & 5.182384 & 9.435051 & 23.732304 \\
\hline 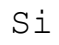 & 5.152041 & 7.305198 & 21.614 \\
\hline & 17.333277 & 21.698580 & 19.520 \\
\hline i & 17.412897 & 23.852463 & 21.663 \\
\hline i & 17.389351 & 11.700600 & 9.489 \\
\hline 2 & 5.102763 & 21.600023 & 7.31 \\
\hline & 5.119513 & 23.742985 & 9.436 \\
\hline & 5.077275 & 9.456413 & 19.52673 \\
\hline & 5.025813 & 11.628504 & 21.7014 \\
\hline i & 1.581733 & 21.623569 & \\
\hline S & 1.596783 & 19.53 & 21.622 \\
\hline S & 1.631010 & 9.4229 & 7.306 \\
\hline 2 & 1.623485 & 7.276068 & 9.43068 \\
\hline 2 & 13.875603 & 21.743244 & 7.18382 \\
\hline & 13.891382 & 19.549791 & 48876 \\
\hline i & 13. & & 1 \\
\hline i & 23.7 & 1 . & 21.62672 \\
\hline 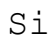 & 21.672604 & 1.535126 & 23.71240 \\
\hline 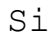 & 23.862417 & 13.852057 & 9.42485 \\
\hline i & 21.7 & 13.774864 & 11.58966 \\
\hline i & 11. & & 00700 \\
\hline & 9.4 & 1. & 11.627533 \\
\hline 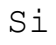 & 11.676083 & $13 . \varepsilon$ & 21.714357 \\
\hline 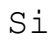 & 9.455441 & 13.778505 & 23.778667 \\
\hline $\mathrm{Si}$ & 23.814110 & 17.34 & 21.70221 \\
\hline & 21.690567 & 17.390808 & 19.55974 \\
\hline $\mathrm{Si}$ & 21.6 & 4 & \\
\hline $\mathrm{Si}$ & 11.6 & 17 & 9.3 \\
\hline Si & 9.463210 & 17.391779 & 7.20445 \\
\hline$\sigma^{-}$ & 11.577285 & 5.157381 & 21.638863 \\
\hline 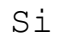 & 9.474861 & 5.11 & 19.50998 \\
\hline $\mathrm{Si}$ & 19.5 & 17.38 & 21.6968 \\
\hline $\mathrm{S}$ & 21. & 17. & 23.82284 \\
\hline$S_{1}$ & 19.5 & 5.15 & 9.49282 \\
\hline $\mathrm{Si}$ & 21.729893 & 5.068294 & 11.65763 \\
\hline $\mathrm{Si}$ & 7.284807 & 17.356339 & 9.35543 \\
\hline $\mathrm{Si}$ & 9.4 & 17. & 11.55252 \\
\hline $\mathrm{Si}$ & & & \\
\hline $\mathrm{Si}$ & 9.4 & 5.1 & 23.77769 \\
\hline Si & 19.5 & 1.638293 & 21.59808 \\
\hline $\mathrm{Si}$ & 21.680130 & 1.608192 & 19.48303 \\
\hline $\mathrm{Si}$ & 19.555132 & 13.829724 & 9.43092 \\
\hline $\mathrm{Si}$ & 7.36 & 1.607949 & 9.43553 \\
\hline $\mathrm{Si}$ & 9.2 & & 7.29451 \\
\hline $\mathrm{Si}$ & 7.252765 & 13.688932 & 21.62478 \\
\hline Si & 21.702948 & 23.744684 & 1.58707 \\
\hline Si & 23.836685 & 21.646389 & 1.61814 \\
\hline $\mathrm{Si}$ & 21.712416 & 11.595248 & 13.78263 \\
\hline $\mathrm{Si}$ & 23.850521 & 9.410534 & 13. \\
\hline $\mathrm{Si}$ & 9.488213 & 23.798088 & 13.82 \\
\hline & & 11.60544 & 1.660 \\
\hline
\end{tabular}




\begin{tabular}{|c|c|c|c|}
\hline $\mathrm{Si}$ & 11.616609 & 9.464666 & 303 \\
\hline $\mathrm{Si}$ & 21.681343 & 23.758036 & 17.386683 \\
\hline & 19.529644 & 21.671633 & 17.39954 \\
\hline & 21.815338 & 11.644768 & 4.882107 \\
\hline & 19.640577 & 9.536519 & 5.008335 \\
\hline & 9.359072 & 23.821878 & 5.161751 \\
\hline & 9.467580 & 11.542572 & 17.299049 \\
\hline & 7.261504 & 9.422671 & 17.368961 \\
\hline & 21.695908 & 19.522604 & 17.34274 \\
\hline & 23.837414 & 21.680130 & 17.34929 \\
\hline & 21.704163 & 7.373652 & 5.12169 \\
\hline & 23.813866 & 9.446703 & 5.13116 \\
\hline $\mathrm{Si}$ & 9.483357 & 19.528671 & $84^{7}$ \\
\hline $\mathrm{Si}$ & 11.570488 & 21.726980 & 5.128737 \\
\hline & 9.434809 & 7.261261 & $17.32211]$ \\
\hline $\mathrm{Si}$ & 11.576799 & 9.366597 & 17.361921 \\
\hline L & 21.697123 & 19.507311 & 1.63440 \\
\hline Si & 19.558529 & 21.628668 & 52129 \\
\hline Si & 21.731592 & 7.234073 & 13.7 \\
\hline $\mathrm{Si}$ & 19.564114 & 9.4 & 13. \\
\hline i & 9.502777 & 19.508282 & 13.79234 \\
\hline SI & 7.345251 & 21.625755 & 13.80520 \\
\hline i & 9.428497 & 7.297673 & 1.61304 \\
\hline $\mathrm{Si}$ & 7.313209 & 9.4 & \\
\hline $\mathrm{Si}$ & 11.662003 & 21.7 & 13. \\
\hline $\mathrm{Si}$ & 11.212923 & 3.403299 & 13.37627 \\
\hline $\mathrm{Si}$ & 20.023388 & 3.292364 & 13.418512 \\
\hline $\mathrm{Si}$ & 7.629263 & 1.210089 & 6615 \\
\hline $\mathrm{Si}$ & 23.374010 & 15.607110 & 17.72458 \\
\hline $\mathrm{Si}$ & 5.446734 & 15.553 & 19.96901 \\
\hline $\mathrm{Si}$ & 3.483405 & 17.7 & 11.2 \\
\hline $\mathrm{Si}$ & 13.797924 & 9.427769 & 19.50876 \\
\hline Si & 23.792992 & 5.133349 & 9.42242 \\
\hline 1 & 15.580894 & 1.281213 & 19.89691 \\
\hline 1 & 15.576768 & 5.4 & 11.20491 \\
\hline 1 & 17.524803 & 9.6 & 7.2 \\
\hline 1 & 9.484086 & 13.520710 & 19.662668 \\
\hline 1 & 7.259804 & 21.632309 & 5.169034 \\
\hline Al & 7.732916 & 17.743519 & 15.422381 \\
\hline Al & 23.371826 & 5.398185 & 3.40863 \\
\hline 1 & 13.455411 & 19.8 & 3.2 \\
\hline Al & 21.793978 & 13.67 & 7.23625 \\
\hline Al & 16.713305 & 5.196706 & $13.77413^{\circ}$ \\
\hline Al & 6.939622 & 18.134338 & 18.08069 \\
\hline 0 & 22.163923 & 0.349069 & 2.866102 \\
\hline 0 & 24.605705 & 24.542105 & 3.95578 \\
\hline 0 & 24.029911 & 2.0 & 2.11747 \\
\hline 0 & 22.847010 & 2.18 & 4.55707 \\
\hline 0 & 22.162224 & 12.472047 & 15.085207 \\
\hline 0 & 24.610559 & 12.475930 & $16.09648^{\circ}$ \\
\hline O & 23.941309 & 14.330267 & 14.329296 \\
\hline 0 & 22.846039 & 14.397265 & 16.771807 \\
\hline 0 & 10.025409 & 0.249543 & 15.17623 \\
\hline O & 12.558706 & 24.691879 & 16.09333 \\
\hline 0 & 11.645011 & 2.102423 & 14.25064 \\
\hline O & 10.707040 & 2.192239 & 16.77933 \\
\hline O & 9.944818 & 12.480785 & 2.94669 \\
\hline O & 12.480785 & 12.472290 & 3.79654 \\
\hline
\end{tabular}




$$
\begin{aligned}
& 11.713951 \\
& 10.782048 \\
& 8.928440 \\
& 6.415291 \\
& 7.077987 \\
& 8.181511 \\
& 9.030151 \\
& 6.404125 \\
& 7.234559 \\
& 8.007948 \\
& 21.305088 \\
& 18.791452 \\
& 19.298307 \\
& 20.480478 \\
& 21.242216 \\
& 18.719114 \\
& 19.283983 \\
& 20.398916 \\
& 8.930868 \\
& 6.401698 \\
& 7.082600 \\
& 8.175927 \\
& 9.018985 \\
& 6.493698 \\
& 7.250094 \\
& 8.254092 \\
& 21.147545 \\
& 18.632696 \\
& 19.297577 \\
& 20.475866 \\
& 21.318924 \\
& 18.707462 \\
& 19.559015 \\
& 20.480722 \\
& 22.257866 \\
& 24.775141 \\
& 24.110989 \\
& 22.759865 \\
& 22.085758 \\
& 24.599634 \\
& 23.934027 \\
& 22.862061 \\
& 10.025652 \\
& 12.557250 \\
& 11.726088 \\
& 10.771853 \\
& 9.877819 \\
& 12.374220 \\
& 11.725117 \\
& 10.617467 \\
& 2.950335 \\
& 3.969868 \\
& 2.098539 \\
& 4.489344 \\
& 2.951549 \\
& \text { o } 3.891218 \\
& \text { O } 4.634021
\end{aligned}
$$$$
\text { o } 2.185199
$$ 


\begin{tabular}{|c|c|c|c|}
\hline & 15.155847 & 22.159554 & 12.463794 \\
\hline & 16.174652 & 24.615900 & 12.456753 \\
\hline & 14.333908 & 24.015104 & 14.247249 \\
\hline & 16.772049 & 22.846283 & 14.401634 \\
\hline & 15.167254 & 9.858643 & 0.342272 \\
\hline & 16.103041 & 12.397281 & 24.524628 \\
\hline & 14.316188 & 11.714922 & 2.037610 \\
\hline & 16.860165 & 10.709952 & 2.112133 \\
\hline & 2.868287 & 8.859015 & 6.407765 \\
\hline & 3.895345 & 6.406552 & 6.478890 \\
\hline & 2.193938 & 6.995939 & 4.550759 \\
\hline & 4.555371 & 8.176898 & 4.566052 \\
\hline & 2.868772 & 21.065983 & 18.706978 \\
\hline & 4.039536 & 18.625900 & 18.622988 \\
\hline & 2.275015 & 19.288839 & 16.775448 \\
\hline & 4.631593 & 20.556944 & 16.865992 \\
\hline & 15.089819 & 8.939849 & 18.616190 \\
\hline & 16.096001 & 6.421117 & 18.710861 \\
\hline & 14.413771 & 7.080657 & 16.775690 \\
\hline & 16.859922 & 8.098248 & 16.850456 \\
\hline & 15.174537 & 21.323536 & 6.324019 \\
\hline & 16.179749 & 18.798977 & 6.485202 \\
\hline & 14.262055 & 19.389578 & 4.733061 \\
\hline & 16.773748 & 20.477566 & 4.553672 \\
\hline & 3.862331 & 8.842994 & 0.184244 \\
\hline & 2.779684 & 6.406795 & 24.620028 \\
\hline & 4.638633 & 7.151539 & 2.100966 \\
\hline & 2.207775 & 8.266715 & 2.103879 \\
\hline & 3.883450 & 21.160410 & 12.556522 \\
\hline & 3.023159 & 18.614977 & 12.559192 \\
\hline & 4.711457 & 19.379141 & 14.417170 \\
\hline & 2.199036 & 20.395761 & 14.333179 \\
\hline & 16.095030 & 8.846878 & 12.398979 \\
\hline & 15.334507 & 6.236387 & 12.816504 \\
\hline & 16.945856 & 7.084056 & 14.338277 \\
\hline & 14.418140 & 8.253364 & 14.404790 \\
\hline & 16.178535 & 21.232992 & 0.340815 \\
\hline & 15.078652 & 18.730524 & 24.614445 \\
\hline & 16.858709 & 19.382540 & 2.109948 \\
\hline & 14.426394 & 20.634138 & 2.102909 \\
\hline & 3.801645 & 22.168535 & 6.491513 \\
\hline & 2.778956 & 24.612261 & 6.414805 \\
\hline & 4.638148 & 24.019472 & 4.635235 \\
\hline & 2.279870 & 22.841913 & 4.487888 \\
\hline 0 & 3.806015 & 9.944575 & 18.629784 \\
\hline ) & 2.861732 & 12.466949 & 18.692411 \\
\hline & 4.625525 & 11.793086 & 16.847542 \\
\hline 0 & 2.121114 & 10.712623 & 16.850212 \\
\hline & 16.023663 & 22.084545 & 18.623714 \\
\hline 0 & 14.924266 & 24.536036 & 18.635124 \\
\hline 0 & 16.846329 & 23.933784 & 16.860893 \\
\hline 0 & 14.408430 & 22.834871 & 16.680290 \\
\hline 0 & 16.094301 & 9.961810 & 6.325232 \\
\hline 0 & 15.169683 & 12.487824 & 6.410193 \\
\hline ) & 16.921337 & 11.802067 & 4.557313 \\
\hline 0 & 14.400663 & 10.788117 & 4.541049 \\
\hline 0 & 0.343971 & 2.868287 & 22.162466 \\
\hline 0 & 24.608860 & 3.964285 & 24.614927 \\
\hline
\end{tabular}




\begin{tabular}{|c|c|c|c|}
\hline 0 & 2.110676 & 2.115046 & 24.016075 \\
\hline 0 & 2.192967 & 4.554643 & 22.837299 \\
\hline 0 & 0.428447 & 15.157545 & 9.949187 \\
\hline 0 & 24.621239 & 16.100613 & 12.476658 \\
\hline O & 2.122086 & 14.253317 & 11.79551 \\
\hline 0 & 2.265548 & 16.768650 & 10.791030 \\
\hline 0 & 12.383930 & 2.939654 & 10.0271 \\
\hline 0 & 12.552638 & 3.874712 & 12.55239 \\
\hline 0 & 14.335608 & 2.111890 & 11.72633 \\
\hline 0 & 14.249433 & 4.556099 & 10.62717 \\
\hline 0 & 12.644396 & 15.086421 & 22.08478 \\
\hline 0 & 12.393397 & 16.093330 & 24.527782 \\
\hline 0 & 14.333179 & 14.323227 & 24.016560 \\
\hline 0 & 14.414500 & 16.782972 & 22.92590 \\
\hline 0 & 6.483260 & 2.877754 & 8.8531 \\
\hline 0 & 6.396842 & 3.954575 & 6.40558 \\
\hline 0 & 4.646401 & 2.041008 & 7.083086 \\
\hline 0 & 4.539350 & 4.482548 & \\
\hline 0 & 6.497824 & 15.082294 & 21.077393 \\
\hline 0 & 6.324261 & 16.193342 & 18.61182 \\
\hline 0 & 4.555129 & 14.333179 & 19.389578 \\
\hline O & 4.642760 & 16.859438 & 20.47829 \\
\hline O & 18.704550 & 2.945480 & 21.12 \\
\hline 0 & 18.797035 & 3.975937 & 3821 \\
\hline 0 & 16.932503 & 2.191511 & 5288 \\
\hline 0 & 16.857737 & 4.622855 & 20.39915 \\
\hline r & 18.706734 & 15.176965 & 9.01898 \\
\hline 0 & 18.802862 & 16.017109 & 6.483746 \\
\hline 0 & 16.933474 & 14.326626 & .706 \\
\hline 0 & 16.924978 & 16.862350 & 2618 \\
\hline 0 & 0.417280 & 3.879567 & 8.95028 \\
\hline 0 & 24.523170 & 2.692296 & 6.564337 \\
\hline 0 & 2.113104 & 4.556828 & 7.00783 \\
\hline 0 & 2.208260 & 2.179374 & 8.256 \\
\hline 0 & 0.422135 & 16.098915 & 21. \\
\hline 0 & 24.619783 & 15.080594 & 18.632938 \\
\hline 0 & 2.258509 & 16.762583 & 19.297092 \\
\hline 0 & 2.111647 & 14.331481 & 20.466400 \\
\hline 0 & 12.393882 & 3.869371 & 21.151188 \\
\hline 0 & 12.470105 & 2.867558 & 18 \\
\hline 0 & 14.243607 & 048 & 19. \\
\hline 0 & 14.327840 & 2.278171 & 20.553787 \\
\hline 0 & 12.566717 & 16.100128 & 8.922615 \\
\hline 0 & 12.483698 & 15.237894 & 6.409223 \\
\hline 0 & 14.420325 & 16.866234 & 7.0840 \\
\hline 0 & 14.410374 & 14.404305 & 394 \\
\hline 0 & 6.404367 & 3.871070 & 22.08575 \\
\hline 0 & 6.422574 & 2.790123 & 24.54210 \\
\hline 0 & 4.637905 & 4.643488 & 23.94495 \\
\hline 0 & 4.484975 & 2.271860 & 22.761806 \\
\hline 0 & 6.332515 & 16.090902 & 9.791645 \\
\hline 0 & 6.583757 & 14.994905 & 12.29848 \\
\hline 0 & 4.724565 & 16.774719 & 11.80522 \\
\hline 0 & 4.621398 & 14.322499 & 10.702428 \\
\hline 0 & 18.541910 & 3.958702 & 9.94457 \\
\hline 0 & 18.873987 & 2.776286 & 12.385629 \\
\hline 0 & 16.944885 & 4.540078 & 12.043357 \\
\hline ? & 16.844387 & 2.194909 & 10.71675 \\
\hline
\end{tabular}




\begin{tabular}{|c|c|c|c|}
\hline & 18.631241 & 16.107168 & 22.160040 \\
\hline O & 18.712803 & 15.083750 & 24.604977 \\
\hline 0 & 16.855553 & 16.858223 & 24.021416 \\
\hline 0 & 16.773262 & 14.406246 & 22.919592 \\
\hline & 2.866587 & 24.693335 & 22.079689 \\
\hline 0 & 0.429175 & 0.421650 & 20.985 \\
\hline 0 & 1.007639 & 22.913036 & 22.852594 \\
\hline U & 2.199279 & 22.771517 & 20.483635 \\
\hline O & 2.957617 & 12.473018 & 9.95064 \\
\hline 0 & 0.504426 & 12.578369 & 8.933781 \\
\hline U & 1.094056 & 10.699272 & 10.708496 \\
\hline O & 2.191025 & 10.617467 & 8.260404 \\
\hline 0 & 15.163371 & 24.693821 & 9.86762 \\
\hline 0 & 12.566717 & 0.509767 & 8.94106 \\
\hline 0 & 13.319715 & 22.929787 & 10.623051 \\
\hline 0 & 14.328082 & 23.010862 & 8.097035 \\
\hline 0 & 15.085692 & 12.562104 & 22 . \\
\hline 0 & 12.558221 & 12.556036 & 21.222069 \\
\hline 0 & 13.315831 & 10.704127 & 22.91837 \\
\hline 0 & 14.252346 & 10.703156 & 20.397217 \\
\hline 0 & 16.111052 & 18.631241 & 22.156397 \\
\hline 0 & 18.622259 & 18.609152 & 21.148516 \\
\hline 0 & 18.036026 & 20.392605 & 22.922504 \\
\hline 0 & 16.853853 & 20.475382 & 20.482906 \\
\hline 0 & 16.187031 & 6.496126 & 10.020311 \\
\hline O & 18.701637 & 6.422331 & 9.007333 \\
\hline O & 18.030445 & 8.345850 & 10.620623 \\
\hline 0 & 16.861622 & 8.169859 & 568 \\
\hline $\mathrm{O}$ & 3.880538 & 18.626627 & 10.028322 \\
\hline 0 & 6.339312 & 18.617161 & 8.936936 \\
\hline 0 & 5.731233 & 20.392847 & 10.777922 \\
\hline 0 & 4.475508 & 20.480722 & 8.351676 \\
\hline 0 & 3.8 & 2 & 22. \\
\hline 0 & 6.3 & 5717 & 21.070351 \\
\hline 0 & 5.814252 & 8.273270 & 22.761320 \\
\hline 0 & 4.552944 & 8.190249 & 20.393333 \\
\hline 0 & 16.182177 & 24.771744 & 21.315041 \\
\hline 0 & 18.7 & 0.416795 & 22.234076 \\
\hline 0 & 17. & 22 . & 20. \\
\hline 0 & 16.938087 & 22.845797 & 22.838514 \\
\hline 0 & 16.093088 & 12.548268 & 8.944704 \\
\hline 0 & 18.530743 & 12.719162 & 10.039489 \\
\hline 0 & 18.034328 & 10.707525 & 8.421344 \\
\hline 0 & 16.853611 & 10.795885 & 10.790302 \\
\hline 0 & 3.814753 & 24.590899 & 8.947374 \\
\hline 0 & 6.323776 & 0.432331 & 9.958411 \\
\hline 0 & 5.723950 & 22.830017 & 8.247052 \\
\hline 0 & 4.631593 & 22.847010 & 10.710439 \\
\hline 0 & 3.716927 & 12.480542 & 21.229836 \\
\hline 0 & 9679 & 5077 & 22.247185 \\
\hline 0 & 5.574176 & 10.704856 & 20.471012 \\
\hline 0 & 4.556342 & 10.698787 & 22.927359 \\
\hline 0 & 2.885522 & 18.702608 & 21.058943 \\
\hline 0 & 0.500299 & 18.536570 & 22.253496 \\
\hline 0 & 7047 & 20.392363 & 20.398916 \\
\hline $\mathrm{O}$ & 2.195880 & 20.489946 & 22.762777 \\
\hline 0 & 2.857120 & 6.327902 & 8.93499 \\
\hline & & & \\
\hline
\end{tabular}




\begin{tabular}{|c|c|c|c|}
\hline 0 & 1.020747 & 8.176413 & 89764 \\
\hline 0 & 2.191025 & 8.248265 & 10.615040 \\
\hline 0 & 15.171138 & 18.630997 & 8.941305 \\
\hline 0 & 12.636143 & 18.626871 & 9.78994 \\
\hline O & 13.393024 & 20.481449 & 8.09897 \\
\hline 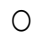 & 14.343133 & 20.471012 & 10.615767 \\
\hline 0 & 15.092731 & 6.333243 & 21.240761 \\
\hline 0 & 12.559435 & 6.335185 & 22.17023 \\
\hline 0 & 13.305635 & 8.164519 & 20.40255 \\
\hline 0 & 14.325412 & 8.188065 & 22.85866 \\
\hline 0 & 22.171206 & 2.777985 & 24.60497 \\
\hline 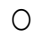 & 21.076178 & 0.332319 & 0.3534 \\
\hline 0 & 22.922504 & 0.921706 & 22.84215 \\
\hline 0 & 20.560099 & 2.187141 & 22.74748 \\
\hline 0 & 22.161495 & 15.081567 & 12.47860 \\
\hline 0 & 21.239546 & 12.552395 & 12.565260 \\
\hline O & 23.001154 & 13.240337 & 10.703642 \\
\hline 0 & 20.486547 & 14.258414 & \\
\hline 0 & 9.962781 & 3.043792 & 12.395339 \\
\hline 0 & 8.937179 & 0.590601 & 12.72207 \\
\hline 0 & 10.622564 & 1.081676 & 10.706312 \\
\hline O & 8.173742 & 2.181801 & 10.702671 \\
\hline O & 9.856459 & 15.098558 & 24.617113 \\
\hline 0 & 8.929897 & 12.550453 & \\
\hline 0 & 10.800255 & 13.295684 & 22.997026 \\
\hline 0 & 8.258946 & 14.239723 & 22.766418 \\
\hline 0 & 22.167078 & 16.090902 & 18.697752 \\
\hline 0 & 21.226681 & 18.618618 & 18.609879 \\
\hline 0 & 22.923717 & 17.954464 & 20 . \\
\hline 0 & 20.476839 & 16.855553 & 20.481207 \\
\hline 0 & 22.147657 & 3.878353 & 6.409465 \\
\hline 0 & 21.147303 & 6.401698 & 6.328874 \\
\hline 0 & 22.919106 & 5.733175 & 8.181753 \\
\hline 0 & 20.485819 & 4.640332 & 8.262 \\
\hline 0 & 9.944090 & 16.100128 & \\
\hline 0 & 9.017771 & 18.618618 & 6.254593 \\
\hline 0 & 10.691748 & 17.933104 & 8.165490 \\
\hline 0 & 8.186366 & 16.870361 & 8.094365 \\
\hline 0 & 9.955013 & 3.787566 & 18.700422 \\
\hline 0 & 8.929411 & 6 . & 18. \\
\hline 0 & 10.692233 & 5.7 & 20. \\
\hline 0 & 8.262102 & 4.632079 & 20.468584 \\
\hline 0 & 21.232992 & 16.180477 & 24.772226 \\
\hline 0 & 22.328989 & 18.626385 & 0.418009 \\
\hline 0 & 20.476353 & 17.948881 & 22.919834 \\
\hline 0 & 22.922504 & 16.773504 & 361 \\
\hline 0 & 21.336401 & 3.811112 & 12.636627 \\
\hline 0 & 22.254953 & 6.323534 & 12.54875 \\
\hline 0 & 20.390421 & 5.566409 & 10.858999 \\
\hline 0 & 22.845068 & 4.552216 & 10.622079 \\
\hline 0 & 9.094964 & 15.929962 & 12.393154 \\
\hline 0 & 9.873693 & 18.450636 & 12.625947 \\
\hline 0 & 8.248752 & 17.786242 & 10.621351 \\
\hline 0 & 10.786660 & 16.932989 & 10.640528 \\
\hline 0 & 8.929169 & 3.803344 & 24.608374 \\
\hline 0 & 9.954285 & 6.326204 & 0.4299 \\
\hline 0 & 8.157722 & 5.731476 & 22.912550 \\
\hline ? & 10.622079 & 4.633050 & 22.83681 \\
\hline
\end{tabular}




\begin{tabular}{|c|c|c|}
\hline 1.24 & 2.864160 & 42639 \\
\hline 2.319281 & 0.425291 & 764 \\
\hline .402800 & 1.020990 & • \\
\hline .830988 & 2.192724 & 20.46154 \\
\hline .393932 & 15.249060 & 6.24633 \\
\hline D 343554 & 12.644882 & 600010 \\
\hline 0.390 & 13.307336 & 8.164 \\
\hline 3.0145 & 14.405761 & 8.194 \\
\hline 8.85270 & 2.953976 & 6.492 \\
\hline 10.02759 & 0.589873 & 6.244 \\
\hline 8.26744 & 1.007396 & \\
\hline 10.6283 & 2.197822 & 8.257 \\
\hline 8.84 & 15.166284 & 18.791 \\
\hline 10.03657 & 12.477387 & 8.452 \\
\hline 8.0125 & 12.989823 & 20.394 \\
\hline 10.6898 & 14.334394 & 20.5550 \\
\hline 4. & 22. & \\
\hline 0.4 & 21. & 0.417 \\
\hline 23.0026 & 22.918377 & 1.0120 \\
\hline 22.8 & 20.488976 & 2.19102 \\
\hline 4. & 4137 & 15. \\
\hline 4669 & 3141 & 12. \\
\hline 22.9 & 10. & \\
\hline 22.9 & 8 . & 14. \\
\hline 12.476 & 22.244757 & 15.0036 \\
\hline 12.638084 & 21.237604 & 12.4885 \\
\hline 10.697816 & 22.921776 & 13.1456 \\
\hline & 20. & 14. \\
\hline 12. & 9 . & 2 . \\
\hline 12.5 & 8149 & 0. \\
\hline 10.70606 & 10.707525 & 1.08871 \\
\hline 10.6 & 8.257976 & 2.1155 \\
\hline 1 & 22.1 & 16. \\
\hline 18.5 & 21. & \\
\hline 20.4 & $22 . \varepsilon$ & 18. \\
\hline 20.4831 & 20.473196 & 16.85725 \\
\hline 18.78441 & 10.031235 & 3.7055 \\
\hline 18.7 & 9.010974 & 6.243 \\
\hline 20 & 10. & \\
\hline 20. & 8. & 4. \\
\hline 6.3 & 22.0 & 3. \\
\hline 6.239058 & 20.981993 & 6.41237 \\
\hline 8.17 & 23.0 & $5.8183^{7}$ \\
\hline 8.3 & 20. & \\
\hline 6 . & 9.8 & 16. \\
\hline 6.3 & 8.5 & 18. \\
\hline 8.172 & 10.695874 & 17. \\
\hline 8.1752 & 8.178597 & 16.8553 \\
\hline 24.8 & 21. & 16 \\
\hline 0.4 & 22.2 & 18.61182 \\
\hline 22. & 20 & 17. \\
\hline 22.862789 & 22.830261 & 16.76937 \\
\hline 24.691879 & 8.844208 & 3.87883 \\
\hline & 10.030993 & 6.3157 \\
\hline 22.915 & 8.27229 & \\
\hline & 10. & 4.39006 \\
\hline 12.47 & 21.408255 & 3.709 \\
\hline & 22.23 & \\
\hline
\end{tabular}




$\begin{array}{rrrr}\mathrm{O} & 10.879633 & 20.318567 & 5.485331 \\ \mathrm{O} & 10.628633 & 22.920805 & 4.636691 \\ \mathrm{O} & 12.479086 & 8.849791 & 16.093817 \\ \mathrm{O} & 12.561133 & 9.871751 & 18.543123 \\ \mathrm{O} & 10.631061 & 8.176898 & 17.957621 \\ \mathrm{O} & 10.628633 & 10.537118 & 16.763796 \\ \mathrm{O} & 18.630512 & 21.244644 & 2.930430 \\ \mathrm{O} & 18.702364 & 22.240631 & 0.421407 \\ \mathrm{O} & 20.389692 & 20.320024 & 1.095027 \\ \mathrm{O} & 20.572237 & 22.759378 & 2.192724 \\ \mathrm{O} & 18.705036 & 8.947618 & 15.166284 \\ \mathrm{O} & 18.546038 & 9.869809 & 12.661145 \\ \mathrm{O} & 20.405228 & 8.093637 & 13.316317 \\ \mathrm{O} & 20.490189 & 10.633002 & 14.315217 \\ \mathrm{O} & 6.582543 & 21.080305 & 15.168955 \\ \mathrm{O} & 6.329602 & 22.162224 & 12.658960 \\ \mathrm{O} & 8.254092 & 20.408140 & 13.218490 \\ \mathrm{O} & 8.264772 & 22.850166 & 14.327597 \\ \mathrm{O} & 6.425729 & 8.940820 & 2.945237 \\ \mathrm{O} & 6.315765 & 9.952343 & 0.491561 \\ \mathrm{O} & 8.175200 & 8.183210 & 1.022932 \\ \mathrm{O} & 8.261617 & 10.630090 & 2.183986 \\ \mathrm{O} & 13.233784 & 5.220010 & 8.259675 \\ \mathrm{O} & 12.467191 & 6.661435 & 5.960871 \\ \mathrm{O} & 11.806194 & 7.754035 & 8.430568 \\ \mathrm{O} & 14.488537 & 7.753550 & 7.588726 \\ \mathrm{O} & 8.349006 & 17.939899 & 19.383511 \\ \mathrm{O} & 6.576717 & 19.708061 & 18.967930 \\ \mathrm{O} & 7.665190 & 19.311899 & 21.920206 \\ \mathrm{H} & 12.684935 & 22.177517 & 3.144774 \\ \mathrm{H} & 15.863208 & 7.983673 & 7.923229 \\ \mathrm{H} & 25.047018 & 6.148756 & 1.711359 \\ \mathrm{H} & 21.949335 & 16.018808 & 6.477920 \\ \mathrm{H} & 13.583095 & 4.993042 & 9.177256 \\ \mathrm{H} & 9.177498 & 15.976085 & 19.256067 \\ \mathrm{C} & 11.868126 & 19.473083 & 20.256424 \\ \mathrm{C} & 11.958881 & 19.131540 & 18.883940 \\ \mathrm{C} & 11.809107 & 19.991346 & 17.858093 \\ \mathrm{C} & 9.289889 & 20.655985 & 20.028486 \\ \mathrm{H} & 12.360384 & 19.284954 & 20.998983 \\ \mathrm{H} & 13.470461 & 20.238705 & 19.972898 \\ \mathrm{H} & 13.618050 & 18.468359 & 20.088202 \\ \mathrm{H} & 12.396067 & 20.906256 & 17.789883 \\ \mathrm{H} & 11.129175 & 19.785011 & 17.030815 \\ \mathrm{H} & 9.639443 & 20.905043 & 19.015993 \\ & 9.845292 & 21.109678 & 20.853823 \\ \mathrm{R} & 12.987638 & 6.884519 & 7.560324 \\ \mathrm{H} & & & \end{array}$

